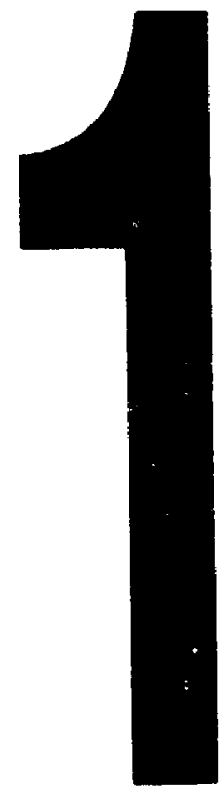

PM-1 3!'z" $\times$ 4" PHOTOGRAPHIC MICROCOPY TARGET NBS 1010 a ANSI/ISO \#2 E.QUIVALENT

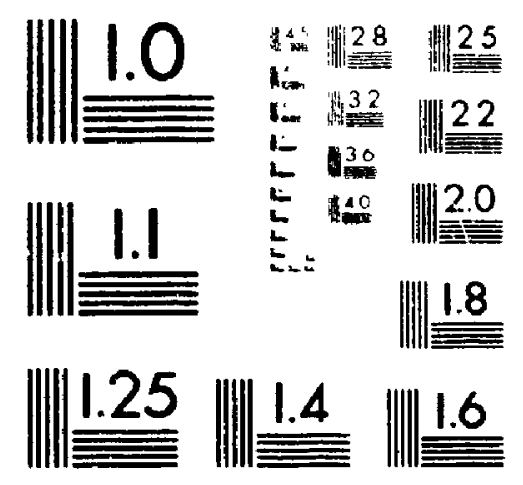

PRECISIONSM RESOLITION TARGETS 
Acquisitions and

Brbliographic Services Branch

395 Wetlington Street

Ottawa. Onlarwo

KIA ONA
Direction des acquisitions et des services bibliographiques

395, rue Wellington

Ottawa (Orilaro)
NOTICE
AVIS
The quality of this microform is heavily dependent upon the quality of the original thesis submitted for microfilming. Every effort has been made to ensure the highest quality of reproduction possible.

If pages are missing, contact the university which granted the degree.

Some pages may have indistinct print especially if the original pages were typed ivith a poor typewriter ribbon or if the university sent us an inferior photocopy.
La qualité de cette microforme dépend grandement de la qualité de la thèse soumise au microfilmage. Nous avons tout fait pour assurer une qualite supérieure de reproduction.

S'il manque des pages, veuillez communiquer avec l'université qui a conféré le grade.

La qualité d'impression de certaines pages peut laisser à désirer, surtout si les pages originales ont été dactylographiées à l'aide d'un ruban usé ou si l'université nous a fait parvenir une photocopie de qualité inférieure.

La reproduction, même partielle, de cette microforme est soumise à la Loi canadienne sur le droit d'auteur, SRC 1970, c. C-30, et ses amendements subséquents. 
STRAIIGRAPHIC AND METAMORPHIC CONSTRAINTS

ON THE TECTONIC EVOLUTION OF THE VALHALLA COMPLEX.

SOUTHERN BRITISH COLLMBIA

by

PETER M. SCHAUBS. B.Sc. (Hons.)

\author{
A thesis submitted to \\ the Faculty of Graduate Studies and Research \\ in partial fulfilment of \\ the requirements for the degree of \\ Master of Science
}

Department of Earth Sciences

Carleton University

Ottawa. Ontario

1995

01995 P. M. Schaubs 
The author has granted an irrevocable non-exclusive licence allowing the National Library of Canada to reproduce, loan, distribute or sell copies of his/her thesis by any means and in any form or format, making this thesis available to interested persons.
L'auteur a accordé une licence irrévocable et non exclusive permettant à la Bibliothèque nationale du Canada de reproduire, prêter, distribuer ou vendre des copies de sa thèse de quelque manière et sous quelque forme que ce soit pour mettre des exemplaires de cette thèse à la disposition des personnes intéressées.

L'auteur conserve la propriété du droit d'auteur qui protège sa thèse. Ni la thèse ni des extraits substantiels de celle-ci ne duivent être imprimés ou autrement reproduits sans son autorisation.

ISBN $\quad 0-612-09030-2$ 
Nam Teter rechaubs

Disimation Abstracts infermational is arranged by braad, genteral subject categories. Please select the one subject which most mearty describes the content of your dissertation. Enter the corresponding four-digit code in the spaces provided.

$$
\frac{\text { Ceo } \log y}{\text { sunatiram }}
$$

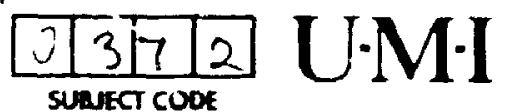

\section{Subinet Cotogories}

\section{THI HUMANmIS AND SOCIAL SCIENCES}

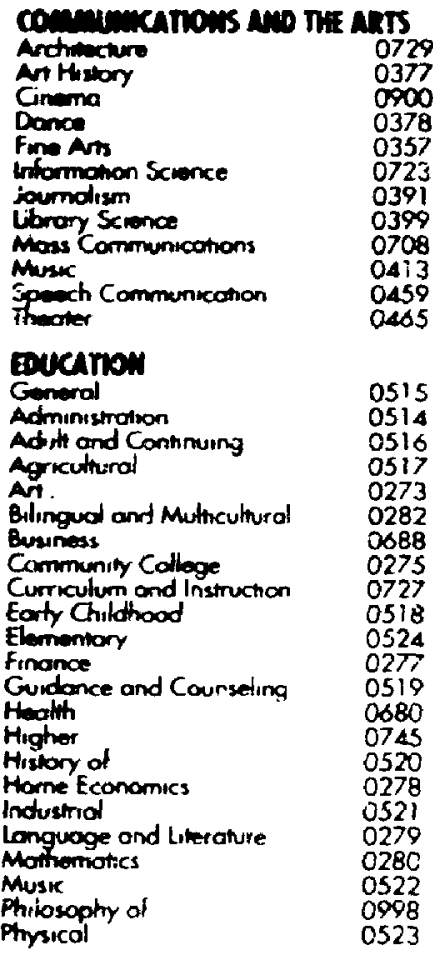

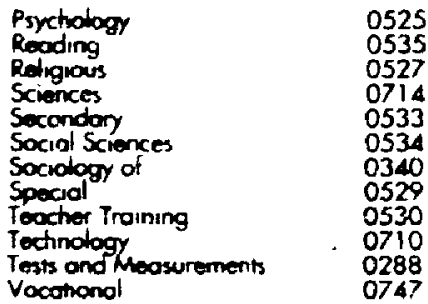

\section{LMGUAGE, UTERATURE AMD} Uinctistics

Language

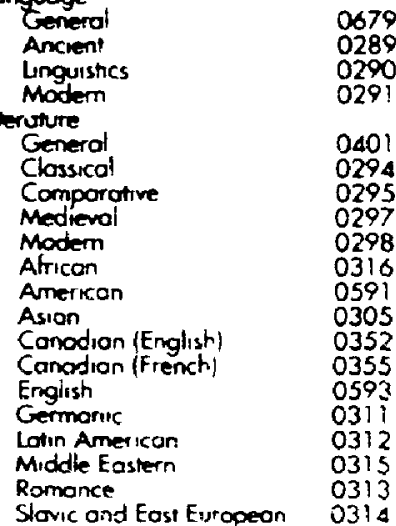

\section{PHILOSOPHY, RELLION ANID}

\section{THEOLOGY}

Philosophy

Religion

Biblicol Snucies

Clergy

Histion of

Philosaphy of

Theology

\section{sogul sofncts}

American Studies

Anthropolog

Aschoedogy

Culural

Business Administrohon

General

Accounting

Bonking

Managemen

Conodion Srudies

Economics

General

Agriculiurol

Commerce-Busines

Finonce

History

Labor

folktore

Geogrophy

Gerontology

History

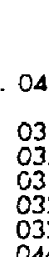

0422

0318

0321

032

0322

0469

0323

0324

0326
0327

0310

0272

0770

0338

0385

0501

0503

0505

0508

0509

0510

0358

0300

$035 \mathrm{i}$

0578

SUAECT CODE

Ancien

Medieval

Modern

Block

African

0331

Asio. Australia and Oxeanio 0332

Conodian 033

Lotin Americon

Middle Eastern

United States

History of Science

Low

Politicai Science

General

hinernotional Low and

Retotions

Public Administrution

Recreation

Social Work

Sociology

Criminology and Penology

Demographr

Ethnic and Rocial Studies

Individual and Family$$
\text { Studies }
$$

Industrial and Labor

Relotions

Pubic and Social Welfare

Social Structure and

Development

Theory and Methods

Transportation

Urban and Regronal Planning

Womens Sirdies
0579

058 !

0582

0332

0335

0333

0337

0585

0615

0616

0617

0814

0452

0626

0627

0631

0628

0629

0630

0700

0344

0709

0453

\section{THE SCIENCES AND ENGINEERING}

\section{moloskal saknas}

Apriculture

\begin{tabular}{|c|}
\hline 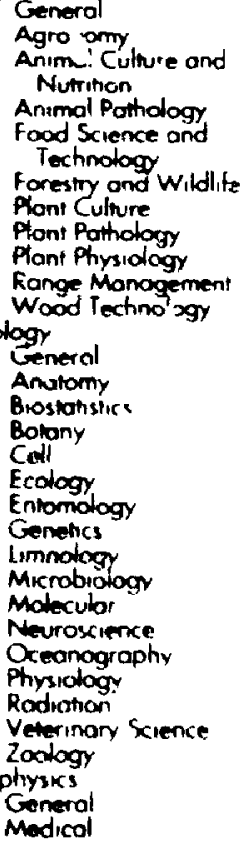 \\
\hline
\end{tabular}

0473

0285

0475

0476

0359

0478

0479

0480

0817

077

0746

0306

0287
0308

0308
0309

0309

0329

0353

0369

0793

0410

0410
0307

0317

0416

04.33

0821

0778
0472

0786

AnTH scatucts

Bisgeortiomisiny

Geochemisiny

\section{Geodesy}

Geology

Geophysiss

Mincrology

Paleobotony

Paleoerology

Paleoniology

Pabmotogy

Physical Geography

Physical Oceanography

HEATH AND ENVIROMUENTAL SaENas

Envircnmentol Sciences

Health Sciences

Generol

Audiology

Dentistiy

Educotion

Hospital Monogement

Human Development

immunoiogy

Medicine and Surgery

Mentol Healith

Nursing

Nutition

Obstertics and Gynecclogy 0380

Decupononal theolth and

Therapy

Ophthalmology

Pathology.

Phormacelogy

Phormoy

Physical therapy

Public Heolith

Rodiology

Recreation
Soeech Pathology Home Economics

\section{PHYSICA SathCES}

Pure Sciences

Chemisiny

General

Agricultura

Biochemisiny

inorgonis

Nuclear

Organic

Phamoceutical

Physical

Rodiotion

Matherncises

Phrsics

General

Acoustics

Astronomy and

Astookhysics

Atrospheric Science

Aromic

Electronics and Electricity

Elemenion Particles and

High Energy

Fluid and Flosma

Molesulot

Nucleor

Opnes

Radiation

Solid Store

Srotishics

Applind Sciences

Applied Mechonics

Computer Science

0450

0383

0.386

0.485

0749

0480

0487

0486

0738
0490

0491

0494

0195

0754

0405

0,05

0986

0606

0608

0748

0607

0798 


\section{Carleton University Ottawa, Canada K1S $5 J 7$}

Thesis contains black and white and/or coloured graphs/tables/pnotographs which when microfilmed may lose their significance. 'I'he hardcopy of the thesis is available upon request from Carleton University Library. 
The u.adersigned recommend to the Faculty of Graduate Studies and Research acceptance of the thesis

\author{
"STRATIGRAPHIC AND METAMORPHIC CONSTRAINTS \\ ON THE TECTONIC EVOLUTION OF THE VALHALLA COMPLEX. \\ SOUTHERN BRITISH COLUMBIA"
}

submitted by Peter M. Schaubs. B.Sc. (Hons.)

in partial fulfilment of the requirements for

the degree of Master of Science

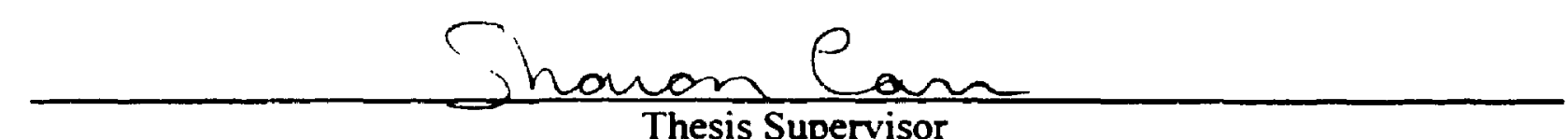

Thesis Supervisor

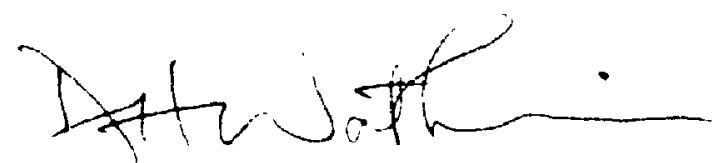

Chair. Department of Earth Sciences

Carleton University

December 1995 


\section{Frontispiece}

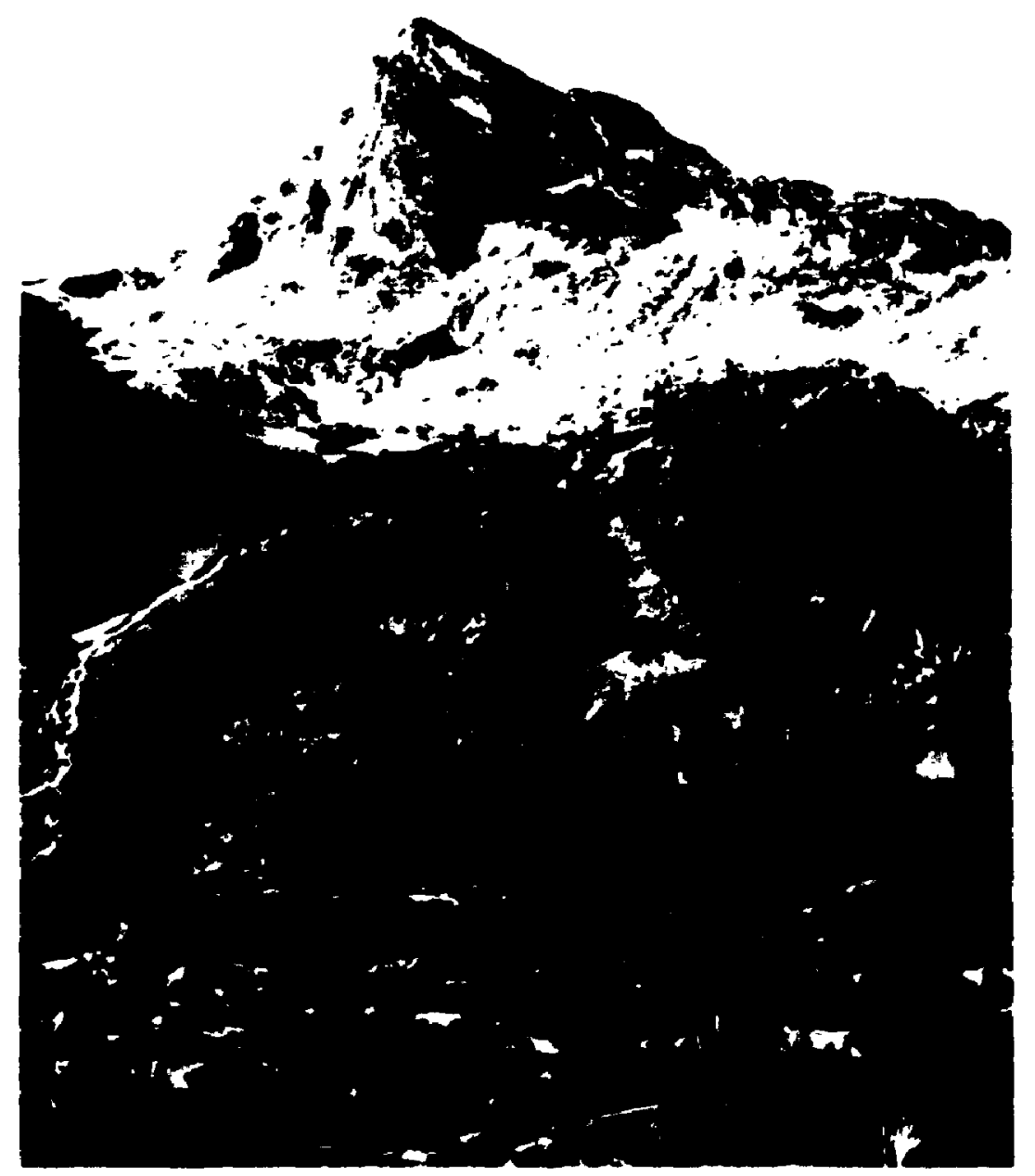

View of Lucifer Peak, looking north across the Gwillim l.akes. 


\begin{abstract}
The Valhalla complex is an exhumed metamorphic core complex situated within the southern (Jmineca belt of the (anadian Cordillera. Sheets of upper amphibolite-facies: paragneiss occur at three structural levels. one of which (lc) lies in the hanging wall of the (iwillim Creek shear zones. while two windous are exposed in the footuall ( $1 \mathrm{a}$ and $\mathrm{lb}$ ). The Valhalla assemblage is a heterogeneous package of metasedimentary rocks which lies in the uppermost paragneiss sheet and includes semipelitic and psammitic schist. marble. calcsilicate gneiss. quartzite. quartzite-cobble metaconglomerate. pelitic schist. amphibolite gneiss and ultramafic boudins. Lithologic similarity of the Valhalla assemblage with rocks in the Goat Ranges. in the Kootenay Arc. permits a tentative correlation with the Badshot Formation. Lardeau Group and Milford Gioup. The Valhalla assemblage comprises the upside down limb of an isoclinally folded $F$, recumbent nappe which was subsequently thinned by up to $80 \%$ as result of east directed compressional tectonics and movement on the Gwillim Creek shear z.ones. Based on similar hanging-wall stratigraphies, the Gwillim Creek shear zones may be correlated with the West Bernard fault. in the Kootenay Arc. It is also possible that the Gwillim Creek shear zones are correlative with a structure that is imaged by seismic reflections and forms the roof of a duplex, east of the Valhalla complex, which is fluored by the basal décollement of the Rocky Mountain fold and thrust belt.
\end{abstract}

Petrographic obs urvations reveal that the peak of metamorphism was synchronous with the dominant $S_{2}$ foliation and that different structural sheets share a common metamorphic history. $S_{:}$fabric development at high structural levels is synchronous with fabrics associated with the Gwillim Creek shear zones, at structurally low levels. The 
Gwillim Creek shear zones were active at the peak of metamorphism $(-75.11$ a) and resurtikce in the southern region of the Valhalla complex near Vallican. Petrographic whervations and thermobarometric results indicate that peak thermal conditions were approximately 775 " $\mathrm{C}$ and $6.5-7$ kbars in the structurally highest domain. : ructurally low levels are characterised by a large variation in thermobarometric data and some anomalously high values, when compared to data trom the structurally highest paragneiss sheet $(1 \mathrm{c})$. The high values recorded by samples in these sheared areas may be the result of disequilibrium caused by the introduction of fluids or the juxtaposition. by shearing. of minerals that were not in chemical equilibrium.

A period of decompression. which occurred during the Late Cretaceous, is recorded by plagioclase moats and different closure temperatures of the GASP and (iRAII. geobarometers. This may be a response to exhumation due to movement along the underlying Monashee décollement. Rare evidence of retrograde metamorphism indicales that paragneisses of the Valhalla complex were exhumed quickly, following the peak of metamorphism. 


\section{List of Tables}

Table

Description

Page

2.1 Summary of geochronology from the Valhalla complex.

56

2.2 Description of major rock types in the Valhalla assemblage.

2.3 Correlation of Valhalla assemblage to other Paleozoic sequences.

3.1 Timing of metamorphic and deformation events in three domains of the Valhalla complex.

3.2 Thermobarometric data from pelitic schists.

3.3 Thermobarometric data from amphibolite gneisses. 


\section{Original Contribution}

This project was based on field work carried out by the author in the last week of August. 1993 and from mid-June to the end of August. 1994. Original contrib:itions include the detailed mapping of a package of paragneisses in the Valhalla complex, which wire subdivided. named and correlated to outlying sequences in the southern Omineca belt. Metamorphic petrology was carried out on semipelitic to pelitic schists. amphibolite gneisses, carbonate rocks and ultramafic schists. Quantitative mineral analyses were obtained for pelitic schists and amphibolite gneisses which were used in thermobarometric calculations. The combination of detailed stratigraphic data. metamorphic and deformational relationships observed in thin-section, and thermobarometric data has resulted in an interpretation of the pre-Eocene tectonic evolution of the Valhalla complex and has also placed constraints on the timing and displacemeni direction of the Gwillim Creek shear zones. 
Acceptance sheet

Frontispiece

Abstract

Acknowledgements

Original Contribution

Table of Contents

List of Tables

I,ist of Figures

List of Plates

CHAPTER 1: Introduction and objectives

CHAPTER 2: Stratigraphy and geologic setting of the Valhalla assemblage

2.1 Introduction

2.2 Regional stratigraphy of the southern Omineca belt

2.3 Geology of the Valhalla complex

2.3.1 General geology

2.3.2 Structure

Folding in domain 1

Fabrics in thin-section

2.4 The Valhalla assemblage $\quad 24$

2.4.1 Introduction $\quad 24$

$\begin{array}{ll}\text { 2.4.2 Lithology } & 25\end{array}$

Paleocene-Eocene leucogranites and pegmatites 25

Ultramafic to mafic intrusive dvkes $\quad 27$

2.4.3 Stratigraphy of metasedimentary units 28

Unit l Ouartzo-feldspathic gneiss $\quad 28$

Unit 2 Hird Lakes marker unit $\quad 29$

Unit 3 Drinnon quartzite 31

Unit 4 Demers marble 33

Unit 5 Hoder Creek composite unit 35

Unit 6 Bannock Burn marble $\quad 37$

Unit 7 Semipelitic schist $\quad 37$ 
L'nit 8 Rinda Ridge composite unit

Lnit 9 Rinda marble

Lnit 10

Linit 11 Lndivided rocks

Slocan Ridge

Perri Ridge

Gwillim Creek windows

2.5 Regional correlation of the Valhalla assemblage

2.5.1 Goat Ranges to

2.5.2 Thor-Odin - Pinnacles area ti)

2.5.3 Riondel area

2.5.4 Nemo Lakes belt

2.5.5 Eagle Bay assemblage il

2.5.6 Scrip nappe

2.5.7 Greenwood - Grand Forks area

2.5.8 Mount Roberts Formation in the Trail area

2.6 Summary

CHAPTER 3: Metamorphism of paragneisses in the Valhalla complex.

3.2 Analytical methods

Domain la Valhalla assemblage (sheet Ic, west side) and domain 16

Textures and mineral compositions

Timing relationships between metamorphism and deformation

Domain 2-Passmore-Vallican-(sheet lcs south end)

Textures and mineral compositions

Timing relationships between metamorphism and deformation

Domain 3-Gwillim Creek windows (sheet la)

Textures and mineral compositions

Timing relationships between metamorphism and deformation 
3.3.2 A r.phibolite gneisses

Textures and mineral compositions

Timing of metamorphism with respect to deformation

3.3.3 Marbles and calc-silicate gneisses

3.3.4 UItramafic schists

3.3.5 Summary

3.4 Thermobarometry, petrology and comparison of structural leveis

3.4.1 Pelitic schist

Domain I

Domain 2

Domain 3

3.4.2 Amphibolite gneiss

124

3.4.3 Summary

125

3,t,t Discussion

3.5 Conclusions

CHAPTER 4: Tectonic interpretation

4.2 Summary of stratiaraphic and metamorphic data

\$.2.1 Stratigraphy

4.2.2 Metamorphic petrology

4.2.3 Timing of deformation in the Valhalla complex

4.3! terpretation

4.3. Inverted stratigraphy

Riondel Nappe

Scrip Nappe

Eagle Bav assemblage

Timing of inversion in the Valhalla complex

4.3.2 Thinning of the Valhalla assemblage

4.3.3 Correlation of the Gwillim Creek shear zones

4.3.4 Decompression

4.3.5 Variation in data within domains 2 and 3 
APPENDIX A: Electron microprobe standards APPENDIX B: Modal mineralogy of pelitic schists APPENDIX C: Mineral analy ses for pelitic schists APPENDIX D: Mineral assemblages for a mphibolite gneisses APPENDIX E: Mineral analy ses for amphibolite gneisses APPENDIX F: Mlineral assemblages for marbles and calc-silicate gneisses

APPENDIX G: Mineral analyses for marbles and calc-silicate gneisses

\section{IN BACK POCKET:}

Cross-sections A,B,C through the Valhalla complex.

Map 1 - Geology of paragneisses in the V'alhalla complex.

Map 2 - Structure of paragneisses in the Valhalla complex. 


\section{List of Tables}

Table

Description

Page

2.1 Summary of geochronology from the Valhalla complex.

2.2 Description of major rock types in the Valhalla assemblage.

2.3 Correlation of Valhalla assemblage to other Paleozoic 60 sequences.

3.1 Timing of metamorphic and deformation events in three domains of the Valhalla complex.

3.2 Thermobarometric data from pelitic schists.

3.3 Thermobarometric data from amphibolite gneisses. 


\section{List of Figures}

Figure

Description

Pagu

1.1 Tectonic assemblage map of the southern ( )mineca belt.

1.2 Geology of the Valhalla comples.

1.3 Generalized cross-section of the Valhalla complex.

2.1 Schematic diagram of the relationships between stratigraphy and terranes in the northern kinotenaly are.

Domain map of paragneisses in the Valhalla complex

2.3 Geology of the Valhalla assemblage.

2.4 Stereonet plots of fold axes and poles to axial surtaces.

2.5 Stratigraphy of the Valhalla assemblage.

2.6 Schematic diagrams of the folded obduction model of the Riondel Nappe.

2.7 Lithoprobe seismic-reflection cross-section of the southern Omineca belt.

3.1 Petrogenetic grid toi pelitic schists showing important melt equilibria.

3.2 Location map for thermobarometry samples.

3.3 Compositional zoning profiles of garnets in pelitic schist.

3.4 Schematic illustrations showing textural relationships between metamorphism and deformation.

3.5 Zoning profiles of garnet from PS-28a-93 (domain 2).

3.6 Zoning profiles for garnet in amphibolite gneiss.

3.7 Petrogenetic grid for ultramafic schists 
3.8 Representative TWEEQL plots for pelitic schists.

3.9 Thermobarometric data for pelitic schists from three domains.

3.11 ('ross-sections through Valhalla complex showing relative position of thermobarometric data.

3.11 Representative TWEEQU plots for amphibolite gneisses

3.12 Compilation of thermobarometric data from amphibolite gneisses

4.1 Summary of petrographic and thermobarometric data in relation to pressure-temperature path. 


\section{List of Plates}

Plate Description

Page

la Strongly folded Demers marble.

Ib Tightly folded quartzo-feldspathic gneiss of unit 1 near Mount Bor.

1c Interference pattern of $F_{\xi}$ refolding $F_{:}$

2a Outcrop-scale east-verging overturned fold in Hird Lakes quartzite-cobble metaconglomerate.

2b Metre-scale east-verging. $z$-fold in anphibolite layer on Rinda Ridge.

2c Typical nature of coarse-grained leucogranite intruding pelituc schist (10b).

2d Metre-sized xenoliths of calc-silicate gneiss (unit 2acs).

3a Typical nature of semipelitic and pelitic (brown) separated by metre thick pegmatites and leucogranites of the Ladybird suite.

3b Centimetre-scale layering in north-east verging. $Z$ folded psammitic gneiss.

3c Quartzite-pebble metaconglomerate (2a-cg) with calcsilicate matrix, near Hird Lakes.

4a Metre scale layering in migmatitic gneiss of unit $3 b$.

4b Millimetre-centimetre straight, gneissic layering of calcareous quartzite (3c-cq). west of Urd Peak.

$4 c \quad$ Tightly folded calc-silicate gneiss of Hoder Creek composite unit (5a).

4d Centimetre-scale interlayered marble and calcareous schist of Rinda Ridge composite unit (8a). 
Sb Beige-buff weathering, clean quartzite layer within

Rinda Ridge composite unit (8a).

5c Interlayered and refolded calc-silicate and arrphibolite gneiss of Rinda Ridge composite unit (8a).

Typical greenish-brown weathering nature of ultramatic pods (8a-u), near Mount Rinda.

Rubbly. metre-scale outcrop of metamorphosed ultramafic rock $(8 a-u)$.

Decimeter scale interlayered marble. calc-silicate gneiss and semipelitic scrist of unit $8 b$.

Typical nature of pelitic schist. amphibolite boudins and pegmatite within undivided units of the Valhalla Assemblage.

Western (upper) Gwillim Creek shear zone.

Ragged garnet porphyroblast with a moat of quartz grains separating it from the surrounding matrix.

$8 b$

Garnet porphyioblast in pelitic schist with quartz embayments interpreted to be a product of garnet breakdown during melting.

8c Garnet porphyroblast with inclusions concentrated in the core and an inclusion free rim.

8d Plagioclase, quartz and biotite inclusions in garnet porphyroblast.

Garnet porphyroblast with inclusions of quartz and sillimanite $\left(S_{1}\right)$. Biotite and sillimanite foliation $\left(S_{:}\right)$ wraps around garnet and occurs in pressure shadows. 
These sillimanite mats in pelitic schist are oriented in the foliation plane but some ery stals within the mat appear to be randomly oriented.

Garnet porph! roblast stirrounded by discontinuous

moat of tine-grained plagioclase.

Advanced stage of garnet consumption with plagioclase. sillimanite and hiotite growing at expensc of garnet in pelitic schist.

Myrmekitic symplectite of quartz and plagnoclase growing at the expense of potassium feldspar.

10c Garnet porphyroblast with pressure shadou of biotitc and ilmenite.

10d $\quad F_{;}$crenulations in pelitic schist tolding sillimanite and biotite.

11a Thin muscovite halo surrounds sillimanite porphyroblast in pelitic schist from sheet $1 \mathrm{a}$.

11b Garnet porphyroblast in amphibolite gneiss contains round hornblende and quartz inclusions.

11c Garnet porphyroblast surrounded by hornblende and biotite.

12a Thin plagioclase rim around garnet porphyroblast in amphibolite gneiss.

12b Symplectite of calcite and plagioclase (quartz?) rimming scapolite porphyroblast in marble.

12c Olivine grain with serpentine filled fractures and surrounded by phlogopite.

12d Orthopyroxene grain partially replaced by tremolite. 


\section{CHAPTER 1: INTRODUCTION AND OBJECTIVES}

The Valhalla complex is a 30 by $90 \mathrm{~km}$ structural culmination which lies in the southern portion of the Omineca belt and is contained within the larger Shuswap metamorphic complex (Fig. 1.1). In the Valhalla complex, arched sheets of paragneiss, granitic rocks and intervening thrust faults (Figs. 1.2, 1.3) were exhumed on the Eocene Slocan Lake fault (SLF) -Valkyr shear zone (VSZ) extensional fault system (Carr et al. 1987). The Gwillim Creek shear zones (GCSZ) in the Valhalla complex are two $50 \mathrm{~m}$ thick horizons of significant ductile strain which occur in the deepest exposed structural levels of the complex The duplication of geology and the apparent easterly-directed shear sense has led to the interpretation that the shear zones are east verging thrust faults (Parrish et al. 1985; Parrish et al. 1988).

The Valhalla complex provides a case study for the evolution of tecianic models of the region. In the 1960 's. Reesor $(1965,1970)$ viewed granitic rocks in the complex as Precambrian basement gneisses and believed that the complex had developed as a mantled gneiss dome. Parrish (1984) recognized the Slocan Lake Fault on the east side of the complex as a major extensional structure, and later Carr (1986), Carr and others (1987) and Parrish and others (1988) revealed that the present configuration of the Valhalla complex is a result of Eocene exhumation and that granitic rocks in the Valhalla complex are no older than Cretaceous. Parrish and others (1988) described the Gwillim Creek shear zones, in the deepest structural levels of the complex, and interpreted them to be Cretaceous or younger easterly directed thrust faults that were possibly linked to the Foreland belt. It has been 
demonstrated that kinematic links exist bc. ween thrust faults in the Omineca belt and those in the Foreland fold and thrust belt (Price 1981: Brown and Read 1983: Brown e't al. 1986: Price 1986; Brown and Journeay 1987: Parrish et al. 1988; Brown et al 1991: Brown el al 1993; Parrish 1995; Carr 1995). Parrish (1995) summarizes U-Pb geochronology studies. and presents data from the southern Omineca belt which indicate that the age of deformation and peak of metamorphism decreases going down structural section. In this model. west verging in-sequence thrusting during the Mesozoic progressively thickened the crust and placed hot thrust sheets onto cool footwalls. Peak thermal ages of - 75 Ma have been estimated by Heaman and Parrish (1991) for the Valhalla complex and it has been inferred that the Valhalla complex lies at mid-crustal levels above the Monashee complex, which records thermal peaks at $\sim 55 \mathrm{Ma}$ (Parrish 1995).

New insights have been gained from geological research programs in the southern Omineca belt including the southern Cordillera Lithoprobe seismic program (Cook et al. 1987; Cook et al. 1988; Cook et al. 1992). Advances in the understanding of the regional geology in the southern Omineca belt as a result of geochronological studies (Archibald $e t$ al. 1983; Archibald et al. 1984; Carr et al. 1987; Parrish et al. 1988; Carr 1992; Scammell 1993), mapping, and structural studies of faults (Höy 1977; 1980. Klepacki 1985; Carr et al. 1987; Corbett and Simony 1984; Simony et al. 1992; Bardoux 1993; Johnson 1994), together with seismic reflection data. have set the stage for new regional reconstructions

The focus of this study is directed toward the pre-Eocene deformation history of the northern part of the complex, and the stratigraphy and metamorphic history of paragneiss sheets that occur at three different structural levels. The aim is to build on the extensive 
geochronological, petrological and structural studies of the Valhalla complex and combine this information with new stratigraphic correlations and thermobarometric data to constrain the timing, magnitude and direction of displacement on the GCSZ, in order to develop a new model for the tectonic evolution of the Valhalla complex. This will constrain future palinspastic reconstructions linking the Omineca and Foreland belts.

In order to consider correlation of the GCSZ to faults in the Foreland fold and thrust belt, the hanging wall - footwall relationships and lithologies must be known. Based on detailed hanging wall stratigraphy and Lithoprobe seismic reflection data. the primary objectives of this study are: 1) to describe the heterogeneous package of paragneisses within the Valhalla complex and to compare them with the regional stratigraphy; 2) to attempt to correlate the Gwillim Creek shear zones with faults to the east in the Kootenay Arc and Purcell Anticlinorium.

The presence of pelitic schists within the paragneiss sheets, which occur at three different structural levels in the Valhalla complex, facilitates a comparison of metamorphic conditions at the three structural levels. The wide range in bulk compositions represented by pelitic schists. mafic gneisses, marbles, calc-silicate gneisses and ultramafic schists and variation within lithologies allows the application of a variety of geothermometers and barometers in order to try to establish pressure-temperature conditions. Careful observations of the relationship between metamorphic minerals, reaction textures and deformation will allow the linkage of quantitative data to the geologic history. Further objectives of this study are, therefore: 1) to characterize the metamorphism of paragneisses at different structural levels and in different domains; 2) to establish pressure-temperature paths, or parts thereof, 
for the paragneisses of the Valhalla complex: 3) to determine if there is any variation in thermal histories across the Gwillim Creek shear zones by examining the differences in pressure or temperature conditions between the upper and lower sheets of paragneiss: and 4) to combine resultant data with published thermo- and geochronological studies. 
Figure 1.1 a) Tectonic Assemblage map of the southern Omineca Belt, simplified from Wheeler and McFeely (1991) and modified after Carr (1991) and Johnson (1994). Important tectonic elements include the Adams Lake-Clearwater-Vavenby area (ACV), the Goat Ranges (GR), the Greenwood-Grandforks area (GGF), then Malton complex (MA), the Monashee complex (MO), the Nemo Lakes belt (NL), the Pinnacies area (P). the Riondel Nappe (RN), the Scrip Nappe (SN), Thor-Odin dome (TO) and the Valhalla Complex (VC). Important plutons include the Fry Creek batholith (FC), the White Creek batholith (WC) and the Whatshan batholith (W). Thrust faults include the Hall Lake fault (HLF), the Monashee décollement (MD), the Moyie fault (MF), the St. Mary fault (SMF) and the West Bernard fault (WBF). Normal faults include the Columbia River fault (CRF), the Okanagan Valley fault (OF), the North Thompson fault (NTF), the Slocan Lake fault (SLF), and the Valkyr shear zone (VSZ).

Figure 1.1 b) Terrane map of the southern Omineca Belt, simplified after Wheeler and others (1991).

Figure 1.1 c) Morphogeological belts of the Canadian Cordillera. 

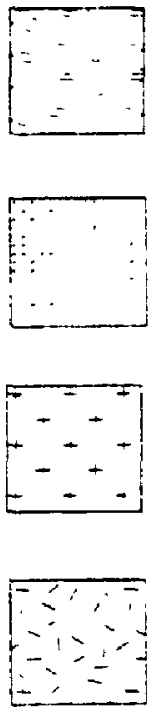

Paleocene - Eocene

granite and evenite

Late Cretaceous granitic rocks

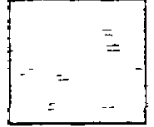

Middle Jurassic granitic rocks

Triassic to Jurassic sedimentary and volcanic rocks of the Slocan and Rossland groups
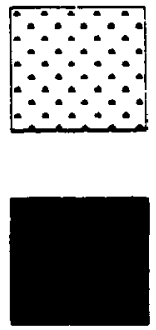

Carboniferous to Triassic volcanic and sedimentary rocks of the Knob Hill, Anarchist and Harper Ransh groups

Permian volcanic and ultramafic rocks of the Kaslo Group

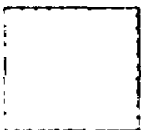

Carboniferous sedimentary and volcanic rocks of the Milford Group

Cambrian to Devonian passive margın sedimentary rocks

Neoproterozoic to Lower Paleozoic clastic and volcanic rocks of the Eagle Bay Assemblage and Lardeau Group

Neoproterozoic to Cambrian sedimentary rocks of the Gog and Hamill groups and Badshot Formation

Neoproterozoic clastic sedimentary rocks of the Windermere Supergroup

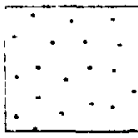

Mesoproterozoic continental margin sedimentary rocks of the Purcell (Belt) Supergroup

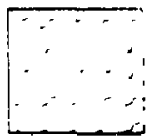

Paleoproterozoic craton related paragneiss and metasedimentary rocks

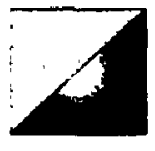

Undivided metamorphic rocks of the Shuswap complex and paragneisses of the Valhalla Complex

Figure 1a. Tectonic assemblage map of the southern Omineca Belt.

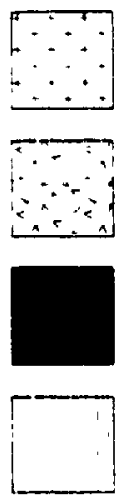

Post-accretionary granitic rocks

Quesnellia

Slide Mounatin

Kootenay

Figure 1b. Terrane map of the southern Omineca Belt. Modified after Wheeler and others (1991).
Kootenay - inferred

North America

Basement rocks of the Monashee complex

Undivided metamorphic rocks 


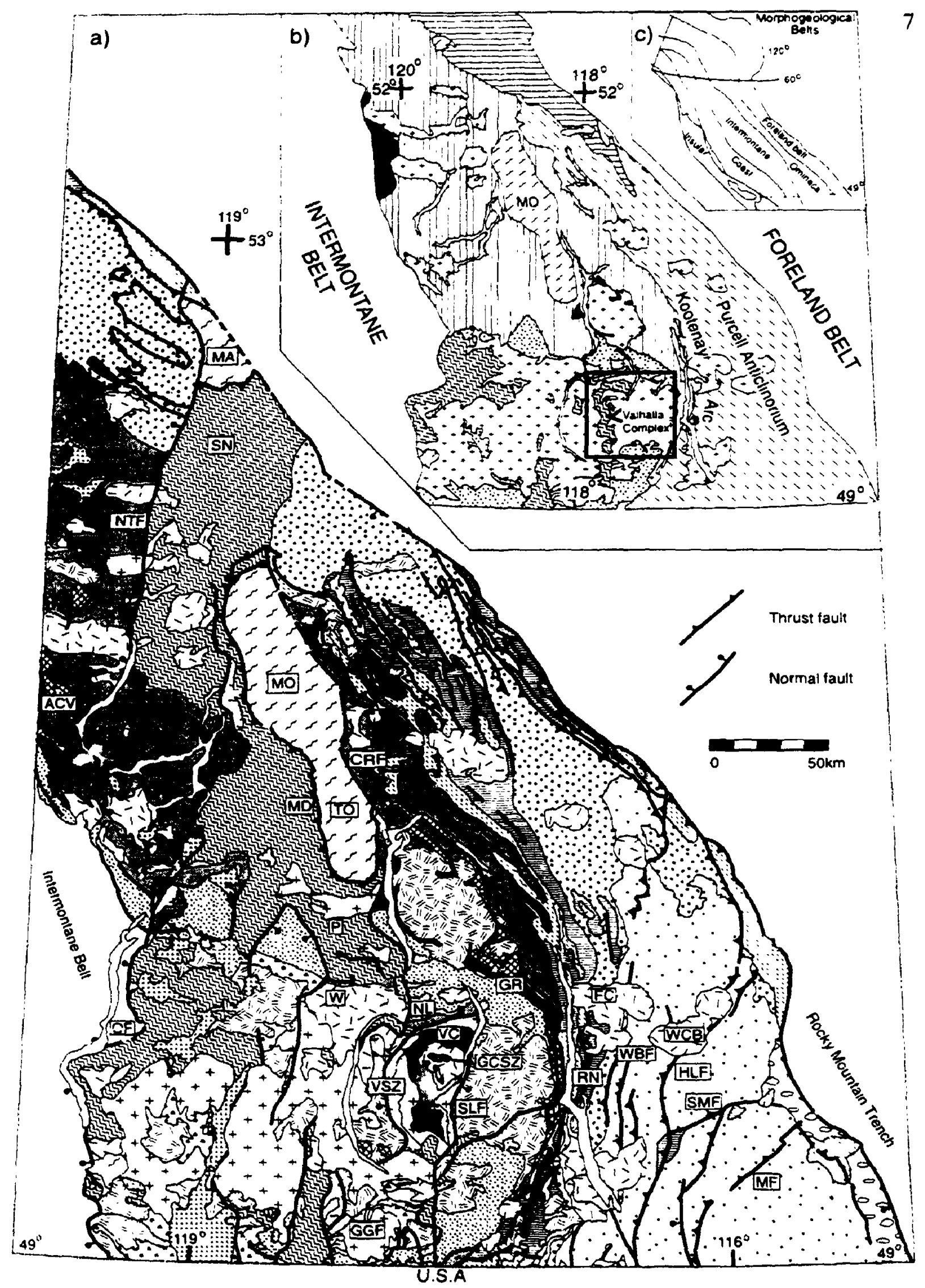


Upper Plate

- Middle Eocene Coryell syenite, granite

$=$

$\times \quad$ Eocene College Creek granite

Late Cretaceous granitic rocks

Middle Jurassic granitic rocks

Middle Paleozoic - Early Mesozoic rocks of allochthonous Quesnallia terrane

- Paragneiss - age uncertain

Valhalla complex (Lower Plate)

Early Eocene Ladybird granite

Paleocene Airy Quartz Monzonite

Late Cretaceous Mulvey granodiorite

Late Cretaceous Kinnaird Gneiss

Middle Devonian Trail Gneiss

Valhalla Assemblage

: Castlegar Gneiss

Thrust fault
Slocan Lake fault
Valkyr shear zone
GCSZ Gwillim Creek shear zones
CRF Columbia River Fault

Steep normal fault

Geologic contact

Figure 1.2. Geology of the Valhalla comple:. Modified after Carr et al. 1987 and P. Simony (unpublished data). 


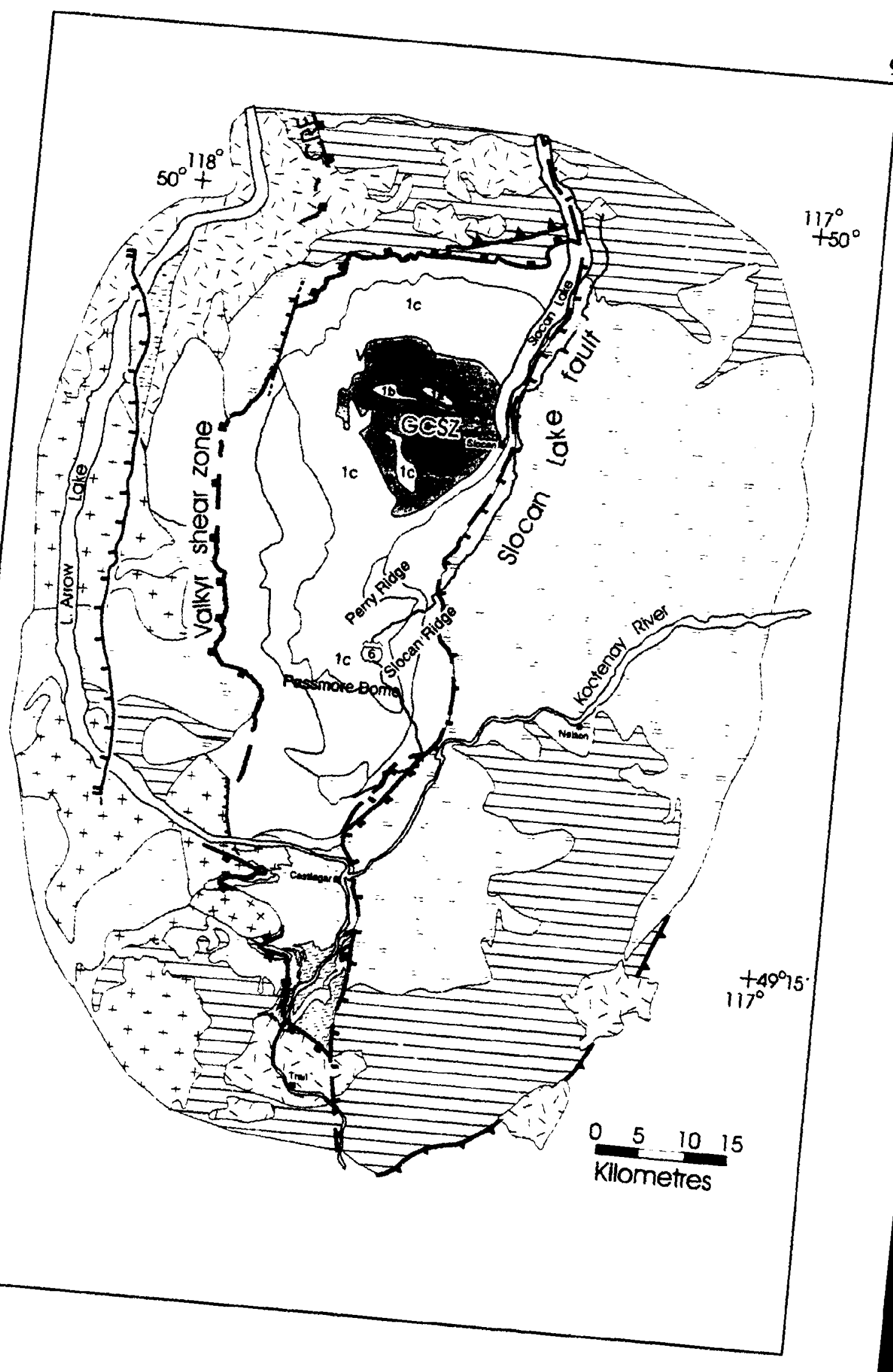



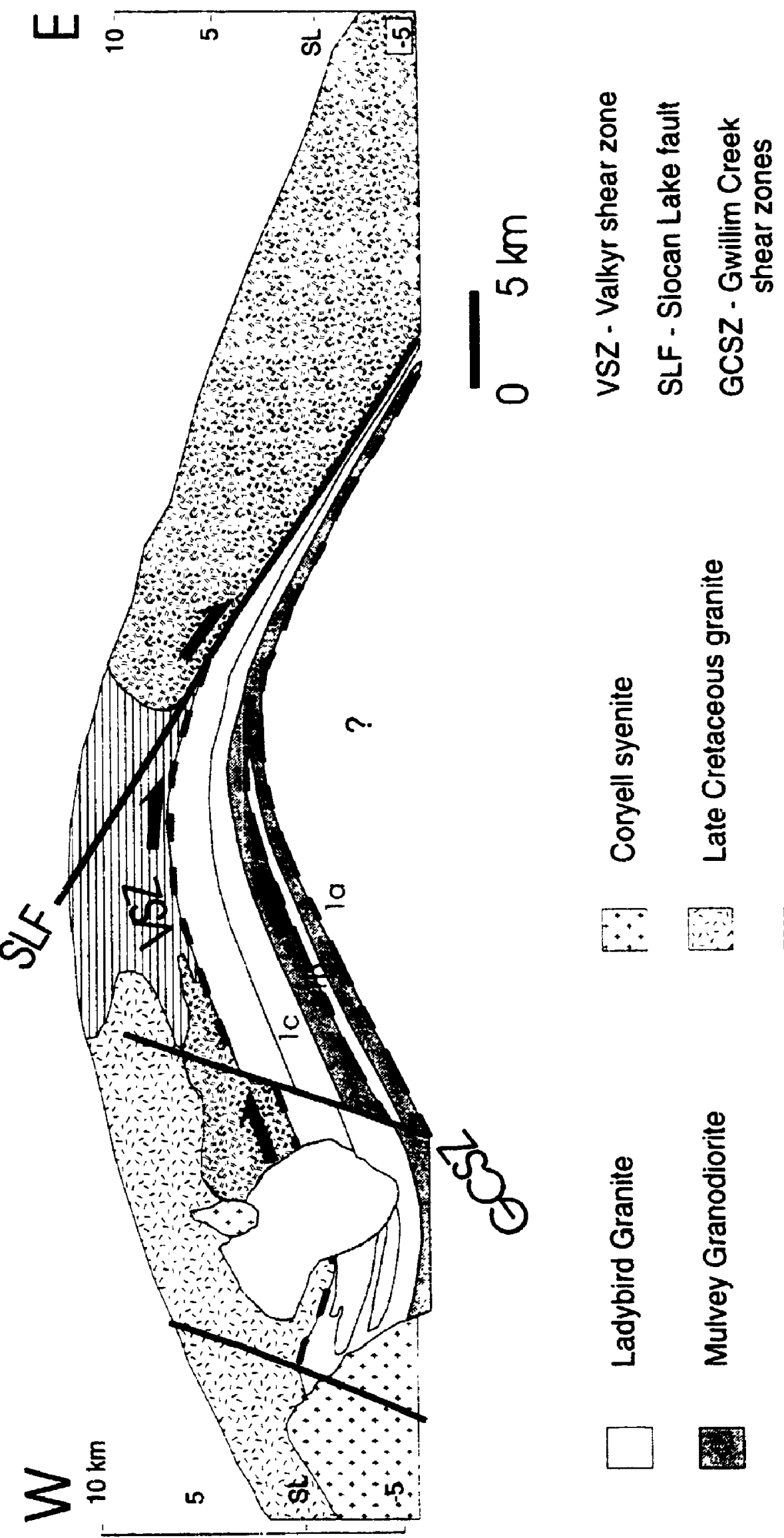

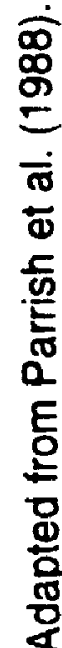

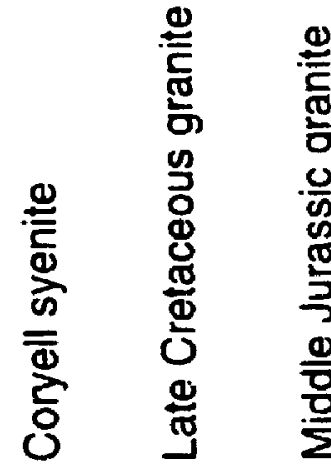

E
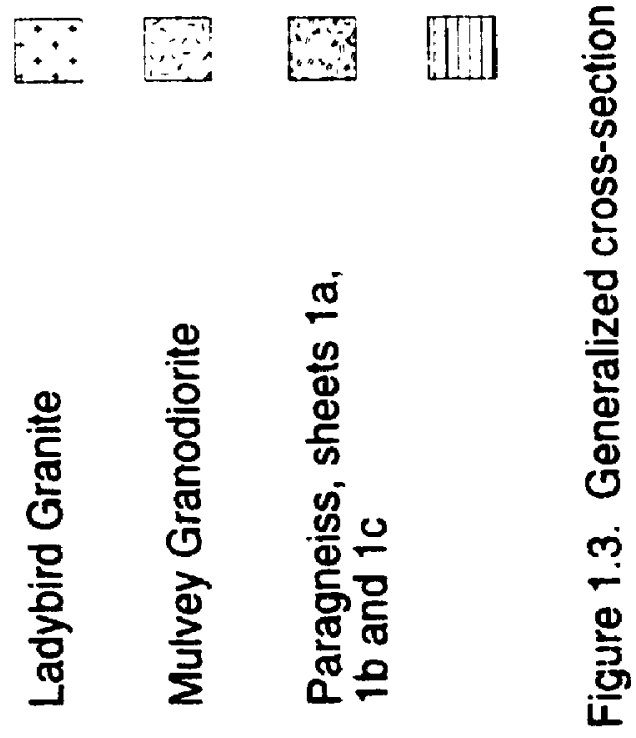
CHAPTER 2: GEOLOGIC SETTING AND STRATIGRAPHY OF THE VALHALLA ASSEMBLAGE

\subsection{Introduction}

The Canadian Cordillera may be described in terms of five morphogeological belts (Monger et al. 1982): 1) the Rocky Mountain foreland thrust and fold belt; 2) the Omineca belt; 3) the Intermontane belt; 4) the Coast plutonic belt; and 5) the Insular belt (Fig. 1.1a). The Omineca belt is the metamorphic and plutonic hinterland of the Foreland thrust and fold belt and was formed in conjunction with the docking of the Intermontane Superterrane to the North American craton during the Mesozoic to Early Cenozoic crustal shortening and thickening (Monger et al. 1982).

The Valhalla complex lies in the southern Omineca belt within the Shuswap metamorphic complex. Geological entities to the east of the Valhalla complex include the Nelson batholith, Kootenay Arc and Purcell Anticlirorium. The middle Jurassic Nelson batholith is bordered on the east and south by the Kootenay Arc (Fig. 1.1b) which is an eastfacing arcuate feature comprising sedimentary and volcanic rocks of the Kootenay terrane and marginal basin rocks of the Slide Mountain terrane (Archibald et al. 1983; Archibald et al. 1984; Roback et al. 1994). The east side of the Kootenay Arc forms the west flank of the Purcell anticlinorium. a north-plunging asymmetrical fold cored by the (Belt) Purcell Group (Gabrielse comp. 1991). On the west side the Valhalla complex, adjacent geology includes Jurassic and Cretaceous plutons, the Thor-Odin - Pinnacles area, and allochthonous metasedimentary and metavolcanic rocks of the Quesnellia terrane, some of which are in the 
upper plate of extensional faults systems (see Parrish et al. 1988).

This chapter presents the regional stratigraphy of the southern Omineca belt in order to provide a framework to compare the stratigraphy and deformation style of paragneisses of the Valhalla complex. Emphasis is placed on the stratigraphy and structural style of the Goat Ranges, Riondel Nappe and the Scrip Nappe (Fig. 1.1). A description of the stratigraphy of paragneisses in the Valhalla complex is followed by correlations to appropriate areas.

\subsection{Regional stratigraphy of the southern Omineca belt}

In the southern Omineca belt the Mesoproterozoic Purcell Supergroup (Fig. 1.1c) is the oldest package of sedimentary rocks and rests upon the North American basement. It comprises a sequence of shallow-marine and non-marine rocks that overlie a sequence of fine-grained, basinal clastic rocks in the west, and platformal clastic and carbonate rocks in the cast (Aitken and McMechan 1991). The Neoproterozoic Windermere Supergroup lies unconformably on the (Belt) Purcell Supergroup and was deposited between 780 and 570 Ma (Gabrielse and Campbell 1991). The presence of rapid facies changes and a large variety of sedimentary rocks has led to the interpretation that the Windermere Supergroup formed adjacent to, and on the rifted western margin of ancestral North America (Stewart 1972; Ross 1991). During the Neoproterozoic and Cambrian, quartzites of the Hamill Group and carbonates of the Badshot Formation were deposited unconformably on the Windermere Supergroup (Reesor 1973; Read and Wheeler 1976; Fritz et al. 1991). These shallow-water platformal rocks make up the upper part of the North American miogeocline (Fig. 2.1). 
The Kootenay terrane was deposited to the west of what has been generally agreed upon to be miogeoclinal rocks. and separates the shallow-water platformal rocks of the miogeocline from accreted rocks of the Intermontane Superterrane (Archibald et al. 1983; Klepacki 1985). The Kootenay terrane consists mostly of coarse clastic and volcanic rocks of the Lardeau Group and part of the Eagle Bay assemblage, which have been interpreted to lie confou mably on the Badshot Formation and are depositionally linked to the miogeocline (Price 1981; Colpron and Price 1995). Due to its similarity to grits in the Windermere Supergroup, Wheeler (1966) suggested that the Lardeau Group is part of an allochthonous slice of Windennere strata. Later. Wheeler and others (1991) proposed that the Kootenay terrane was suspect and therefore it should be designated a pericratonic terrane. Smith and Gehrels (1992a) suggested that the Lardeau Group was tectonically inverted and thrust over the Badshot Formation, based on correlations with the lithologically similar Covada Group in the southem Kootenay arc. Finally, Colpron and Price (1995) propose that the change from shallow-water sedimentary rocks of the miogeocline to coarse clastic and mafic volcanic rocks of the Lardeau Group repteser:ts $€$ pisodic periods of extensional rifting which occurred during the early Paleozoic.

The Kootenay terrane also includes the Davis and Keen Creek assemblages of the Carboniferous Milford Group (Fig. 2.1), which lie unconformably on, but are depositionally linked to the Lardeau Group (Klepacki 1985; Roback et al. 1994). The Slide Mountain terrane is a marginal basin that, together with the overlying accreted Quesnellia terrane, was obducted onto the Kootenay terrane and miogeocline (Monger 1977; Monger et al. 1982; Klepacki 1985). In the southern Omineca belt, the Slide Mountain terrane consists of the 
McHardy assemblage of the Milford Group and overlying Permian volcanic rocks of the Kaslo Group (Klepacki 1985). The Keen Creek and Davis assemblages of the Milford Group are included in the Kootenay terrane and are in fault contact with the McHardy assemblage (Klepacki 1985). The presence of the Milford Group in both the Kootenay and Slide Mountain terranes depositionally links the two (Fig. 2.1) and limits the distance of the Slide Mountain terrane from ancestral North America (Klepacki 1985; Roback et al. 1994). Kaslo Group volcanics were extruded within an ocean ridge, which was proximal to the margin of North America, and probably also zepresents the eastern margin of a Permian marginal basin (Roback et al. 1994).

Permian sedimentary and volcanic rocks of the Mount Roberts Formation and Triassic sedimentary rocks of the Slocan Group lie unconformably on the Kaslo Group. belong to the Quesnellia terrane and represent a thick section of deep-water sedimen'. which were deposited in a marginal basin.

\subsection{Geology of the Valhalla complex}

\subsubsection{General geology}

In the Valhalla complex, arched sheets of upper amphibolite-facies paragneiss, granitic rocks and intervening thrust faults (Gwillim Creek shear zones) were exhumed due to extensional faulting (Carr et al. 1987; Parrish et al. 1988). The Gwillim creek shear zones (GCSZ) have been interpreted to be part of a thrust fault system that links the Omineca belt and the Foreland belt (Cook et al. 1988, 1992; Parrish et al. 1988). The Valhalla complex 
was exhumed from mid-crustal depths by approximately $15 \mathrm{~km}$ of extensional displacement on the Slocan Lake (SLF) -Valkyr (VSZ) fault system during Early Eocene time (Figs. 1.2, 1.3; Carr et al. 1987). The lower plate high-grade rocks of the Valhalla complex were juxtaposed against upiper plate greenschist to amphibr.ite-facies metasedimentary rocks of the Quesnellia terrane and granitic rocks of the Nelson bathclith (Fig. 1.2). The 159-175 Ma Nelson batholith (Parrish and Wheeler 1983) in the hanging wall of the SLF cooled below $\sim 500^{\circ} \mathrm{C}$ during the Jurassic and late Cretaceous and was generally below $300^{\circ} \mathrm{C}$ when the Valhalla complex was at its metamorphic peak (Carr et al. 1987).

Three sheets of paragneiss (Figs. 1.2.1.3) in the Valhalla complex are separated by sheets of granitoid rocks. The lowest paragneiss sheet ( 1 a) is exposed in a window at the deepest structural level of the complex in the footwall of the GCSZ. The middle sheet (1b) is under- and overlain by the $-110 \mathrm{Ma}$ Mulvey granodiorite gneiss. The lower contact is intrusive and the upper contact is the upper strand of the GCSZ. The uppermost sheet (1c) is sandwiched between the Mulvey granodiorite gneiss below and the $63 \mathrm{Ma}$ Airy quartz monzonite and the 59-56 Ma Ladybird granite above (Carr et al. 1987). The uppermost sheet has been polydeformed by at least three periods of folding (Parrish et al. 1985: Carr et al. 1987). The southern part of the complex (Fig. 1.2) consists of heterogeneous quartzofeldspathic Castlegar paragneiss. Late Cretaceous leucocratic hornblende \pm biotite Kinnaird orthogneiss. and the Devonian Trail amphibolitic gneiss (Simony 1979; Halwas and Simony 1986: Simony and Armstrong 1990).

The GCSZ constitute sheared contacts between the two windows of paragneiss and the overlying Mulvey granodiorite gneiss. with an apparent easterly sense of displacement 
(Parrish et al. 1988). The GCSZ are imaged in subsurface seismic reflection data and may be correlative with east verging structures that are imaged to the east within the Kootenay Arc and Purcell Anticlinorium, and to the west below the Pinnacles area and Vernon Arch (Cook et al. 1988, 1992; Varsek and Cook 1994; Cook and Van der Velden 1995; Carr 1995). The age of movement on the GCSZ is bracketed between 51 and 100 Ma Parrish (1995). Igneous rocks cross-cutting the GCSZ give U-Pb zircon ages of 50-51 Ma (Parrish et al. 1988) and $52 \mathrm{Ma}$ (Parrish 1995), and the $100 \mathrm{Ma}$ Mulvey granodiorite is sheared by the GCSZ (Carr et al. 1987). Based on thermal history studies. Parrish and others (1988) and Heaman and Parrish (1991) suggested that the GCSZ were active at the thermal peak of metamorphism, at approximately 70-80 Ma.

The complex is roofed by the arched ductile Valkyr shear zone (VSZ) and is bounded on the eastern margin by the ductile - brittle Slocan Lake fault (SLF), both of which deform 59-55 Ma Ladybird granite and pegmatite. The VSZ has a maximum thickness of $2-3 \mathrm{~km}$ and is exposed around the northern, southern and eastern periphery of the complex. It was active at amphibolite-facies metamorphism, between 59 and $54 \mathrm{Ma}$ (Carr et al. 1987). The SLF is an easterly dipping normal fault that truncates the VSZ and was active between 54 and approximately $45 \mathrm{Ma}$ (Carr et al. 1987). The fault was active through the ductile-brittle transition and final motion occurred at greenschist-facies metamorphism as indicated by synkinematic albite, chlorite and muscovite within the 100-800 $\mathrm{m}$ thick fault zone (Carr et al. 1987). Muscovite with a $\mathrm{Rb}-\mathrm{Sr}$ age of $54.5 \pm 1.5 \mathrm{Ma}$, grew at temperatures below the closure temperature of Sr in feldspar and dates the greenschist facies movement on the SLF (Parrish et al. 1988). The SLF and VSZ are genetically related; movement along the SLF outlasted 
the VSZ during the Early to Middle Eocene (Carr et al. 1987).

The Slocan Lake fault and Valkyr shear zone juxtapose the high-grade rocks of the Valhalla complex against low-grade rocks in the upper plate. The upper plate (Fig. 1.2) consists of Pennsylvanian low-grade metasedimentary rocks of the Mount Roberts Formation and Triassic volcanic rocks of the Rossland Group, both belonging to the accreted Quesnellia terrane, Middle Jurassic Nelson Batholith and equivalent plutonic rocks to the west, and late Cretaceous two mica granites $(\sim 100 \mathrm{Ma})$ and intrusives of the $79 \mathrm{Ma}$ Watshan Batholith. To the north, middle-high grade metasedimentary rocks of the Nemo Lakes belt are thought to be in part equivalent to the Paleozoic Lardeau Group as well as the Triassic Slocan Group (Parrish 1981).

Rocks within the Valhalla complex were exhumed rapidly along the SLF and VSZ, preserving sillimanite - potassium feldspar grade conditions and yielding Eocene $\mathrm{KJAr}$ cooling ages (Carr et al. 1987). Retrograde metamorphism is rare and indicates that rocks of the Valhalla complex were exhumed rapidly. Cooling rates of the Valhalla complex are estimated to be between $15-20^{\circ} \mathrm{C} / \mathrm{Ma}$ (Heaman and Parrish 1991), however Spear and Parrish (in prep.) suggest even faster, periodic cooling rates up to $1000^{\circ} \mathrm{C} / \mathrm{Ma}$ over a 0.1 Ma period.

Paragneisses of the Valhalla complex are characterized by their heterogeneity and abundant migmatites, leucosome and cross-cutting granitic rocks. Granitic pegmatites and leucogranites belonging to the Ladybird suite occur throughout the complex (Carr et al. 1987: Parrish et al. 1988). The absolute age of the paragneisses in the Valhalla complex is only broadly constrained. Detrital zircon ages of $\sim 1400,1700$ and $2700 \mathrm{Ma}$ were obtained 
from two sample localities in the upper paragneiss sheet $(\mathrm{l})$ and place a maximum age of $1400 \mathrm{Ma}$ on the deposition of at least part of the protoliths of the paragneisses (Parrish 194) 1 : Ross and Parrish 1991). A minimum age of deposition of the paragneisses is constrained h! the intrusive Mulvey granodiorite. which has a maximum age of - 110 Ma (Carr ef al 1987: Parrish et al. 1988; Parrish 1992). A summary of geochronological data from the Valhalla complex is presented in Table 2.1 .

\subsubsection{Structure}

The Valhalla complex is a fault bounded culmination which has gentrally outward dipping foliations which define its domal nature. Within the large scale culmination, there are two northern and southern subculminations which Reesor (1965) termed the Valhalla and Passmore domes. respectively (Fig. 1.2, cross-section A; see also the structural geology map and cross-section in Reesor 1965). A third subculmination occurs in the southern portion of the complex near Castlegar and Trail (Simony 1979). The major domal shape of the complex has been interpreted to be a result of tectonic denudation due to exhumation along Eocene normal faults (Carr et al.1987; Parrish et al. 1988), however the reason tor the secondary subculminations is uncertain.

Three domains are delineated within the Valhalla paragneisses on the basis of structural level and the presence or absence of transposed fabrics (Fig. 2.2 cross-section A.B.C). Domain 1 constitutes sheet $l c$ at high structural levels and is divided into northern (la) and southern ( $1 \mathrm{~b})$ subdomains. Domain 2 is also contained within sheet $1 \mathrm{c}$ but is at a deeper structural level and contains a transposition fabric that is interpreted to be related to 
the (iCS7. Domain 3 occurs in sheet la in Gwillim Creek and contains a transposition fabric related to the GCSZ which forms the upper boundary of this domain. Domain 1 offers the best exposure and continuous stratigraphy, whereas rocks are not well exposed in domains 2 and 3. Nomenclature of structural sheets $(1 \mathrm{a}-1 \mathrm{c})$ is retained because of previous use.

Fabrics related to $D_{1}$ are virtually absent in the field and only recognized in thinsection as quartz inclusions trai!s in garnet. At the highest structural level of paragneisses within sheet $1 \mathrm{c}$ on the west side of the complex (domain $1 \mathrm{a}$ ). $S_{2}$ is the predominant foliation and is parallel to the west flank of the complex and the contacts of geologic units. $S_{2}$ transposes bedding which is defined by variations in the relative amounts of calcite and quartz in marble (Plate la) and compositional layering in all other rock types. In pelitic and semipelitic schists, $S_{2}$ is defined by platy minerals such as biotite and discrete layers of sillimanite. In calc-silicate gneisses and amphibolite gneisses, it is defined by the segregation of amphiboles and quartzo-feldspathic minerals and is parallel to compositional layering. $S_{2}$ contains the dominant lineation. $L_{2}$, a mineral lineation. Lineations are defined by sillimanite needles, quartz ribbons and rare aligned biotite aggregates in semipelitic and pelitic schists and most commonly by amphibole in calc-silicate and amphibolite gneisses. The $S_{2}$ fabric is folded by abundant $W$ to $N W$ plunging $F_{3}$ folds. On the east side of the complex, sheet $1 c$ is significantly thinner and folds are absent due to transposition and attenuation resulting from motion on the SLF and possibly the GCSZ. Also on the east side, mineral lineations are oriented down the dip of the SLF (east plunging) and are inferred to have been rotated to this position during faulting. The same fabric development is observed in the southern portion of the paragneisses near Airy Creek and Slocan Ridge and constitutes 
domain $1 \mathrm{~b}$.

The GCSZ project toward the southerly areas (cross-section $A$ ) at the structural level of domain 2 and are consistent with interpretations of seismic datia that suggest that the GCSZ resurface in the Passmore-Vallican area (Cook et al. 1988). In domain 2 in the Slecian River Valley, the fabric is more intense with respect to domain $1 \mathrm{~h}$ and preexistung fibrics are transposed. Although this transposition fabric is generally parallel to $S$, in higher structural levels. it is uncertain if it is a result of the same structural event. and is therefore designated $\mathrm{S}_{\mathrm{T}}$. Folds are rare. but where present, are rootless or have sheared limbs. Sheath tolds have fold axes parallel with the lineation. Leucogranites and pegmatites that are clearly discordant in domain $1 \mathrm{~b}$ become transposed in domain 2 where they are not obviously cross-cutting. Sillimanite forms a strong stretching lineation with fractures oriented perpendicular $t_{1} S_{1}$.

At deep structural levels beneath the GCSZ (sheets la. domain 3). a transposition fabric predominates $\left(\mathrm{S}_{\mathrm{T}}\right)$ that is interpreted to have developed during motion on the ( $;(\mathrm{S} /$. As in domain 2, folds are rare and where present occur as sheath folds. The (iCS/. are 50) $m$ thick horizons of ductile strain and grain size reduction which reduced the normally coarse $\mathrm{K}$-feldspar megacrystic Mulvey granodiorite to a biotite schist. In thin-section, quartz forms discrete bands. The GCSZ are parallel to $S_{r}$. however it is uncertain if this transposition fabric and $S_{2}$ in domain 1 resulted from the same detormation event.

Local areas on Perry Ridge contain a strong fabric with no apparent strain gradıent into adjacent areas. It is possible that the mylonitic fabric defines a shear zone that is contiguous with the GCSZ, but it is also possible that it delineates another shear rone of unknown magnitude or displacement. Fold axes on Peiry Ridge are shallowly plunging and 
trend in a general $W$ to $\mathrm{XW}$ direction. Axial surfaces are also subhorizontal and dip to the west.

\section{Folding in Domain I}

Evidence for $D_{1}$ structures in the Valhalia complex include rare $F_{1}$ isoclinal folds and inv rted stratigraphy inferred from regional correlations presented later in this chapter. The stratigraphic and structural relationships in the area between Lucifer Col and Black Prince (Fig. 2.3) are uncertain but suggest the presence of a tirst phase fold hinge that is obscured by large volumes of granitic rocks. Parrish and others (1985) reported a large first phase isoclinal fold in the Gwillim Lakes area but evidence to support this was not observed by this author. The timing of first phase folding is not weil constrained in the Valhalla complex. The -110 Ma Mulvey granodiorite contains the dominant $S_{2}$ fabric. This tabric is interpreted to be synchronous with the peak of metamorphism at $-75 \mathrm{Ma}$ (Heaman and Parrish 1991. see discussion of timing of metamorphism and deformation in chapter 3 ). Therefore. the absolute minumum age for $F_{1}$ folding is $\sim 75 \mathrm{Ma}$. There is no reported evidence to suggest that the Mulvey cross-cuts $F_{1}$ folds and the maximum age is constrained by the age of strata that is deformed (see section 2.5 on regional correlations). Carr (1991) presented a model which divides the crust into three crustal zones. Parrish (1995) builds on this model and divides the crust into five levels where he proposes that the age of deformation and metamorphism decreases with deeper sticictural levels. The upper zone lies in the hanging walls of Locene normal faults and has deformusev and cooling ages which are primarily Jurassic. At deep crustal levels in the Monashee complex, ages of metamorphism and 
detormation are essentially Paleocene-Eocene (Parrish 1995). In these models, the Vallabllat complex should have Cretaceous deformation ages. hut age constraints from previous geochronological studies and this study do not constrain the maximum age fo (retaceus. Jurassic $D_{1}$ deformation ( $F$ folding) in the Valhalla complex cannot be ruled out.

$F_{2}$ folds in domain 1 are overturned. tight asymmetric tolds which are retolded hy $f$ : folds (Plate $1 \mathrm{c}$ ). $\mathrm{F}_{2}$ folds are difficult to distinguish from $F$; folds. due to their coaxiality. and $D_{2}$ and $D_{3}$ may represent one protracted folding event. $S_{2}$ axial planar eleavages are rarc. $F$ : folds leucosome, biotite and sillimanite. and therefore posidates the peak of metamorphism.

Third phase folds are the dominant fold generation in domain 1 (Plate $/ b$ ). They are open to close tolds that vary from centimetre- to metre-scale structures ((Plate la and Plate 2a, b, repsectively). Fold hinges have a variable orientation, but generally plunge moderately to shallowly $W$ to $N W$ (Fig. 2.4). F, axial surfaces are $W$ to $S W$ dipping and $F_{3}$ folds are 1 : to $\mathrm{NE}$ verging. $\mathrm{F}_{3}$ folds peak metamorphic minerals of sillimanite, biotite and concentrated layers of garnet.

Evidence for a fourth phase of deformation is in the occurrence of rare broad, tens of metre-scale open folds with steep axial planes (Fig. 2.4). F, folds are best exposed in the Hela Peak area where a large Z-fold (looking NW) occurs near the contact with the Ladybird granite (Fig. 2.3). Crenulations in semipelitic to pelitic schists may also be related to I) deformation.

Faults are rare within the Valhalla complex. One north-south striking, nearly vertical, minor normal fault occurs near Urd Peak. and offsets the Demers marble by approximately 
$10 \mathrm{~m}$. Steep. north-south oriented joints also occur near the Demers Lakes. These brittle features may be related to similarly oriented ultramafic dykes in this area.

\section{Eabrics in thin-section}

In thin-section. $S_{2}$ is defined by the preferred alignment of biotite and sillimanite and discrete layers of quartz. K-feldspar and plagioclase. The peak mineral assemblage of garnet. biotite, sillimanite. K-feldspar. ritile and ilmenite is contained within this foliation and also occur in the pressure shadows of garnet. Garnets contain an internal schistosity defined by quartz which is oblique to the dominant external $S_{2}$ fabric Sillimanite inclusions occur near garnet rims and are often parallel to the external foliation $\left(S_{2}\right)$. Quartz inclusion trails are interpreted to represent $S_{\mathrm{f}}$ which was overgrown by garnets, implying that garnet growth began following $D_{1}$. The main fabric $\left(S_{2}\right)$ began to develop at high temperatures and pressures during garnet growth. which is indicated by the occurrence of sillimanite inclusions within garnet that can be traced into the external $S_{2} . S_{2}$ is interpreted to have developed during the thermal peak because the peak minerals (sillimanite, potassium feldspar. biotite) occur with pressure shadows associated with garnet. Deformation outlasted garnet growth hecause these same minerals wrap around the garnet porphyroblasts. $F_{3}$ folds deform the $S_{2}$ main fabric and all peak metamorphic minerals, and therefore postdate the peak of metamorphism. Rare muscovite grains oriented sub-parallel to $S_{2}$ are crenulated by $F_{3}$ and indicate that third phase folding occurred after the rocks had cooled substantially. A complete discussion of the relationships between metamorphism and deformation is presented in chapter 3 . 


\subsection{The Valhalla assemblage}

\subsubsection{Introduction}

The paragneisses within the Valhalla complex comprise a heterogeneous package of metasedimentary rocks. They were originally mapped as the Hybrid Gneiss (Unit 1) by Reesor (1965 p. 15), who described the unit as "intimately interlayered rocks consisting of a metasedimer:ary fraction with leucogranite-gneiss and pegmatitic interlayers". Reesor (1965) also mapped out the major carbonate marker units within the Hybrid Gneiss, however no detailed mapping of stratigraphy or correlation was attempted. Parrish and others (1985) described a quartz-pebule conglomerate in the Gwillim Lakes area and suggested that it may be correlative with the Carboniferous Milford Group. Mapping for this project was carried out throughout the complex, including the lower window in Gwillim Creek; however, attention was concentrated in the area of best exposure, the west-central flank of the complex between Mount Rinda and the Demers Lakes (Fig. 2.3). This area lies in the uppermost paragneiss sheet (1c) and in the hanging wall of the GCSZ. A laterally continuous stratigraphy was identified and is referred to as the Valhalla assemblage.

Distinctive marble, calc-silicate - quartzite-cobble metaconglomerate and ultramafic units were used as stratigraphic markers. A mappable sequence of lithologies including semipelitic to pelitic schist and gneiss, psammitic gneiss, quartzite, marble, calc-silicate gneiss, quartz-pebble to cobble metaconglomerate, amphibolite gneiss, and ultramafic boudins occur in the Valhalla assemblage. The Valhalla assemblage is characteristically lacking appreciable amounts of amphiholite and orthogneiss. The predominant siliciclastic 
rocks are semipelitic schists and gneisses and psammitic gneisses, with lesser amounts of pelitic schist. All rock types are characterized by the presence of ubiquitous leucogranites and pegmatites (Plate 2c, d), which consist of multiple generations of concordant to discordant stocks and dykes ranging from strongly deformed to totally undeformed. In many cases, these granitic rocks make up $75 \%$ of any particular outcrop. The stratigraphy of metasedimentary rocks within the Valhalla complex is preserved despite the large volume of these young granitic rocks (Plate 3a). Map units are not always continuous, but can be followed along strike, where screens or schlieren of paragneisses are normally present within the leucogranites.

\subsubsection{Lithology}

A variety of rock types occur within the Valhalla assemblage. The dis tinguishing characteristics and mineralogy of each are described in Table 2.2 in order to avoid repetition in the subsequent section characterizing the Valhalla assem' 1 lage.

\section{Paleocene-Eocene leucogranites and pegmatites}

A considerable amount of the stratigraphy in the Valhalla complex is intruded by both concordant and discordant granites, which comprise leucocratic biotite-bearing pegmatite, coarse-grained granite and weakly foliated to undeformed, locally garnetiferous, medium-grained leucogranite (Plate 3a). The gametiferous granite occurs as small stocks and sheets that often form mountain peaks and are up to tens of metres thick. Biotite clots and knots are common, often replacing garnet porphyroblasts. In many cases, the granitic 
rocks do not constitute mappable bodies. but occur as metre-scale thick layers that often make up 50 to $75 \%$ of outcrops and ridges (Plate 3a). Pegmatites and coarse-grained granites are thought to be Ladybird granite equivalents that have been dated at 59-56 Ma (Carr et al 1987) and are the product of crustal melting (Carr 1992).

In the southern, structurally higher areas south of Perry Ridge (Fig. 1.2), granitoid rocks occur as sub-concordant to clearly discordant dykes up to $3 \mathrm{~m}$ thick which make up to $75 \%$ of unit 11 . The peak-forming homogeneous bodies of garnet-bearing granite stocks that abound in the area between Mount Rinda and Hela Peak are absent in the southern area. In contrast, pegmatites and leucogranites within the paragneisses of the Gwillim Creek windows have been transposed and do not obviously cross-cut the main $\mathrm{S}_{\mathrm{T}}$ fabric.

Up to five graniioid phases may be present in one outcrop, as was mapped at station 130 (Map 1), at the head of Bannock Bum. They are described in order from oldest to youngest:

1) Thin layers ( $<1 \mathrm{~cm}$ wide) of plagioclase-quartz leucosome are oriented parallel to the predominant $\left(S_{2}\right)$ foliation in pelitic, scrnipelitic and migmatitic schists (Plate $3 b$ ).

2) Boudins up to $1 \mathrm{~m}$ thick of fine- to medium-grained, well foliated granite contain biotite - rutassium feldspar - plagioclase \pm homblende \pm garnet. The hornblende content varies from $5-15 \%$. The amount of fabric is also variable in these granitic rocks and ranges from weakly to well foliated.

3) Medium-grained granite that contains 3-5\% biotite clots and no homblende. These 10-20 cm wide Jands show pinch and swell textures and are commonly weakly foliated (Plate 1b). 
4) Undeformed to weakly-deformed, discordant, medium-grained granite and pegmatite are up to $1 \mathrm{~m}$ thick.

5) Sugary textured undeformed aplite dykes which cross-cut pegmatites of (4) are rare and are up to $30 \mathrm{~cm}$ thick.

This description is representative of the area between Mount Rinda and Hela Peak. Aplite dykes may be a younger phase of the undeformed granite and pegmatite (\#4) and are not always present.

\section{Ultramafic and mafic intrusive dykes}

Metre-wide, near vertically dipping mafic pyroxene-plagioclase \pm olivine dykes are the youngest rocks in the Valhalla complex. They are traceable for 1 to $2 \mathrm{~km}$ and have little offset along strike. These dykes intrude both the upper and lower plates, as well as the Coryell syenite and are younger than $51.5 \mathrm{Ma}$ (Bevier 1987). These dykes were described by Reesor (1965 p. 40) as basaltic dykes.

South of Woden Peak, a fine-grained, very hard and clinkery weathering, olivinebearing ultramafic dyke was observed. On the weathered surface it is pitted due to the weathering of some unknowir mineral which gives it a vesicular appearance. In the areas north of the Hird Lakes and surrounding the western Demers Lakes, numerous $0.5-1.5 \mathrm{~m}$ wide. near vertical, north-south trending ultramafic to mafic dykes are spaced $200-500 \mathrm{~m}$ apart. Mafic mineral content varies from $60-90 \%$. Some dykes contain $20 \% 1-2 \mathrm{~mm}$ laths of plagioclase, while others contain $20-30 \%$ serpentinized (?) phenocrysts of olivine. Still other dykes appear to contain litte plagioclase or relict olivine phenocrysts, and instead 
contain diopside. Zoned phenocrysts of plagioclase are observed in thin-section and no metamorphic textures are observed. The presence of rare chilled margins suggests that these dykes intruded the Valhalla complex after it had cooled.

\subsubsection{Stratigraphy of metasedimentary units}

Map units are ordered from structurally lowest to highest and the terms "underlying" and "overlying" are used in a structural context; however. this does not imply stratigraphic ordering (Fig. 2.5). It is possible that the entire Valhalla assemblage is inverted, and that unit 1 is the youngest stratigraphic unit. This will be discussed in the section entitled "Regional correlation of the Valhalla assemblage". There is a strong transposition foliation within the Valhalla assemblage and therefore unit thicknesses reported here are not the original thicknesses of unmetamorphosed and unstrained protoliths. An estimate of the present thickness of the metasedimentary package is between $1.5-2 \mathrm{~km}$. The reader is referred to Figure 2.2 and Map 1 for the locations of the following units and Figure 2.4 for the order of units.

\section{Unit 1. Ouartzo-feldspathic gneiss}

Unit 1 is a light-grey weathering quartzo-fe!dspathic gneiss which is commonly tightly folded (Plate $1 \mathrm{~b}$ ). Psammite and semipelitic schist occur as rare $10 \mathrm{~cm}$ layers and make up less than $5 \%$ of the unit. At the crest of the ridge joining Mount Bor and Black Prince, a $10 \mathrm{~m}$ thick homblende-plagioclase amphibolite gneiss is present and is most likely contained within unit 1 . Unit 1 is the structurally lowest unit mapped in the uppermost 
paragneiss sheet, carried in the hanging wall of the GCSZ and is underlain by the Mulvey gneiss. It has a minimum thickness of $50 \mathrm{~m}$. At the basal contact over an interval of approximately $10 \mathrm{~m}$, there is a significant increase in the volume of leucogtanite and pegmatite approaching the Mulvey granodiorite. There is no apparent strain gradient at the contact and it is interpreted to be an intrusive contact. The contact between units 1 and 2 is not often observed. In the area between Mount Bor and Lucifer Col, unit 1 occurs amongst the leucogranite and pegmatites which make up approximately $75 \%$ of the $\sim 1 \mathrm{~km}$ square flat glaciated area. The stratigraphic and structural relationships in the area between Lucifer $\mathrm{Col}$ and Black Prince (Fig. 2.3) are unclear, but it is possible that a first phase fold hinge lies here and is concealed by the large volumes of granitic rocks.

\section{Unit 2. Hird Lakes marker}

The Hird Lakes unit is a 10-20 m thick marker that varies along strike between a dark-green weathering, calc-silicate gneiss (2a-cs) and quartzite-pebble to cobble metaconglomerate (2a-cg), with either a calc-silicate or psammitic matrix. Marble (2b) occurs at the same structural level and is included within this map unit.

\section{2a-cs. calc-silicate gneiss}

The calc-silicate gneiss (2a-cs) varies from area to area, commonly contains discrete, biotite-rich layers, and has a thickness on the order of $10 \mathrm{~m}$. In the Gwillim basin, the unit is a diopside-bearing calc-silicate (Table 2.2) with discrete biotite layers. Near Black Prince (Fig. 2.3), fine- to medium-grained marble is interlayered with calc-silicate gneiss on a scale 
of 1-3 m, and makes up 50\% of the unit. Marble and calc-silicate gneiss layers are cross-cut by $\sim 2-10 \mathrm{~cm}$ wide actinolite veins that are a late feature. At the Gwillim Lakes and the head of Gwillim Creek, the unit is predominantly calc-silicate gneiss and occurs as xenoliths within coarse grained granite and pegmatite (Plate 2d). Near the head of Gwillim Creek. there is a distinctive metre-wide layer of foliated magnetite-calcite-diopside calc-silicate within the marble and calc-silicate gneiss.

In the Hird Lakes area. the calc-silicate gneiss is less massive than in the Gwillim basin. The content of phlogopite is higher giving the rock a more schistose texture. Coarsegrained, white weathering scapolite occurs as masses up to $10 \mathrm{~cm}$ thick.

\section{2a-cg. quartzite-pebble metaconglomerate}

A distinctive quartzite-pebble to cobble metaconglomerate, with calc-silicate or semipelitic matrix (2a-cg), first described by Parrish and others (1985), occurs in the vicinity of the most eastern Gwillim Lake, south of Hird Lakes and near Rocky Lakes. The metaconglomerate is generally calc-silicate matrix supported with elliptical rounded and occasionally blocky clasts of relatively clean quartzite and vein quartz which range in size from $1 \times 2$ to $5 \times 10 \mathrm{~cm}$ (Plate $3 \mathrm{~b}$ ). The metaconglomerate is under- and overlain by $5-10 \mathrm{~m}$ thick dark-green weathering calc-silicate schist or gneiss. Metaconglomerate layers are approximately $30 \mathrm{~cm}$ to $2 \mathrm{~m}$ thick in the Gwillim Lakes area and up to $20 \mathrm{~m}$ in the Rocky Lakes area, perhaps due to thickening in a fold hinge. In the Rocky Lakes area. a $20 \mathrm{~m}$ thick, clast suppo 
argillaceous quartzite (Plate 4a). Clasts are rounded and up to $10 \mathrm{~cm}$ in diameter. This change in matrix composition may be the result of variations in protolith.

\section{2b. marble.}

A $10 \mathrm{~m}$ thick marble outcrops on the ridge separating the headwaters of Bannock Burn and Drinnon Lake (Fig. 2.3) and is typical of marbles in the Valhalla complex (Table 2.2). Based on structural projections, it may correlate along strike with the Hird Lakes conglomerate. The presence of marbles interlayered with the calc-silicate (2a-cs) near the Gwillim Lakes suggests that variations in protolith are possible along strike. For this marble to correlate to unit $2 \mathrm{a}$, a change in protolith and depositional environment is required.

\section{Unit 3. Drinnon quartzite}

\section{3a. quartzite}

A 150-200 m thick clean, homogeneous, rusty to pinkish, buff-grey weathering quartzite with minor biotite-rich millimetre- to centimetre-scale layers outcrops south of Mount Gregorio and north of Drinnon Lake (Fig. 2.3). Contact relationships are unclear due to poor exposure. but no compositional gradation is observed near the top or base of the unit.

\section{3b. psammitic gneiss - migmatitic gneiss - semipelitic schist}

This package of rocks is approximately $150-200 \mathrm{~m}$ thick and consists of rusty weathering semipelitic schist, psammitic gneiss, migmatitic gneiss and quartzite interlayered 
on a scale of metres with minor of pelitic schist and amphibolite gneiss. These siliciclastic rocks are predominantly quartzo-feldspathic in composition with widely varying amounts of biotite (5-50\%). In the more biotite-rich pelitic layers, sillimanite and garnet are common. On the ridge to the south of the Black Prince. crenulated sillimanite-rich semipelitic to pelitic schists are interlayered with migmatitic gneiss on a scale of tens of centimetres to metres. Migmatitic gneiss contains $50 \%$ quartzo-feldspathic leucosome and $50 \%$ millimetre to centimetre thick biotite layers. On the west side of the Gwillim Lakes, above the Hird Lakes metaconglomerate ( $2 \mathrm{a}-\mathrm{cg})$, unit $3 \mathrm{~b}$ is predominantly migmatitic schist with up to metre-thick discordant and concordant leucogranites and pegmatites (Plate 4b).

\section{3c. quartzo-feldspathic gneiss - pelitic schist}

In the vicinity of the Rocky Lakes and south of the Hird Lakes, a $100 \mathrm{~m}$ thick package of quartzo-feldspathic gneiss and pelitic schist is characteristic of most of the unit. Rare, $5 \mathrm{~m}$ thick layers of garmet-sillimanite schist are also present. The quartzo-feldspathic gneiss is interlayered, on the millimetre to centimetre scale, with quartzo-feldspathic leucosome.

\section{3c-cq. calcareous quartzite}

A 75-100 m thick calcareous quartzite (3c-cq) is present locally in the Hird Lakes area where it overlies the quartzo-feldspathic gneiss and pelitic schist of unit 3c. This subunit consists of calcareous or pitted quartzite interlayered, on the metre scale, with lesser amounts of calc-silicate gneiss and semipelitic schist. West of Urd Peak, a pitted, white to 
buff weathering calcareous quartzite is interlayered, on the centimetre scale, with diopsidebearing calc-silicate gneiss. Layering is straight, with little variation in thickness (Plate 4c). Minor coarse-grained marble occurs as less than $1 \mathrm{~m}$ thick layers and make up less than $5 \%$ of the unit.

This unit (3c-cq) is not present is the north of Urd Peak. Near the Demers Lake, semipelitic schists and crenulated garnet-sillimanite schists occur at the same structural level. Along strike from south to north, there is a dramatic facies change from clean quartzite (3a) to semipelitic and pelitic schist ( $3 b)$ and then to pitted quartzite (3c-cq). The variation within unit 3 may represent facies changes or structural complications such as faulting. However, the continuity of the underlying Demers marble ( $4 \mathrm{~b}$, described below), which has no apparent offset. disputes both of these ideas. Another hypothesis is that this unit (3c) is actually an enigmatic thickened portion of the Demers marble (4b). There are no structural reasons to attribute thickening to folding.

\section{Unit 4. Demers marble}

4a. marble - calc-silicate

A coarse-grained, commonly siliceous marble unit, at most 10 metres thick occurs discontinuously $3 \mathrm{~km}$ along strike on the ridge south of Drinnon Lake, west of Wicca Lake and west of the Gwillim Lakes (Fig. 2.3). It is not as prominent as other marble marker units. Minor calc-silicate is associated with the marble and occurs as metre-sized boudins and discontinuous layers. Contact relationships are uncertain due to the presence of 
leucogranite and scarce outcrop.

\section{4b. marble - calc-silicate - siliceous marble}

North of the Hird Lakes. a 5-10 m thick marble is characterized by $30 \mathrm{~cm}-1 \mathrm{~m}$ interlayers of marble, calc-silicate, calcareous quartzite and psammitic gneiss. Lesser amounts of calc-silicate and siliceous marble are interlayered with the marble. on a centimetre scale. Contacts of unit $4 b$ with units $3 c-c q$ and $5 b$ are conformable and no mineralogical or grain-size change is observed towards them. North of the Hird Lakes, the marble is closely associated with calcareous quartzite (3c-cq). It is possible that units $4 b$ and $3 c-c q$ belong to the same package, although calcareous quartzite is not observed along strike in either direction. Further west along strike. thin marble (4b) is clearly distinguishable from surrounding siliciclastic rocks ( $3 c$ and $5 c$ ) and is characteristically thin. Unit $4 b$ is observed discontinuously along $5 \mathrm{~km}$, from Urd Peak to the ridge north of the western Demers Lake. Blocks of marble lie at the base of a steep cliff near the Rocky Lakes where Reesor (1965) mapped a carbonate layer. This is interpreted to be continuous with unit $4 \mathrm{~b}$. Based on structural projections and the under- and overlying lithology, this marble unit (4b) is correlated with thin marbles west of the Gwillim Lakes and at the head of Bannock Burn (4a).

\section{4c. marble - siliceous marble}

A $20 \mathrm{~m}$ thick marble marker was observed west of Hela Peak in the northern part of the complex, and contains layers of diopside calc-silicate and locally $1-2 \mathrm{~cm}$ wide quartz rich 
layers (Plate 2b). It is tentatively correlated with the marble observed north of Demers Lakes (4b), as weil as unit 4a, south of Drinnon Lake. This correlation is based on structural projections, relative thickness and the fact that it is more siliceous than other marbles.

\section{Unit 5. Hoder Creek composite unit}

\section{5a. semipelitic schist - quartzite - marble - calc-silicate - amphibolite}

A 75-100 m thick heterogeneous package of rocks consists of 1-4 metre thick layers of psammitic to semipelitic schist. argillaceous quartzite. marble. calc-silicate gneiss, and aimphibolite gneiss which are exposed along a steep slope in the headwaters of Hoder Creek. near the parking lot accessing Valhalla Park (Fig. 2.3). Marble layers are $30 \mathrm{~cm}$ to $2 \mathrm{~m}$ wide and are spaced 20-30 $\mathrm{m}$ apart. Three metre wide diop.ide-rich (20\%, more diopside than normal) calc-silicate gneiss (Plate $4 \mathrm{~d}$ ), overlies $30 \mathrm{~m}$ of argillaceous quartzite, garnetvillimanite pelitic to semipelitic schist and amphibolite gneiss. Boudins of medium-grainec. $100 \%$ diopside are common within marble and psammitic gneiss layers. Amphibolite gneiss orcurs as 0.5 to $2 \mathrm{~m}$ thick layers, as well as metre-scale boudins. A quartzite - calc-silicate metaconglomerate with a biotite-quartz-feldspar matrix, also occurs within the section south of the Hoder Creek parking lot. Clasts are smaller than those near the Gwillim Lakes (unit 2a-cg) and approxim:tely $50 \%$ of the clasts art light-green weathering calc-silicate gneiss, while the remainder are made up of quartzite and white vein quartz. The relative position of the metaconglomerate within this unit does not aid in the interpretation of stratigraphic younging. There does not appear to be any apparent rhythm to the ordering of siliciclastic 
and carbonate units. nor is there any indication of gradation in primary grain size from top to bottom.

To the south-east. the area near the headwaters of Bannock Burn is characterized by an increase in the amount quartzite and carbonate rocks and a decrease in the amount of amphibolite gneiss. Pitted calc-silicate and clean quartzite are interlayered on the centimetre scale in this area. Locally this area contains $10-30 \mathrm{~cm}$ thick discontinuc us psammitic gneiss layers.

\section{5b. Semipelitic schist}

A greater than $100 \mathrm{~m}$ thick, rusty to dark-brown weathering homogeneous, crumbly weathering semipelitic to pelitic schist forms the ridge west of Urd Peak and is characterized by many crags and pillar-like blocks. The actual thicksess of this unit is unknown, since its upper contact projects ahove a ridge. 1 he semipelitic schist is friable with many fractures. It is an aluminum-poor unit, lacking garnet and sillimanite and is homogeneous throughout.

\section{5c. semipelitic schist - psammitic gneiss}

A light-brown to grey weathering, homogeneous, $100-200 \mathrm{~m}$ thick unit of semipelitic and psammitic gneiss to argillaceous quartzite is observed in the northern area west of Hela Peak, above the Demers marble (4c). True pelitic schist is rare, and garnet is completely absent. Semipelitic schist, locally contains distinctive knots of sillimanite, $1-2 \mathrm{~cm}$ in size. which were not observed anywhere else in the Valhalla complex. The lack of amphibolite gneiss and pelitic schist, as well as the association with a carbonate unit (4c), makes the 
correlation of units $5 c$ and $5 b$ feasible.

\section{Unit 6. Bannock Burn marble}

A 5-10 m thick, homogeneous marble marker was observed in the Bannock Burn valley on the northern flank of Rinda Ridge. It commonly has a black, weathering colour. Contact relationships are sharp, as there appears to be no gradation in mineralogy or grainsize near the top or base of the marble. Calc-silicate interlayers and boudins are rare.

\section{Unit 7. Semipelitic schist}

Unit 7 is a 30 to $40 \mathrm{~m}$ thick. rusty to black weathering sequence consisting of semipelitic schist with minor amounts of pelitic schist and psammitic gneiss interlayered on a scale of tens of centimetres to metres. It is observed immediately below unit 8 on the north flar. $k$ of Rinda Ridge, at the base of steep cliffs. It is generally an aluminum-poor section although garnet and sillimanite occur locally. Where present, garnet occurs as s $3 \mathrm{~mm}$ porphyroblasts and sillimanite occurs as fibrous mats and glassy needles. In more pelitic areas, this schist is characterized by a distinctive yellow, weathered surface.

\section{Unit 8. Rinda Ridge composite unit}

8a. semipelitic schist - marble - calc-silicate gneiss - quartzite - amphibolite gneiss

A heterog cneous sequence may be described from structurally lowest to highest as:

1) 1-2 m thick marble layers with intervening semipelitic to pelitic schist and psammite;2) 
discontinuous pods of ultramafic (marker unit 8a-u). with associated minor coarse-grained metagabbroic anorthosite; 3) amphibolite, interlayered amphibolite and calc-silicate and marble; 4) psammite, marble. calc-silicate gneiss and calcareous schist (Plate 5a) and quartzite (Plate 4c); and 5) semipelitic to pelitic schist. psammite (Plate 3b) and argillaceous quartzite. A 2-5 m thick amphibolite (Plate 2b) layer on Rinda Ridge, grades downward into centimetre-scale interlayered amphibolite and calc-silicate gneiss (Plate $5 c$ ) and is unique to this area. Relatively continuous amphibolite gneiss layers of this thickness are uncharacteristic in the uppermost paragneiss sheet (sheet 1c. Fig. 1.2). between Mount Rinda and Hela Peak.

\section{8a-u - ultramafic schists}

A number of 4-6 $\mathrm{m}$ wide metamorphosed olivine-diopside-tremolite ultramafic pods (Plate 6a) which form a discontinuous, yet concordant stratigraphic marker (Plate 6b) were observed on Rinda Ridge. Hornblende-plagioclase-quartz \pm garnet amphibolite gneiss and coarse-grained gabbroic anorthosite were observed in association with ultramafic rocks along strike. The fresh surface is a pistachio-green colour due to the presence of tremolite, diopside and olivine. Along the same ridge, but to the northwest, an ultramafic boudin was observed above the Rinda marble (unit 9) and consists of nearly $100 \%$ tremolite (station 146. Map 1). At roughly the same stratigraphic level to the west, a dark-green to black weathering rock containing phenocrysts of olivine along with phlogopite and probable chromite occurs, but no visible tremolite or diopside is present (station 136, Map 1). This is possibly a young dyke similar to those occurring near the Hird Lakes, as it does not 
contain the distinctive tremolite composition. In the same vicinity (station 156, Map 1), boulders of ultramafic that are probably outcrop (or near outcrop), contain much phlogopite, minor serpentine and possibly pyroxene and brucite. Whether the ultramafics above the Rinda marble are reliable stratigraphic markers or are the same as those seen on Rinda Ridge is unclear. Those near Mount Rinda appear to be a reliable stratigraphic marker due their relatively continuous nature.

\section{8b. marble - calc-silicate gneiss - semipelitic schist}

A $100 \mathrm{~m}$ thick carbonate-rich package of rocks consists of centimetre to metre-scale interlayered coarse-grained marble, calc-silicate gneiss and semipelitic schist to psammitic gneiss. Marble makes up the smallest component of the unit as 3 to $4,<1 \mathrm{~m}$ thick layers spaced at $30-40 \mathrm{~m}$ intervals. The rest of the section consists of $1 \mathrm{~cm}$ to $<1 \mathrm{~m}$ thick layers of green-grey weathering calc-silicate gneiss and psammite (Plate 6c). There is an increase in the ratio of semipelitic layers to carbonate layers downwards and an apparent gradational contact with unit 7. The occurrence and thinly interlayered nature of the marble, calc-silicate and psammite is distinctive. Though both units $8 \mathrm{a}$ and $8 \mathrm{~b}$ occur at the same structural level and both contain metre wide marble layers, there seems to be a lateral, southward increase in the amount of amphibolite and ultramafic rocks within unit 8a.

\section{Unit 9. Rinda marble}

This 20-40 m thick, homogeneous, coarse-grained granoblastic marble is the most distinctive and easily mappable layer within the Valhalla assemblage (Plate 7a). The 
thickness of $30 \mathrm{~m}$ is relatively constant near Bannock Burn and along Rinda Ridge but the unit thins to $20 \mathrm{~m}$ towards Woden Peak (Fig. 2.3). Layering, where present, is defined by thin graphitic layers. Diopside-rich calc-silicate gneiss boudins and discontinuous interlayers are $30 \mathrm{~cm}$ to $1 \mathrm{~m}$ thick, making up less than $5 \%$ of the unit. Although not always observable. the lower contact with unit $8 \mathrm{~b}$ appears conformable. No apparent compositional gradation is present on either the upper or lower contact. and contacts appear conformable and sharp. Sporadically occurring plagioclase-rich chunks and dismembered layers are $1-10 \mathrm{~cm}$ wide and commonly outline $F_{2}$ and/or $F_{3}$ folds, where no other layering is visible. These are probably dismembered pegmatites that were emplaced prior to $F_{2}$ or $F_{3}$ folding.

\section{$\underline{\text { Unit } 10}$}

\section{Unit 10a. Quart-ite - psammitic gneiss}

A $>10 \mathrm{~m}$ thick, rusty weathering, coarse-grained, homogeneous quartzite is observed above the Rinda marble and outcrops discontinuously along strike for $3 \mathrm{~km}$ along Rinda Ridge at the headwaters of Bannock Burn. It often has a massive appearance and the rusty weathering is likely to have been due to minor amounts of hematite. In other areas, a flaggy, nusty brown weathering, argillaceous quartzite to psammitic gneiss overlies the marble. The grain size and variation from quartzite to psamnitic gneiss is not systematic but is in sharp contact with the Rinda marble.

Unit 10b. Psammitic gneiss - argillaceous quartzite 
A $>50 \mathrm{~m}$ thick package of psammitic gneiss, argillaceous quartzite and garnet sillimanite schist (Plate 2c) with minor calc-silicate and clean quartzite occurs above the Rinda marble. Layers are 5-10 m thick and are interlayered on this scale. Locally, above the Rinda marble, a white weathering quartzite with $1-2 \mathrm{~cm}$ wide calcareous layers containing diopside and calcite was observed. Minor amphibolite gneiss occurs as boudins and discontinuous layers of metre thickness.

A fine- to medium-grained gametiferous hornblende-bearing granodiorite gneiss with a moderately strong $S_{2}$ fabric occurs above the Rinda marble (unit 9). Hornblende makes up $20-25 \%$ and biotite is rare. Due to strong $S_{2}$ gneissic fabric, which is absent from other granitic rocks, this granodiorite gneiss appears to be the oldesi granitoid in the Valhalla complex.

Unit 11. Undivided rocks: semipelitic to pelitic schist - migmatitic gneiss-amphibolite gneiss

A heterogeneous group of rocks, of uncertain thickness, consist of pelitic schist, semipelitic schist and gneiss, migmatite and amphibolite gneiss. It occurs in the southern area of paragneisses on the west side of domain $1 \mathrm{~b}$ in the Passmore Dome (Fig. 1.2). The gneisses weather a light-grey to rusty colour, schists commonly weather yellow and amphibolites are black to dark-grey weathering (Plate 7b). Locally, pelitic schists are quite sillimanite-rich and may contain up to $30 \%$ sillimanite. Coarse- to medium-grained hornblende-bearing, felsic granitic gneiss occurs locally and is more common than in the other structurally underlying units. Amphibolite gneiss occurs as metre-scale discontinuous 
layers and boudins that constitute $25-75 \%$ of a typical outcrop. This unit has a significantly higher proportion of amphibolite gneiss than any others in the Valhalla assemblage. Pelites almost always contain sillimanite and garnet. $30-50 \%$ biotite and are migmatitic. In many cases, a distinct segregation between biotite restite and plagioclase-quartz leucosome is visible. Elsewhere in the Valhalla assemblage, melt pods and discontinuous leucosome layers are common, but gamet-biotite restite of this nature is not. Restite occurs in layers up to $50 \mathrm{~cm}$ thick and may comprise as much as $90 \%$ biotite and $20 \% 2-20 \mathrm{~mm}$ diameter garnet. Garnets are commonly altered to retrograde biotite and/or chlorite in both restite and paleosome. Leucosome is coarse-grained and consists of plagioclase, quartz \pm potassium feldspar \pm garnet. Rock types other than semipelitic to pelitic schist and amphibolite are rare in this unit but are observed on the east side of the complex along Highway 6 (Fig.1.2). Clean, buff weathering quartzite, calc-silicate gneiss and marble occur in minor amounts as less than $3 \mathrm{~m}$ thick layers. Boudins of dark-green to black weathering ultramafic up to $1 \mathrm{~m}$ in diameter occur north of Slocan. The litholugic associations are not apparently systematic and no distinguishing characteristics or stratigraphic relationships are apparent.

\section{Slocan Ridge}

On Slocan Ridge (Fig. 1.2), there is an abundance of leucosome within pelitic schist and gamet-homblende-plagioclase amphibolite, making up to $50 \%$ of the unit. Near the base of the ridge along Highway 6, sillimanite-rich schists and garnet-hornblende amphibolite gneisses predominate. At $\sim 975 \mathrm{~m}$ elevation, there is a decrease in the amount of amphibolite and argillaceous quartzites and garnet-bearing semipelitic to pelitic schists are 
common.

\section{PerruRidge}

Perry Ridge (Fig. 1.2) consists of psammite, semipelitic to pelitic schist, quartzite, marble, calc-silicate gneiss and amphibolite gneiss much the same as units 7-9 in the Bannock Bum valley and along Rinda Ridge. On the north side of the ridge, units are difficult to follow due to scarce and discontinuous outcrop along logging roads. On the south side of the ridge, two marble layers are present, and the lower one has a thickness of at least $20 \mathrm{~m}$. This marble layer is probably correlative to a marble layer napped on the north side of the ridge. The only other marble of this thickness is the Rinda marble, however they are not correlative because there is no similarity with surrounding rock types and they occur at different structural levels. The presence of two relatively thick marbles in close proximity to each other might suggest that these are two limbs of an overturned isoclinal fold but there was no evidence for this, nor was isoclinal folding of this scale observed anywhere else in the Valhalla assemblage. The relationship between the rocks on Perry Ridge and the rest of the sequence in the Valhalla complex remains uncertain.

\section{Gwillim Creek windows}

The paragneisses in the Gwillim Creek windows consist primarily of argillaceous quartzite, quartzo-feldspathic gneiss. semipelitic and pelitic schist and minor amphibolite gneiss and are similar to rocks of unit 11 (Plate $7 \mathrm{~b} \mathrm{c}$ ). Rare $2-3 \mathrm{~cm}$ garnet and $2 \times 5 \mathrm{~mm}$ sillimanite porphyroblasts occur in pelitic schist. but generally the rocks have meager 
amounts of aluminosilicate minerals. Carbonate units were not observed, although Reesor (1965 p. 22) noted them. The only other distinguishing characteristic is the presence of more argillaceous quartzite relative to semipelitic and pelitic schist. The lack of any good marker units makes a correlation between the Gwillim Creek windows (paragneiss sheet la and $1 \mathrm{~b}$. Figs. 1.2, 1.3) and the structurally higher main sheet $1 \mathrm{c}$ of paragneisses difficult. Rocks in the Gwillim Creek windows mav not correlate to any other units of the Valhalla assemblage due to a large displacement on the Gwillim Creek shear zones.

\subsection{Regional correlations of the Valhalla assemblage}

On the basis of mappable sequences of partially transposed stratigraphy containing some distinctive marker horizons, a regional correlation of the Valhalla assemblage is proposed. This is done bearing in mind that: 1) the rocks are at upper-amphibolite facies and often migmatitic; 2) the original thickness of units is uncertain due to possible transposition and other structural complications, such as cryptic thrusts; 3) there are no primary sedimentary structures or fossils preserved; and 4) that Paleocene - Eocene leucogranites and pegmatites may constitute up to $75 \%$ of any one outcrop. Despite these significant complications, the persistence of markers and distinctive packages along strike indicate that a relict stratigraphy has been preserved. Distinctive units include the Rinda marble (unit 9), Hird Lakes calc-silicate gneiss - metaconglomerate (unit 2a-cs, 2a-cg) and the ultramafic boudins (unit 8a-u) of the Rinda Ridge composite unit.

The age of the Valhalla assemblage is older than the $100 \pm 5 \mathrm{Ma}$ igneous crystallization age of Mulvey granodiorite gneiss (Carr et al. 1987). Ross and Parrish (1991) 
suggested that the quartzite-cobble metaconglomerate (2a-cg) is Late Proterozoic to Middle Paleozoic. Detrital zircons from a quartzite layer from the vicinity of the metaconglomerate yielded ages between $\sim 1400-2759 \mathrm{Ma}$ (Ross and Parrish 1991). These dates only reveal the age of the source area and place a maximum age of $-1400 \mathrm{Ma}$ on the sediment deposition.

The Valhalla assemblage is a heterogeneous sequence of rocks that contains conglomerates, calc-silicate gneisses and marbles interlayered with 50-100 m thick units of aluminum poor semipelitic to pelitic schists at its structurally lowest level (units 1-3). Proceeding up in structural direction, the sequence becomes more carbonate rich with metre thick marbles and calc-silicates gneisses, interlayered with quartzites and slightly more aluminum-rich pelitic rocks (units 4-8). There is also an increase in amphibolite gneisses and ultramafic rocks which are absent from the structurally lower sections. This is overlain by a thick marble and quartzite layers, respectiveiy (units 9-10). At the structurally highest level, a thick unit of pelitic schist and amphibolite gneiss predominates (unit 11).

A primary environment for the Valhalla assemblage may be described as a shallowwater, nearshore environment that was changing often and influenced by terrestrial sediments and minor volcanism as evidenced by the interlayering of quartzites, carbonates, pelitic rocks and amphibolite gneisses. The Valhalla assemblage is unlike the thick, homogeneous, deepwater. marginal-basin type sediments of the Slocan Group, nor is it indicative of an islandarc setting like the Kaslo Group. The lack of thick quartzites and conglomerates also makes it unlike terrestrial sedimentary rı kss of some parts of the Purcell Supergroup.

The correlation of the Valhalla assemblage proposed in this study is based on a comparison of descriptions of Neoproterozoic to Paleozoic rocks exposed in the Goat Ranges 
(Klepacki 1985), Thor-Odin - Pinnacles area (Carr 1991), the Riondel area (Höy 1977. 1980), the Kootenay Arc (Fyles and Eastwood 1962: Fyles 1964; Read 1973: Reesor 1973), the Greenwood - Grandforks area (Little 1983; Fyles 1990), the Adams Lake - Clearwater Vavenby area (Schiarizza and Preto 1987) and the area of the Scrip Nappe (Raeside and Simony 1983; Scammell 1991, 1993). Based on literature describing the Paleozoic strata in the Omineca belt, the most reasonable interpretation is that the Valhalla assemblage is correlative with part of the Neoproterozoic and Eocambrian to Carboniferous stratigraphy of North America and the Kootenay terrane (Table 2.3). In this interpretation. the Valhalla assemblage is inverted, for which the implications are presented in chapter 4 .

\subsubsection{Goat Ranges}

The Goat Ranges located to the northeast of the Valhalla complex (Fig. 1.1) consist of Paleozoic to Triassic low-grade metasedimentary and metavolcanic rocks of the Hamill to Slocan Group and are described by Klepacki (1983), Klepacki and others (1985), Klepacki and Wheeler (1985) and Klepacki (1985).

Based on thickness, potential correlatives of the Rinda marble (unit 9) are either the Barishot Formation or the Basal Limestone member of the Davis assemblage of the Milford Group. The former is favoured because the sequence of interlayered marbles, calc-silicate gneisses and pelitic schists (unit 8), marble (unit 9), and quartzite (unit 10) is best correlated with the Index Formation, Badshot Formation and Hamill Group, respectively. This implies an inverted sequence. If the Valhalla assemblage is not inverted and the Rinda marble is correlative to the Badshot Formation it would require units 8 and below be in part correlative 
to the Hamill Group. The small amount of quartzite in unit 8 is not consistent with this.

The Rinda marble is not correlative with the Basal Limestone member of the Davis assemblage because it would require that either 1) the sequence is upright, in which case units 10-11 would in part be correlative to the Milford Group, or 2) the sequence is inverted, in which case units 8-1 would be in part correlative to the Milford Group. Both cases are unlikely correlations because units 10-11 lack the carbonates and conglomerates of the Milford Group, and unit 8 contains ultramafic rocks which have not been documented in the Milford Group.

Conglomerates are often distinctive and less abundant than most other rock types in the Omineca belt. Possible correlatives of the Hird Lakes metaconglomerate (unit 2a-cg) are contained within the Keen Creek assemblage of the Milford Group, the Cooper Conglomerate of the McHardy assemblage, within the Milford Group, and the Marten Conglomerate (Klepacki 1985). The most viable correlative of the Hird Lakes metaconglomerate ccuirs in the area of Wilson Creek in the Goat Ranges and is described by Klepacki (1985). This conglomerate is contained within the Siliceous Wacke Member of the Keen Creek assemblage (Klepacki 1985) within the Milford Group, and has an age of Upper Mississippian - Lower Pennsylvanian. Klepacki (1985) describes plagioclaseactinolite granulite and quartz-cobble conglomerate channel deposits that lie at the base of the overlying Banded Limestone Member, which is a $10-30 \mathrm{~m}$ buff weathering hard calcsilicate (quartz-zoisite-tremolite) and silicified limestone. Conglomerate clasts are comprised of granular quartz cobbles and foliated quartzite. Klepacki $(1985$, p. 87) described the contact between a "rusty mica schist and marble ur" ts that is gradational with 
1-20 m of intervening intermediate composition: quariz-felus/ $v$-dolomite-biotite-actinolite sc'ist or grey quartz-plagioclase-biotite-granulite (calc-sch.sı)". Both the clast and matrix compnsitions of the conglomerate in the Wilson Creek area appear to be compatible with the Hird Lakes metaconglomerate: however, there is no marble in contact with the Hird Lakes metaconglomerate equivalent to the Banded Limestone Member. The Hird Lakes calcsilicate (2a-cs) may be equivalent to the associated calc-schist described by Klepacki (1985).

The Cooper Conglomerate consists of metadiorite, mafic vuicanic and quartz. monzonite clasts within a matrix of fine-grained quartz-biotite-plagioclase-sphene, or a rusty weathering quartz-cobble conglomerate with clasts of sugary quarzite (Klepacki 1985). Since the Hird Lakes metaconglomerate contains only quartzite clasts, this is not a likely correlation. Parrish and others (1985) suggest that the Cooper conglomerate and the Hird Lakes metaconglomerate are correlative due to the absence of appreciable quartzites as sociated with the Hird Lakes metaconglomerate. However, the matrix of Cooper Conglomerate is not the same as the calc-silicate matrix of the Hird Lakes metaconglomerate and a correlation is not tenable.

The Permian Marten Conglomerate lies unconfonnably on the Kaslo Group a. $d$ is overlain unconformably by the Slocan Group (Klepacki 1985). It is composed of greens'one, diorite and serpentinite clasts, in a rusty weathering, green, calcareous phyllite matrix. Beds of limestone are also present. The limestone layers and the matrix composition of the Marten sanglomerate could represent the protolith of the matrix of the Hird Lakes metaconglomerate. However, none of the clast types mentioned by Klepacki (1985) are observed in the Hird Lakes metaconglomerate. 


\subsubsection{Thor-Odin - Pinnacles area}

The Thor-Odin - Pinnacles area (Fig. 1.1) consists of a heterogeneous package of metasedimentary and mafic rocks (Carr 1991) similar to those of the Valhalla assenblage. Carr (1991) has divided the area into two stratigraphic groups: the Gold Range assemblage (Hamili Group to Slcican Group correlatives) and the Fawn Lake assemblage (Horsethief Creek (rroup correlatives). Based on stratigraphic ordering and lithologic similarity, unit 10, the Rinda marble (unit 9), the Rinda Ridge cor:posite unit (unit 8a, 8a-u) and the Hird Lakes metaconglomerate are correlated with the Mount Symonds quartzite-bearing unit, the Empress marble, the Lower Mount Fosthall composite unit and the Vidler calc-silicate gneiss of the Gold Range assemblage, respectively. The distinctive sequence of psammites, calcsilicates and marbles (Lower Mount Fosthall Composite unit). marble (Empress marble), and quartzites (Mount Symonds quartzite-bearing unit) has been correlated o the Index Formation of the Lardeau Group, Badshot Formation and Hamill Group, respectively, by Carr (1991). Both unit 8 of the Valhalla assemblage and the Lower Mount Fosthall romposite unit contain interlayered psammites, calc-silicate gneisses, marbles and ultramafic rocks, which is a suitable correlation.

Ultramafic pods in the Thor-Odin - Pinnacles area, occur at two different stratigraphir, levels. Unit 7a (the lower Mount Fosthall composite unit) contains phlogopiteorthopyroxene-clinopyroxene-tremolite-olivine-calcite bcaring ultramafic gneiss as layers, hlocks and pods. Carr (1991) has suggested that these are high-grade equivalents of talc schists which occur in the Lardeau Group (Sears 1979; Zwanzig 1973) and has correlated the 
Mount Fosthall Composite Unit with the Index Formation of the Lardeau Group. based in part on this correlation. This is a possible correlative to unit 8a-u of the Valhalla as. 'mblage. based on similar composition and nature of the pods, as well as the association with marbles. calc-silicates gneisses, amphibolites gneisses, pelitic schists and quartzites.

The Vidler calc-silicate gneiss of the Gold Range assemblage consists of calcareous quartzite, marble and minor psammitic cobble metaconglomerate, which Carr (1991) has conclated with the siliceous argillite member of McHardy assemblage of the Milford Group. as described by Klepacki (1985).

The Fawn Lake assemblage consists of migmatitic semipelitic and psammitic paragneiss and amphibolite gneiss. Unit 11 of the Valhalla assemblage is comparable to this assemblage as well as the semipelite-amphibolite (SPA) of the Horsethief Creek Group, due to its lack of carbonate and mature siliciclastic rocks.

\subsubsection{Riondel area}

The Riondel area is located in the Kootenay Arc to the east of the Valhalla complex (Fig. 1.1). Höy $(1977,1980)$ has correlated units H, B and L of the Riondel Nappe to the Hamill Group, Badshot Formation and Lardeau Group. respectively. Höy's (i977) description of unit L2 as "diopside-rich calc-silicate layers, thin rusty-weathering calcite marble layers, and less commonly hornblende-biotite gneiss and micacecus quartzite" is similar to unit $8 \mathrm{~b}$ of the Valhalla assemblage. Höy (1977) also describes a marble layer (L3iii) that is possibly correlative to the $F \cdot$, nock Burn marble (unit 6). A corrclation of unit 8 to unit L2 and the Bannock Burn marble to unit L3iii is consistent with an inverted 
Valhalla assemblage.

\subsubsection{Neme Lakes belt}

The Nemo Lakes belt, located immediately north of the Valhalla complex (Fig. 1.1), consists of sillimanite-grade metasedimentary rocks, including semipelitic and caicareous schists, marbles, amphibolites and ultramafic rocks. This belt of rocks is correlative to parts of the Lardeau and Slncan group, on the basis of lithologic similarity and $\mathrm{Rb}-\mathrm{Sr}$ whole rock ages (Parrish 1981). Ultramafic rocks in the Nemo Lakes belt are described by Parrish (1981) as pod-like, lenticular bodies up to $800 \mathrm{~m}$ in length, composed of anthophyllite, forsterite, enstatite, talc, chlorite, clino ar:phibole and serpentine. These ultramafics are enclosed by homblende-plagioclase amphibolite and are interpreted as alpine-peridotites (Parrish 1981). They are bounded on both contacts by predominantly pelitic schists with intervening layers of calc-schist and marble. A correlation between the Nemo Lakes belt and the Valhalla assemblage is not reasonable because surrounding lithologies are dissimilar. The former has characteristically large volumes of pelitic schist, while the Valhalla assemblage has a heterogeneous nature.

\subsubsection{Eagle Bav assemblage}

The Eagle Bay assemblage in the Adams Lake-Clearwater-Vavenby area contains a succession of rocks ranging from possibly Windermere Supergroup to Milford Group (Schiarizea and Preto 1987). The area lies on the western edge of the Omineca belt (Fig. 1.1) and comprises low grade metavolcanic and metasedimentary rocks including the distinctive 
up to $1000 \mathrm{~m}$ thick Tshinakin limestone (EBGt) which is correlative with the Badshot Formation.

There are four different conglomerate bearing units within the Eagle Bay assemblage. two of which resemble the conglomerates in the Valhalla assemblage. Unit EBGcg is a polymictic conglomerate which occurs at the base of unit EBG, and consists of a fine-grained light- to medium-green calcareous. chlorite-sericite-quartz schist matrix with quartzite, limestone, dolostone, chlorite schist and vein quartz clasts (Schiarizza and Preto 1987). Unit EBSc is a quartz-pebble to boulder conglomerate with predominantly quartzite clasts and often has a calcareous matrix. Unit EBS has been correlated to the Lardeau Group. Schiarizza and Preto (1987) mention that it often underlies volcanics of unit EBM and this is not the case in the Valhalla complex.

None of the units in the Eagle Bay assemblage of the Adams Lake-ClearwaterVavenby area have a st iking similarity to marker units in the Valhalla assemblage except the EBSc conglomerate, which is insufficient to make a viable correlation. In the Eagle Bay assemblage, mafic volcanic rocks under - and overlie the Tshinakin limestone (Schiarizza and Preto 1987). This relationship is absent in the Valhalla assemblage, and therefore a confident correlation between the Eagle Bay assemblage in the Adam Lake-Clearwater-Vavenby area and the Valhalla assemblage is not possible.

\subsubsection{Scrip Nappe}

The Scrip Nappe. located north of the Monashee complex (Fig. 1.1) consists of inverted Horsethief Creek Group strata which have been metamorphosed to amphibolite- 
facies (Raeside and Simony 1983; Scammell 1990, 1993). The Horsethief Creek Group has been divided into 5 members: the lower clastic, lower carbonate, semipelite-amphibolite, middle marble and upper clastic member (Wheeler 1963; Reesor 1973; Brown et al. 1978; Poulton and Simony 1980; Raeside and Simony 1983; Pell and Simony 1987). Overall, proportions of lithologies are similar between the Valhalla assemblage and rocks in the Scrip and Seymour ranges. There are numerous marble layers and the amount of amphibolites and metavolcanics is relatively low (Scammell 1990, 1993). Scammell (1990) describes quartzcobble to boulder conglomerate in the southern Scrip and northern Seymour ranges; however, quartzite-pebble metaconglomerates with calc-silicate matrix like the Hird Lakes metaconglomerate are absent in the Scrip Range (Scammell pers. comm. 1995).

Ultramafic rocks of similar composition and nature also occur in the Scrip Nappe. They are present throughout the section, whereas in the Valhalla assernblage, ultramafics appear to be concentrated within two stratigraphic units (8 and 10). Although both the Valhalla assemblage and the Scrip Nappe have similar rock types, the ordering of units is not compatible, and theiefore there is no compelling reason to correlate the Valhalla assemblage with rocks of the Scrip Nappe.

\subsubsection{Greenwood-Grand Forks area}

The Greenwood-Grand Forks area located to the southwest of the Valhalla complex (Fig. 1.1) is underlain by greenschist-facies Late Paleozoic and Mesozoic volcanic and sedimentary rocks that are m-ssibly correlative to the Carboniferous Milford Group (Preto 1970; Little 1983; Fyles 1990). The Knob Hill and Attwood groups contain limestone with 
Permian aged fauna and are overlain by the Triassic Brooklyn conglomerate (Fyles 1990). Paleozoic rocks consist of chert, greenstone, diorite and serpentinite of the Knob Hill Group and grey argillite, limestone and minor volcanic rocks of the Attwood Group (Fyles 1990). The only rocks that are similar to those in the Valhalla complex are serpentinites within the Knob Hill Group, which are interpreted to have Carboniferous or Permian ages and to be tectonically emplaced along structures at various times during the Mesozoic and Tertiary (Fyles 1990). The Greenwood-Grand Forks area does not contain the same volume of marble or limestone or any marker units that have comparable thicknesses to the Rinda marble. The amount of chert and volcanics and the angularity and type of clasts within the Knob Hill Group conglomerates is dissimilar to the Hird Lakes metacongomerate. The distinct differences between the rocktypes and primary environments of the Greenwood Grand Forks area and the Valhalla assemblage does not allow a correlation.

\subsubsection{Mount Roberts Formation in the Trail area}

In the Trail area to the south of the Valhalla complex, sedimentary rocks thought to be part of the Mount Roberts formation contains siltstone, slate, greywacke and comglomerate (Simony 1979). Pebble conglomerate contains clasts of quartzite, chert, siltstone and volcanic rock. These rocks are thought to be of Pennsylvanian age (Little 1978). The wide range in clast types of conglomerate and the lack of carbonate layers does not enable a correlation between the Mount Roberts Formation near Trail and the Valhalla assemblage to be made. 


\subsection{Summary}

Detailed mapning of stratigraphy in the Valhalla complex has recognized a heterogeneous package of metasedimentary rocks named the Valhaila assemblage (Figs. 2.3, 2.5). The Valhalla assemblage contains conglomerates, calc-silicate gneisses and marbles interlayered with 50-100 m thick units of aluminum poor semipelitic to pelitic schists at its structurally lowest level (units 1-3). Proceeding up in structural direction, the sequence becomes more carbonate rich with metre thick marbles and calc-silicates gneisses, interlayered with quartzites and slightly more aluminum-rich pelitic rocks (units 4-8). There is also an increase in amphibolite gneisses and ultramafic rocks which are absent from the structurally lower sections. This is overlain by a thick marble and quartzite layers, respectively (units 9-10). At the structurally highest level, a thick unit of pelitic schist and amphibolite gneiss predominates (unit 11).

Based on lithologic similarity and relative ordering of the distinct Rinda marble (unit 9). Hird Lakes metaconglomerate (unit $2 a$ ) and ultramafic pods (8a-u), a correlation of the Valhalla assemblage to units ranging from Horsethief Creek Group to the Keen Creek assemblage of the Milford Group can be made (Table 2.3). Key elements of this correlation are the unique quartzite clast - calcareous matrix of the Hird Lakes metaconglomerate, concentration of the ultramafic rocks within a particular structural horizon, the lack of significant quartzites below the Rinda marble and the relative abundance of carbonates (high) and amphibolites (low) below the Rinda marble. These correlations indicate that the Valhalla assemblage is inverted of which the tectonic implications are discussed in chapter 4. 


\section{Age (Ma) Event}

52

Aplite dykes cross-cut Gwillim Creek shear zones

54-45 Movement along the Slocan Lake fault

58-47 K/Ar dates from homblende, muscovite and biotite record temperature decrease from $500-280^{\circ} \mathrm{C}$

59-54 Movement on the Valkyr shear zone

59-55 Ladybird granite suite intrudes during extensional fal

60 Growth of metamorphic zircon in amphibolite

Titanite cools below $600^{\circ} \mathrm{C}$ closure temperature

63 Intrusion of Airy quartz monzonite, minimum age for $S_{z}$ development

$<65$ Cooling and uplift (U-Pb thermochronology) caused by uplift and erosion

-65 Melting and growth of igneous zircons (formed after thermal peak by decompression melting)

70-65 Pegmatites containing igneous zircon intruded metasedimentary rocks

80-75 Thermal peak; closure of zircon and monazite: GCSZ and $S_{2}$ proposed to be active at this time

100-<92 Oldest age possible for Gwillim Creek shear zones (Mulvey gneiss is thoroughly sheared)

Crystallization of Mul vey granodiorite and Kinnaird gneiss, maximum age for $\mathrm{S}_{2}$

Age of first phase folding implied from stratigraphic correlations (post Mississippian) and age of Mulvey granodiorite

$>110-1400$

Absolute ages constraints for deposition of sedimentary protoliths followed by or synchronous with minor igneous events (mafic dykes, volcanic?, granitic intrusion)

-1400 ,

1700,2700 Crystallization of igneous rocks later eroded and provided zircon and monazites ihat were deposited in sedimentary rocks of Valhalla assemblage.

Table 21 . Compilation of geo- and thermochronology and structural and stratigraphic constraints for tectonic events in the Valhalla complex from Carr and others (1987), Parrish and others (1988), Parrish (1990), Heaman and Parrish (1991), Ross and Parrish (1991), Parrish (1995) and this study. 


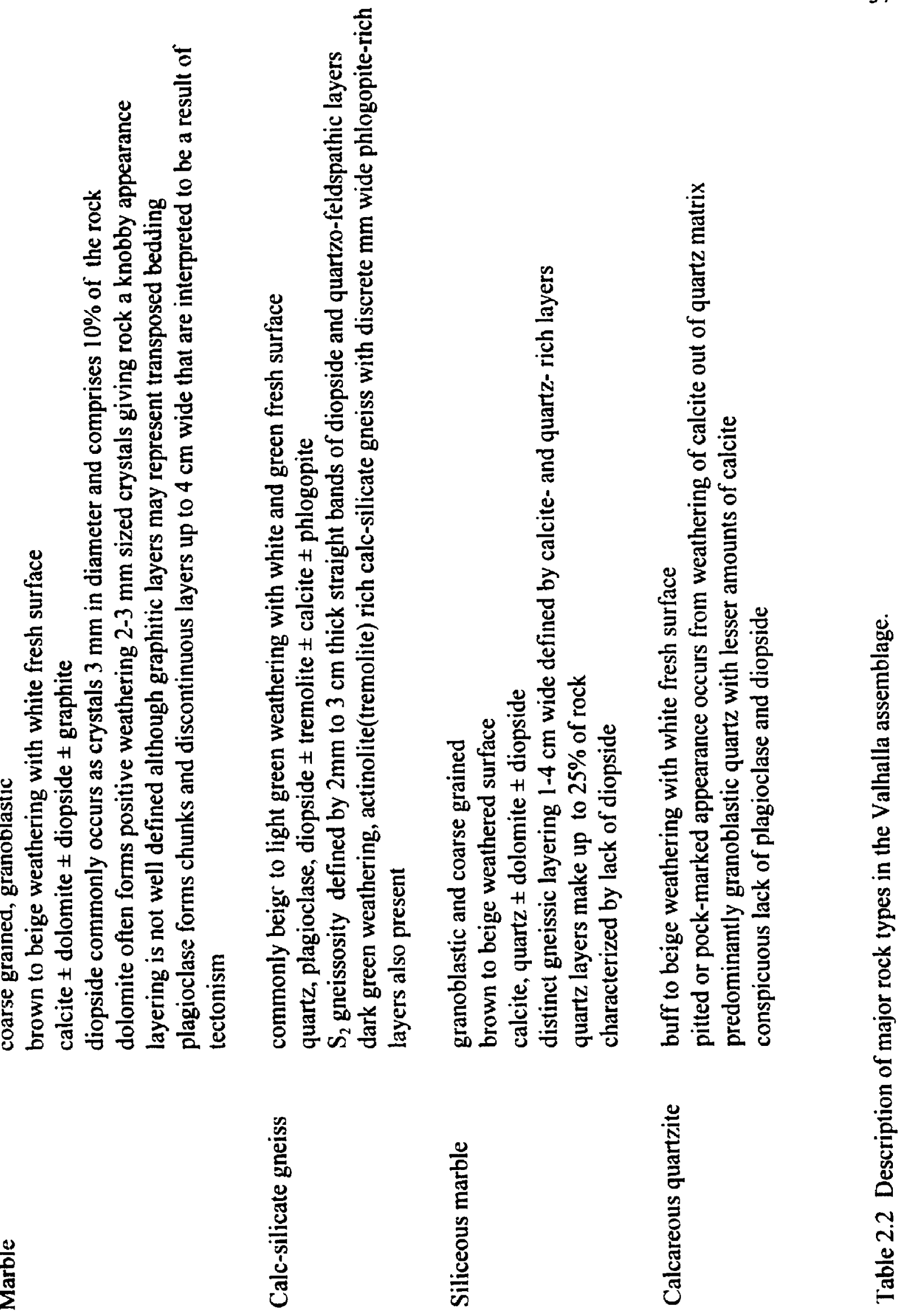




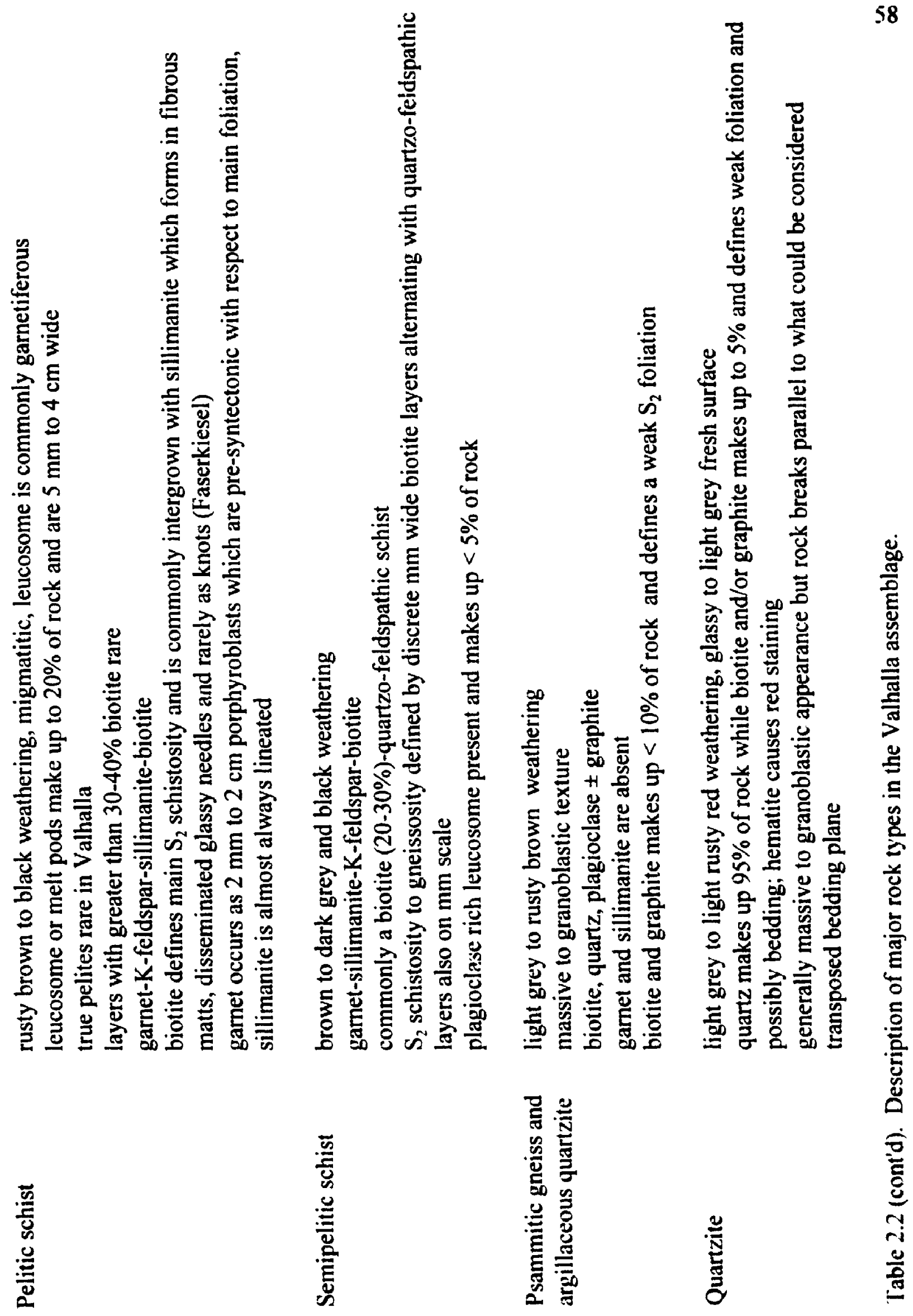



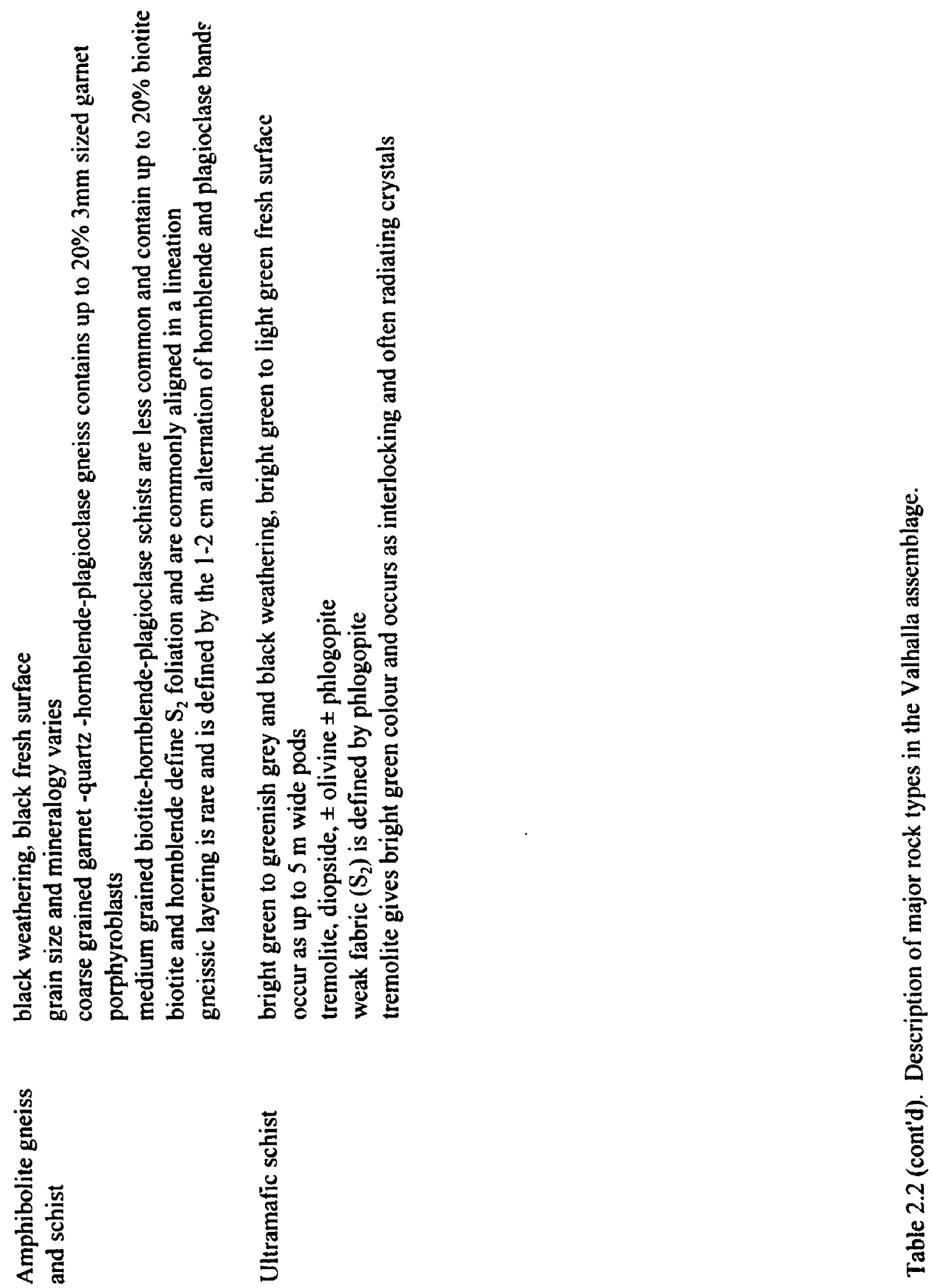

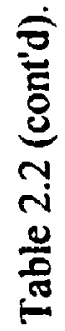




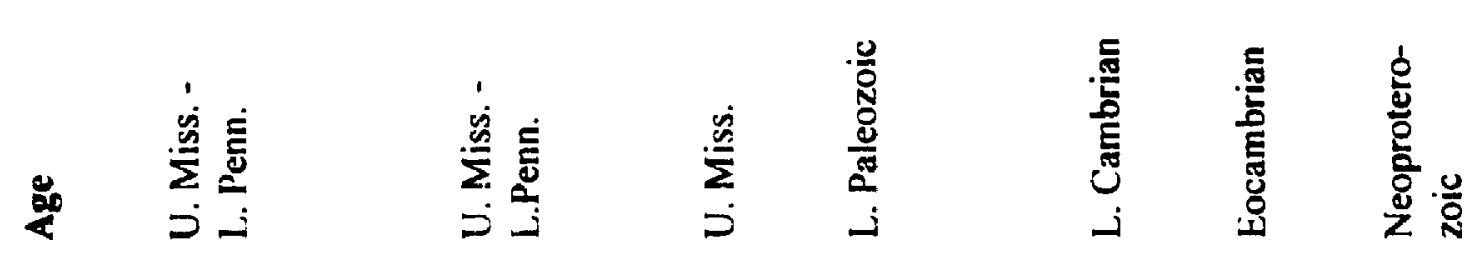

60
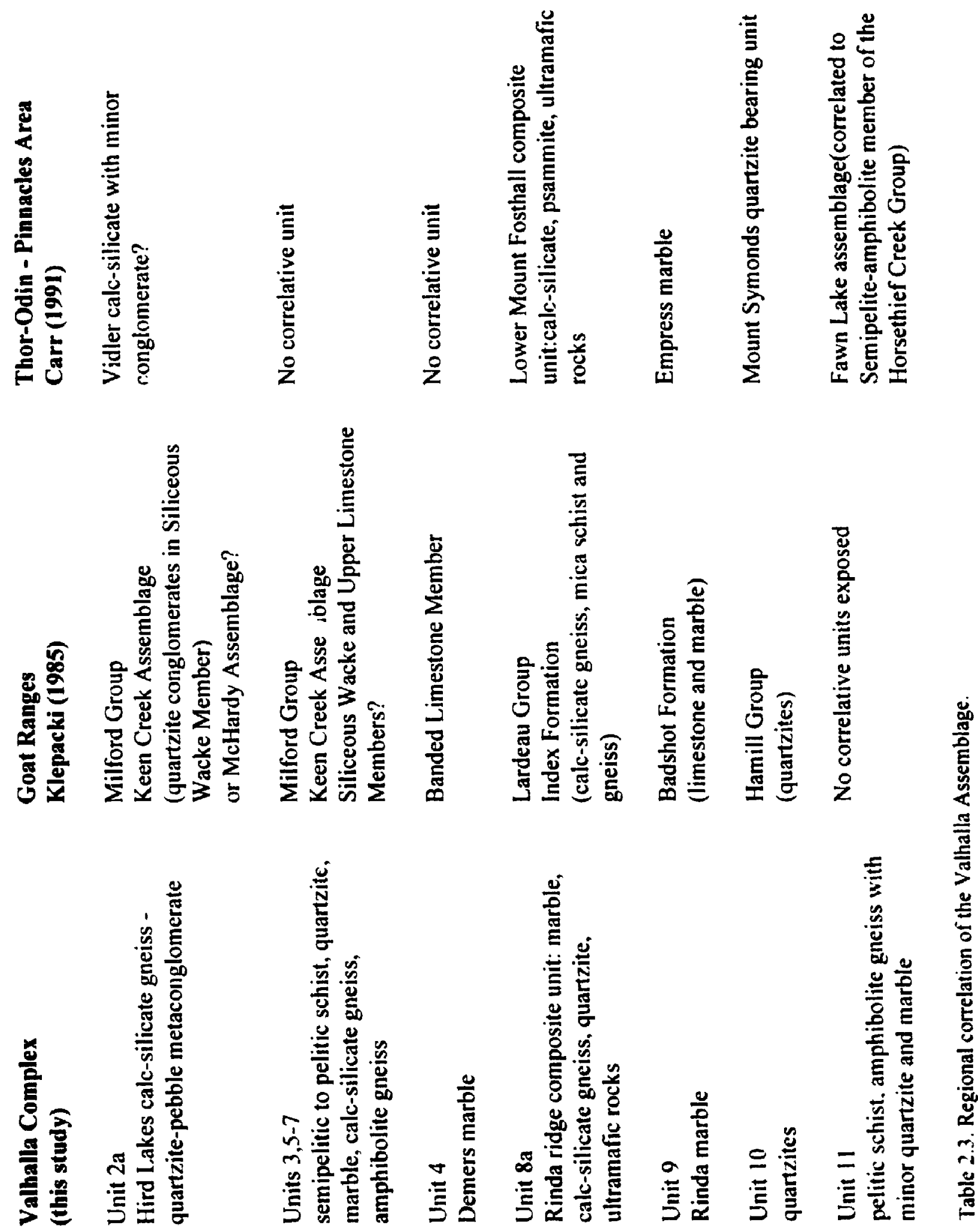


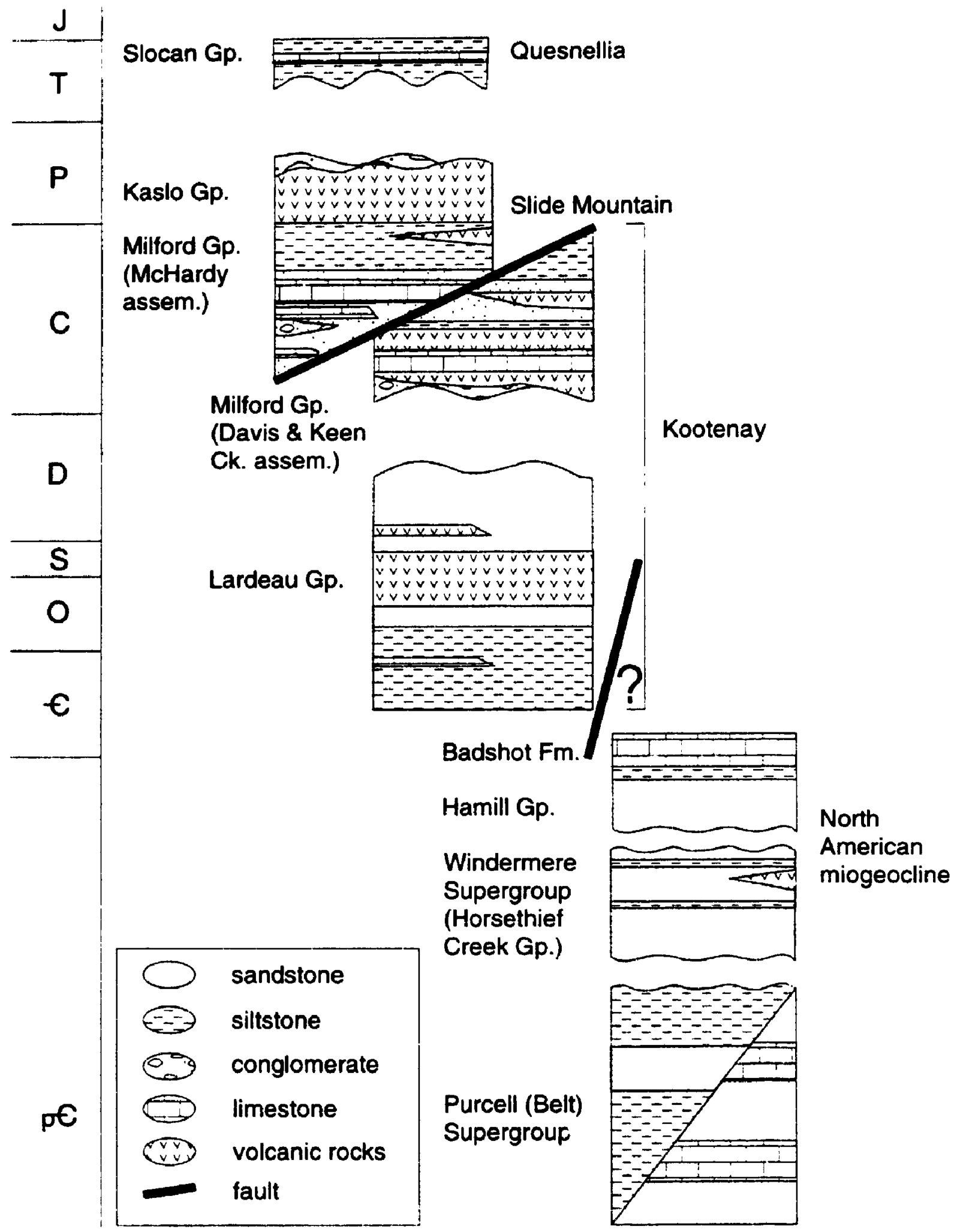

Figure 2.1. Schematic diagram of relationships between stratigraphy and terranes in the northern Kootenay arc. Modified from Klepacki (1985) and Roback et al. (1994). 


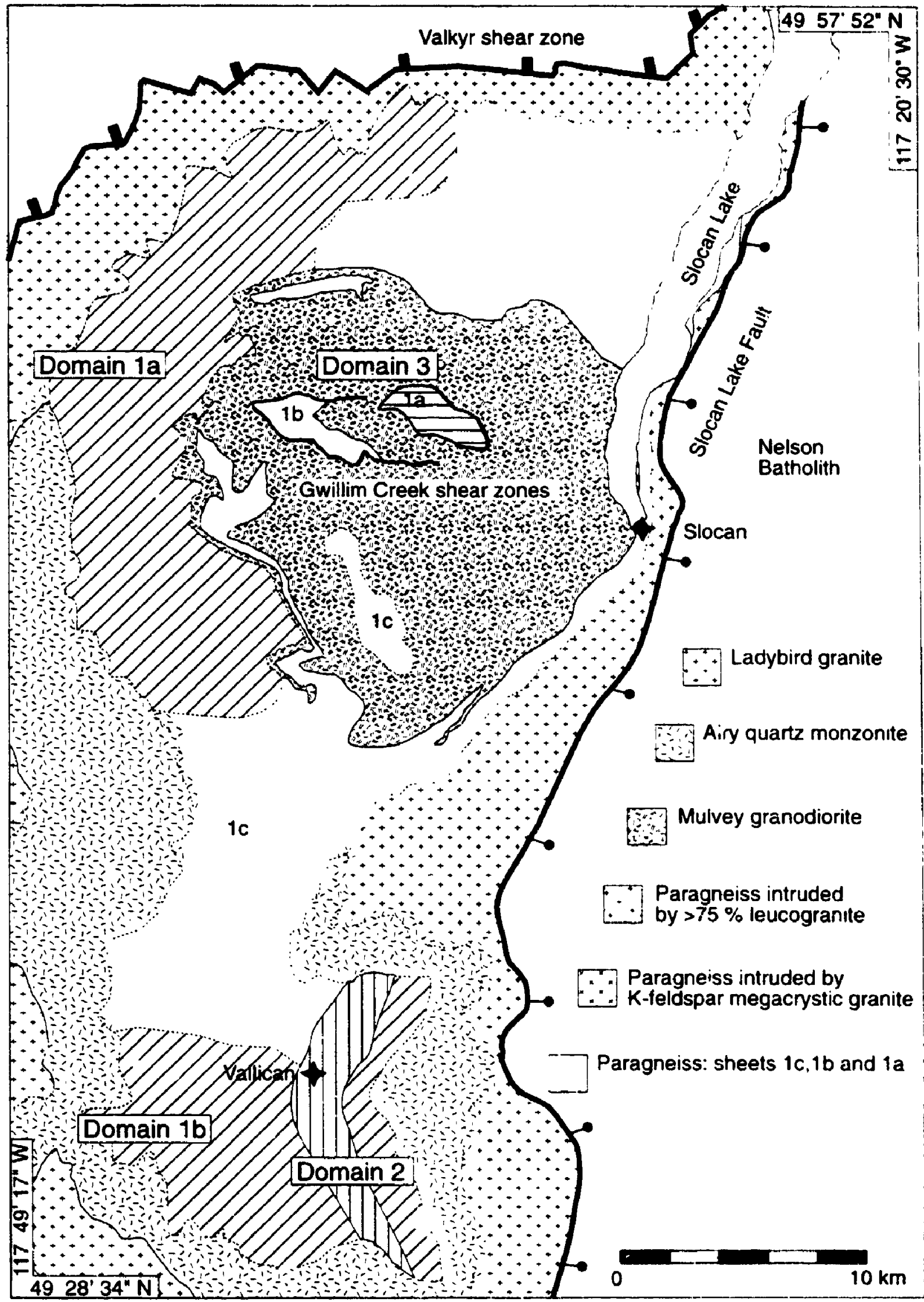

Figure 2.2. Map showing limit of domains in paragneisses of the Valhalla complex. 
Early Eocene Ladybird granite

Paleocene Airy quartz monzonite

$\mathrm{Lic}^{4} \mathrm{e}$ Cretaceous Mulvey granodiorite

mioidle Jurassic granitic rocks

Valhalla Assemblage intruded by K-feldspar megacrystic granite

Valhalla Assemblage cornprising $>75 \%$ leucogranite

Valhalla Assemblage

10a Quartzite, psammite

$10 \mathrm{~b}$ Semipelitic, schist, psammite, argillaceous quartzite

9 Rinda marble

8a Ririda Ridge composite unit: marble, calc-silicate gneiss, psammite, semipelitic to pelitic schist, quartzite, amphibolite gneiss, ultramafic rocks $(8 \mathrm{a}-\mathrm{u})$

8b Marble, calc-silicate gneiss, semipelitic schist, psammite

7 Semipelitic to pelitic schist, psammite

6 Bannock Burn marble

5a Hocer Creek composite unit: psammite, pelitic schist, argillaceous quartzite, marble, calc-silicate gneiss, amphibolite gneiss

$5 \mathrm{~b}$ Semipelitic to pelitic schist, psammite

5c Semipelitic schist, psammite

4a Marble, calc-silicate gneiss

4b Demers marble

4c Marble, silicecus marble

3a Quartzite

3b Semipelitic to pelitic schist, migmatite, argillaceous quartzite

3c Quartzo-feidspathic gneiss, peltic schist, calcareous quartzite (3c-cq)

2a Hird Lakes calc-silicate (2a-cs) - quartzite cobble metaconglomerate (2a-cg)

2b Marble

1 Quartzo-feld'spathic gneiss

Figure 2.3. Geulogy of the Valhalla assemblage. 
Figure 2.3 


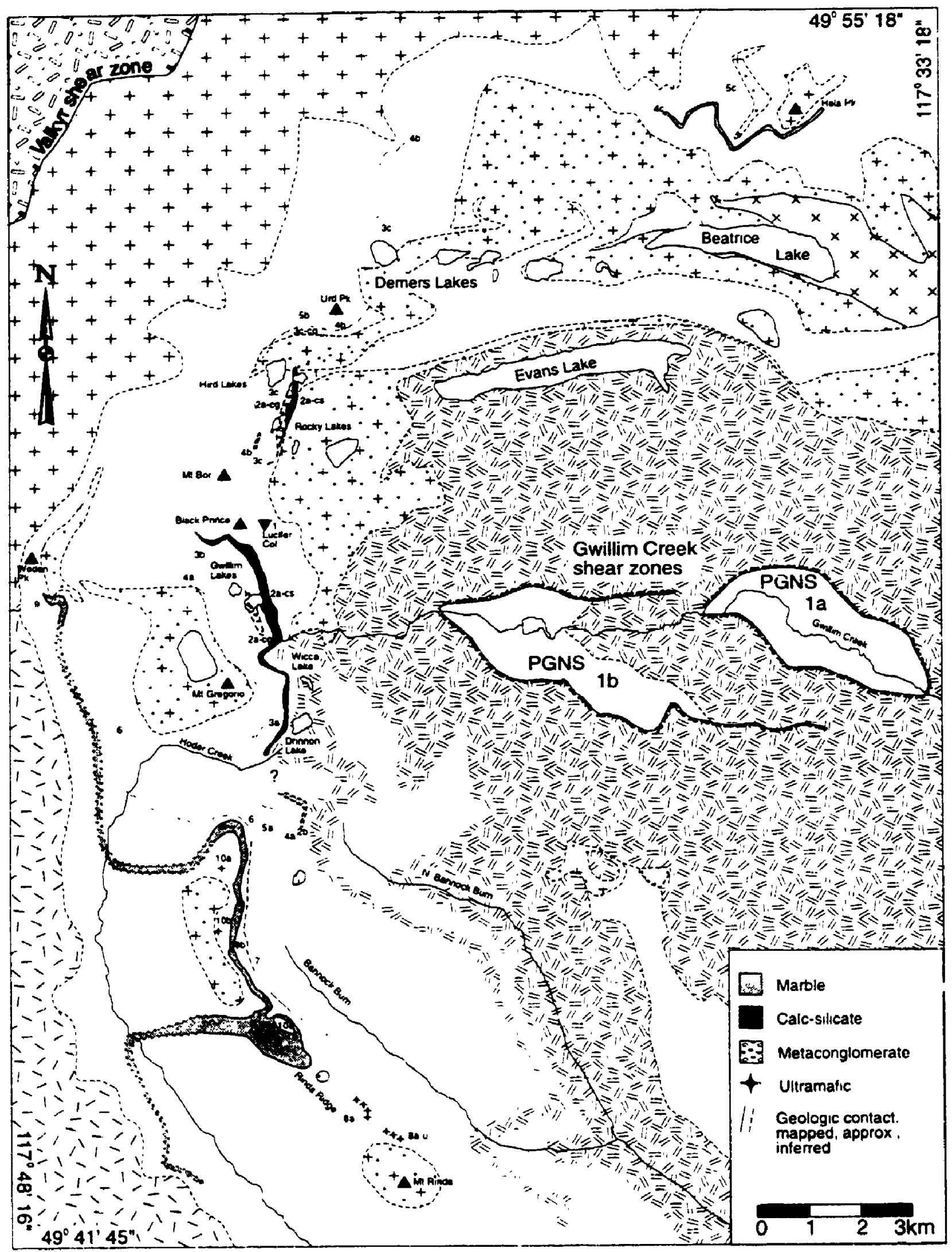




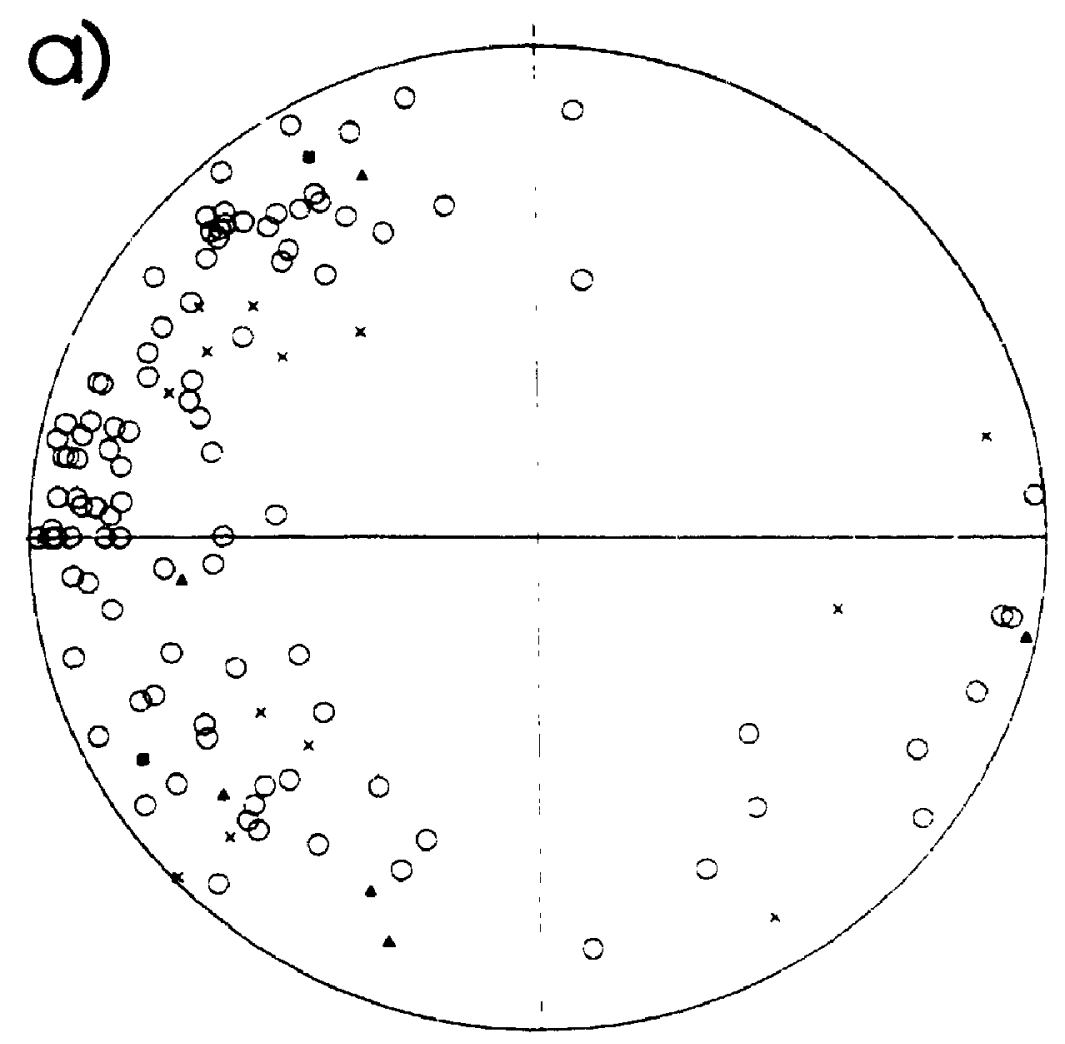

b)

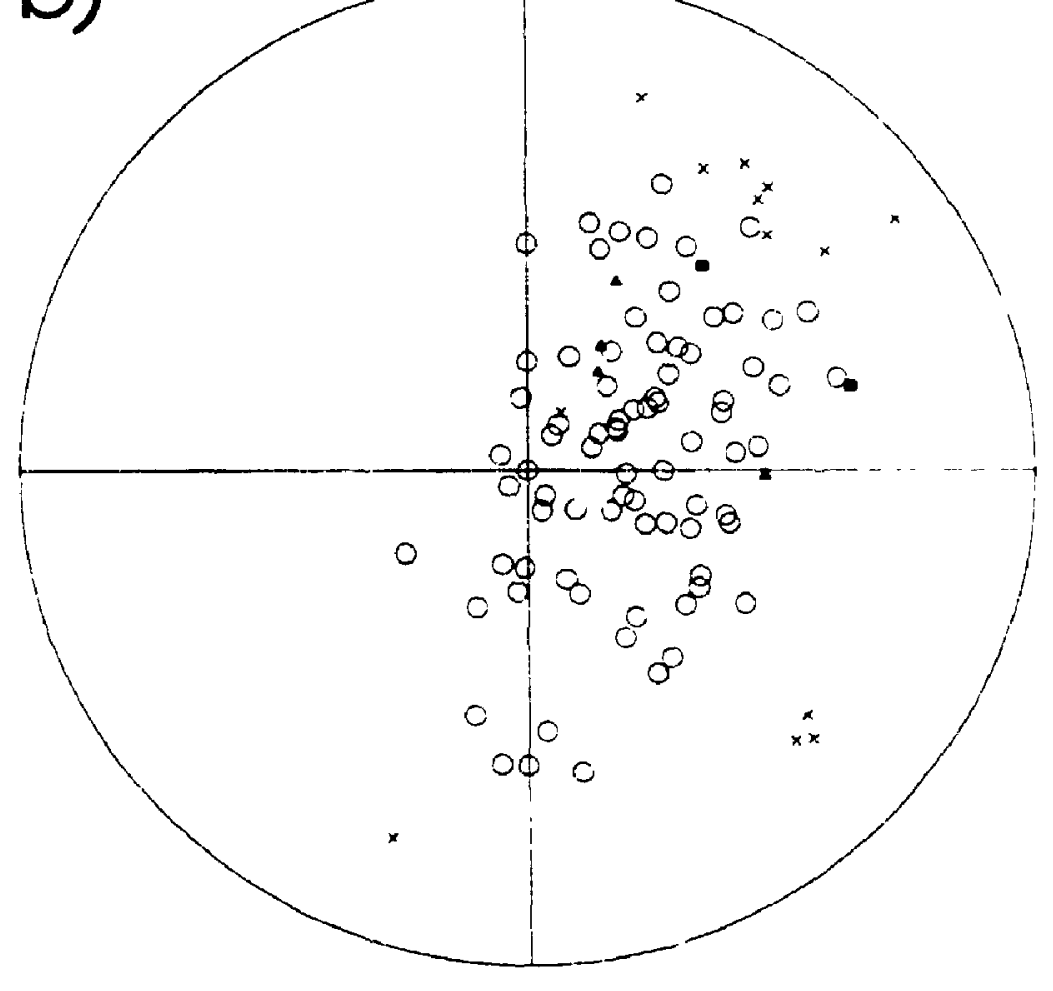

- $\mathrm{Fl} \cdot \mathrm{F} 2$ - $\mathrm{F3} \times \mathrm{F4}$

Figure 2.4. a) Fold axes b) poles to axial surfaces. 


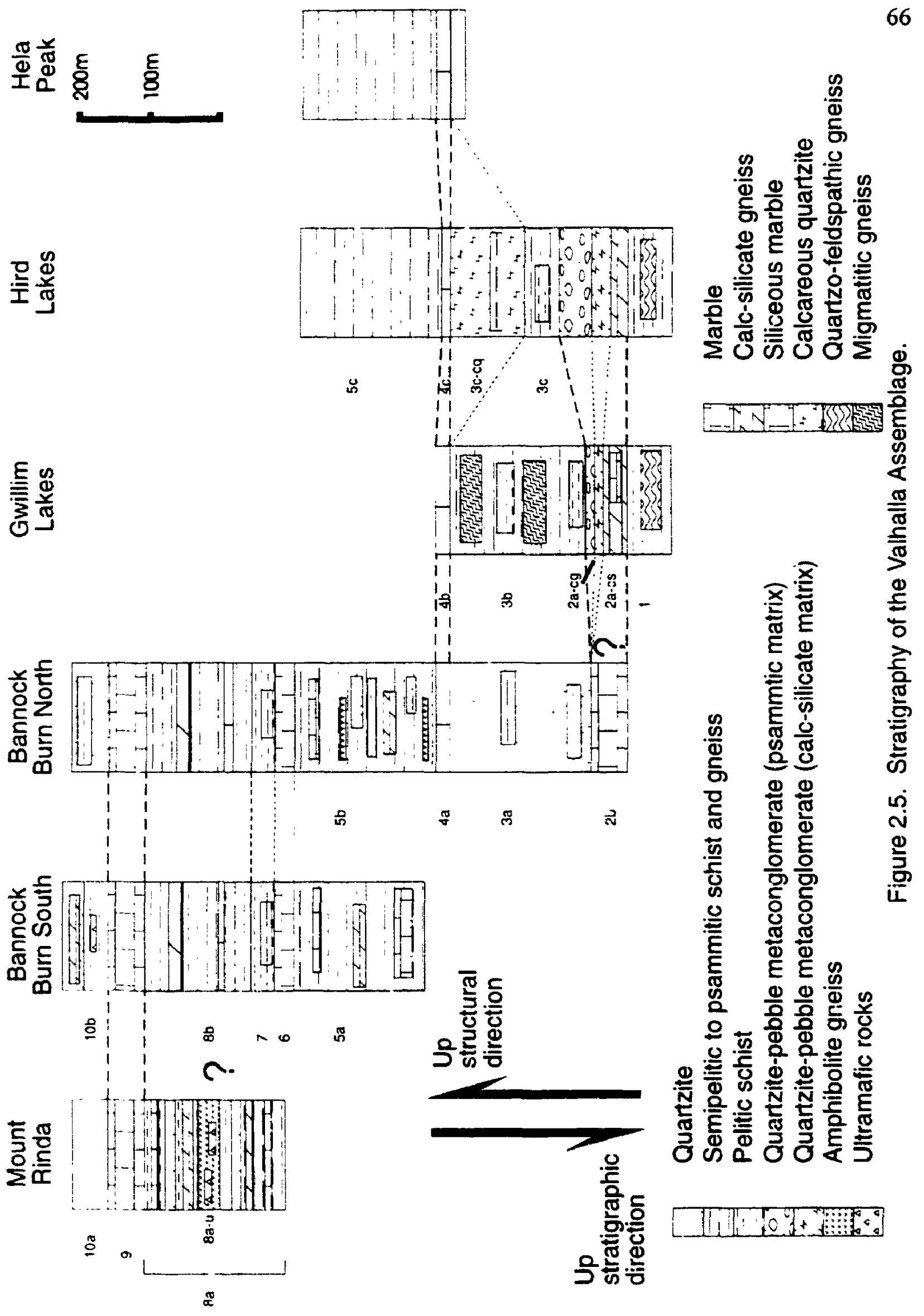



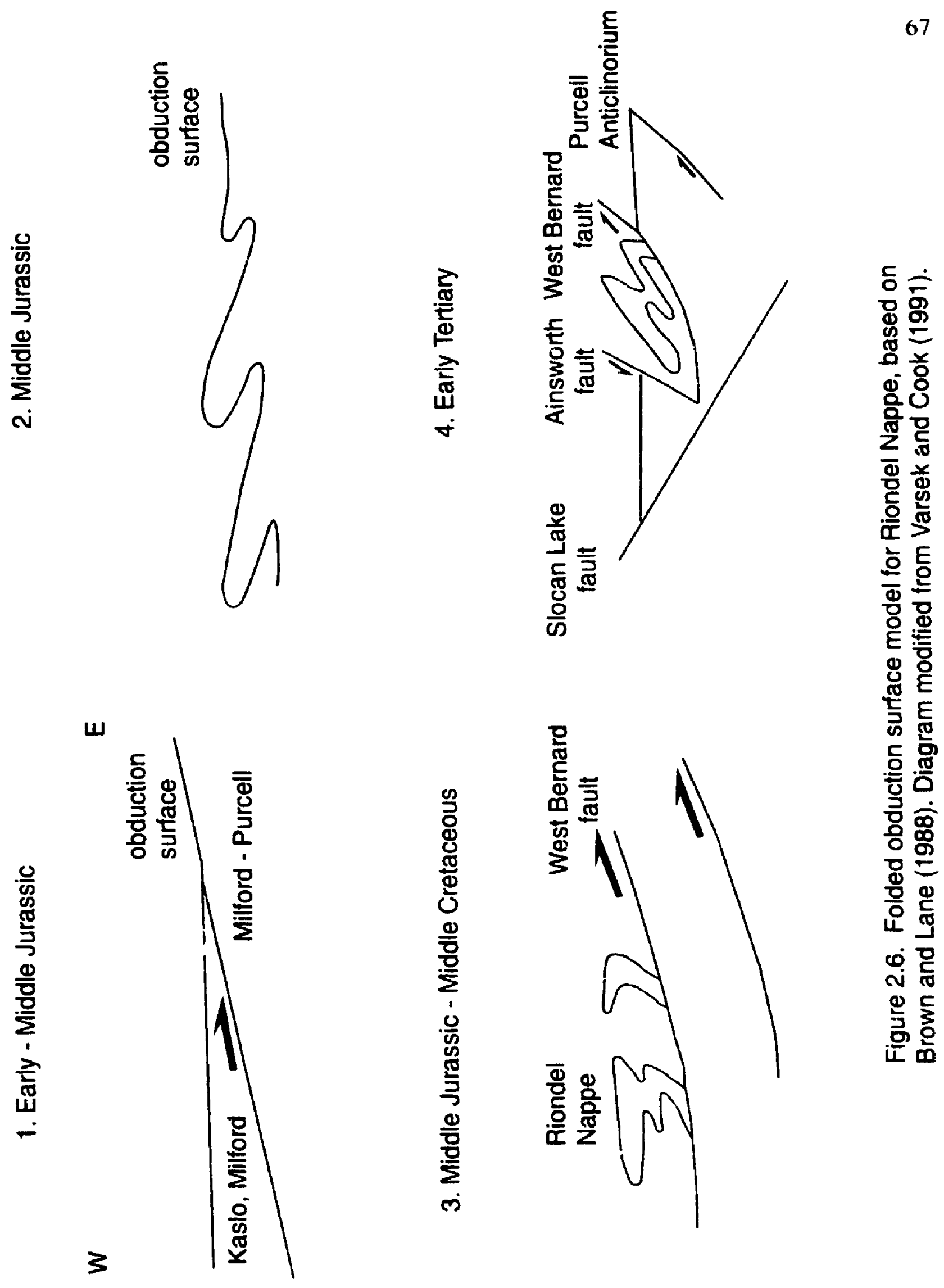
Figure 2.7 


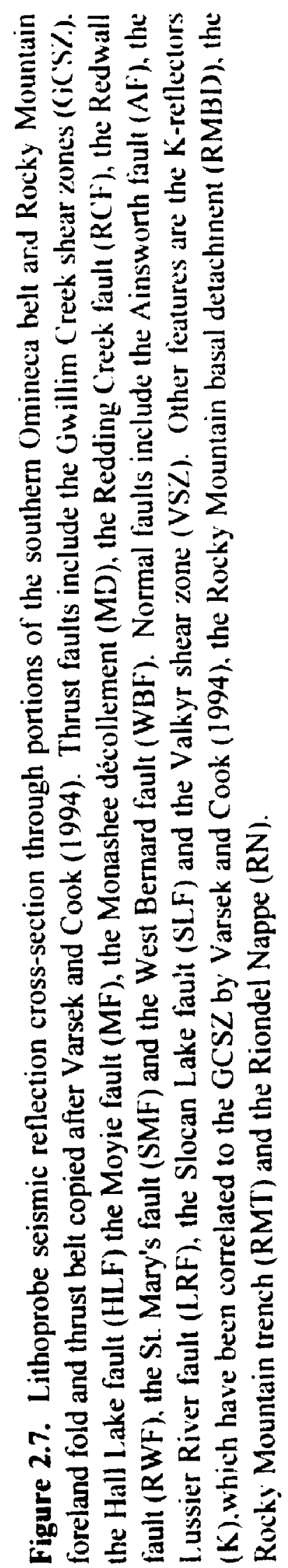




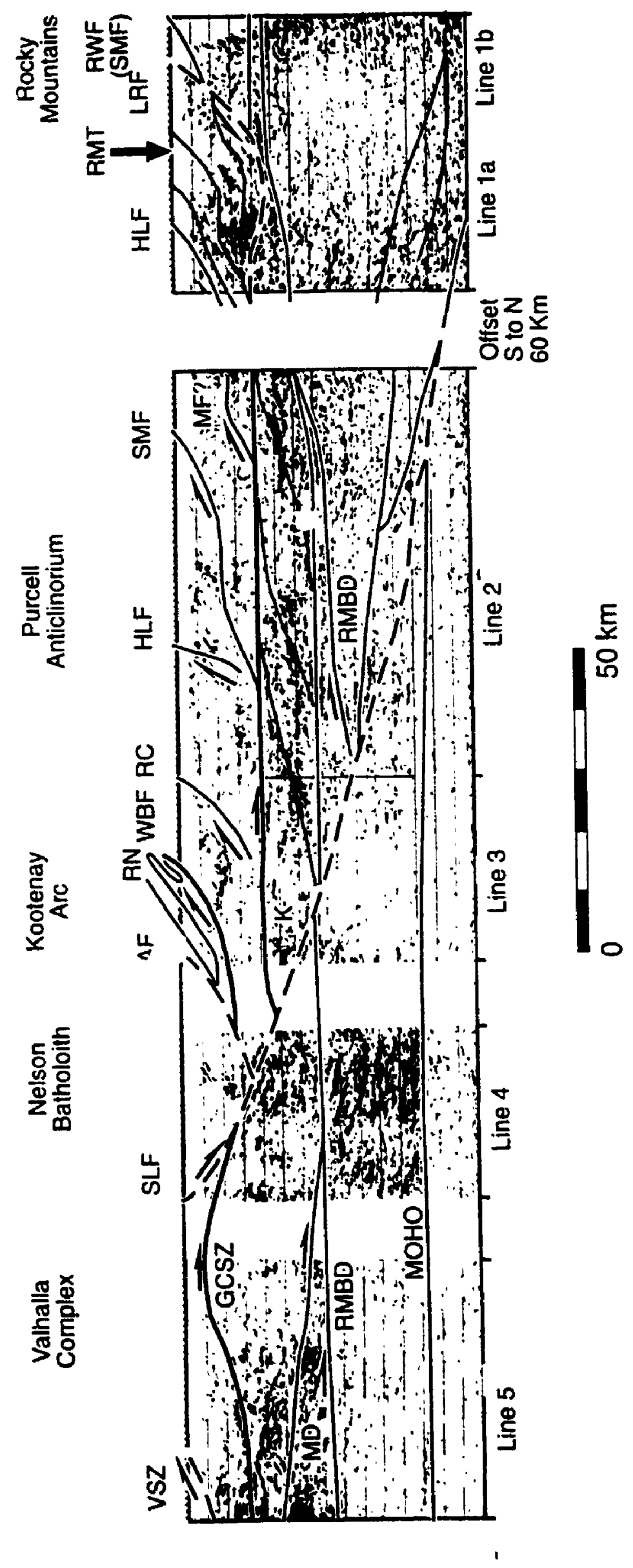




\section{Plate 1}




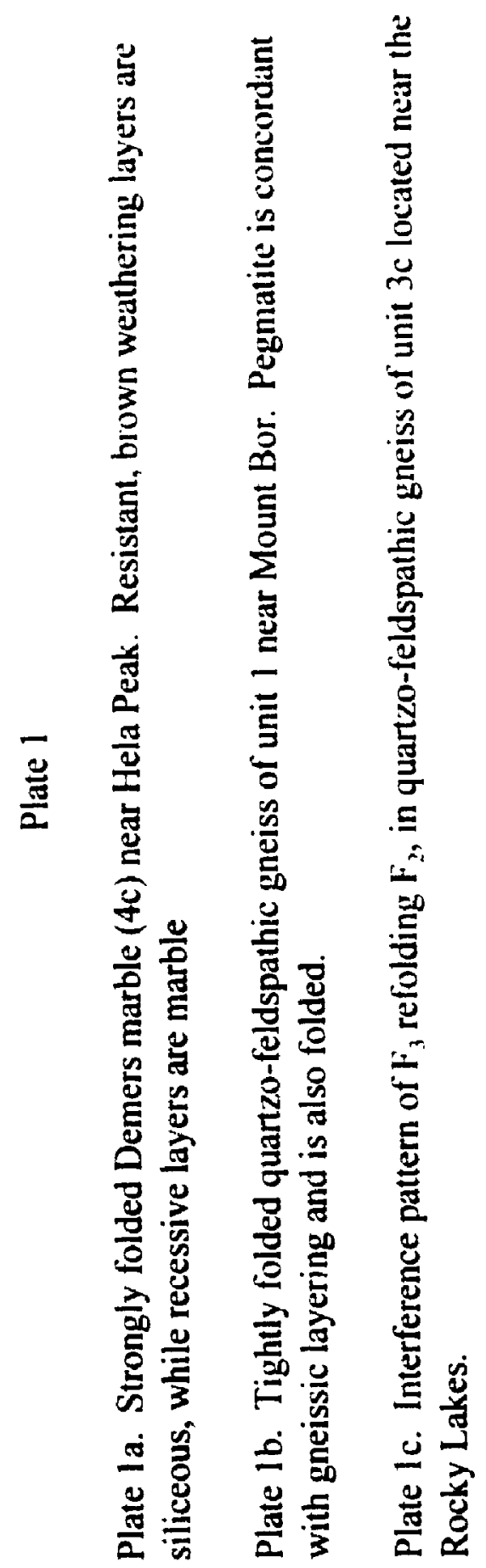




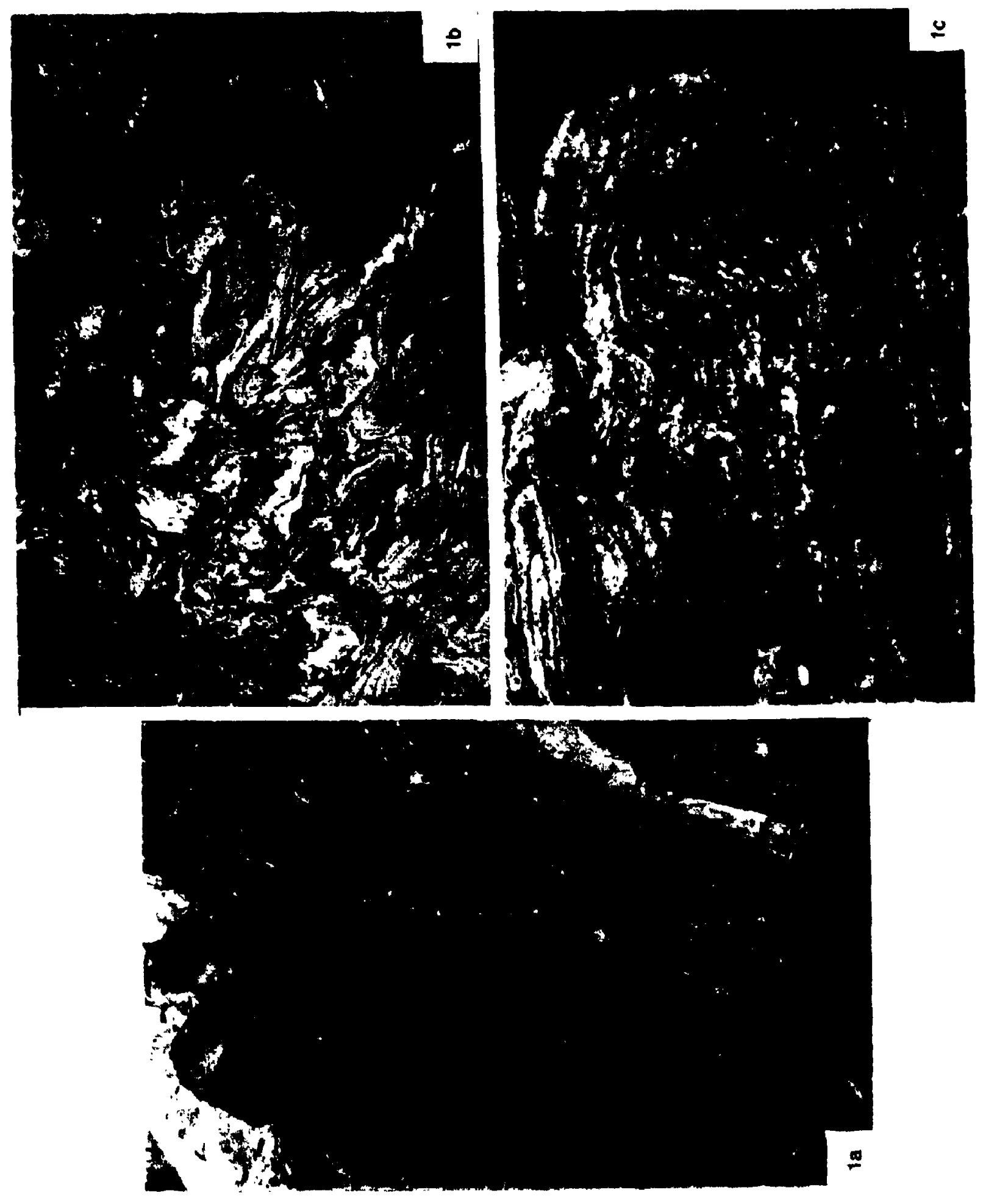


Plate 2 


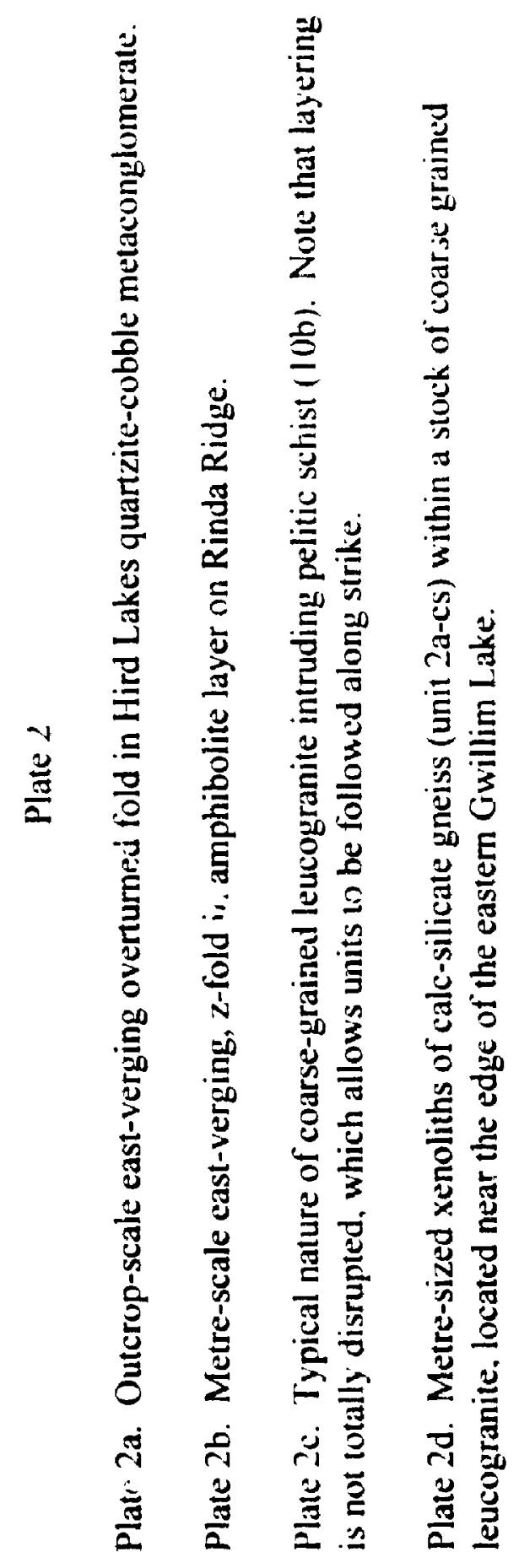




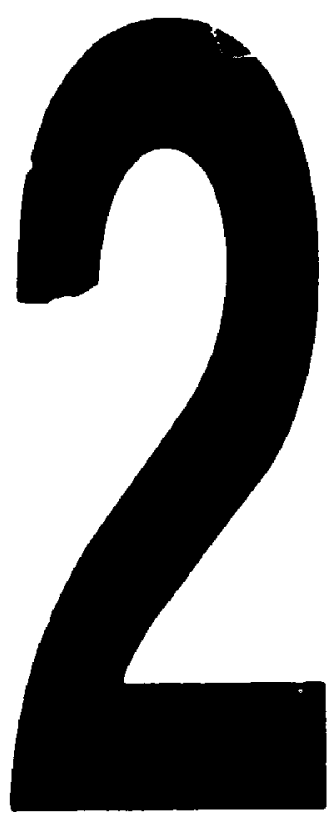

PM.1 31/2"X4" PHOTOGRAPHIC MICROCOPY TARGET NBS 1010a ANSI/ISO \#2 EOUIVALENT

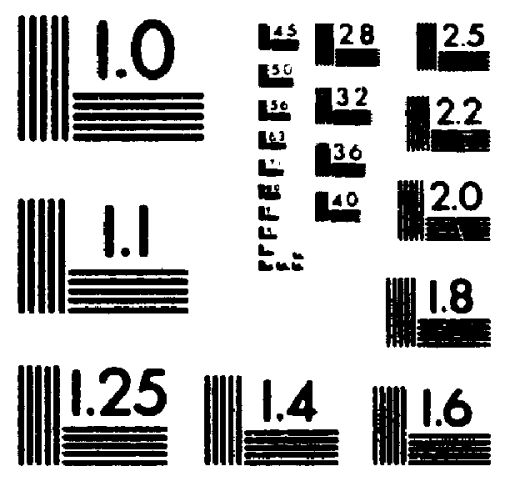

PAECISIONEM RESOLUTION TARGETS 

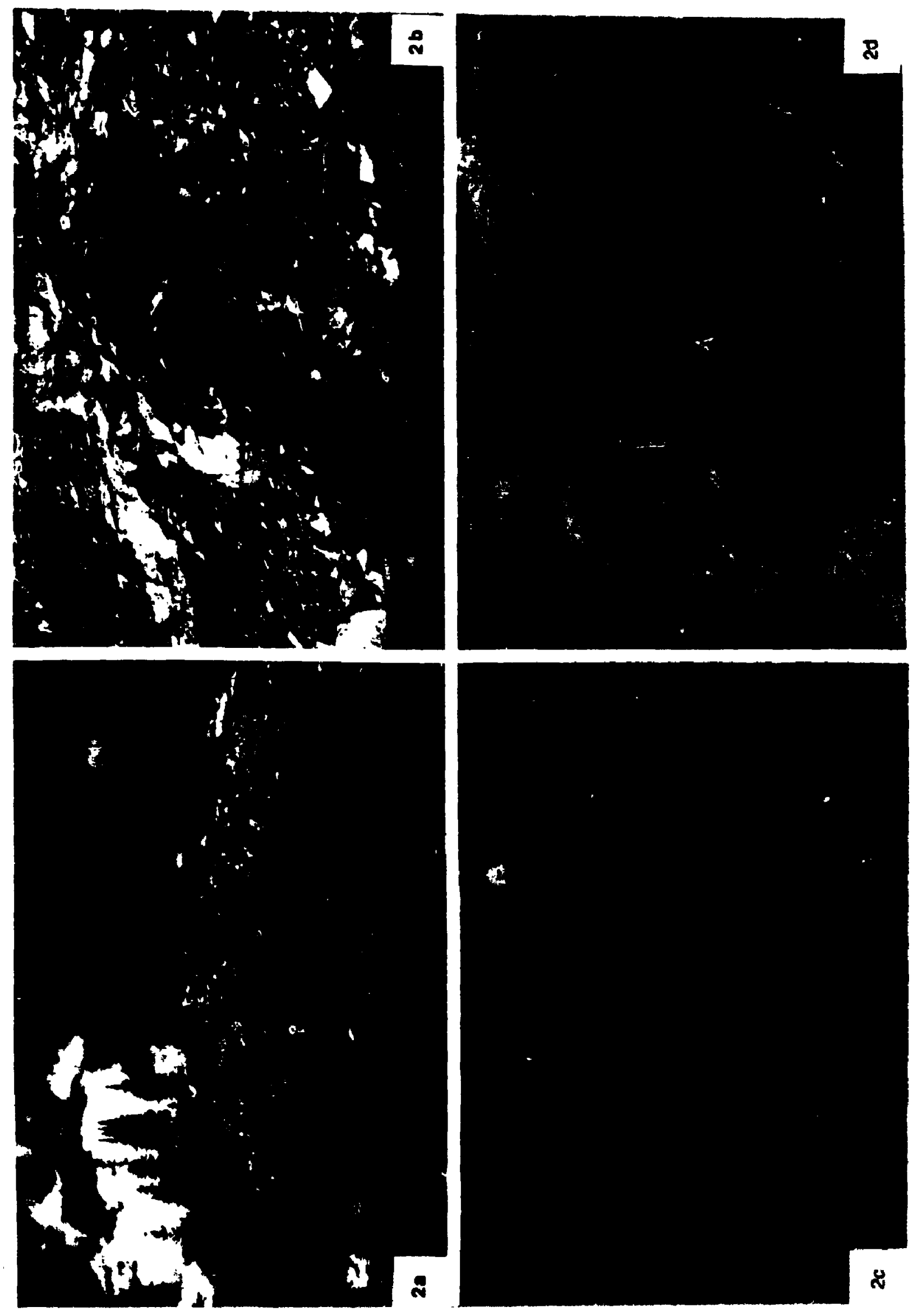
Plate 3 


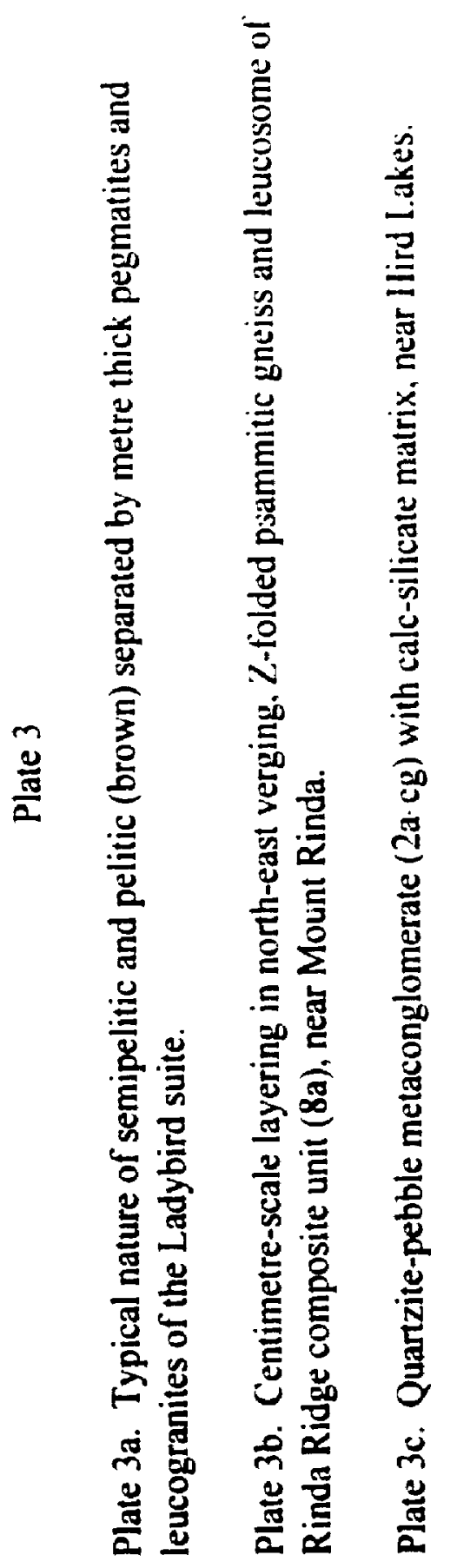



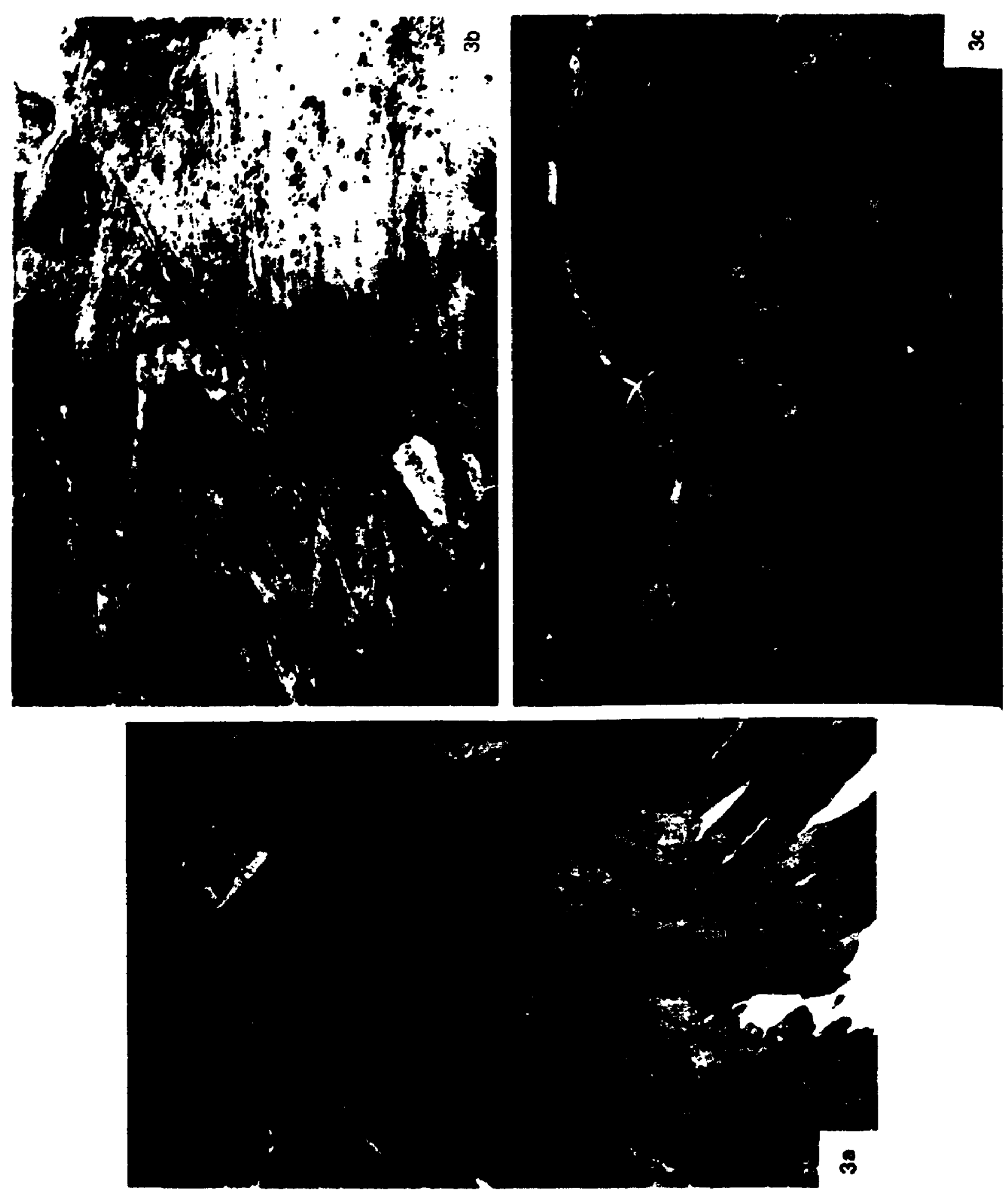
Plate 4 


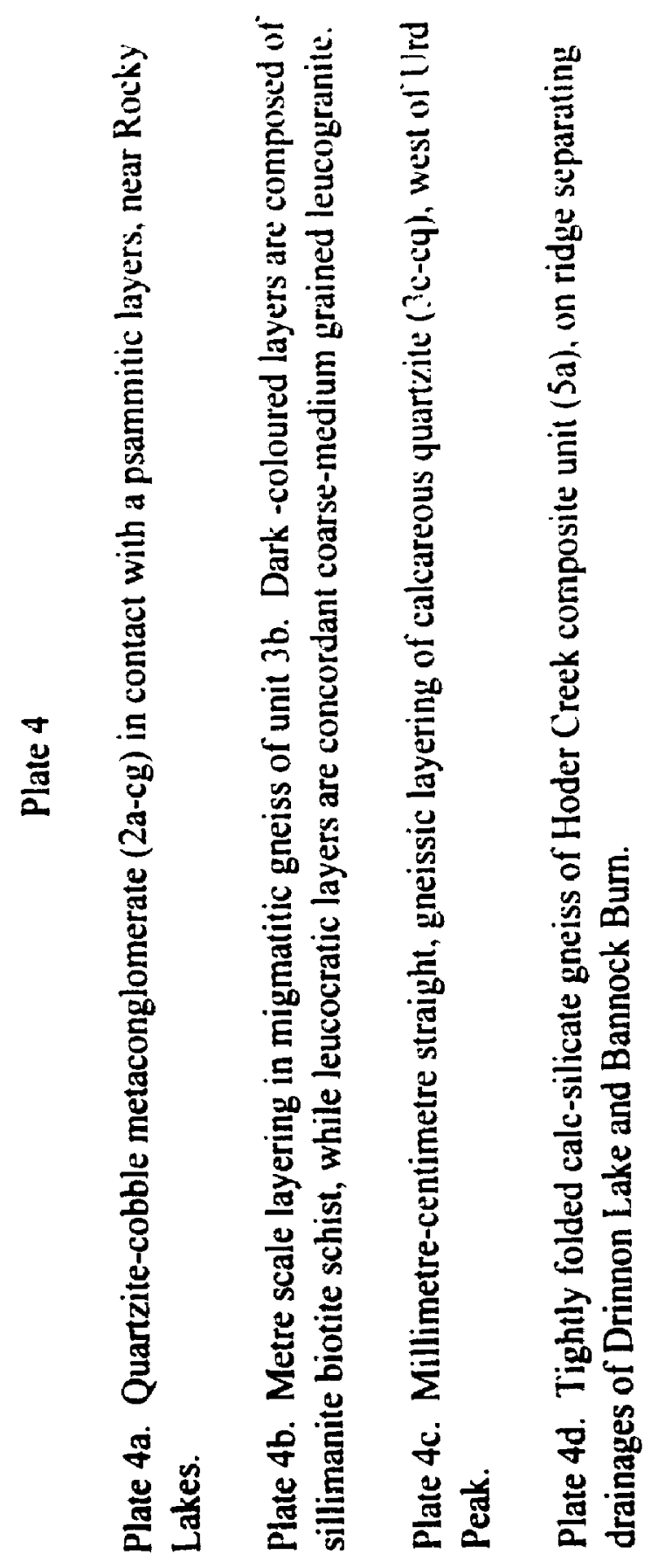




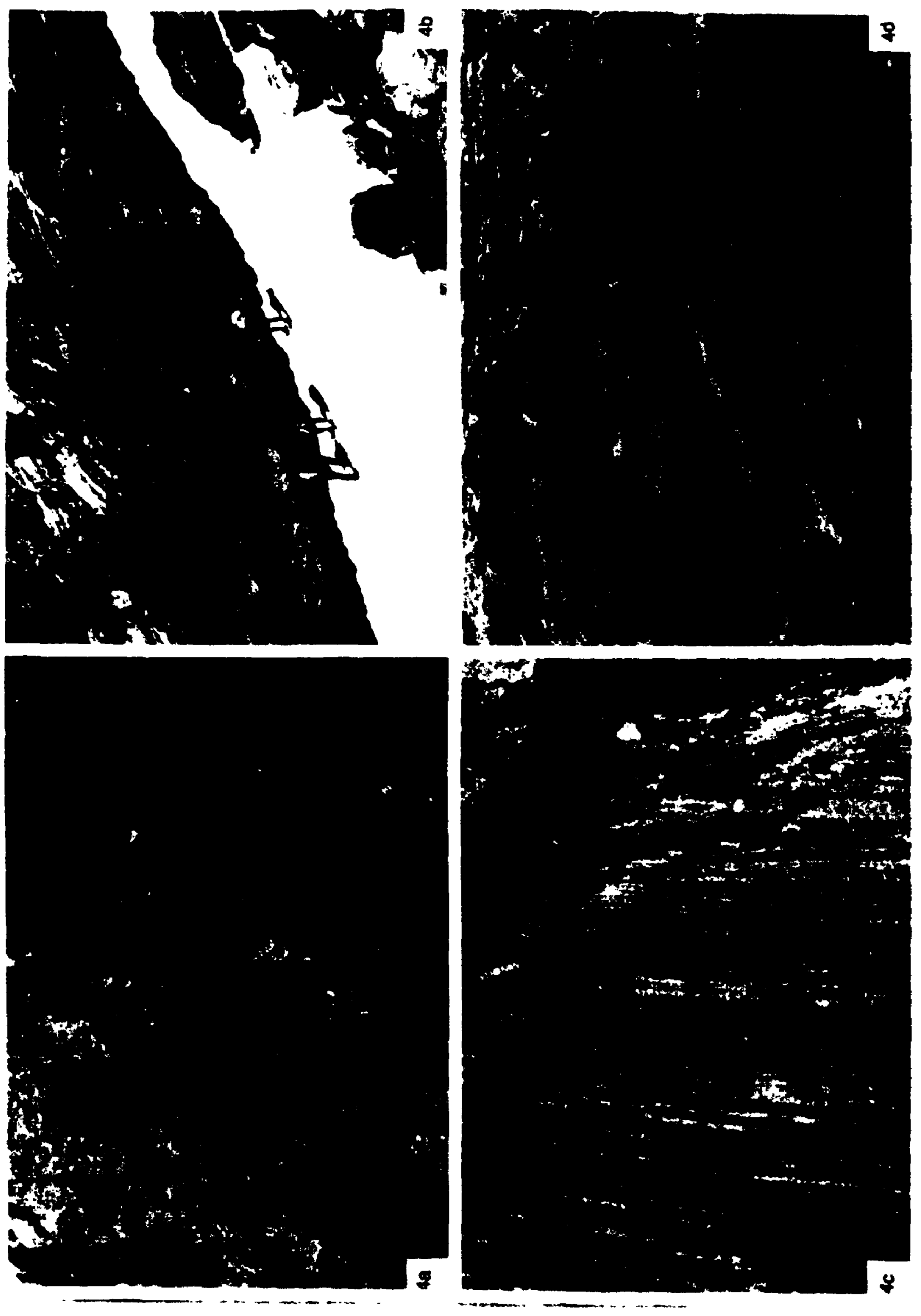




\section{Plate 5}




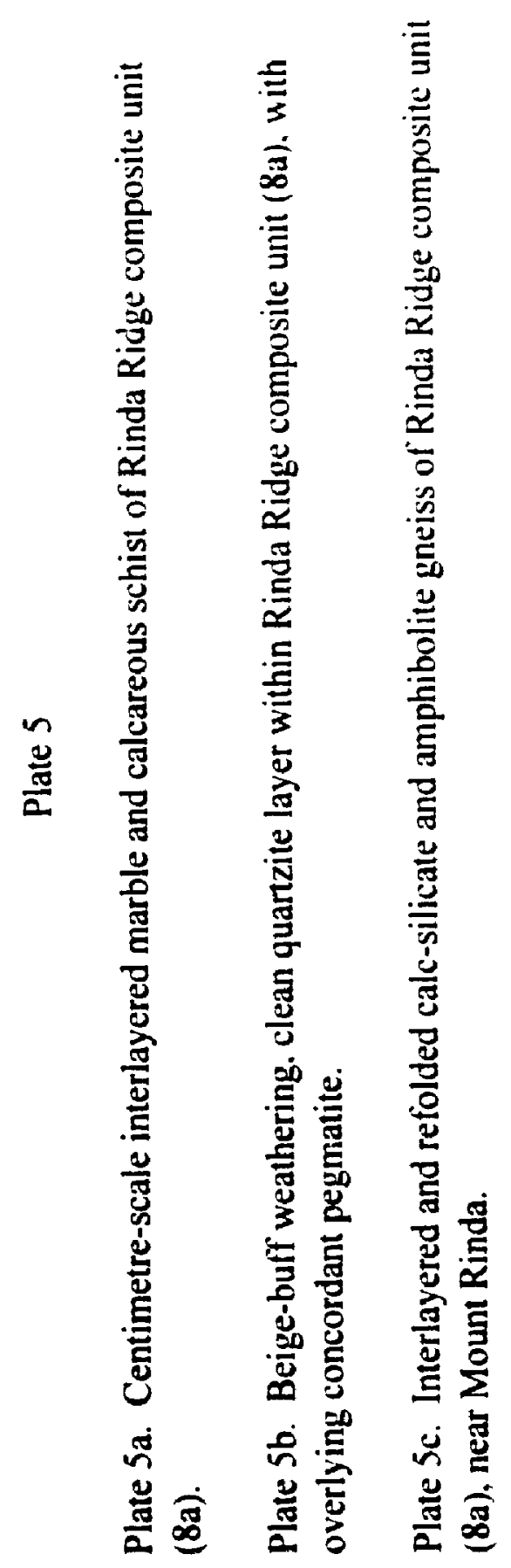




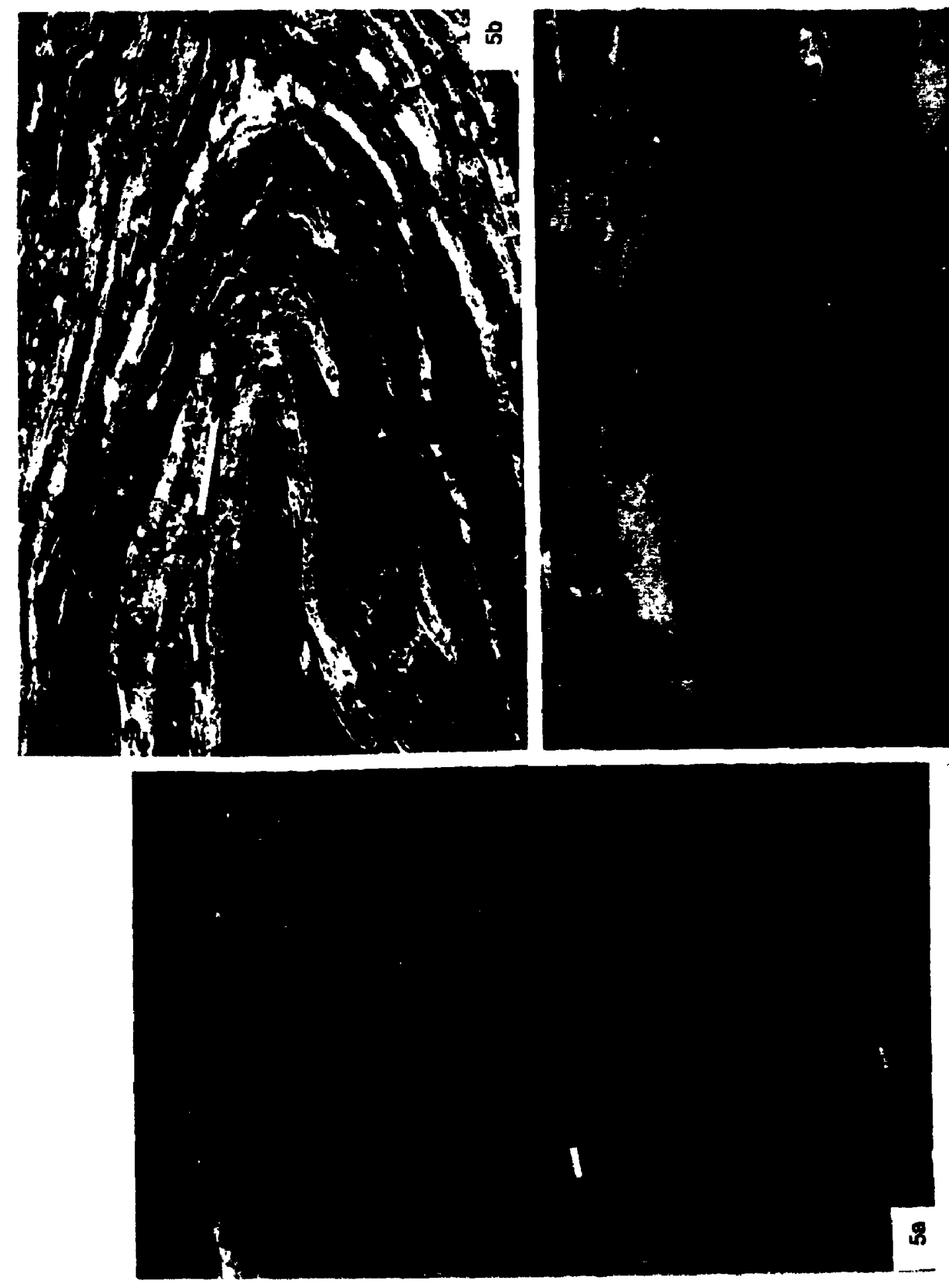


Plate 6 


\section{Plate 6}

Plate 6a. Typical greenish-brown weathering nature of ultramafic pods (8a-u), near Mount Rinda. In lower left. amphibolite gneiss associated with ultramafics is visible.

Plate 6b. Rubbly, metre-scale outcrop of metamorphosed ultramafic rock (8a-u), near Mount Rinda.

Plate 6c. Decimeter scale interlayered marble, calc-silicate gneiss and semipelitic schist of unit 8b, on north flank of Rinda Ridge. 


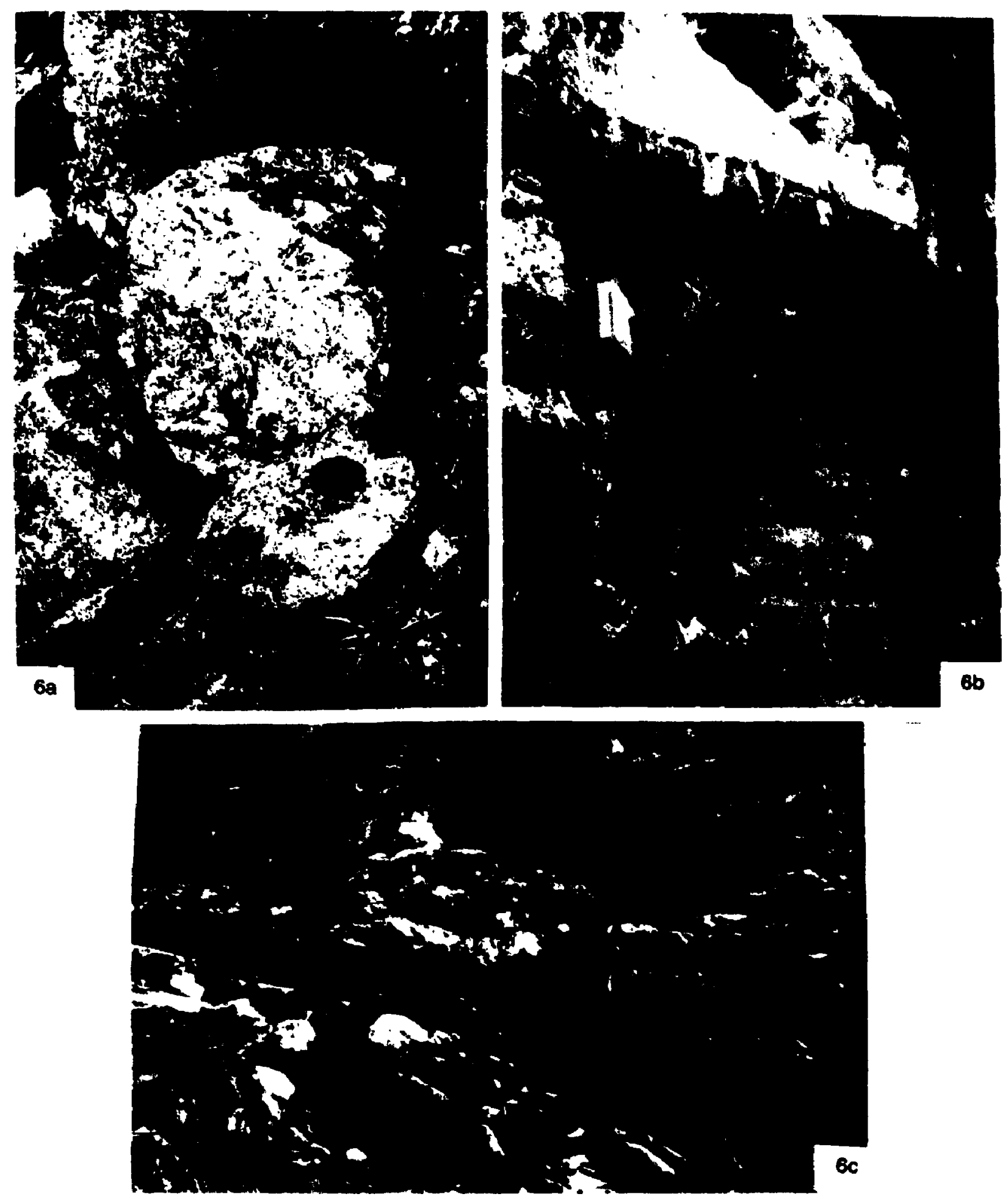


Plate 7 


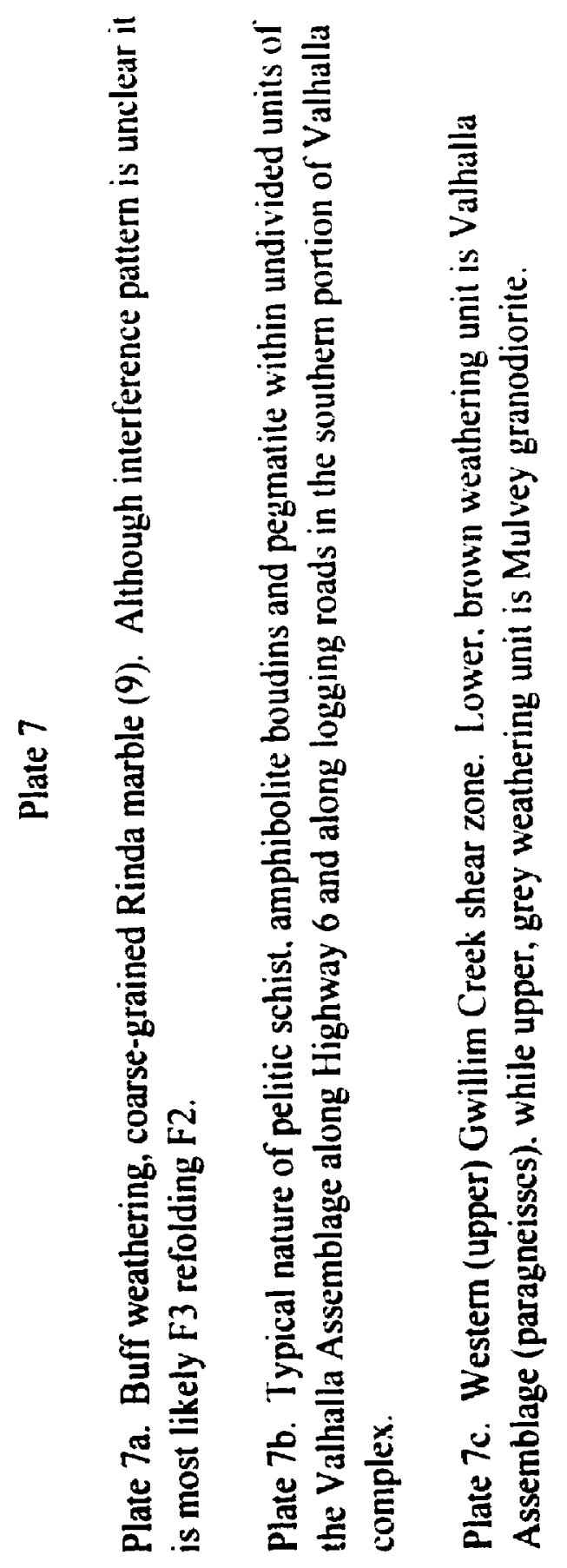



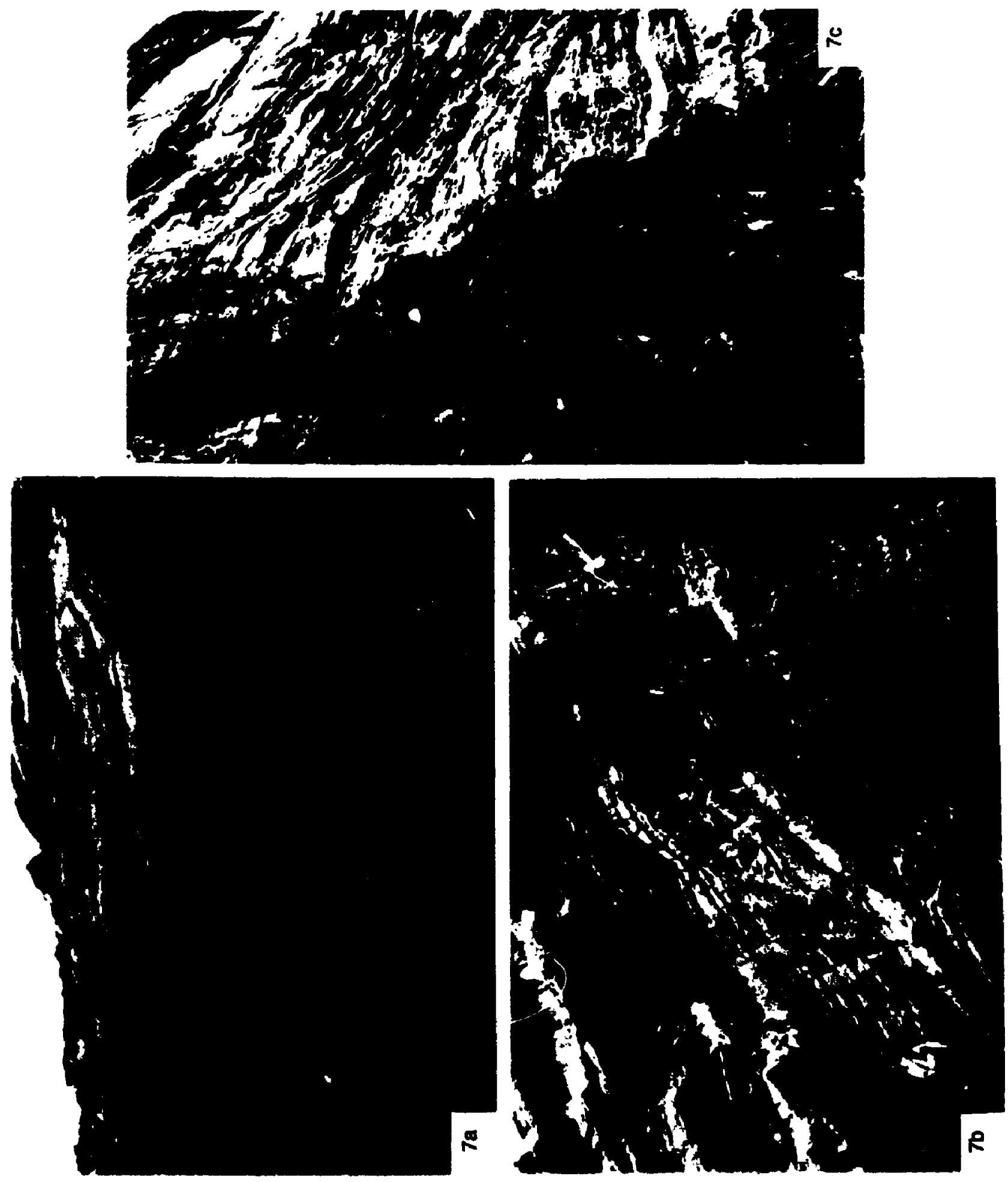

COMPLEX

\subsection{Introduction}

Stratigraphic correlations place few constraints on the magnitude of displacenent or vergence of the GCSZ. and do not give any indication of whether the shear sones are thrust or extensional faults. The aim of this chapter is to characterize and compare the metamorphic history of paragneisses in the hanging wa!l and footwall of the GCSK. lexlural evidence is used to determine the relative timing of metamorphism with respect to deformation, and thermobarometry is used to deduce differences in pressure-temperature conditions of paragneisses across the GCSZ and at different structural levels within the Valhalla complex. Integration of the textural study with thermobarometry is used to infer parts of the pressure-temperature paths from the different structural levels in the Valhalla complex.

The wide range of bulk compositions in the Valhalla complex facilitates independent determinations of pressure-temperature conditions. Mineral assemblages and textures in marbles, calc-silicate gneisses and ultramafic schists were not studied in detail: however. they do provide general constraints using a petrogenetic grid and are consistent with conditions determined using thermobarometry. Thermobarometry was carried out on pelitic schists, amphibolite gneisses and one sample of marble. Textural observations and chemical data in pelitic schists were used to relate the timing of metamorphic reactions to deformation. Zoning patterns and chemical data obtained from microprobe and SEM analyses were used 
to help decipher the reaction history of pelitic schists. These textures and chemical data were used as a guide to suggest which minerals were in chemical equilibrium and which portions of the pressure-temperature path were represented by the compositions that were used in thermobarometric calculations.

\subsection{Analutical methods}

Sixty samples of pelitic to semipelitic schist. twelve samples of amphibolite gneiss. nineteen samples of marble and calc-silicate gneiss, and five samples of ultramafic schist were examined using the petrographic microscope to determine metamorphic assemblages and textures. Minerals from thirteen samples of pelitic schist and five samples of amphibolite gneiss were analysed using the electron microprobe at Carleton University. Two samples (PCA-93-84. PCA-200-85) of pelitic schist collected by Sharon Carr were analysed by Marc St. Onge at the Geological Survey of Canada and the data have been incorporated into this study. The Cambridge Microscan 5 electron microprobe was coupled to an ORTEL Si energy dispersive $x$-ray detector, which was operated at $20 \mathrm{kV}$ accelerating potential, with a 3.0 nanoampere beam current measured on a Faraday cup. The counting time of all elements was 120 seconds live time and raw X-ray spectra were calculated into oxide weight percent using the EDDI computer program (Pringle 1989). The calculation of $\mathrm{Fe}^{-3}$ in ilmenite was carried out using the EDDI program, which uses a stoichiometric method modelled after Droop (1987).

In pelitic schists and amphibolite gneisses, ai least two garnet-bearing domains were analysed within each thin-section. Quantitative mineral analyses from the microprobe and 
$\mathrm{X}$-ray maps from the SEM were used to detect compositional zoning and its relationship to texture. Reconnaissance analyses taken on two samples of marble and one sample of calc-silicate included scapolite. plagioclase. diopside, hornblende and forsterite.

Quantitative data for garnet, biotite, plagioclase and ilmenite were obtained for pelitic schists. In each thin-section, up to five different spatially related biotites were analysed: I) grains in contact with garnet rims: 2) proximal biotite grains $-0.1-0.5 \mathrm{~mm}$ away from the garnet rim and separated by non-ferro-magnesium minerals: 3) grains in garnet pressure shadows: 4) biotite inclusions in garnet; and 5) biotite grains enclosed by wormlike quartz in garnet (armoured biotite grains). Biotite grains that are in the matrix but near garnet rims and are not in direct contact with garnet are herein referred to as proximal biotite grains. Plagioclase inclusions in garnet, plagioclase grains in contact with garnet. and plagioclase grains in the matrix as close as possible to garnet without actually touching it were also analysed. As ilmenite was not observed in contact with garnet, ilmenite grains as near as possible to the garnet were analysed. In amphibolite gneisses. hornblende, biotite, and plagioclase were analysed to observe differences between grains in contact with garnet and those within 1-2 mm of the garnet. Hornblende inclusions in garnet were analysed where present.

Thermobarometric data were obtained from minerals in contact with garnet and also those minerals that were in the matrix but within the same thin-section domain. In pelitic schists, inferred post peak temperatures and pressures ( $T 1, \mathrm{P} 1)$ were obtained from garnet rims adjacent to non-ferromagnesium minerals, and from biotite and plagioclase grains less than $1 \mathrm{~mm}$ away from gamet. The compositions of these proximal matrix biotite grains were 
used to avoid the effects of the latest $\mathrm{Fe}-\mathrm{Mg}$ exchange between garnet and biotite. In samples that did not contain plagioclase in contact with garnet. compositions from matrix plagioclase grains as close as possible to the garnet rim were used in calculations. Temperatures and pressures representative of a lower pressure-temperature part of the pressure - temperature path (T2, P2) were obtained from garnet rims in direct contact with biotite and plagioclase grains in contact with the garnet rims. Since no pattern in composition was observed between plagioclase grains in contact with garnet and those in the matrix. $\mathrm{P} 1$ and $\mathrm{P} 2$ pressures are virtually the same. $\mathrm{P} 2$ pressures that intersect $\mathrm{T} 1$ are an artifact formed as a result of moving down the same GASP isopleth calculated for P1.

Garnet compositions used in thermobarometric calculations were obtained from the average of 1-4 data points from a similar textural location. and were within 1-2 mole $\%$ of each other. Biotite and plagioclase compositions were obtained by averaging at least two data points from each mineral. In some instanres, one analysis of biotite was used due to small grain size. The same procedures were applied to garnet, hornblende, plagioclase and biotite in amphibolite gneisses.

Pressures and temperatures were generated using the computer program TWEEQL (Berman 1991) which calculates temperatures and pressures from equilibria calculated using the thermodynamic database of Berman $(1988,1990)$ and Mader and Berman (1992) and is internally consistent because it uses the same thermodynamic data for all phases and the same solution models for the appropriate mineral in each equilibrium. Empirical calibrations of geothermometers and geobarometers often only give reasonable data when a particular solution model is applied. This program allows the user to calculate as many equilibria as 
possible, both stable and metastable. however. not all of these are independent. The degree to which more than 2 independent equilibria intersect at a single pressure-temperature point is used as a test for the relative equilibration state of all minerals. assuming that the mineral systems closed at the same time. This method assumes that mineral analyses. activity solution models and thermodynamic data are all reliable. In this study. only the most robust thermometers and barometers were applied; those with extensive and well constrained thermodynamic data and with relative insensitivity to errors in input data. For this reason equilibria involving pargasite, Fe-pargasite and Fe-tschermakite were excluded in amphibolite gneisses because thermodynanic data are less certain tor these end-members than they are for other amphibole end-members. All reactions presented in this study are listed with the high temperature or pressure side on the right hand side.

Thermometry of pelitic schists was based on the garnet - biotite exchange equilibrium (e.g. Ferry and Spear 1978);

(1) $\mathrm{Fe}_{3} \mathrm{Al}_{2} \mathrm{Si}_{3} \mathrm{O}_{12}+\mathrm{KMg}_{3} \mathrm{AlSi}_{3} \mathrm{O}_{10}(\mathrm{OH})_{2}=\mathrm{Mg}_{3} \mathrm{Al}_{2} \mathrm{Si}_{3} \mathrm{O}_{12}+\mathrm{KFe}_{3} \mathrm{AlSi}_{3} \mathrm{O}_{14}(\mathrm{OH})_{2}$ almandine phlogopite $\quad$ pyrope $\quad$ annite. Barometry was based on the GASP (e.g. Ghent 1976) net-transfer equilibrium;

(2) anorthite $=$ grossular + sillimanite + quartz,

and the GRAIL (Bohlen et al. 1983) equilibrium;

(3) sillimanite + quartz + ilmenite $=$ almandine + rutile.

In pelitic schists, some assemblages allowed the application of both the GASP and GRAIL barometers. This allowed for a test of the relative equilibrium of the assemblage garnet-biotite-plagioclase-sillimanite-quartz-ilmenite-rutile. The various equilibria used in 
thermobarometric calculations operate by different microprocesses and therefore it might be expected that different equilibria would "close" or "freeze in" at different temperatures. Readjustment of the GASP equilibrium involves breaking of tetrahedral Al-Si bonds in plagioclase and a change in garnet-plagioclase proportions. The GRAIL equilibrium involves $\mathrm{Fe}-\mathrm{Mg}$ exchange and therefore a lower closure temperature may be expected for GRAIL than for the GASP equilibrium. If Fe-Mg exchange outlasts the operation of nettransfer reactions these two barometers might be expected to record two points along a pressure-temperature path. Alternatively. intersections of the garnet-biotite thermometer and GASP and GRAIL barometers at a single pressure-temperature point. would suggest that all phases last equilibrated at the same pressure-temperature conditions.

Thermobarometry of amphibolite gneisses was carried out using TWEEQU to compute the positions of the garnet - homblende - plagioclase - quartz barometer of Kohn and Spear (1990);

(4) tremolite + anorthite $=$ tschermakite + grossular + pyrope + quartz .

the garnet - homblende thermometer (e.g. Graham and Powell 1984);

(5) almandine + tremolite $=$ Fe-tremolite + pyrope,

and the garnet-biotite thermometer (reaction 1). To gain a better understanding of the prograde history of amphibolite gneisses, reaction (5) was applied to samples with homblende inclusions in garnet. Plagioclase inclusions are absent in gamet and therefore reaction (4) could not be applied. The solution models of Berman (1990), McMullin and others (1991), Fuhrman and Lindsley (1988), and Mader and Berman (1992) were used for garnet. biotite, plagioclase and hornblende, respectively. Fixed activities for ilmenite were 
calculated assuming ideal mixing.

Thermobarometry was carried out on one sample of marble. for which the equilibrium:

(6) anorthite + calcite $=$ meionite $(\mathrm{Ca}$-scapolite $)$

was used to constrain peak temperatures. This equilibrium was also generated with TWEEQU using the solution model of Oterdoom and Gunter (1983) for scapolite.

\subsection{Petrology of naragneisses}

\subsubsection{Pelitic schist}

Pelitic schists in the Valhalla complex contain the assemblage quartz - biotite plagioclase \pm garnet \pm sillimanite \pm potassium feldspar (see Appendix B for modal abundances), which places them in the upper-amphibolite metamorphic facies above the breakdown of muscovite. Accessory minerals include ilmenite, rutile, pyrite. zircon, apatite and monazite. Mineral assemblages for individual samples are presented in Appendix B. Kyanite was observed in one sample by Carr (pers. comm. 1994), but has not been found in any other samples. Peak conditions are constrained by the presence of sillimanite, potassium feldspar and abundant melt, and by the absence of prograde muscovite and kyanite (Fig. 3.1). Metamorphic conditions are further constrained by the absence of orthopyroxene, which is indicative of granulite facies metamorphism (Harley 1988, Fig. 3.1). Retrograde metamorphism is generally restricted to late faults and fractures. However, it is rarely penetrative and is represented by randomly oriented chlorite that replaced biotite in garnet 
pressure shadows and fractures, and by non-aligned radiating muscovite and sericite that occurs in plagioclase (Appendix B).

Pelitic schists are described in terms of the three domains presented in chapter 2 . The first area (domain 1a) is the structurally highest, and occurs on the west side of the complex between Mount Rinda and Hela Peak in the hanging wall of the GCSZ (Fig. 3.2). Domain $1 \mathrm{~b}$ occurs in the southern area of the paragneisses at higher structural level than domain 2 and has the same structural characteristics as domain la (see section 2.2. cross-section A). Domain 2 occurs in the southern portion of the paragneisses in the area of Vallican. below domain 1b. Domain 3 occurs in the lower Gwillim Creek window (sheet 1a, Fig. 3.2, section 2.2). Textural, chemical and thermobarometric data, as well as textures which relate metamorphic reactions to deformation for each of the three domains are presented in the following sections. Within each domain, the textures and chemical data will be presented for individual mineral species in relation to each other.

\section{Domain L-Valhalla assemblage (sheet lc, west side)}

\section{Textures, mineral compositions and reaction histories}

Pelitic schists of the Valhalla assemblage, between Mount Finda and Hela Peak (Fig.

2.3) may be divided into two bulk compositions based on the relative proportions of sillimanite and plagioclase. The first rock type is a strongly foliated pelitic schist, which has a foliation defined by biotite and discrete layers of sillimanite, and quartz and feldspar. Garnet porphyroblasts are common. The second rock type is also a pelitic schist, based on 
biotite content, but has a moderately to weakly developed foliation. Biotite does not wrap around garnet porphyroblasts. but abuts them. and is commonly randomly oriented. Sillimanite is rare or absent. and plagioclase is abundant. These sillimanite poor schists have ragged, angular garnet porphyroblasts with uneven rims and many fractures (Plate 8a).

\section{Garnet}

Garnet occurs as subhedral to anhedral porphyroblasts and poikiloblasts, ranging from $0.5 \mathrm{~mm}$ to $2 \mathrm{~cm}$ in size (Plate 8a, 8b). Small and closely spaced quartz grains appear to represent true inclusions that have been incorporated into the garnet crystal during its growth. Commonly, inclusions are concentrated in the core of the garnet and show no preferred orientation (Plate 8c). There is no compositional difference between areas of the garnet core with high and low numbers of inclusions. The inclusion-free rim texture may be either the result of: 1) a second phase of garnet growth, or 2) a slower rate or a fluid enhanced phase of garnet growth allowing included phases to be consumed or excluded from the crystal, rather than frozen in. Thus garnet growth may have been achieved by reaction:

(7) biotite + quartz + sillimanite $=$ garnet + potassium feldspar $+\mathrm{H}_{2} \mathrm{O}$

or

(8) sillimanite + biotite + plagioclase $=$ garnet + potassium feldspar + melt $($ Fig. 3.1)

If reaction (7) were operative, then the high concentration of quartz inclusions in the core of certain garnets may indicate an excess of quartz that could not be consumed by the garnet forming reaction. Reaction (8) would explain the scarcity of plagioclase in some samples, although this is more likely to be a function of bulk composition. Garnets also contain many 
fractures which are commonly oriented at nearly $45^{\circ}$ to the foliation. and are interpreted to be caused by stress applied to the coherent garnet grains.

Garnet often has many large quartz embayments (Plate 8 b) which are interpreted to represent the final consumption of garnet following the peak of metamorphism. Quartz occurring as embayments, wormlike inclusions and halos around garnet (Plate 8a) may be the result of the garnet consuming reaction (7) proceeding in the opposite direction as described in the previous paragraph. Sillimanite and biotite are not commonly in close proximity to these quartz embayments and therefore reaction (7) may not be responsible for the production of quartz; however if it is, then biotite and sillimanite may increase in size rather than nucleating new grains.

Inclusions in gamet include quartz, biotite, plagioclase, sillimanite, apatite, ilmenite and rarely rutile (Plate $8 \mathrm{~d}$ ). Inclusions may make up to $40 \%$ of the garnet. Where inclusion trails of quartz are aligned they are at a high angle to the external foliation (Plate 9a). Quartz inclusion trails are usually straight and cannot be traced to the external foliation, suggesting that the garnet overgrew a preexisting foliation $\left(S_{1}\right)$.

$\mathrm{S}_{2}$ contains the peak metamorphic mineral assemblage. The initiation of gamet growth occurred prior to $S_{2}$ and the onset of coarse grained minerals. Sillimanite inclusions often occur within the garnet rim and are generally parallel to the external foliation (Plate 9b). This texture suggests that garnet growth continued during prograde metamorphism at sillimanite grade and the onset of $\mathrm{S}_{2}$ foliation development. Peak minerals aligned within $\mathrm{S}_{2}$ wrap around garnet porphyroblasts and indicate that deformation outlasted metamorphism. Peak metamorphic minerals (biotite. sillimanite and $\mathrm{K}$-feldspar) occur in the pressure 
shadows. indicating that the peak of metamorphism was synchromous wth $S:$.

\section{Garnet zoning}

Microprobe analyses show the typical diffusion zoning patterns (ty pe 3) described by Tracy (1982). Relatively flat protiles of Fe. Mg. Ca. and Mn are observed in the cores of all garnets (Fig. 3.3). Garnet rims in contact with biotite have fe/Fe+Mg ratios that are 5-8 mole percent higher relative to the core (Fig. 3.3). Garnet rims in contact with either quartz, plagioclase or potassium feldspar have lower $\mathrm{Fe} / \mathrm{Fe}+\mathrm{Mg}$ ratios than those rims in contact with biotite. but also show a -5 mole $\%$ increase relative to the core. Increases in Fe and decreases in $\mathrm{Mg}$, in the same sample. at rims in contact with biotite are attributed to Fe-Mg exchange via reaction (1). The other common ferro-magnesium mineral in pelitic schists of the Valhalla complex is ilmenite, but it is rarely observed in contact with garnet and it is therefore unlikely that exchange between ilmenite and garnet is responsible for increased $\mathrm{Fe}$ levels in garnet rims. If exchange reactions were the only reactions taking place. increased Fe levels would only be present where biotite is in contact with the garnet rim, and this is not the case. The fest that garnet rims have higher Fe/Fe+Mg relative to the core, regardless of whether the garnet is in contact with biotite or not, suggests that both net-transfer and exchange reactions were taking place. Garnet may have been consumed via reaction (7) or (8), producing both a garnet rim and new biotite grains with an increased Fe content. $\mathrm{Fe} / \mathrm{Fe}+\mathrm{Mg}$ ratios are also elevated where garnets contain biotite inclusions (Fig. 3.3). This is interpreted to be a result of the Fe-Mg exchange between garnet and biotite (reaction 1) during the cooling portion of the pressure temperature path. 
Ca content remains relatively constant from garnet core to rim (Fig. 3.3). inciuding most of the samples where the garnet rim is in direct contact with plagioclase. Mn zoning is slight in most cases. Some samples exhibit sharp increases of 1-2 mole $\%$ spessartite towards the rim which may be attributed to retrograde conditions during cooling (e.g. rardley 1977).

The flat zoning protiles of garnet cores in Valhalla complex are interpreted to be a result of diffusion. At temperatures above $675^{\circ} \mathrm{C}$. diffusion rates in garnet are fast enough that garnet is able to equilibrate to ambient $\mathrm{P}-\mathrm{T}-\mathrm{X}$ conditions. thus homogenizing gamet compositions from rim to core and erasing all previous grouth zoning (Yardley 1977).

\section{Sillimanite}

Sillimanite is present in most pelitic schists of the Valhalla complex making up to $2^{e r} \therefore$ of the rock. The large amount of sillimanite. the presence of potassium feldspar and the lack of muscovite in the peak metamorphic assemblage indicates that these rocks lie on the high temperature side of the second sillimanite isograd:

(9) quartz + muscovite $=$ potassium feldspar + sillimanite $+\mathrm{H}_{2} 0$.

Sillimanite is aligned in the main transposition fabric and occurs in the pressure shadows of garnet. Sillimanite rarely occurs as radiating non-aligned masses suggesting that some sillimanite growth either outlasted deformation in some areas or grew in areas of low strain (Plate 9c).

Plagioclase 
Plagioclase makes up $5-20^{\circ}$ o of most samples. Porphy roclasts occur within discrete layers of partially recrystallized quartz. Deformation twins are common. Plagioclase was observed, in some cases. forming narrow moats around garnet (Plate $9 \mathrm{~d}$ ). The moats are discontinuous and are likely to have been to be the result of garnet consuming reactions that occurred during decompression (reaction 2). In one case. plagioclase replaces garnet and the relict shape of the garnet contains sillimanite and biotite aligned in $S_{2}$ (Plate 10a). This appears to be an example of the advanced stage of reaction (8). Most plagioclase moats around garnet are interpreted to be a result of reaction (2) because plagioclase moats are solid and intergrowths of plagioclase, sillimanite and biotite are absent. These symplectites might be expected if reaction (8) were responsible for the formation of plagioclase moats around garnet.

Matrix plagioclase grains range in composition from albite to labradorite between samples (An 19 to An 48. Appendix C), however grains within a sample do not vary more than $\sim 5-10$ mole \%. Grains in contact with garnet have a slightly narrower range in composition, from An 22 to An 49, and no systematic difference in An content is related to textural location. Plagioclase inclusions in garnet have an An content that ranges from 26 to 30, and are elevated in Ca relative to plagioclase that occurs in the matrix and adjacent to garnet in the same sample (An $23-25$, Appendix C). Although An contents may vary within a plagioclase grain, coherent or concentric compositional zoning is absent.

Quartz

Quartz makes up to $30 \%$ of pelitic schists and most uften occurs as aggregates of 
grains and ribbons. In quartz ribbons recovery is not advanced. Grain size is quite variable and many subgrains are present. Grain boundaries are generally seriate and $120^{\circ}$ grain boundaries are rare. suggesting that strain rates and temperature conditions were such that minerals did not recover and reorganize strain features such as subgrains. Quartz is more abundant in sillimanite poor rocks.

Myrmekite is observed as vermicular symplectites of quartz and plagioclase (Plate $10 \mathrm{~b})$ and is attributed to the breakdown of potassium feldspar:

(10) muscovite + plagioclase + quartz $=$ potassium feldspar $+\mathrm{Al}_{2} \mathrm{SiO} 5+\mathrm{H}_{2} \mathrm{O}$

(Evans and Guidotti 1966).

Retrograde muscovite is often associated with myrmekite.

Biotite

Biotite makes up to $40 \%$ of pelitic schists. It defines the foliation in most samples and is often found intergrown with illimanite, suggesting simultaneous growth via reaction (7) or (8). It occurs as disseminated grains in samples where its modal abundance is lower $(15-20 \%)$. Biotite is commonly concentrated in the pressure shadows of garnet (Plates $8 \mathrm{c}$. 10c). Contacts with gamet are usually sharp, bui biotite also occurs as embayments in garnet and, in this case, is interpreted to have formed during garnet consumption. The normally sharp grain boundaries are consistent with relative equilibrium between the garnet and biotite grains.

Biotite exhibits compositional variations that are related to the five different spatial associations with respect to garnet, as described previously (Appendix C). Biotite inclusions, 
within quartz inclusions in garnet. and matrix grains of biotite have the highest li content (up to 10 inole \%). Armoured inclusions are 10rmally found in "inclusions" of yuartz that have been interpreted to be a product of consumption. These quartz "inclusions" embay garnet from outside the plane of section. Unarmoured biotite inclusions and grains in contact with, and in the pressure shadows of garnet have the lowest $\mathrm{Fe} / \mathrm{Fe}+\mathrm{Mg}$ ratios $(-0.45)$. while matrix grains have the highest $\mathrm{Fe} / \mathrm{Fe}+\mathrm{Mg}$ ratios $(-0.5)$. The high $\mathrm{Fe}$ levels of biotite grains in direct contact with garnet and unarmoured biotite inclusions is interpreted to be a result of Fe-Mg exchange occurring during cooling along a retrograde pressure-temperature path.

\section{Ilmenite and rutile}

Ilmenite occurs in most samples and rutile is less common. but the two are never found together in samples within domain $1 \mathrm{a}$ in the hanging wall of the GCSZ. In rare instances, ilmenite occurs as inclusions in garnet. The absence of rutile might indicate low pressure or increased Fe bulk compositions. Ilmenite occurs in the pressure shadows of garnet indicating its presence at the peak of metamorphism (Plate 10c).

In samples PS-332-94, from domain 1b, rutile overgrows ilmenite. There are two net-transfer reactions that may be responsible for this; either the GRAIL reaction ( 3 ) or; (11) ilmenite + anorthite + quartz $=$ grossular + almandine + rutile.

In sample PS-332-94, ilmenite in contact with rutile contains higher Mg levels than those grains elsewhere in the sample. $\mathrm{Mn}$ and $\mathrm{Mg}$ make up minor amounts substituting for $\mathrm{Fe}^{{ }^{2}}$ (Appendix C). The absence of rutile may be attributed to an Fe-rich bulk composition. 


\section{Retrograde minerals}

Prograde muscovite is not observed in any samples and is evidence that these rocks overstepped the second-sillimanite isograd (reaction 10). The reaction

(12) muscovite + quartz $+\mathrm{Na}$-plagioclase $=$ sillimanite $+\mathrm{Na}$-orthoclase $+\mathrm{Na}$-poor plagioclase + melt (Thompson 1982)

might also explain the production of melt as well as the consumption of muscovite and plagioclase. However, pelitic schists in the Valhalla complex do not contain Na-poor plagioclase. For this reason, it is more likely that reaction (10) resulted in the consumption of muscovite and plagioclase, and the production of potassium feldspar and sillimanite.

Retrograde minerals are post- $\mathrm{S}_{2}$ and are not aligned in this foliation. Retrograde muscovite occurs as radiating starburst shaped clusters and also as sericitic alteration in plagioclase. In samples where $F_{4}$ crenulations are observed, muscovite appears to be subparallel with the $S_{2}$ fabric that is folded. This suggests that the muscovite grew after $D_{2}$. but prior to $D_{3}$ or $D_{4}$. Sillimanite has rare thin rinds of muscovite, which is probably a retrograde product of reaction (9). Muscovite is commonly found in contact with or within the same thin-section domain as potassium feldspar which is consistent with reaction (9).

Retrograde chlorite replaces biotite and is sometimes associated with rutile. It occurs along fractures within garnet and shows a distinct anomalous blue colour under crossed nichols.

The presence of only local retrograde minerals indicates that these rocks cooled quickly and did not have a sufficient water content to form new hydrous minerals. Retrograde metamorphism is rare however, and its general absence is consistent with rapid 
exhumation and cooling rates in the Valhalla complex.

\section{Timing relationships between metamorphism and deformation}

The textural and chemical relationships described above provide evidence for a relative chronology of metamorphic and deformation events (Fig. 3.4). The earliest fabric recognized in thin-section occurs as inclusion trails of quartz in garnet which represent $S$, (Fig. 3.4a). These inclusion trails are aligned at an angle to the external foliation and cannot be traced from the garnet to the matrix. Garnets are interpreted to have gro: n over this older foliation, indicating that garnets started to grow after $D_{1}$ (Fig. 3.4b). Peak metamorphic minerals including sillimanite, biotite and $\mathrm{K}$-feldspar are aligned in the main $\mathrm{S}_{2}$ fabric and occur in the pressure shadows of garnet. Sillimanite inclusions in garnet are generally parallel to the external foliation and occur within garnet rims (Fig. 3.4c). This texture demonstrates that sillimanite began to grow during the formation of $S_{2}$ foliation and that garnet was growing before and during the early stages of sillimanite growth and $S_{2}$ formation. Since peak metamorphic minerals are aligned parallel to $S_{2}$ and wrap around garnet, it is interpreted that $S_{2}$ deformation outlasted garnet growth (Fig. 3.4d). Garnets are commonly elongate and lozenge shaped, and oriented at oblique angles to $\mathrm{S}_{2}$. Preferential garnet consumption and resorption on faces parallel to the elongation direction of garnet occur in areas of high stress and occurred during $\mathrm{S}_{2}$ deformation to account for these shapes (Fig. 3.4d).

Gamets are homogeneous except for $\sim 5-10$ mole $\% \mathrm{Fe} / \mathrm{Fe}+\mathrm{Mg}$ zoning at their rims. This rim is concentric and consistent regardless of adjacent phases (Fig. 3.4e). This suggests 
that net-transfer reactions involving the consumption of garnet and production of biotite. were occurring while temperature decreased and are responsible for the increased Fe levels in gamet rims relative to the garnet core rather than $\mathrm{Fe}-\mathrm{Mg}$ exchange via reaction (1). The lack of symplectitic intergrowths of plagioclase. sillimanite and biotite suggests that the GASP reaction (2) is a more likely means of forming plagioclase moats. Increased Fe levels of garnet rims where garnet is not in contact with ferro-magnesium minerals cannot be attributed to the GASP equilibrium. Quartz embayments around garnet are attributed to the consumption of garnet, but a specific reaction for the formation of this texture is lacking. Since $D_{2}$ deformation outlasted the thermal peak of metamorphism, and quartz related to garnet consumption cross-cuts textures attributed to this deformation, it is possible that decompression caused melting. Quartz embayments truncate concentric $\mathrm{Fe} / \mathrm{Fe}+\mathrm{Mg}$ zoning patterns, which suggests that two successive reactions are responsible for zoning followed by garnet consumption (Fig. 3.4e). The first resorbed the garnet, causing concentric zoning (reaction 7), while the second involved consumption and final breakdown of the garnet. The presence of solid (not symplectitic) plagioclase moats suggests that reaction (2) was responsible for the production of these moats, as a result of decompression.

Textures indicative of cooling and retrograde metamorphism, following deformation and decompression, include randomly oriented muscovite that formed as a result of the breakdown of potassium-feldspar, and chlorite that replaced biotite and grew along gamet fractures. Third- and fourth-phase folding events occurred after metamorphism since all minerals are folded including retrograde muscovite (Plate 10d).

Textures and chemical data in samples from domain $1 \mathrm{~b}$ are nearly identical to 
samples from domain la. but may be differentiated by the presence of rutile in some samples

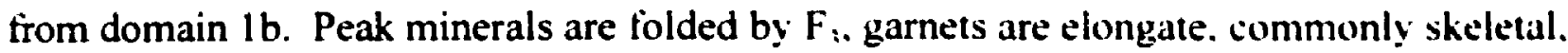
and quartz embayments cross-cut zoning patterns.

\section{Domain 2-Passmore-Vallican}

\section{Textures and mineral compositions}

Domain 2 occurs structurally below domain $1 \mathrm{~b}$ in the Slocan River Valley near Vallican and Passmore. Five samples were studied and analyses were obtained from three of these samples (Fig. 3.2). At this low structural level. samples contain a more intense fabric than samples in domain 1 , which is reflected by the segregation of leucosome layers into thin semi-continuous bands, a strong sillimanite lineation and el gate garnet porphyroblasts around which the transposition fabric is wrapped. Along with these textures observed in hand specimen. samples from domain 2 may be differentiated from domain 1 by the presence of significant plagioclase moats around garnet and by calcium zoning in garnet (Plates 9d, 11).

\section{Garnet zoning}

Garnets are subhedral, commonly elongate porphyroblasts to poikiloblasts, containing fractures oriented $45^{\circ}$ to the extension direction. Garnet in sample PS-28a-93 (Fig. 3.2) has higher grossular content $\left(X_{C_{2}}=0.078-0.102\right)$ than all other samples within the Valhalla complex and is more complexly zoned than other samples. Garnets in sample 
PS-28a-93 exhibit are Ca zoning: from rim to core. grossular content decreases $\sim 5$ mole $\%$ into a trough and then increases 5 mole \% to level off in the core. and finally increases -1 mole \% towards the other rim (Fig. 3.5). Gamet also exhibits increased $\mathrm{Fe} / \mathrm{Fe}+\mathrm{Mg}$ levels of 10 mole $\%$ from core to rims where in contact with plagioclase. Rare symplectites of biotite and quartz are observed in contact with garnet rims in sample PS-28b-93, which would suggest that garnet was being consumed as a result of net-transfer reaction (7), while sillimanite, quartz and biotite were produced. This reaction (7) may also have produced increased $\mathrm{Fe}$ levels in garnet rims not in contact with biotite, but widespread textural evidence is lacking. Solid moats of plagioclase that are not symplectitic may be attributed to the breakdown of garnet via the GASP reaction (2).

In sample PS-28b-93, $\mathrm{Mn}$ is enriched and $\mathrm{Ca}$ is depleted near a $0.5 \mathrm{~mm}$ wide biotite inclusion (Fig 3.3a). This may be due to the introduction of fluids along the fracture in this particular sample. since biotite is not a Ca rich phase. In other samples, $\mathrm{Mn}$ and $\mathrm{Ca}$ are not affected by biotite inclusions (Fig. 3.3c).

\section{Plagioclase}

Plagioclase makes up to $15 \%$ of samples in this southern region and often forms discontinuous moats around garnet rims. The An content of plagioclase moats varies from 37 to 51, within one sample. Portions of the moats that occur in the pressure shadows of the garnet have higher An (51) than portions that are at gamet rims parallel with the elongation direction (An 38). There is no spatial relationship between An content within the moat and distance away from the garnet. Matrix plagioclase in the same sample is An 50 - An 52 . 
Sample PS-28a-93 also contains quartz inclusions that have a thin rim of plagioclase with an An content of 48. which is similar to both matrix plagioclase grains and plagioclase moats in the same sample. This texture also suggests that garnet and quartz were breaking down to form plagioclase via reaction (2). The variability of plagioclase compositions in moats around garnet suggests that growth occurred at different times. in different areas around the garnet.

Sillimanite, quartz and biotite

Sillimanite is abundant in samples from the southern domain making up to $30 \%$ of the rock and occurring as intergrown mats with biotite. Quartz ribbons are partially annealed and seriate grain boundaries and subgrains are common. Quartz rarely occurs as teardrop shaped grains in quartz-biotite symplectites. Biotite makes up to $30 \%$ of the rock and is concentrated in garnet pressure shadows.

\section{Titanium phases}

Rutile and ilmenite are found together in two samples and in contact with each other in one sample. Textures suggesting the timing of formation of ilmenite or rutile relative to one another are absent. Both phases are elongate and aligned parallel to $S_{2}$, and often occur in the pressure shadows of garnet which might indicate their presence at the peak of metamorphism. 
Retrograde metamorphism is represented by randomly oriented muscovite grains that are in close contact with K-feldspar and sillimanite, suggesting the reverse of reaction (9). Chlorite is also present and grew at the expense of biotite. Rutile is commonly associated with chlorite replacing biotite. Chlorite is more common in samples at this structural level (domain 2) and suggests that this area cooled less quickly than domain 1 or that more fluids were present than in other domains, which would have allowed retrograde metamorphism to take place. The introduction of fluids along shear zones may also be responsible for the abundance of retrograde minerals.

\section{Timing of metamorphism with respect to deformation}

Timing relationships between metamorphism and deformation are slightly different than those observed in domain $1 . \mathrm{S}_{\mathrm{T}}$ contains the peak metamorphic minerals of gamet. sillimanite, biotite, $\mathrm{K}$-feldspar, rutile and ilmenite. This fabric wraps around garnet porphyroblasts and the peak metamorphic minerals occur in garnet pressure shadows. Evidence that garnet overgrew a preexisting fabric is rare in domain 2, but some samples do contain aligned quartz inclusions that are oriented obliquely to the external transposition fabric. Sillimanite inclusions in garnet occur near the rim and are commonly parallel to the external transposition foliation $\left(\mathrm{S}_{\mathrm{T}}\right)$. The presence of these two inclusion varieties indicates that garnet overgrew an existing $S_{1}$ fabric, represented by quartz inclusions, and continued to grow during $\mathrm{S}_{\mathrm{T}}$, represented by sillimanite inclusion. Plagioclase moats around garnet are common in this domain and were formed during resorption and consumption of garnet following the thermal piak of metamorphism. Deformation outlasted plagioclase formation 
and the thermal peak of metamorphism because plagioclase is concentrated in garnet pressure shadows and appears to have been consumed along garnet rims parallel to the extension direction. Quartz embayments and textures inferred to be a result of garnet breakdown are less common in domain 2 than in domain 1, and may reflect differences in the ability of reactions to occur. perhaps controlled by kinetics. It may also reflect slight bulk compositional differences or different pressure-temperature conditions in domain 2. Randomly oriented retrograde muscovite is common in domain 2 and suggests that this area remained at a temperature sufficient to grow muscovite. whereas samples from domain 1 cooled more rapidly or had an increased amount of fluids relative to domain 1.

\section{Gwillim Creek windows (sheet la-Domain 3 and sheet (b)}

\section{Textures and mineral compositions}

Four samples of pelitic schist were studied from sheets $1 \mathrm{a}$ and $\mathrm{b}$, the Gwillim Creek windows. These samples are characterized by the strong alignment of minerals within the transposition fabric and by extensive consumpticn of garnet and replacement of biotite by chlorite.

\section{Garnet}

Garnet in the Gwillim Creek windows is commonly elongate, up to $2 \mathrm{~cm}$ long with quartz occupying embayments. Higher $\mathrm{Mg}$ levels in garnet cores and biotite grains from domain 3 (5-10 mole $\%$ higher, $\sim 0.28 \mathrm{X}_{\mathrm{Mz}}$, Appendix $\mathrm{C}$ ), than samples from domains 1 or 
2, reflect a more $\mathrm{Mg}$-rich bulk composition. Garnet cores are homogeneous and rims are elevated in $\mathrm{Fe}(\sim 5 \mathrm{~mole} \%)$ and lower in $\mathrm{Mg}(\sim 5$ mole \%) relative to the core. Ca content in garnet is fairly consistent ( $\sim 5$ mole \%) throughout the core but decreases to less than 5 mole $\%$ at the rim. Ca levels also decrease near quartz (melt?) inclusions within the garnet. by $<5$ mole \% suggesting that modification of garnet rim composition and formation of quartz at the expense of garnet resulted from the same net-transfer reaction, or that two reactions were occurring simultaneously.

Plagioclase, sillimanite, biotite and quartz

Plagioclase makes up 5-15\% of samples from the Gwillim Creek window and has a consistent An composition (32-36). A discontinuous plagioclase moat in sample PCA-20085 , occurs around only one garnet (in the pressure shadow) and has a variable An content of 38 to 45 .

Sillimanite occurs as porphyroblasts up to $2 \mathrm{~cm}$ long. Sillimanite inclusions in garnet are strongly aligned and parallel to the external foliation (Plate $9 b$ ).

Biotite grains located with particular spatial relationships to garnet show a systematic variation in composition. Biotite inclusions in garnet and biotite grains in contact with garnet rims have the highest Fe levels $\left(\mathrm{X}_{\mathrm{Fe}_{\mathrm{e}}} \sim 0.7\right)$. Ti levels in biotite are highest in matrix grains and armoured biotite inclusions. Matrix grains, however, have a variable Ti content $\left(X_{T_{1}}=0.05-0.10\right.$, Appendix $\left.D\right)$, which may not be explained by relative distance away from the garnet. 


\section{Ilmenite and rutile}

Ilmenite and rutile were observed in direct contact in sample PCA-200-85. in a similar relationship as that observed in sample PCA-93-84 from domain 2. Hoth rutile and ilmenite are elongate and aligised in the transposition tabric. In sample PĆA-200-85. rutile is cored by ilmenite. This suggests an increase in pressure, since rutile is on the high pressure side of reaction (3). This texture may be a relict texture preserving part of the prograde path. In sample PS-348-94, also from domain 3, there is abundont rutile, hut ilmenite is absent. Gamets cores in sample PS-348-94 have Fe levels up to 4 mole $\%$ louer than garnet in PCA-200-85 and therefore the absence of ilmenite may be a function of bulk composition.

\section{Retrograde metamorphism}

Retrograde metamorphism is common in samples from the Gwillim Creek window and quite extensive in some samples. Miuscovite halos surround sillimanite porphyroblasts in samples from sheet $1 \mathrm{~b}$ (Plate $11 \mathrm{a}$ ), and chlorite replaced biotite and occurs along garnet fractures in samples from both sheets $1 \mathrm{a}$ and $1 \mathrm{~b}$. The abundance of retrograde minerals in domain 3 is interpreted to be a result of fluid introduction along the GCSZ which allowed retrograde reactions to take place at these deep structural levels.

\section{Timing of metamorphism with respect to deformation}

Aligned quartz inclusions in garnet are absent and therefore evidence for $D_{1}$ is not observed in domain 3 , in contrast to domains 1 and 2. The main fabric in this domain $\left(S_{1}\right)$ 
wraps around garnet porphyroblasts and contains the peak metamorphic mineral assemblages of sillimanite, K-feldspar and biotite. which also occur in garnet pressure shadows. Sillimanite inclusions in garnet occur near the garnet rim and are continuous with sillimanite grains in pressure shadows, suggesting that garnet growth began prior to the development of $S_{1}$ and continued during this deformation period. Rare plagioclase moats around garnet suggest that these rocks underwent a period of decompression following the thermal peak. Consumption of garnet is more extensive than in domains 1 and 2. as exhibited by near skeletal garnets and the abundance of quartz embayments. Retrograde metamorphism occurs as the replacement of biotite by chlorite and is more prevalent in domain 3, relative to domains $I$ and 2. The main fabric in samples from the Gwillim Creek windows is a transposition fabric $\left(\mathrm{S}_{\mathcal{T}}\right)$ associated with the GCSZ. It is uncertain if $S_{2}$ in domain $\mathbf{I}$ and the GCSZ are synchronous. $\mathrm{S}_{\mathrm{T}}$ transposes all folds, contains the peak metamorphic mineral assemblage a: 1 outlasts metamorphism. $S_{T}$ in domain 3 could be a result of the same $D_{2}$ deformation period which occurred in domain 1, or it could be a result of shearing along the GCSZ which realigned metamorphic minerals.

\subsubsection{Amphibolite gneiss}

\section{Textures and mineral compositions}

Amphibolite gneisses have $\approx$ ariable range in mineral assemblage, grain size and textur, reflecting different bulk compositiotis inid different possible protoliths. All samples of amphibolite gneiss were collected from sheet $l c$ in domains 1 and 2 . They contain the 
assemblage hornblende - plagioclase - quartz \pm garnet \pm biotite \pm ilmenite \pm titanite \pm rutile \pm clinopyroxene. Clinopyroxene was observed in only one tine-grained sample which is devoid of garnet. Some samples contain euhedral hornblende and lack biotite. while others contain ragged homblende and biotite. The presence of hornblende, plagioclase and garnet is indicative of amphibolite-facies metamorphism. The presence of pyroxene (diopside) in one sample also containing hornblende and plagioclase. suggests that these rocks may have reached lower granulite-facies metamorphism. The presence of pyroxene may also be a function of a more Ca rich bulk composition, allowing clinopyroxene to form. The textural and mineralogical variation in amphibolite gneisses is reflected in the wide range of analysed mineral compositions.

\section{Garnet}

Garnets are rounded, often skeletal, and embayed by quartz and are up to $6 \mathrm{~mm}$ in diameter (Plate 11b). Poikiloblastic gamets contain up to $50 \%$ inclusions of quartz, hornblende and ilmenite. Inclusions are often concentrated in garnets cores as they are in pelitic schists (Plate 11c). Plagioclase commonly forms coronas around garnets and may be a result of the reaction (Plate 12a):

(13) tschermakitic-homblende + garnet + quartz $=$ tremolitic-homblende + plagioclase.

The formation of plagioclase moats around garnet in mafic gneisses may be similar to the formation of plagioclase as a result of garnet breakdown in pelitic schists.

Garnets have $\sim 10$ mole $\%$ lower almandine component and $\sim 10-15$ mole $\%$ higher grossular component than garnets in pelitic schists. The mole \% of almandine, pyrope, 
grossular and spessartite do not vary more than $\sim 5$ mole $\%$ within garnet (Fig. 3.6). Some samples exhibit slight decreases in $\mathrm{X}_{\mathrm{CA}_{4}}$ towards the rim and then a sharp increase at the rim (Fig. 3.6). The general decrease in Ca towards the rim may be attributed to plagioclase moat growth around the garnet porphyroblasts. where plagioclase grew at the expense of garnet. The final increase of $\mathrm{Ca}$ at garnet rims may be attributed to late fluid infiltration or possibly an increase in pressure. Fe also increases gradually from core to rim and drops sharply at the rim.

\section{Plagioclase and quartz}

Plagioclase and quartz make up $20-35 \%$ of the rock and are usually subequal in modal abundance. Systematic differences in composition between matrix grains and grains in contact with garnet are not observed. Plagiociase occurring as moats around garnet have An contents ranging from $35-60$ and have no spatial pattern. This wide range probably represents the continuing growth of plagioclase around garnet rims during decompression or continued heating following the peak of pressure. There are no sympathetic changes of the grossular content in garnet with respect to An content in plagioclase moats.

\section{Hormblende}

Most mafic gneisses in the Valhalla complex contain up to $65 \%$ homblende. In plane polarized light homblende varies from light brown, to olive green and dark green. Grains are equigranular and have sharp grain boundaries with adjacent grains of plagioclase. quartz, biotite and garnet. Homblende is usually evenly distributed within a sample but rarely forms 
discrete bands. alternating with quartz and feldspar. Fe/Fe+Mg ratios in both matt $\mathrm{x}$ grains and those in contact with garnet range trom $-0.45-0.58$, in all samples. Inclusions in sample PS-26-93 have a lower Fe level $(\sim 41$ mole \%) than matrix homblende grains $(-45$ mole \%) in the same sample (Appendix D).

\section{Biotite}

' iotite makes up to $30 \%$ of mafic gneisses. In some samples, biotite and hornblende are equal in proportion and both are aligned in the foliation plane. The large modal amounts of biotite in some mafic gneisses may be attributed to a sedimentary origin. In other samples, biotite is a younger phase that overgrows homblende and along fractures in garnet. Biotite was analysed in three samples, and although intersample variance is large (Appendix D). grains within a sample are relatively homogeneous and do not vary more than 0.036 $\mathrm{Fe} / \mathrm{Fe}+\mathrm{Mg}$ per sample.

\section{Rutile, ilmenite and titanite}

Titanium bearing phases include ilmenite, titanite and rutile. Ilmenite constitutes up to $5 \%$ of the mafic gneisses as equigranular, disseminated grains and as vermicular inclusions in garnet. Rare vermicular intergrowths of ilmenite (possibly magnetite) and an unknown mineral (possibly orthopyroxene) may be similar to symplectites described by Barker (1990, p. 56) and Mackenzie and others (1982, p. 53), but their significance is unknown. Titanite and rutile are less common. In sample PS-27b-93, an inclusion of carbonate in garnet contains an inclusion of rutile, but its significance is also unknown. 
Plagioclase forms rare coronas around ilmenite inclusions in garnet. as well as embayments in garnet.

\section{Retrograde metamorphism}

Retrograde minerals are sparse in amphibolite gneisses of the Valhalla complex. Chlorite commonly replaces biotite, and plagioclase commonly contains retrograde muscovite (sericite) along fractures.

\section{Timing of metamorphism with respect to deformation}

The peak metamorphic mineral assemblage in amphibolite gneisses includes garnet. hornblende, biotite, and plagioclase and is aligned in $\mathrm{S}_{2}$. Biotite. homblende and plagioclase have sharp grain boundaries when in contact with garnet and are found in the pressure shadows of garnet, indicating that the peak of metamorphism was synchronous with deformation $\left(D_{2}\right)$. Inclusions in garnet are not aligned und therefore the timing of events before $S_{2}$ is not recorded in amphibolite gneisses. Garnet is often skeletal and contains the same sort of quartz embayments that some garnets in pelitic schists do. These embayments are interpreted to have formed during defornation, but following or during the thermal peak of metamorphism.

\subsubsection{Marbles and calc-silicate gneisses}

Mineral assemblages in carbonate rocks of the Valhalla complex are in general agreement with metamorphic conditions recorded by pelitic schists and amphibolite gneisses. 
Due to the absence of suitable mineral assemblages and the fact that carbonate rocks do not generally exhibit strong fabrics. marbles and calc-silicate gneisses in the Valhalla complex are not exceptionally suitable for thermobarometry or deciphering the relationships between metamorphism and deformation. Marbles in the Valhalla assemblage contain the assemblage calcite \pm phlogopite \pm diopside \pm forsterite \pm titanite \pm scapolite \pm tremolite \pm graphite \pm apatite. Rutile and amphibole (hornblende?) occur in one sample, while chlorite and plagioclase occur as retrograde minerals. Calc-silicate gneisses contain the assemblage quartz - diopside - titanite \pm scapolite \pm phlogopite \pm plagioclase \pm potassium feldspar \pm tremolite \pm spinel. Green amphibole occurs as a retrograde mineral replacing diopside. Granoblastic textures predominate, although diopside often forms blocky irregular shaped masses, as opposed to disseminated grains. Titanite is present in almost all samples ds rounded grains, making up to $5 \%$ of the rock.

Reesor (1965, p. 50) suggested that the preserice of scapolite- and forsterite-bearing assemblages in the Valhalla complex indicates lower granulite facies. Typical granulite assemblages contain wollastonite, scapolite and grossular (Harley i988), huwever wollastonite and grossular are absent in the Valhalla complex.

Scapolite breaks down via the fluid independent reaction:

(6) anorthite + calcite $=$ meionite $($ Ca-scapolite $)($ Goldsmith and Newton 1977).

In one sample, rims of plagioclase and symplectites of plagioclase and calc: 4 cur around scapolite porphyroblasts (Plate 14b). In other samples, the breakdown of scapolite is more advanced and plagioclase and calcite replace half of the original scapolite grain. This is interpreted to be a retrograde effect of reaction (6) and implies significant cooling, either 
isobaric or polybaric, since the reaction curve has a very steep pressure-temperature slope. Scapolite porphyroblasts in marbles and calc-silicates are virtually the same in composition with equivalent anorthite contents $(\mathrm{EqAn}=[(\mathrm{Al}-3) \times 100] / 3)$ contents of $64-74$. Sulphur is absent and chlorine ranges from $\mathrm{X}_{\mathrm{Cl}}=0.12-0.20$. Fixed activities were calculated using the solution of model of Oterdoom and Gunter (1983) which is based on the experimental data of Goldsmith and Newton (1977). Using this activity model in conjunction with TWEEQU, temperatures of approximately $800{ }^{\circ} \mathrm{C}$ were calculated. Symplectites of plagioclase and quartz (myrmekite) are also present in marbles and calc-silicates.

Diopside is ubiquitous in calc-silicate gneisses comprising up to $4 . \%$ of the mode, but it is less abundant in marbles. Retrograde amphibole (tremolite) commonly replaces diopside in a haphazard fashion. Diopside is more iron-rich in calc-silicate gneisses $(\mathrm{Fe} / \mathrm{Fe}+\mathrm{Mg}=0.393-0.526)$ than in marbles $(\mathrm{Fe} / \mathrm{Fe}+\mathrm{Mg}=0.014)$. Forsterite occurs as up to $2 \mathrm{~mm}$ sized porphyroblasts. The presence of forsterite has been attributed to the dedolomitization of marbles in prograde metamorphism (Spear 1993, p. 455) and is consistent with the absence of dolomite in marble samples. Mg levels in forsterite are approximately $\mathrm{X}_{\mathrm{F}_{0}}=96$. Non-pleochroic amphibole occurs in marbles and has high magnesium levels $(\mathrm{Fe} / \mathrm{Fe}+\mathrm{Mg}=0.030)$.

\subsubsection{Ultramafic schist}

Ultramafic schists are restricted to unit 8 of the Valhalla assemblage which occurs in the structurally highest sheet (1c, domain 1) and one outcrop on the east side of the complex near Slocan and the SLF (Map 1). Ultramafic schists in the Valhalla complex 
contain the assemblage tremolite - olivine - orthopyroxene \pm clinopyroxene \pm phlogopite \pm serpentine \pm chromite. Olivine is highly fractured and altered to serpentine (Plate 12c). Diopside is overgrown by tremolite. Tiemolite is the main component in some samples. making up $90 \%$ of the rock. Grains are randomly oriented. coarse-grained and radiating. Phlogopite overgrows all minerals except serpentine (Plate 12c).

The first appearance of tremolite and the disappearance of diopside may have been achieved by the reaction:

(14) diopside + antigorite $=$ forsterite + tremolite $+\mathrm{H}_{2} \mathrm{O}$.

however, as Spear (1993, p. 472) notes. the assemblage diopside - forsterite reappears at higher temperatures. Enstatite occurs as broken up grains separated by phlogopite which has grown along fractures. Enstatite appears at approximately $675{ }^{\circ} \mathrm{C}(-7 \mathrm{kbars})$, via the reaction:

(15) anthophyllite + forsterite $=$ enstatite $+\mathrm{H}_{2} \mathrm{O}$ (Fig.3.7)

and is stable to higher temperatures. Orthopyroxene is also partially replaced by tremolite (Plate 12d) which might suggest that the reaction:

(16) forsterite+tremolite $=$ enstatite + diopsicie $+\mathrm{H}_{2} \mathrm{O}$

has taken place (Fig. 3.7). The absence of anthophyllite does not constrain peak temperatures even though it has a relatively low stability field $\left(600-800{ }^{\circ} \mathrm{C}\right.$, Spear 1993, p. 470). The lack of anthophyllite does not constrain pressure-temperature conditions well because reaction (15) consumes anthophyllite at $\sim 650-700^{\circ} \mathrm{C}$ at $7 \mathrm{kbars}$ (Fig. 3.7) in quartz free mineral assemblages.

Metamorphic mineral assemblages in ultramafic schists are more temperature 
dependent than pressure dependent because most reactions curves are quite steep in the system $\mathrm{MgO}-\mathrm{CaO}-\mathrm{SiO}_{2}-\mathrm{H}_{2} \mathrm{O} \pm \mathrm{CO}_{2}$. Metamorphic conditions in ultramafic schists are not well constrained because the minerals present have a wide stability and there are few reactions indicating the breakdown of these minerals. Mineral assemblages in ultramafic schists indicate general agreement with mineral assemblages and thermobarometry of pelitic schists and amphibolite gneisses and are consistent with amphibolite facies metamorphism.

\subsubsection{Summary}

From the petrographic and chemical observations discussed in the previous sections, it may be concluded that peak metamorphic mineral assemblages from four different bulk compositions and three different domains are consistent with upper amphibolite facies metamorphism. Peak metamorphic minerals (sillimanite, K-feldspar, biotite) in pelitic schists are aligned in $S_{2}$, in domain 1 , and $S_{\mathrm{T}}$ in domains 2 and 3 , and also occur in the pressure shadows of garnet, indicating that metamorphism was synchronous with deformation in all three domains. In detail, some reaction textures are observed in domains 2 and 3 that allow a distinction from domain 1. Plagioclase moats around garnet and Ca zoning in garnet is more pronounced in domain 2, while elongate garnets, extensive quart? embayments in garnet, and retrograde chlorite are typical of domain 3. Domain 3 also has a more $\mathrm{Mg}$ rich pelitic bulk composition than domains 1 or 2 , which is reflected in up to 15 mole \% higher $\mathrm{Mg}$ levels in both garnet and biotite in domain 3.

\subsection{Thermobarometry petrolory and comparison of structural levels}


Petrographic observations suggest that metamorphism was synchronous with deformation in all three domains. Petrography also indicates that peak metamorphic conditions are similar at different structural levels with a peak metamorphic mineral assemblage of biotite. garnet, sillimanite. K-feldspar. ilmenite and rutile in pelitic schists. Thermobarometry was employed to quantify the metamorphic conditions of domains 1. 2. and 3. at different structural levels. Mineral textures and chemical data are interpreted to link compositions and pressure-temperature conditions of mineral pairs to portions of the pressure-temperature path.

\subsubsection{Pelitic schist}

Only portions of the pressure-temperature path are preserved in pelitic schists of the Valhalla complex. Estimates of the peak metamorphic conditions can be made from petrographic observations, but thermobarometric data are not representative of the peak because garnet and biotite compositions are thought to have been modified by the effects of diffusion and retrograde reactions. Plagioclase and biotite in the matrix are interpreted to have been modified during or following the thermal peak, by garnet-consuming reactions ( 7 ) or (8) and are not in equilibrium with garnet cores. Net-transfer reactions are thought to have been active in some samples during cooling and exhumation due to the presence of continuous and concentric $\mathrm{Fe} / \mathrm{Fe}+\mathrm{Mg}$ zoning in garnets, bearing no relationship to other ferro-magnesium minerals. Biotite grains formed as a result of these reactions have higher $\mathrm{Fe} / \mathrm{Fe}+\mathrm{Mg}$ ratios than biotite present at the peak of metamorphism, and therefore produce anomalously high temperatures when combined with data from garnet cores $(\sim 850-1000$ " $\mathrm{C})$. 
Since biotite grains have a wide range in composition, it was assumed that only grains proximal to garnet rims, with lower Fe levels than biotite grains in contact represent near peak compositions and have not been affected by later Fe- $\mathrm{Mg}$ exchange. Proximal biotite grains record conditions near but following the thermal peak of metamorphism (T1). Garnet rims in contact with biotite yield the lowest temperatures (T2) due to Fe-Mg exchange. as a result of cooling.

Plagioclase shows little variation between grains in the matrix and those in contact with garnet, and therefore pressure calculations are problematic. If plagioclase grains in the matrix had lower An contents than plagioclase moats around garnet it might be suggested that matrix grains are representative of peak pressures and plagioclase moats represent part of the decompression path. The same might be said if matrix plagioclase grains were zoned and had lower An content in the core than at the rim. These relationships do not exist in samples from the Valhalla complex because no zoning patterns or systematic relationships between An content and textural variety of plagioclase were observed. For this reason, plagioclase grains in contact with garnet (as moats or otherwise) are believed to be in chemical equilibrium with garnet and produce pressures interpreted to be near- to post-peak conditions. Where plagioclase is in contact with garnet, it usually occurs as moats, suggesting that it formed as a result of a decompression reaction such as (2). If the plagioclase moats and the proximal biotite grains were both formed as a result of garnet consumption, then all three minerals should be in relative chemical equilibrium and produce genuine data. This will produce quantitative data for near-peak temperatures and part of the decompression path of pelitic schists in the Valhalla complex. 
When applying the GRAIL barometer. chemical equilibrium was assumed between gamet rims and proximal matrix biotite. and ilmenite. Since the GRAIL. equilibrium is Fe dependent. any increase in $\mathrm{Fe}$ in garnet rims due to $\mathrm{Fe}-\mathrm{Mg}$ exchange between garnet and biotite will give an erroneously higher pressure. where it intersects with the garnet-biotite thermometer.

Domain li la - The Valhalla assemblage (sheet lc, west side) and 16 -Slocan Ridge, Airy Creek

Thermobarometry was carried out on 6 samples from domain la and 2 samples from domain $1 \mathrm{~b}$. The intersection between the gamet-biotite thermometer and GASP barometer was employed in all samples and the GRAIL barometer was used in one sample from domain lb. In domain la, on the west side of the Valhalla complex average post peak temperatures (T1) and pressures $(\mathrm{P} 1)$ range from $736 \pm 10$ to $777 \pm 50^{\circ} \mathrm{C}$ and $5.8 \pm 0.3$ to $7.1 \pm 1$ kbars. respectively (Table 3.2, Figs. 3.8-3.9). The magnitude of the error represents the standard deviation among the number of calculations carried out in one sample (See Table 3.2). In domain lb, garnet-biotite temperatures ( $\mathrm{Tl}$ ) and GASP pressures $(\mathrm{Pl})$ range from $790 \pm 26$ to $764 \pm 21^{\circ} \mathrm{C}$ and $6.5 \pm 0.7$ to $7.5 \pm 0.3$ kbars respectively. The GRAIL barometer intersects the garnet-biotite thermometer at nearly the same pressure $(7.3 \pm 0.3 \mathrm{kbars})$ as the GASP barometer, implying equilibration of all minerals at the same pressure and temperature (Fig. 3.8, 3.9, Table 3.2). These results are consistent with the metamorphic mineral assemblage and with petrographic observations suggestive of textural equilibrium. Later and lower post-peak temperatures on the cooling path (T2, Table 3.2) of $600 \pm 16$ to $721 \pm 13^{\circ} \mathrm{C}$ 
are recorded by biotite grains in contact with garnet rims. Since no systematic difference in composition was observed between plagioclase grains in direct contact with gamet or matrix plagioclase grains, reliable pressures cannot be associated with these temperatures (T2). Pressures produced are merely a function of moving down the same GASP isopleth used for the calculation of $\mathrm{Pl}$ to where it intersects the lower temperature (T2) (Fig. 3.8). In sample PS-277-94. discontinuous plagioclase rims have a variable An content. yield pressures from 4.7 to $8.3 \mathrm{kbars}$, and have likely recorded a period of decompression.

Biotite inclusions and garnet cores yield temperatures of $600-730^{\circ} \mathrm{C}$ (Table 3.2) which are lower than estimates for the thermal peak. These inclusions appear to have been affected by retrograde Fe-Mg exchange and therefore do not record peak temperature or any part of the prograde pressure-temperature path.

Armoured biotite inclusions are biotite inclusions enclosed in quartz inclusions contained within garnet. Spear and Parrish (in prep.) pair armoured biotite inclusion compositions with gamet core compositions and interpret the temperature to represent apparent thermal peak. Armoured biotite inclusions from this study yield temperatures of approximately $\sim 850^{\circ} \mathrm{C}$ (Table 3.2 ). These data are interpreted to represent peak thermal conditions.

The simultaneous increase in Fe levels of both garnet and biotite may be attributed to reaction (7) proceeding with decreasing temperature. Normal $\mathrm{Fe}-\mathrm{Mg}$ exchange via reaction (1) would result in increased $\mathrm{Fe}$ in garnet and decreased $\mathrm{Fe}$ in biotite as temperatures decreased. The simultaneous progress of reactions (1) and (7) causes Fe to increase in both garnet and biotite. The assemblage biotite-garnet-sillimanite- $\mathrm{K}$-feldspar-quartz- $\mathrm{H}_{2} \mathrm{O}$ is 
divariant in the KFMASH system. forming a $T-X(F e)$ loop at constant pressure in which the $\mathrm{Fe}$ in both garnet and biotite increase as temperature decreases and garnet is consumed while biotite is produced. In sample PS-4-94 plagioclase is ahundant $(-20 \%)$, sillimanite is rare and biotite is commonly randomly oriented. This might suggest that hiotite formed via reaction (8) after the peak of metamorphism and possibly after deformation had ceased (or at least decreased). From chemical analyses. it is not apparent that sample PS-4-93 has a different bulk composition than samples with abundant sillimanite and little plagioclase.

Plagioclase inclusions were analysed in two samples and their compositions record pressures of 4.6 to 5.8 kbars (at fixed temperature -800 "C. Table 3.2) when paired with compositions from garnet cores. Pressure calculations using garnet core compositions and plagioclase inclusions were done assuming that sillimanite was present. These pressures are lower than pressures calculated from garnet rims and matrix plagioclase grains in the same sample even at temperatures of $\sim 800^{\circ} \mathrm{C}$ (Table 3.2). These plagioclase inclusions mav have been enclosed by the garnet prior to the thermal peak. before the garnet was compositionally homogenized. The relatively low diffusion rate of $\mathrm{Ca}$ would have minimized cation exchange between garnet and plagioclase. and it is unlikely that garnet cures and plagioclase inclusions re-equilibrated after gamet homogenisation.

Thermobarometric data from all samples in domains $\mathrm{la}$ and $\mathrm{lb}$ are consistent with each other and with petrographic observations, peak metamorphic mineral assemblages and mineral reactions. These data indicate temperatures and pressures of $\sim 750^{\circ} \mathrm{C}$ and $\sim 6.5 \mathrm{kbars}$. It is uncertain if biotite inclusion compositions in gamet reflect conditions representative of the prograde pressure-temperature path when paired with the compositions of garnet cores. 
The low temperatures recorded by biotite inclusions and garnet cores $\left(-650^{\circ} \mathrm{C}\right)$ suggest that biotite inclusions underwent $\mathrm{Fe}-\mathrm{Mg}$ exchange during cooling. Plagioclase inclusions were are interpreted to have been frozen in the garnet during the prograde path an's had no apparent mechanism to reequilibrate with the garnet core which was compositionally homogenised during the thermal peak of metamorphism.

\section{Domain 2 - Vallican area}

Samples near Vallican (domain 2) are structurally deeper and record near-peak temperatures $(\mathrm{Tl})$ and pressures $(\mathrm{Pl})$ from $819 \pm 48$ to $935 \pm 27^{\circ} \mathrm{C}$ and $9.8 \pm 1.5$ to $11.5 \pm$ 0.5 kbars. respectively. Pressures and temperatures fall partly into stability fields indicated by mineral assemblages and textures, however they also overlap the kyanite and orthopyroxene stability fields. Kyanite has been reported in one sample from this domain by Carr (pers. comm. 1994). GRAIL data intersect the garnet-biotite thermometer at substantially lower pressures $(7.1 \pm 0.2$ to $7.6 \pm 0.3 \mathrm{kbars}$, Fig. 3.9. Table 3.2$)$ than the C 4 SP barometer. This difference will be addressed in the discussion section (3.4.3). Biotite rims in contact with gamet rims record lower temperatures (T2) than $\mathrm{T} 1$ but they are higher than those of domains la and $\mathrm{lb}$ at higher structural levels.

\section{Domain 3 - shee' la (lower Gwillim Cree; window)}

Thermobarometry was carried out on two samples from the GCW (sheet la) and record garnet-biotite temperatures (T1) and GASP pressures (P1) of $849 \pm 50$ to $901 \pm 60^{\circ} \mathrm{C}$ and $8.5 \pm 1$ to $9.2 \pm 1.4$ kbars, respectively (Table 3.2, Fig. 3.9). The GRAIL barometer 
intersects the garnet-biotite thermometer at lower pressures $(6.3 \pm 0.1 \mathrm{kbars})$ than the GASP barometer does (Table 3.2. Fig. 3.9). Biotite grains in contact with garnet rims (T2) record temperatures of $690 \pm 58$ and $796 \pm 1{ }^{\circ} \mathrm{C}$ (Table 3.2).

\subsubsection{Amphibolite gneiss}

Thermobarometric data from homblende inclusions in garnet. garnet-biotite pairs and garnet-hornblende pairs in amphibolite gneisses record a large portion of the pressure temperature path. Temperatures and pressures recorded by amphibolite gneisses are in general agreement with those calculated from garnet rims and proximal biotite grains in pelitic schists (Table 3.3). Garnet in amphibolite gneisses exhibits only subtle zoning patterns, and therefore pressures and temperatures do not vary much between pairs of 11 garnet rims in contact with ferro-magnesiunn mirırals (biotite and hornblende), 2) garnet rims in contact with quartz or plagioclase and proximal biotite or hornblende or, 3) the average of all data from the garnet and garnet paired with grains of hornblende and biotite in direct contact with garnet or within the matrix. Three samples were studied from domain la, one sample from domain $\mathrm{lb}$, and one sample from domain 2 (Figs. 3.11, 3.12. Table 3.3). Garnet-hornblende-plagioclase equilibria (reactions 4 and 5) in domain la record temperatures and pressures that range from ó $91 \pm 12$ to $780 \pm 11{ }^{\circ} \mathrm{C}$ and $6.9 \pm 0.5$ to $8.0 \pm$ $0.3 \mathrm{kbars}$, respectively. Samples from domain $\mathrm{lb}$ record temperatures and pressures from $755 \pm 22$ to $789 \pm 32{ }^{\circ} \mathrm{C}$ and $8.5 \pm 0.6$ to $10.3 \pm 1.7$ kbars.

PS-52-94 from domain $1 \mathrm{~b}$ has a wider range in temperature $\left(750-848{ }^{\circ} \mathrm{C}\right)$ and pressure (8.4 - $13.4 \mathrm{kbars})$ than all other samples, reflecting the variable composition of 
plagioclase moats surrounding gamet (Appendix D). Inclusions of homblende in gamet give slightly lower temperatures $\left(642-682{ }^{\circ} \mathrm{C}\right)$ compared to grains of hornblende in either the matrix or in direct contact with garnet (Table 3.3). Since plagioclase inclusions in garnet were absent in samples with homblende inclusions. no pressure data were obtained. If hornblende inclusions are paired with matrix plagioclase, assuming that plagioclase compositions did not change, then pressures are in the range of $6.5 \mathrm{kbars}$, and are consistent with a clockwise pressure-temperature path.

The intersection of garnet-biotite pairs. using reactions (1) and (4), record higher temperatures than gamet-hormblende temperatures (Figs. 3.11, 3.12). These data range from $842 \pm 7$ to $856 \pm 23^{\circ} \mathrm{C}$ in domain la and $827 \pm 27^{\circ} \mathrm{C}$ in domain 2, and are higher than garnet biotite temperatures (T1) recorded by pelites at the same structural level.

\subsubsection{Summary}

Tr. mobarometric data from the three domains are summarized in Figures 3.9. 3.10 and 3.12 and Tables 3.2 and 3.3. The main conclusions that may be drawn from textural and thermobarometric data are: (i) samples from the structurally highest domains ( $1 \mathrm{a}$ and $\mathrm{lb}$ ) record the lowest and most consistent inter- and intra-sample pressures and temperatures; (ii) GRAIL data are consistent with GASP data in domain 1; (iii) domain 2 contains significant plagioclase moats and $\mathrm{Ca}$ zoning in garnet: (iv) samples in domain 3 have a $\mathrm{Mg}$ bulk composition higher in $\mathrm{Mg}$ than samples from domains 1 or 2 and contain abundant retrograde chlorite and entensive quartz embayments in gamet; ( $v$ ) domains 2 and 3 have a wide range in temperature and pressure within and between samples. some of which overlap data from 
domain 1: (vi) GRAIL data are consistently lower than GASP data in domains 2 and $3:$ and (vii) the similarity of textures and thermobarometric data between domain 2 (Vallican area) and domain 3 (lower Gwillim Creek window - sheet la) is consistent with the structural projections (Cross-section A) and the interpretation that the Gwillim Creek shear zones resurface in the Passmore-Vallican area (Cook et al. 1988).

\subsubsection{Discussion}

In domains 2 and 3 pressures generated using the intersection between the GRAIL. barometer and the garnet-biotite thermometer are generally lower than those of GASP (Figs.3.8. 3.9). GRAIL pressures are fairly constant when calculated using garnet rims and biotite grains in direct contact with garnet. garnet rims and proximal bistite grains, and garnet cores and matrix biotite grains. GRAIL data are $\sim 3$ kbars lower than GASP data in both domain 2 and domain 3. Variation in An content of plagioclase occurs within plagioclase moats surrounding garnet in all three domains, but does not result in a significantly wide range ( $\sim 9-12 \mathrm{kbars})$ in pressures using the GASP equilibrium within the same sample except for one sample (PS-28a-93, Fig. 3.9). The difference in data from these two barometers may be a result of either different closure temperatures, or disequilibrium.

The consistently $3 \mathrm{kbar}$ lower values recorded by the GRAIL equilibrium suggests that, on the scale of the sample, minerals involved in the GASP and GRAIL equilibria did not equilibrate at the sanie pressure and temperature, but that on a local scale, minerals involved in the individual equilibria were in equilibrium. The interpretation that GRAIL pressures represent a later point on the pressure-temperature path at which mineral rims were 
in equilibrium is consistent with the GRAIL equilibrium involving $\mathrm{Fe}-\mathrm{Mg}$ exchange. which is easier to activate than the breaking of $\mathrm{Ca}-\mathrm{Si}-\mathrm{Al}$ bonds required for the GASP reaction to proceed. Since the GRAIL barometer and the garnet-biotite thermometer both involve FeMg exchange. it is assumed that they represent the same point on the pressure-temperature path. No temperature may be confidently associated with the GASP pressure data in domains 2 and 3. GASP pressures are likely to have had a higher temperature associated with them. The observation of kyanite in one sample by Carr (pers. comm. 1994) and the fact that some GASP pressure data plot in the kyanite stability field suggest that these data are representative of near peak pressures.

The wide variation in temperature data from domains 2 and $3\left(-775-980^{\circ} \mathrm{C}\right)$ may be the result of increased Fe levels due to the downward progression of reaction (7). This reaction may not have proceeded in domain 1 because of rapid cooling and quenching at higher structural levels, whereas domains 2 and 3 may have remained at high temperatures longer allowing reaction (7) to proceed.

Alternatively, the wide range in plagioclase moat compositions (An 38-52) and calculated pressures, and the fact that GRAIL and GASP data do not intersect may be attributed to disequilibrium. Disequilibrium may be the a result of two processes: 1) fluids which were introduced along shear zones, and 2) shearing itself. Strain fabrics are quite strong in some samples and the effects of mechanical transport or enhanced diffusion are unknown. Biotite grains that may have never been in chemical equilibrium with garnet may now be in relatively close proximity, due to deformation.

Temperatures calculated using the garnet-biotite thermometer in amphibolite gneisses 
yield temperatures up to $150^{\circ} \mathrm{C}$ higher than those calculated using the gamet-hornblende thermometers. at the same pressure (Table 3.3). The large variation between garnet-biotite data in amphibolite gneisses and pelitic schists may be a function of more Mg rich bulk compositions (amphibolites) recording higher temperatures. Mg rich bulk compositions generally record higher temperatures. This is exemplified by reaction (1) where higher temperatures are recorded by pyrope-rich - annite-rich pairs. Garnet rims in direct contact with biotite grains produce high temperatures $\left(-850^{\circ} \mathrm{C}\right)$ and show little evidence of $\mathrm{Fe}-\mathrm{Mg}$ exchange during cooling. Temperatures recorded by garnet-biotite pairs in amphibolite gneisses are in agreement with temperatures recorded by armoured biotite inclusions and garnet cores in pelitic schists which suggests that peak temperatures were approximately 850 ${ }^{\circ} \mathrm{C}$.

The variation (up to $150^{\circ} \mathrm{C}$ ) between garnet-hormblende and gamet-biotite temperatures within amphibolites may be a result of the two equilibria recording different portions of the pressure-temperature path. Garnet-biotite pairs record an earlier portion of the pressure-temperature path close to the thermal peak, while garnet-homblende pairs record part of the cooling path. These data are consistent with petrographic observations and the peak metamorphic mineral assemblage in pelitic schists and amphibolite gneisses (Fig. 3.12). Alternatively, garnet-homblende temperatures may be less reliable due to variable oxidation states of homblende relative to the thermodynamic calibration and therefore produce lower temperatures than the gamet-biotite equilibrium.

The fact that GRAIL pressures are consistently lower than GASP data in the structurally low domains ( 2 and 3 ) suggest a period of decompression following the peak of 
metamorphism. GRAlL data at higher structural levels (domain 1) do not record this period of exhumation because they were exhumed early and the mineral system cooled quickly. Samples at deeper structural levels (domains 2 and 3) remained hot for a longer period.

\subsection{Conclusions}

Textural observations and thermobarometric data are summarized in Figures 3.4, and 3.8-3.12. respectively. Subtle textural differences observed in thin-section characterize the three domains of paragneisses at three different structural levels in the Valhalla complex. The thermal peak of metamorphism was synchronous with the main $D_{2}$ fabric forming deformation period in all three structural levels and all geographic areas. Peak minerals include biotite, sillimanite. K-feldspar, rutile and ilmenite, all of which are aligned in the main fabric $\left(\mathrm{S}_{2}\right.$ and $\left.\mathrm{S}_{\mathrm{T}}\right)$ and occur in the pressure shadows of garnet porphyroblasts. This fabric represents $D_{2}$ in sheet $1 \mathrm{c}$, shearing on the GCSZ in the lower reaches of the Slocan River Valley and in the lower Gwillim Creek window. Textural and thermobarometric data indicate that shearing in the Vallican area and the Gwillim creek windows was synchronous with $\mathrm{D}_{2}$ in sheet 1 . GRAIL pressures are consistently 3 kbars lower than GASP pressures within samples from domains 2 and 3 and are interpreted to record lower post-peak portions of a decompression path as a result of different closure temperatures of the two equilibria. Samples in the Vallican area also contain plagioclase moats which record a wide range of pressures ( $7-11$ kbars) attributed to continued plagioclase growth during this period of decompression. GRAIL and GASP data are nearly identical at higher structural levels (Figs. 3.8. 3.9) and may not have recorded this period of decompression because rapid exhumation 
"froze" both equilibria. In domains 2 and 3 the GASP reaction was "frozen", while minerals involved in the GRAIL reaction continued to reequilibrate along the decompression path (Figs. 3.8. 3.9). Consumption of garnet and retrograde metamorphism which occurred during cooling was more pronounced in the Gwillim Creek windows. due possibly to the introduction of fluids which facilitated melting reactions.

Amphibolite gneisses also record a portion of the cooling and decompression pressure-temperature path. Garnet-biotite equilibria record near thermal peak conditions and garnet-hornblende pairs record post-peak temperatures along the cooling path (Fig. 3.12). Garnet-biotite temperatures recorded by amphibolite gneisses are consistent with garnetarmoured biotite inclusion temperatures recorded by pelitic schists and are representative of peak thermal conditions $\left(\sim 850^{\circ} \mathrm{C}\right)$. Marbles record temperatures of at least 800 " $\mathrm{C}$, as indicated by the breakdown of scapolite to plagioclase and calcite.

Textures relating metamorphism and deformation and thermobarometry do not clearly record a clockwise pressure-temperature path for the Valhalla complex. In domains 2 and 3 pressures recorded by the GRAIL equilibrium are $\sim 3 \mathrm{kbars}$ lower than data recorded by the GASP equilibrium. Garnet-biotite temperature data are interpreted to be in equilibrium with the GRAIL pressure data and therefore temperatures associated with the GASP pressure data are likely to have been higher. The compositions of hornblende inclusions in garnets of amphibolite gneisses suggest that they were enclosed along the prograde portion of a clockwise pressure-temperature path. Pressures calculated from plagioclase inclusions in garnet of pelitic schists record low pressures (6.5 kbars), which possibly suggest that rocks underwent a counter-clockwise pressure temperature path. 
Reliable pressure-temperature data could not be obtained from these inclusions because mineral assemblages did not allow it. The presence of ilmenite coring rutile in pelitic schist might also suggest a counterclockwise pressure-temperature path and more work must be done to determine the prograde pressure-temperature trajectory of the Valhalla complex. A clockwise pressure-temperature $r$ th would imply that the overall tectonic setting for the Valhalla complex was compressional (England and Thompson 1984). 

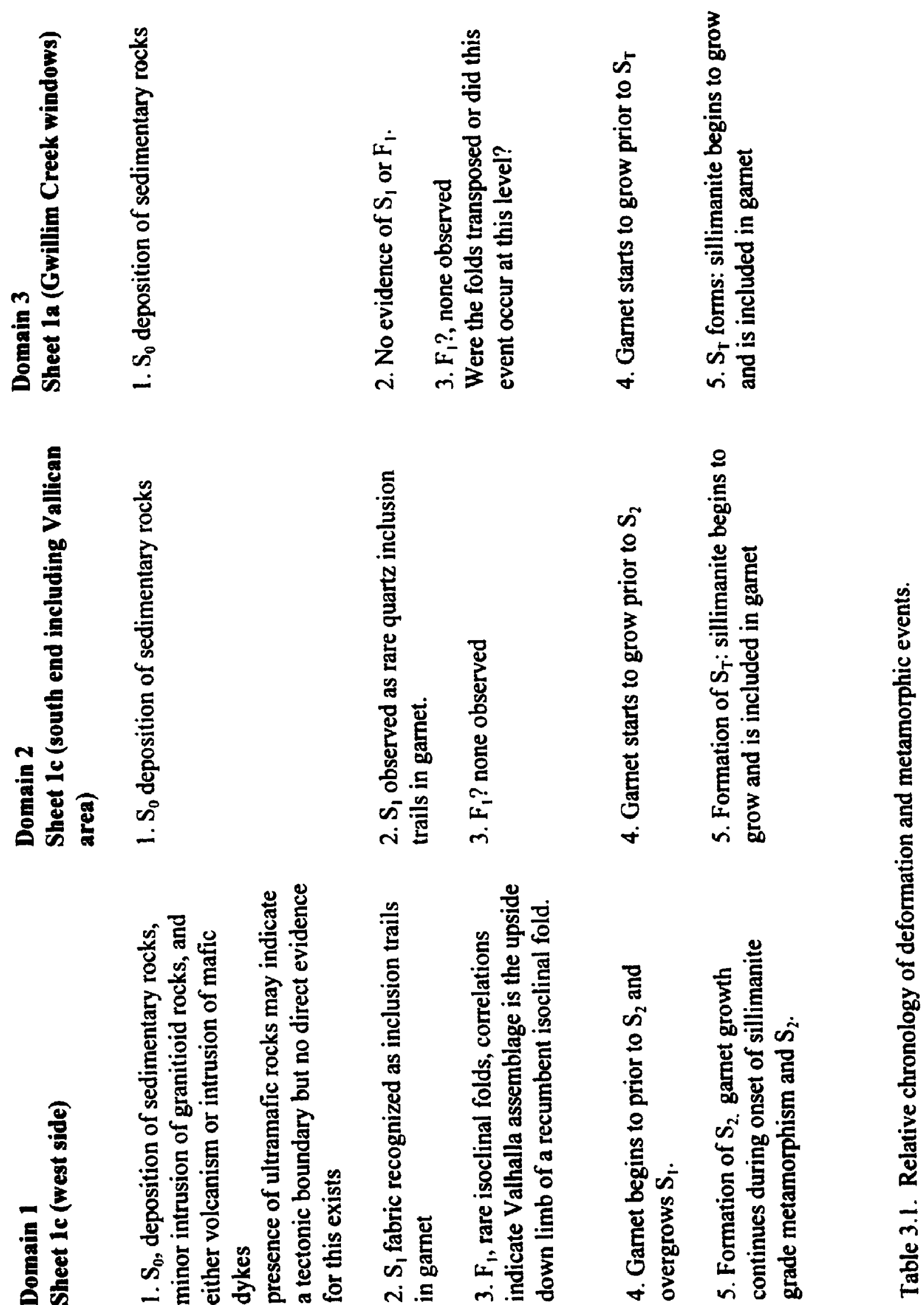

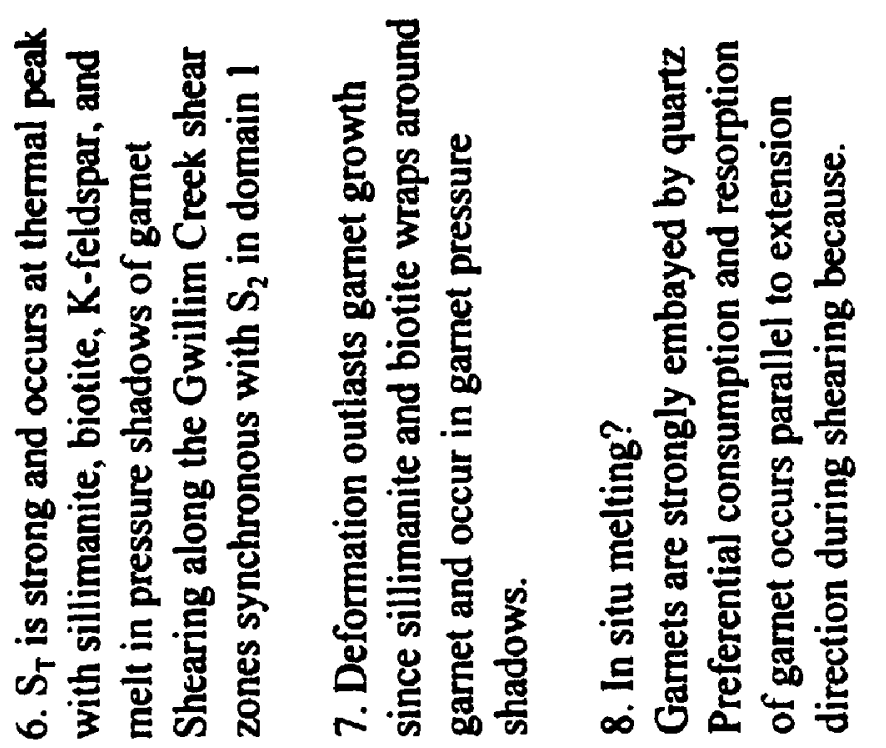

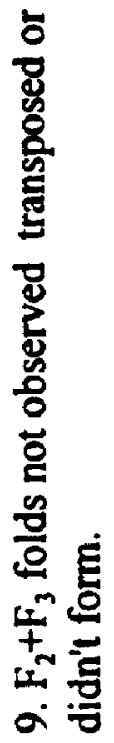
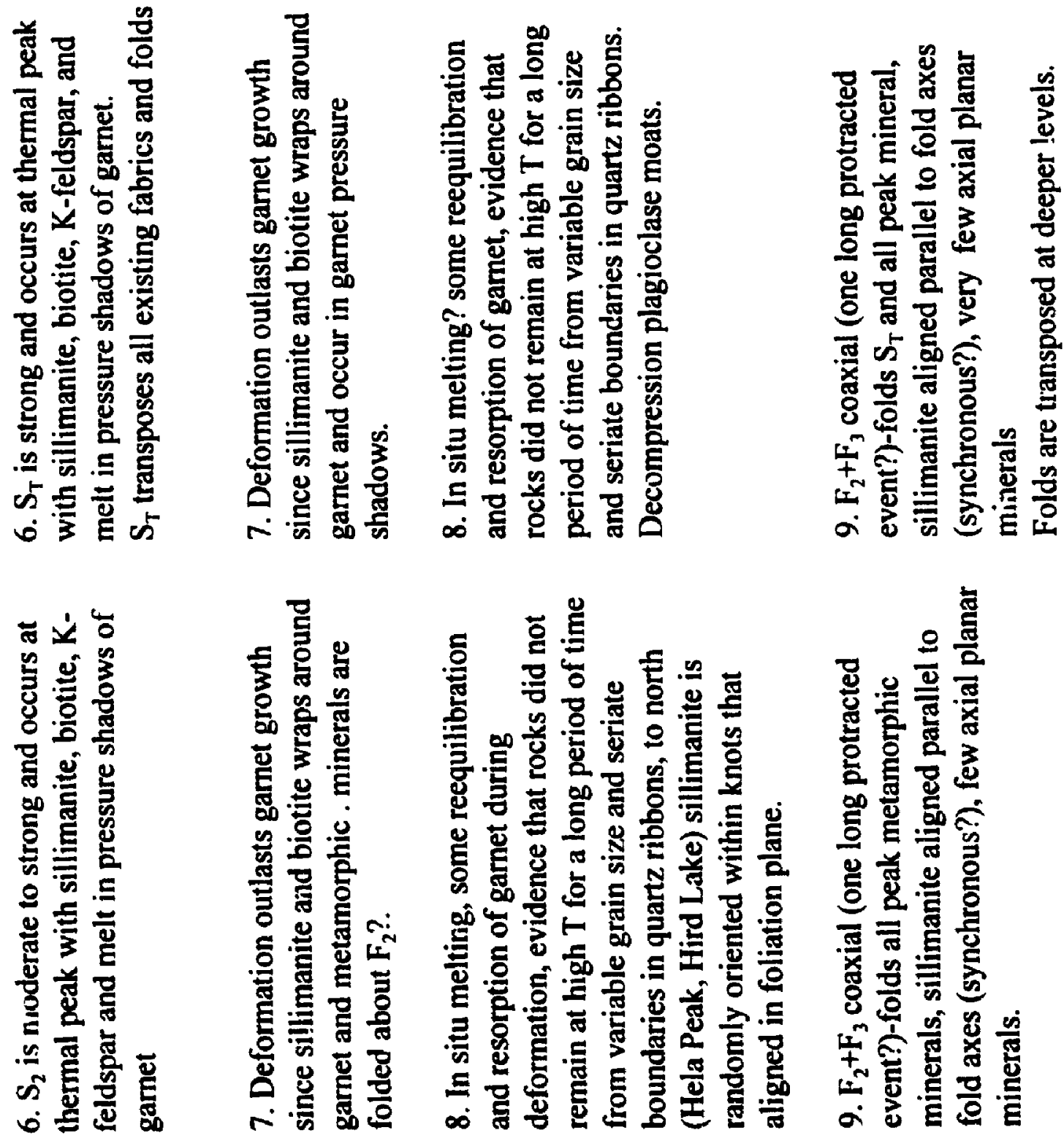

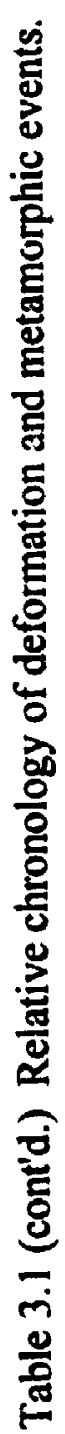



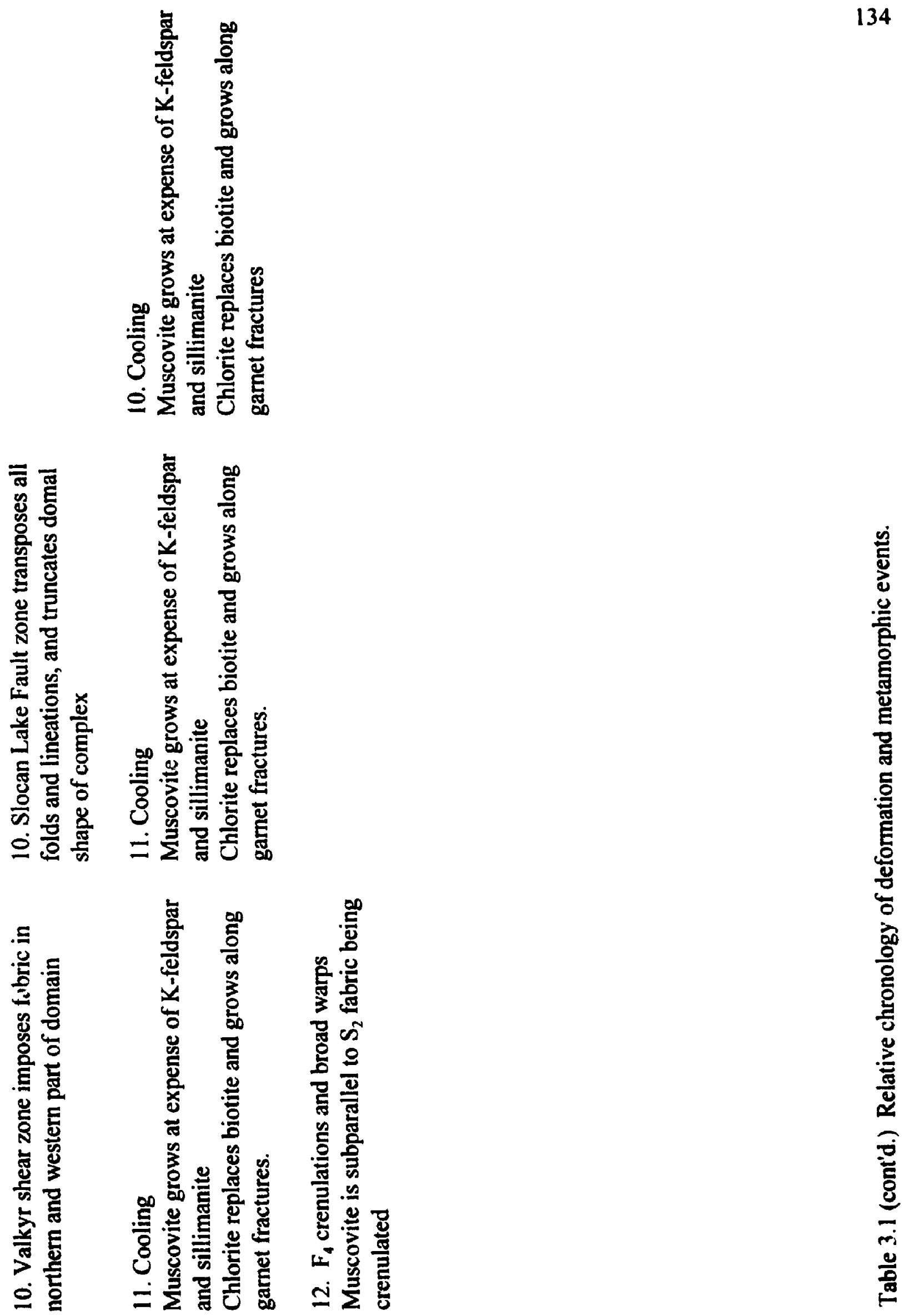
Table 3.2 
Table 3.2. Thermobarometric data from pelitic schists.

$\mathrm{Tl}$ - Post peak temperatures and pressures calculated from garnet-proximal biotite pairs ( l!) separated by plagioclase. quartz or potassium feldspar: and plagioclase in contact with the garnet rim (P1), where possible.

T2 - Temperatures calculated from garnet rims in direct contact with hiotite reflecting near closure temperacures for reaction 1. Pressures are not reliable since they were calculated with the same plagioclase compositions used in $\mathrm{Tl}$ and are merely a function of moving down the GASP isopleth to were it intersects the garnet - biotite thermometer at T2.

GASP - anorthite $=$ sillimanite + grossular + quartz

GRAIL - almandine + rutile $=$ ilmenite + sillimanite + quartz

The numbers in the first coldumn are for cataloguing composition combinations. The second. third, and fourth columns refer to the garnet, biotite and plagioclase compositions used to calculate the temperatures and pressures. These data may be found in Appendix C. CAASP data are found in the fifth and sixth columns. while GRAIL data are found in the sreventh and eighth columns. Average temperatures and pressures and standard deviations are locited at the bottom of the columns. 
Domain la - sheet lc, west side

\begin{tabular}{|c|c|c|c|c|c|c|c|c|c|c|c|c|c|c|c|}
\hline \multicolumn{3}{|c|}{ PS-4-93 } & \multicolumn{3}{|c|}{ GASP } & & & \multicolumn{3}{|c|}{ PS-9-93 } & \multicolumn{3}{|c|}{ GASP } & & \\
\hline $\mathrm{T} 1$ & gl & bt & $\mathrm{pl}$ & $\mathrm{T}$ & $P$ & & & $\mathrm{~T} 1$ & gl & bt & $\mathrm{pl}$ & $\mathrm{T}$ & $\mathbf{P}$ & & \\
\hline 40 & & $\mathbf{g}$ & $a$ & 737 & 6.1 & - & - & 10 & $c$ & c & $\dot{b}$ & 721 & 5.4 & - & - \\
\hline 41 & & $g$ & b & 738 & 6.3 & - & - & 11 & $c$ & c & a & 724 & 5.9 & - & - \\
\hline 42 & & i & d & 846 & 8.0 & - & - & 13 & & d & b & 743 & 5.5 & - & - \\
\hline 43 & $\mathrm{~h}$ & i & $\mathrm{c}$ & 844 & 7.7 & - & - & 14 & e & e & b & 740 & 5.8 & - & - \\
\hline 44 & & h & a & 803 & 7.2 & - & - & 15 & $\mathrm{e}$ & e & a & 745 & 5.9 & - & - \\
\hline 45 & & h & d & 808 & 8.1 & $\cdot$ & - & 16 & f & d & a & 742 & 6.2 & - & - \\
\hline 46 & & $\varepsilon$ & $\mathbf{a}$ & 720 & 5.5 & - & - & & & & & 736 & 5.8 & & \\
\hline 47 & c & $g$ & b & 721 & 5.7 & $\cdot$ & - & & & & & 10 & 0.3 & & \\
\hline & & & & 777 & 6.8 & & & $\mathrm{~T} 2$ & & & & & & & \\
\hline $\mathrm{T} 2$ & & & & 50 & 1.0 & & & 1 & $\mathbf{a}$ & a & b & 583 & 3.0 & - & - \\
\hline 24 & & & & & & & & 2 & a & a & $\mathbf{a}$ & 585 & 3.3 & - & - \\
\hline $\begin{array}{l}24 \\
25\end{array}$ & a & e & $\mathbf{a}$ & (i? & 4.2 & - & - & 3 & b & b & b & 616 & 3.5 & - & - \\
\hline 25 & $\mathbf{a}$ & e & b & 634 & 4.4 & - & - & 4 & b & b & $\mathbf{a}$ & 617 & 3.8 & - & - \\
\hline 26 & & d & b & 772 & 6.6 & - & - & & & & & 600 & 3.4 & & \\
\hline 27 & b & d & a & 770 & 6.4 & - & - & & & & & 16 & 0.3 & & \\
\hline 28 & f & b & c & 565 & 3.4 & - & - & & & & & & & & \\
\hline 29 & f & b & d & 566 & 3.5 & - & - & & & & & & & & \\
\hline .80 & & c & d & 678 & 5.4 & - & - & & & & & & & & \\
\hline 31 & & c & c & 677 & 5.2 & - & - & & & & & & & & \\
\hline 32 & & J & c & 702 & 5.9 & - & - & & & & & & & & \\
\hline 33 & $k$ & $\mathrm{j}$ & d & 703 & 6.1 & $\cdot$ & $\cdot$ & & & & & & & & \\
\hline & & & & 670 & 5.1 & & & & & & & & & & \\
\hline & & & & 69 & 1.1 & & & & & & & & & & \\
\hline PS-1 & $36 c-9$ & & & GASP & & & & PS-16 & $9-94$ & & & GASP & & & \\
\hline $\mathrm{T} 2$ & $\mathrm{gt}$ & bt & $\mathrm{pl}$ & $T$ & & & & $\mathrm{~T} 1$ & $g t$ & bt & pl & $T$ & & & \\
\hline 23 & b & c & a & 675 & 4.5 & - & - & 41 & i & I & d & 834 & 7.4 & - & - \\
\hline 24 & b & c & $\mathbf{b}$ & 682 & 5.7 & - & - & 42 & i & j & d & 781 & 6.6 & - & - \\
\hline 25 & $\mathbf{a}$ & b & a & 744 & 6.6 & - & - & 43 & h & k & d & 723 & 54 & - & - \\
\hline 26 & a & b & $\mathbf{b}$ & 736 & 5.3 & - & - & 45 & a & $\mathrm{m}$ & f & 769 & 6.0 & - & - \\
\hline 27 & e & e & $\mathbf{a}$ & 658 & 4.0 & - & $\cdot$ & 46 & $\mathbf{a}$ & n & f & 780 & 6.7 & - & - \\
\hline 28 & $\mathbf{e}$ & e & $\mathbf{b}$ & 665 & 5.2 & - & . & & & & & 777 & 6.5 & & \\
\hline & & & & 662 & 4.6 & & & & & & & 35 & 0.7 & & \\
\hline & & & & 4 & 0.6 & & & $\mathrm{~T} 2$ & & & & & & & \\
\hline & & & & & & & & 30 & g & f & $\mathrm{d}$ & 663 & 4.8 & - & - \\
\hline & & & & & & & & 32 & $f$ & $g$ & $d$ & 607 & 3.6 & - & - \\
\hline & & & & & & & & 35 & d & a & $\mathrm{f}$ & 689 & 5.0 & - & - \\
\hline & & & & & & & & $\$ 7$ & c & t & $\mathbf{f}$ & 667 & 4.6 & - & - \\
\hline & & & & & & & & 39 & & 0 & $f$ & 747 & 5.2 & - & - \\
\hline & & & & & & & & & & & & 675 & 4.6 & & \\
\hline & & & & & & & & & & & & 45 & 0.6 & & \\
\hline
\end{tabular}

Table 3.2. Thermobarometric data for pelitic schists. 


\begin{tabular}{|c|c|c|c|c|c|c|c|}
\hline \multicolumn{3}{|c|}{ PS-277-94 } & \multicolumn{3}{|c|}{ GASP } & & \\
\hline T1 & gt & $b_{t}$ & $\mathrm{pl}$ & $T$ & $\mathbf{P}$ & & \\
\hline 1 & $\mathbf{a}$ & b & a & 714 & 4.7 & . & . \\
\hline 4 & $\mathbf{a}$ & d & a & 719 & 4.8 & - & . \\
\hline 6 & & f & c & 745 & 7.9 & - & . \\
\hline 7 & & g & c & 768 & 8.3 & - & . \\
\hline 23 & & b & d & 749 & 6.8 & - & . \\
\hline 24 & & d & d & 754 & 6.9 & - & . \\
\hline 25 & & g & e & 724 & 5.4 & - & . \\
\hline & & & & 748 & 7.1 & & \\
\hline & & & & 14 & 1.0 & & \\
\hline $\mathrm{T} 2$ & & & & & & & \\
\hline 9 & b & $c$ & $\mathbf{a}$ & 688 & 5.3 & - & . \\
\hline 12 & c & $\mathbf{a}$ & $\mathbf{a}$ & 644 & 5.7 & - & . \\
\hline 14 & e & e & $c$ & 691 & 5.4 & - & . \\
\hline & & & & 674 & 5.5 & - & . \\
\hline & & & & 21 & 0.2 & & \\
\hline
\end{tabular}

\begin{tabular}{cccccccc} 
PS-344-94 & \multicolumn{9}{c}{ GASP } & & & \\
T! gt be & pl & T & P & & \\
$36 \mathrm{k}$ & 1 & d & 736 & 6.0 &. & - \\
$37 \mathrm{~h}$ & j & d & 786 & 6.7 & - & - \\
$38 \mathrm{c}$ & $\mathrm{k}$ & $\mathrm{e}$ & 804 & 6.4 & - & - \\
& & & & 775 & 6.4 & & \\
& & & & 29 & 0.3 & &
\end{tabular}

T2

$\begin{array}{llllllll}43 & \mathrm{a} & \mathrm{d} & \mathrm{e} & 706 & 4.7 & . & - \\ 44 \mathrm{~b} & \mathrm{c} & \mathrm{e} & 724 & 5.1 & - & - \\ 47 \mathrm{e} & \mathrm{f} & \mathrm{e} & 707 & 5.2 & \cdot & - \\ 48 \mathrm{f} & \mathrm{g} & \mathrm{e} & 729 & 5.1 & - & - \\ 51 \mathrm{~g} & \mathrm{e} & \mathrm{e} & 738 & 5.6 & - & - \\ & & & 721 & 5.1 & & \\ & & & 13 & 0.3 & & \end{array}$

Domain $1 \mathrm{~b}$ - sheet 1c. Slocan Ridge, Airy Creek

\begin{tabular}{|c|c|c|c|c|c|c|c|c|c|c|c|c|c|c|}
\hline PS-61-94 & \multicolumn{4}{|c|}{ GASP } & & & \multicolumn{3}{|c|}{ PS-332-94 } & \multicolumn{2}{|c|}{ GASP } & \multicolumn{3}{|c|}{ GKAIL } \\
\hline $\mathrm{Tl} \mathrm{gt}$ & bt & pl & $\mathbf{T}$ & $\mathbf{P}$ & & & $\mathrm{T} 1$ & gt & $b t$ & pl & $\mathbf{T}$ & $\mathbf{P}$ & $\mathrm{T}$ & $\mathbf{P}$ \\
\hline $35 c$ & j & a & 800 & 6.5 & . & - & 40 & $g$ & $\mathbf{k}$ & $a^{2}$ & 780 & 7.6 & 780 & 7.6 \\
\hline $36 \mathrm{~d}$ & $\mathrm{j}$ & $\mathbf{a}$ & 747 & 5.5 & - & - & 42 & a & j & $\mathbf{a}$ & 734 & 7.1 & 733 & 0.5 \\
\hline $37 \mathrm{~d}$ & $\mathbf{k}$ & a & 753 & 5.6 & - & - & 44 & $d$ & $i$ & c & 778 & 7.7 & 776 & 7 \\
\hline $38 \mathrm{c}$ & k & a & 807 & 6.7 & . & - & & & & & 764 & 7.5 & 763 & 7.3 \\
\hline $39 \mathrm{~h}$ & $\mathrm{~g}$ & b & 798 & 6.5 & - & - & & & & & 21 & 0.3 & 21 & 0.3 \\
\hline $40 j$ & $\mathrm{~h}$ & $b$ & 814 & 7.4 & - & - & $\mathrm{T} 2$ & & & & & & & \\
\hline \multirow[t]{3}{*}{$41 j$} & $i$ & $b$ & 808 & 7.3 & - & - & 28 & c & f & b & 650 & 4.7 & 662 & 7 \\
\hline & & & 790 & 6.5 & & & 30 & k & e & b & 731 & 6.4 & 736 & 7 \\
\hline & & & 26 & 0.7 & & & 33 & i & d & d & 691 & 60 & 697 & 7 \\
\hline $\mathrm{T} 2$ & & & & & & & 34 & f & $\mathbf{a}$ & a & 712 & 5.9 & 721 & 7 \\
\hline $21 \mathrm{~b}$ & a & a & 690 & 4.3 & . & . & & & & & 696 & 5.8 & 704 & 7 \\
\hline $24 a$ & b & a & 652 & 4.0 & - & - & & & & & 30 & 06 & 28 & 0 \\
\hline $26 \mathrm{f}$ & $\mathrm{d}$ & $\mathrm{b}$ & 742 & 5.6 & - & - & & & & & & & & \\
\hline \multirow[t]{3}{*}{$21 \mathrm{~g}$} & $e$ & $b$ & 708 & 5.3 & - & - & & & & & & & & \\
\hline & & & 698 & 4.8 & & & & & & & & & & \\
\hline & & & 32 & 0.7 & & & & & & & & & & \\
\hline
\end{tabular}

Table 3.2. Thermobarometric data for pelitic schists. 
Domain 2 - Sheet 1c, Vallican

\begin{tabular}{|c|c|c|c|c|c|c|}
\hline \multicolumn{2}{|c|}{ PS-28a-93 } & \multicolumn{3}{|c|}{ GASP } & & \\
\hline & bt & pl & $T$ & P & & \\
\hline $1 \mathrm{c}$ & a & a & 858 & 11.0 & - & - \\
\hline $15 \mathrm{~b}$ & a & d & 775 & 8.4 & - & - \\
\hline $16 g$ & f & $c 5$ & 868 & 11.7 & - & - \\
\hline $17 \mathrm{~g}$ & $\mathrm{f}$ & cl & 862 & 10.6 & - & - \\
\hline $18 \mathrm{~g}$ & b & h & 917 & 10.6 & - & - \\
\hline $19 \mathrm{~h}$ & b & h & 894 & 10.1 & - & - \\
\hline $20 \mathrm{i}$ & $b$ & $\mathrm{~g}$ & 873 & 8.5 & - & - \\
\hline $21 \mathrm{j}$ & b & $f$ & 804 & 7.1 & - & - \\
\hline & & & 856 & 9.8 & & \\
\hline & & & 43 & 1.5 & & \\
\hline $\mathrm{T} 2$ & & & & & & \\
\hline $4 \mathrm{e}$ & d & c & 913 & 11.9 & - & - \\
\hline $5 \mathrm{f}$ & e & c & 814 & 10.4 & - & - \\
\hline & & & 854 & 11.2 & & \\
\hline & & & 50 & 1.0 & & \\
\hline PCA-93- & & & GASP & & GRAIL & \\
\hline $\mathrm{Tl} \quad \mathrm{gt}$ & $\mathrm{bt}$ & $\mathrm{pl}$ & $\mathrm{T}$ & P & $T$ & $\mathbf{P}$ \\
\hline $32 \mathrm{e}$ & $\mathbf{g}$ & $c$ & 841 & 11.1 & 819 & 7.4 \\
\hline $33 \mathrm{f}$ & $\mathrm{g}$ & d & 728 & 9.6 & 714 & 7.1 \\
\hline $34 a$ & e & b & 870 & 11.4 & 844 & 7.0 \\
\hline $35 \mathrm{~b}$ & f & b & 856 & 11.6 & 830 & 6.9 \\
\hline $37 \mathrm{~g}$ & $\mathbf{g}$ & b & 832 & 10.2 & 815 & 7.3 \\
\hline $38 j$ & h & e & 786 & 9.2 & 775 & 7.1 \\
\hline & & & 819 & 10.5 & 800 & 7.1 \\
\hline & & & 48 & 0.9 & 44 & 0.2 \\
\hline
\end{tabular}

$\mathrm{T} 2$

$\begin{array}{lllllll}18 \mathrm{~h} & \mathrm{a} & \mathrm{a} & \mathbf{8 7 8} & 21.9 & 800 & 7.4\end{array}$ $\begin{array}{lllllll}21 \mathrm{c} & \mathrm{d} & \mathrm{b} & 744 & 9.4 & 731 & 7.0\end{array}$

Domain 3 - sheet 1a, lower Gwillim Creek window

\begin{tabular}{|c|c|c|c|c|c|c|c|c|c|c|c|c|c|c|}
\hline $\mathrm{PCA}-90$ & & & GASP & & GRAII & & PS -348 & $8 a-9$ & & & jASP & & & \\
\hline TI $g$ & bt & $\mathrm{pl}$ & $T$ & P & $\mathrm{T}$ & $\mathbf{P}$ & $T 1$ & gt & bt & pl & $\mathrm{T}$ & P & & \\
\hline $27 \mathrm{a}$ & a & $\mathbf{a}$ & 844 & 8.3 & 833 & 6.4 & $36 c$ & & $q$ & a & 875 & 8.7 & - & - \\
\hline $30 \mathrm{a}$ & a & b & 844 & 8.3 & - & - & $37 \mathrm{c}$ & & p & a & 901 & 9.9 & - & - \\
\hline $28 \mathrm{f}$ & e & d & 970 & 10.5 & 946 & 6.4 & $38 \mathrm{r}$ & h & $\mathrm{n}$ & c & 851 & 8.4 & - & - \\
\hline $29 \mathrm{f}$ & e & c & 972 & 10.8 & - & - & $39 \mathrm{i}$ & & o & c & 768 & 7.1 & - & - \\
\hline $35 \mathrm{f}$ & c & d & 947 & 10.1 & 924 & 6.2 & & & & & 849 & 8.5 & & \\
\hline $36 \mathrm{f}$ & c & c & 949 & 10.5 & - & - & & & & & 50 & 1.0 & & \\
\hline 37 a & a & e & 836 & 6.9 & $\begin{array}{ll}- & -1 \\
\end{array}$ & - & & & & & & & & \\
\hline 38 a & a & f & 842 & 8.0 & - & - & $\mathrm{T} 2$ & & & & & & & \\
\hline & & & 901 & 9.2 & 901 & 6.3 & $30 a$ & & h & a & 750 & 6.4 & - & - \\
\hline & & & 60 & 1.4 & 49 & 0.1 & $31 \mathrm{~b}$ & b & i & a & 745 & 7.0 & - & - \\
\hline & & & & & & & $32 \mathrm{f}$ & & k & c & 621 & 4.4 & $=$ & - \\
\hline Г2 & & & & & & & $33 g$ & $g$ & $\mathbf{m}$ & c & 643 & 5.5 & - & - \\
\hline $33 \mathrm{~d}$ & d & c & 796 & 7.8 & 785 & 5.9 & & & & & 690 & 5.8 & & \\
\hline $34 \mathrm{~d}$ & d & d & 795 & 7.6 & - & - & & & & & 58 & 1.0 & & \\
\hline
\end{tabular}

Table 3.2. Thermobarometric data for pelitic schists.

\begin{tabular}{clllrlll} 
PS-28b-93 & \multicolumn{3}{c}{ GASP } & \multicolumn{3}{c}{ GRAII } \\
T1 & gt & bt & pl & T & P & \multicolumn{1}{c}{ T } & P \\
36 & c & g & b & 959 & 12.2 & 959 & 7.9 \\
37 & d & g & b & 920 & 11.2 & 899 & 7.6 \\
38 i & h & a & 913 & 11.1 & 891 & 7.3 \\
39 & i & h & b & 913 & 11.2 & - & - \\
40 & c & g & a & 983 & 12.0 & - & - \\
41 & d & g & a & 919 & 11.0 & - & - \\
& & & & 935 & 11.4 & 916 & 7.6 \\
& & & & 27 & 0.5 & 30 & 0.3 \\
T2 & & & & & & & \\
26 & b & c & a & 885 & 10.1 & 867 & 7.0 \\
27 & b & c & b & 885 & 10.2 & - & - \\
28 & a & b & b & 853 & 7.5 & 853 & 7.5 \\
29 & a & b & a & 852 & 7.4 & - & - \\
& & & & 869 & 8.8 & 860 & 7.3 \\
& & & & 16 & 1.4 & 7 & 0.2
\end{tabular}


unarmoured biotite inclusions in gamet at fixed P (8Kbars)

Domain la

PS- $136 c-94$

PS-169-94

21

22

23

PS-344-94

$$
23
$$

24

Domain Ib

PS-61-94

13

PS- $332-94$

20

j

$\begin{array}{ll}\mathrm{e} & \mathrm{e} \\ \mathrm{e} & \mathrm{d} \\ \mathrm{c} & \mathrm{c}\end{array}$

60080

6108.0

6358.0

$\begin{array}{llll}\text { d } & b & 680 & 8.0\end{array}$

$\begin{array}{llll}d & a & 700 \quad 8.0\end{array}$

Domain ?

PS-28b-93

$\begin{array}{lllll}16 & f & a & 905 & 8.0 \\ 17 & \text { g } & \text { e } & 818 & 8.0\end{array}$

16

Domain 3

PS-348-94

$\begin{array}{lllll}3 & 1.2 .3 & \text { a } & 670 & 8.0 \\ 4 & 1.4,5 & \text { c } & 720 & 8.0 \\ 5 & 1.6,7 & \text { d } & 680 & 8.0 \\ 6 & 1.8,9 & \text { e } & 6 / 0 & 8.0\end{array}$

$730 \quad 8.0$

$765 \quad 8.0$

8188.0
Armoured biotite inclusions in garnet at tixed P ( 8 Kbars)

Domain $1 \mathrm{~b}$
PS-61-94
$1:$

PS-332-94

19

21

Dumain 3

PS-348-94
$1.24 \mathrm{t}$

$1.25 \mathrm{~g}$
865 885
80

8.0 
Table 3.3 
Table 3.3. Thermobarometric data for amphibolite gneisses.

GT-HN, TR-TS is the intersection between the garnet - tremolite thermometer (reaction 5) and the garnet-hornblende-plagioclase barometer (reaction 4). GT-BT, TR-TS is the intersection between the garnet-biotite thermometer (reaction 1) and the garnet-hornblendeplagioclase barometer (reaction 4). Columns are arranged in the same manner as Table 3.2. Mineral compositions used for calculations are listed in Appendix D. 
Domain la - sheet 1c, west side

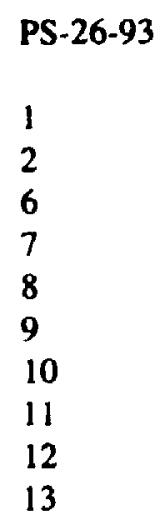

\begin{tabular}{llllllllll} 
& & & \multicolumn{3}{c}{ GT-HN } & \multicolumn{2}{c}{ GT-BT } \\
RIM-T & GT & HN & PL & BT & TR-TS & \multicolumn{2}{c}{ TR-TS } & \\
& C & A & A & - & $\mathbf{6 8 8}$ & 7.3 & - & - \\
ALL-T & D & B & A & - & 702 & 7.4 & - & - \\
& B & E & E & C & 692 & 6.6 & 901 & 9.7 \\
& F & A & A & - & 693 & 7.4 & - & - \\
& F & B & A & - & 701 & 7.4 & - & - \\
ALL-M & F & E & A & - & 734 & 7.8 & - & - \\
& B & J & C & E & 679 & 7.1 & 854 & 9.8 \\
& B & J & C & D & 679 & 7.1 & 836 & 9.5 \\
& F & F & B & A & 706 & 7.3 & 850 & 9.4 \\
& F & F & B & B & 706 & 7.3 & 841 & 9.3 \\
& & & & & 698 & 7.3 & 856 & 9.5 \\
& & & & & 15 & 0.3 & 23 & 0.2
\end{tabular}

PS-115-94

1
2
3
4
5
6
7
8
9
10
11
12
13

$$
\begin{array}{llll}
\text { B } & \text { F } & \text { B } & \text { A } \\
\text { A } & \text { C } & \text { E } & \text { D } \\
\text { A } & \text { C } & \text { F } & \text { E } \\
\text { A } & \text { C } & \text { G } & \text { B } \\
\text { B } & \text { D } & \text { A } & \text { C } \\
\text { B } & \text { D } & \text { C } & - \\
\text { B } & \text { D } & \text { D } & - \\
\text { B } & \text { E } & \text { D } & - \\
\text { B } & \text { E } & \text { C } & - \\
\text { B } & \text { E } & \text { A } & - \\
\text { B } & \text { F } & \text { A } & - \\
\text { B } & \text { F } & \text { C } & - \\
\text { B } & \text { F } & \text { D } & - \\
& & &
\end{array}
$$

\section{1}

783

784

794

792

783

793

786

785

786

767

766

766

780

11

PS-248-94

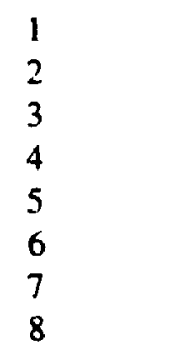

$\begin{array}{llll}\text { D } & \text { A } & \text { D } & - \\ \text { B } & \text { C } & \text { A } & - \\ \text { D } & \text { A } & \text { D } & - \\ \text { C } & \text { C } & \text { A } & - \\ \text { F } & \text { B } & \text { C } & - \\ \text { F } & \text { B } & \text { D } & - \\ \text { C } & \text { D } & \text { A } & - \\ \text { C } & \text { D } & \text { B } & -\end{array}$

694

717

677

676

696

698

690

683

691

$\begin{array}{rrr}7.0 & 847 & 6.3 \\ 8.2 & 829 & 6.8 \\ 8.3 & 844 & 7.0 \\ 8.4 & 840 & 9.0 \\ 8.2 & 850 & 9.0 \\ 8.2 & - & - \\ 8.3 & - & - \\ 8.2 & - & - \\ 8.0 & - & - \\ 8.2 & - & - \\ 7.9 & - & - \\ 7.8 & - & - \\ 7.9 & - & - \\ 8.0 & 842 & 7.6 \\ 0.3 & 7 & 1.2\end{array}$

12

7.3

7.9

6.6

6.5

6.5

6.7

7.0

6.6

6.9

0.5

Table 3.3 Thermobarometric data for amphibolite gneisses. 
Domain lb

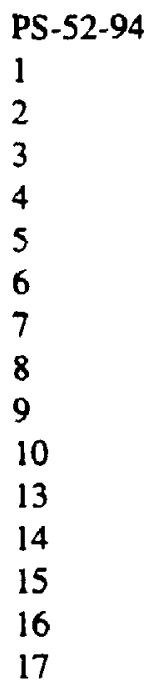

$\begin{array}{llllrr}\text { B } & \text { B } & \text { A } & - & 776 & 8.7 \\ \text { B } & \text { B } & \text { B } & - & 807 & 13.4 \\ \text { D } & \text { C } & \text { B } & - & 788 & 13.1 \\ \text { D } & \text { C } & \text { A } & - & 759 & 8.6 \\ \text { G } & \text { F } & \text { H } & - & 843 & 10.6 \\ \text { G } & \text { F } & \text { I } & - & 848 & 11.4 \\ \text { F } & \text { G } & \text { I } & - & 785 & 10.0 \\ \text { F } & \text { G } & \text { H } & - & 781 & 9.3 \\ \text { A } & \text { E } & \text { A } & - & 753 & 8.8 \\ \text { A } & \text { E } & \text { B } & - & 782 & 13 \\ \text { B } & \text { B } & \text { J } & - & 778 & 9 . \\ \text { D } & \text { C } & \text { J } & - & 761 & 8.9 \\ \text { A } & \text { E } & \text { J } & - & 750 & 8.4 \\ \text { G } & \text { F } & \text { E } & - & 847 & 11.2 \\ \text { F } & \text { G } & \text { G } & - & 782 & 9.4 \\ & & & & 789 & 10.3 \\ & & & & 32 & 1.7\end{array}$

PS-29A-93

1
2
3
4
5
6
7
8

$\begin{array}{llll}\text { A } & - & - & \text { B } \\ \text { C } & - & - & \text { A } \\ \text { D } & \text { B } & \text { A } & \\ \text { E } & \text { C } & \text { A } & \\ \text { F } & \text { A } & \text { B } & \\ \text { B } & \text { B } & \text { A } & \\ \text { G } & \text { C } & \text { A } & \text { A } \\ \text { G } & \text { B } & \text { A } & \text { B }\end{array}$

7.6

8.8

8.8

Hornblende inclusions in garnet

$\begin{array}{ccccccc}26-3 & \text { A } & \text { H } & - & - & 647 & 9.7 \\ -4 & \text { E } & \text { D } & - & - & 646 & 8.7 \\ -5 & \text { E } & \text { C } & - & - & 682 & 9.6 \\ 52-12 & \text { C } & \text { D } & - & - & 712 & 8.6 \\ 248-11 & & & - & - & 667 & 7.8 \\ -12 & & & - & - & 698 & 5.8\end{array}$

Table 3.3 Thermobarometric data for amphibolite gneisses. 
Figure 3.1 
Figure 3.1. Petrogenetic grid for pelitic schists showing important melt equilibria moditied after Yardley (1989), Spear and Cheney (1989) and Spear (1993). Those equilibria labelled with numerals correspond to those used in the text.

A) albite + potassium feldspar + quartz $+\mathrm{H}_{2} \mathrm{O}=$ melt (Luth et al 1964$)$

B) muscovite + potassium feldspar + quartz $+\mathrm{H}_{2} \mathrm{O}=$ melt (Thompson 1982)

C) muscovite + plagioclase + quartz $=$ potassium feldspar + aluminosilicate + liquid (LeBreton and Thompson 1987)

8) biotite + plagioclase + aluminosilicate + quartz $=$ garnet + potassium feldspar + liquid (LeBreton and Thompson 1987)

10) muscovite + quartz $=$ potassium feldspar + aluminosilicate + melt (Thompson 1982)

D) biotite + quartz + plagioclase $=$ orthopyroxene + potassium feldspar + liquid $($ Clemens and Wall 1981)

E) biotite + sillimanite $=$ gamet + cordierite ++ potassium feldspar $($ Spear and Cheney 1989 ) 


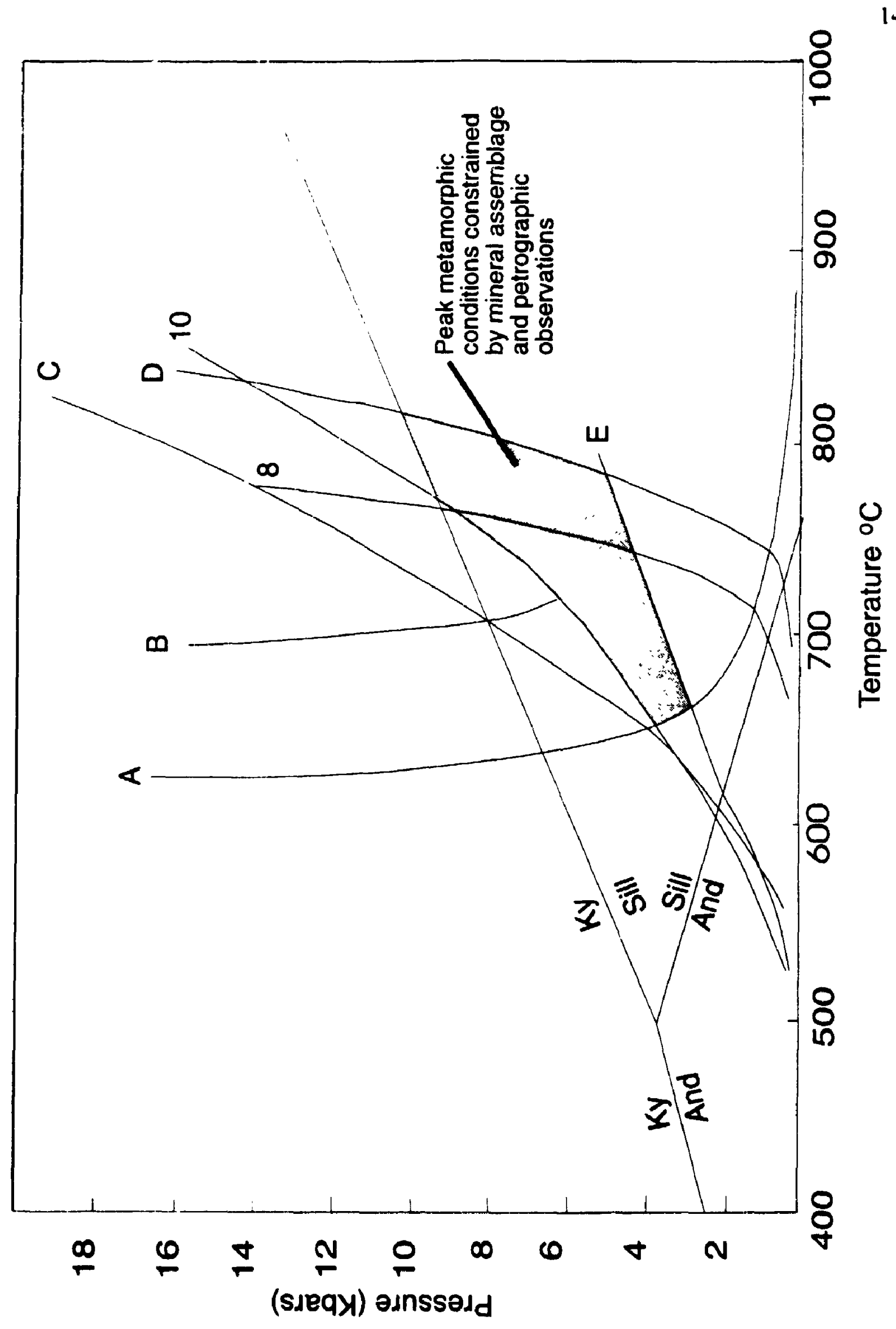




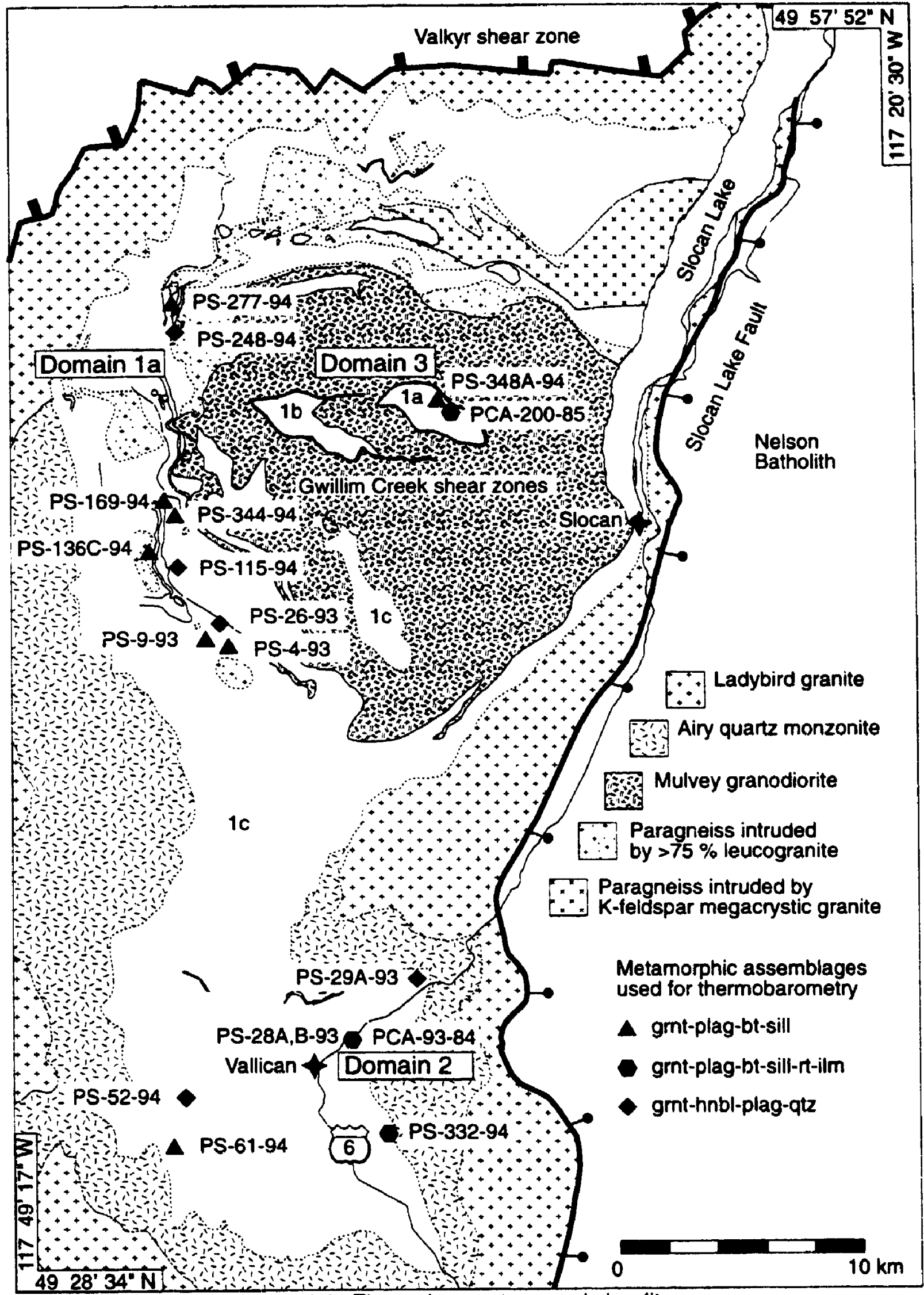

Figure 3.2. Thermobarometry sample locality map. 
Figure 3.3 


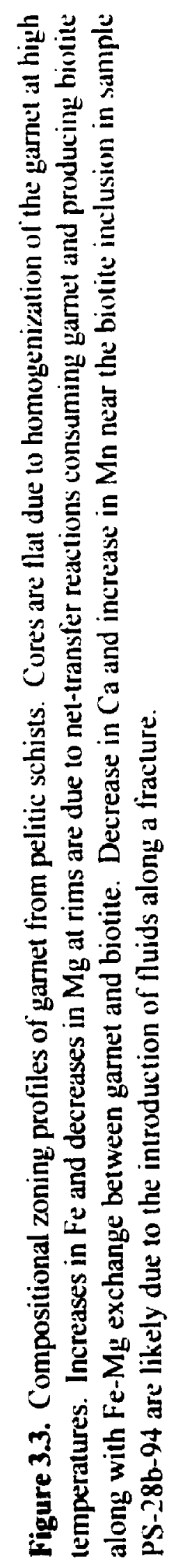




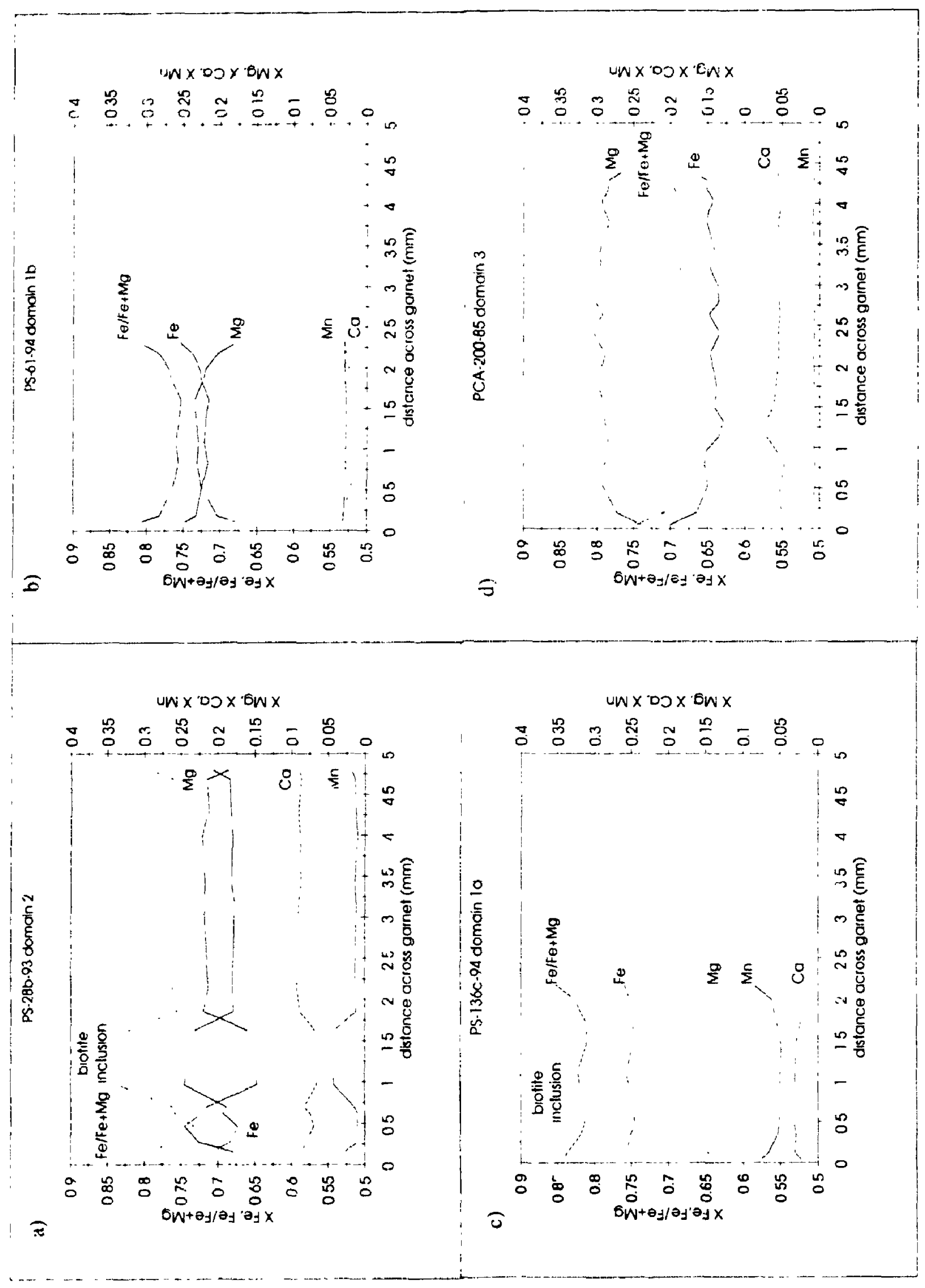


Figure 3.4 
Figure 3.4. Schematic illustrations outlining chronology of metamorphic and deformation events as observed from petrographic and chemical data.

a) $S_{1}$ formed and is defined by quartz inclusions..

b) Gamet overgrew $S_{1}$ fabric.

c) Garnet included sillimanite oriented in $S_{2}$ indicating that $S_{z}$ began to form during prograre metamorphism and garnet growth continued during part of $S_{2}$ formation.

d1) Garnet growth stopped and preferential resorption of garnet occurred giving elongate shape to garnet while $S_{2}$ continued and wrapped around garnet porphyroblasts.

d2) Garnet consumption created increased $\mathrm{Fe} / \mathrm{Fe}+\mathrm{Mg}$ levels about rim of garnet while plagioclase moats formed around garnet.

e) Melt producing reactions formed vermicular quartz which cross-cuts $\mathrm{Fe}$ zoning. Plagioclase moats are diminished along garnet rims parallel to $S_{2}$ fabric due to continued deformation. 


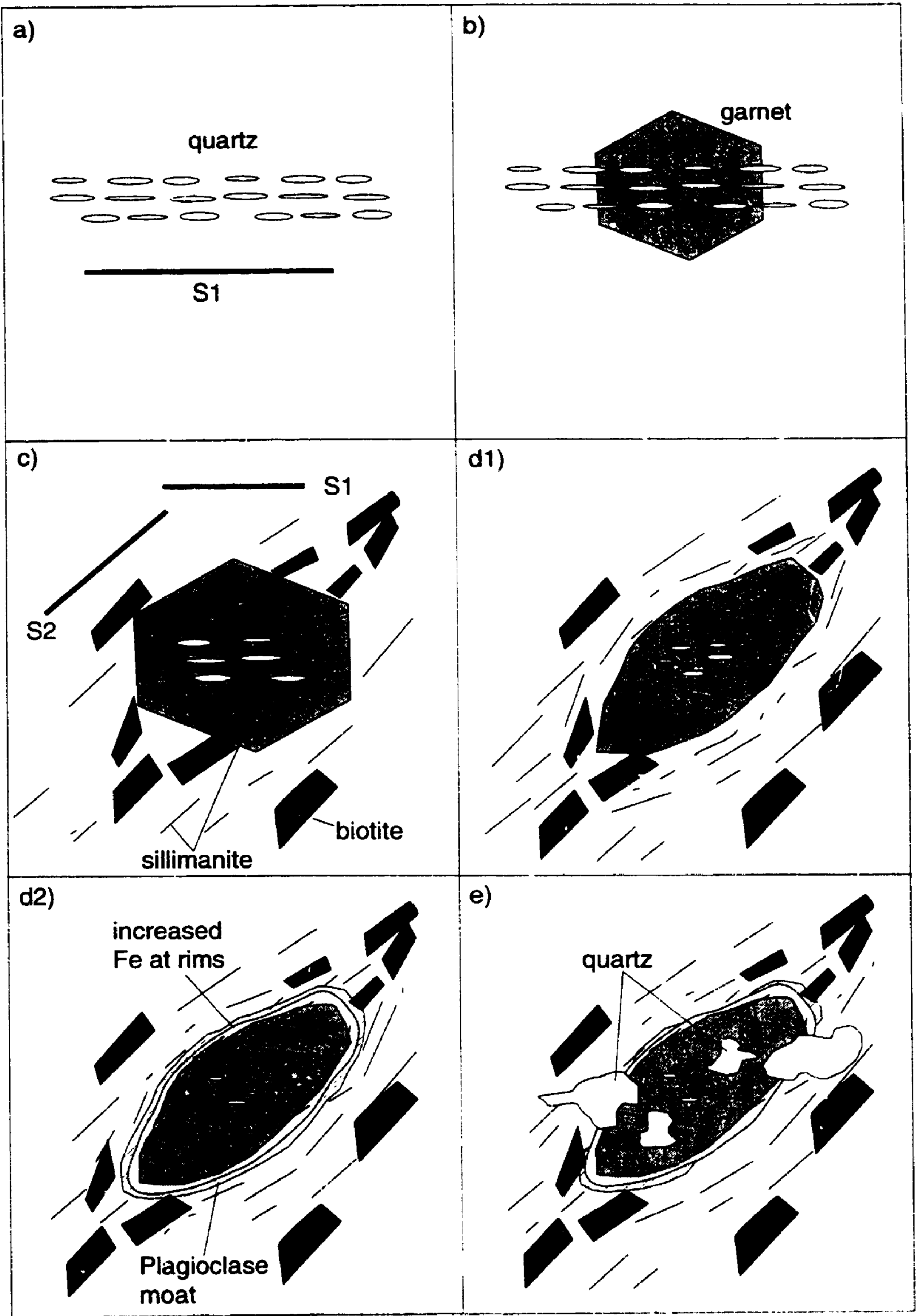


Figure 3.5 
Figure 3.5. Zoning profiles of garnet in sample PS-28a-93 (domain 2), located near Vallican. This sample shows rare Ca zoning and is attributed to decompression reactions consuming garnet and precipitating plagioclase as moats around the garnet. $\mathrm{Fe} / \mathrm{Fe}+\mathrm{Mg}$ increases toward the rim are also attributed to net-transfer reactions consuming gamet. 


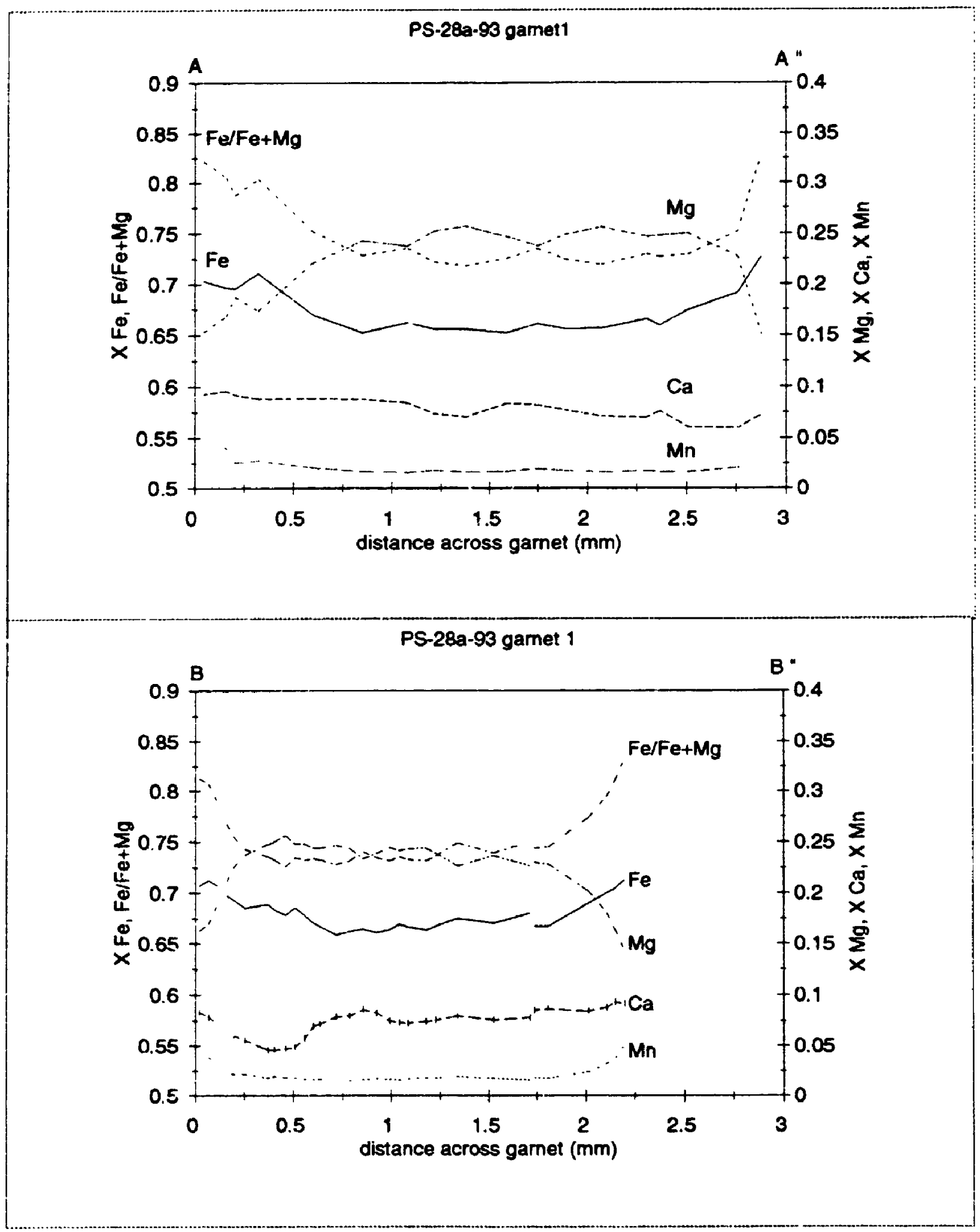


Figure 3.6 


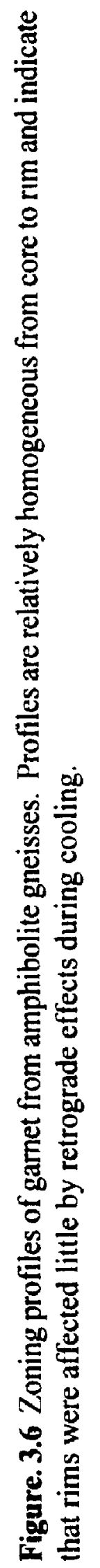




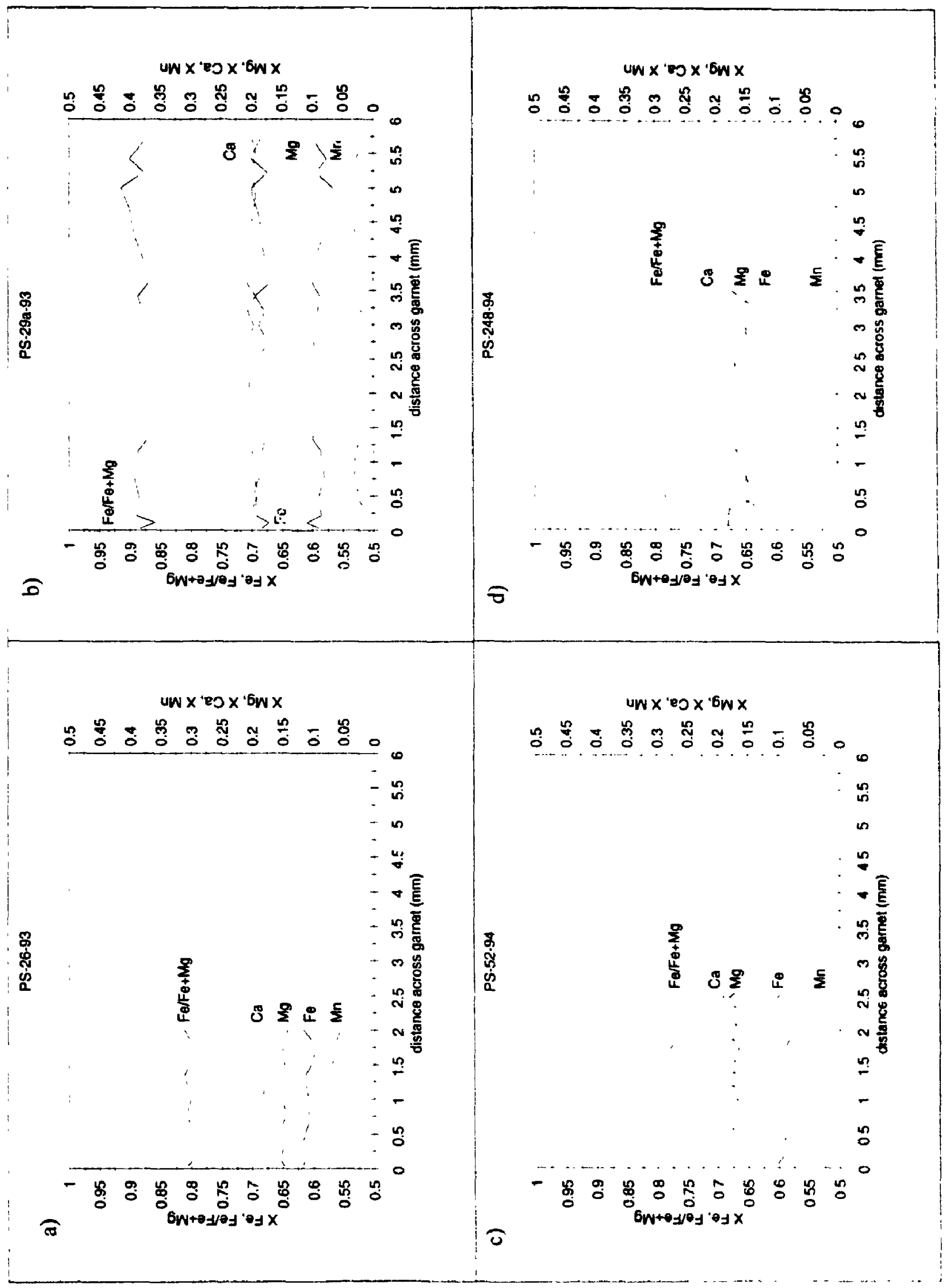




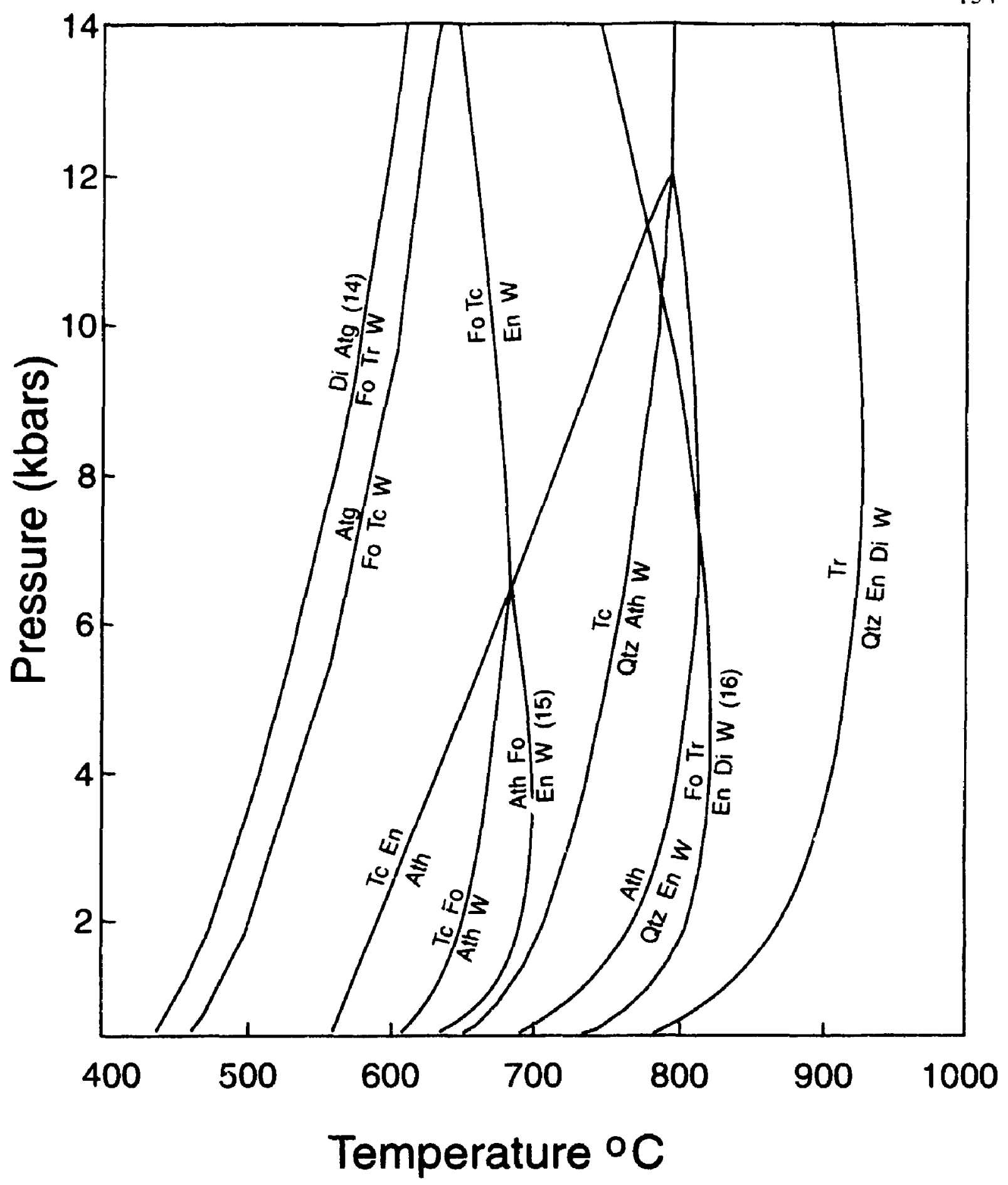

Figure. 3.7 Pelrogenetic grid for ultramafic schists calculated using TWEEQU computer program (Berman 1991). Ath - anthophyllite, Atg antigorite, Di - diopside, En - enstatite, Fo - forsterite, Qtz - quartz, Tc - talc, $\mathrm{Tr}$ - tremolite, $\mathrm{W}$ - water. 
Figure 3.8 
Figure 3.8. Representative plots for $\mathrm{Tl}, \mathrm{P} 1$ data from pelitic schists, generated using the computer program TWEEQU. Multiple lines for individual equilibria indicate that more than one mineral pair was used.

Equilibria:

1) almandine + phlogopite $=$ pyrope + annite

2) anorthite $=$ grossular + sillimanite + quartz $($ GASP $)$

3) sillimanite + quartz + iimenite $=$ almandine + rutile $($ GRAIL $)$

a) PS-9-93 - domain la, Rinda Ridge.

b) PS-332-94 - domain 1b, Slocan Ridge.

c) PCA-93-84 - domain 2, Vallican.

d) PCA-200-85 - domain 3, lower Gwillim Creek window (sheet la) 

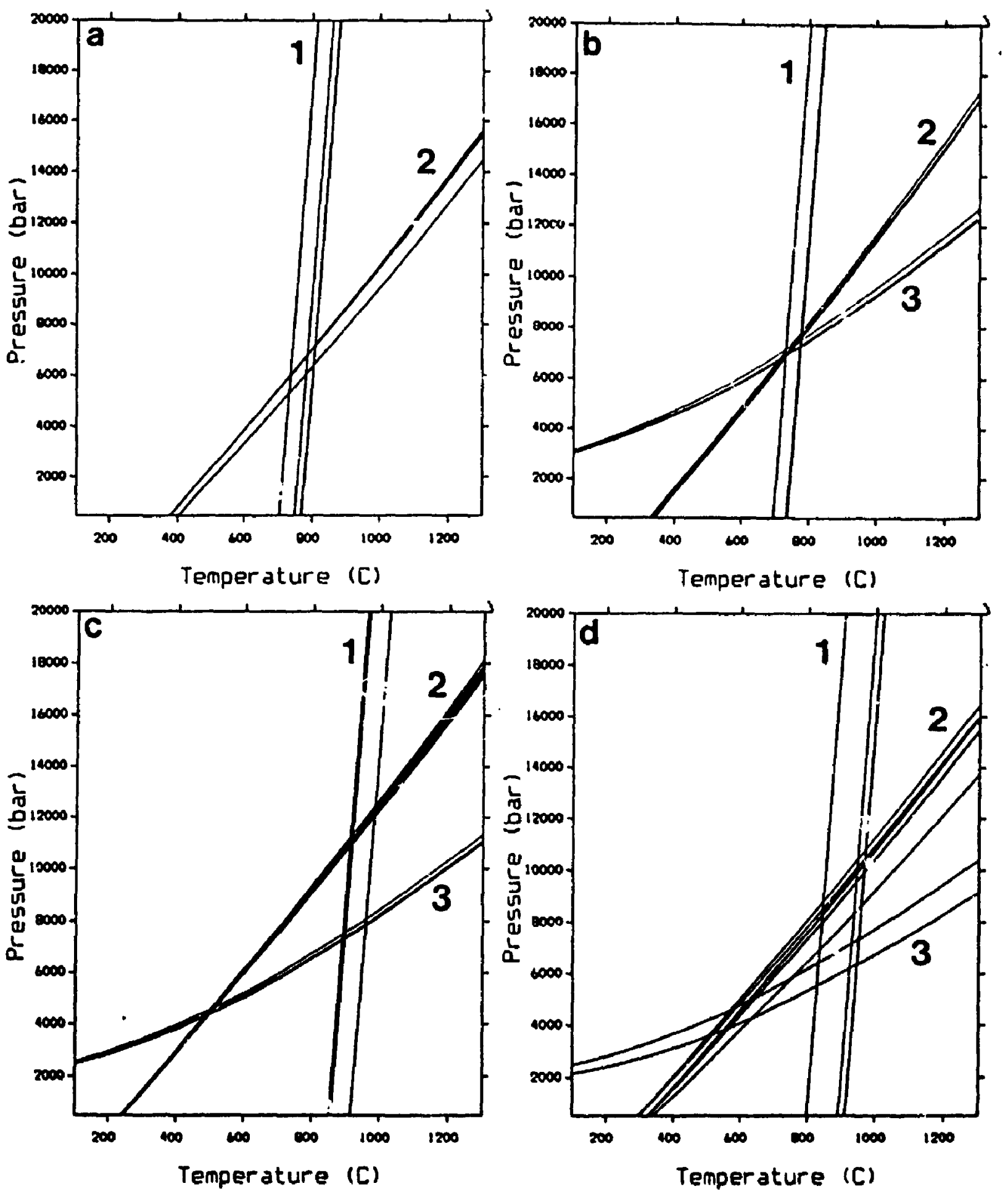


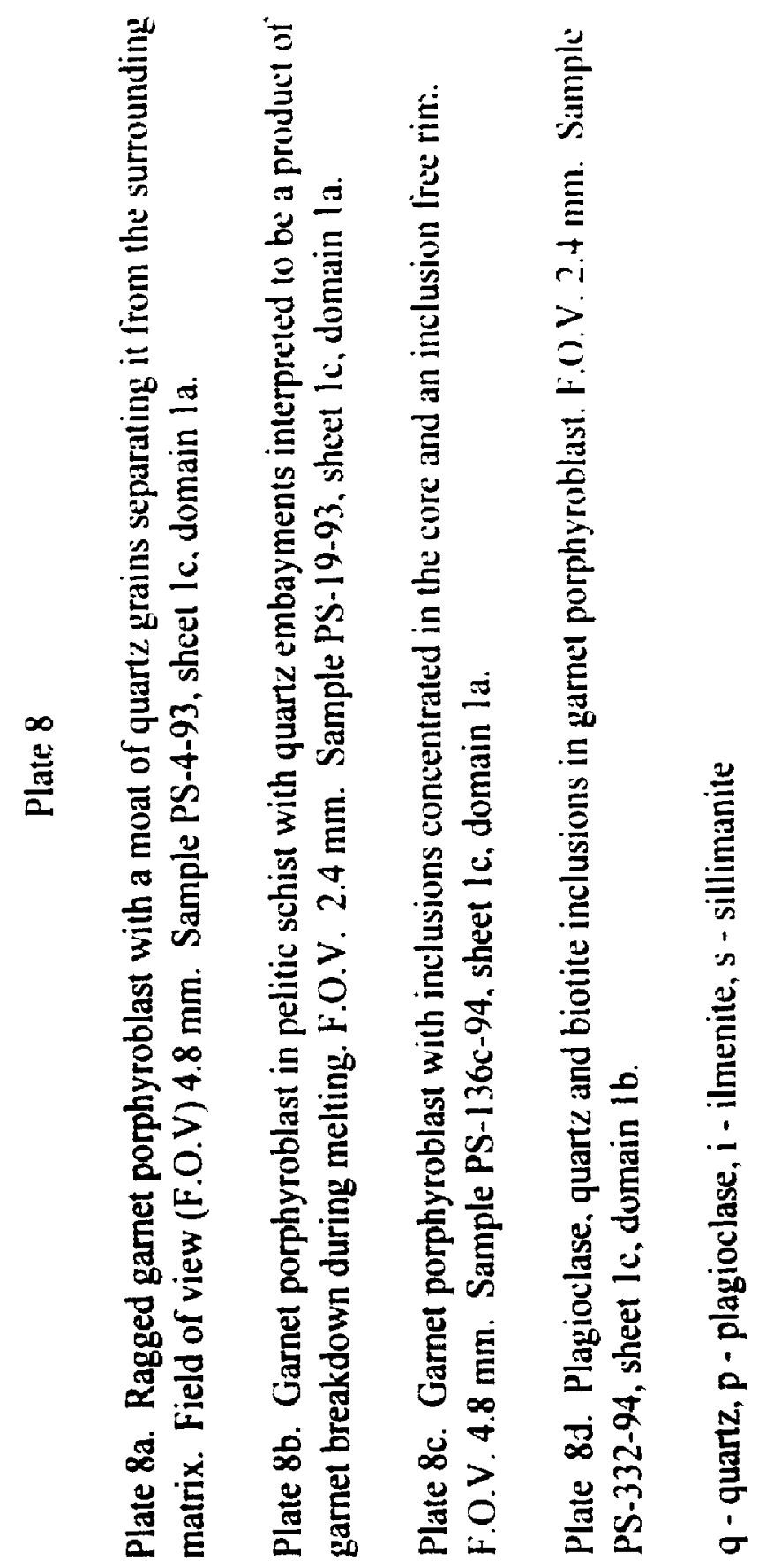


157

Figure 3.9 
Figure 3.9. Petrogenetic grads lor pelitic schists and thermobarometric data results from the $V$ alhalla complex. Data represents post but near-peak pressures and temperatures calculated trom garnet rims. proximal biotite: and plagioclase in contact $w$ ith the garnet rim. where possible. I hese data are representative of the temperature at whict reaction (8) closed. Rhombohedr; sri: generated from maximum and minimum pressures and temperatures from I H. H:Ol plots s presented in figure 3.7. Shaded areas are the intersection range for (iRAll. and the garnet-biotite thermometer. while unshaded areas are the intersection range for ( $A$ ASP and the garnet biotite thermometer.

I. quilibria:

A) albite + potassium feldspar + quartz $+\mathrm{H}_{2} \mathrm{O}=$ melt

B) muscorite - potassium feldspar + quartz $+11,0=$ melt

( ) muscovite + plagioclase + quartz = potassium feldspar - aluminosilicate - liquid

8) biotite + plagioclase + aluminosilicate + quartz $=$ garnet + potassium feldspar - liquid

10) muscovite + quart $z=$ potassium feldspar + aluminosilicate + melt

D) biotite + quartz + plagioclase $=$ orthopyroxene + potassium feldspar + liquid

a) Samples PS-4-93, PS-9-93. PS-169-94. PS-277-94 and PS-344-94 from the west side of sheet $l$ (domain la) and samples PS-61-94 (Airy Creek) and PS-332-94 (Slocan Ridge. domains $(\mathrm{b})$. Data is in agreement with petrographic observations.

1) Samples PS-28a-93. PS-28b-93, and PCA-93-84 from the area of Vallican (domain 2) in the Slocan River Valley. These samples show a large variation in pressure and temperature and large difference between GASP and GRAIL data. Sample 28a has variable compositions of plagiociase rimming garnet which indicate different portions of a decompression period (large trapezoid).

c) Samples PCA-200-85 and PS-348-94 from the lower Gwillim Creek window (sheet 1 a. domain 3). These samples record a large variation and higher temperatures than sheet $1 \mathrm{c}$ but have similar pressures. 
a)

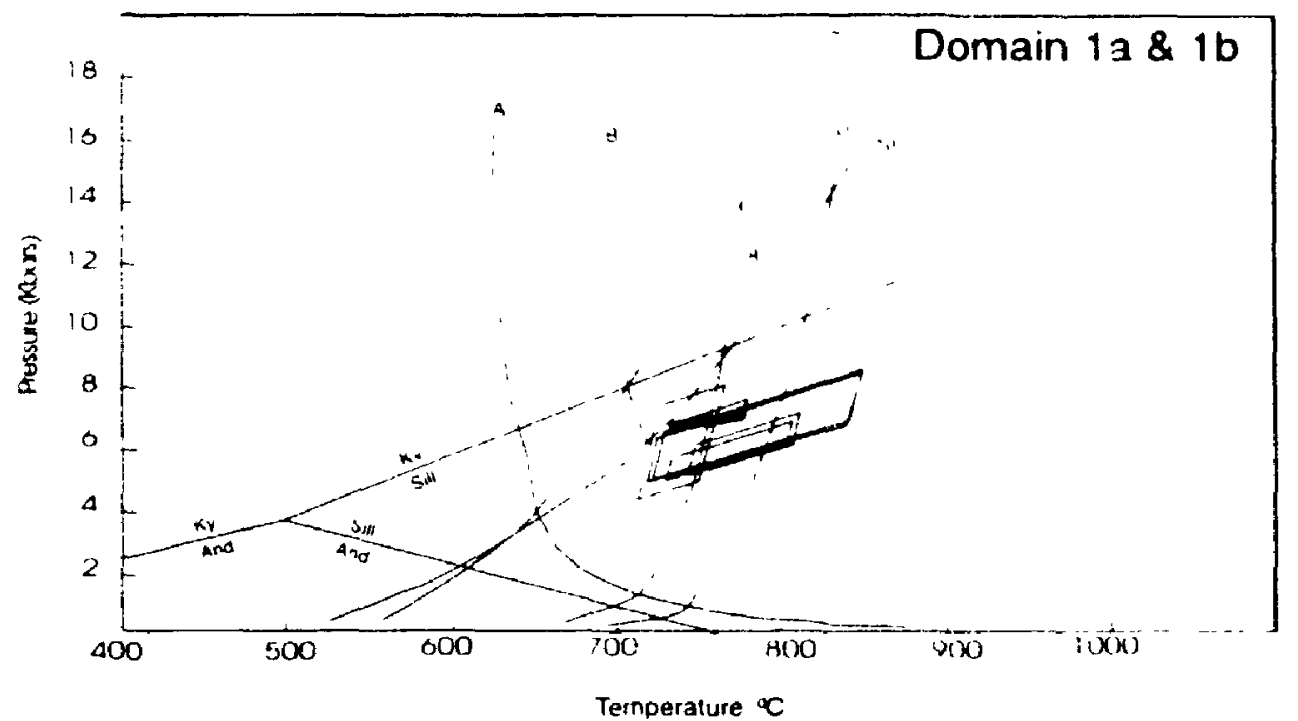

b)

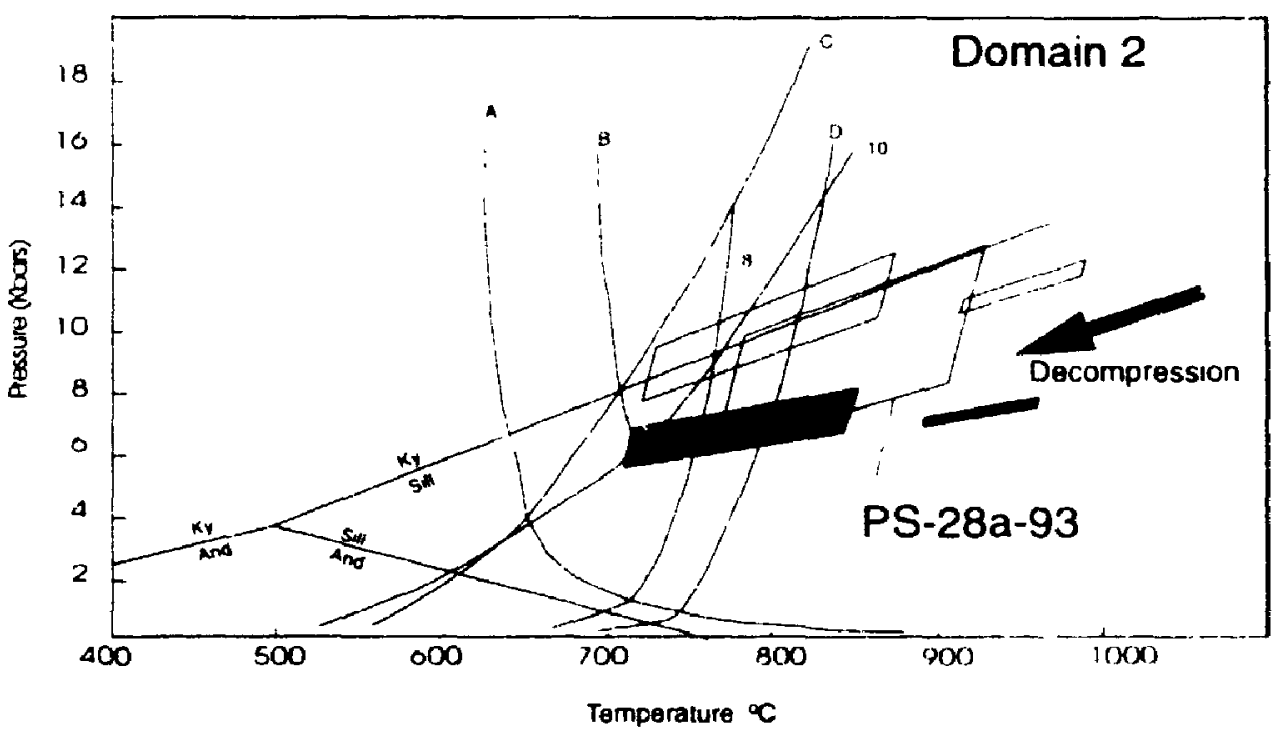

c)

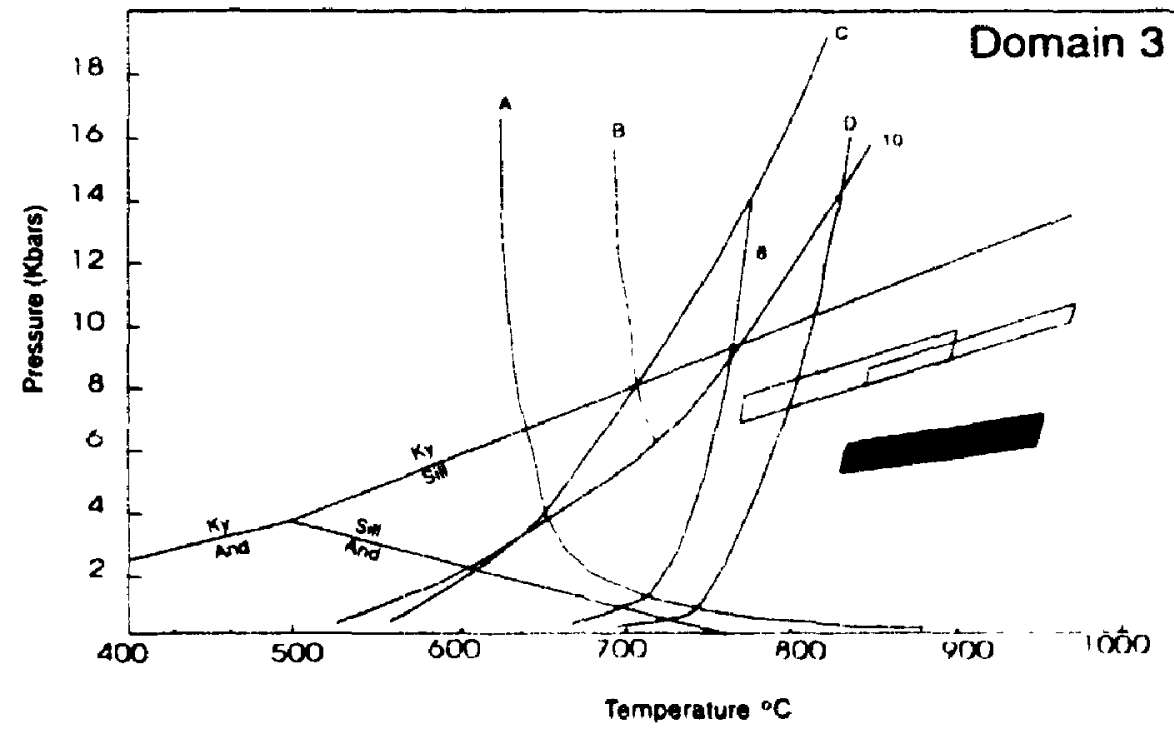



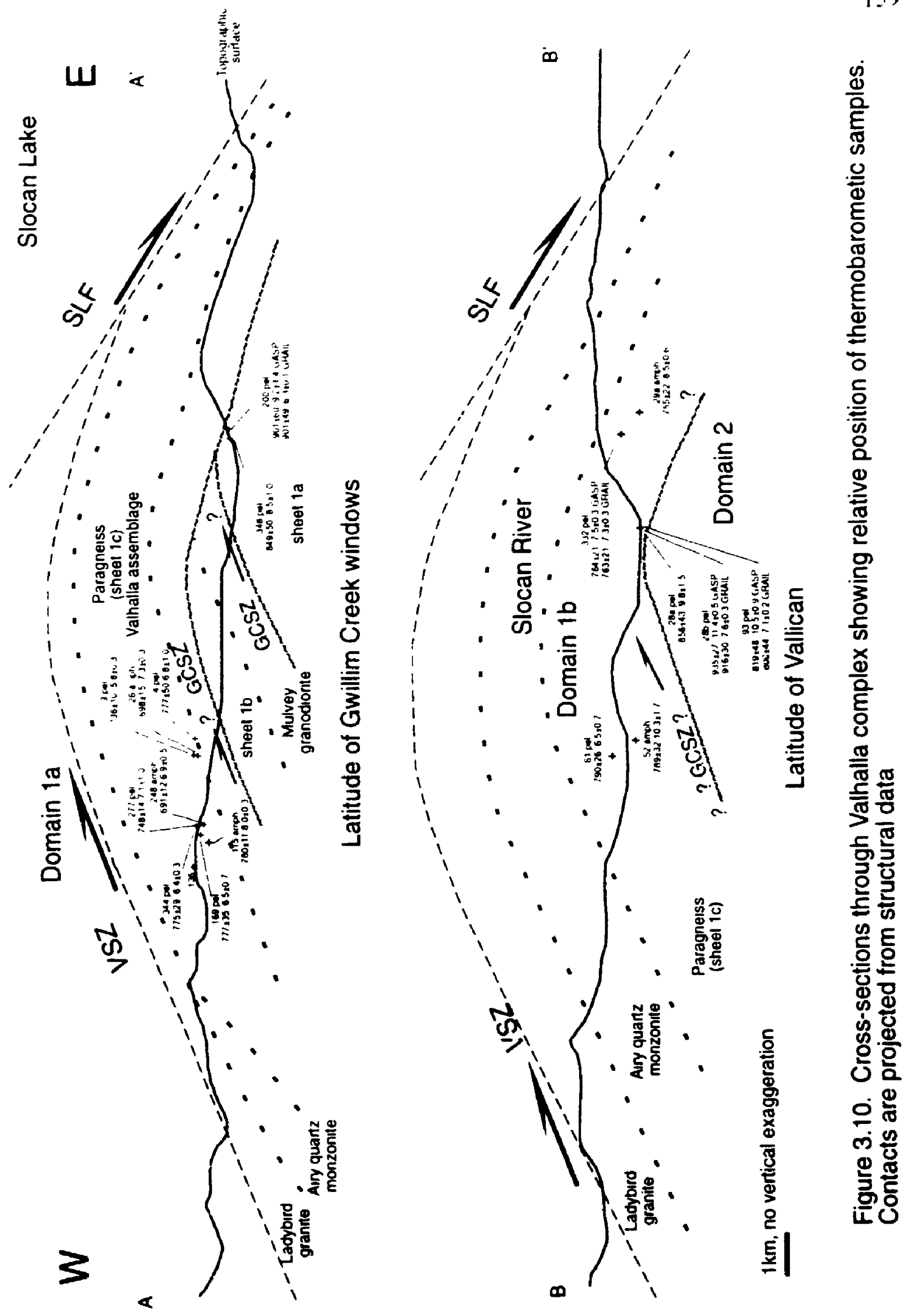
Figure 3.11 
Figure 3.11. Representative plots of equilibria for amphibolite gneisses generated using the computer program TWEEQU.

1) tremolite + anorthite $=$ tschermakite - grossular - pyrope - quartz

2) almandine + tremolite $=$ Fe-tremolite + pyrope

3) almandine + phlogopite $=$ pyrope + annite

a) PS-26-93 - de nain la. Rinda Ridge

b) PS-52-94 - domain 1b. Airy Creek

c) PS-248-94 - domain la, Hird Lakes

d) PS-115-94 - domain la. Rinda Ridge 

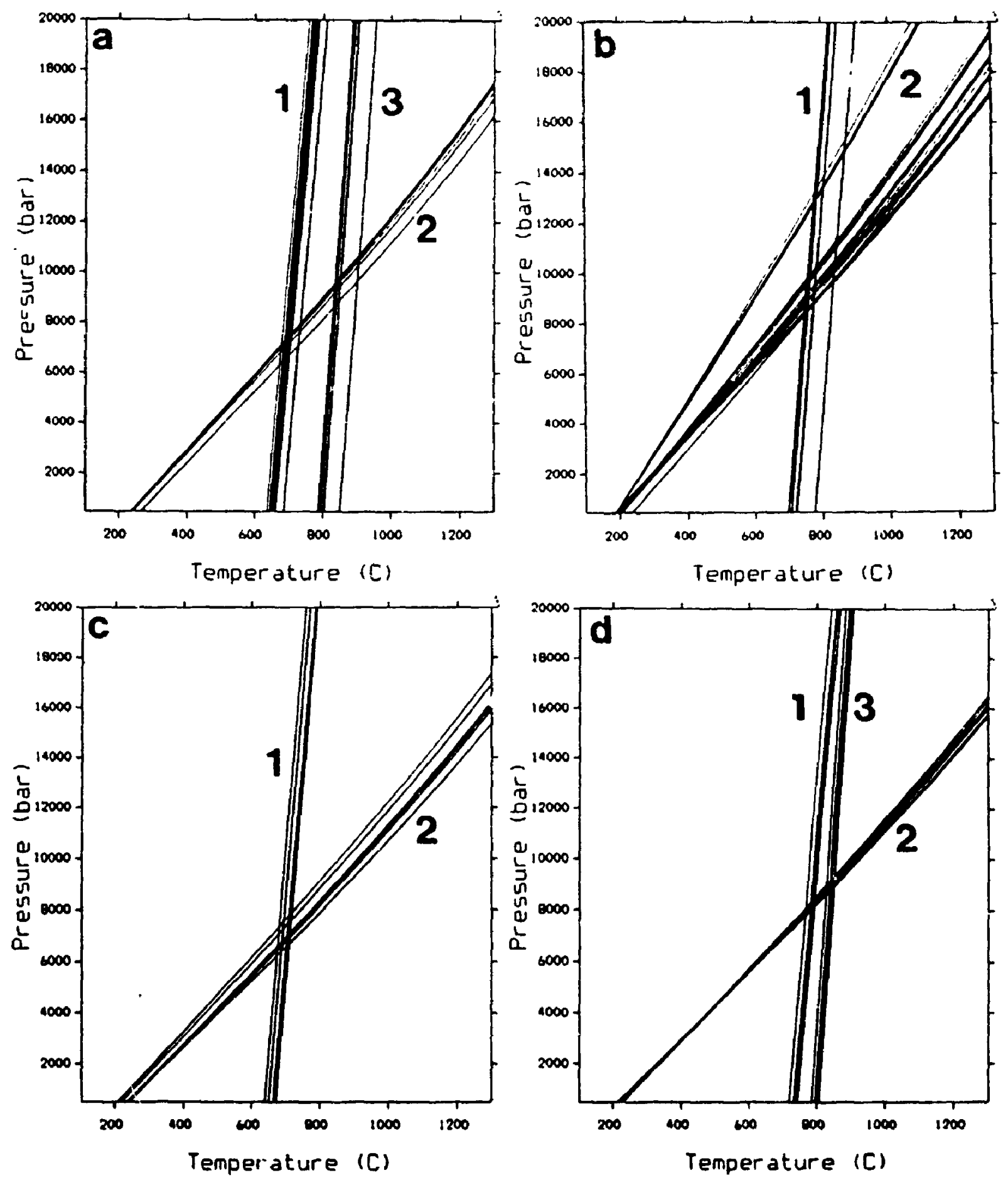


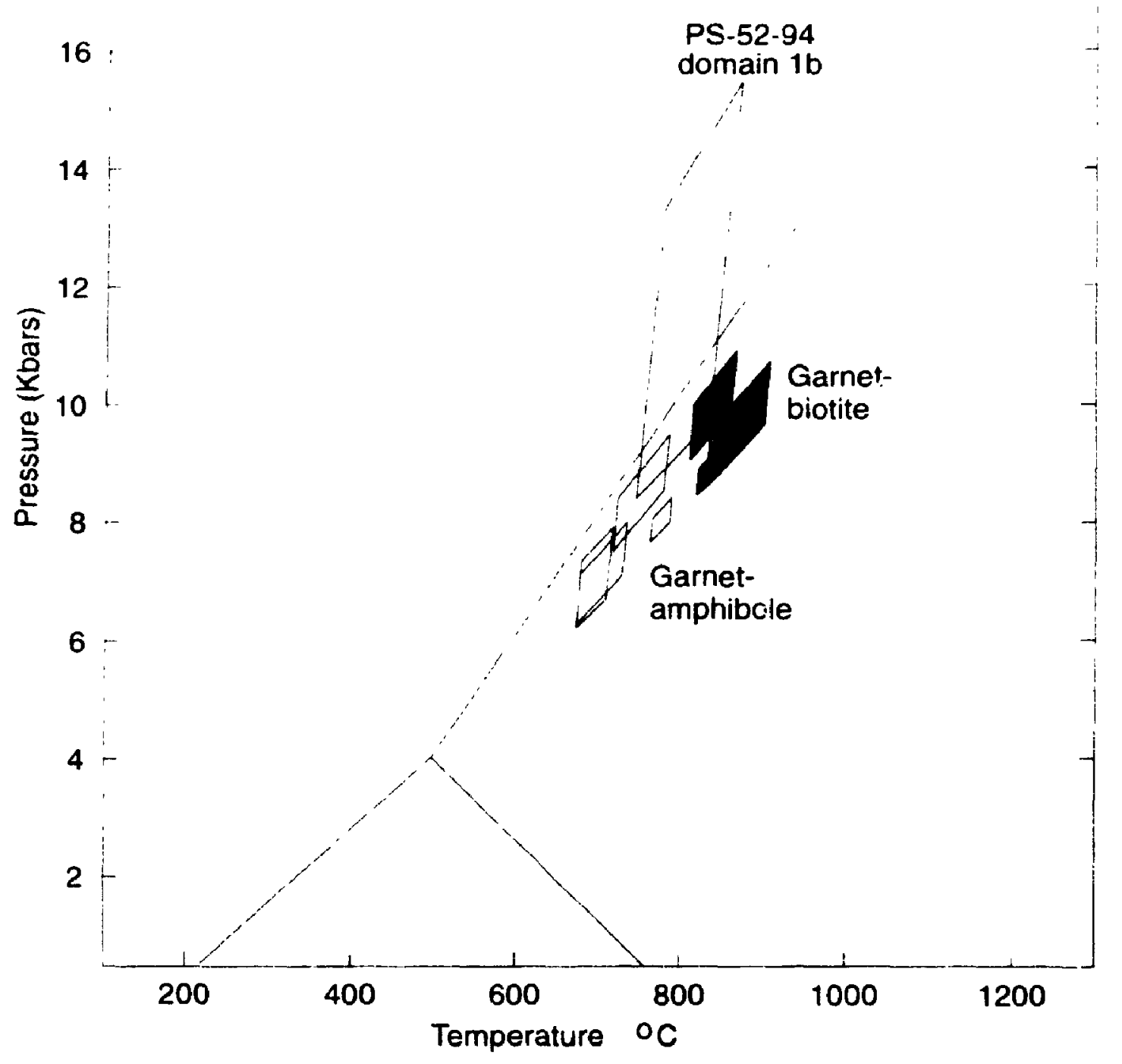

Figure 3.11. Compilation of thermobarometric data from amphibolite gneisses. Boxes represent maximum and minimum pressure and temperature presented in Table 3.3. Unshaded areas represent the intersection of the garnet-amphibole thermometer (reaction 5) and the garnet-plagioclase-amphibole barometer (reaction 4). The shaded area is the intersection area of the garnet-biotite thermometer (reaction 1) and reaction 4 . The aluminosilicate equilibria are shown for reference. Anomalously high pressures recorded by PS-52-94 are a result of local disequilibrium between garnet and plagioclase moats. 
$16:$

Plate 8 


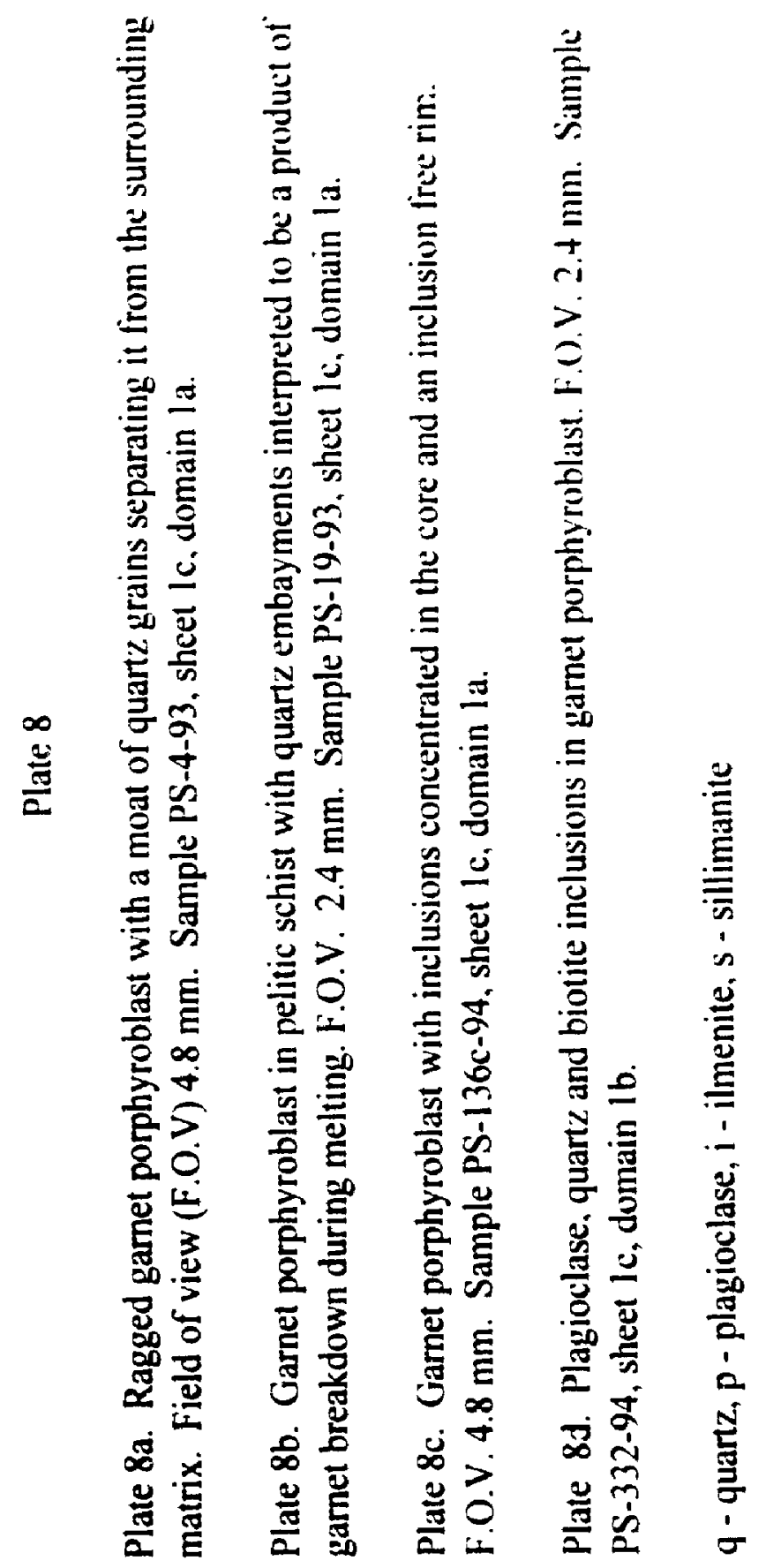




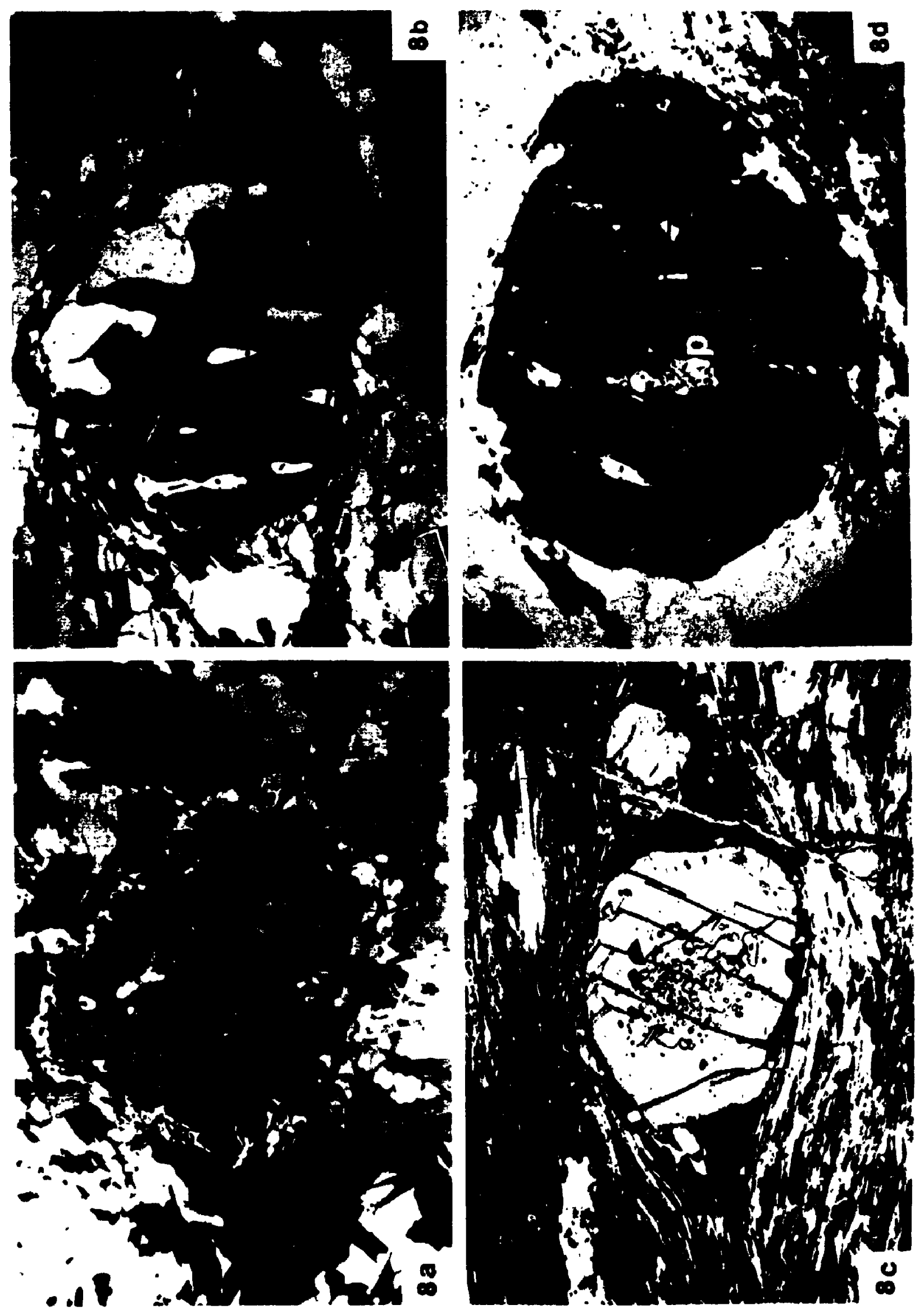


Plate 9 


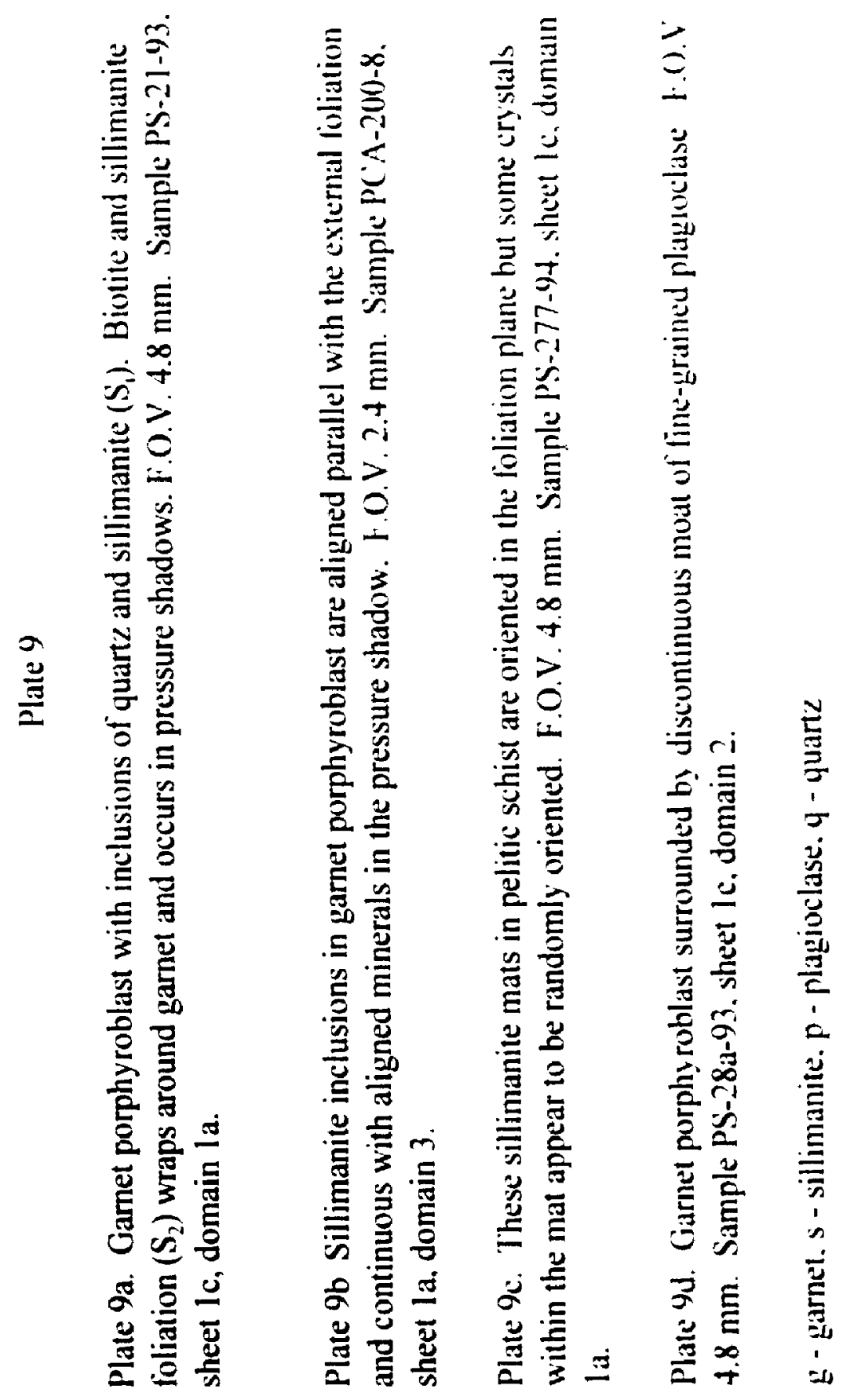




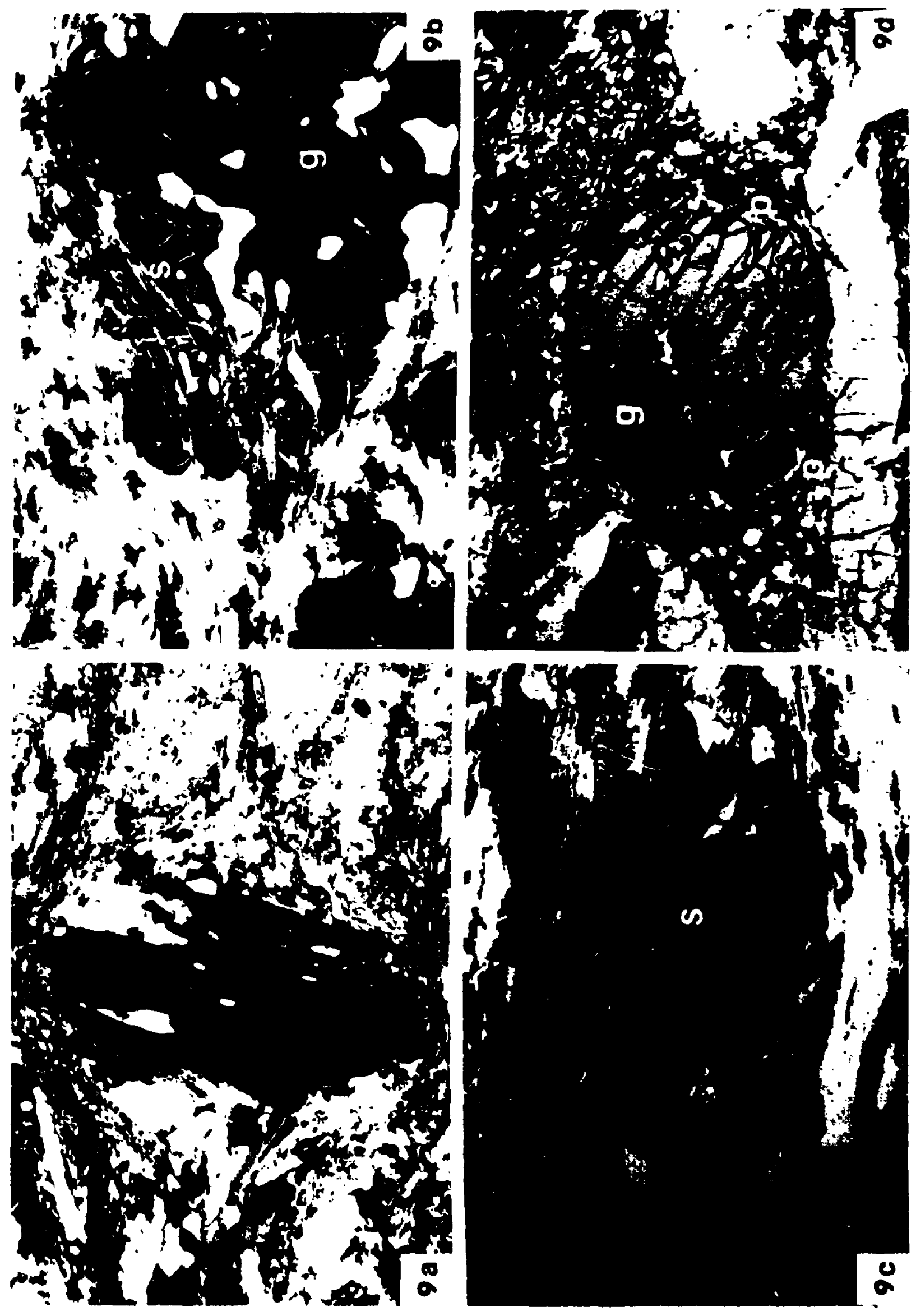


$16 ?$

Plate 10 


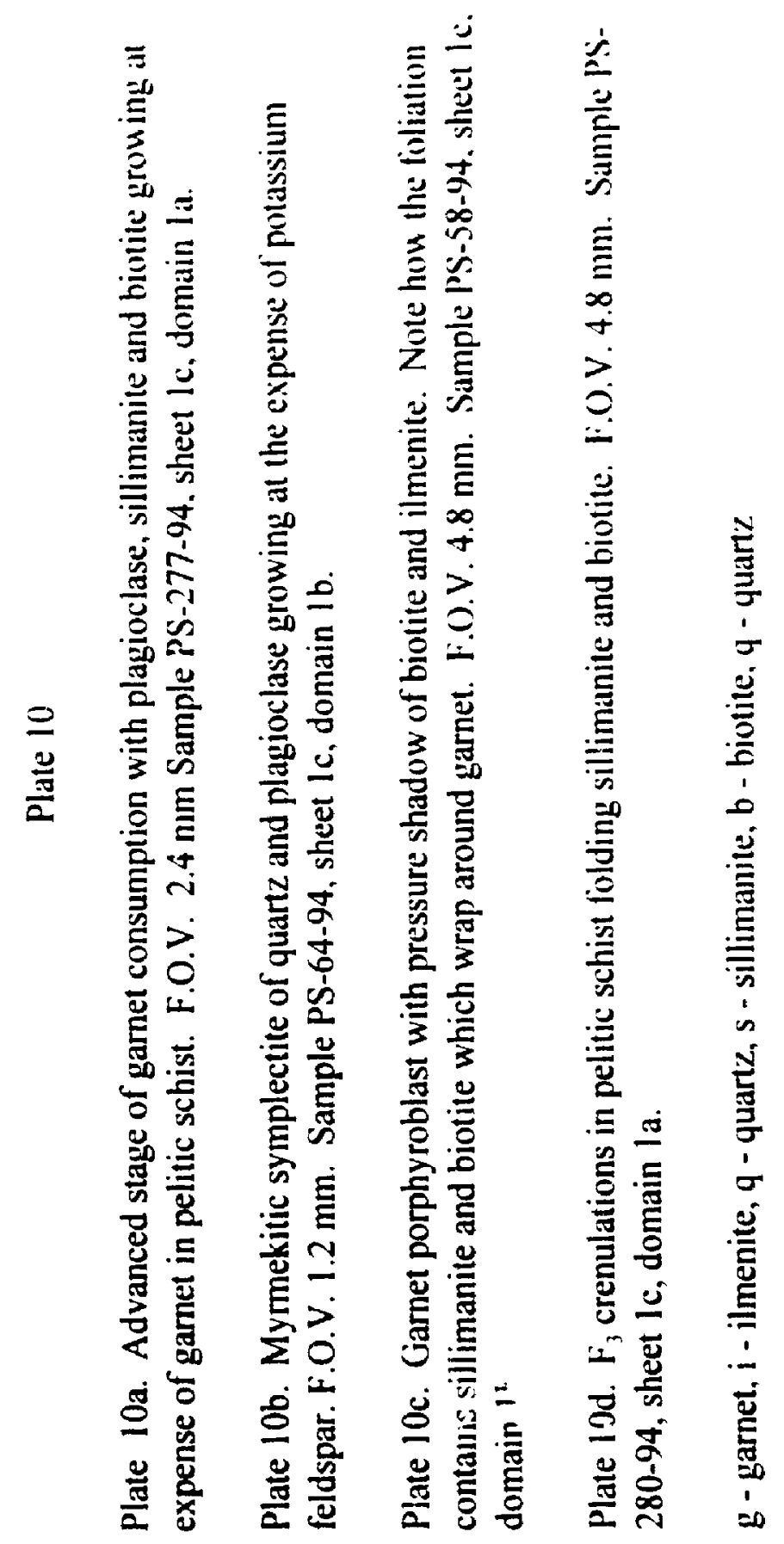



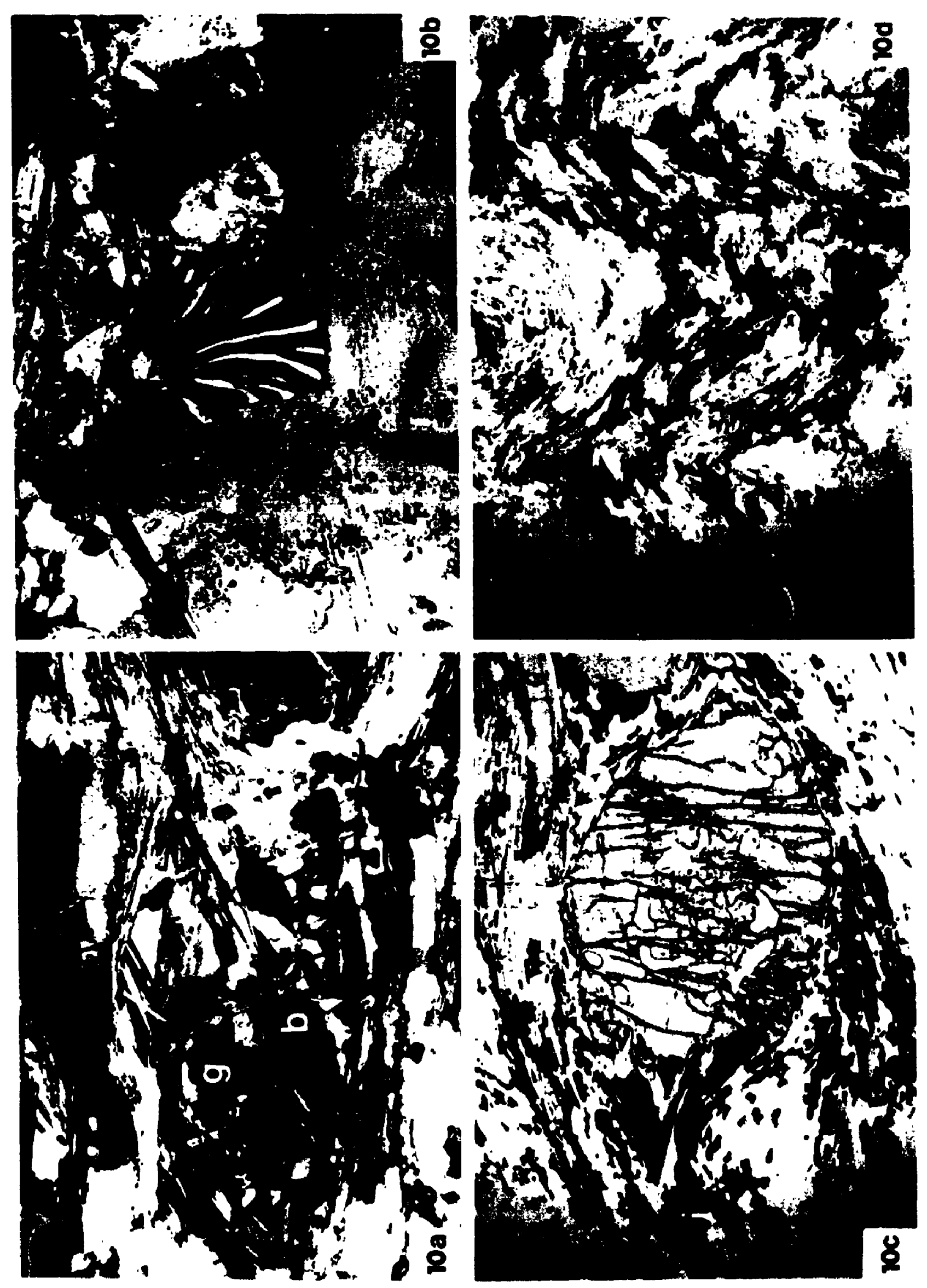

ivf

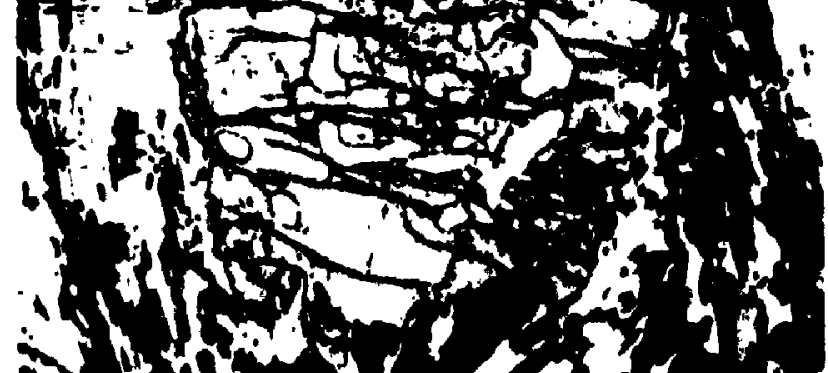
$x+y+y, y+a$

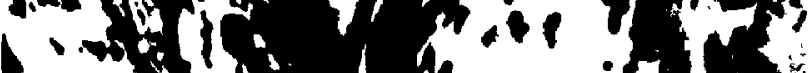

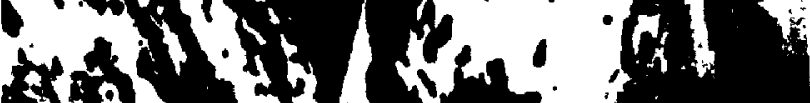

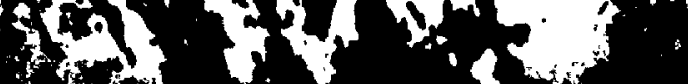

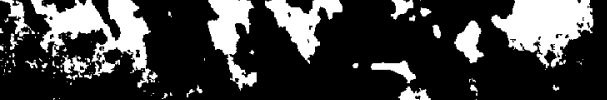


Plate 11 


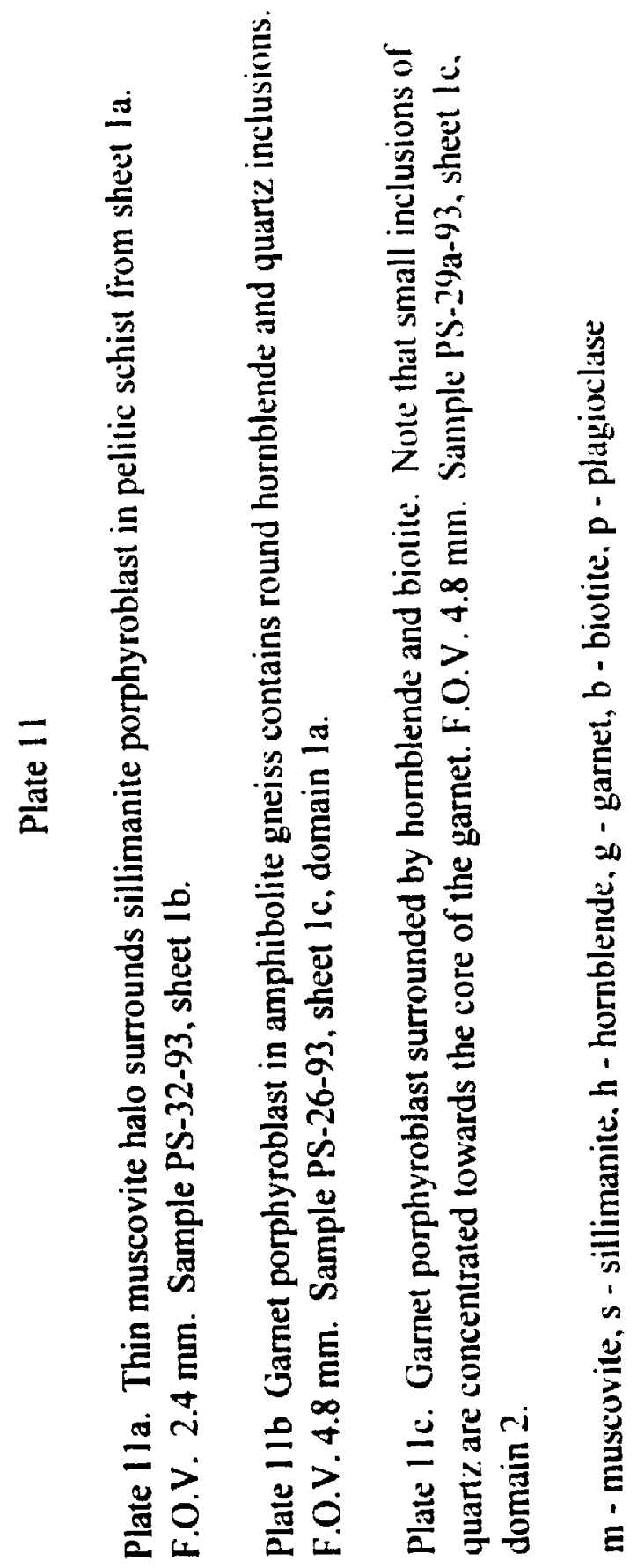




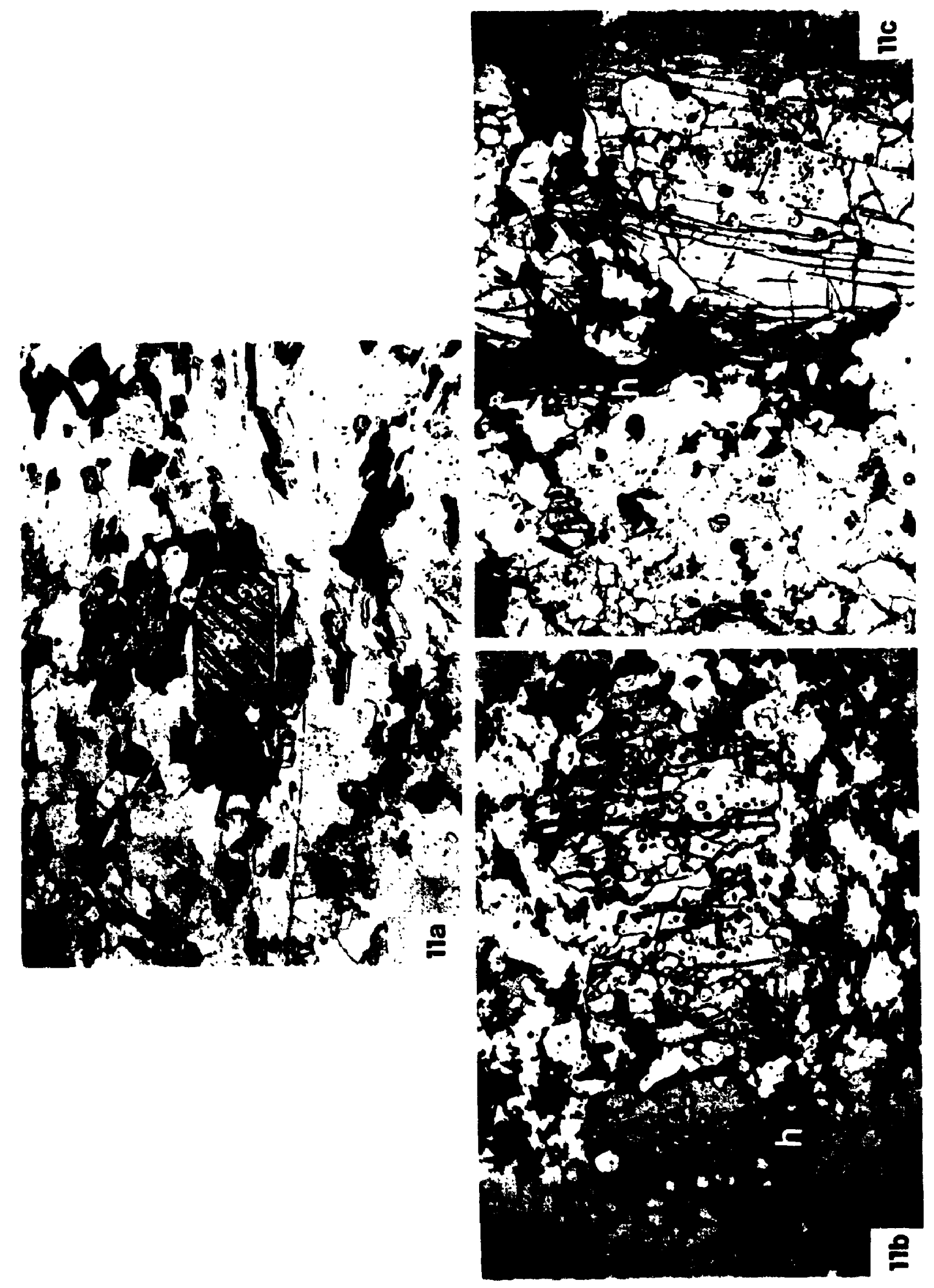




\section{Plate 12}




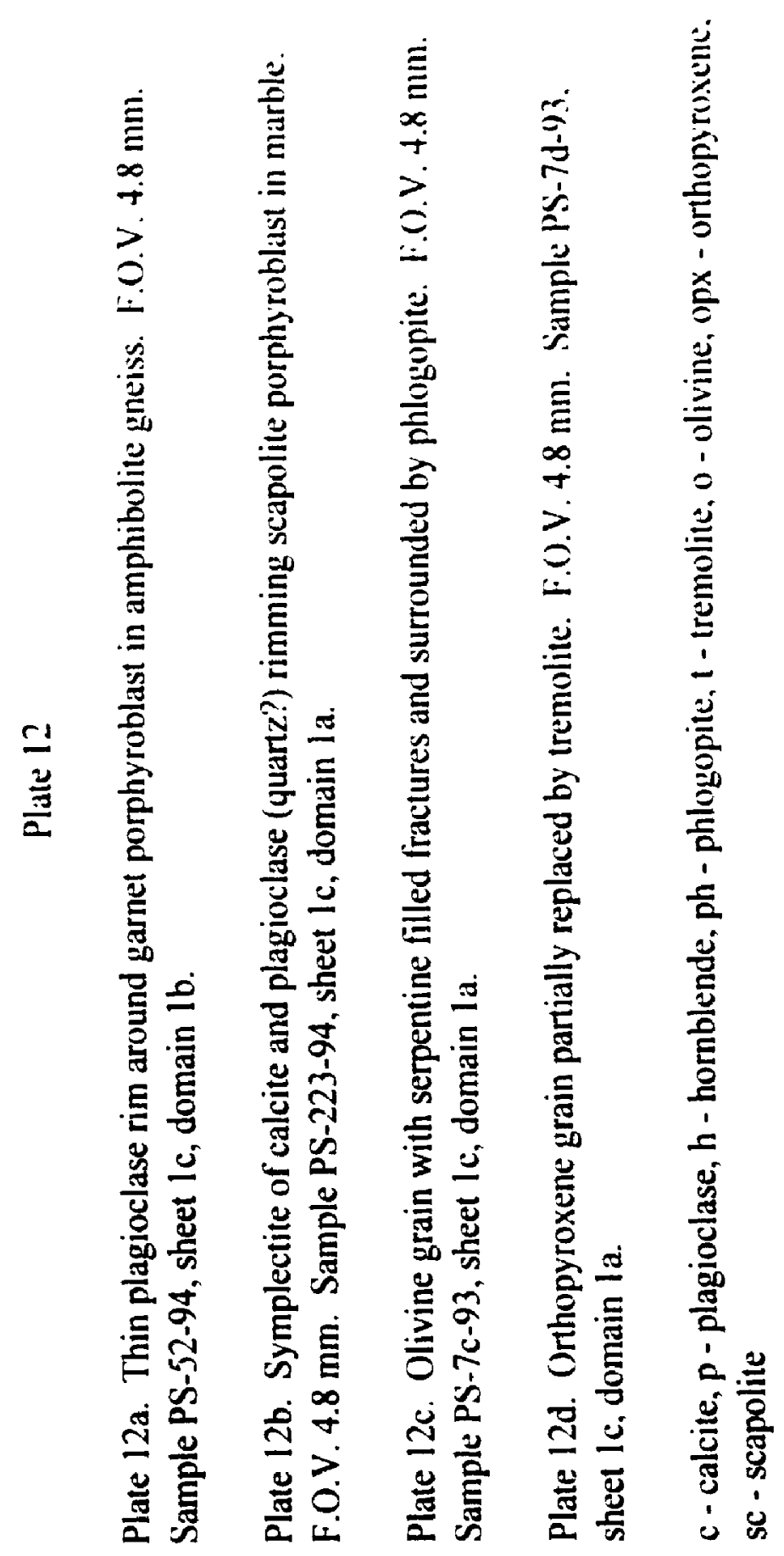



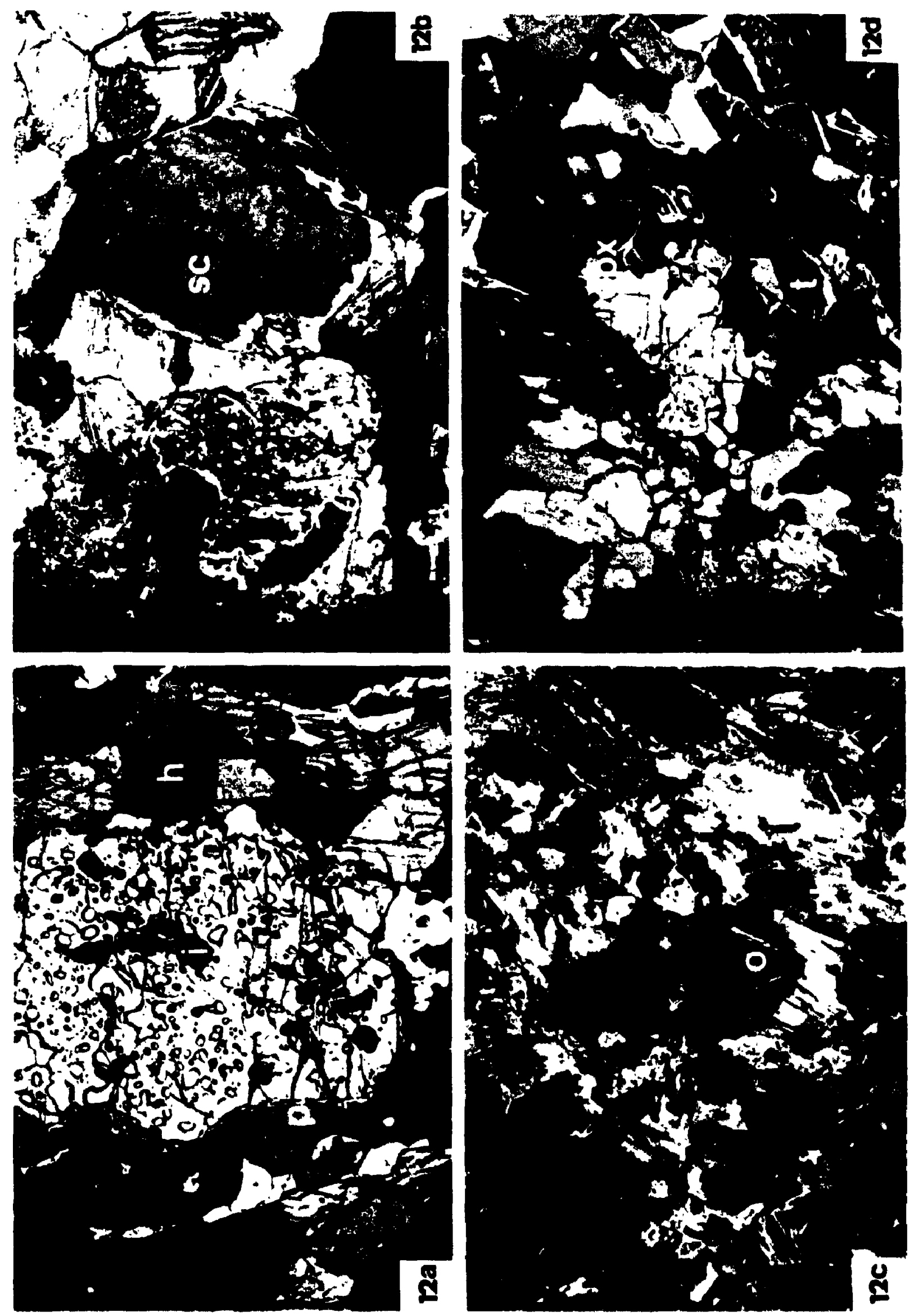


\section{CHAPTER 4: TECTONIC INTERPRETATION}

\subsection{Introduction}

Previous work in the Valballa complex has emphasized the geochronology of the granitic rocks and faults bounding the complex (Carr et al.1987; Parrish et al. 1988; Carr 1995; Parrish 1995). This study brings forth new stratigraphic and thermobarometric data which elucidate the evolution of the Valhalla complex. Based on data presented in this study together with data from previous studies, the timing of compressional deformation and metamorphic events were used to place constraints on the tectonic evolution of the Valhalla complex and the Gwillim Creek shear zones (Tables 2.1,3.1). Tectonic interpretations based on the conclusions made in chapters 2 and 3 are summarized in a geologic history of the Valhalla complex.

\subsection{Summary of stratigraphic and metamorphic data}

\subsubsection{Stratigraphy}

Paragneisses in the Valhalla complex occur at three structural levels separated by Cretaceous to Eocene granitic rocks and the Gwillim Zreek shear zones. The uppermost paragneiss sheet (lc) is composed of a heterogeneous package of pelitic schists, marbles, calc-silicate gneisses, metaconglomerates, amphibolite gneisses and ultramafic schists, termed the Valhalla assemblage (Figs. 2.3, 2.4). At the base of the sheet (units 1-3), there is a sequence of conglomerates, calc-silicate gneisses, and marbles interlayered with 50-100 
$m$ thick units of aluminum-poor semipelitic to pelitic schist. Procecding up in structural direction, the sequence becomes more carbonate-rich, with metre-thick marbles and calcsilicates gneisses interlayered with quartzites and slightly more aluminum-rich pelitic rosks (units 4-8). There is also an increase in amphibolite gneisses and ultramafic rocks. which are absent in the structurally lower sections. This sequence is overlain by thick marble and quartzite layers, units 9 and 10 , respectively. In domains $1 \mathrm{~b}$ and 2 in the southern area of the paragneisses, a thick unit of pelitic schist and amphibolite gneiss predominates (unit 11 ).

The Valhalla assemblage is correlated with rocks from the Neoproterozoic Horsethief Creek Group, Hamill Group, Badshot Formation, Lardeau Group and Milford Group (Table 2.3 ) and was thinned by up to $80 \%$. Stratigraphic correlations indicate that the Valhalla assemblage has been inverted. These correlations have a bearing on three aspects of the tectonic evolution of the Valhalla complex: 1) the timing and mechanism for tectonic inversion of the Valhalla assemblage; 2) the significance and timing of thinning of the Valhalla assemblage; and 3) correlation of the GCSZ. possibly linking the (Omineca belt to the Foreland belt.

\subsubsection{Metamorphic Petrology}

Textures relating the timing of metamorphism to deformation, coupled with thermobarometric data from various bulk compositions and mineral equilibria from three domains at successively deeper structural levels, place constraints on the timing and sense of displacement of the GCSZ. A period of near thermal peak decompression is inferred, firstly, from reactions responsible for plagioclase moats, and secondly, by the difference 
between pressures determined from GRAIL and GASP equilibria, which are interpreted to represent a variation in closure temperature. The prograde pressure-tel perature path of the Valhalla complex is not well constrained. If the Valhalla complex recorded a clockwise pressure-temperature path it would indicates that the complex was in a compressional tectonic setting (England and Thompson 1984. Harley 1988). which would be consistent with the interpretation of the GCSZ being compressional structures. ithe presence of rutile rimming ilmenite and the compositions of plagioclase inclusions in garnets of pelitic schists suggests that these rocks underwent a counter-clockwise P-T path. however. more data is required to confirm this.

Textural observations and thermobarometry indicate that the peak of metamorphism was synchronous with $D_{2}$ in sheei $1 c$, and movement on the GCSZ in the GCW and the Passmore dome. The peak of metamorphism has been interpreted to be $75 \mathrm{Ma}$ (Heaman and Parrish 1991) and therefore the GCSZ were active at this time. The fact that thermobarometric data indicates that temperatures and pressures increase with structural level within the limits of normal thermal and pressure gradients implies that sheets $I c$ and la share the same thermal history. If metamorphism had totally predated the main deformation event $\left(D_{2}\right)$ and movement on the GCSZ was east verging then one would expect to find hot rocks placed on cold rocks, and possibly an inverted thermal gradient. This is not the case.

\subsubsection{Timing of deformation in the Valhalla complex}

The youngest rocks of the Valhalla assemblage are correlated with the Carboniferous 
Milford Group, therefore the deformation event that inverted them must be post Mississippian. The minimum age for first phase folding is constrained by the -75 Ma peak of metamorphism and synchronous $S_{2}$ development. The idea that the first phase of folding or urred after the intrusion of the $\sim 110$ Mulvey granodiorite cannot be dismissed because field relationships between the Mulvey granodiorite and $F_{1}$ folds were not observed. Although age constraints for $F_{1}$ folding are fairly broad $(>75-\sim 290 \mathrm{Ma})$, the timing of this deformation is inferred to be pre-Mulvey granodiorite because $S_{1}$ fabrics are not observed in the Mulvey granodiorite.

The Mulvey granodiorite is interpreted to be structurally duplicated by the GCSZ. The Miulvey granodiorite contains the dominant $S_{2}$ fabric present in the Valhalla assemblage and therefore at least part of the $S_{2}$ fabric development is younger than $110 \mathrm{Ma}$. The development of $S_{2}$ is interpreted to be synchronous with movement on the GCSZ which means that the GCSZ are also at least in part younger than $110 \mathrm{Ma}$. $\mathrm{S}_{2}$ is interpreted to be synchronous with metamorphism which peaked at 75-80 Ma (see section 2.2) and therefore at least part of the $S_{2}$ development occurred before $75 \mathrm{Ma}$. It is possible that $S_{2}$ development and movement on the GCSZ began while the Mulvey granodiorite intruded. $S_{2}$ has a minimum age of $>63 \mathrm{Ma}$ because the Airy quartz monzonite does not contain this fabric. $\mathrm{F}_{3}$ folds leucosome containing the $S_{2}$ fabric, sillimanite and biotite indicating that this folding event was syn- post metamorphic peak. These folding events are $<75 \mathrm{Ma}$ because they are younger than the peak of metamorphism, which is inferred to be this age (Heaman and Parrish 1991). 


\subsection{Interoretations}

\subsubsection{Inverted stratigraphy}

The Valhalla assemblage is interpreted to be the upside down limb of a large first phase recumbent fold similar to other recumbent nappes in the southern Omineca belt such as the Scrip Nappe (Raeside and Simony 1983), the Riondel Nappe (Höy 1977, 1980), and the Carnes Nappe (Brown and Lane 1988). The following section will evaluate the timing relationships of deformation in the Valhalla complex, based on stratigraphic correlations and published geochronological data. and compare this data with models for the timing of first phase folding in the Riondel and Scrip Nappes.

The timing, origin and tectonic setting of large scale recumbent nappes in the Kooienay Arc and eastern Omineca belt have been the subject of debate for some time. Inverted stratigraphy in the Omineca belt has been attributed to isoclinal $F_{1}$ folding of a surface related to the obduction of a marginal basin during the Early to Middle Jurassic (Brown et al. 1986). In this scenario, west verging $F_{1}$ folds resulted from the delamination of the North American crust from the mantle lithosphere about an east-dipping subduction zone (Brown et al. 1993). East-verging folds that overprint $F_{1}$ were formed as a result of east directed thrusting during the Middle Jurassic to Late Cretaceous (Brown et al. 1986; Price 1986; Brown and Journeay 1987; Brown et al. 1993).

\section{Riondel Nappe}

The Riondel Nappe, to the east of the Valhalla complex (Fig. 1.1), is a first phase 
west verging fold which deforms Hamill to Lardeau group (and possibly Milford) strata. The nappe was refolded by east verging $I_{2}$ folds and coaxial $F_{3}$ folds (Höy 1977, 1980). Although first phase folding has been interpreted to be Paleozoic in some parts of the Kootenay Arc (Read and Wheeler 1976), it has been demonstrated that the lack of a cleavage in the Milford Group is a function of structural level (Struik 1981: Murphy 1987). Brown and Lane (1988) presented evidence that first phase folding in the Carnes nappe is Jurassic and is related to obduction of the Quesnellia terrane and subsequent backthrusting. Brown and others (1993) suggested that west verging folds in the southern Omineca belt are a result of folding of the obduction surface between the Quesnellia and Kootenay terranes due to delamination of the crust during east directed subduction of the mantle lithosphere.

In the Riondel Nappe, it is uncertain if the Carboniferous Milford Group was folded by first phase folding, however seismic reflection data suggest that it might have been (Varsek and Cook 1991). Cook and Varsek (1991) present two models for the formation of the Riondel Nappe. Model 1 is based on the model of Brown and Lane (1988). In the Riondel area, the Milford Group does not surface on the east limb of the Riondel Nappe. It is possible that the Milford Group is folded by first phase deformation and is truncated on the east limb of the Riondel Nappe by the West Bernard fault (Cook and Varsek 1991).

The second model of Cook and Varsek (1991) is based on the mapping of Read and Wheeler (1976) and Klepacki (1985), and suggests that west-directed folds were possibly related to the Paleozoic docking of the Kootenay terrane to the miogeocline. This raises another question concerning the boundary between the Kootenay terrane (Lardeau Group) and the North American miogeocline. Colpron and Price (1995) present evidence that the 
Lardeau Group lies unconformably on and is depositionally linked to the miogeocline. This contradicts the "Lardeau shear zone" theory of Smith and Gehrels (1992b), which implies that the Kootenay terrane is allochthonous and was juxtaposed against the miogeocline by faulting. The inference that the Kootenay terrane and the miogeocline are depositionally linked would suggest that a Paleozoic deformation was not significant.

\section{Scrip Nappe}

The Scrip Nappe, located north of the Monashee complex (Fig. 1.1a), exhibits evidence for a Jurassic age for the first two phases of deformation in the southern Omineca belt. It contains a $50 \mathrm{~km}$ long inverted, southwest verging, $F_{1}$ limb of Horsethief Creek Group strata, which rocts in the east and is refolded by later $F_{2}$ and $F_{3}$ coaxial deformation events (Raeside and Simony 1983). The first phase of folding was initiated with the onset of metamorphism in the Jurassic, followed by Middle to Late Jurassic $F_{2}$ and Early Cretaceous $\mathrm{F}_{3}$ folds (Raeside and Simony 1983). The structure of the Scrip Nappe is consistent with the model of Brown and Lane (1988).

\section{Eagle Bay assemblage}

In the Adams Lake-Clearwater-Vavenby area, Schiarizza and Preto (1987) found that the dominant structural pattern is defined by Mesozoic deformation and they concluded that there is no clear evidence to suggest Paleozoic deformation in the Eagle Bay assemblagc. This is consistent with observations in the Valhalla complex which suggest that Carboniferous aged Milford equivalents were involved in first phase folding and that 
Paleozoic deformation was not responsible for isoclinal folding.

\section{Timing of inversion in the Valhalla complex}

Stratigraphic correlations indicate that the Valhalla assemblage is an inverted sequence of Horsethief Creek to Milford Group strata, and may have been inverted as a result of isoclinal folding, similar to other recumbent nappes such as the Riondel Nappe (Höy 1977. 1980) and Scrip Nappe (Raeside and Simony 1983). These nappes are inverted by primarily Jurassic deformation which has not been documented at the mid-crustal levels of the Valhalla complex. Although timing constraints place first phase folding between $\sim 75 \mathrm{Ma}$ (peak of metamorphism $-\mathrm{S}_{2}$ development) and $\sim 290 \mathrm{Ma}$ (age of Milford Group correlatives) which are inverted, a pre-Mulvey granodiorite age of Early Cretaceous age is favoured for first phase folding in the Valhalla complex. This is consistent with the model of Parrish (1995) which suggests that deformation and metamorphism is younger at deeper structural levels in the southern Omineca belt. Although Jurassic first phase folding has not been documented at the mid-crustal structural level of the Valhalla complex, it cannot be ruled out because timing constraints are not narrow enough.

The inferred Early Cretaceous age of first phase folding in the Valhalla complex is consistent with the model of Brown and Lane (1988) and with seismic data from the Riondel area (Varsek and Cook 1991) which suggest that the present structural framework in the southern Omineca Belt was controlled by Jurassic or younger deformation.

\subsubsection{Thinning of the Valhalla assemblage}


The present thickness of the Valhalla assemblage is at most $1.5 \mathrm{~km}$ which implies that the sequence was thinned by up to $80 \%$. if correlations presented in this study are correct. Together, the Hamill to Milford strata alone are upwards of $4 \mathrm{~km}$ thick in the Goat Ranges where they are less deformed than the Valhalla assemblage and metamorphosed to greenschist - lower amphibolite facies. $S_{2}$ fabrics are parallel to the contacts of units within the Valhalla assemblage and this fabric forming development is interpreted to be synchronous with movement on the GCSZ. Structural thinning during east directed compressional deformation and shearing associated with the GCSZ are likely to have been the cause of this thinning. since there are no kinematic or structural data to support extensional deformation prior to the Eocene.

Alternatively, thinning of the Valhalla assemblage may be synchronous with $F_{1}$ folding, where the bottom limb of the recumbent nappe was dragged out and thinned while the upper limb retained its original thickness.

\subsubsection{Correlation of the Gwillim Creek shear zones}

It has been proposed that the Gwillim Creek shear zones are major faults linking the Omineca belt to the Foreland fold and thrust belt (Parrish et al. 1987; Cook et al. 1988). This is based on correlative seismic reflection packages beneath the Kootenay Arc and Purcell Anticlinorium (Varsek and Cook 1994: Cook and Van der Velden 1995). The duplication of units in the Valhalla complex (Fig. 1.3, Cross section B), the easterly fold vergence in the Valhalla assemblage, as well as minor kinematic indicators suggesting top to the east displacement (Parrish et al. 1985) are evidence that the GCSZ are thrust faults. 
No detailed structural study has been carried out on these shear zones. The correlation of the Valhalla assemblage to Paleozoic strata in the Kootenay Arc has implications for the correlation of the GCSZ to faults in this same area. A comparison of available structural. geochronological and seismic data from adjacent areas to the Valhalla complex in the Kootenay Arc and Purcell Anticlinorium and timing constraints on the GCSL (this study) and published geochronological data has resulted in several possibilities for the correlation of the GCSZ and faults in the Kootenay Arc and Purcell Anticlinorium. The possible correlations are with either the West Bernard fault in the Riondel area (Fig. 1.1). the Moyie fault in the Purcell Anticlinorium or structures imaged by the subsurface K-reflectors below the Purcell Anticlinorium described by Varsek and Cook (1994).

The timing of displacement on the GCSZ is younger than the -110 Ma deformed Mulvey granodiorite and older than 52 Ma cross-cutting aplite dykes (Parrish 1995). Parrish and others (1988) and Heaman and Parrish (1991) presented preliminary thermochronologic data which suggest that the thermal peak of metamorphism was at 75-80 Ma. Metamorphism is synchronous with fabrics associated with the GCSZ and therefore movement on the GCSZ. is syn-metamorphic and occurred at approximately $75-80 \mathrm{Ma}$.

There are four thrust faults in the Kootenay Arc and the Purcell Anticlinorium that are possibly correlative to the GCSZ (Figs. 1.1a. 2.6). The West Bernard fault (WBF), in the Riondel area. carries Hamill to Milford Group strata in its hanging wall and is cross-cut by the Late Cretaceous (?) Fry Creek batholith (Reesor 1973; Archibald et al. 1984). Further to the west, the Hall Lake fault (HLF) is cut by the $111-129 \mathrm{Ma}$ White Creek batholith (Wanless et al. 1968; Price 1981; Archibald et al. 1984), and the St. Mary fault (SMF) is 
plugged by the 94 Ma Reade Lake stock (Höy and Van der Heyden 1988). The Moyie fault (MF) cuts across Devonian aged gneisses but its age is not further constrained. excluding later cross-cutting Tertiary normal faults. The HLF. SMF and MF all carry (Belt) Purcell Supergroup with minor Windermere Supergroup and Hamill Group strata in their hanging walls. The $129 \mathrm{Ma}$ White Creek batholith cross-cuts the HLF and correlation with the GCSZ is inconsistent with a $<100$ Ma movement on the GCSZ.

Both the WBF and the GCSZ carry inverted Paleozoic stratigraphy in their hanging walls. Timing of displacement is not well constrained for the WBF. The Fry Creek batholith truncates the WBF (Höy 1980) and K'Ar dates gathered by Reesor (1973) give ages of 76-86 Ma from biotite and 83-97 Ma from muscovite. Archibald and others (1984) found K/Ar ages to be 50-58 $\mathrm{Ma}$, however these ages are related to cooling rather than emplacement. The present age data on the Fry Creek batholith constrain the age of displacement of the WBF to be between $\sim 100$ and $50 \mathrm{Ma}$. The age constraints on the GCSZ are between 110 and $52 \mathrm{Ma}$ and therefore it is permissible to correlate the GCSZ and the WBF. Cook and Van der Velden (1995) discuss the possible correlation of the WBF with the GCSZ, but note that the WBF has been interpreted to be associated with the Jurassic accretion of the Kootenay Arc with the North American craton (Varsek and Cook 1991).

In the Midge Creek area. Leclair (1984) has correlated the Seeman Creek fault with the WBF. The Seeman Creek fault is cross cut by the $117+4 /-1$ Ma Baldy pluton (Leclair et al. 1993) which suggests a pre-Early Cretaceous age for the WBF. Although age data and similar hanging wall stratigraphy permit a correlation between the GCSZ and the WBF. more geochronological data must be collected from the Fry Creek batholith to constrain the age 
of the WBF. If the correlation of the Seeman Creek tault to the WBF is correct (l.eclair 1984) then the WBF is probably Early Cretaceous in age and therefore does not correlatc to the GCSZ.

The GCSZ may also be correlative with the Moyie fault, whict: has a relatively: unconstrained age. The MF cuts Devonian gneisses (Leech 1962: Benvenuto and Price 1979) and is cut by Tertiary normal faults. Since the strata in the hanging wall of the $\mathrm{MF}^{\circ}$ belongs to the Purcell Supergroup, it would require the GCSZ to cut down-section, eastward.

Varsek and Cook (1994) have correlated the GCSZ across the SLF with a series of sub-horizontal reflections, termed the "K-reflectors" (Fig. 2.7). "K - reflectors" form the root" thrust of a duplex, which is bounded on the bottom by the combined Rocky Mountain basal detachment (RMBD) - Monashee décollement (MD) reflections. These "K - reflectors" are also interpreted to truncate thrust faults in the Purcell Anticlin . ium (HLF, SMF, MF) and merge with the RMBD-MD near the Rocky Mountain trench. The Monashee decollement was in motion in the Late Paleocene and had ceased by $58 \mathrm{Ma}$ (Carr 1992; Scammell 1993 ). If the "K-reflectors" merge with the RMBD in the east (Varsek and Cook 1994), then it is likely that they do not project to the surface, and a correlation is not possible using similar hanging wall stratigraphy. It is also possible that the GCSZ occur as blind thrusts below the Purcell Anticlinorium.

In the Pinnacle Peaks area, to the west of the Valhalla complex a subsurface structure imaged by seismic reflection data lies in the hanging wall of the Arrow Park thrust (Carr 1991, 1995; Parrish 1992). The Arrow Park thrust is interpreted to be -77 Ma based on the $79 \pm 2$ Ma age of the cross-cutting Watshan batholith. The Arrow Park thrust also lies in the 
hanging wall of the > $58 \mathrm{Ma}$ Monashee décollement. The seismic reflection in the hanging wall of the Arrow Park thrust has been interpreted to be correlative with the GCSZ. which is consistent with a break-forward sequence where the age of displacement on thrust faults decreases in age with depth (Carr 1995).

The GCSZ and the WBF. both carry inverted Paleozoic strata in their hanging walls. however a lack of solid age constraints on the WBF does not permit a correlation of the two faults. If they are correlative. either the GCSZ are older than the estimate of 70-80 Ma (Parrish et al. 1988) or the WBF is younger than the maximum age limits of $\sim 100 \mathrm{Ma}$. A correlation of the GCSZ to the WBF might also indicate that the WBF is younger than the Jurassic age proposed by Varsek and Cook (1991). More geochronological work needs to be done to refine the age of the Fry Creek batholith and thereby more closely constrain the age of the WBF. Alternatively, the GCSZ may be part of the duplex that merges with the Rocky Mountain basal detachment beneath the Purcell Anticlinorium.

\subsubsection{Decompression}

The wide range in An content ( $-38-52)$ of plagioclase in moats around garnet and the difference between pressures calculated from GASP and GRAIL equilibria in domains 2 and 3 are interpreted to record a period of decompression which occurred after the metamorphic peak. Plagioclase moats formed as a result of decompression reactions and are thinner along garnet rims parallel to the $\mathrm{S}_{\mathrm{T}}$ fabric than they are in garnet pressure shadows. This suggests that they were formed during or shortly after the thermal peak and before $\mathrm{S}_{2}$ and $\mathrm{S}_{\mathrm{T}}$ fabric development had ceased. The thermal peak of metamorphism has been 
interpreted to be $\sim 75 \mathrm{Ma}$ (Heaman and Parrish 1991). Thermochronology also records a period of increased cooling rates at $-65 \mathrm{Ma}$ (Heaman and Parrish 1991: Parrish 1995). Since decompression textu-es are truncated by the $S_{Z}$ fabric and $S_{Z}$ is interpreted to be synto post metamorphic. then decompression is constrained to $<75 \mathrm{Ma}$ and occurred before exhumation along the Paleocene-Eocene Slocan Lake-Valkyr normal fault system. Decompression may be the result of rapid exhumation of the Valhalla complex at $-75 \mathrm{Ma}$ in response to thickening due to movement along the underlying Monashee décollement, as suggested by Parrish (1995). This is consistent with variable An in plagioclase moats and consistently lower GRAIL than GASP pressure data.

\subsubsection{Variation in thermobarometric data within domains 2 and 3}

The wide range of thermobarometric data from domains 2 and $3(-775-980$ ' $\mathrm{C} .7 .1$ 12.2 kbars) compared with the consistent data of domain 1 does not easily discriminate between either a thrusting or extensional model for displacement on the GCSZ. Domain 1 and domains 2 and 3 are separated by $\sim 1 \mathrm{~km}$ of structural thickness (Fig. 3.10. Cross-section B, C). Thermobarometric data from domains 2 and 3 overlap the data of domain $1(\sim 750$ ${ }^{\circ} \mathrm{C}, 6.5$ kbars) but some temperatures and pressures are up to $135^{\circ} \mathrm{C}$ and $3 \mathrm{kbars}$ higher than domain 1. If there were no structural breaks in the section, the difference between the temperatures $\left(\sim 850^{\circ} \mathrm{C}\right)$ and pressures $(\sim 9 \mathrm{kbars})$ recorded by domains 2 and 3 and the $\sim$ $750^{\circ} \mathrm{C}$ and $6.5 \mathrm{kbar}$ conditions of domain 1 indicate temperature and pressure gradients of $\sim 100^{\circ} \mathrm{C} \mathrm{km}^{-1}$ and $\sim 2 \mathrm{kbars} \mathrm{km}^{-1}$. Normal thermal and pressure gradients in continental crust

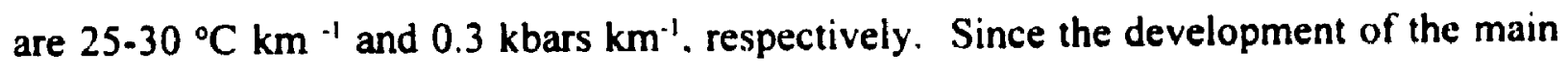


fabric is interpreted to be synchronous with metamorphism in the Valhalla complex. these normal gradients are expected. Some high temperatures and pressures may be the result of disequilibrium caused by the introduction of fluids.

Decompression textures and thermobarometry implying decompression may be explained by either thrust or normal faulting of the GCSZ. The pressure-temperature trajectory for pelitic schists and amphibolite gneisses is not well constrained for the Valhalla complex because data is lacking for the prograde portion of the pressure-temperature path. Plagioclase inclusion compositions in pelitic schist and the presence of ilmenite coring rutile suggest that rocks of the Valhalla complex underwent a counter-clockwise pressuretemperature path. However, GASP pressures from plagioclase-garnet data may not reliable because it is unknown if sillimanite was present at the time of plagioclase inclusion. A clockwise pressure-temperature path is implied for the Valhalla complex from garnet rims and proximal biotite grains, and garnet rims and biotite grains in direct contact in pelitic schists (Fig. 3.9). This implies an overall compressional tectonic setting for the Valhalla complex which suggests that the GCSZ had a thrust displacement and that movement of these shear zones was synchronous with the thermal peak of metamorphism. Clockwise pressure-temperature paths have initially $d P / d T>1$ and are interpreted to be the result of compressional deformation. Counter-clockwise pressure-temperature paths have initial $d P / d T<1$ and are the result of extensional tectonism or plutonism (England and Thompson 1977. Harley 1988). This does not imply that extension within individual thrust sheets could not have taken place. 


\section{$\underline{\text { t.t Conclusions }}$}

1. The Valhalla assemblage, the uppermost paragneiss sheet in tike Valhalla complex. is correlated to Neoproterozoic to Paleozoic strata of the North American miogecocline and Kootenay terrane. including the Horsethief Creek Group. Hamill Group. Badshot Formation. Lardeau Group and Milford Group (Table 2.3).

2. Based on this correlation. the Valhalla assemblage is interpreted to be the upside limb of an $F_{1}$ isoclinally folded recumbent nappe, where $F_{1}$ folding is younger than the Mississippian aged Milford correlatives which it folds. and older than the - 75 Ma peak of metamorphism and $S_{2}$ development (Heaman and Parrish 1991: chapter 3). Based on the data presented by Parrish (1995) which suggests that the deformation at the mid-crustal level is Cretaceous, the age of inversion and $F_{1}$ folding in Valhalla complex is interpreted to be Cretaceous.

3. Textures observed in thin section indicate that deformation was synchronous with the peak of metamorphism at all structural levels. Metamorphism began prior to $D_{2}$ in the uppermost sheet (sheet $1 \mathrm{c}$ - domain $\mathrm{la}$ and $\mathrm{lb}$ ) and also prior to shearing in the (jwillim Creek windows (sheet la - domain 3) and in the area of Vallican (domain 2). Deformation also outlasted metamorphism at all structural levels. The metamorphic peak is inferred to be $\sim 75 \mathrm{Ma}$ (Heaman and Parrish 1991) and therefore the GCSZ were active at this time. Evidence of shearing, increased strain relative to higher structural levels, and similar thermobarometric data of domains 2 and 3 (sheet $1 \mathrm{a}$ - Gwillim Creek window) suggests that 
the (iwillim Creek shear zones resurface in the Passmore Dome (Fig. 1.2. Cross-section A).

4. Stratigraphic correlations indicate that the Valhalla assemblage may have been thinned by up to $80 \%$ when compared to less deformed equivalents in the Goat Ranges to the northwest of the Valhalla complex (Fig, 1.1). Thinning is attributed to $D_{2}$ east directed compressional deformation and simultaneous shearing on the Gwillim Creek shear zones.

5. Although timing constraints are broad for the West Bernard fault. the Gwillim Creek shear zones are best correlated with this fault. because they both carry Paleozoic inverted strata in their hanging walls. Further geochronological constraints on the West Bernard fault may prove that it has an Early Cretaceous age of displacement. Other researchers have suggested that the Gwillim Creek shear zones are correlative with the "K-reflectors" that form the roof of the duplex thai merges with the Rocky Mountain basal detachment beneath the Purcell Anticlinorium (Cook and Varsek 1994: Cook and Van der Velden 1995).

6. Thermobarometric data from domain 1 is consistent between different bulk compositions and different equilibria. Near peak conditions of $\sim 850^{\circ} \mathrm{C}$ are recorded by gamet-biotite pairs in amphibolite gneisses and armoured biotite inclusions in gamets of pelitic schists. The breakdown of scapolite to plagioclase and calcite in marbles, indicates that peak temperature conditions were in excess of $800^{\circ} \mathrm{C}$. Post to near-peak temperatures recorded by garnet-biotite pairs in pelites and garnet-homblende pairs are estimated at -750 " $\mathrm{C}$ in the structurally high area of domain 1. Both GASP and GRAIL equilibria record 
pressures of -6.5 kbars in domain 1. Also in domain 1. - 6001 "C temperatures representattus of the cooling path are recorded by garnet and biotite pairs in direct contact with each other

7. Domains 2 and 3 are - $1 \mathrm{~km}$ structurally below domain 1 and record a wide range in garnet-biotite temperatures and associated (iASP pressures $(-775-980$ " $(.7 .1-12.2$ kbars) some of which overlap data from domain 1. Averaged temperatures ( 850 " "(') and pressures ( $\sim 9$ kbars) recorded by samples from domains 2 and 3 , taken with 750 " ${ }^{\prime}$ and 6.5 kbar conditions of domain 1 . indicate pressure and thermal gradients of - 100 " $\left(\mathrm{C}^{\prime} \mathrm{km}\right.$ ' and -2 kbars $\mathrm{km}^{-1}$. Some anomalously high temperatures $(-150$ " $(\mathrm{c})$ recorded by sheared samples in domains 2 and 3 are likely to have been the result of the introduction of lluids and the juxtaposition. by shearing, of minerals which were not in equilibrium. High temperatures in domains 2 and3 may also be a result of increased Fe levels due to the downward progression of reaction (7). GRAIL pressures recorded by samples trom domains 2 and 3 are in equilibrium with ga: net-biotite temperatures and consistent wi:h pressures in doillan 1. GASP are interpreted to represent near-peak pressure conditions.

8. Textures and thermobarometry indicate that a period of decompression followed the thermal peak of metamorphism as exhibited by 1 ) the consistent (iRAll, pressures that are - 3 kbars lower than GASP pressures, and the variation of plagioclase moat compositions in pelitic schists; and 2) the variation in plagioclase composition of moats around garnet in amphibolite gneisses, which produces a wide range in pressures. (iASP and (iRAIl. pressures record two points on the decompression path because of different closure 
temperatures. Plagioclase moats attributed to the (jASP reaction (2) which occurred during decompression are truncated by fabrics associated with $S_{2}$ and are therefore interpreted to be pre-locene. Evidence of retrograde metamorphism is scarce and so rocks in domains 2 and 3 are interpreted to have cooled rapidly after the thermal peak of metamorphism. Therefore. decompression occurred shortly after $75 \mathrm{Ma}$. This may be attributed to exhumation as a result of movement along the underlying Monashee décollement.

9. The prograde pressure-temperature path followed by rocks of the Valhalla complex is not well constrained. Pressures calculated from plagioclase inclusions in garnets of pelitic schists and rutile rimming ilmenite suggests that rocks underwent a counterclockwise pressure path. Reliable temperature data associated with pressures calculated from plagioclase inclusions is lacking and more work is required to constrain the prograde pressure-temperature path direction for the Valhalla complex.

\subsubsection{Geologic history}

The Valhalla complex is a domal shaped culmination in which arched sheets of upper amphibolite-facies paragneiss, granitic rocks, and intervening thrust faults were exhumed due to extensional faulting. Paragneisses in the highest structural level (sheet 1c, domain 1) consist of a heterogeneous package of metasedimentary rocks and metaplutonic rocks of the Valhalla assemblage. Based on stratigraphic correlations the sedimentary protoliths of Valhalla assemblage were liksly to have been deposited during the Neoproterozoic to Mississippian with intervening periods of mafic plutonism and/or vulcanism. The presence 
of metamorphosed ultranatic rocks in the Valhalla assemblage mal represent obducted oceanic crust: however. the primary relationships with surrounding rocks are unclear.

First phase isoclinal folding resulted in the inversion of the Valhalla assemblage during the Early Cretaceous and is constrained by the age Mulvey granodiorite $(-110 \mathrm{Ma}$ ) which is interpreted to postdate $F_{1}$ folding. Timing constraints for tirst phase folding are between $75 \mathrm{Ma}$ (peak of metamorphism - $\mathrm{S}_{2}$ development and - $290 \mathrm{Ma}$ (inferred age of Milford correlatives). Other nappes in the southern Omineca belt have been inverted by primarily Jurassic deformation (Raeside and Simony 1983: Brown and Lane 1988). A Cretaceous age of inversion in the Valhalla complex is consistent with data presented by Parrish (1995), which suggests a Cretaceous age of deformation and metamorphism for midcrustal levels of the Valhalla complex. Structural duplication of the Mulvey granodiorite and paragneisses along the GCSZ was synchronous with metamorphism and occurred during the middle of the Late Cretaceous (75-80 Ma). The GCSZ may be related to the WBF fault based on similar inverted Paleozoic hanging wall stratigraphy, however age constraints on the WBF must be refined. It has been proposed that the GCSZ are correlative to structures imaged by the "K-reflectors" beneath the Purcell Anticlinorium (Varsek and Cook 1994; Cook and Van der Velden 1995).

Textural observations indicate that metamorphism was simultaneous with deformation in all structural domains. Thermobarometric data in domains 2 and 3 record a wide range in temperatures $\left(775-980^{\circ} \mathrm{C}\right)$ and pressures $(7.1-12.2 \mathrm{kbars})$, some of which overlap data from domain $1\left(\sim 775^{\circ} \mathrm{C}, 7 \mathrm{kbars}\right)$. This indicates that the different structural levels share the same thermal history prior to and during movement along the GCS\%. D, in 
the structurally highest sheet (1c - domain 1) was synchronous with movement on the GCSZ and outlasted metamorphism. $F_{3}$ and $F_{4}$ folds in the Valhalla assemblage fold all metamorphic minerals and are rare at deeper structural levels near the GCSZ. This suggests that either movement on the shear zones outlasted this deformation. or that all folds and deformation are related to shortening associated with the GCSZ as one protracted event.

Exhumation as a result of movement on the underlying Monashee décollement may have occurred at $\sim 70-60 \mathrm{Ma}$ as recorded by decompression textures (this study). thermobarometry (this study) and thermochronology (Heanıan and Parrish 1991). The Airy quartz monzonite intruded at $\sim 63 \mathrm{Ma}$ and places a minimum age on $\mathrm{S}_{2}$ development (Carr et al. 1987). This was followed by the intrusion of the L.3dybird granite (59-55 Ma). Eocene extension and exhumation of the complex began with movement on the VSZ which occurred during and after the intrusion of the Ladybird granite. Movement on the Slocan Lake fault began at $~ 58 \mathrm{Ma}$ at which time it merged with the Valkyr shear zone and continued to denude the complex until $45 \mathrm{Ma}$ (Carr et al. 1987).

\subsection{Sugaested further work}

Due to the limited outcrop in the Gwillim Creek windows and the lowest portion of the Slocan Valley, the data is sparse in these areas. Now that it has been established that transposition fabrics in the southern portion of the complex at low structural levels are not related to Eocene faulting, detailed structural studies documenting strain in the Passmore Dome (Fig. 1.2) should be pursued to determine if the GCSZ are present in this area. U.Pb geochronology of folded pegmatites and granitic dykes would constrain the age of folding. 
Further detrital U-Pb geochronological studies of paragneisses aight help to deduce the provenance of these rocks and comparison with other studies may further constrain correlations. Palinspastic cross-sections across the southern Omineca belt based on stratigraphic correlations made in this study. would indicate the magnitude of displacement on the GCSZ.

The idea that net-transfer reactions involving sillimanite increased fe levels at garnet rims may be tested by analysing samples of pelitic schist lacking sillimanite. Further study of the metamorphic petrology. kinematics and structure in the Gwillim Creek windows may help to refine a model for the evolution of the Valhalla complex and the role of the GCS\%. Only two samples were studied from sheet $1 \mathrm{a}$ and both are in close proximity to the lower GCSZ. The wide range in temperatures may be due to the influx of fluids or juxtaposition of minerals in chemical disequilibrium causing local disequilibrium. Samples from areas further away from the GCSZ may yield better data. Amphibolite gneisses appear to be less affected by retrograde reactions and shearing, and therefore samples from the Gwillim ('reek windows might place better constraints on the metamorphic conditions. No thermobarometric data was collected from sheet $\mathrm{lb}$, and although the pelitic schists are highly regressed and sheared, data from this area might also help to constrain conditions at the different structural levels of the complex. Peak temperatures are estimated to be 75-80 Ma in the Passmore-Vallican area (Parrish 1991, 1995). Thermochronologic studies and cooling data in domain (west side of sheet $1 \mathrm{c}$ ) and the Gwillim Creek windows will demonstrate if the thermal peak occurred at the same time throughout the complex. 


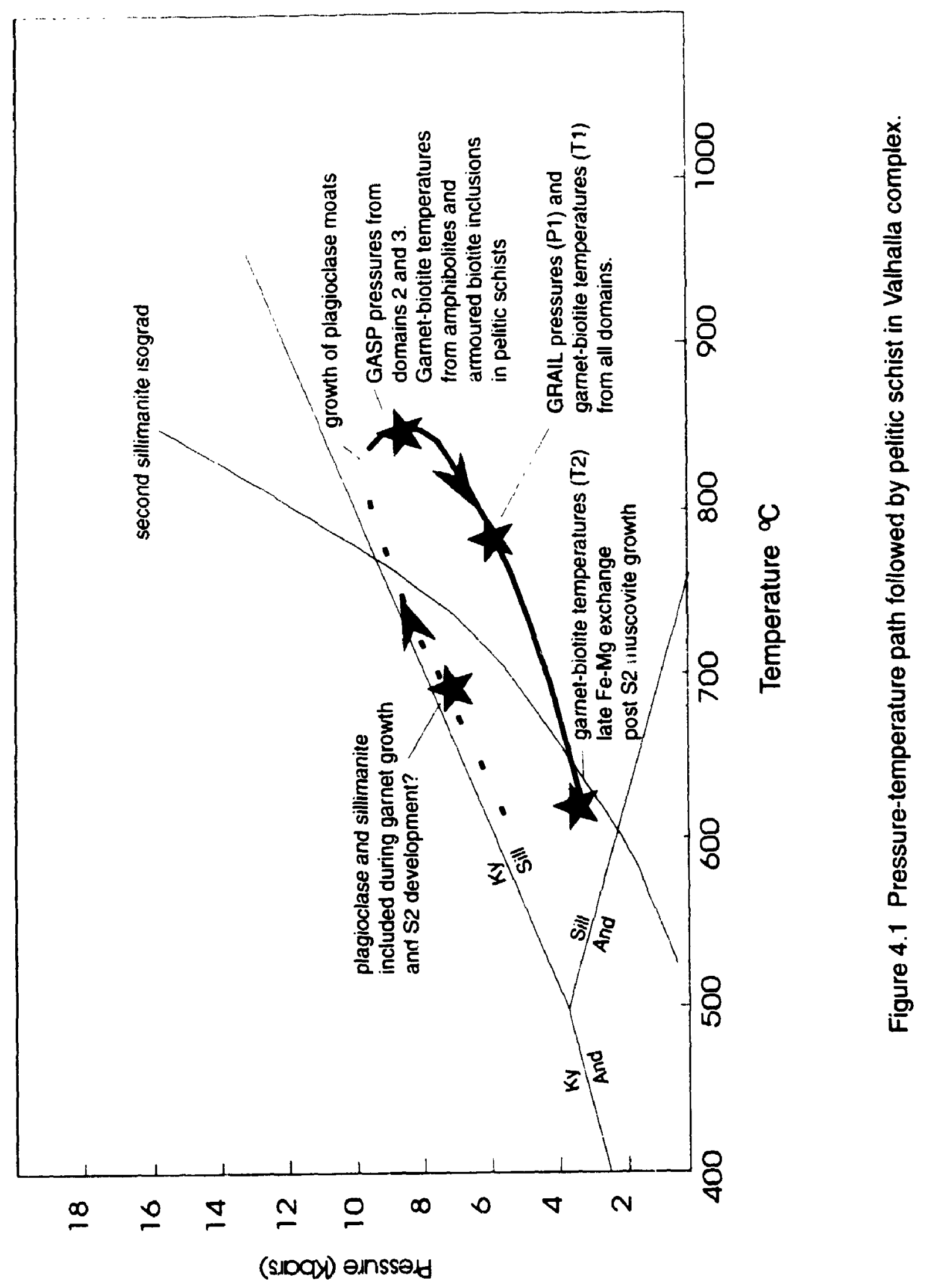




\section{REFERENCES}

Aitken, J.D. and McMechan, M.E. 1991. Middle Proterozoic assemblages, Chapter 5. In Geology of the Cordilleran Orogen in Canada. Edited by H. Gabrielse and C.J. Yorath. Geological Survey of Canada, Geology of Canada, no.4. p. 97-124.

Archibald, D.A., Glover, J.K., Price, R.A., Farrar, E., and Carmicheal, D.G. 1983. Geochronology and tectonic implications of magmatism and metamorphism, southern Kootenay Arc and neighbouring regions, southeastem British Columbia. Part I: Jurassic to mid-Cretaceous. Canadian Journal of Earth Sciences, 20: 1891-1913.

Archibald, D.A., Krogh, T., Armstrong, R., and Farrar, E. 1984. Geochronology and tectonic implications of magmatism and metamorphism, southem Kootenay Arc and neighbouring regions, southeastern British Columbia. Part II: Mid-Cretaceous to Eocene. Canadian Journal of Earth Sciences, 21: 567-583.

Bardeaux, M. 1993. The Okanogan Valley normal fault from Penticton to Enderby, Southcentral British Columbia. Unpublished Ph.D. thesis, Carleton University, Ottawa, Ontario

Barker, A.J. 1990. Introduction to metamorphic textures and structures. Blackie. 170p.

Benvenuto, G. and Price, R. 1979. Structural evolution of the Hosmer thrust sheet, southeastern British Columbia, Bulletin of Canadian Petroleum Geology, 27: 360-394.

Berman, R.G. 1988. Internally-consistent thermodynamic data for stoichiometric minerals in the system $\mathrm{Na}_{2} \mathrm{O}-\mathrm{K}_{2} \mathrm{O}-\mathrm{CaO}-\mathrm{MgO}-\mathrm{FeO}-\mathrm{Fe}_{2} \mathrm{O}_{3}-\mathrm{Al}_{2} \mathrm{O}_{3}-\mathrm{SiO}_{2}-\mathrm{TiO}_{2}-\mathrm{H}_{2} \mathrm{O}-\mathrm{CO}_{2}$. Journal of Petrology, 29: 445-522.

Berman, R.G. 1990. Mixing properties of Ca-Mg-Fe-Mn garnets. American Mineralogist, 75: $328-344$.

Berman, R.G. 1991. Thermobarometry using multiequilibrium calculations: a new technique with petrologic applications. Canadian Mineralogist, v. 29, 833-855.

Bevier, M.L. 1987. A Pb isotspic study of the Valhalla complex and its surrounding rocks, southeastern British Columbia: preliminary interpretations. In R adiogenic Age and Isotopic studies: Report 1, Geological Survey of Canada, Paper 87-2: :-3:\%.

Bohlen, S.R., Wall, V.J. and Boettcher, A.L. 1983. Experimental investigations and the geological application of equilibria in the system FeO-TiO2-Al2O3-SiO2-H2O. American Mineralogist, 68: 1049-1058. 
Brown, R.L., and Journeay, J.M. 1987. Tectonic denudation of the Shuswap metamorphic terrane of southeastern British Columbia. Geology, 15: 142-146.

Brown, R.L., and Lane, L.S. 1988. Tectonic interpretation of west-verging folds in the Selkirk Allochthon of the Southern Cordillera. Canadian Journal of Earth Sciences, 25: 292 300 .

Brown, R.L. and Read, P.B. 1983. Shuswap Terrane of British Columbia: a Mesozoic "core complex". Geology, 11: 164-168.

Brown, R.L., Tippett, C.R., and Lane, L.S. 1978. Stratigraphy. facies changes, and correlations in the northern Selkirk Mountains, southern Canadian Cordillera. Canadian Journal of Earth Sciences, 15: 1129-1140.

Brown, R.L., Journeay, J.M., Lane, L.S., Murphy, D.C., and Rees, C.J. 1986. Obduction, backfolding and piggyback-thrusting in the metamorphic hinterland of the southeastern Canadian Cordillera. Journal of Structural Geology, 8: 255-268.

Brown, R.L., Carr, S.D., Johnson, B.J., Coleman, V.J., Cook, F.A., and Varsek, J.L. 1992. The Monashee decollement of the southern Canadian Cordillera: a crustal-scale shear zone linking the Rocky Mountain Foreland Belt to lower crust beneath accreted terranes. In Thrust Tectonics. Edited by K.R. McClay. Chapman \& Hall, London, 357-364.

Brown, R.L., Beaumont,C., and Willet, S.D. 1993 Comparison of the Selkirk fan structure with mechanical models - implications for interpretation of the southem Canadian cordillera. Geology, 21: 1015-1018

Carr, S.D. 1986. The Valkyr shear zone and the Slocan Lake fault zone: Eocene structures that bound the Valhalla gneiss complex, southeastem British Columbia, M.Sc. thesis, Carleton University, Ottawa, Ont.

Carr, S.D. 2991 . Three crustal zones in the Thor-Odin - Pinnacles area, southern Omineca Belt, British Columbia. Canadian Journal of Earth Sciences, 28: 2003-2023.

Carr, S.D. 1992. Tectonic setting and U-Pb geochronology of the Early Tertiary Ladybird leucogranite suite, Thor-Odin - Pinnacles area, southem Omineca Eelt, British Columbia. Tectonics, 11: 258-278.

Carr. S.D. 1995. The southern Omineca belt, British Columbia: New perspectives from the Lithoprobe Geoscience Program. Canadian Journal of Earth Sciences, in press. 
Carr, S.D., Parrish, R.R., and Brown. R.L. 1987. Eocene structural development of the Valhalla complex, southeastern British Columbia. Tectonics, 6: 175-196.

Clemens, J.D., and Wall, V.D. 1981. Origin and crystallization of some peraluminous (Stype) granitic magmas. Cianadian Mineralogist, 19: 111-131.

Colpron, M., and Price, R.A. 1995. Tectonic significance of the Kootenay terrane. southeastern Canadian Cordillera: An alternative model. Geology, 23: 25-28.

Cook, F.A., and Van der Velden, A.J. 1995. Three-dimensional crustal structure of the Purcel! Anticlinorium in the Cordillera of southwestern Canada. Geological Society of America Bulletin, 107: 642-664.

Cook, F.A., Simony, P.S., Coflin, K.C., Green, A.G., Milkereit, B., Price, R.A.. Parrish, R.R., Patenzude, C., Gordy, P.L., and Brown, R.L. 1987. LITHOPROBE southem Canadian Cordilleran transect: Rocky Mountain thrust belt to Valhalla gneiss complex. Geophysical Journal of the Royal Astronomical Society, 89: 91-98.

Cook, F.A., Green, A.G., Simony, P.S., Price, R.A., Parrish, R.R., Milkereit, B., Brown, R.L., Coflin, K.C., and Patenaude, C. 1988. Lithoprobe seismic reflection structure of the southeastern Canadian Cordillera: Initial results. Tectonics, 7: 157-180.

Cook, F., Varsek, J., Clowes, R., Kanasewich, E., Spencer, C., Parrish, R., Brown, R., Carr, S., Johnson, B., and Price, R. 1992. LITHOPROBE crustal reflection cross section of the southern Canadian Cordillera, 1, Foreland thrust and fold belt to Fraser River fault. Tectonics, 11: 12-35.

Corbett, C.R. and Simony, P.S. 1984. The Champion Lake Fault in the Trail-Castlegar area of southeastern British Columbia. Geological Survey of Canada, Paper 84-1A: 103-1047.

Droop, G.T.R. 1987. A general equatrion for estimating $\mathrm{Fe}+3$ concentrations in ferromagnesiail silicates and oxides from microprobe analyses, using stoichiometric criteria. Mineralogical Magazine, 51: 431-435.

Eaton, D.W.S., and Cook, F.A. 1990. Crustal structure of the Valhalla complex, British Columbia, from LITHOPROBE seismic-reflection and potential field data. Canadian Journal of Earth Sciences, 27: 1048-1060.

England, P.C., and Thompson, A.B. 1984. Pressure - temperature - time paths of regional metamorphism, Part I: Heat transfer during the evolution of regions of thickened continental crust. Journal of Petrology, 4: 1-30. 
Evans. B.W. 1965. Application of a reaction rate method to the breakdown equilibria of muscovite plus quartz. American Journal of Science. 262: 21 5-226.

Evans, B.W.. and Guidotti. C.V. 1966. The sillimanite-potash feldspar isograd in western Maine. U.S.A. Contributions to Mineralogy and Petrology. 12: 25-62.

Ferry. J.M., and Spear. F.S. 1978. Experimental calibration of partitioning of Fe and Mg between biotite and garnet. Contributions to Mineralogy and Petrology. 66: 113-117.

Fritz, W.H. Cecile. M.P.. Norford, B.S., Morrow.D.. Geldsetzer. H.H.J. 1991. Cambrian to Middle Devonian assemblages: In Geology of the Cordilleran Orogen in Canada. Edited hy H. Gabrielse and C.J. Yorath. Geological Survey of Canada. Geology of Canada. no.t. p. 151-218.

Fuhrman. M.L. and Lindsley. D.H. 1988. Ternary-feldspar modeling and thermometry. The American Mineralogist, 73: 201-216.

Fyles. J.T. 1964. Geology of the Duncan Lake area. Lardeau District, British Columbia. British Columbia Department of Mines and Petroleum Resources, Bulletin 49.

Fyles. J.T. 1990. Geology of the Greenwood-Grand Forks area. British Columbia. British Columt ad Ministry of Energy, Mines and Petroleum Resources. Open File 1990-25.

Fyles, J.T., and Eastwood, C.E.P. 1962. Geology of the Ferguson area, Lardeau District. British Columbia. British Columbia Department of Mines and Petroleum Pesources. Bulletin 45 .

Gabrielse, H., (comp.). 1991. Structural styles, Chapter 17. In Geology of the Cordilleran Orogen in Canada. Edited by H. Gabrielse and C.J. Yorath. Geological Survey oi Canada. Geology of Canada, no.4, p. 571-675.

Gabrielse, H. and Campbell, R.B. 1991. Upper Proterozoic assemblages, Chapter 6. In Geology of the Cordilleran Orogen in Canada. Edited by H. Gabrielse and C.J. Yorath. Geological Survey of Canada. Geology of Canada. no.4, p.125-150.

Goldsmith, J.R. and Newton, R.C. 1977. Scapolite-plagioclase stability relations at high pressures and temperatures in the system NaAlSi3O8 - CaAl2Si2O8 - CaCO3 - CaSO4. American Mineralogist 62: 1063-1081.

Graham. C.M., and Powell, R. 1984. A garnet-homblende geothermometer: calibration, testing and application to the Pelona Schist. southern California. Journal of Metamorphic Geology, 2: 13-21. 
Halwas. D.. and Simony. P.S. 1986. The Castlegar gneiss complex. southern British Columbia. In Current research. part A. Geological Survey of Canada. Paper 86-1 A: 58 3-587

Harley. S.L. 1988. The origins of granulites: a metamorphic perspective. (icological Magazine. 126: 215-331.

Harrison. T.M. 1985. Thermal history of the Nelson batholith. British Columbia. Cesologicla Society of America Abstract Programs. 17: 260.

Heaman. L., and Parrish. R. 1991. Uranium-lead geochronology of accessory minerals: In Applications of radiogenic-isotope system to problems in geology, short course ser.. Vol 19. Edited by L. Heaman and J.P. Ludden. Mineralogical Association of Canada. Ottawa. Ontario, pp. 82-102.

Höy, T. 1977. Stratigraphy and structure of the Kootenay Arc in the Riondel area. southeastern British Columbia. Canadian Journal of Earth Sciences. 14: 2301-2315.

Höy. T. 1980. Geology of the Riondel area. Central Kootenay Arc, southeastern British Columbia. British Columbia Department of Mines and Petroleum Resources. Bulletin 73.

Höy. T. and van der Heyden, P. 1988. Geochemistry, geochronology, and tectonic implications of two quartz monzonite intrusions, Purcell Mountains, southeast British Columbia, Canadian Journal of Earth Sciences, 25: 106-115.

Jarosewich, E., Nelen, J.A.. and Norberg. J.A. 1979. Electron microprobe reference samples for mineral analyses. Smithsonian Contriburions to Earth Sciences, Edited by R.F. Fudali. No. 22: .

Johnson, B.J. 1994. Structure and tectonic setting of the Okanagan Valley fault system in the Shuswap lake setting, southern British Columbia. Unpublished Ph.D. thesis. Carleton University, Ottawa, Ontario. 266p.

Joumeay, J.M. 1986. Stratigraphy, internal strain and thermo-tectonic evolution of northern Frenchman's Cap dome: an exhumed duplex structure, Omineca hinterland, S.E. Canadian Cordillera. Ph.D. thesis, Queen's University, Kingston, Ont.

Klepacki, D.W. 1983. Stratigraphic and structural relations of the Milford. Kaslo and Slocan groups, Roseberry Quadrangle, Lardeau map area. British Columbia. In Current research. part A. Geological Survey of Canada, Paper 83-1 A: 229-233.

Klepacki, D.W., Read, P.B. and Wheelor, J.O. 1985. Geology of the headwaters of Wilson Creek, Lardeau map area, southeastern British Columbia. In Current research, part $\Lambda$. Geological Survey of Canada, Paper 85-1 A: 273-276. 
Klepacki, D.W. and Wheeler, J.O. 1985. Stratigraphic and structural relations of the Milford, Kaslo and Slocan Groups, Goat Range, Lardeau and Nelson map areas, British Columbia. In Current research, part A. Geological Survey of Canada, Paper 85-1A: 277-300.

Klepacki, D.W. 1985. Stratigraphy and structural geology of the Goat Ranges area, southeastern British Columbia. Ph.D. thesis. Massachusetts Institute of Technology, Boston, MA.

Kohn, M.J. and Spear, F.S. (1990). Two new barometers for garnet amphibolites with applications to southeastern Vermont. American Mineralogist, 75: 89-96.

Le Breton, N., and Thompson, A.B. 1987. Fluid-absent (dehydration) melting of biotite in metapelites in the early stages of crustal anatexis. Contributions to Mineralogy and Petrology, 99: 226-237.

Leclair, A. 1984. Comparison of stratigraphy and structure across the Seeman Creek fault, a major tectonic boundary in the central Kottenay arc, southeast B.C. Geological Association of Canada/Mineralogical Association of Canada. Annual Meeting program with abstracts, London, Ontario, p.83.

Leclair, A.D.. Parrish, R.R. and Archibald, D.A. 1993. Evidence for Cretaceous deformation in the Kootenay Arc based on U-Pb and 40Ar/39Ar dating, southeastern British Columbia. In Current research, part A. Geological Survey of Canada, Paper 93-1 A: 207-220.

Leech, G. 1962. Structure of the Bull River valley near latitude $49 \circ 35^{\prime}$. Journal of the Alberta Society of Petroleum Geologists. 10: 396-407.

Little, H.W. 1983. Geology of the Greenwood map-area, British Columbia. Geological Survey of Canada, Paper 79-29.

Luth. W.D., Jahns, R.H. and Tuttle, O.F. 1964. The granite system $\approx$ pressures of 4 to 10 kbars. Journal of Geophysical Research, 69: 659-773.

Mackenzie, W.S. Donaldson, C.H. and Guilford, C. 1982. Atlas of igneous rocks and their textures. Longman Scientific and Technical. 148p.

McMullin. D., Berman, R.G., and Greenwood, H.J. 1991. Calibration of the SGAM thermobarometer for pelitic rocks using data from phase equilibrium experiments and natural assemblages. Canadian Mineralogist, 29: 889-908.

Monger. J.W.H. 1977. Upper Paleozoic rocks of the western Canadian Cordillera and their bearing on Cordilleran evolution. Canadian Journal of Earth Sciences, 14: 1832-1859. 
Monger, J.W.H., Price, R.A., and Templeman-Kluit, D.J. 1982. Tectonic accretion and the origin of the two major metamorphic and plutonic welts in the Canadian Cordillera. Geology, 10: $70-75$.

Murphy, D.C. 1987. Suprastructure/infrastructure transition , east-cental Cariboo Mountains, British Columbia: geometry, kinematics and tectonic implications. Journal of Structural Geology,

Oterdoom, W.H., and Gunter, W.D. 1983. Activity models for plagioclase and $\mathrm{CO}_{3}$ scapolites - an analysis of field and laboratory data. American Journal of Science. 283A: 255-282.

Parrish, R.R. 1981. Geology of the Nemo Lakes belt, northern Valhalla Range, southeast British Columbia. Canadian Journal of Earth Sciences. 18: 944-958.

Parrish, R.R. 1990. U-Pb dating of monazite and its application to geological problems. Canadian Journal of Earth Sciences, 27: 1431-1450.

Parrish, R.R. 1992. U-Pb ages of Jurassic-Eocene plutonic rocks in the vicinity of Valhalla complex, southeast British Columbia. In Radiogenic Age and Isotope Studies, Report 5. Geological Survey of Canada, Paper 91-2: 115-134.

Parrish, R.R. 1995. Thermal evolution of the southeastern Canadian Cordillera. Canadian Journal of Earth Sciences, in press.

Parrish, R.R., Carr, S.D., and Brown, R.L. 1985. Valhalla gneiss complex, southeast British Columbia: 1984 Field work. In Current research, part A. Geological Survey of Canada, Paper 85-1A, pp. 81-87.

Parrish, R.R., Carr, S.D., and Price, R.A. 1987. Geological Association of Canada/Mineralogical Association of Canada, Annual Meeting program with abstracts,

Parrish, R.R., Carr, S.D., and Parkinson, D.L. 1988. Eocene extensional tectonics and geochronology of the southem Omineca Belt, British Columbia and Washington. Tectonics, 7: 181-212.

Pell, J.A., and Simony, P.S. 1987. New correlations of Hadrynian strata, south-central British Columbia. Canadian Journal of Earth Sciences, 24: 302-313.

Poulton, T.P., and Simony, P.S. 1980. Stratigraphy, sedimentology, and regional correlation of the Horsethief Creek Group (Hadrynian, Late Precambrian) in the northern Purcell and Selkirk Mountains, British Columbia. Canadian Journal of Earth Sciences, 17: 1708-1724. 
Preto, V.A. 1970. Structure and petrology of the Grand Forks Group, British Columbia. Geological Survey of Canada, Paper 69-22.

Price, R.A. 1981. The Cordilleran foreland fold and thrust belt in the southern Canadian Rocky Mountains. In Thrust and nappe tectonics. F.dited by K.R. NicClay and N.J. Price. Geological Society of London, Special Publication No. 9: 427-488.

Price, R.A. 1986. The southeastem Canadian Cordillera: thrust faulting, tectonic wedging, and delamination of the lithosphere. Journal of Structural Geology, 8: 239-254.

Price, R.A. and Mountjoy, E.W. 1970. Geologic structure of the Canadian Rocky Mountains between Bow and Athabasca Rivers - A progress report. Geological Association of Canada, Special Paper, 6: 7-25.

Pringle, G.J. 1989. A Fortran computer program to produce corrected microprobe analyses of minerals using an energy dispersive x-ray spectrometer. Geological Survey of Canada Open File Report 2127.

Raeside, R.P., and Simony, P. 1983. Stratigraphy and deformational history of the Scrip Nappe, Monashee Mountains, British Columbia. Canadian Journal of Earth Sciences, 20: 639-650.

Read, P.B. 1973. Petrology and structure of the Poplar Creek map area, British Columbia. Geological Survey oi Canada, Bulletin 193.

Read, P.B., and Wheeler, J.O. 1976. Geology of Lardeau west-half map area. Geological Survey of Canada, Open File Map 432.

Reesor, J.E. 1965. Valhalla gneiss complex, British Columbia. Geological Survey of Canada, Bulletin 129.

Reesor, J.E. 1970. Some aspects of structural evolution and regional setting in part of the Shuswap metamorphic complex. The Geological Association of Canada, Special Paper, 6: 73-86.

Reesor. J.E. 1973. Geology of the Lardeau map-area. east-half, British Columbia, Geological Survey of Canada. Memoir 369.

Roback. R.C., Sevigny, J.H., and Walker, N.W. 1994. Tectonic setting of the Slide Mountain terrane, southem British Columbia. Tectonics, 13: 1242-1258.

Ross, G.M. 1991. Tectonic setting of the Windermere Supergroup revisited. Geology, 19: 1125-1128. 
Ross, G.M., and Parrish. R.R. 1991. Detrital zircon geochrnnology of metasedimentary rocks in the southern Omineca Belt, Canadian Cordillera. Canadian Journal of Earth Sciences. 28: 1254-1270.

Scammell, R.J. 1990. Preliminary results of stratigraphy, structure and metamorphism in the Scrip and northern Seymour ranges, southern Omineca Belt. British Columbia. In Current research, part E. Geological Survey of Canada, Paper 90-E, pp. 97-106.

Scammell, R.J. 1993. Mid-Cretaceous - Tertiary thermotectonic history of former midcrustal rocks, southem Omineca Belt, Canadian Cordillera. Unpublished Ph.D. thesis. Queen's University, Kingston. Ontario. 576p.

Schiarizza, P. and Preto. V.A. 1987. Geology of the Adams Plateau - Clearwater - Vavenby area. British Columbia Ministry of Energy, Mines and Petroleum Resources. Paper 1987-2.

Sears, J.W. 1979. Tectonic contrasts between the infrastructure and suprastructure of the Columbia orogeny, Albert Peak area, western Selkirk Mountains. British Columbia. Ph.D. thesis, Queen's University, Kingston, Ont.

Simony, P.S. 1979. Pre-Carboniferous basement near Trail, British Columbia. Canadian Joumal of Earth Sciences. 16: 1-11.

Simony, P.S. and Armstrong, R.L. 1990. Geochronometryof a Devonian pluton in the Trail gneiss north of Trail, British Columbia: Basement of Quesnellia. Geological Association of Canada, Abstract and Program, 15: A122.

Simony, P.S., Carr, S.D., and Corbett, C.R. 1992. Large lateral, south-facing ramps with southward decrease in displacement; Eocen extensional Valkyr shear zone, southem British Columbia, Canada. Lithoprobe Southern Cordillera Transect Workshop, University of Alberta, Edmonton, Alberta, report 24: 123-125.

Smith, M..., and Gehrels, G.E. 1991. Detrital zircon geochronology of Upper Proterozoic to lower Paleozoic continental margin strata of the Kootenay Arc: implications for the early Paleozoic tectonic development of the eastern C'anadian Cordillera. Canadian Journal of Earth Sciences, 28: 1271-1284.

Smith, M.T., and Gehrels, G.E. 1992a. Stratigraphic comparison of the Lardeau and Covada groups: Implications for revision of the stratigraphic relations in the Kootenay arc. Canadian Journal of Earth Sciences, 29: !320-1329.

Smith, M.T., and Gehrels, G.E. 1992b. Structural geology of the Lardeau Group near Trout Lake, British Columbia: implications for the structural evolution of the Kootenay Arc. Canadian Journal of Earth Sciences, 29: 1305-1319. 
Spear, F.S. 1993. Metamorphic phase equilibria and pressure-temperature-time paths. Mineralogical Society of America Monograph 799p.

Spear, F.S. and Cheney, J.T. (i989). A petrugenetic grid for pelitic schists in the system $\mathrm{SiO} 2-\mathrm{Al}_{2} \mathrm{O} 3-\mathrm{FeO}-\mathrm{MgO}-\mathrm{K} 2 \mathrm{O}-\mathrm{H} 2 \mathrm{O}$. Contributions to Mineralogy and Petrology. 101: $149-164$.

Spear, F.S., and Parrish. R.R. (in prep.). Petrology and petrologic cooling rates of the Valhalla complex, British Columbia. Canada.

Stewart. J.H. 1972. Initial deposits of the Cordilleran geosyncline: Evidence of the late Precambrian ( $<850 \mathrm{~m} . y$.) continental separation. Geological Society of America Bulletin. 83: 1345-1360

Struik. L.C. 1981. A re-examination of the type area of the De zono-Mississippian Cariboo Orogeny, central British Columbia. Canadian Journal of Earth Sciences. 18: 1767-1775.

Thompson, A.B. 1 182 . Dehydration melting of pelitic rocks and the generation of $\mathrm{H}_{2} \mathrm{O}-$ undersaturated granitic liquids. American Journal of Science. 282: 1567-1595.

Tracy, R.J., Robinson, P., and Thompson, A.B. 1976. Garnet composition and zoning in the determination of temperature and pressure of metamorphism. central Massechusetts. American Mineralogist. 61: 762-775.

Varsek. J.. and Cook, F. 1991. Seismic reflection geometry of a folded and detached accretionary complex: Kootenay Arc, British Columbia. Geology. 19: 159-162.

Varsek. J., and Cook, F. 1994. Three-dimensional crustal structure of the eastern Cordillera. southwestern Canada and northwestern United States. Geological Society of America Bulletin. 106: 803-823.

Wanless, R.K., Loveridge.W.D. and Mursky, G. 1968. A geochronological study of the White Creek batholith, southeastern British Columbia. Canadian Journal of Earth Sciences. 5: $375-86$.

Wheeler, J.O. 1963. Rogers Pass map area. British Columbia and Alberta. Geological Survey of Canada. Paper 62-32.

Wheeler, J.O. 1966. Eastern tectonic belt of western Cordillera in British Columbia. In Tectonic history and mineral deposits of the western Cordillera: Canadian Institute of Mining and Metallurgy Special Volume 8: 27-45. 
Wheeler. J.O.. Brookfield. A.J. Gabrielse. H.. Monger. J.W.H., lipper. H.W.. and Woodsworth. G.J., compilers. 1991. Terrane map of the Canadian C'ordillera: (ieological Survey of Canada Map 1713A. scale 1:2000000.

Yardley. B.W.D. 1977. An empırical study of diffusion in garnet. American Mineralogist. 62: $793-800$.

Zwanzig. H.V. 1973. Structural transition between the foreland zone and the core sone of the Columbian orogeny. Selkirk Mountains. British Columbia. Ph.D. thesis. Queen's I niversity. Kingston, Ont. 


\section{Appendix A: Electron microprobe standards}

Garnet

Fe. Si. Al - Ingamells garnet $\mathrm{R} 1598$

Ca. Mg - Kakanui hornblende. United States National Museum 143965

Cr - chromite, United States National Museum 117075

$\mathrm{Mn}$ - synthetic tephroite $\left(\mathrm{Mn}_{2} \mathrm{SiO}_{4}\right)$

Biotite

Si, Al. Ti, Mg, Na - Kakanui hornblende. United States National Museum 143965

$\mathrm{K}$ - synthetic phlogopite

Fe - Ingamells garnet R1598

$\mathrm{Cr}$ - chromite. United States National Museum 117075

$\mathrm{Mn}$ - synthetic tephroite $\left(\mathrm{Mn}_{2} \mathrm{SiO}_{4}\right)$

$\mathrm{Cl}$ - Tugtupite

Plagioclase

Si. Al. $\mathrm{Ca}$. Na - natural. pure albite and synthetic anorthite

K - microcline, United States National Museum 143966

Fe - Kakanui hornblende. United States National Museum 143965

Hornblende

$\mathrm{Si}, \mathrm{Al}$. Ti. Fe, Mg, Ca, K, Na - Kakanui hornblende, United States National Museum 143965

$\mathrm{Cr}$ - chromite, United States National Museum 117075

$\mathrm{Cl}$ - Tugtupite

Ilmenite

$\mathrm{Fe}, \mathrm{Mn}, \mathrm{Ti}$ - ilmenite, United States National Museum 96189

$\mathrm{Mg}, \mathrm{Cr}$ - chromite, United States National Museum 117075

Pyroxene

Fe,Mg. Ca, Si . Al - Kakanui augite. United States National Museum 143965

Olivine

$\mathrm{Fe}, \mathrm{Mg}, \mathrm{S}_{1}$ - San Carlos olivine. United States National Museum 111312

$\mathrm{Ni}-\mathrm{NiO}$

Scapolite

Si. Al, Ca. Na - scapolite, United States National Museum R6600-1

$\mathrm{Cl}$ - Tugtupite

K - Kakanui homblende, United States National Museum 143965 
A number of other natural minerals were analy sed as internal standards during each analytical session. These are listed in Jarosewich and others (1979):

e.g.

Garnet. United States National Museum 87375

Labradorite. United States National Museum 115900 


\section{Appendix B: Modal mineral abundances of pelitic schists}

See map 1 in pocket for sample localities.

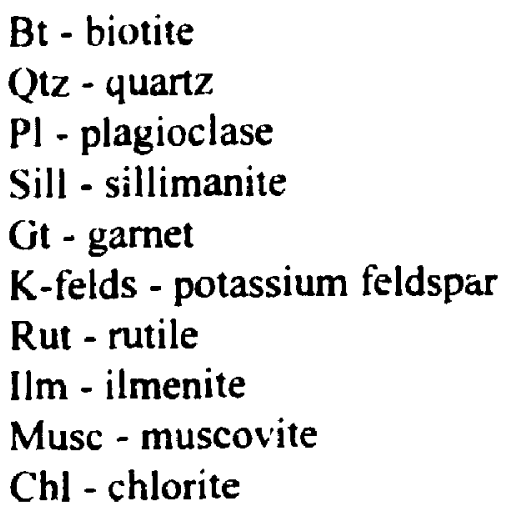

\begin{tabular}{|c|c|c|c|c|c|c|c|c|c|c|c|c|}
\hline Sample & $\mathrm{Bt}$ & Qtz & $\mathrm{PI}$ & Sill & Gt & Kfelds & Rut & $\mathrm{Ilm}$ & Musc & $\mathrm{Chl}$ & Textures D & $\begin{array}{c}\text { Domai } \\
\text { n }\end{array}$ \\
\hline PCA-93-84 & 25 & 35 & 6 & 10 & 10 & 10 & 1 & 2 & & 1 & -pl. moat & 2 \\
\hline PCA-109-84 & 40 & 25 & 5 & 15 & 10 & 5 & & & & & & $1 \mathbf{a}$ \\
\hline PCA-200-85 & 30 & 20 & 15 & 15 & 10 & 5 & 1 & 1 & 1 & 2 & $\begin{array}{l}\text {-pl. moat } \\
\text {-rut. cor } \\
\text { ilm }\end{array}$ & 3 \\
\hline PCA-204B-85 & 25 & 30 & 15 & 5 & 10 & 10 & 1 & 2 & 1 & 1 & & la \\
\hline PS-4-93 & 30 & 20 & 30 & 5 & 10 & 5 & & & & & & la \\
\hline PS-9-93 & 30 & 25 & 15 & 15 & 10 & 5 & & & & & & la \\
\hline PS-10-93 & 30 & 30 & 10 & 20 & 5 & 5 & & & & & & $1 a$ \\
\hline PS-21A-93 & 30 & 35 & 25 & 2 & 8 & & & & & & & la \\
\hline PS-22-93 & 35 & 25 & 30 & 1 & 3 & 5 & & & 1 & & & $1 a$ \\
\hline PS-24-93 & 20 & 50 & 20 & 5 & 5 & & & & & & & la \\
\hline PS-27B-93 & 30 & 25 & 10 & 15 & 15 & 3 & & 2 & & & & 2 \\
\hline PS-27C-93 & 25 & 35 & 5 & 15 & 10 & 5 & & 2 & & 3 & & 2 \\
\hline PS-28A-93 & 35 & 20 & 5 & 15 & 15 & 5 & & 3 & 2 & & pl. moat & 2 \\
\hline PS-28B-93 & 35 & 15 & 5 & 20 & 15 & 5 & 1 & 2 & 2 & & pl. moat & 2 \\
\hline PS-29B-93 & 35 & 35 & 10 & 5 & 8 & 5 & 1 & & & 1 & & 2 \\
\hline PS-29C-93 & 20 & 35 & 15 & 10 & & 20 & & & & & & 2 \\
\hline PS-31-93 & 35 & 33 & 15 & & 10 & & & & 2 & 5 & & \\
\hline PS-32-93 & 20 & 40 & 20 & 10 & & 10 & & & & & & \\
\hline PS-6A-94 & 35 & 45 & 10 & & 5 & & & 2 & & 3 & & la \\
\hline PS-8B-94 & 30 & 35 & 20 & & & & & 5 & & 10 & & la \\
\hline PS-22-94 & 37 & 30 & 20 & & 10 & & & 3 & & & & la \\
\hline PS-32-94 & 35 & 40 & 20 & & & & & & 5 & & & la \\
\hline PS- $47-94$ & 35 & 30 & 30 & & 5 & & & & & & & 16 \\
\hline PS-48A-94 & 15 & 55 & 20 & $s$ & 5 & & & & & & & lb \\
\hline
\end{tabular}




\begin{tabular}{|c|c|c|c|c|c|c|c|c|c|c|c|}
\hline PS-50A-94 & 15 & 50 & 15 & 5 & 5 & 9 & & & 1 & & ith \\
\hline PS-56A-94 & 5 & 70 & 5 & 5 & 10 & 5 & & & & & Ib \\
\hline PS-57A-94 & 20 & 55 & 10 & 5 & 5 & & 2 & 3 & & & 16 \\
\hline PS-58-94 & 25 & 50 & & 10 & 10 & 5 & & & & & $1 \mathrm{~b}$ \\
\hline PS-61-93 & 30 & 34 & 10 & 15 & 10 & & & & & 1 & lb \\
\hline PS-62B-94 & 25 & 35 & 10 & 10 & 10 & 10 & & 1 & & & Ib \\
\hline PS-64-94 & 40 & 15 & 5 & 20 & 20 & & & & & & Ib \\
\hline PS-76-94 & 35 & 30 & 10 & & 15 & & 2 & 3 & & 5 & \\
\hline PS-77-94 & 15 & 40 & 35 & & 10 & & & & & & \\
\hline PS-86D-94 & 30 & 25 & 10 & 25 & 8 & & & 2 & & & \\
\hline PS-91-94 & 35 & 15 & 30 & & 15 & & & 5 & & & \\
\hline PS-99-94 & 30 & 35 & 25 & & 5 & & & & & 5 & \\
\hline PS-103-94 & 30 & 40 & 25 & 2 & 2 & & & & 1 & & la \\
\hline PS-108-94 & 15 & 45 & 10 & 15 & 5 & 10 & & & -1 & & la \\
\hline PS-1 10-94 & 20 & 55 & 20 & 5 & & & & & & & la \\
\hline PS- $111 \mathrm{~B}-94$ & 40 & 25 & 25 & & 10 & & & & & & la \\
\hline PS- $111 \mathrm{C}-94$ & 35 & 30 & 20 & & 15 & & & & & & la \\
\hline PS-134-94 & 40 & 25 & 25 & & 9 & & 1 & & & & Ia \\
\hline PS-136c-94 & 45 & 10 & 5 & 30 & 10 & & & & & & la \\
\hline PS-143-94 & 30 & 40 & 20 & & 5 & 5 & & & & & la \\
\hline PS-144-94 & 30 & 20 & 5 & 25 & 10 & 9 & & & 1 & & la \\
\hline PS-169-94 & 30 & 30 & 15 & 10 & 10 & & & 5 & & & $1 \mathrm{a}$ \\
\hline PS-172-94 & 35 & 30 & 20 & 2 & 5 & 8 & & & & & la \\
\hline PS-179-94 & 40 & 25 & & 30 & & & & & 5 & & la \\
\hline PS-237-94 & 30 & 35 & 25 & 1 & 8 & & & & & 1 & la \\
\hline PS-267B-94 & 30 & 30 & 15 & 20 & & 4 & 1 & & & & Ia \\
\hline PS-277-94 & 35 & 30 & 10 & 15 & 5 & 5 & & & & pl. moat & la \\
\hline PS-280-94 & 35 & 35 & 5 & 20 & 5 & & & & & & la \\
\hline PS-302-94 & 30 & 38 & 2 & 20 & & 10 & & & & & $1 \mathbf{a}$ \\
\hline PS-319-94 & 35 & 30 & 25 & & 10 & & & & & & la \\
\hline PS-332-94 & 30 & 25 & 10 & 15 & 10 & 5 & & 2 & 3 & $\begin{array}{l}\text { rut. cor. } \\
\text { ilm }\end{array}$ & $\mathbf{l b}$ \\
\hline PS-344-94 & 30 & 25 & 15 & 15 & 15 & & & $<1$ & & & la \\
\hline PS-348-94 & 25 & 32 & 5 & 15 & 10 & 10 & & 3 & & & 3 \\
\hline
\end{tabular}


Appendix C: Mineral analyses for pelitic schists 


\section{Appendix C}

Abbreviations used in first row of garnet analyses are as follows

RI - garnet rims in contact with biotite.

R2 - garnet rims in contact with non-ferromagnesian minerals

C - garnet cores

I - points in garnet core adjacent to biotite inclusions

Abbreviations in first row of biotite and plagioclase analyses are as follows:

incl. - occurs as inclusion in garnet

cont. - is in contact with garnet rim

Letters in second row of all analyses refer to point or average of points used in thermobarometric calculations which are refered to in Table 3.2.

Numbers in third row of all analyses refer to number of points used to obtain average value used in thermobarometric calculations.

Calculations to determine $\mathrm{Fe}^{+3}$ content in ilmenite were carried out because it commonly makes up a large part of this mineral. $\mathrm{Fe}^{+3}$ estimates are less reliable for garnet and do not make a substantial difference in thermobarometric calculations. $\mathrm{Fe}^{-3}$ calculations were not possible for biotite because of the variable $\mathrm{H}_{2} \mathrm{O}$ content. 
N9-887ニ

- กิ กั

-

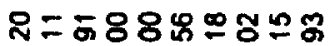

-的只OONNTN ํํำํํㅇำำ

-

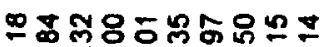

-

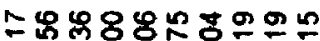

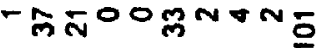

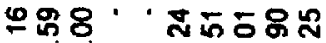

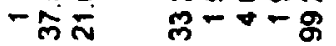

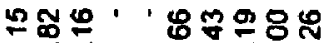

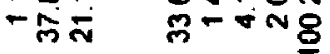

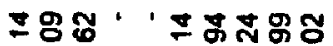

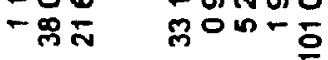

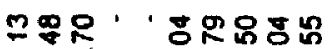

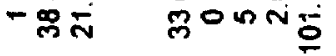

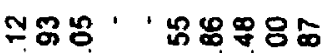

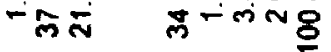

二罗品・的却品

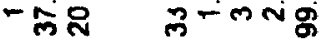

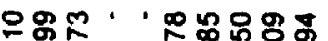

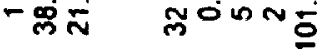

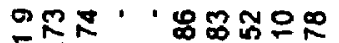

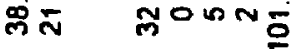

명' '

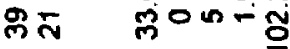

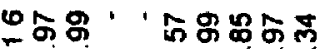

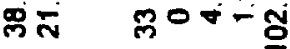

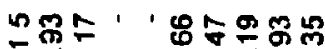

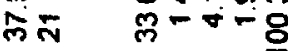

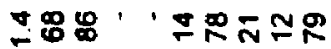

塄 MONN

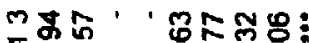

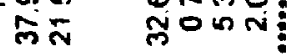

쬼ำ

ำล NON-g

క- ' '

的

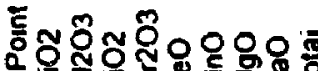

กี้ำㅇํㅇ

जo

훙용

ஸि 6

능요

no 0

$\overline{8}$

nio:

용웅요

no 0

앵양유

no

行 융유

웅용응

000

홍응

जio 0

ㅎํㅇ용

in 0

풍융훙

000

훙용

600

品品器

000

뭉용풍

000

ติธoิ

ตั0

$5 \overline{5} \overline{0}$

000

ริํㅛㅇ

606

F⿱亠䒑口阝

000

解店

जio

웅응

000

ำำ

둥ㅇํ
సั.ర్రిళ్రి

ल유

గ్ర్ర유유.

ल00ल

영융요

ल00\%

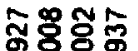

ल00

융용

ल००ण

串

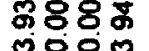

L.

m

8 ' ' 8

i

జ' ' జ

(i)

品 '

i)

肯' '

m +0000

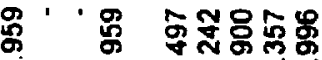

$\checkmark 0000$

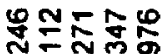

vo 0 - 0 in

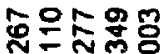

$\checkmark 0-00$

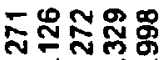

$\rightarrow 0-0$.

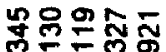

प0

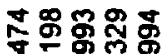

-000 in

농호용요

둥ํํㅇ

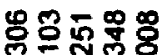

व 0 - 0

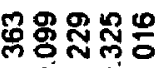

$\checkmark 0-00$

论面品虽

-000

둘율쬬

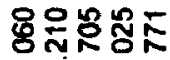

00000

్ㅇㅇ휴융융

00000

్ㅇㅇ으웧웡

00000

号틍ㅎㅇ

00000

品Nㅠㅇ융

00000

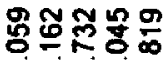

00000

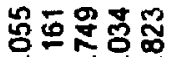

$0000 \%$

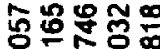

0ํํㅇㅇํㅇ

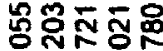

Оㅇํㅇ응

员는돈

0000

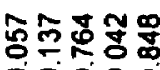

00000

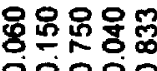

00000

象풍훙옹

00000

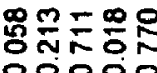

00000

นํํㅇำํํำ

00000

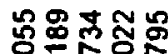

0ㅇํㅇㅇ

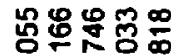

00000

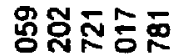

00000

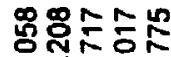

00000

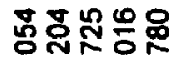

00000

냉응뮤융명

00000

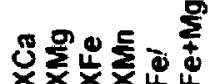




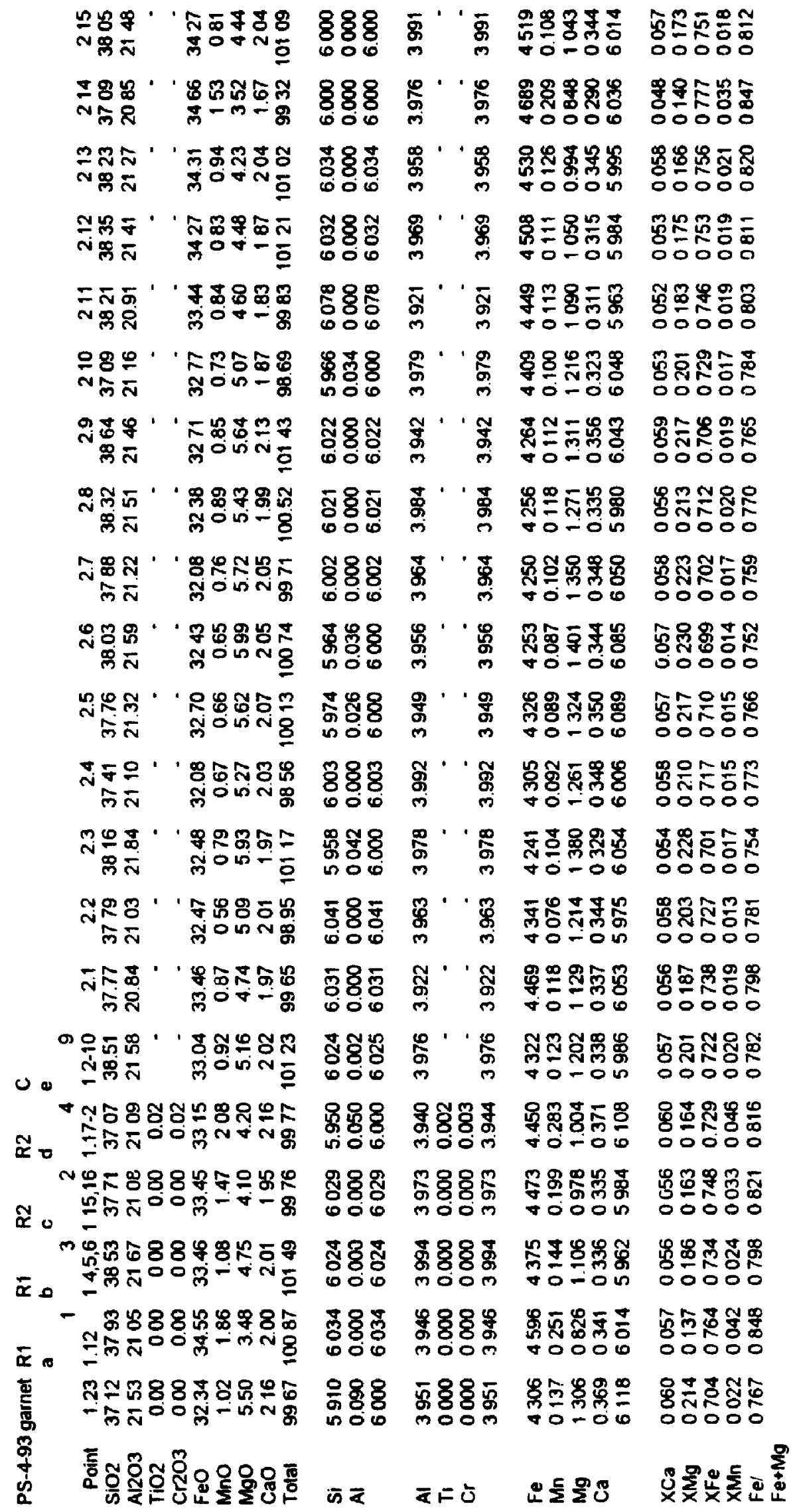




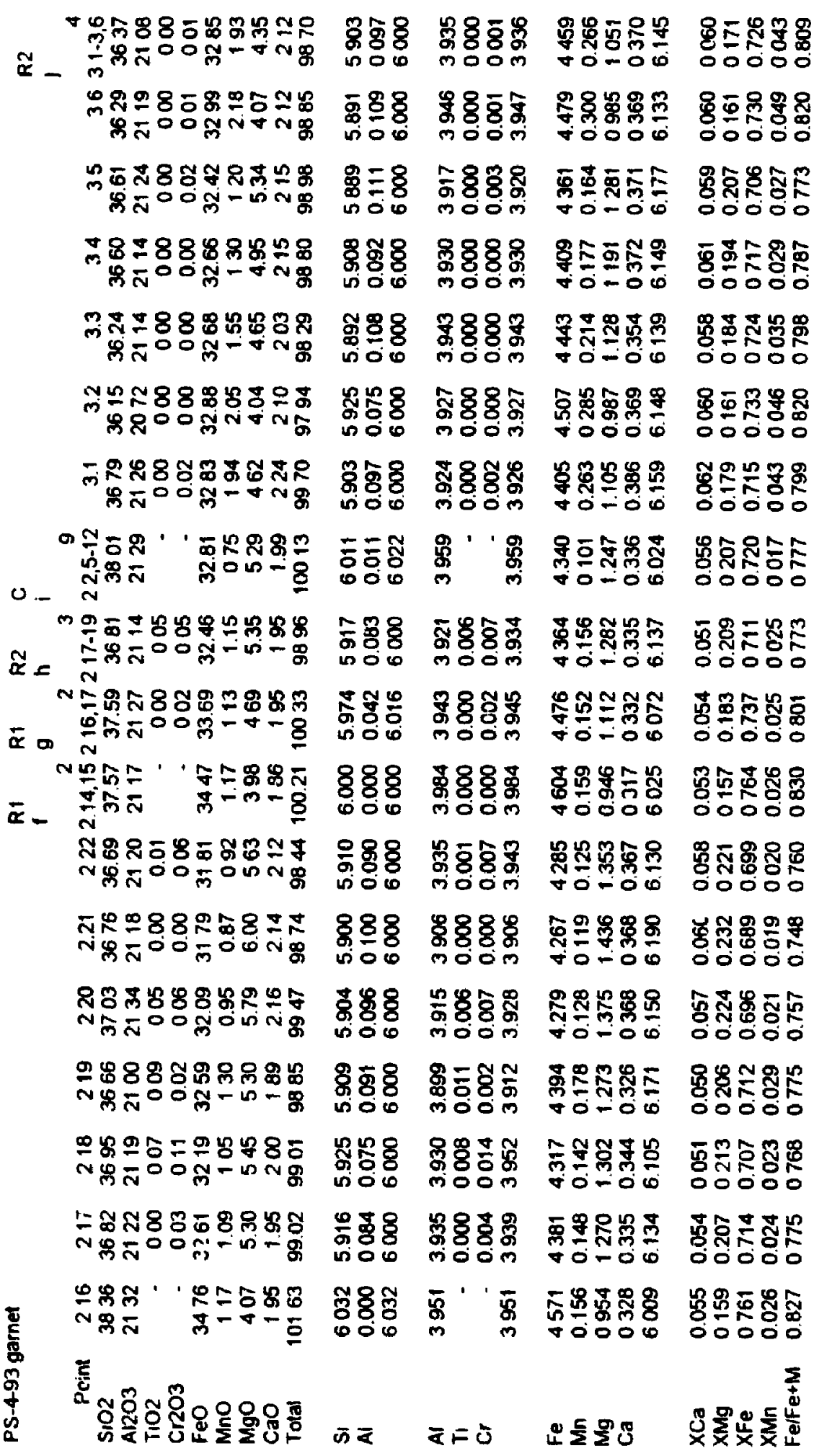




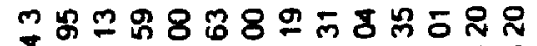

స్ 유잉

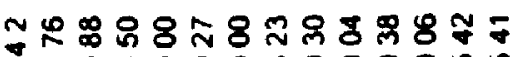

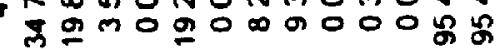

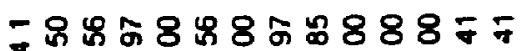

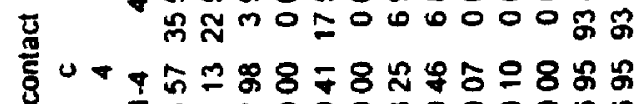

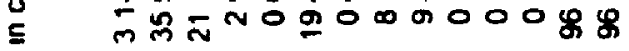

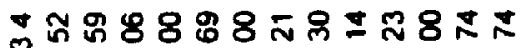

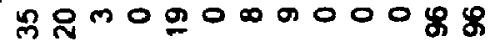

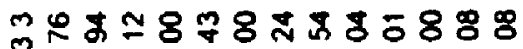

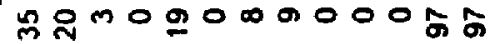

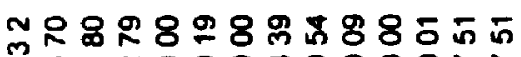

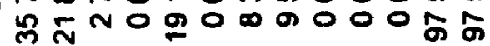

帘ニ 8 \%

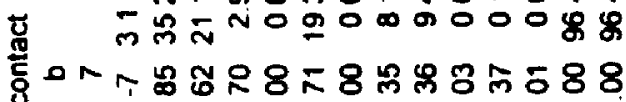

त̄

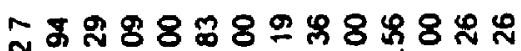

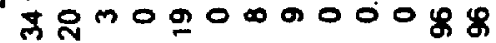

ํํำ ส

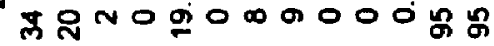

Nํำ

茫 NO

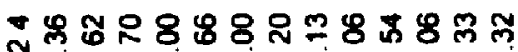

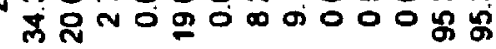

นำส

मूं

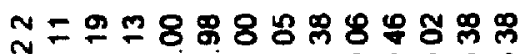

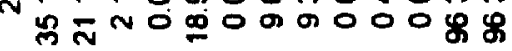

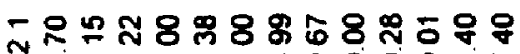

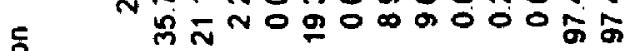

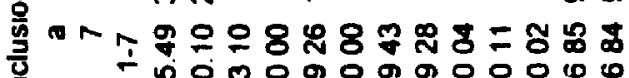

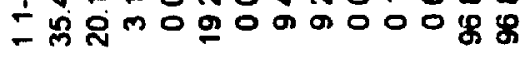

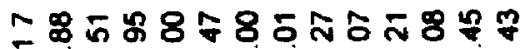

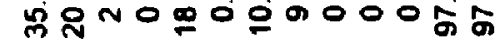

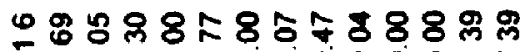

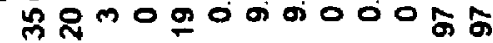

ㄷำ

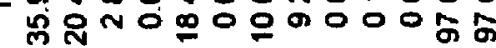

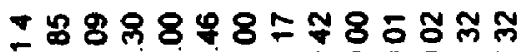

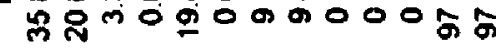

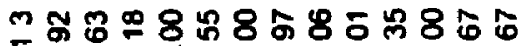

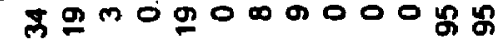

⿻ำ

लำ

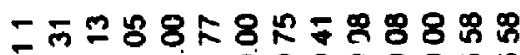

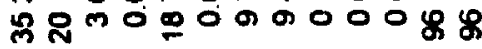

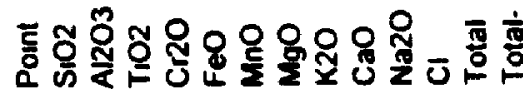

ำกำ

- 0 O O N O

赵市家

$\operatorname{s\infty }$

兽 ్ㅇㅇ

ก

员융

in $N$

要 8

in $\sim$

ले

Un

N

Un $\infty$

品

ก

용 -

ก N

$\stackrel{2}{N} 8$

ก $N$ 我

용용

in $\infty$

웅용

जi $N$

罚行 8

U $N$ D

규용ㅇㅇ

in i

융ㅇㅇ

जN

్ㅏ용요

กN

发豆

以N

융용

in

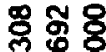

in

능용

Un⿻

눙요

in

융ㅇㅇ

in N

节 굥

กิ

กิะี

in $N$

फ ₹

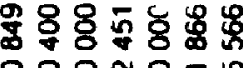

000nO-

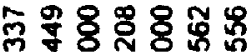

-00No-

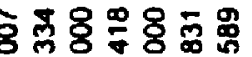

-00 No-4

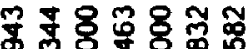

OOOnO-

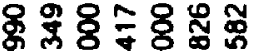

ODOnO-m

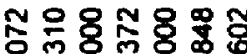

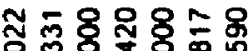

-0ONO-

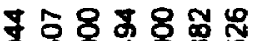

응응

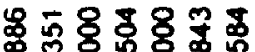

OOONOT-

要品 800

OOONO-n

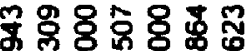

OดONO-

- 8480

sono

- Noñ

o o onon

ळ స్లం

OOONON

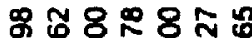

$18.8 \%$
-00No-n

으는

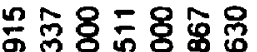

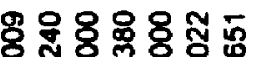

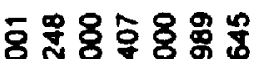

-00no-n

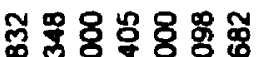

요 $888 \%$

000non

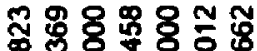

O으은

용요요요용

₹8용

o. ONON

OOONONU

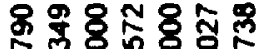

엉유유

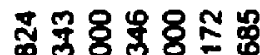

OOONON

舟骂 8

品势的等

- $00-000000$

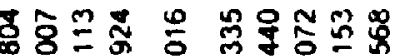

- O०-

00000

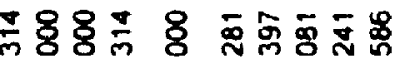

-00-000000

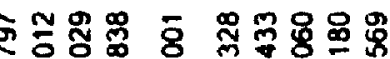

- $00-00000$

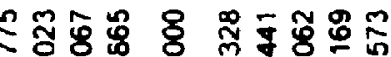

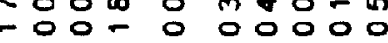

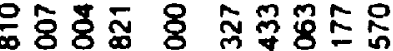

क-

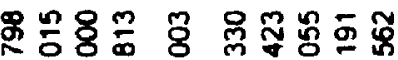

-OO-0 00000

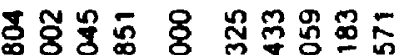

-00-0 00000

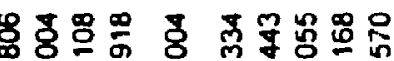

- 응 0 0 0000

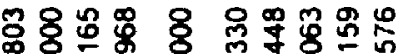

-00- 000000

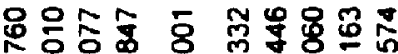

-00-0 00000

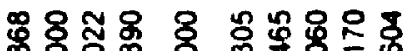

-00-0 00000

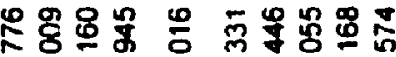

- O0- 000000

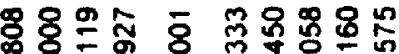

-

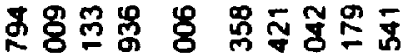

- 000- 0 00000

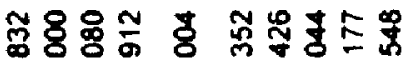

-00-000000

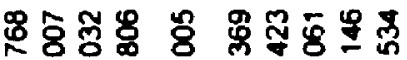

- $00-000000$

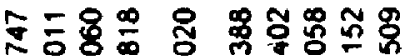

- O0- 00000

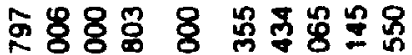

- $00-$

융요용

- $00-$

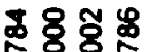

- $00-$

กำ

- $00-$

กํㄷㅎㅇㅇㅛ

$=80=$

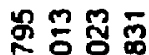

- 0 -

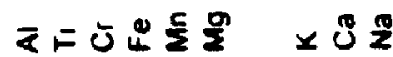

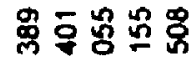
00000

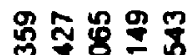
00000

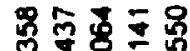
00000

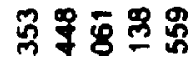

00000

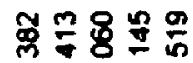
00000

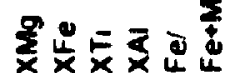

ㅇoㅇㅇ 


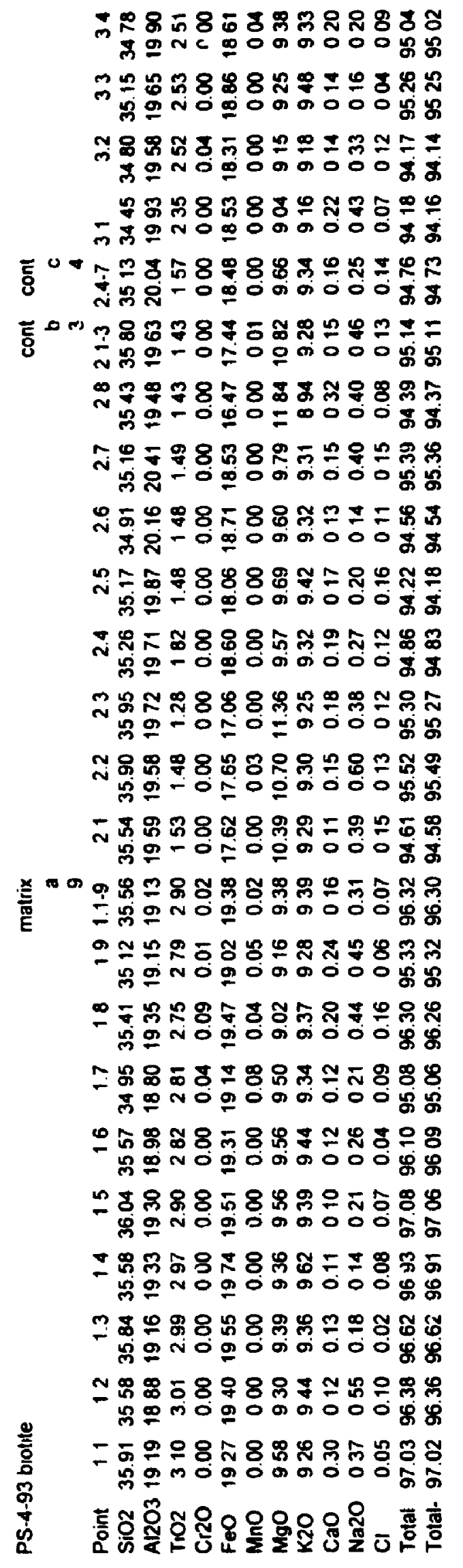

胥 8 क त ริ ஸा त 鬲 8 जल Б용 जN

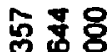
ज为 学需8 นก 递聶 两夜 กิ๊

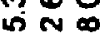
量要号 กิ

造要 N $\infty$ 뉴요 in 궁요 ชก๊ 고요용 บก 해용 ธก

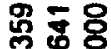
แ $⿻$ 话。 我亦 용용요 in ₹ เก 웅융응 ธत这 중응 in

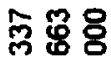
ต 융요 ธत

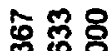
กㄴ. 윰융용 un $\infty$ $\bar{n} \overline{2}$

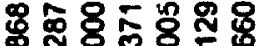
-0N에

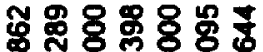
000 에 n

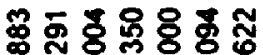
OOONON

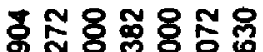
OOONON

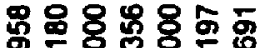
-0№ñ

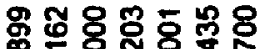
o o ononn

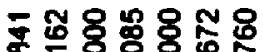

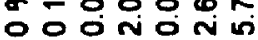
도웅ㅇㅁㅇㅇㅇㅇㅇㅇㅛ

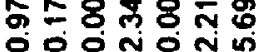

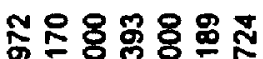
OOONONE

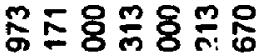

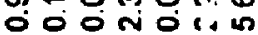
영용요용 क N óonon

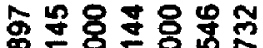

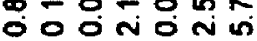

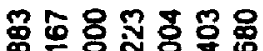
o o ONON Un

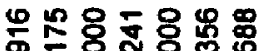
ó on 0 in m

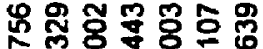
o o onon m

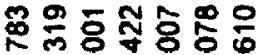

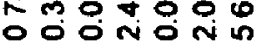

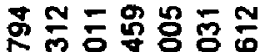

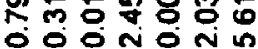

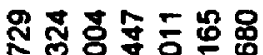
- OONON

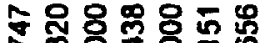
o o on ơ ñ Nㅗㄱㅇㅇ용요용

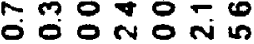

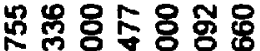
o o on 옹융용용 ్ㅗㅇ ó óñ

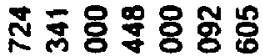
óo OñN

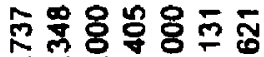
odonon テたす兄旁
꿍용

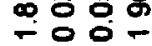
๙ - 0 '

옹영 용 - 00 홍ㅁㅇㅇ -00$\infty$ ิㅗㅇ 융융

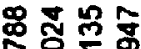
$\div 05 \%$

동 - 0 웅

g옹 ־잉잉 훙ㅁㅇ용 - 0 요

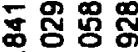
$\div 00-$

증 ๑잉잉

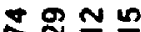

중 -00 흉용 잉 융뭉 - 00 -

ํㅠㅇㅠㅛ 종 - 0 O -

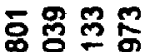
융후

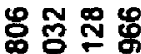
- 0 0 동용용 -0 융용 禹递 曲융 ㅎㅇㅇㅇㅛ - 00 -

অ它安 - 00 -

옹 苫 - 00 등ㅎㅇㅇㅛ - 0 웅

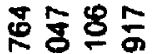
- 00 $\times 32$

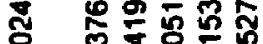
○。0 00

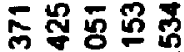
○ 0000 Nㅜㅇㅎㅇ ○ 0000

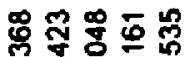
00000 喿 00000

ํㅛ용 잉ㅇㅇ

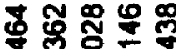
○ 0。 0 0 용요요 뜽

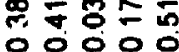
눙요 กั 00000 앴용용됴 00000 蛋台它望 00000

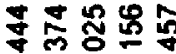
o O C 00 ㄱํㅇ묘욤 - 0000 해유 00000 需罗点 00000

용娄乐是

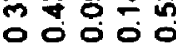

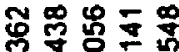
00000

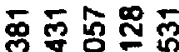
-0000

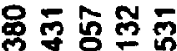
- 0000

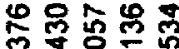
잉ㅇㅇㅇ 옹요ㅇㅛㅛ ○。ㅇํㅇ

点栾号㖞 ○००00

추ㅇㅛㅛ ○ 0000

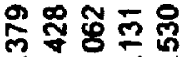
00000

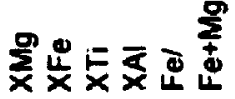




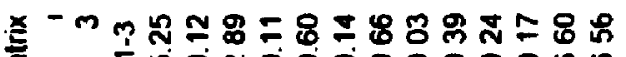

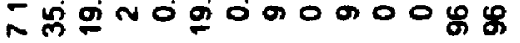

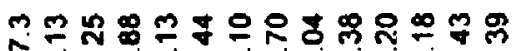

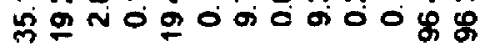

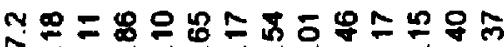

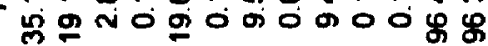

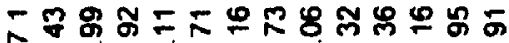

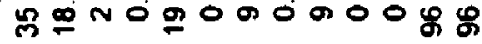

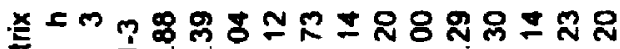

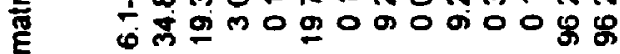

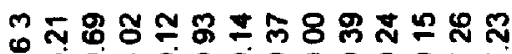

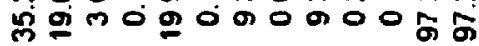

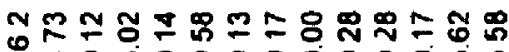

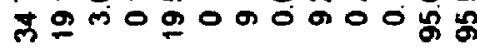

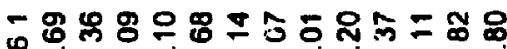

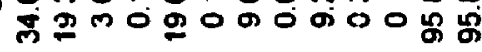

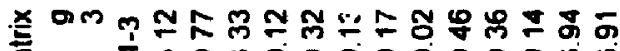

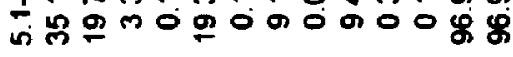

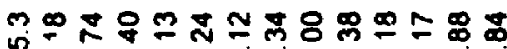

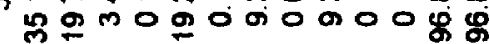

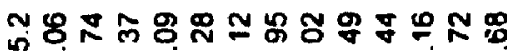

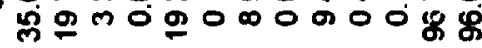

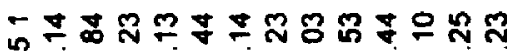

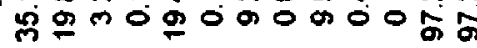

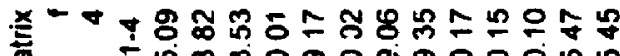

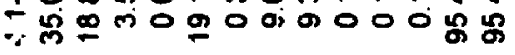

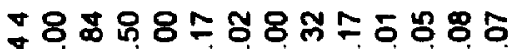

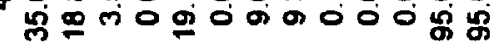

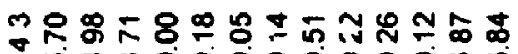

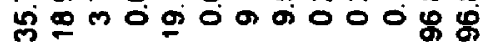
พNE

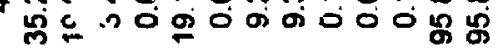

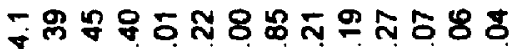

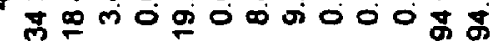

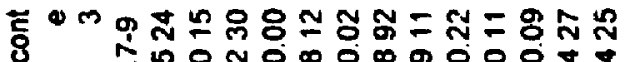

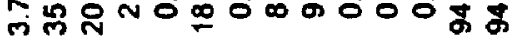

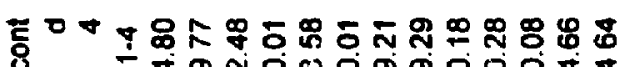

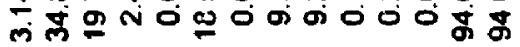

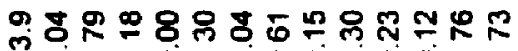

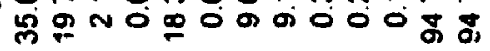

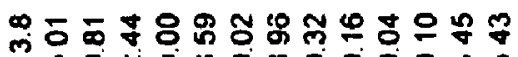

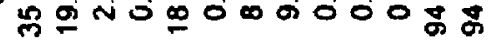

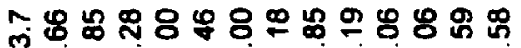

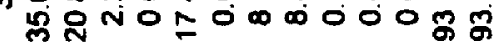

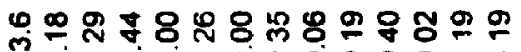

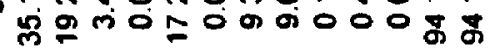

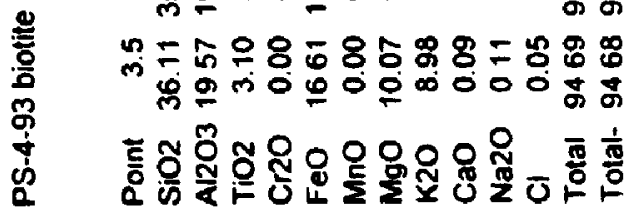

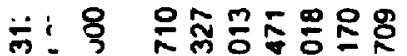
ni, 000non 总 in $N$

急喿 8 i $N$

స్లై

in

离 $\frac{9}{1} 8$

in $\mathrm{N}$ 舟

空员号

का N

马융

in

츄요

in $N$

包负吕

n. $4 \alpha$

ลูกํํㅇ

in $\infty$

กํㅇ

in w

密员

in $N \infty$

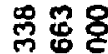

in

울융용

in

옹용ㅇㅇ

क ⿻ 丨

58:

in i

్ํㅇㅇㅇㅇ

กำ

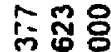

的

象 蒫

i

융

in $N$

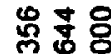

的

羿莒 잉

ก

区융유

in $N$

จู่์

in $\infty$

$\bar{s} \bar{\alpha}$

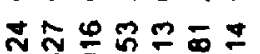

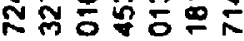
OOONON

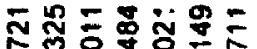
OOONON

品扈둥용요

O응요

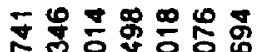
응웡ㅇㅇㅇㅇ

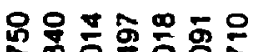
OOONON Un

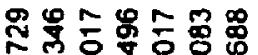
OOONONU

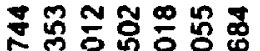
OOONON

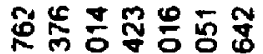
o OOU一N

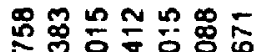
幽훙ㅇㅇㅇ OOONON

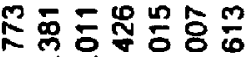
oOOnON

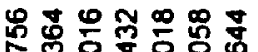
OOCLN

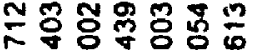
ㅇํㅇ영영

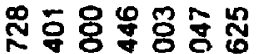
o OOnON

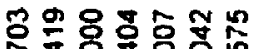
O O ONON

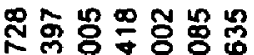
OOONON

용 000 N $\mathrm{N}$ in

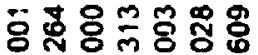
- OONON

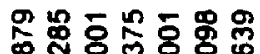
OOONON

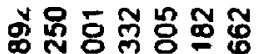
OOONON U

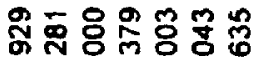
OOONON

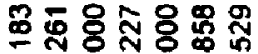
- 00 N 0 - in

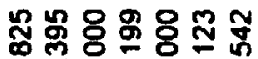
OOONON In

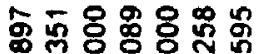
OOONON

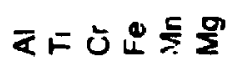

웅ㅎㅇ용

- 00 -

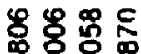

-

퐁용요

응 -

过용용

- 00 .

홍ㅇㅇㅇㅇㅇㅇㅇㅇ

홋옹영

- 00 -

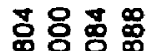

- 00 -

몽응용

โ-80-

중응

- 00 -

욧용명

- 00 -

ธูญ융

- $00-$

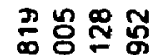

- 00 -

엉 沚㔚

-00-

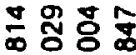

- 00 -

的零

- 00 -

농옹요

$\infty 00$

$\infty$ 용요

๘

- 00 -

令管合离

- 00 -

二零品

- 00 -

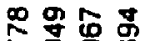

등응

오응요

중응

-

충응 웅

응은

这突至

- 00 -

궁용

등요

$\times 80 \frac{\pi}{2}$

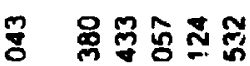
00000

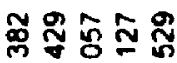

00000

央战里

잉ㅇㅇ

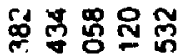

00000

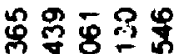

00000

ษ学变紊

00000

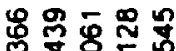

00000

芦昌䔎的早

00000

若守点点

00000

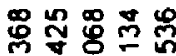

00000

号

00000

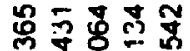

00000

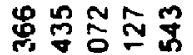

00000

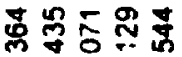

001200

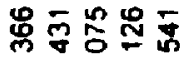

00000

우웡요요

응ㅇㅇㅇ

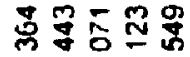

00000

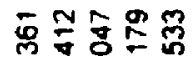

00000

동요ㅇㅛㅛ

00000

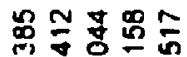

०0ㅇㅇㅇ

号羿号品

00000

峞解致

잉ㅇㅇ

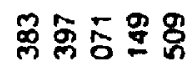

00000

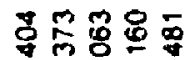

00000

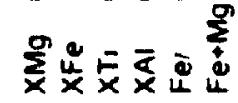




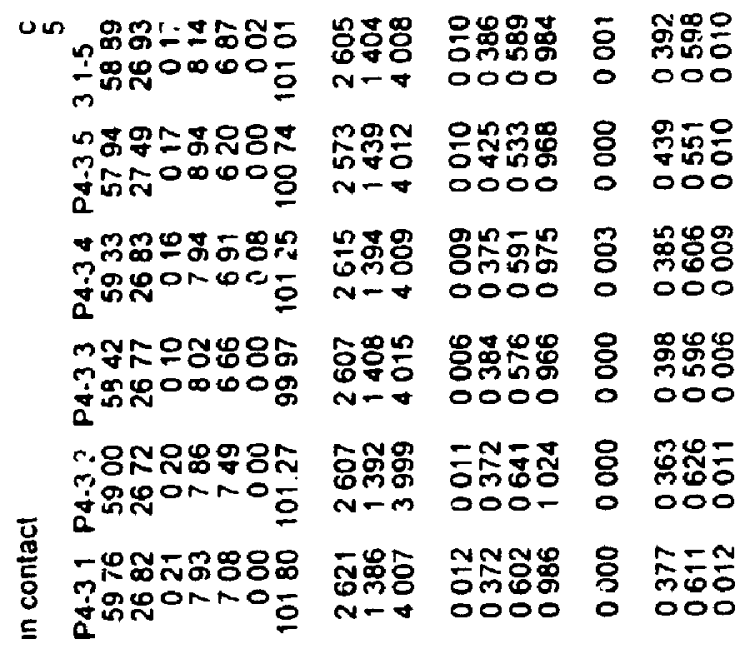

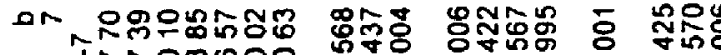

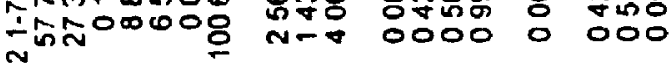

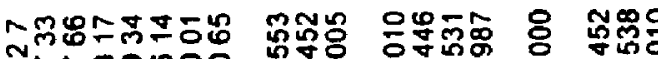

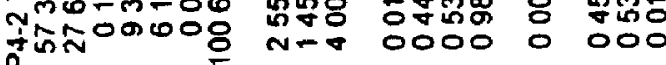

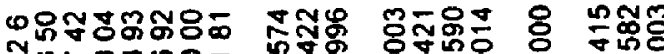

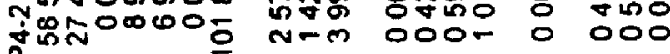

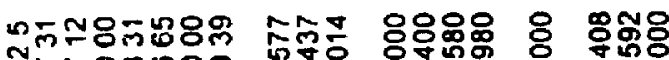
tíñ

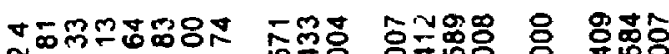

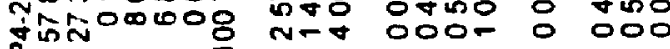

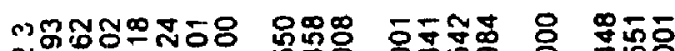

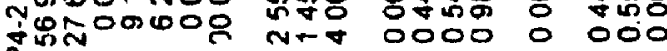

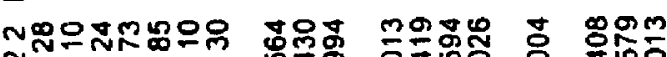

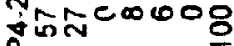
वरु०

-

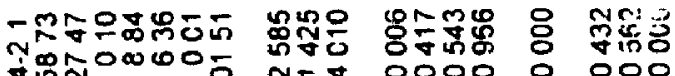
à

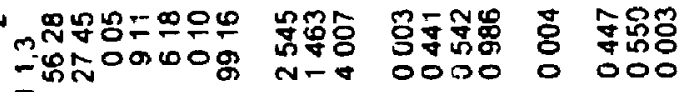

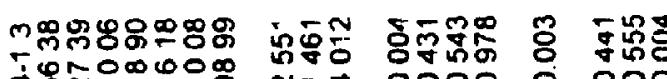

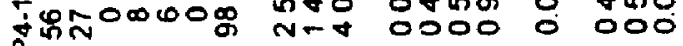

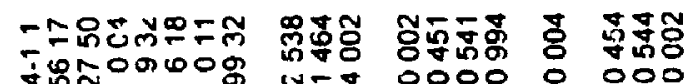$$
\text { 兽 }
$$

- $803=\div 8 \div$ iñก̃

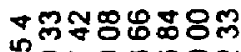

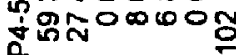

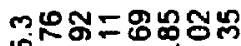
प⿺辶卬

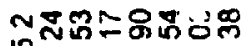

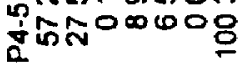

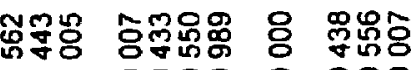
NT 00000000

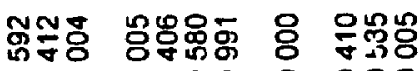
nT- 0000000

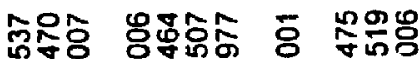
N-F 00000000

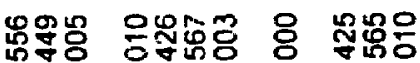
n-v 000 0 000

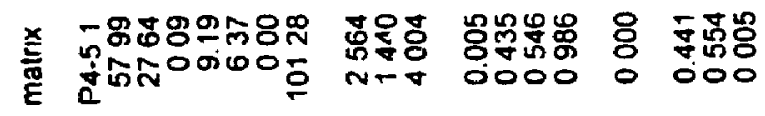

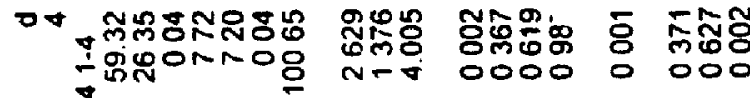

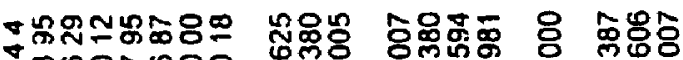

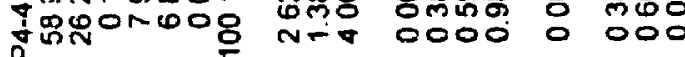
అగำ

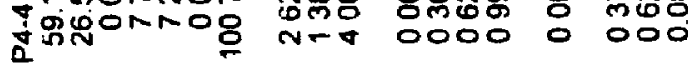

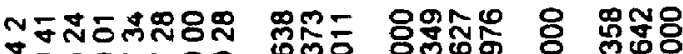

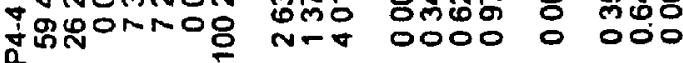
:

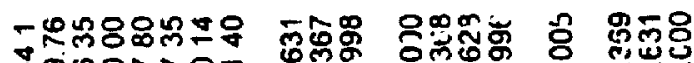
İ⿴囗十⺝

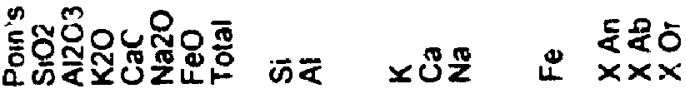




\begin{tabular}{|c|c|c|c|c|c|c|c|}
\hline م & 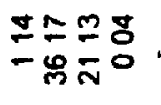 & 兽品范号品 & $\begin{array}{l}5008 \\
500 \\
50\end{array}$ & 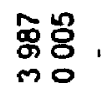 & $\underset{m}{\mathscr{乃}}$ & 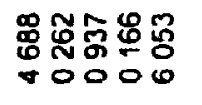 & 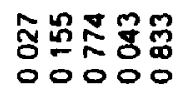 \\
\hline \multirow{20}{*}{ 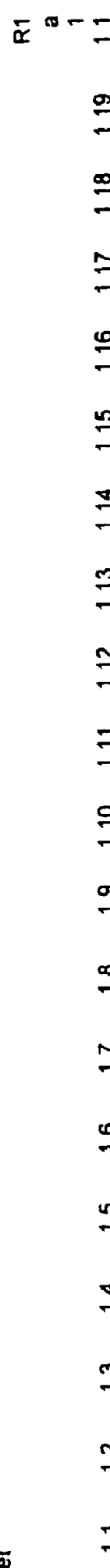 } & =5贯总 & 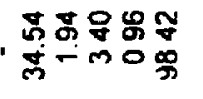 & 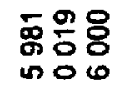 & 형응. & $\frac{\pi}{8}$ & 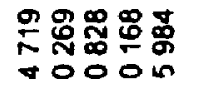 & 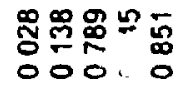 \\
\hline & 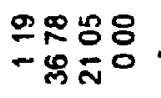 & 乐禺蛋怘 & 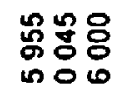 & $\begin{array}{l}\text { 穴各. } \\
\text { mo. }\end{array}$ & $\underset{\substack{\pi \\
m}}{\bar{m}}$ & 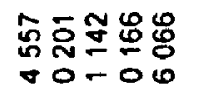 & 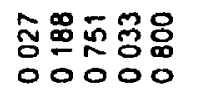 \\
\hline & 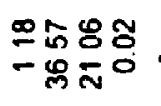 & 융 & 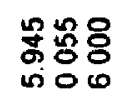 & 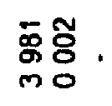 & 恕 & 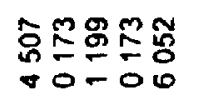 & 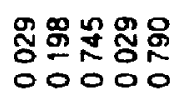 \\
\hline & 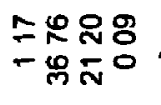 & 勿志罗号 & 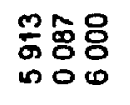 & 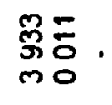 & 哥 & 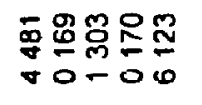 & 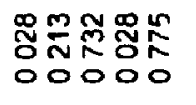 \\
\hline & $\begin{array}{l}\text { 용영 } \\
\text { 一용요. }\end{array}$ & 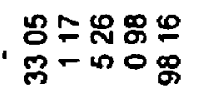 & 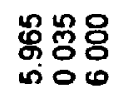 & 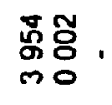 & 惫 & 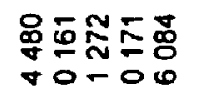 & 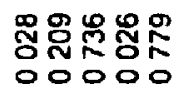 \\
\hline & $\begin{array}{l}\simeq \bar{m} \\
-6 \bar{m}\end{array}$ & 象怘吕吕 & 형용 & 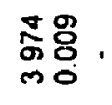 & 心 & 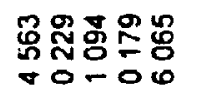 & 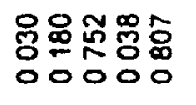 \\
\hline & 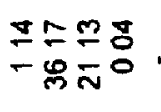 & 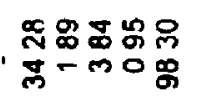 & 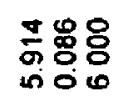 & 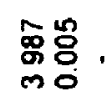 & 怘 & 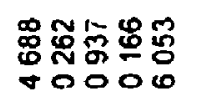 & 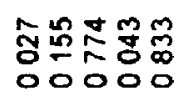 \\
\hline & 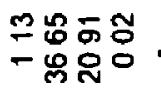 & 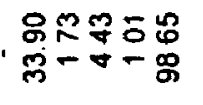 & 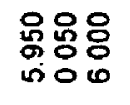 & 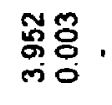 & 总 & 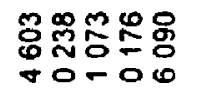 & 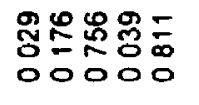 \\
\hline & 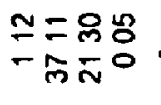 & எ & $\begin{array}{l}\text { 형요 } \\
500 \\
500\end{array}$ & 罣导 & . & 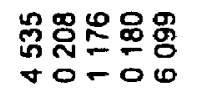 & 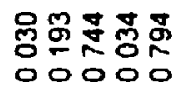 \\
\hline & 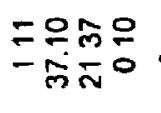 & 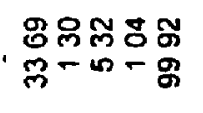 & 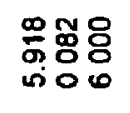 & 勇 & . & 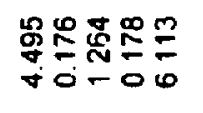 & 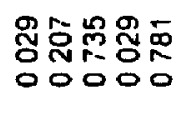 \\
\hline & 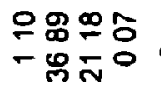 & 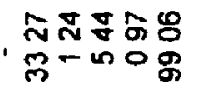 & 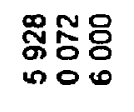 & 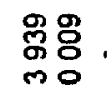 & . & 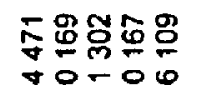 & 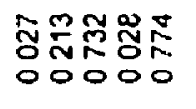 \\
\hline & 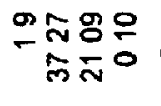 & 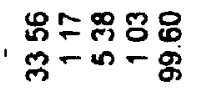 & $\begin{array}{l}\text { 공용 } \\
\text { nᄋㅇㅇ }\end{array}$ & 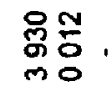 & . : & 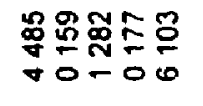 & 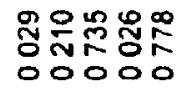 \\
\hline & 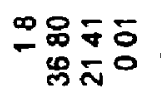 & 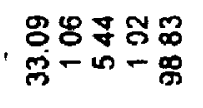 & 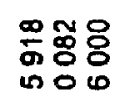 & 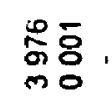 & . & 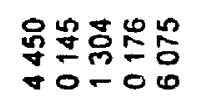 & 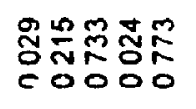 \\
\hline & 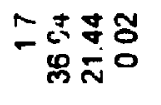 & 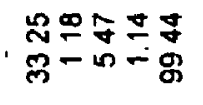 & 웅융유 & 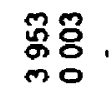 & . & 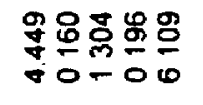 & 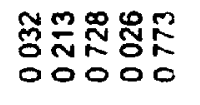 \\
\hline & 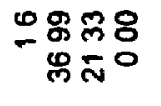 & 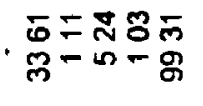 & $\begin{array}{l}588 \\
580 \\
600\end{array}$ & 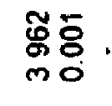 & . & 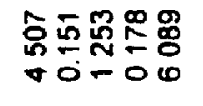 & 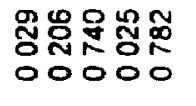 \\
\hline & 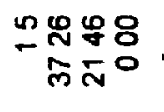 & 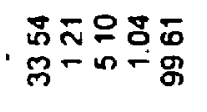 & 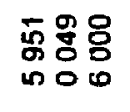 & 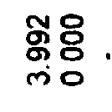 & 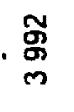 & 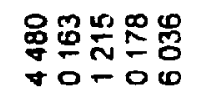 & 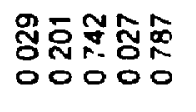 \\
\hline & 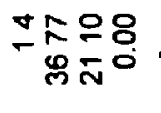 & 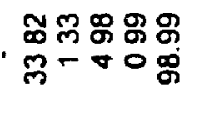 & $\begin{array}{l}358 \\
888 \\
000\end{array}$ & 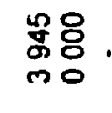 & . 号 & 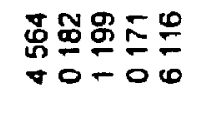 & 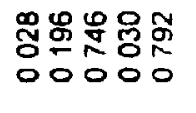 \\
\hline & 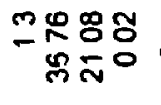 & 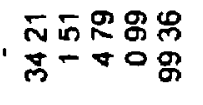 & 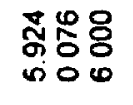 & 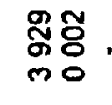 & . & 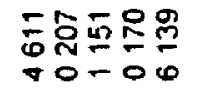 & $\begin{array}{l}\text { 幽占点品品 } \\
000000\end{array}$ \\
\hline & $\begin{array}{l}\text { 퓽ㅇㅇ } \\
\text { 号융 }\end{array}$ & 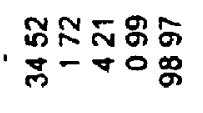 & 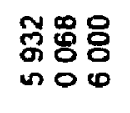 & 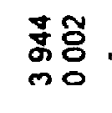 & . 褱 & 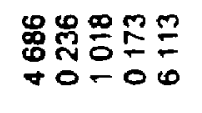 & 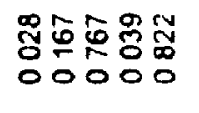 \\
\hline & - & 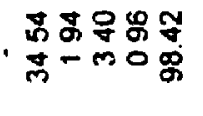 & 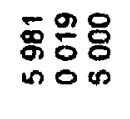 & $\begin{array}{l}\text { 훙응. } \\
\text { 응 }\end{array}$ &.$\frac{7}{0}$ & 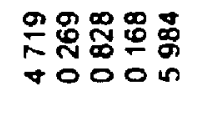 & 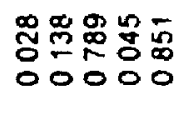 \\
\hline & 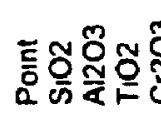 & & $\bar{n}$ & Q & & น돌 & \\
\hline
\end{tabular}




\begin{tabular}{|c|c|c|c|c|}
\hline 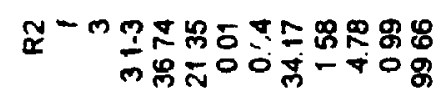 & 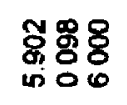 & 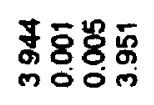 & 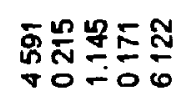 & 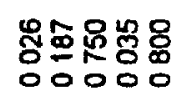 \\
\hline 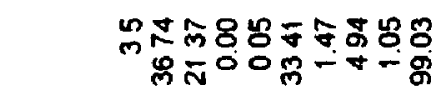 & 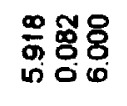 & 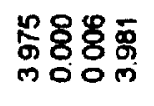 & 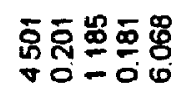 & 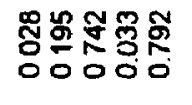 \\
\hline 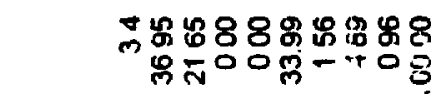 & 总舍宫 & 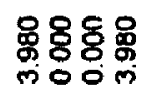 & $\underset{8}{8}$ & 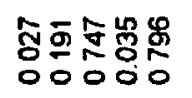 \\
\hline 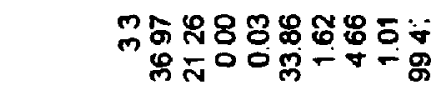 & $\begin{array}{l}8 \\
\end{array}$ & 落 & 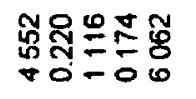 & 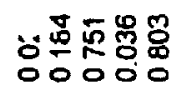 \\
\hline 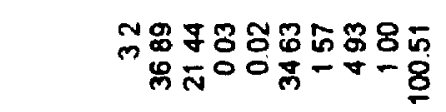 & 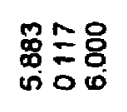 & $\overline{\tilde{\pi}}$ & $=\frac{2}{0} \frac{2}{6}$ & 䐱 \\
\hline - & 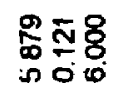 & 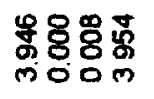 & 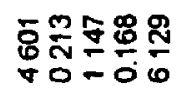 & 络总 \\
\hline 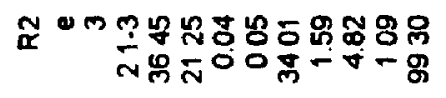 & 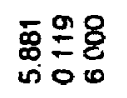 & 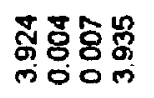 & & \\
\hline 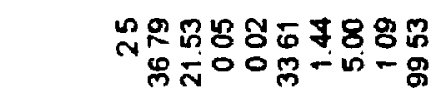 & 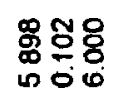 & 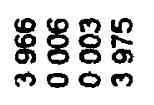 & & 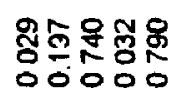 \\
\hline 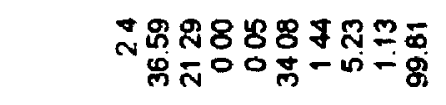 & 惫 & 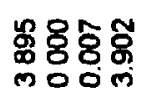 & $\underset{\substack{N \\
o}}{\stackrel{m}{ }}$ & 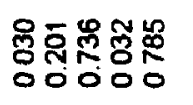 \\
\hline 象兽 & 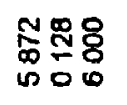 & 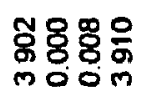 & 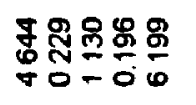 & 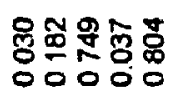 \\
\hline 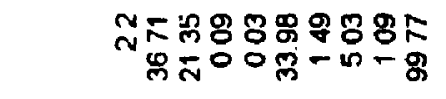 & 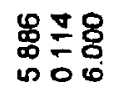 & 홍유융용 & 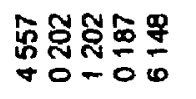 & 疍: \\
\hline ヘ & 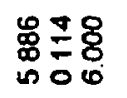 & 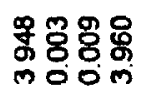 & 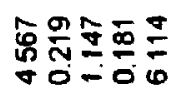 & 00 \\
\hline 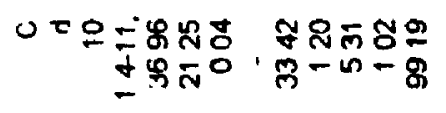 & 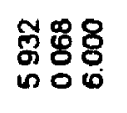 & 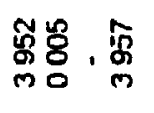 & & r \\
\hline 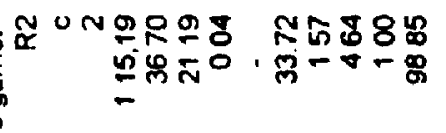 & 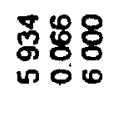 & 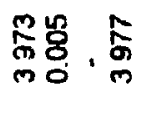 & 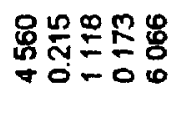 & :్రి \\
\hline 룽연욘 & & & & \\
\hline
\end{tabular}




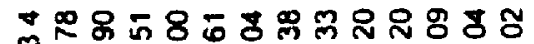

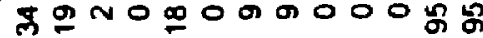

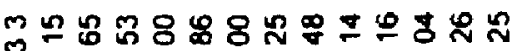

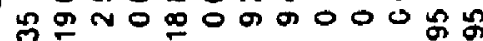
ก

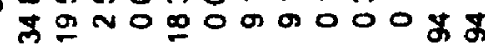

ๆ

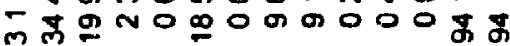

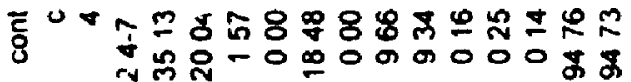
ㄷำㅇำ

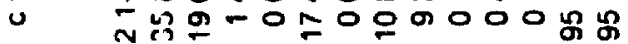

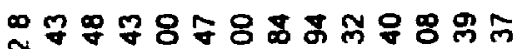

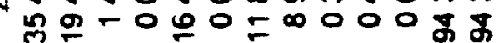

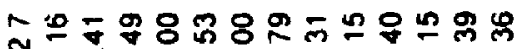

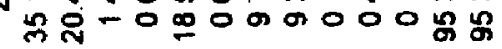

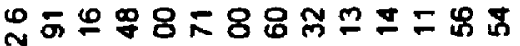

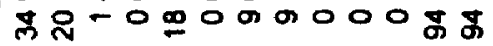

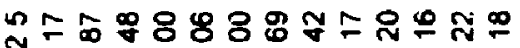

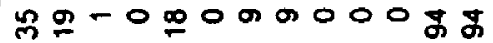
ลักล์

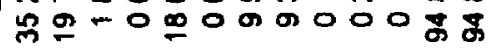

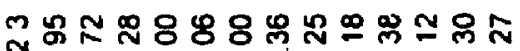
号 $9-0=0=0000$ ณू

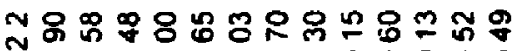

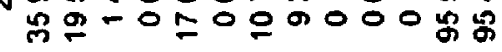

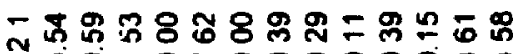

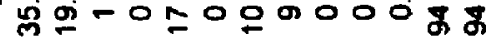

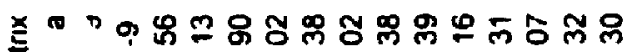

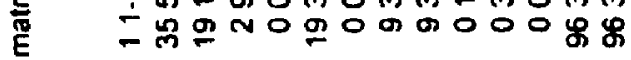

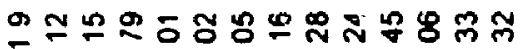

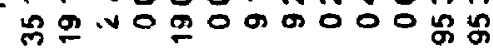

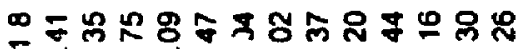

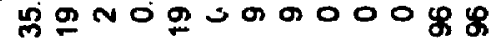

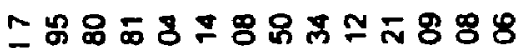

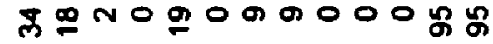

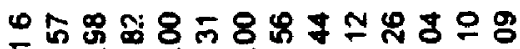

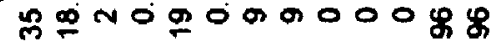

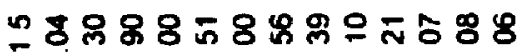

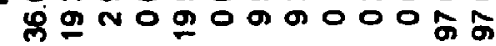

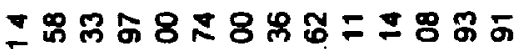
"

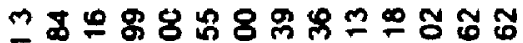

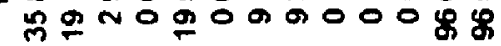

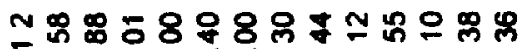

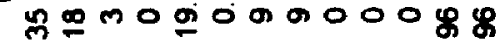

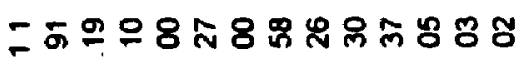

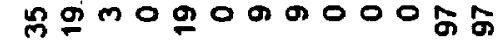

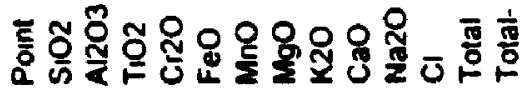

$\mathscr{H}$ ก $\sim$ 承员 mis 豆范 in $\infty$

蛋员这

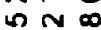

部客

กิ

늉용ㅇㅇ

in $\infty$

总苨8

in

సิํํㅇㅇㅇ in $N$

要 $\overline{8}$ in $\infty$

通 in 禺范 un $\infty$

领 n $\infty$ 웡용 जn $N$ 효용 जN 总安造 in 产员 in $\infty$

응용 แ N

พั้ 8 लख 웅융ㅇㅇ i ก แ $N$ क

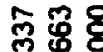
ஸN 뭉 8 in $\infty$ क्ष in $8 ㅇ ㅛ ㅇ$ ก $\infty$ कर

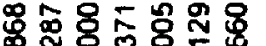
000 N

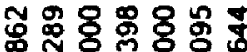
OOONON

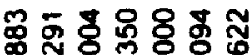
OOONON

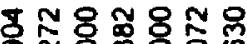

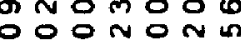

品8 88 向可

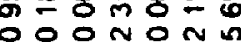

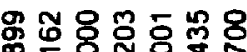
00 0 non

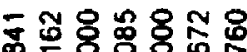
도용항요용 óOONON

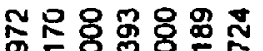
OOONON 중요요용

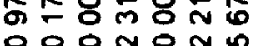
융영중용웅

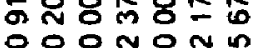

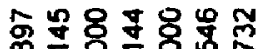
o O OnONm 옹용용융 OO유에

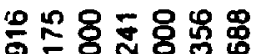

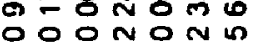

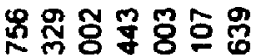
oOdnon 3옹 충용응 OOONONU 耳도요유.

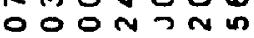

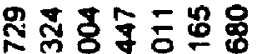
000 on

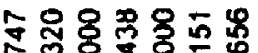

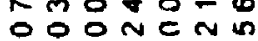

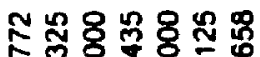
OOONON

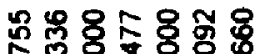
OOONON

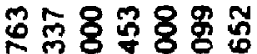
ว

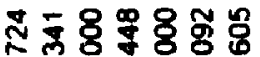
OOONON

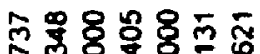
OOONON

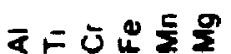

잉영 -00-

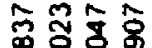
-00\% ํํㅇ용 - 00 -

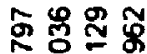
- 00 $\infty \tilde{N} \approx$

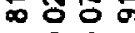
思导告京 응-

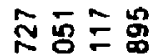
- $00-$ 兽染志 - 00 동푱 - 0 응

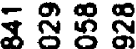
- $00-$

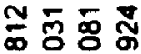
- $00-$ 융ำ - 00 -

战象 - $00-$ 옹요 -응응

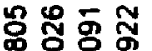
- 00 -

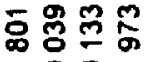
- 00 埋瓷号 - $00-$

총ㅎㅀ - $00-$ 0욤 -0ㅇㅇㅇ

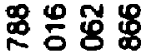
- $00-$

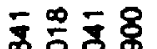
- $00-$

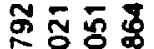
응-

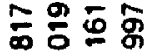
- 00 -

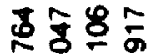
- 00 $\times 0 ึ 2$

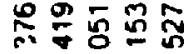
00000

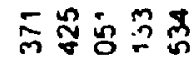
0000 ำํำ 00000

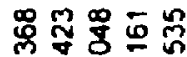
응ㅇ

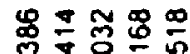
잉영

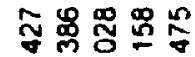
00000

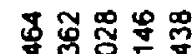
03000 品융옹는 00000 궁융요 중 00000

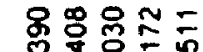
영응

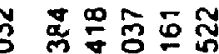
00000

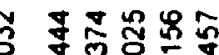
00000 雨总员要 00000

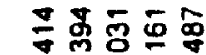
00000

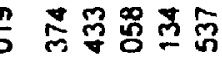
00000

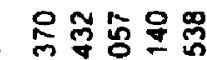
00000

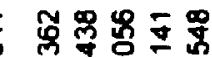
000000

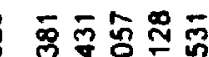
00000

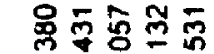
- 00000

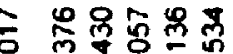
00000 옹 密总 - 00000

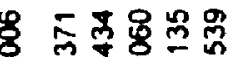
- 00000 盆缶要旁 - 00000

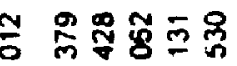
- 00000

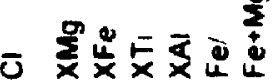




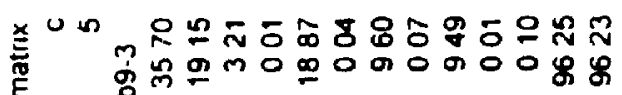

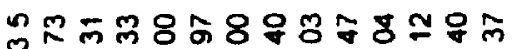

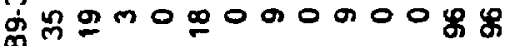

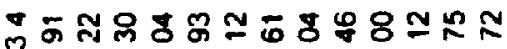

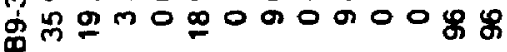

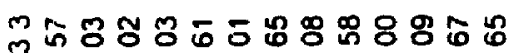

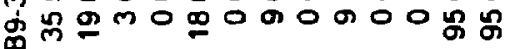

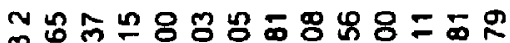

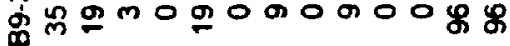
-

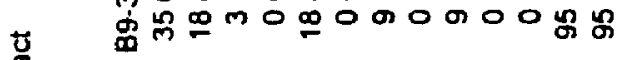

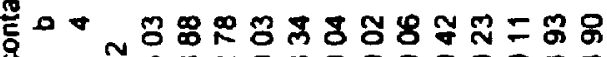

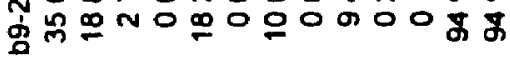

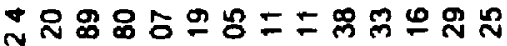

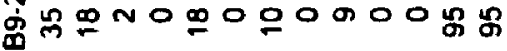

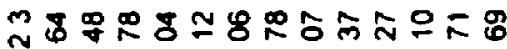

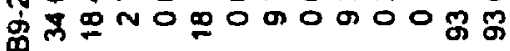

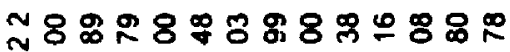

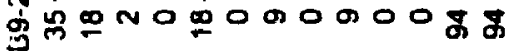

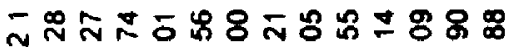
贾员은 范心

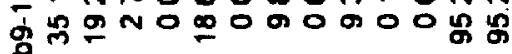

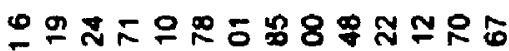

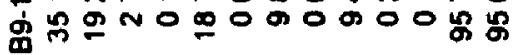

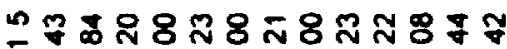

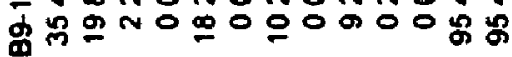

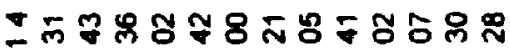
要号乐N

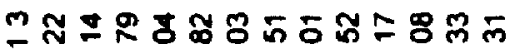

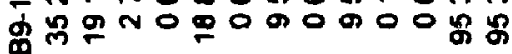

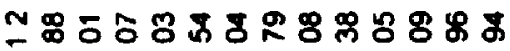

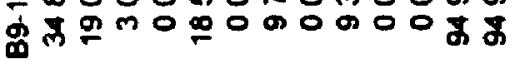
こ亩ニ ะ

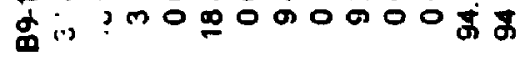

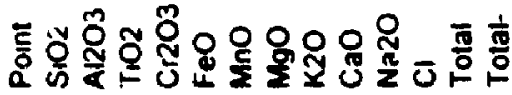

足 宓 in

角色宫 in

兽宮 in $N$ 施号 $\sim \sim \infty$

㧀产 in $\infty$

올은웅 的少

号范 ก

今్ in

80 in $\infty$

눙용 แ

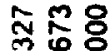
ज N

공웅 กำ

88 in $N$

起落 की $\infty$

동 ก त 然各 事

ำ 8 纱

\% in $\infty$ $\bar{n} \bar{\alpha}$

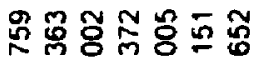
o Oonon

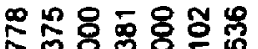
OOONON

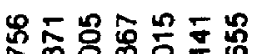
OOONON

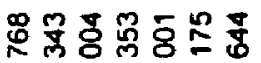
OOONON

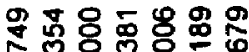

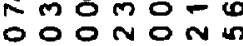

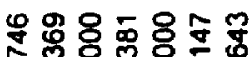
OOONON

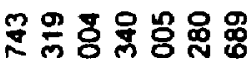
OOONON

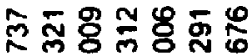
OOOOOONON

유ㄹㅠㅛㅇㅎㅇ O유엥

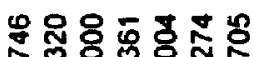
OOONONM

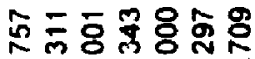
OOONON

品= 웅중용 OOOONON

ํํㅇㅎํ요용 OOONON

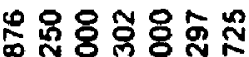
OOONON

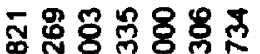

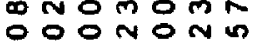

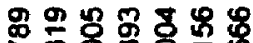
○ㅇํ윰ำ

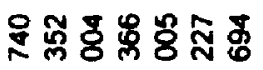
oOONON

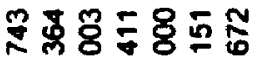
oOd non 뜽 ㄴㅇ-

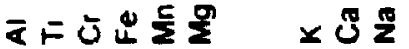

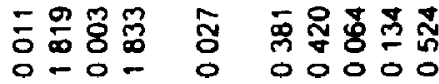

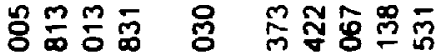
O-O- 0 O००००0

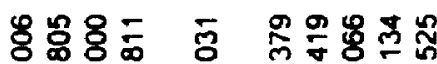
0-0- 0 00000

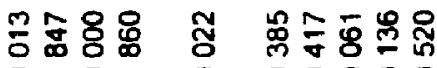
0-0- 0 00000

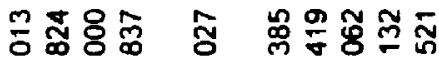

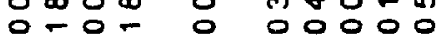

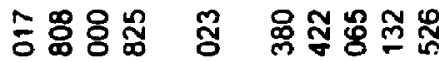
$0-0-000000$

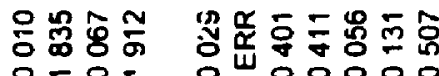

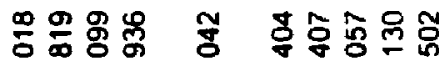

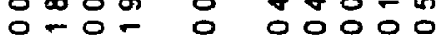

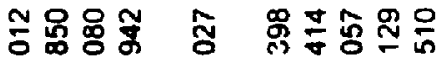
- - 0 - 00000

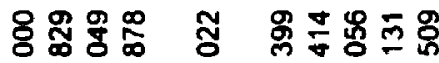
O-0- 0 00000

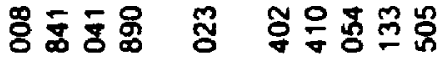
O-0- 0 00000

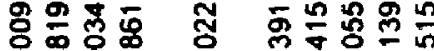

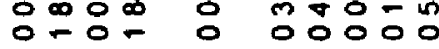

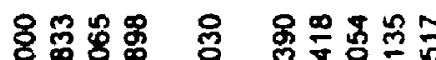
O-0- 000000

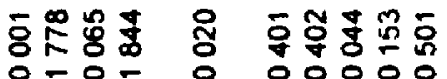

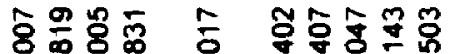
ㅇㅇㅇ 영ㅇㅇㅇ응

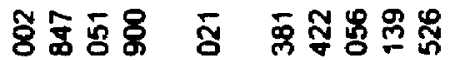
O- 0 - 0 00000

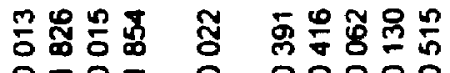
O-O- 0 00000 웅

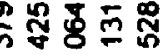
00000

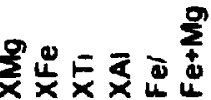




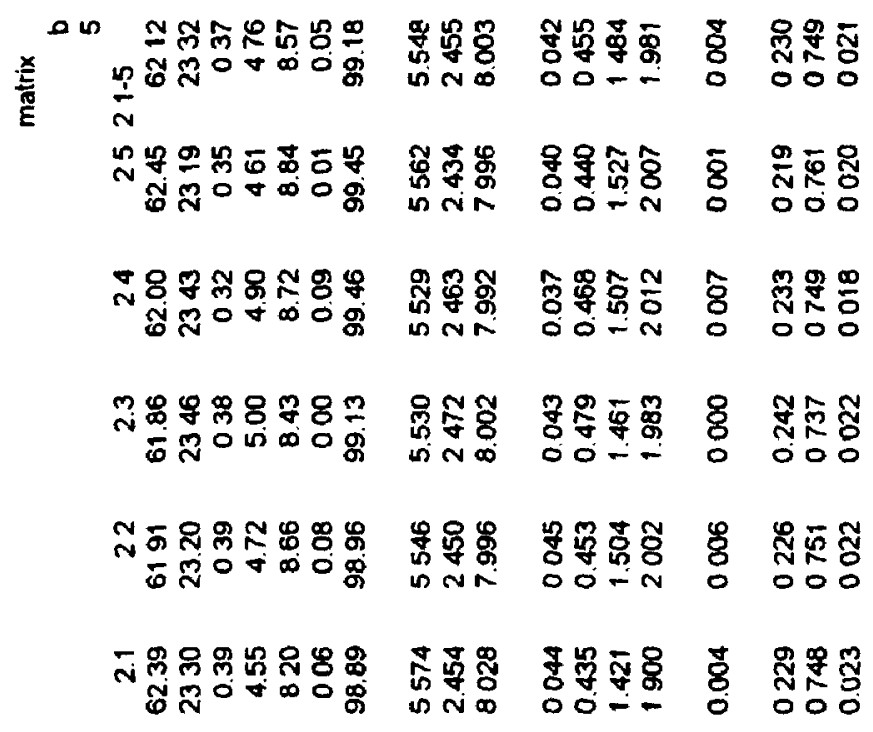

\begin{tabular}{|c|c|c|c|c|c|}
\hline & 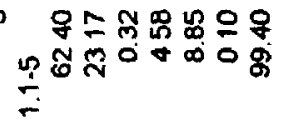 & 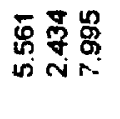 & 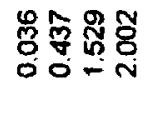 & $\hat{8}$ & 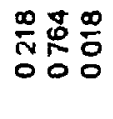 \\
\hline & 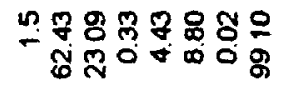 & 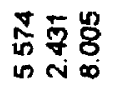 & 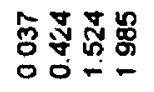 & ళ̊ & 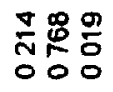 \\
\hline & 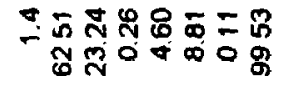 & 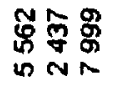 & 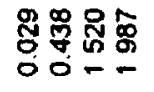 & $\$_{0}^{\infty}$ & 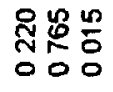 \\
\hline & 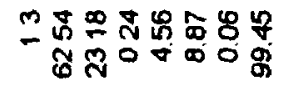 & 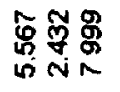 & 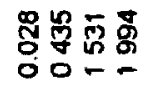 & $\begin{array}{l}\text { Zे } \\
8 \\
0\end{array}$ & $\begin{array}{l}\infty \\
\stackrel{\infty}{*}: \\
0 \\
0\end{array}$ \\
\hline & 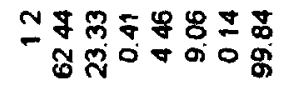 & 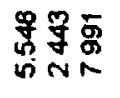 & 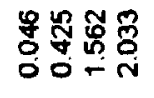 & 웅 & 胥: \\
\hline & 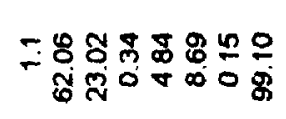 & 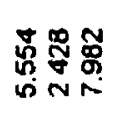 & 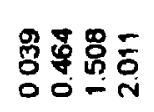 & $\overline{\overline{0}}$ & $\begin{array}{l}\text { 矛员是 } \\
000\end{array}$ \\
\hline & 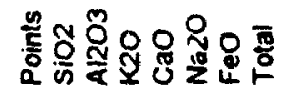 & $\bar{n}$ & & & \\
\hline
\end{tabular}


구응

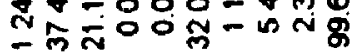

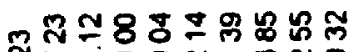
ㅅ⿵冂卄二⿺辶万人

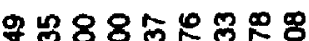

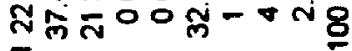
- 988 出 $\cong 8 \%$

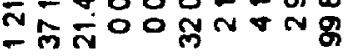

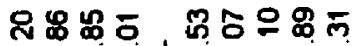
- 罗只 - N

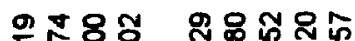

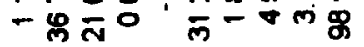

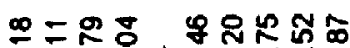

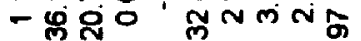
요요

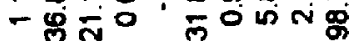

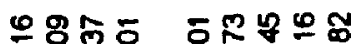
-

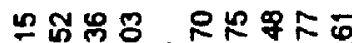

-

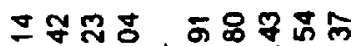
一的品品以

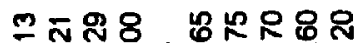

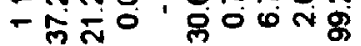
푼

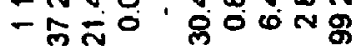

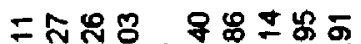

- M̃

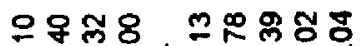

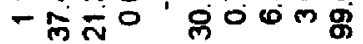

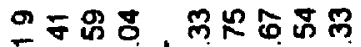
的。 品 ๓888 员

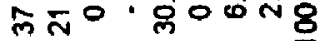

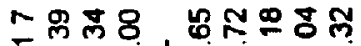

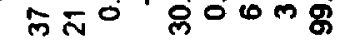

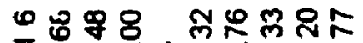
ผิ त० แ กิ - ำ ำกำ ตำ

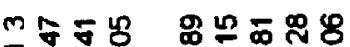
के त०

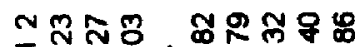

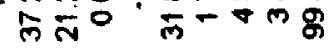

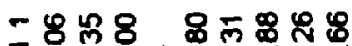

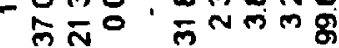

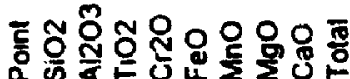

号

ஸ०००

$\overline{\mathrm{g}}$

जi 0

웅융요

जi 0

궁용

กิ 0

品엉

ino 0

总 ㅃ⿺ㅇ

in 0

중

in 0

용요

no 0

융영

赵象

内i.

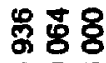

जo

응 8

no 6

过范

is 0

\%ั ชิ

is 0

왕용ㅇㅇㅇ

ज००

영웅

๓ 06

步㖞号

응

范 :

in 0

可总郭

in 0 io

훙ㅇㅇㅇ

in 00

最 8

is 0

융ㅇㅇㅇ

in 00

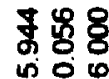

$\sim \infty 8$

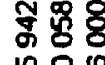

in 0

ऊ $\bar{\alpha}$
$8=8 \%$

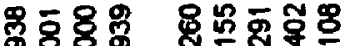

- $00 \mathrm{~m}$

웅 \&

mo

요요

ํㅣㅇㅇㅛ

용요

응요

횽용

mo

员

mं लं

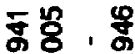

mi m

总䒹 $\cdot \frac{2}{\sigma}$

iो ल

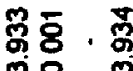

묭,

m

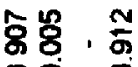

mo m

용요 ․ㅛㅇ

mo

क욜.

mo

융항

mo i

त్ర్ . \%

ल이 ल

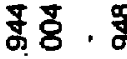

\$ 8 ,

लं

综思罗

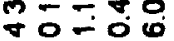

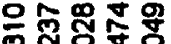

- 0 - 0

急点象总号

$\checkmark 0000$

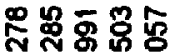

ชั0ㅇㅇ

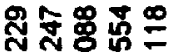

$\checkmark 0-00$

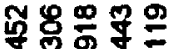

ن 000

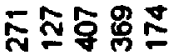

ช0 -06

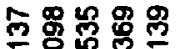

- 응

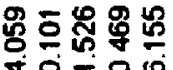

ธㄷำ

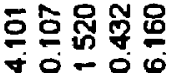

둥휴웡

$+0-06$

守舒学量

ن 0 - 00

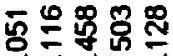

$\checkmark 0-00$

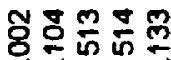

ن० 000

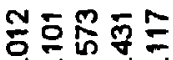

ن 0 - 00

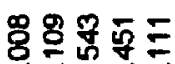

్ㅐ용. 요

ช0-06

费三余幽。

00000

동응ㅎㅇ용

०0000

오음용ㄷㅇ

엉ㅇㅇㅇㅇㅇㅇ

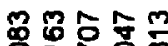

00000

象声名安

엉ㅇㅇㅇ영

홍응요융

엉ㅇㅇㅇ

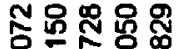

응ㄷㅇㅇㅇㅇㅇ

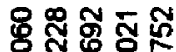

00000

居员过옹

ธ。ㅇㅇㅇㅇㅇ

응 是量응

ㅇㅇㅇㅇㅇ

옹워응응

응ㅇㅇ

동앵응옹

00000

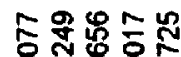

00000

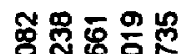

00000

症余舟

0.000

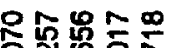

ㅇํㅇㅇ

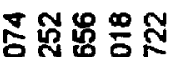

00000

ल० ल ब

용요용

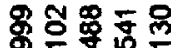

mo m

mo- 00

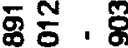

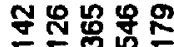

范兽额 응

O융ㅇㅇㅇ

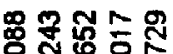

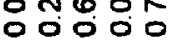

吅ำํํㅇํㅇ

00000

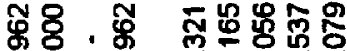

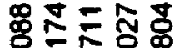

mo m

- 0 - 0

00000

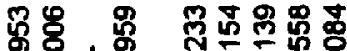

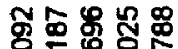

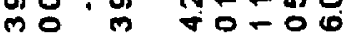

00000

సै.

员응형영

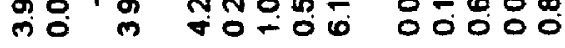

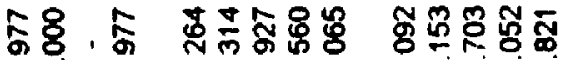

ल० $\mathrm{m}$ च००००

00000

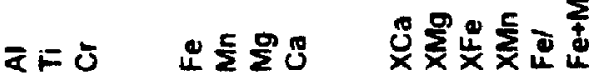




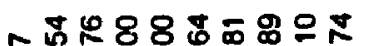

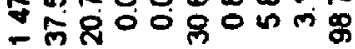

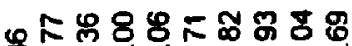

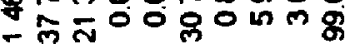

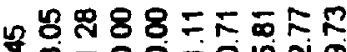
-

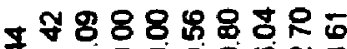
的方品品心

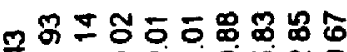

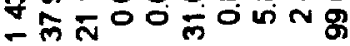

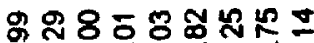

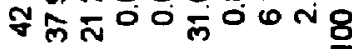

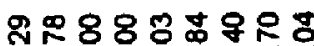

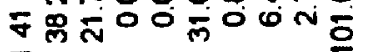

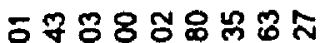

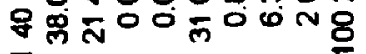

ด กัน

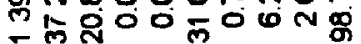

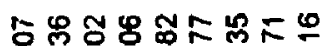

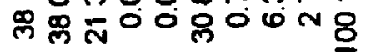

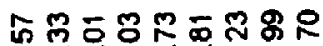

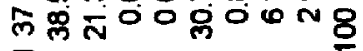

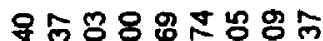

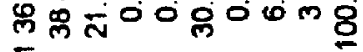

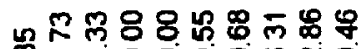

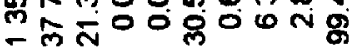

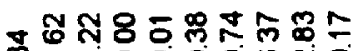

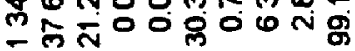

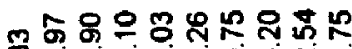

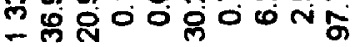

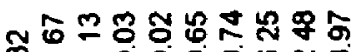

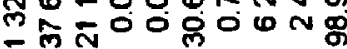

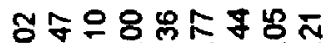

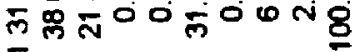

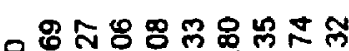

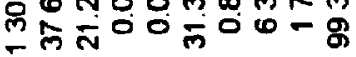

出此8

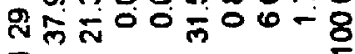

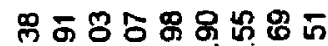

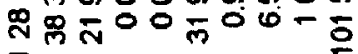

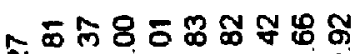
는

8용요

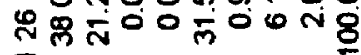
4

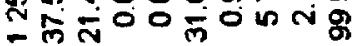

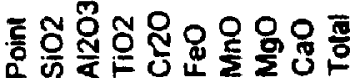

8. 8

is 0

\&

(2)

잉 용ㅇㅇ

006

눙용

in 0

$\overline{8} 8 \overline{8}$

606

ํํㅇ

แ0

용용

जि 6

象号

no 0

둉ㅇㅇㅇㅇㅇ

ํํㅇ응

옹ㅎㅇㅇㅇㅇ

ज० 0

ㅎํㅇ융유

000

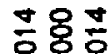

io 0

号芦

in 0

多志宫

的品

品 8

is 0

罗言号

in 0

동융

no 0

뉴융유

웅융용

in 0

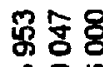

no

般号

888 000

品聖啹

no

कर
양용ㅇㅇ영

mo요

웅용요

mo $0 \mathrm{~m}$

용요

moOm

웅용ㅎㅇ

त० $0 \mathrm{~m}$

ㄱํㅇㅇㅇㅇㅇํㅇ

ल०0m

ูํㅇㅎㅇ

ल०ठ

นึ. 8

mo o

\%马용요

mo $\mathrm{m}$

究

mo

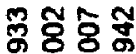

MOO

్ํㅇ유용

लㅇㅇํ

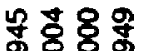

ल이요

㑒吾市

강융

局

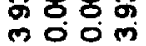

형응 웅영

m응

过 858

m०0

곻웅

mo

号额응 응음

劳审实哭 moon

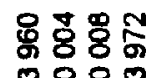

moOn

궁융

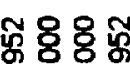

mo

下莤炙察

mo $0 \mathrm{~m}$

$\bar{E}=\bar{U}$

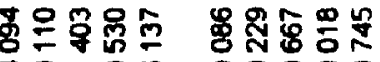

प0-06 00000

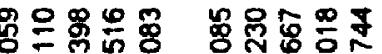

-0-00 00000

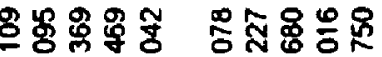

$\checkmark 0-0000000$

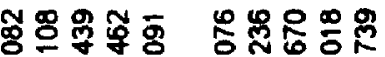

寸0 - 0000000

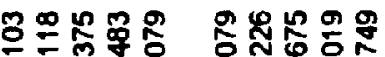

จ0-00 00000

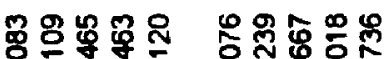

प0 0600000

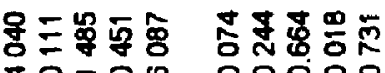

- 0 - 0000000

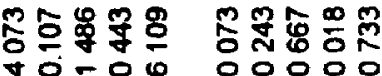

过罗出可 $m=0$

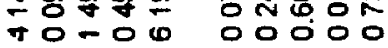

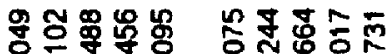

प엉ㅇㅇ 00000

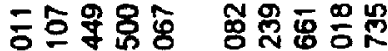



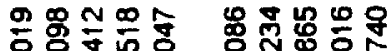

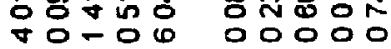

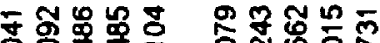

응 00000

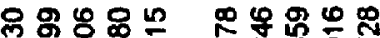

ชํํㄴㄷㅡ

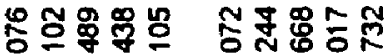

จ응 00000

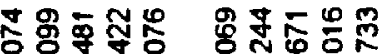

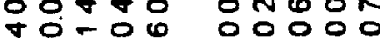

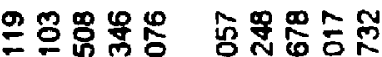

于융유

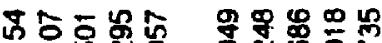

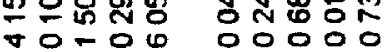

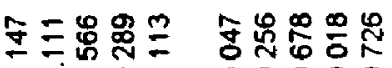

จ 0 - 0000000

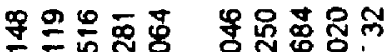

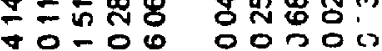

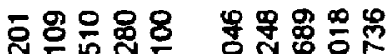

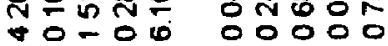

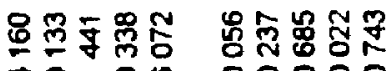

+0-0000000

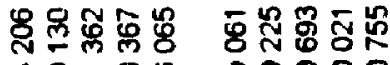

प0-00 00000

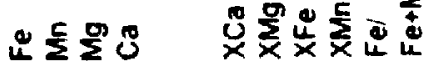




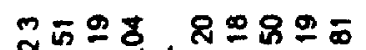

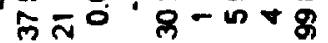

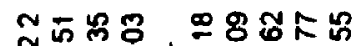

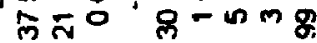
-

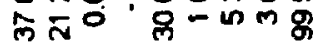

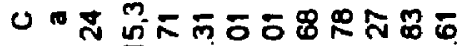

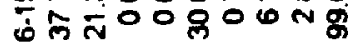

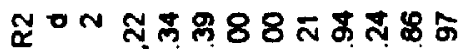

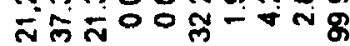

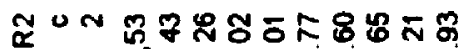

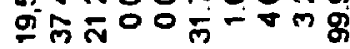

ฉคN

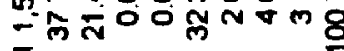

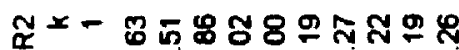

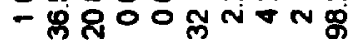

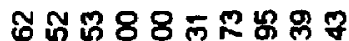

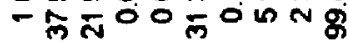

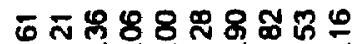

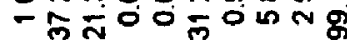

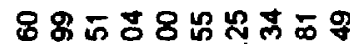

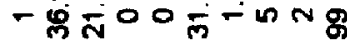

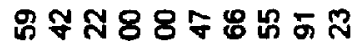

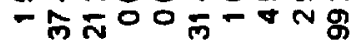

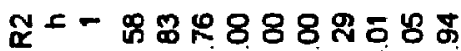
- 万人ั

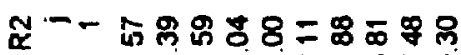
- 公云O

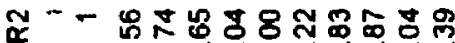

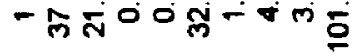

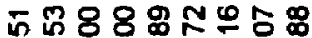
岁界市

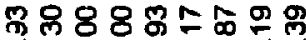

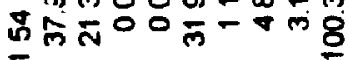

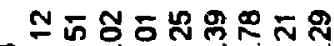

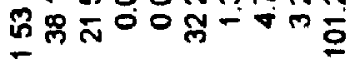

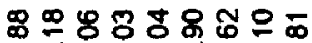

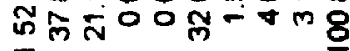

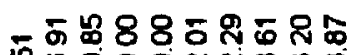

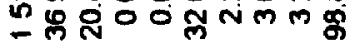

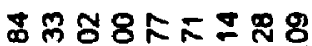

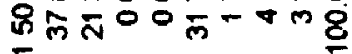
ธิ $858 \%$ 뜡ำ

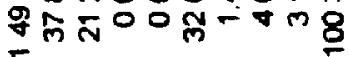

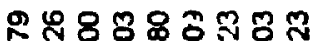

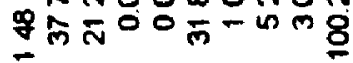

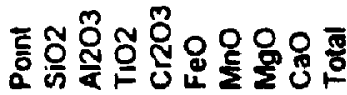

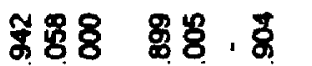

जi 0

过范 8

no 0

ํํㅇㅇㅇㅇ

is 0

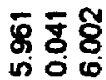

48

ज० 0

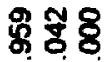

जo 6

형옹

is 0

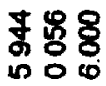

员员 8

은

可

ज.

名

no 0

品눙융

no 6

㶽 8

in 0 io

స్.

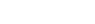

ส్๊듕్ㅠ

융양융

유:

:용요

no 6

ริ్ㅎㅇㅇㅇ

जi 0

弯

4 0

ำำ

no 6

웅웅응

000

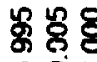

no 0

융융

no 0

\& 8 .

mo

옹 용 용

융 요유.

(

\%8 8 \%

mo

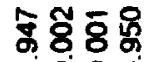

ल००

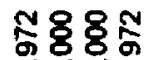

लिठठ

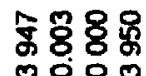

요용

i००。

응효용

io

궁요

mo

양용용요

mo $0 \mathrm{~m}$

영용영

mo

웅효용혀

लं०

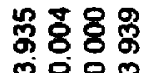

乌ु용

mo

앵 8 용용

io

岁额要

ल०० 0

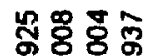

ल0 $0 \mathrm{~m}$

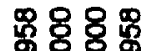

ल० $0 \mathrm{~m}$

ร용용

ल०0

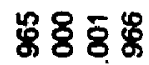

mo

总尔 궁

लं०

$\overline{\text { ๙ }}$

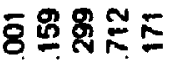

ช0ำ

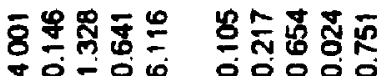

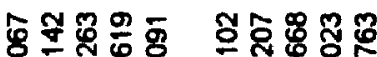

ช0-00 00000

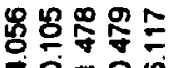

$-0-06$

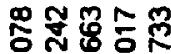

00000

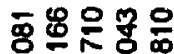

00000

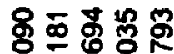

00000

용는옹농응

00000

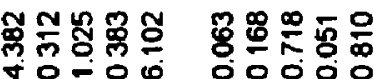

กำㄴำ

†영

ำํํำ

영-0

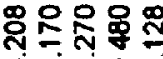

$\forall 0-0 \infty$

ำ ํํㅇํํㅇํㅇ

$\checkmark 0-00$

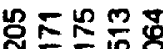

ज० - 0

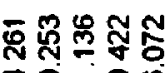

$\checkmark 0-00$

踏需的号

$\checkmark 0-00$

มี่

$\forall 0006$

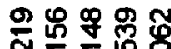

N-

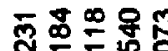

$\checkmark 0 \div 0$

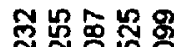

$\rightarrow 000$

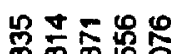

ช0 000

응요

त的品\%

$\checkmark 000$ is

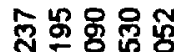

$\checkmark 0-0 \%$

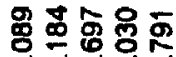

웅ㅇㅇㅇ

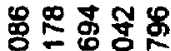

o o o o o

ㅇํㅇำ

00000

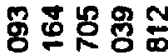

0엉응

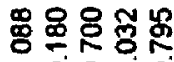

00000

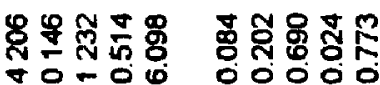

$\frac{2000}{80}$

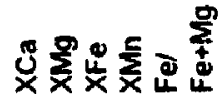




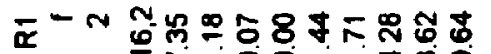

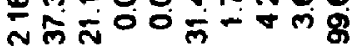

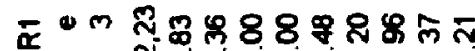

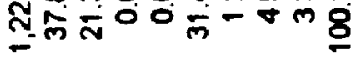
त

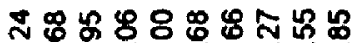
N

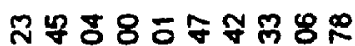
సल्ल

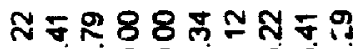

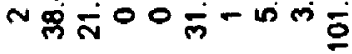

స

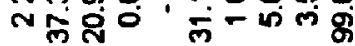

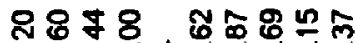

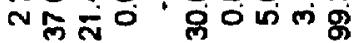

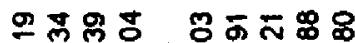

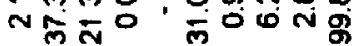

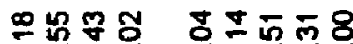

Nลूं

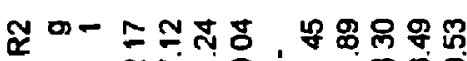

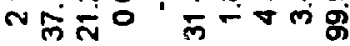

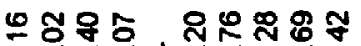

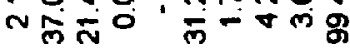

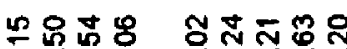

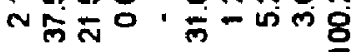

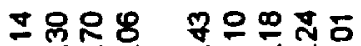

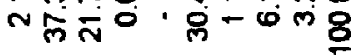

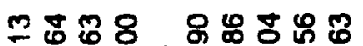

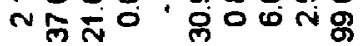

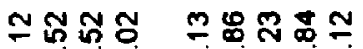

Nलिं

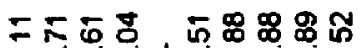

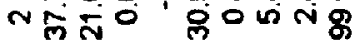

으뭉요응

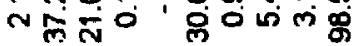

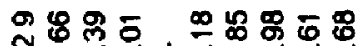

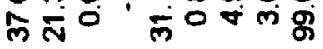

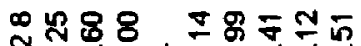

边

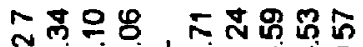

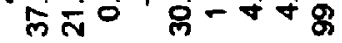

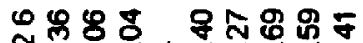

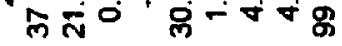

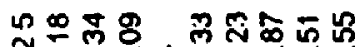

অัّ

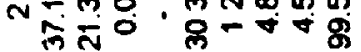

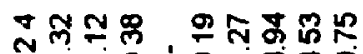

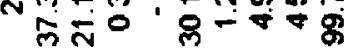

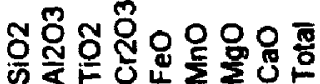

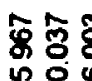

事웅 8

is 0

88

00

多등용

जio

罗舍号

sio

\%영

iํㅇ

芯㗅。

二용요

in 00

豆琞8

is 0

ธิ

ก0 6

กิะ

no 6

ธิกีั

in 0

怘

sio

용용요

no 0

홍용

is 0

ธ๊

no 0

옹융용

in 0

용

iิ 0

象禺右

जi 0

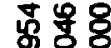

넝

$\overline{\$}$.

is 0

용영

แo 0

กิับํㅇㅇ

nิ응

क
幽䒹

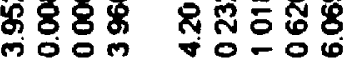

ชิ8 8

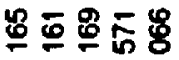

$\checkmark 0-00$

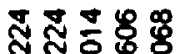

응응

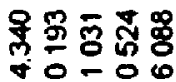

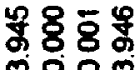

ริ \& \& \%

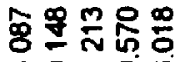

寸o-0.

효용

용

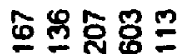

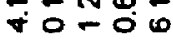

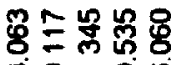

(

ن 0 - 0

용용. 용

吕 충용

$\checkmark 0-00$

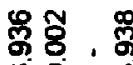

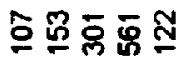

ल $\mathrm{m}$

员罂, 员

\&

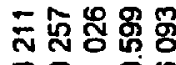

$\checkmark 0-00$

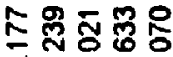

mo $n$ ष०

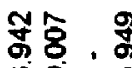

등 충

$\div 0-00$

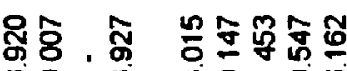

mo $m$

品 80.8

mo

咠쏭

+ $0-00$

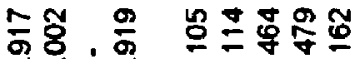

$+0-06$

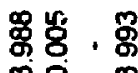

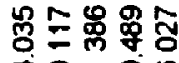

ن 0 - 00

₹ำ. भू⿵

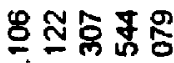

$\checkmark 0-06$

స్ $\overline{8} \cdot \frac{\infty}{6}$

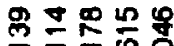

등ㅇㅇ

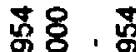

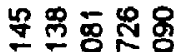

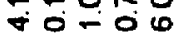

过合

i⿱ 口)

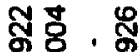

$\checkmark 0-06$

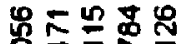

드응

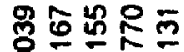

믄응

no

澏岁。兽

등요운전

T $=0 \%$

4 동용

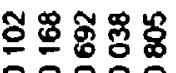

0000

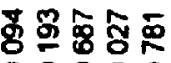

00000

8눙요용

등ㅇㅇ

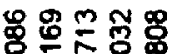

00000

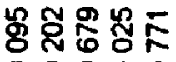

00000

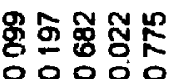

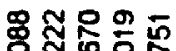

00000

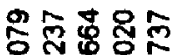

00000

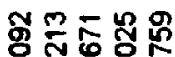

00000

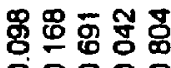

00000

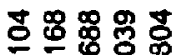

00000

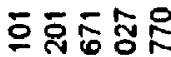

00000

品品芯芯怘

00000

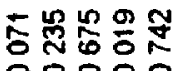

00000

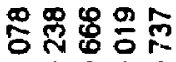

00000

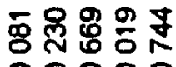

00000

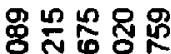

00000

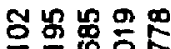

00000

올용영

00000

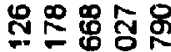

$\circ 0000$

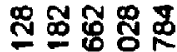

00000

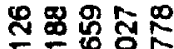

등ㅇㅇㅇ

赵互员颉

응ㅇㅇ

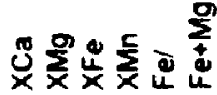




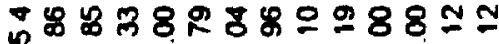

只

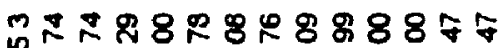

出

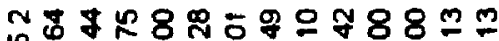

品

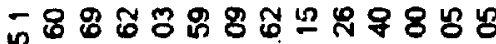

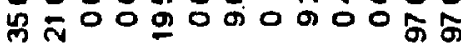

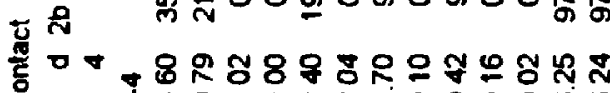

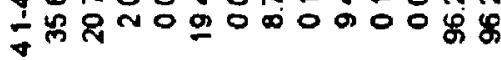

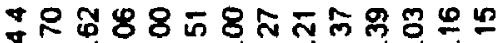

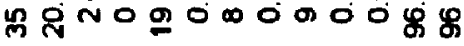

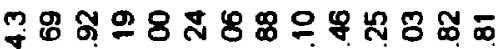

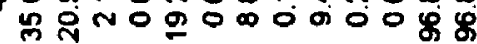

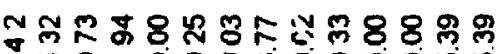

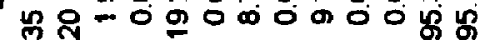

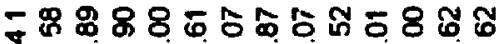

品-

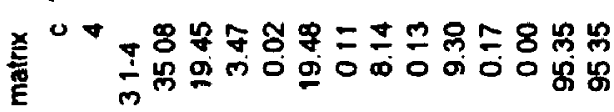

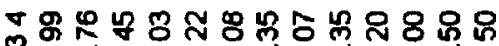

से 200900000 边

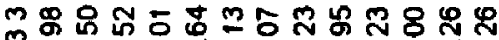

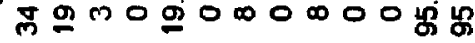

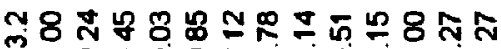

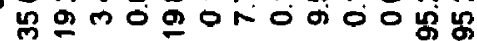

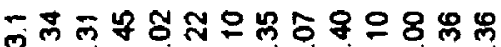

出

崖

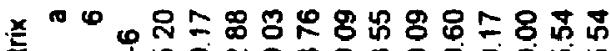

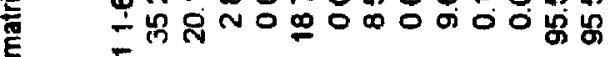

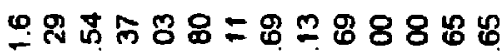

品

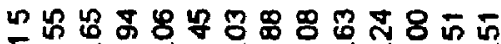

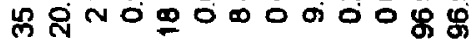

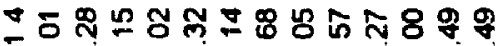

पूर

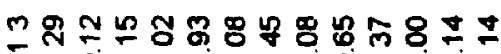

मू प्रं

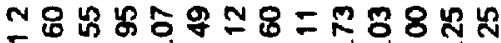

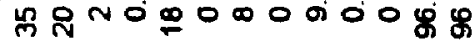

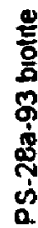

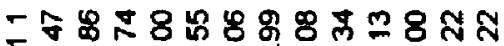

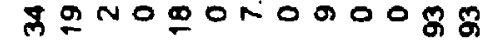

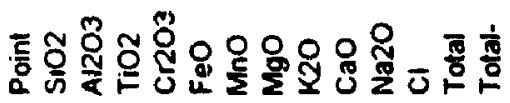

$\frac{0}{98}$

अ 8 \% 8 जิ

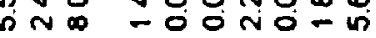

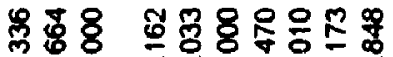

कN

寻盟 8

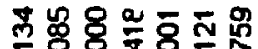

is $\approx$ -

888

in

电范 8

in $\infty$

동ํำ

ஸे

$\mathscr{8} 8$

i

某号客

ถั

888

is $N$

查安 \&

แก

응 8

แn $\mathrm{N} \infty$

\%용

जiํㅇ

ธำ 웅

is

菺 $\overline{8}$

जi $N$

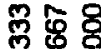

ज方

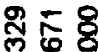

กิ

象迢

แ

도용

ज त

- 88

(1)

용

แ

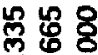

in

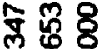

क्षं

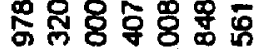

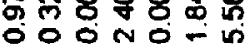

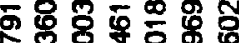

$000 n+0$

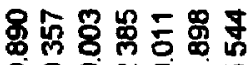

ОOONOT

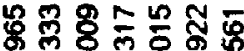

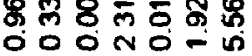

(1)

ธํ⿺ㅇ

-0-

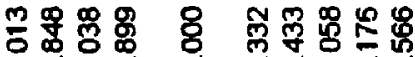

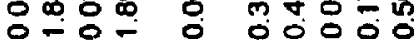

응용용 \&

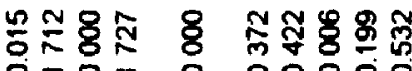

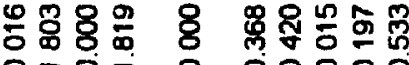

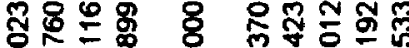

- $0-0$ 00000

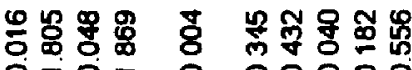

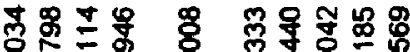

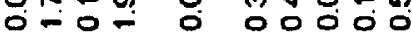

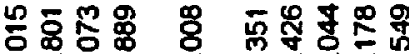

- - - 0 - 0000

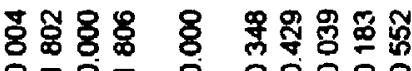

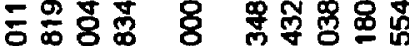

$0-0=0000$

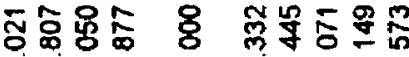

끙응명용

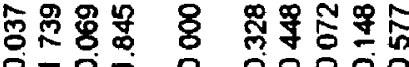

ก

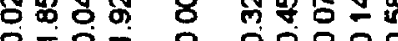

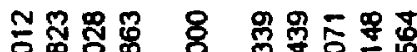

$0-0$ - 00000

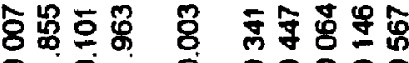

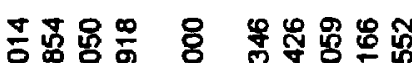

$0-0-000000$

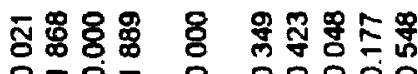

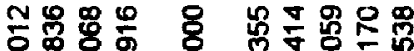

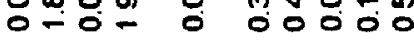

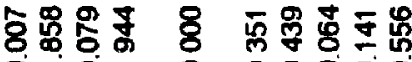

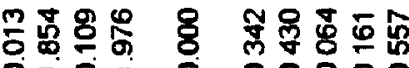

$0-0-000000$

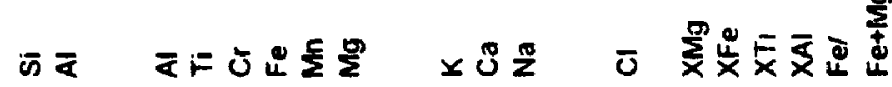




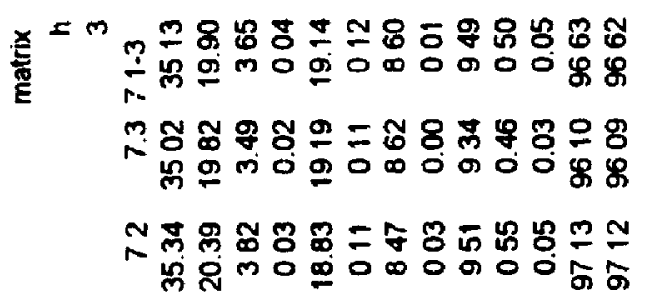

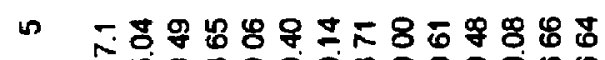

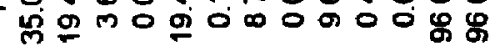

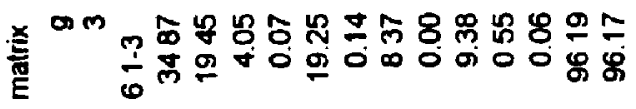

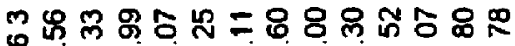

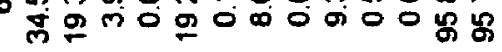

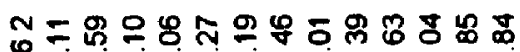

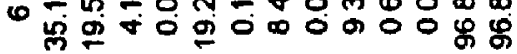

- ธ。ำำ

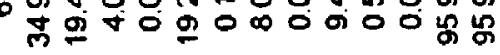

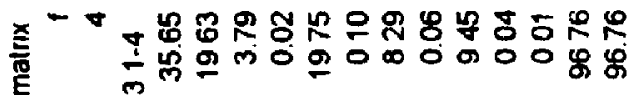

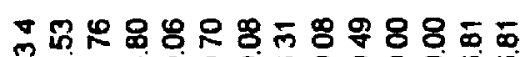

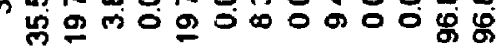

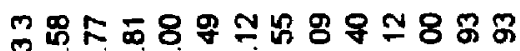

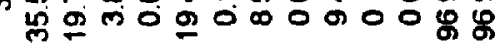

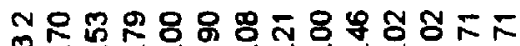

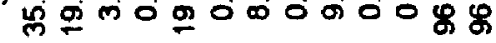

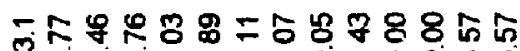

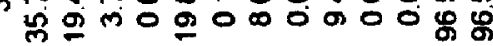

兽

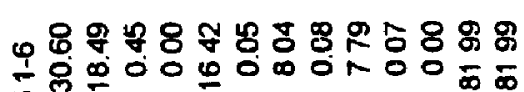

फल का का

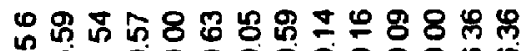

出

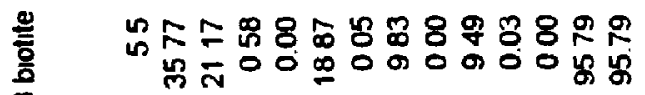

ลกำ

in $N$

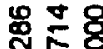

की त

t:

in $N$

满

in $N$

ำ ํํㅇ

जN

号员各

in $N$

兽

in $N$

总 ริㅇㅇㅇㅇ

แ $N$ 嗢

学哭然 ज小 $\infty$

중용

un $N$ 冓

站 : :

in $\mathbb{0}$

总

in $N$

동용

i

융 贸害

$\rightarrow N \infty$

శ్లై 용

is $N$

象苋各 융

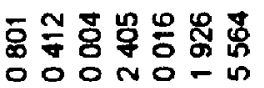

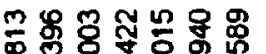

क哭

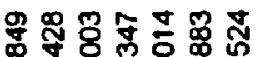

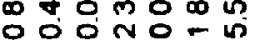

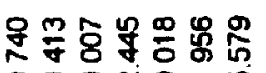

OO ONO-N

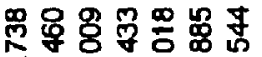

OOONO-m

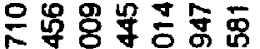

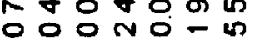

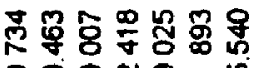

OOONO-

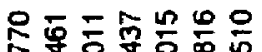

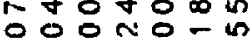

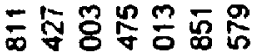
0

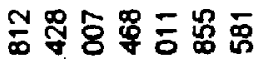
OOONO- in

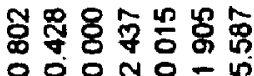

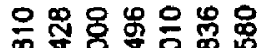

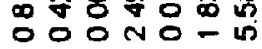

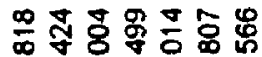
OÓn

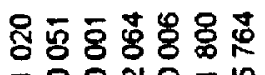

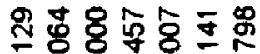
$=00 \mathrm{n}$ 的

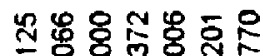
- OONON in

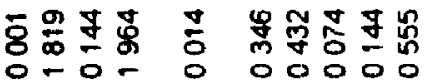

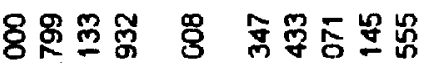
O-0- 0 00000

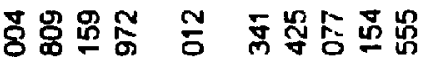
0-0- 000000

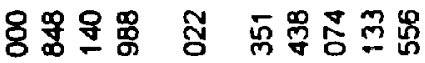
$0-0-000000$

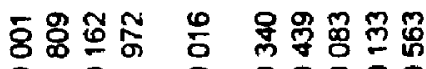
О-0- 0 00000

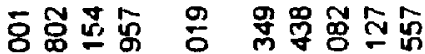
0-0- 0 00000

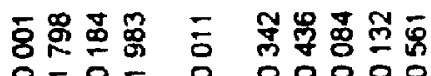

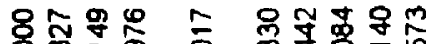

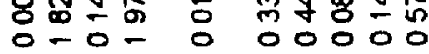

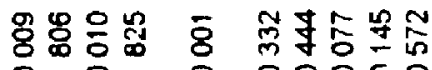

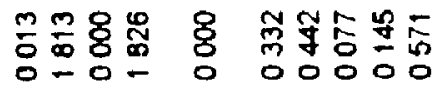

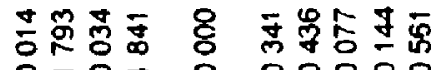
O- - 0 00000

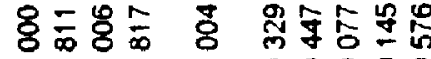
0-0- 0 00000

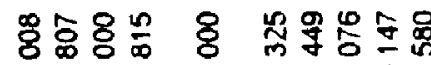
0-0- 000000

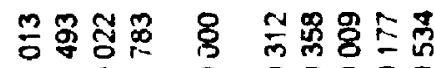
$0-0-000000$

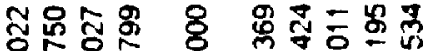
등응 영응영

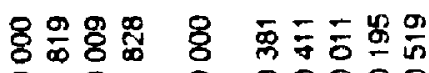
0 - 0 - 000000

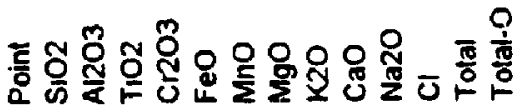

कंष

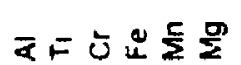

$x \pi$

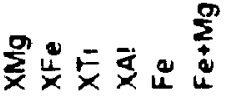




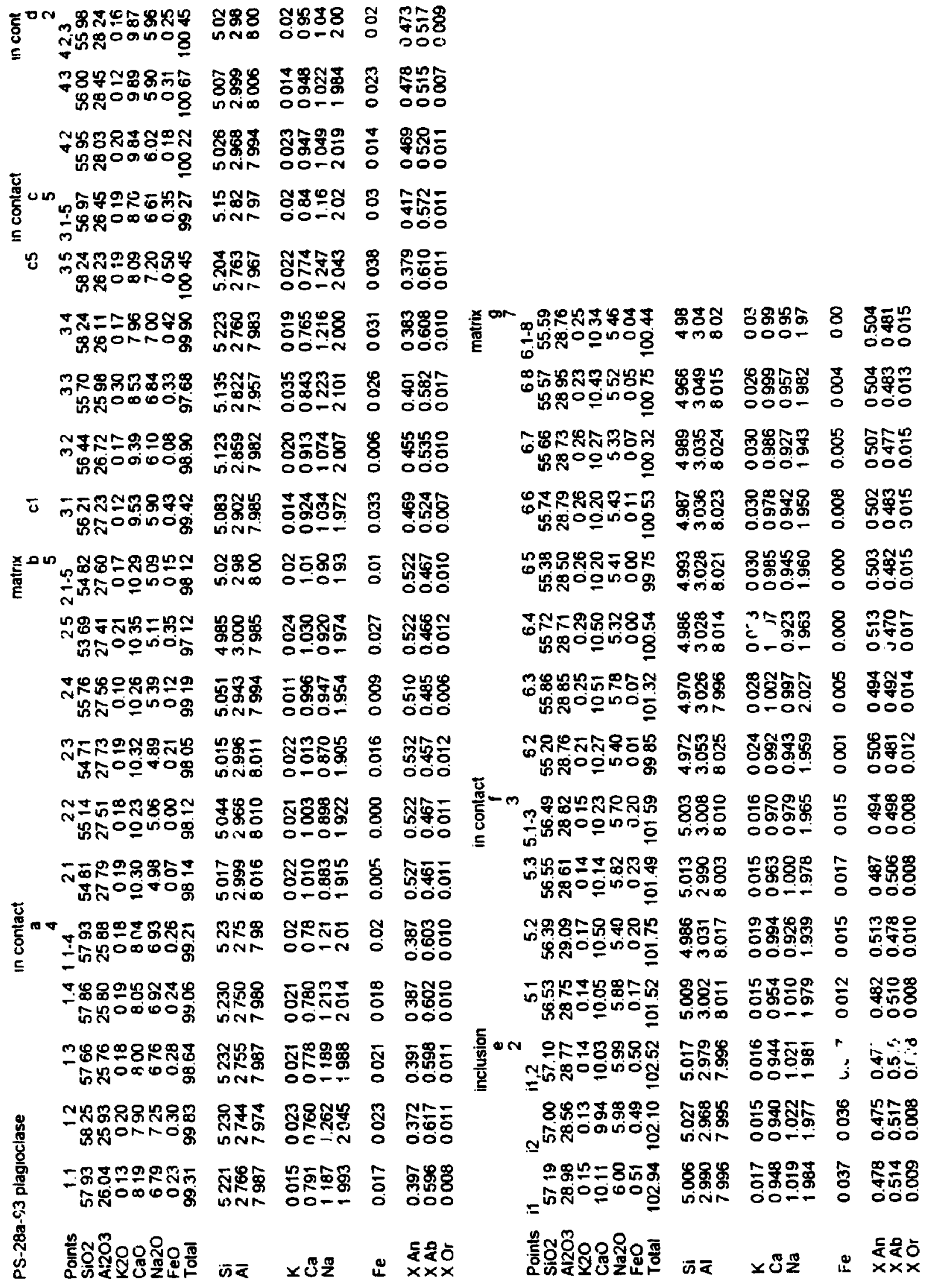




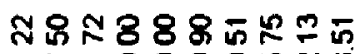

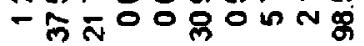

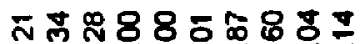

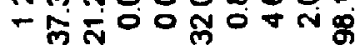

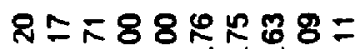

- 罗

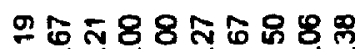

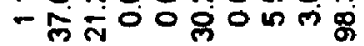

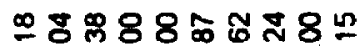

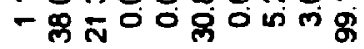

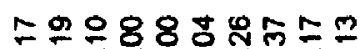

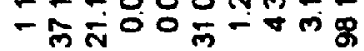

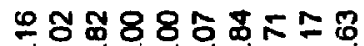

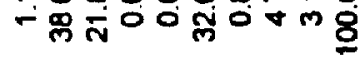

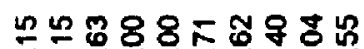

- 每

士9

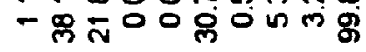

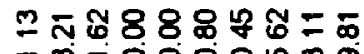

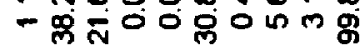

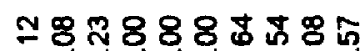

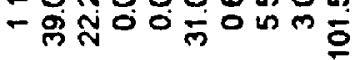

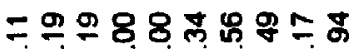

= बूं

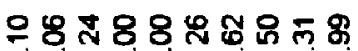

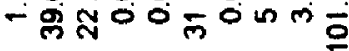

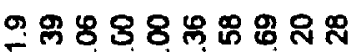

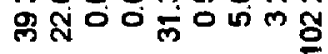

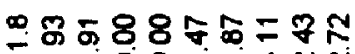

需穴०

₹䑻 $588 \%$ 웅

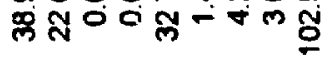

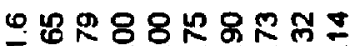

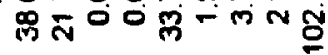

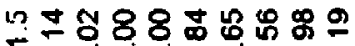

只

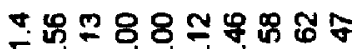

网

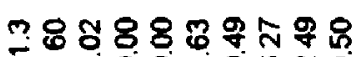

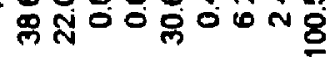

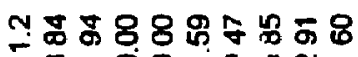

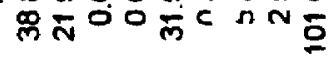

$\therefore$ ะ

ติ

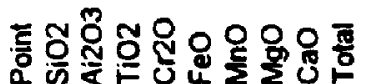

중

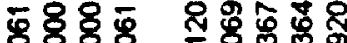

no०

\&్రి용염

600

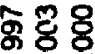

no

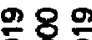

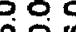

通号骂

000

\& \& \&

600

要웅용

ஸㅇㅇㅇ

్ํ융

000

ธิ8े

000

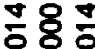

유.

융용

000

\&:

600

ํㅜㅇ용 웅

000

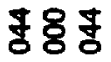

000

중용

00

웅용

공용

000

용 8

000

ิํㅇํำ 응

888 006

88

000

ㅎํㅇㅎㅎㅎ

क

궁웜

$588 \mathrm{~A}$

잉음

啘8 8

moom

용용

moom

용요

응ㅇ

\%ั8

400

ํㅐㅇ웅용

$40 \%$

988

004

응웅웅음

잉영

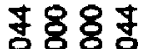

408

윔웅웅울

잉영

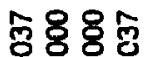

404

용요요

món

훙흏ㅎㅁ

$\checkmark 00 \mathrm{~V}$

훙영형

훙용

ช००

然客客兽

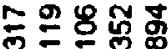

$70=0 \mathrm{0}$

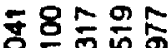

+0-0

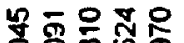

ro-0

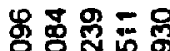

ro-0

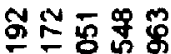

उo- 0 in

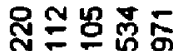

으응

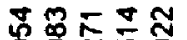

그음

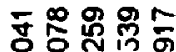

ㄱㅇㄱㄷํ

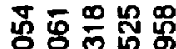

ro-0

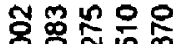

긍ㄷำ

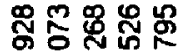

กํㅇำ

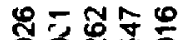

영 동

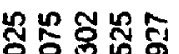

To-0w

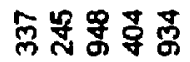

พ000

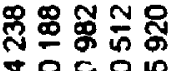

○com

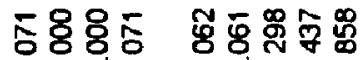

단

To-on

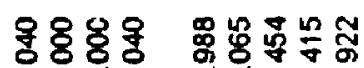



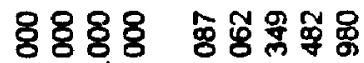

अ०त4

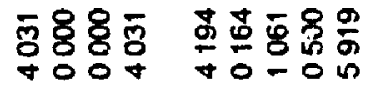

द्र

นั $\overline{8} \overline{2} \frac{8}{8} \overline{0}$

00000

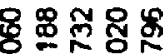

00000

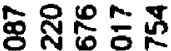

응ำ

象을

00000

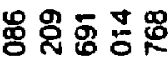

00000

กำำ

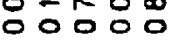

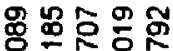

00000

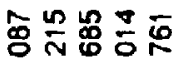

0000

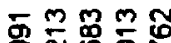

00000

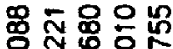

00000

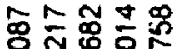

00000

옹 옹

00000

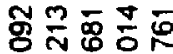

00000

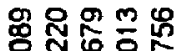

00000

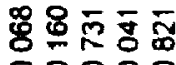

00000

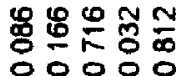

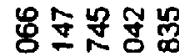

응ㅇㅇ응

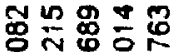

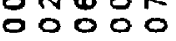

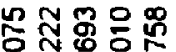

00000

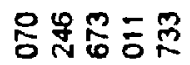

00000

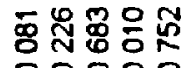

00000

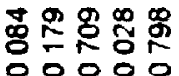

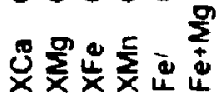




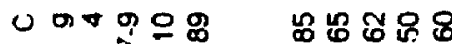
लंg

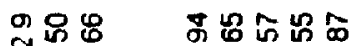
㝦的. '

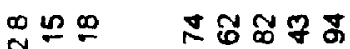

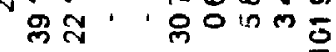

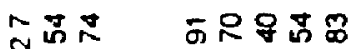

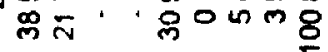
$\mathscr{N}=9$ 品

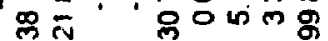

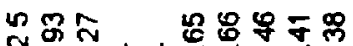

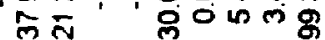

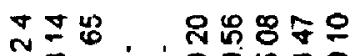

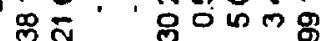
융 零'

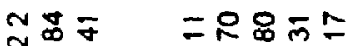
眾

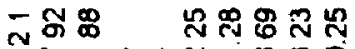

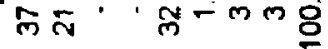

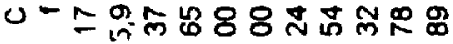

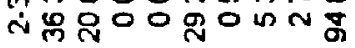

- แ

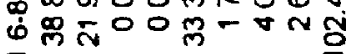

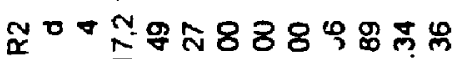
-

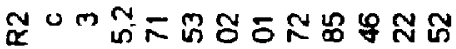
=网

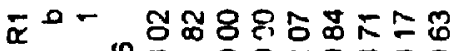

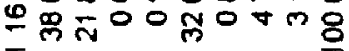
反 $\pi-$ -

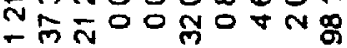

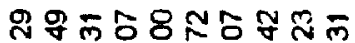

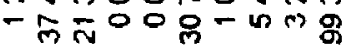

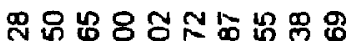
- 的空 00 잉

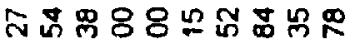

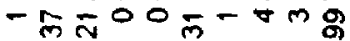

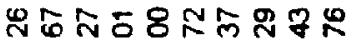

- ले त̄ O

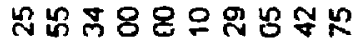

- लिं

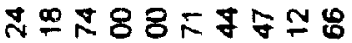

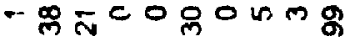

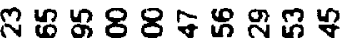

- M

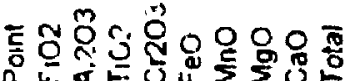

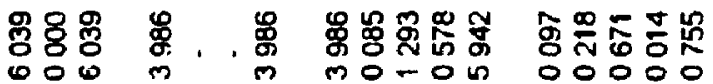

兽总

$00: 0$

\%ัฒ.

10048

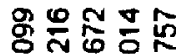
mo-co 00000

융 $\frac{8}{0} \frac{\pi}{0}$

尽. .

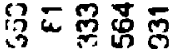

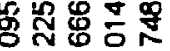

m

106

兽 ,

苟密婴号

- o- 0 in

동웅웅

$\omega 00$

응 웅응

606

용 용 용

006

형웅형

006

용 용

용.

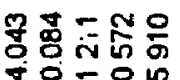

䒺, 恋

옹영윤용

- 0 - 00

₹. . छ

용요ㅇㅛㅛ

m- 0 is

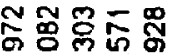

mo-o in

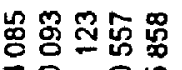

จ 0 - 0 in

용용

芯

กำ类

$\forall 000$ in

8 용

in 0

용 006

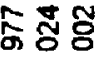

जo 0

品要

แ० 0

훙용

in 0 ०

్ํㅇ융융

000

界骂 ร马

in 0

苟安

nᄋㅇㅇㅇ

号望

us 30

꽁웅

in 0

옳융융 ్ㅗㅇ m0 0 요

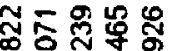

mo잉

웅웅웅형 $\checkmark 00 \%$

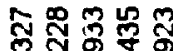
प0 00 นn

융용용 m००

适 8 io $0 \mathrm{~m}$

웡옹웅్ㅕㅇ $\checkmark 00$.

형용영

०००

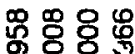

moon

용용 요

लㅇㅇㅇ영

웅궁용요 mo

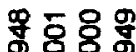
mo $0 \mathrm{~m}$

号勇号

w 50

융용요용

m $00 \mathrm{~m}$

능 $\frac{5}{5}$

006

용응

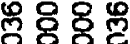

$\checkmark 00 \%$

용용 శ్

ल $00 \mathrm{~m}$

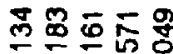

प 0 - 06

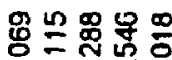

प0 - 0

웛느ㅇㅛㅛ

प 0 - 0 in

동으용

จ0-0 a

要吉员员

ن 0 - 0

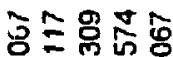

女 0 -

용요용

- 0

농윰용

จ

을쏭용

४ $0=06$

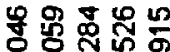

$\checkmark 0-0$ i

농유ㅇㅝㅛ용

以 0

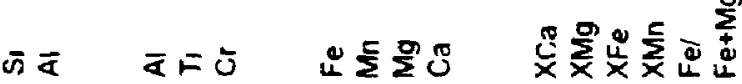

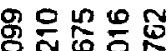

00000

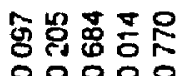

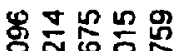

00000

으ㅇㅛㅠ용

00000

发융뭉

00000

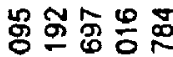

00000

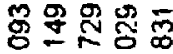

00000

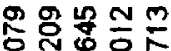
00000

옹 00000

芦 今ั 00000

可六象号号 00000

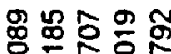
00000

ஜฒㅛ용 OOC.OO

各尔志芯 00000

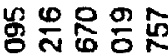
잉응

형요용용 - jo0

号뭉용융요 00000

녕 옹 옹 둥ㅇㅇ

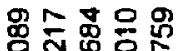
ㅇํㅇㅇㅇㅇㅇㅇ

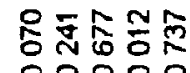

00000 


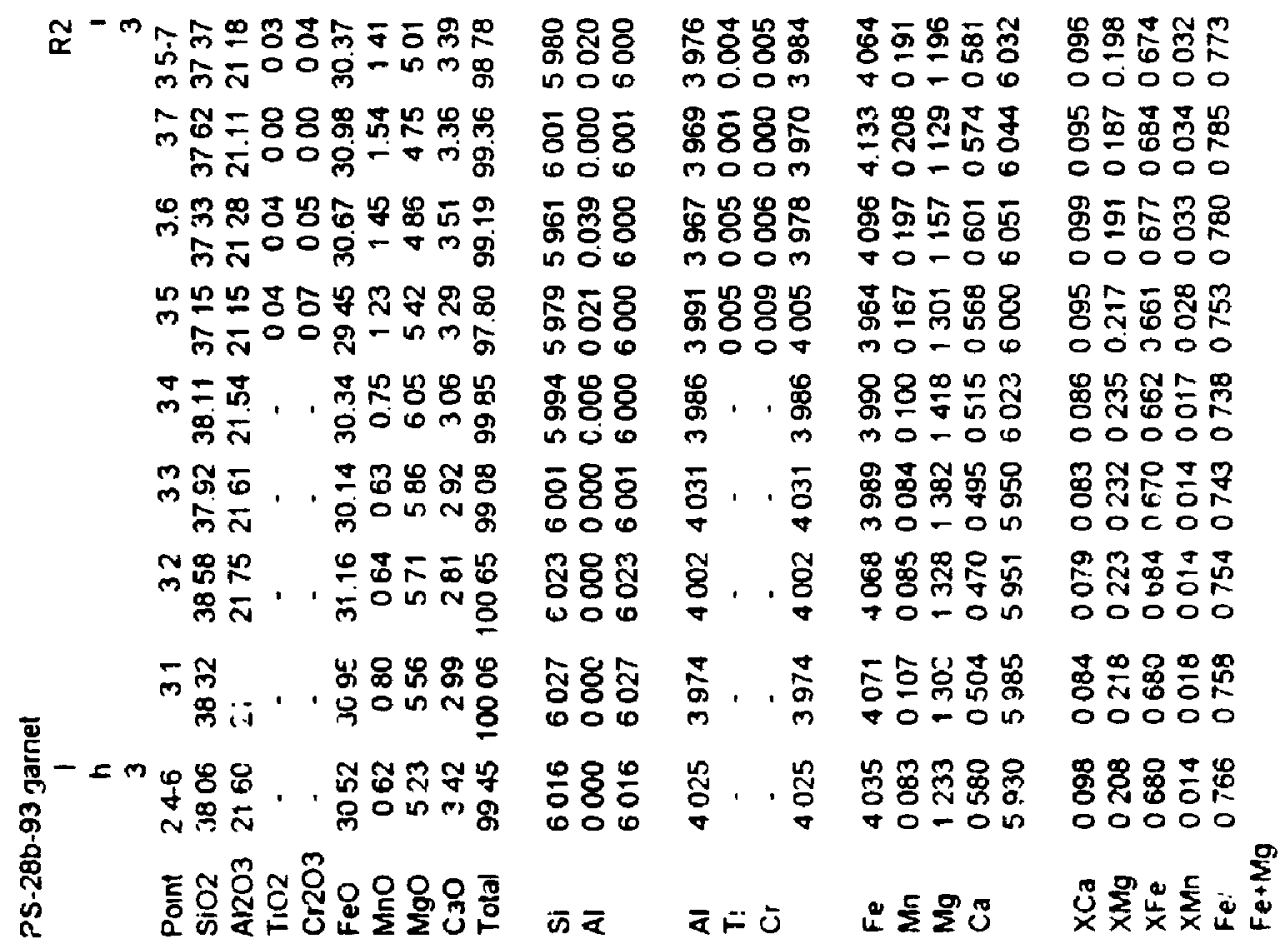




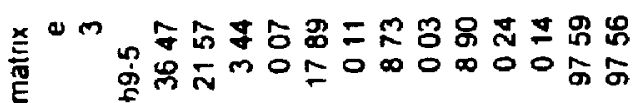

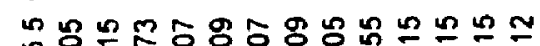

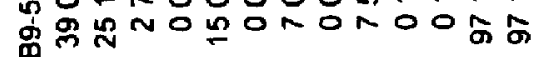

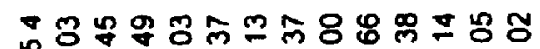

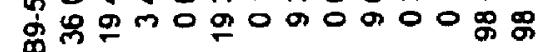

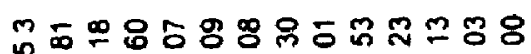

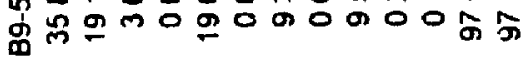
N

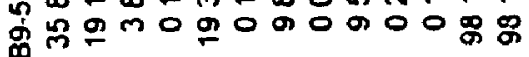

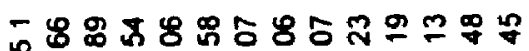

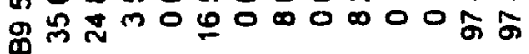

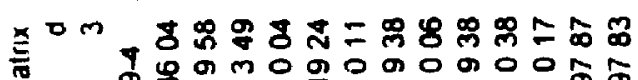

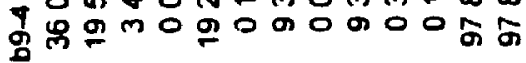

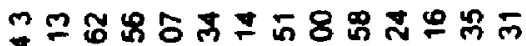

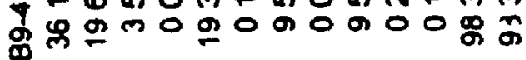

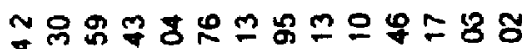

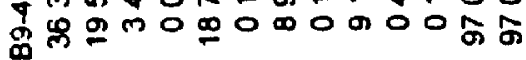

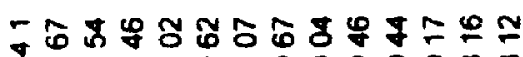

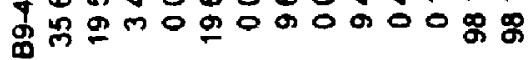

今ે in

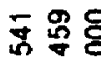
시 政: 川 $\infty$ 5 in

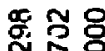
ind

总最客 i $N \infty$

啹需号 in $\sim \infty$

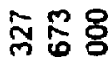
ก $N$

馬 in $\infty$

퐁용 in $\sim \infty$

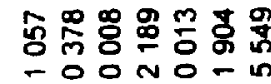

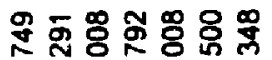

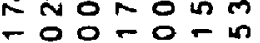
공용영둥옹응 $000 \mathrm{NON}$

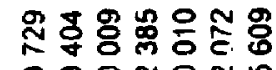
OO OnO N in

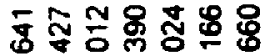
OOOONON 象总 - 0 O N 0 -

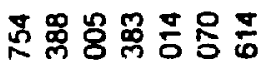
OOONON

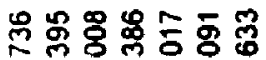

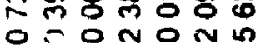

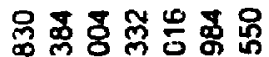
융융요

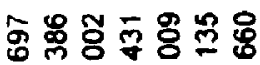
OOONON
늉용용

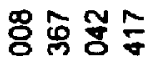
$0-0-$

융 융용 O-O용요요용 응홍ㅇㅇㅇ ㄷ-

巨大용 0-0용용옹 웅 용 罗吊 - - 0 -

엉 ్ㅠ욣 응发怘哭

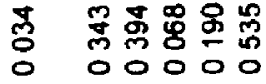

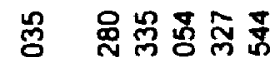
กิ०0

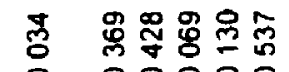
- 00000

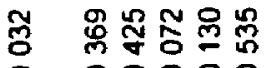
- 00000

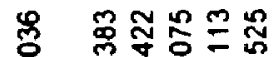
- 00000

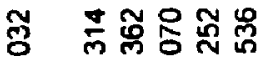
00000

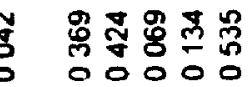

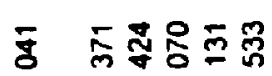
$\circ$ ०००000

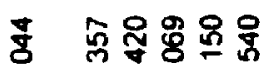
- 00000

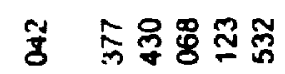
०0000 


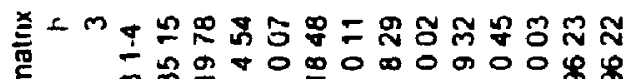
的-

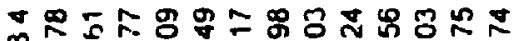

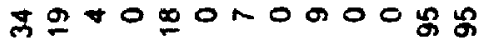

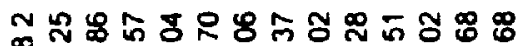
约田

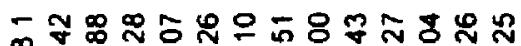

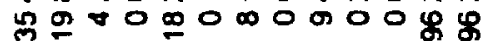

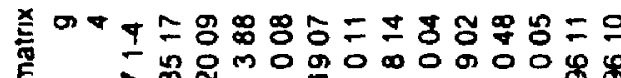

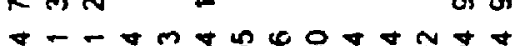

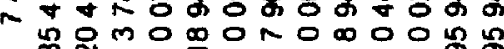

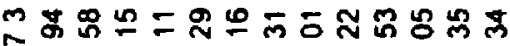

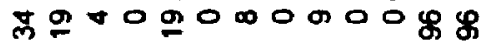

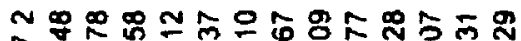

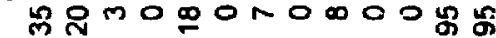

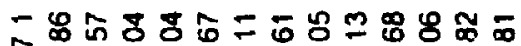

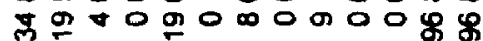

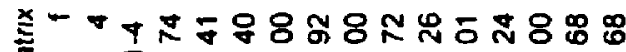

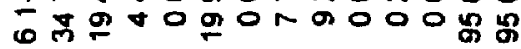

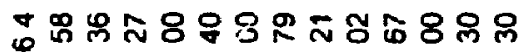

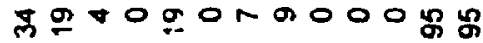

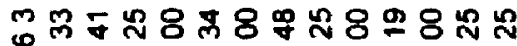
范田

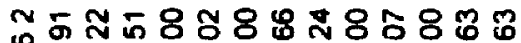

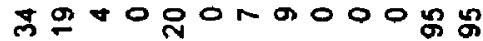

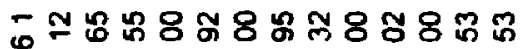
出

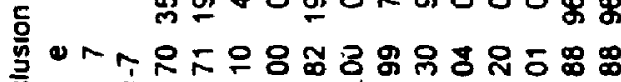
的节 $090 \% 0000 \%$

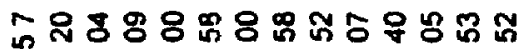
号穴

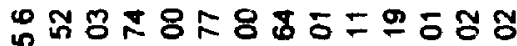

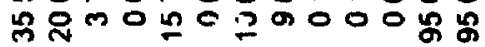

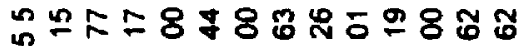
只 $900000000 \%$

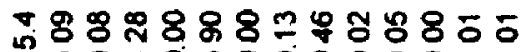

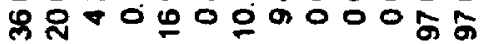

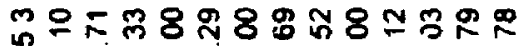

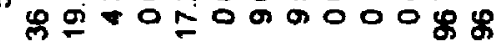

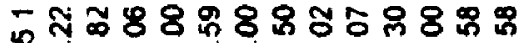
సं ำกำธ

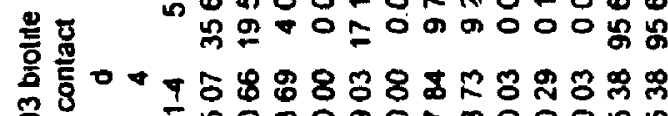

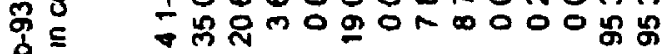
总

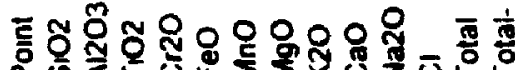

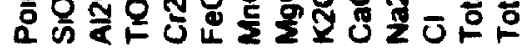

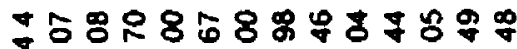

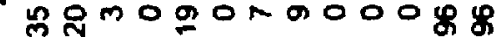

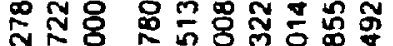
กิก 娄市象 in $N$

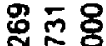
ก N 领各 un $N$ 负 웅 แก $N$ 용ㅇㅇ in

鱼苍 in $N \infty$

जे กㄴ 웅ㅇㅇㅇ in $N \infty$

需是员 ก $N$

№ ก

品 $\approx 8$ Nñ⿻ 芦 용 \& in $N$ क 可采品 in $N \infty$ ฟู่ n $N$

స్ำ in $\infty$

용 8 n N

斥 in $\infty$ ำ: n $N$ 구용ㅇㅇ

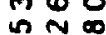

ㅇ户용ㅇㅇ in $N$

奠 in

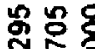
n $\infty$

$\bar{n} \bar{x}$

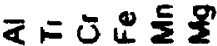

空市員 n $\infty$

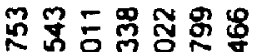
OOONO-

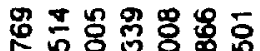
응 은

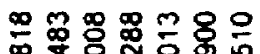

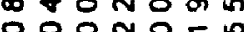

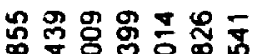
O O ONO-

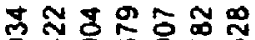
000N0-

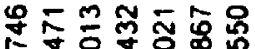
OOOONO-

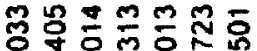
음으 -

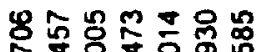
000 인

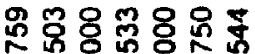
OOONO- n

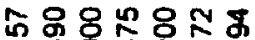
000 No-

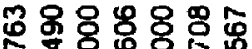
o o ono-n

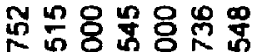
OOONO- -

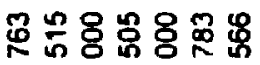
oOONOF ธ。 O OOONON

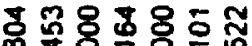
O0№n

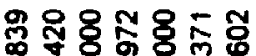
$00000 \mathrm{~m}$

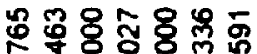
OOONON Wn

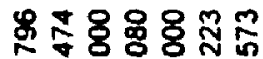
000 non ळ : \% OOONON

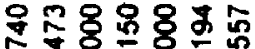
OOONON

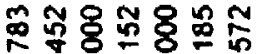
OOONON

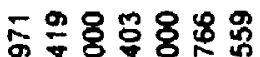
$000 \mathrm{NO}$ क웅 O잉ㅁㅇ

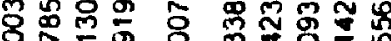
0-0- 0 00000

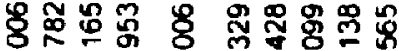
O-0응 00000

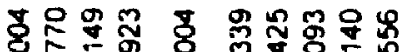
O-0- 000000

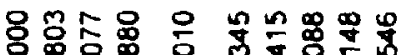
0 - 0 - 000000 岁只王总 0-0 - 000000

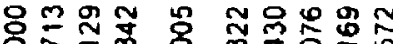

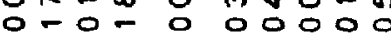

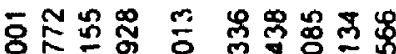
0-5응 00000

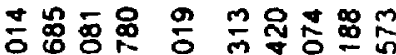
0-0- 0 o 0000

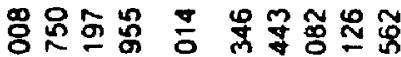
$0-0-000000$

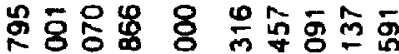
- $00-000000$

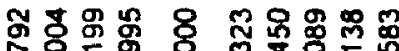
- $00-000000$

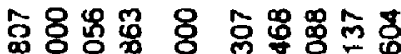
-

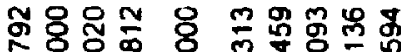
- $00-0$ - 00000

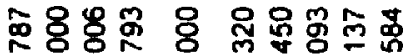
- $00-000000$

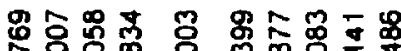
- $00-000000$

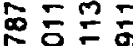
- $00-$ 옹혀용 -00 -

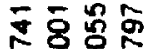
-00칭응 - 00 -

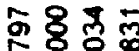
- 00 -

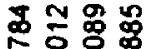
- 0 a -

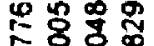
- 0 0 -

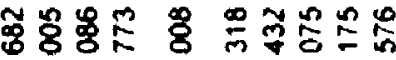
-.00- 000000

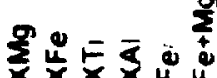

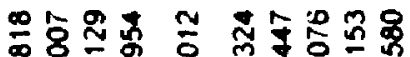
- $00-0$ - 00000 


\section{突 N

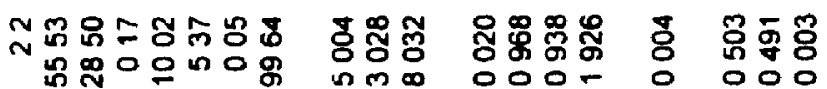

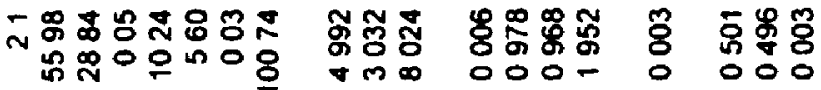

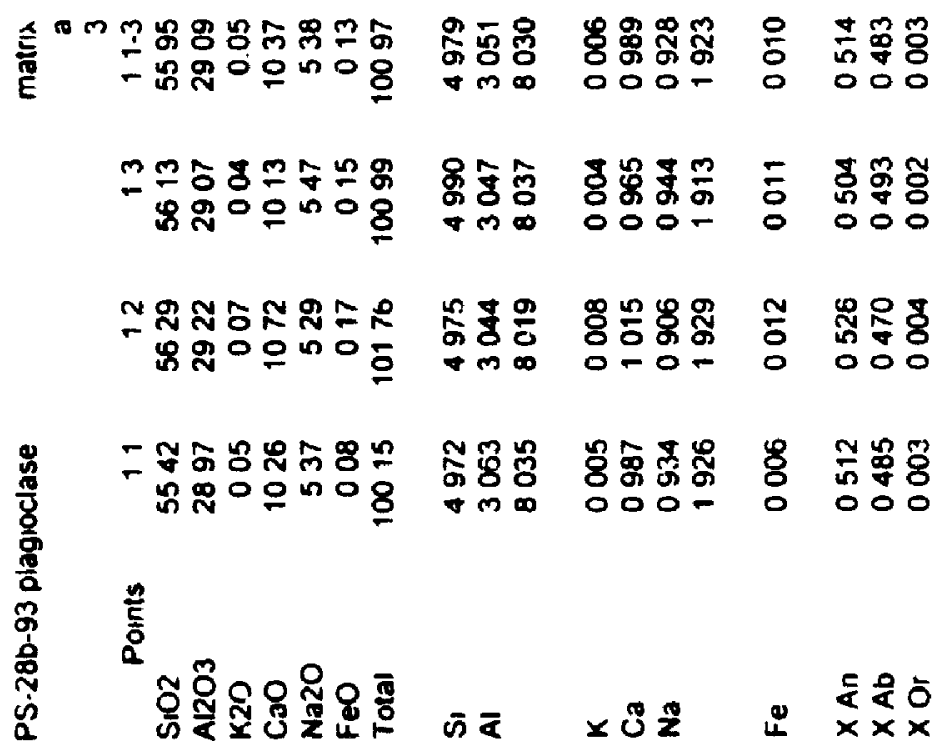




\begin{tabular}{|c|c|c|c|}
\hline 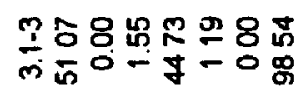 & 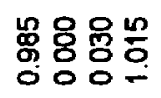 & 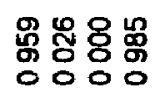 & 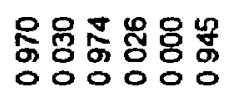 \\
\hline 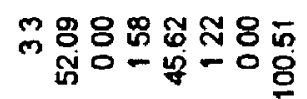 & & & 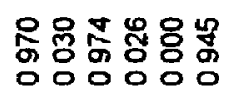 \\
\hline 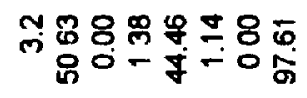 & \& & $\mathbb{Z}$ & 5용용 \\
\hline 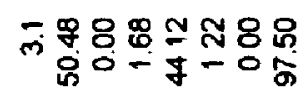 & $\tilde{\sigma}$ & 㔛 & \\
\hline
\end{tabular}

\begin{tabular}{|c|c|c|c|}
\hline $0 \div 8=08$ & $\frac{2}{5}$ & 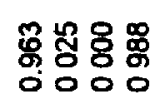 & $\begin{array}{l}\text { g } \\
0\end{array}$ \\
\hline $80-7$ & & & \\
\hline $80-7-$ & & & \\
\hline $\begin{array}{l} \pm 8 \approx 5= \\
\frac{1}{6} 0 \div \frac{5}{7}\end{array}$ & & & \\
\hline
\end{tabular}

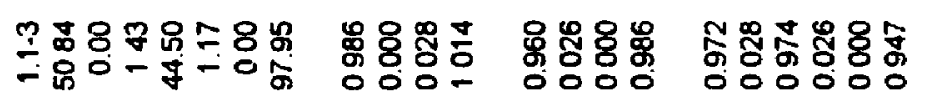

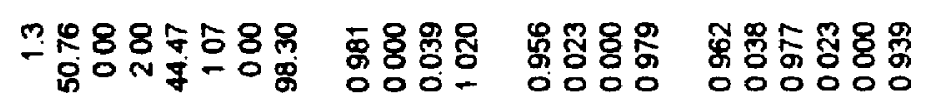

\begin{tabular}{|c|c|c|c|c|}
\hline & 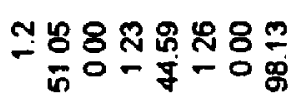 & 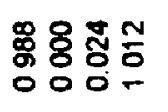 & 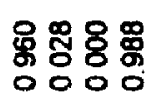 & 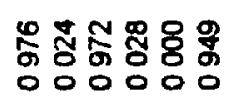 \\
\hline & $=\pi 85798=$ & 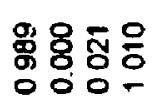 & 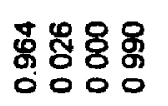 & 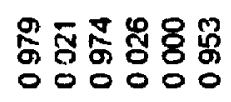 \\
\hline & 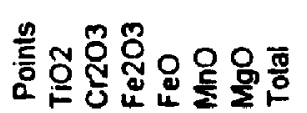 & F心 & 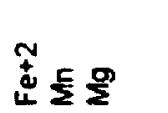 & \\
\hline
\end{tabular}




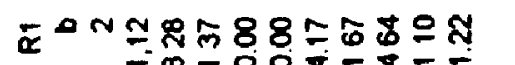

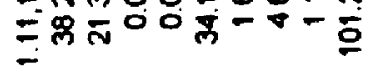

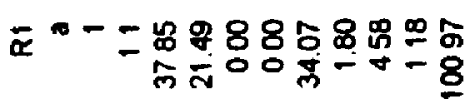

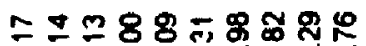

- कित 으윰유.

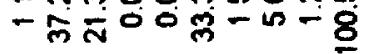

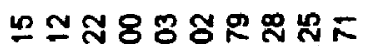

-

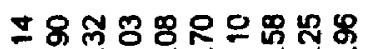

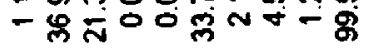

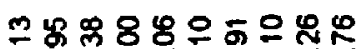

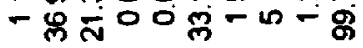

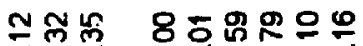

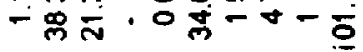

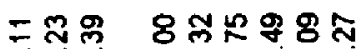

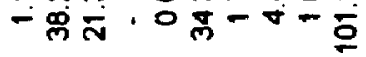

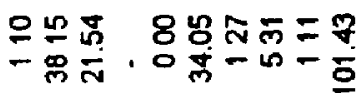

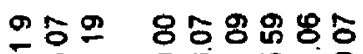

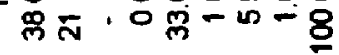

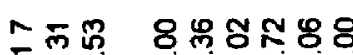

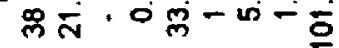

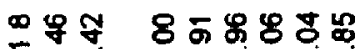

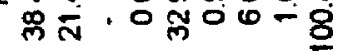

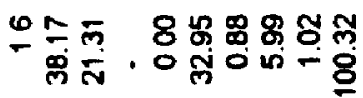

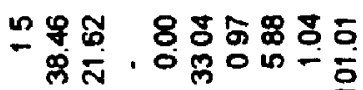

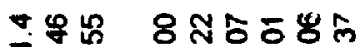

品

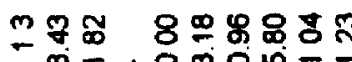

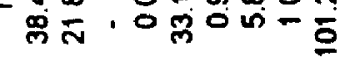

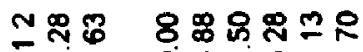

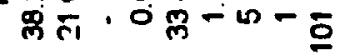

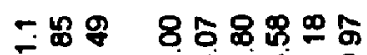

欲・嗵-4-8

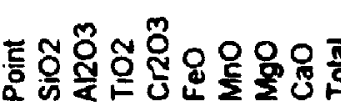

कृष

응

ஐ응

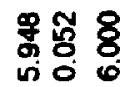

业要

जo 6

声蔗 8

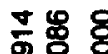

응

형융 웅

ก0

5용 흥

60

홍융

刕言兽

잉융 : ్ㅠㅇ

88

○. 0

₹

000

훙융

000

동융

훙용

挅亩

芯柿

炙

no

必 88

moom

问 $8=9$

강흉

낭 중ํํำ

ल००

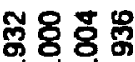

लि0

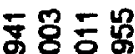

ल잉

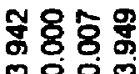

逭

సิ.

.

今.

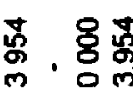

怘.

㞼.

弯, 各另

就

ลิ. 8 ธิธ जi०

m.

惫, 啰杰

\%

喝

(8)

券. 8 \%

m.

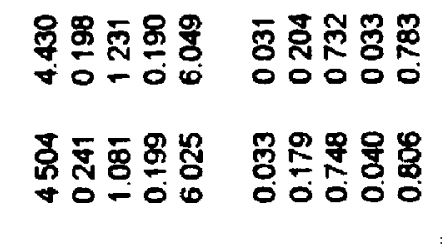

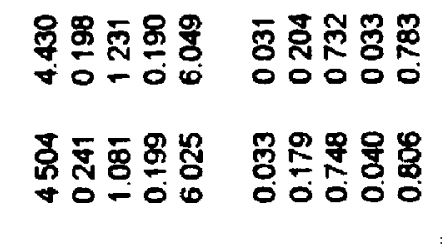

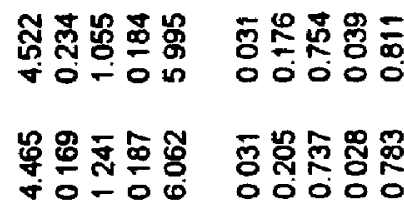

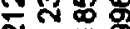

\% 0 - 0 i

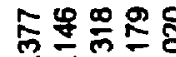

$\checkmark 0-0$

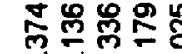

象产告起

\% 0

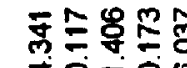

윰종종

$\forall 0-0$ us

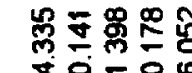

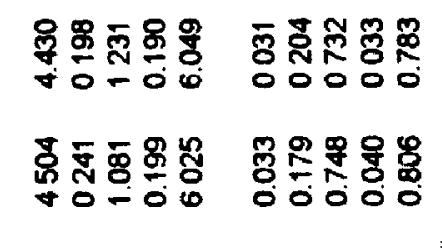

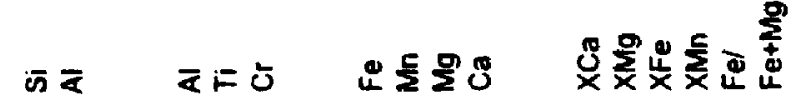


ニ

N命. 0 ภु०

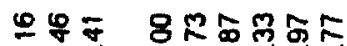

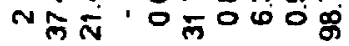

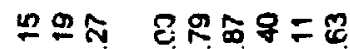

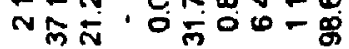

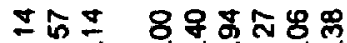

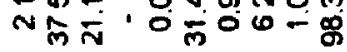

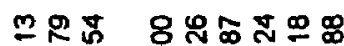

ำกㄴ.

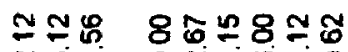

N命, O

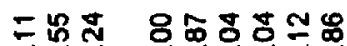

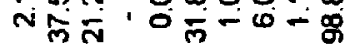

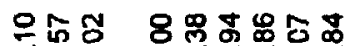

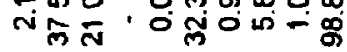

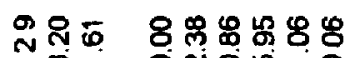

需, 0

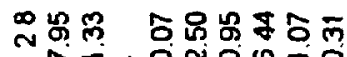

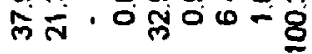

กิธุ

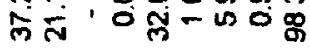

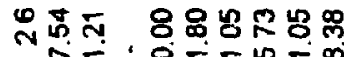

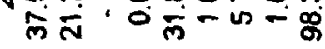

饮苜

लें

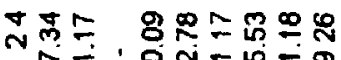

लेंत्र तु-以 -

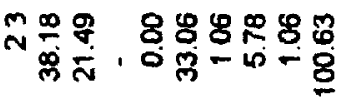

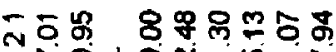

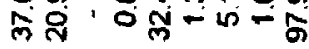

กัญ

满六, O

U

空

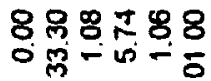

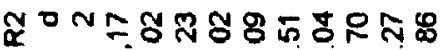

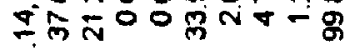

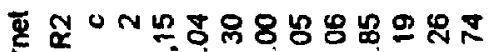

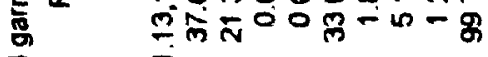

\$

$\frac{1}{\varphi}$

ç

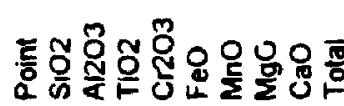

卷 $\overline{8} 8$

ถู่ิ

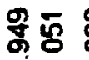

no 6

88

œ 0

皮白

ஸ 0

兽产

\%̊ํㅇㅇ

iि 0

$8 \%$

00

흉용

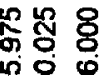

क्षे 웅

둥용

웅영

00

悉高

8

용응

สํํㅇ

600

$\sum_{0} 8$

홍용

no 0

ชู่禺

ㅇํㅇ
\% 8 \%

(8)

总. 잉 兽

昌. 8 용

$90 \mathrm{~m}$

惫, 8

옹. 8

m. ठठ

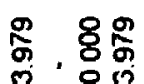

离. 8 8.

ก 0 m

路.

$\overline{0}, 8 \overline{0} \overline{0}$

总 8 音

. .

落.

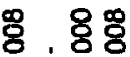

$+0$

总. 8

0 om

昫. 홍용

\% $8 \%$

. $0 \mathrm{~m}$

总.

\%, 需

.

5.

ल.

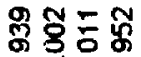
ल००

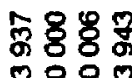

速尽粂庹司

- -06

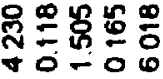

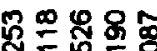

-

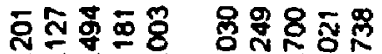
प0 -06 0000

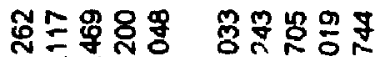
ช0.00 00000

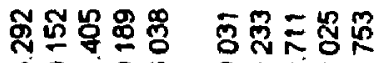
प0-00 00000

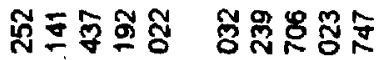
Т०-00 00000

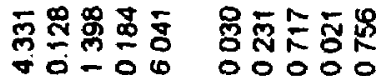

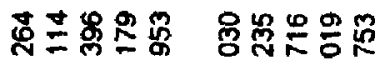

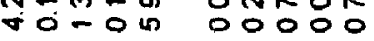

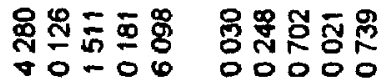

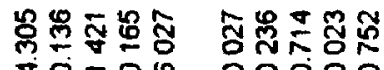
(1)

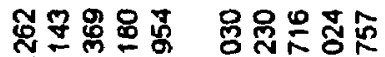

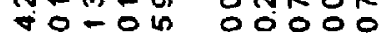

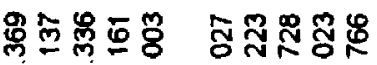
- 0 -O 00000

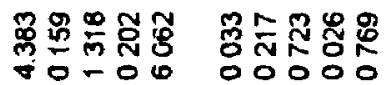

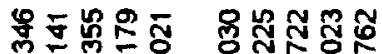
ช O-

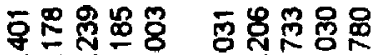
+0-00 0000

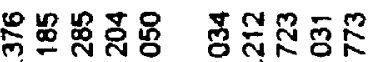
- 0-00 00000

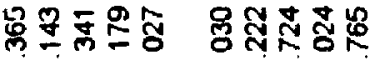

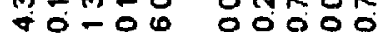

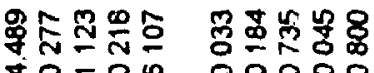

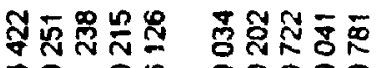
०0-0600000

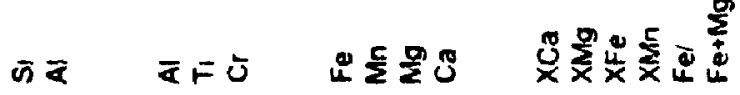




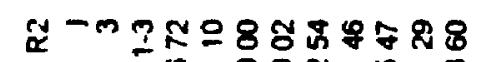

-

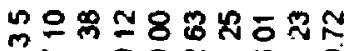

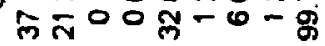

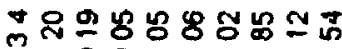

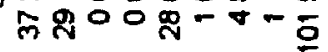

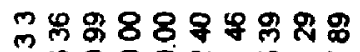

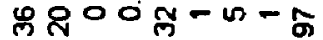

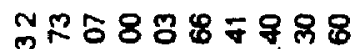

边

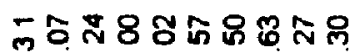

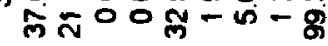

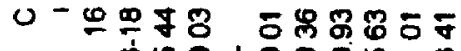

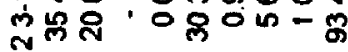

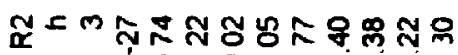
官号 i

$\bar{x}^{\circ-8}$

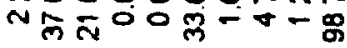

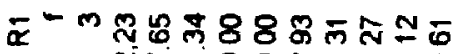
สูก

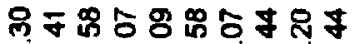

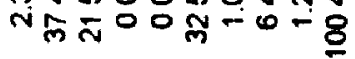

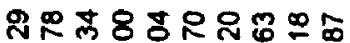
N留OO

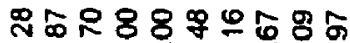

N品

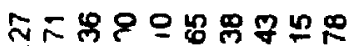

ํㅐㅇㄱ

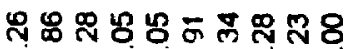

N品 స 0 लै

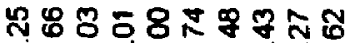

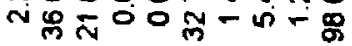

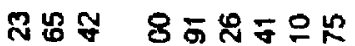

त的.

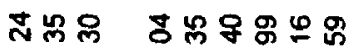

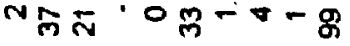

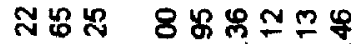

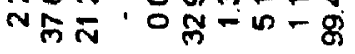

สิ8 8 8

พริ

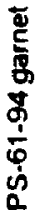

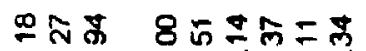

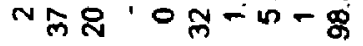

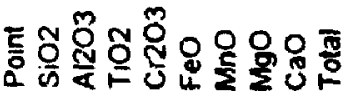

ัㅇㅇ용



发旁 8

융

แn 0

잉잉

$\checkmark 00 \forall$

푱

in 0

乐

응ㅇㅇㅇ

\%ั.

nio 0

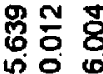

용 8

웅

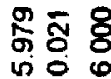

多 ¿̊ ఫ

in

\% 88

递客

ल.

융 8 욯

ल००

声.$\overline{8} \frac{9}{\sigma}$

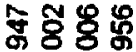

को०

兽

लं०

\%

ल 0 \%

马ㅇㅀㅇ ర్రి

is 0

费贯 동

ज००

웅영 웅

궁

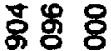

is 0

웅

ii

芯里 8

is 6

ธิธ

जि 0

쟁융농형

लि०

용용용용

융웅응

응용

号号曹怘

ल0

충ㅎㅇㅇㅇㅇㅇ

ल0

范응

in 0

惫. 옹另

옹융 웅

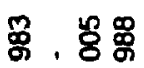

100 0 om

양ㅇㅇ 웅

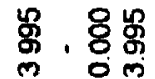

용ㅈำ

ज० 0 क

$88 \%$

00 ल

कंष बहப
-

- O-O O

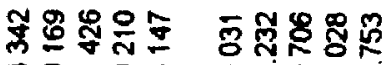

उ०-06 00000

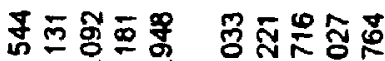

mo-0 영영

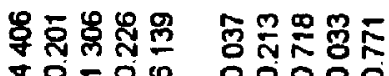

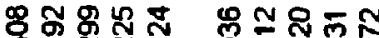

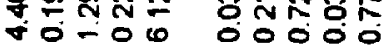

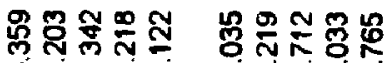

- O-O 00000

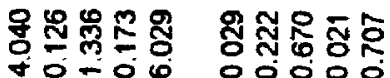

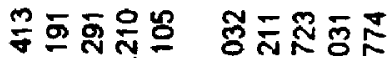
O-O

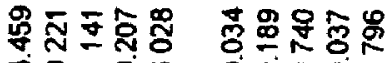
ช0-00 0000

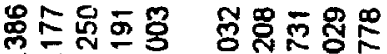
प으응

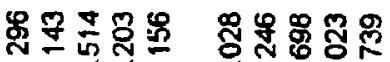
\%

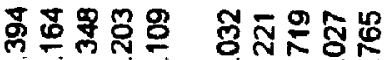
- ODO

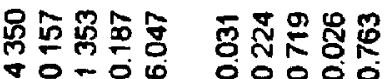

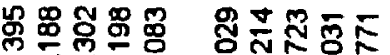

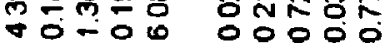

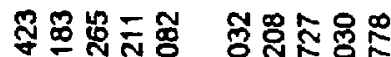
$\checkmark 0-0 \% 0000$

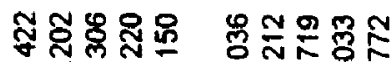
$\checkmark 0$-00 00000

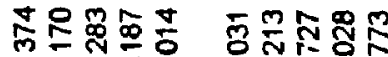

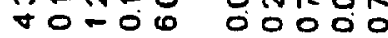

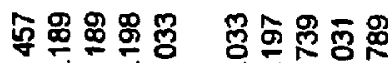
ง

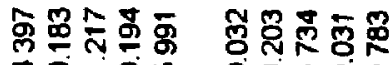

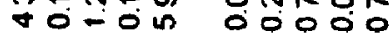

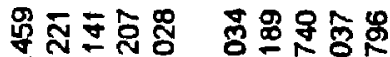
$\checkmark 0=06$ O50ㅇํㅇ

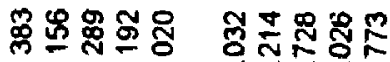

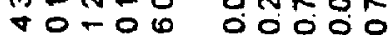

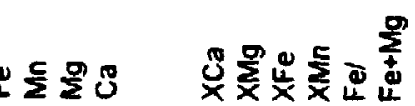




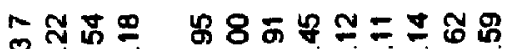

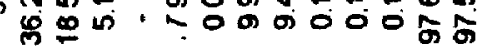

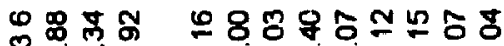

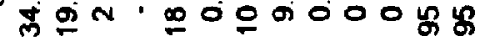

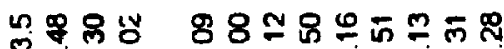

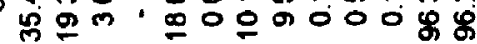

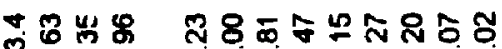

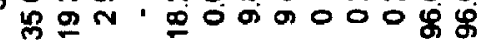

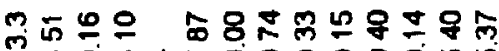

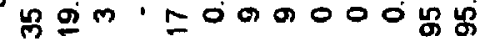

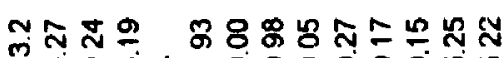

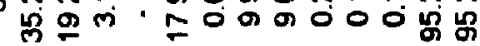

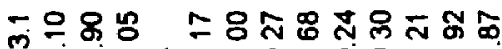

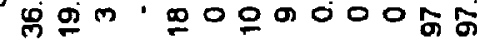

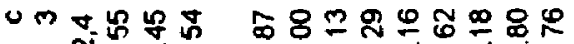
ปู่

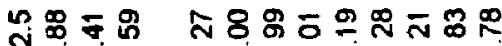

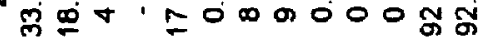

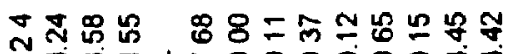

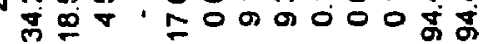

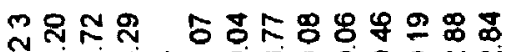

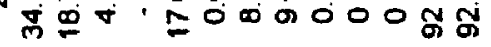

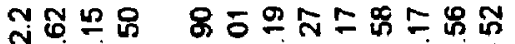
品娄

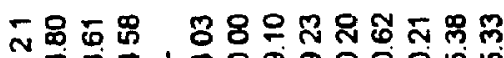

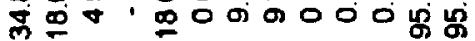

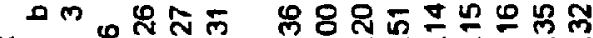

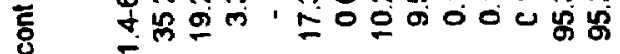
“ m กิกั

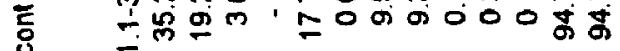

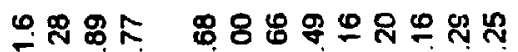

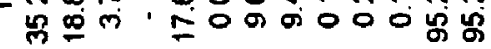

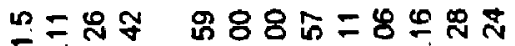
出은

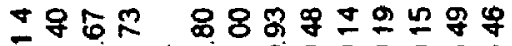

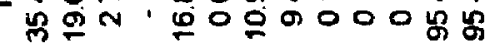

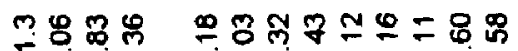

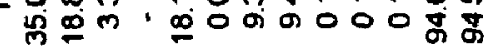

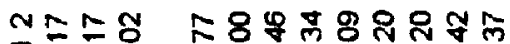
边可 స000000

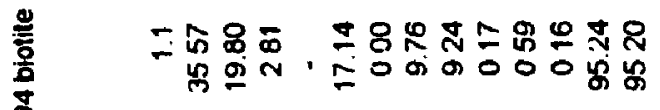

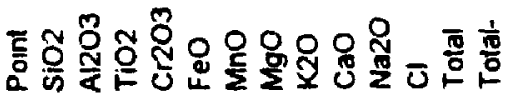

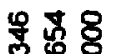
in

ㄴ户ㅇ in

发范苔 in N

昜 宮 un

总范 in $N$

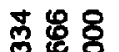

๓

त్లై

in $N$

品芐号

in $\mathrm{N} \infty$

오용 $\cos$

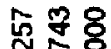

被

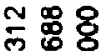

in $\sim$

స要 जi

总 แก

议 in $\infty$

号范 in

声员 is $N$

동 in $\mathrm{N}$

్ㅠㅇ용ㅇㅇ แ $N$

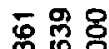
ज त

空果号

象迢 in

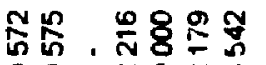

O NONU

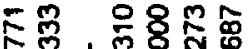

웡. 용 N

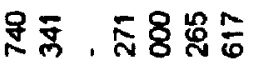

OO NON

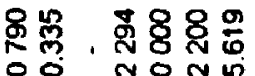

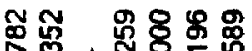

DO NONL

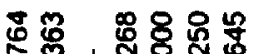

O NONU

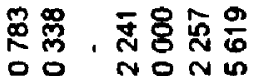

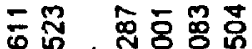

O०口. NON

屯ํ.

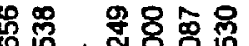
OO NON

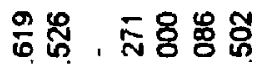

O NON

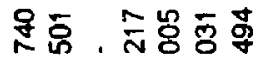

OO NON

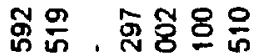

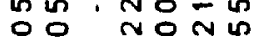

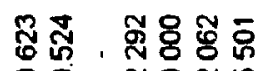

o Non

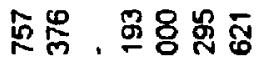

०0 NoN

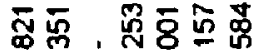
○ㅇ. NON

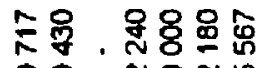

OO NONU

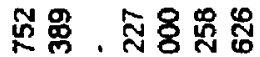

O NONL

总启, 三各罢它

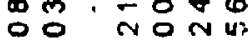

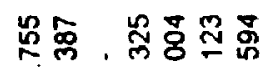

OO NONL

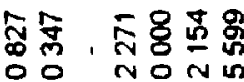

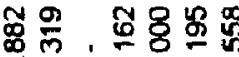

ó non

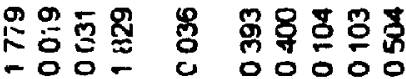

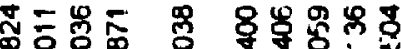

- $00-0$ 00ㅇํ

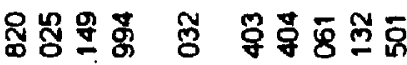

- $00-000000$

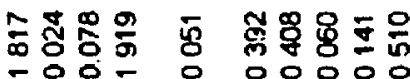

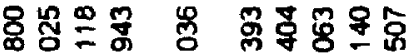
- 00 0 0 0000

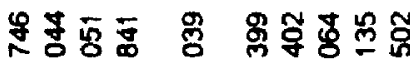

- 00 - 000000

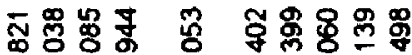
- $00-0$ 00000

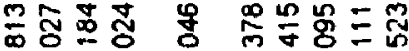
-0ㅇㅇㅇ 00000

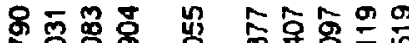
ㄷㅇㅇ- 0 응ㅇㅇㅇ

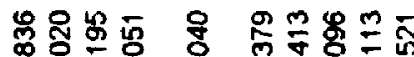
- OON O 00000

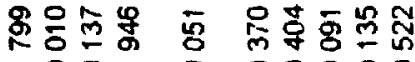
- 00 o 00000

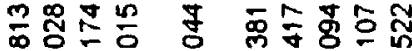

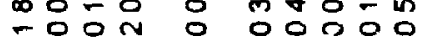

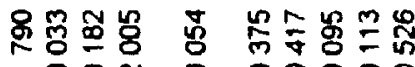
- 00 o 00000

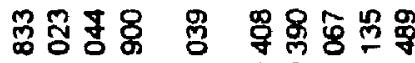
- $00-0$ 00000

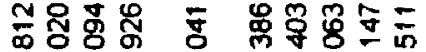
영응 잉ㅇㅇㅇ

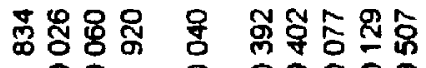
- $00-000000$

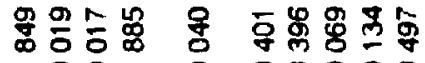
- 0 - 0 00000

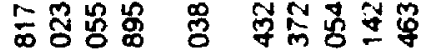

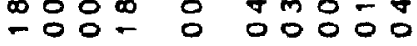

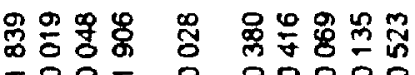
- $00-000000$

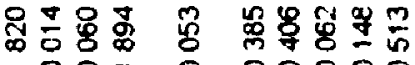
- $00-0$ 00000

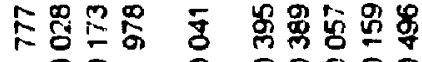

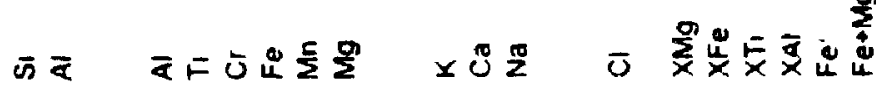




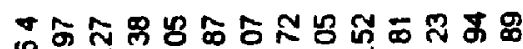

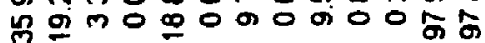

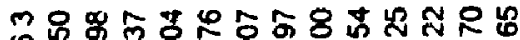

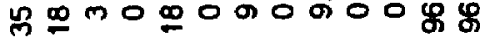

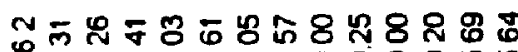

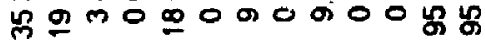

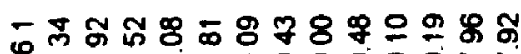

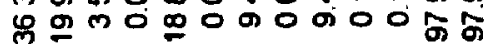

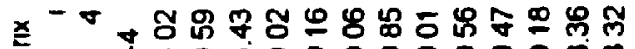

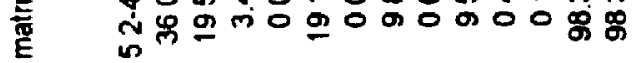

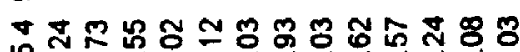

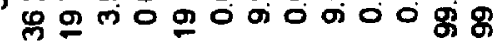

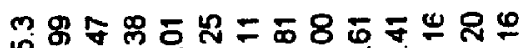

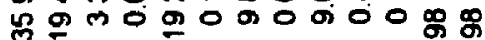

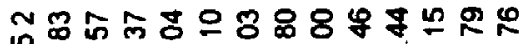

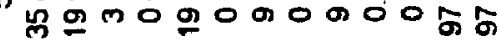
肴 고용 每 ล

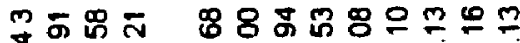
只席以

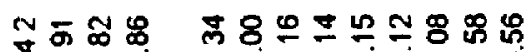

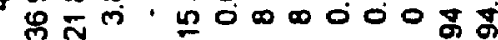

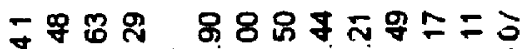
出里 $=000000 \%$

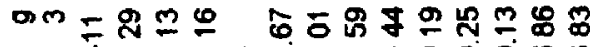
ভ

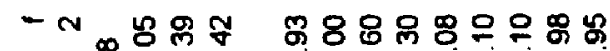
उ ₹ व

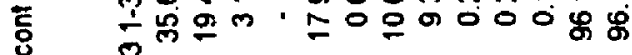

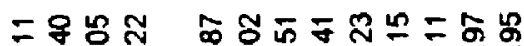
m邑只

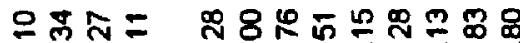
以迤品.

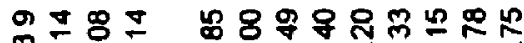
只只

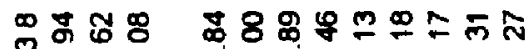

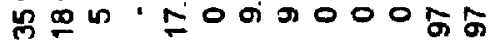

勇 8 in $\sim$

స్లి जi $\infty$

象哭 8 in $\sim$

号志 in $\infty$

용 in $\infty$

遍 in तi

옹용 的

응용용 is 品

畓8 แก

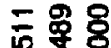
ธत

骂总 ธ

ชิ 觉 8 ज त

ริ- 8 SN

可 8 용요 ㄴ- $\infty$

융융요 in

承总 8 in

品 8 जा क

解要 的些

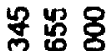
की

议芯 8 जิ के

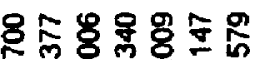
00어에

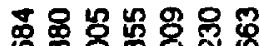
응어유

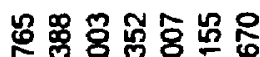
000NON

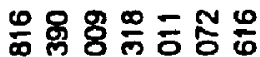
ด0อกำ

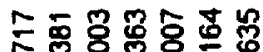
응슨

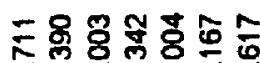
00№N

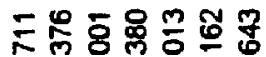
○次舟品 응에용

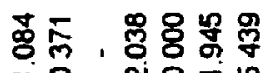
-

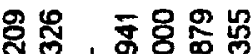
$-0.9-0$

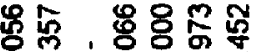
응.

速染 . $-0.0-6$

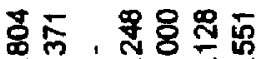
。O Non

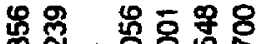
엉. 잉

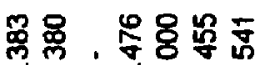
$00-0-4$

ํํำ. . . ㅇำ

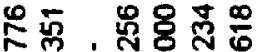
C) 密这 ०० Non

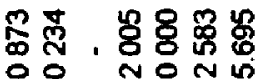

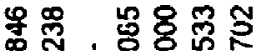

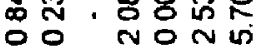

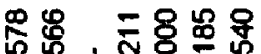

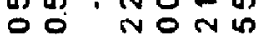

츙유ㅇㅛㅛ -Oㅇ-

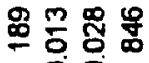
- 0 - -

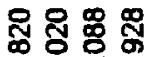
-0ㅇ-

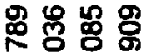
$=8: 0$

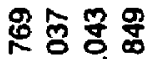
- 0 -

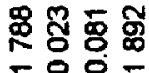

志致费要 공응

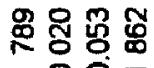
$-80-$

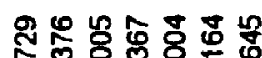

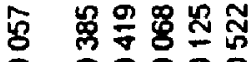

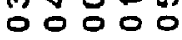

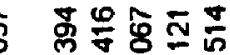

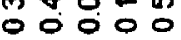

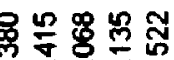
으응

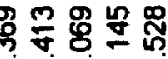
.0.

产無骂 00000

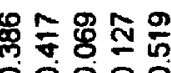
00000

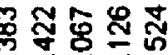
0000

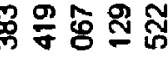
00000 路器尔 00000

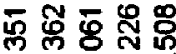
00000

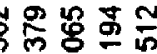
0000

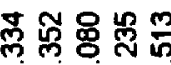
00000

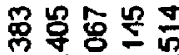
응으응

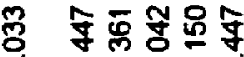
00000

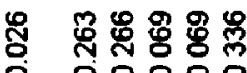
00000

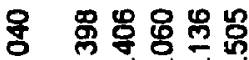
00000

罢 ㅇํㅇ으음

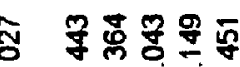
00000

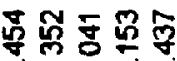
00000

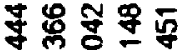
응응

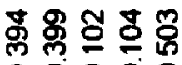
00000

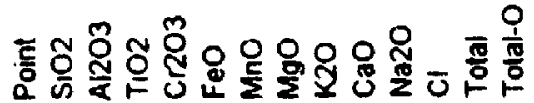


肴

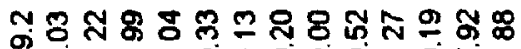

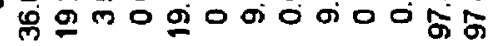

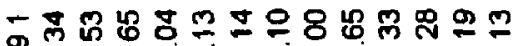

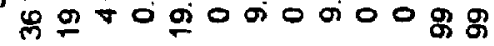
ำ

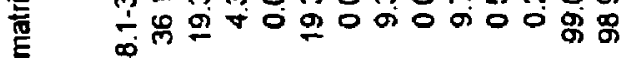

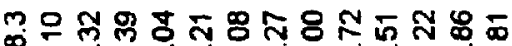

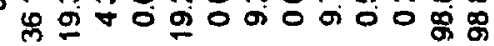

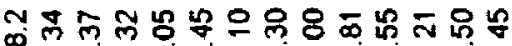

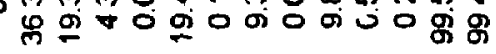

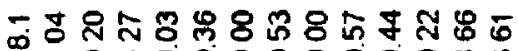
员 9 จ

$x^{\times N}$ บ

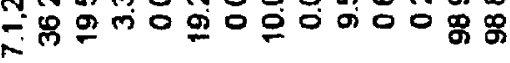

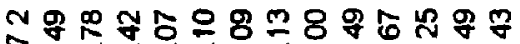

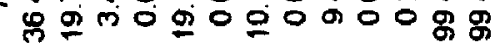

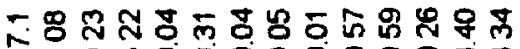
员

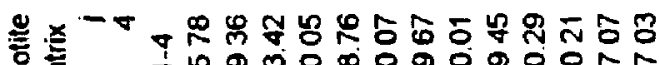

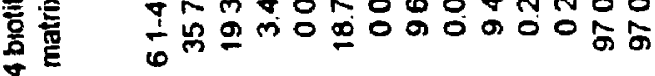
के 官

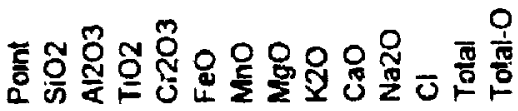

\%ำ용 แล

웅용. is $N$ क

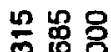
에

용용 i $\infty$

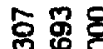
in 영용 in $N$

\section{응용요} in $\sim \infty$

\%ำ in $N$

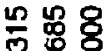
in

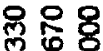
थ $N$

융용 n

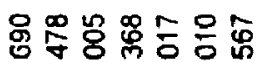

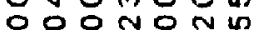

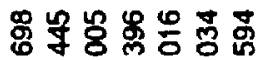
OOONONU

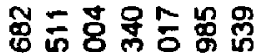

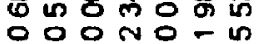

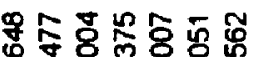

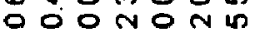

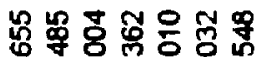
000 No

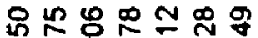
\% 8 品 000 NO n

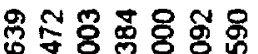

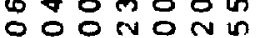

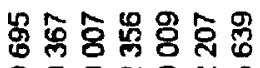

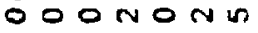

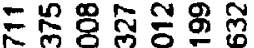
०0융

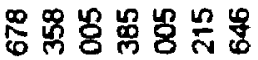
000 Non

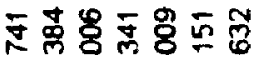
मо०तon

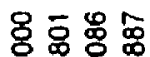
ํ-

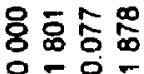

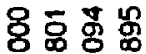
음

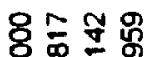
8.

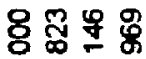
- $-0-$

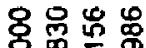
잉웅윰쬬 드음

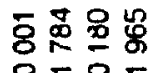
-

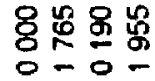
ริธ

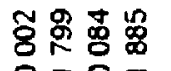
- - O -

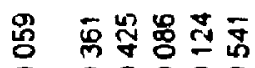
000000

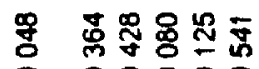
으음

영 哭 $\approx$ 졍 . 00000

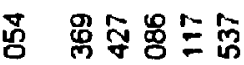
. 00000

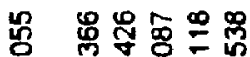
응 0050

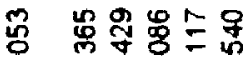

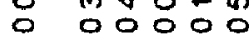

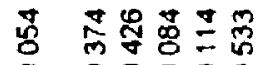
응oㅇ응

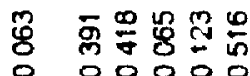

- 895 $\circ 00000$

岁 귬

No

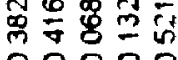

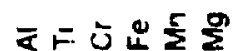

$x \stackrel{000}{2}$

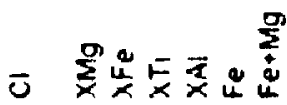




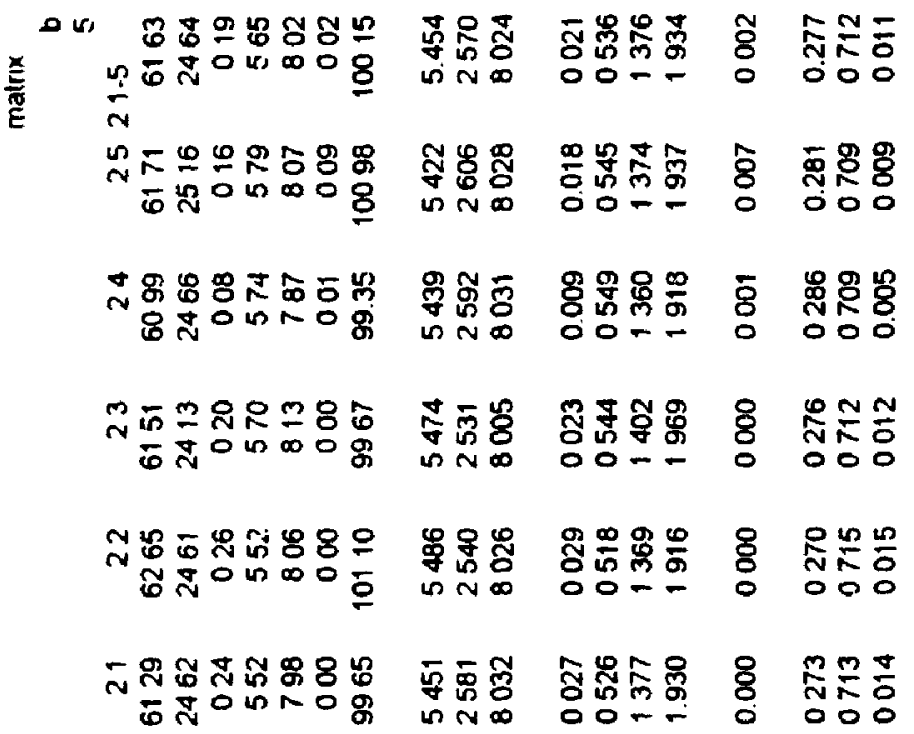

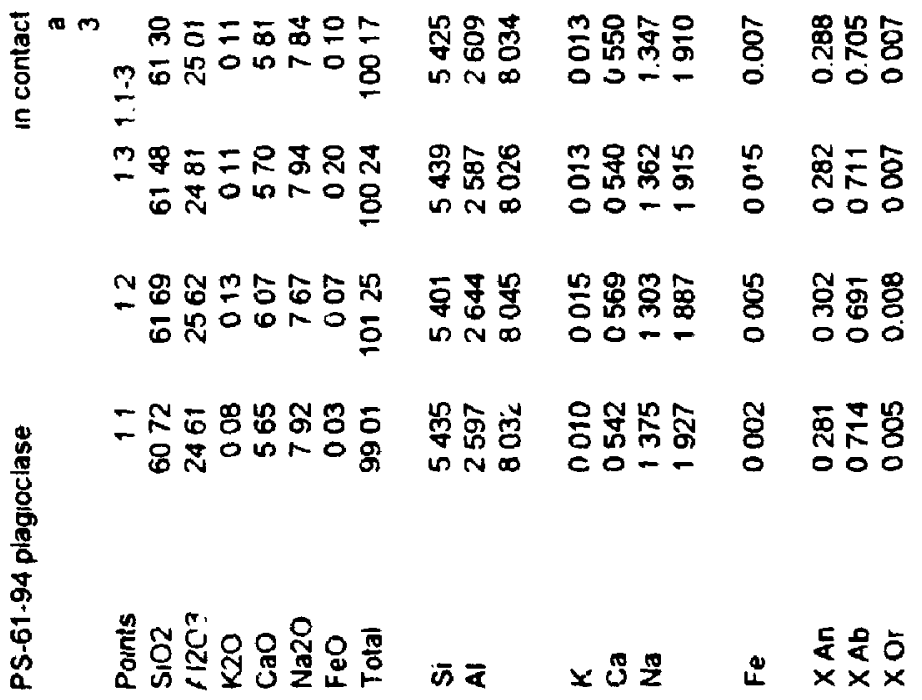




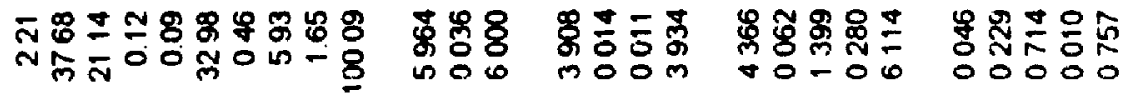

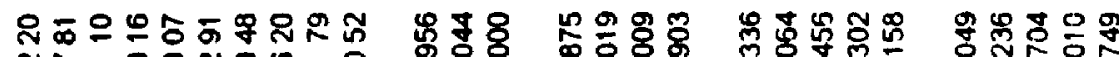

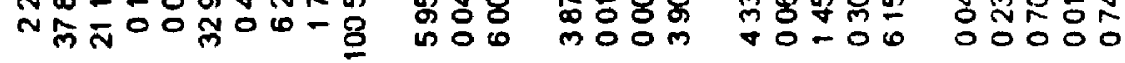

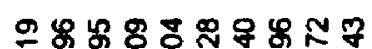

N命O

पू⿱乛⿻上丨

응유

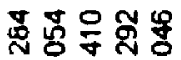

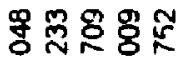

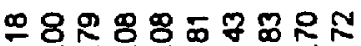

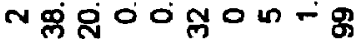

实 8

喿응응

$+0-06$

00000

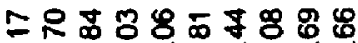

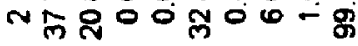

웅으용

moOm

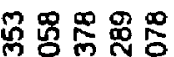

罗突능응

8ㄷㅇ

$\checkmark 0-00$

00000

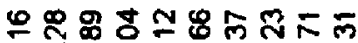

N"ू

$\$ 8$

mo

总晶尔器昌

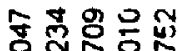

- O0́ 00000

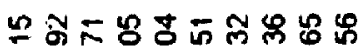

స్̄

000

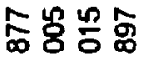

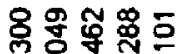

옹옹융

능 웅응

จ 00

00000

サேニ

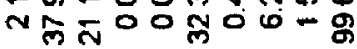
106

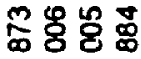

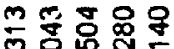

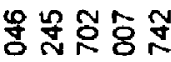

\&్ㅇㅇㅇㅇ

$100 \mathrm{~m}$

- $0-00$

00000

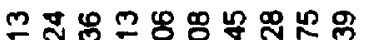

ง

男安

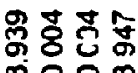

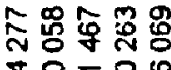

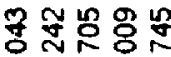

00000

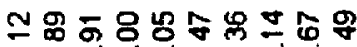

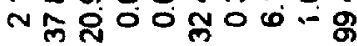

ㅇํㅇํํㅇํㅇ

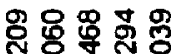

옹

뭉용풍

m๐

$\checkmark 0-06$

00000

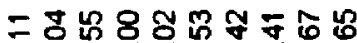

N品只

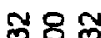

중용

$\frac{N}{\sigma} 8 \frac{8}{8} \frac{9}{6}$

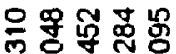

守忿员罗思

ल० $0 \mathrm{~m}$

$\checkmark 0-00$

00000

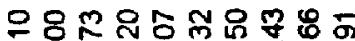

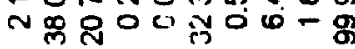

용응

₹ิ융용

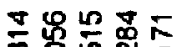

왕 용 mo

西的里

00000

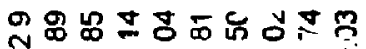

分只이잉

앙융유.

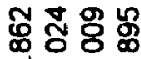

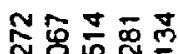

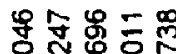
mo $0 \mathrm{~m}$

$\checkmark 0-00$

00000

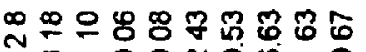

塄

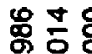

곻ㄷㅎㅇ영

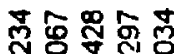

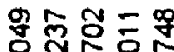
लं००

$\checkmark 0-06$

00000

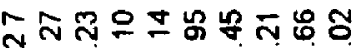

雨

แั

용응응

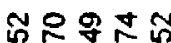

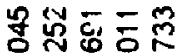

moom

$\checkmark 0-06$

00000

思芒号

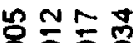

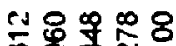

눈송응뭉

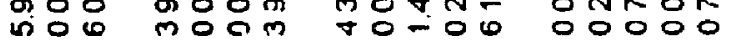

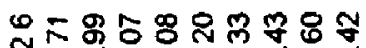

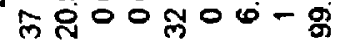

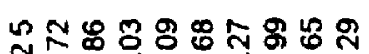

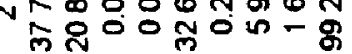

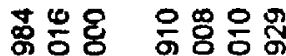

군제

용요용 10

ल०0m

NON

ग ल

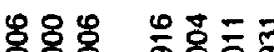

论

$000 \mathrm{moOm}$

प0-00

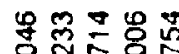

00000

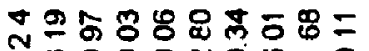

ลิธัช

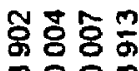

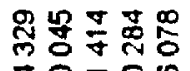

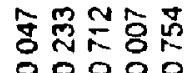

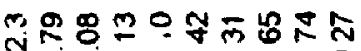

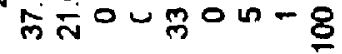

옹종

moOm

Т०-0

00000

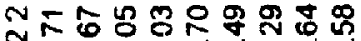

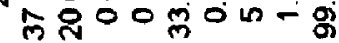

ก00

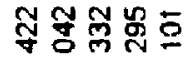

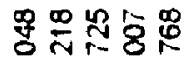

중호

ธ్రె

00000

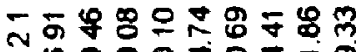

006

ल0 0 m

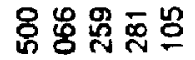

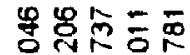

怘骨家

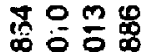

00000

㞸

n 06

m 000

迢要

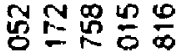

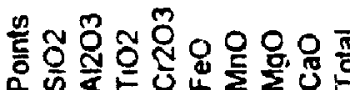

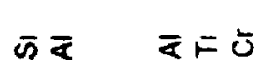

แั

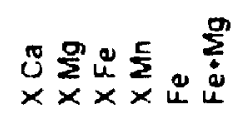




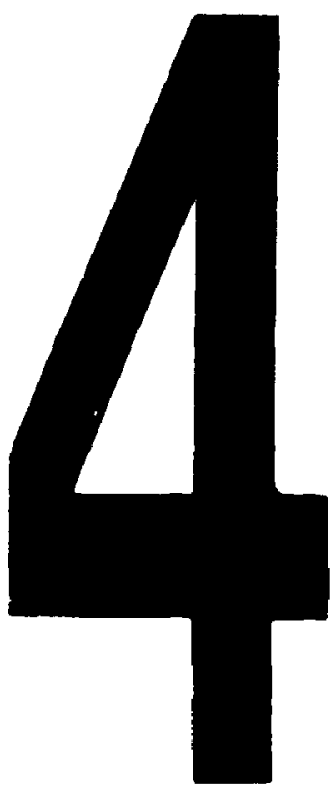

PM-1 3\%" $\times$ " PMOTOGRAPHIC MICROCOPY TARGET NBS 1010 ANSI/ISO "2 EOUIVALENT

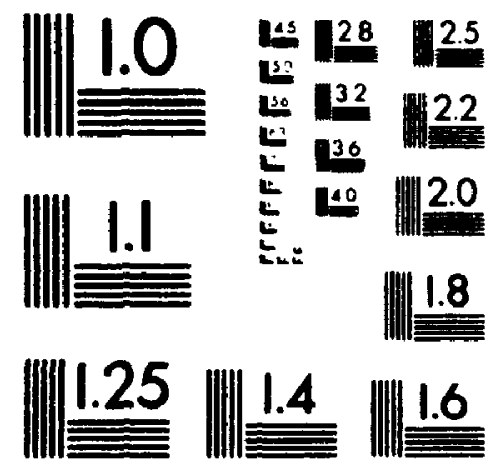

PRECISIONSM RESOLUTION TARGETS 


\begin{tabular}{|c|c|c|c|c|}
\hline 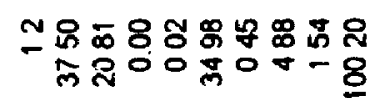 & 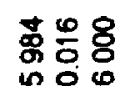 & 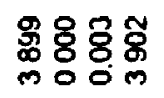 & 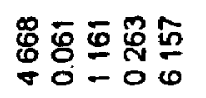 & 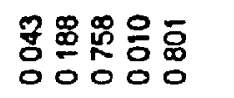 \\
\hline 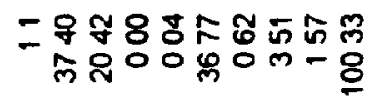 & 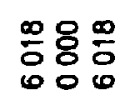 & 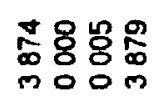 & 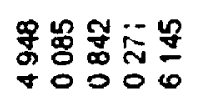 & 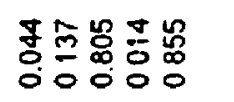 \\
\hline 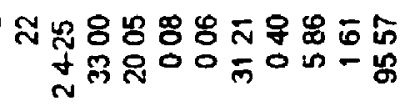 & $\begin{array}{l}9 \\
\text { 용ㅇㅇㅇ } \\
\text { no }\end{array}$ & 융용혀 & 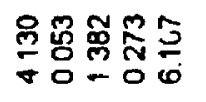 & 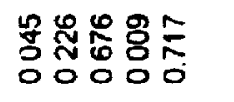 \\
\hline 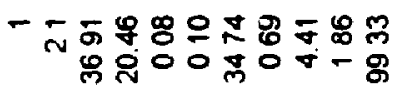 & 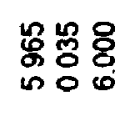 & 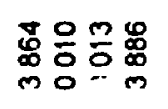 & 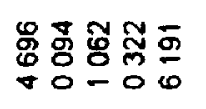 & 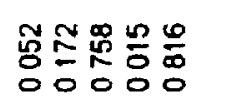 \\
\hline 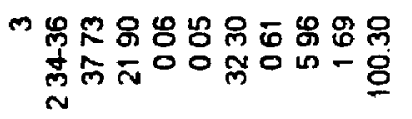 & 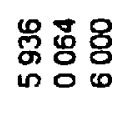 & 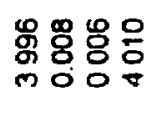 & 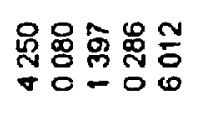 & $\begin{array}{l}\text { 罢点员员 } \\
00000\end{array}$ \\
\hline 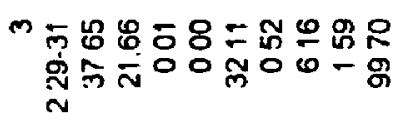 & $\begin{array}{l}\text { 鬲肯 } 8 \\
\text { in } 0\end{array}$ & 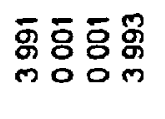 & 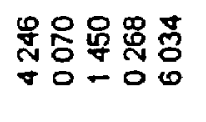 & 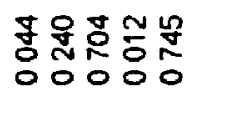 \\
\hline 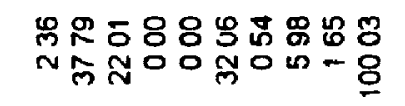 & 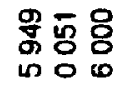 & 웅용영융 & 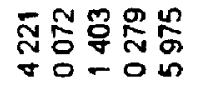 & 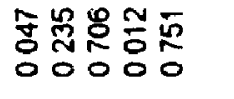 \\
\hline 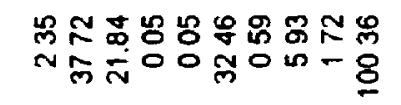 & 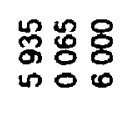 & 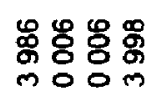 & 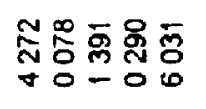 & 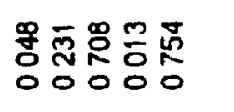 \\
\hline 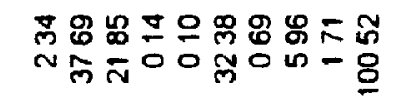 & 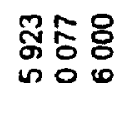 & 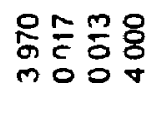 & 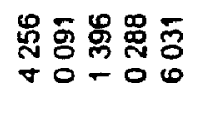 & 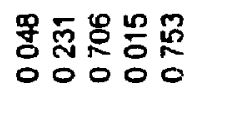 \\
\hline 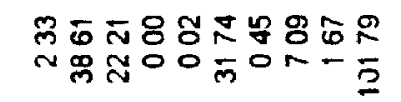 & 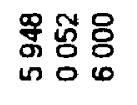 & 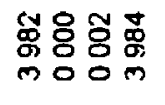 & 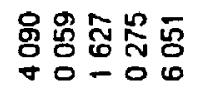 & 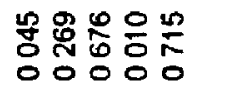 \\
\hline 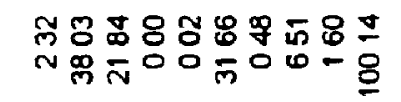 & 怘皆 & $\begin{array}{l}8.8080 \\
80\end{array}$ & 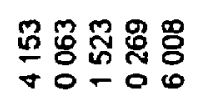 & 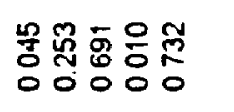 \\
\hline 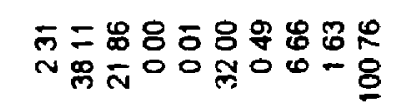 & $\begin{array}{l}\text { 앵용ㅇㅇㅇ } \\
\text { 잉 }\end{array}$ & 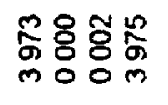 & 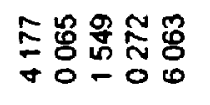 & 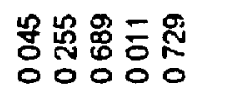 \\
\hline 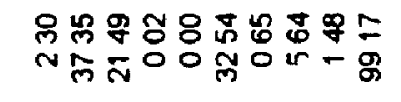 & 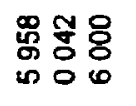 & 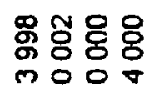 & 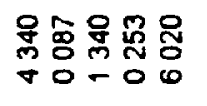 & 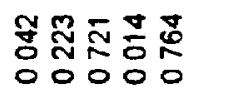 \\
\hline 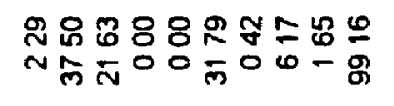 & 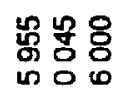 & $\begin{array}{l}808080 \\
80\end{array}$ & 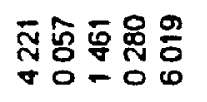 & 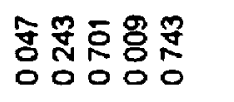 \\
\hline 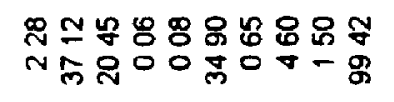 & 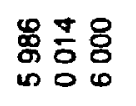 & 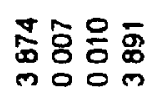 & 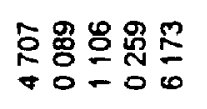 & 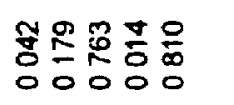 \\
\hline 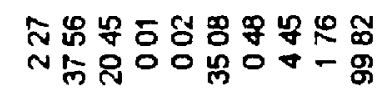 & 융요용 & 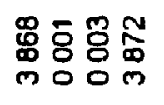 & 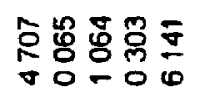 & 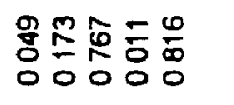 \\
\hline 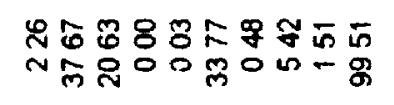 & 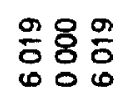 & 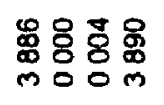 & 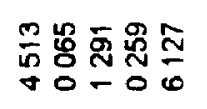 & 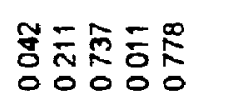 \\
\hline 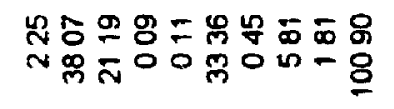 & 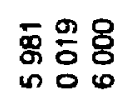 & 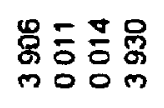 & 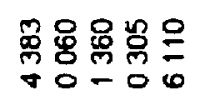 & 유ㅇㅝㅠ응ㅇㅇㅇㅇㅇㅇ \\
\hline 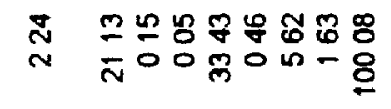 & 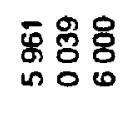 & $\begin{array}{l}m \\
\bar{\sigma} \\
m \\
0\end{array}$ & 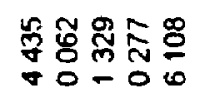 & 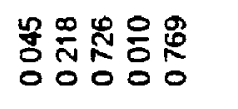 \\
\hline 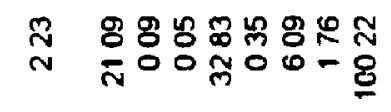 & 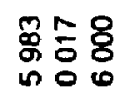 & 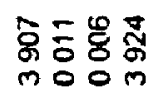 & 四 তे : & 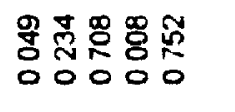 \\
\hline 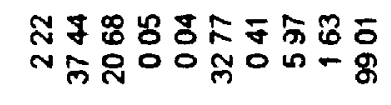 & 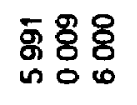 & 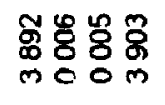 & 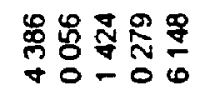 & 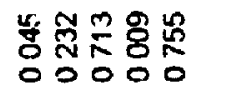 \\
\hline 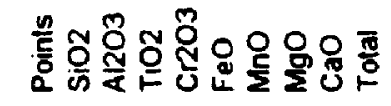 & $\bar{\omega}$ & & \& & 出点市竞 \\
\hline
\end{tabular}




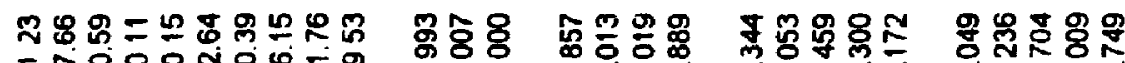

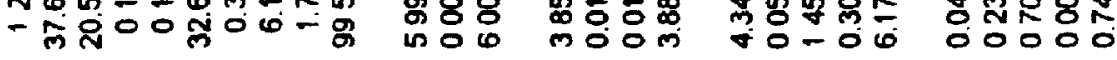

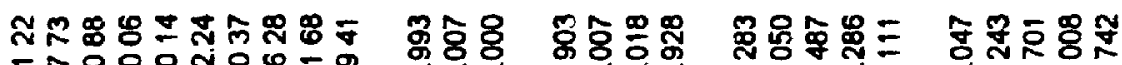

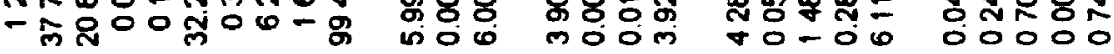

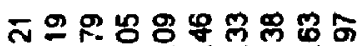

- 塄

\$ัชำ

$8: 0$

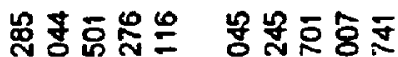

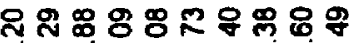

- 掣

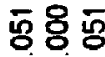

mo

$\checkmark 0-06$

00000

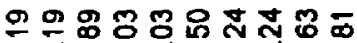

-

용요

最最=

吉莒总六路

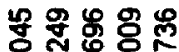

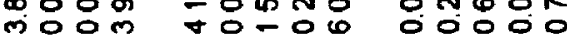

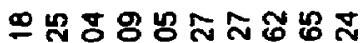

-

ஸ 0

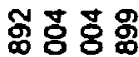

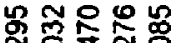

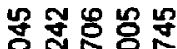

mo 0 m

$\checkmark 0 \div 06$

00000

응영응

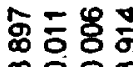

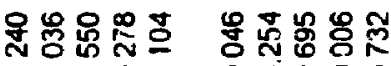

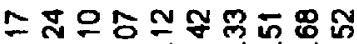

웅웅용

员骂咢哭

จ $0-06$

00000

- 罢

000

moom

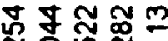

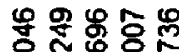

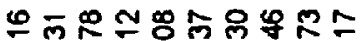

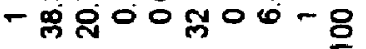

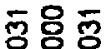

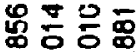

$\checkmark 0-00$

00000

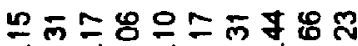

10 0

moom

웡웅응 오ㅇㅡㅡ

罗罢余战

ㄱำ-0

-

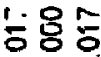

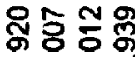

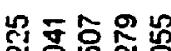

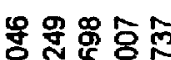

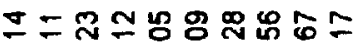

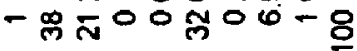

$\circ$ 웅

moom

ช०-0

00000

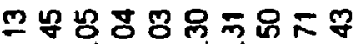

영응

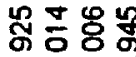

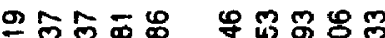

in 0

m००

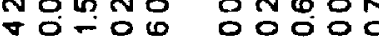

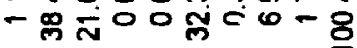

융웅용

ฐึํํㅇ용

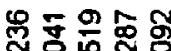

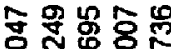

600

moO

จ $0-00$

00000

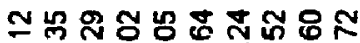

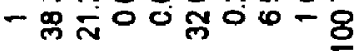

Б̄ํํ요

సิธิ์

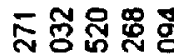

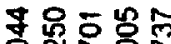
100

ก००

응- 06

$0000 \mathrm{C}$

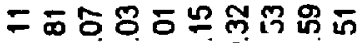

器응

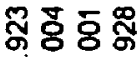

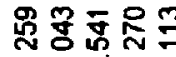

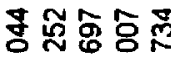

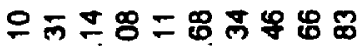

5용

ल००

ช०-00 00000

- 每

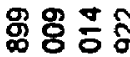

은 ๓.

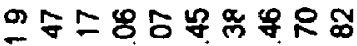

옹음

moom

$70-50$

염엉영영

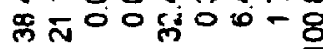

ริํํㅇํำ

害号总总量

会禺品 긍ㅇ

จ0-0

응응응

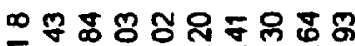

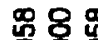

ริำ

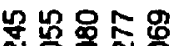

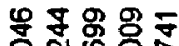
00

舟吕范

T응

00000

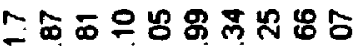

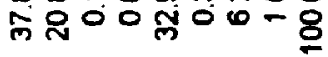

영응 8

동ㄷㅎㅇ융

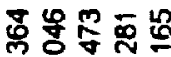

웅

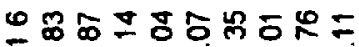

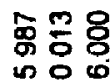

贾言驾

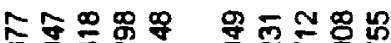
万人

$58 \%$

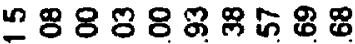

88.

岁

궁ำ은

00000

D

mo

果部总

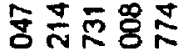
+

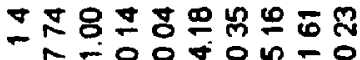

홍요

สักิัตับ

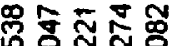

녹뭉 moㅇ

จ응

00000

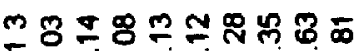

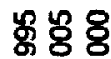

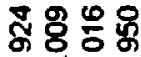

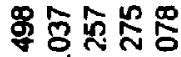

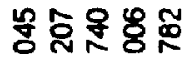

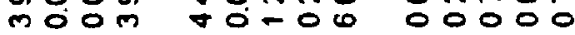

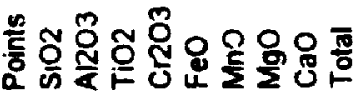

कर रह

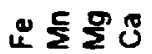

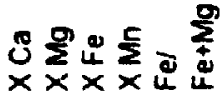




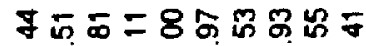

-

ชณ®

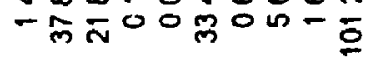

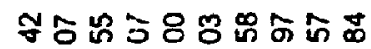

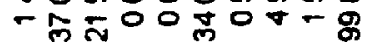

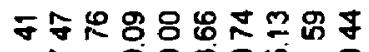

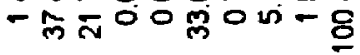

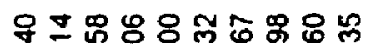

一命

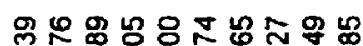

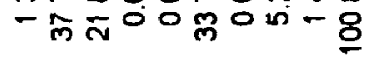

只テঢ

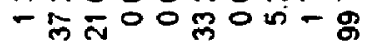

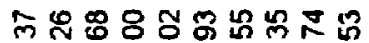

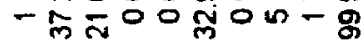

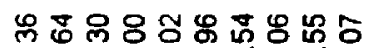

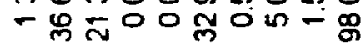

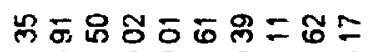

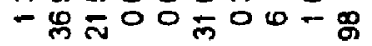

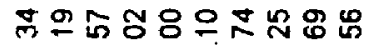

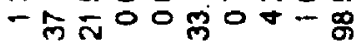

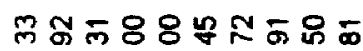

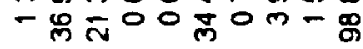

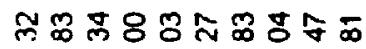

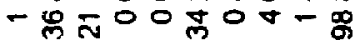

ติธีกัน

- ลิกับ0

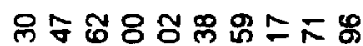

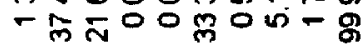

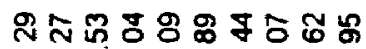

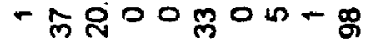

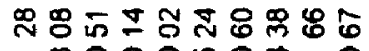

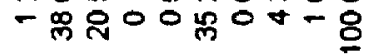

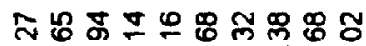

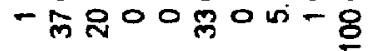

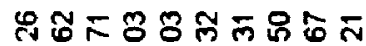

-

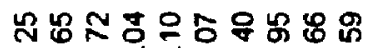

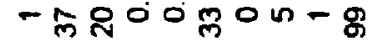

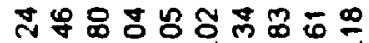

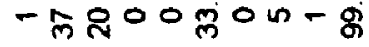

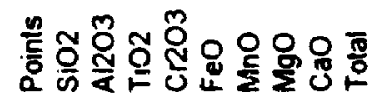

눙용 แกㅇำ

앵중용

으

눙용

ก० 6

융ㄷㅇㅇㅇㅠ

ज० 0 is

命 䔩

공ํㅇㅇㅇ

เ5 06

究吉号

ริ혀용

in 0 o

劳

in 0 o

乐范号

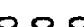

융응

150

종ำ

ตั.

5:

若器

is 0

앵융 8

ज० 0

ริํํㅇํำ

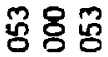

10 :

密言实

in 0

웅응

100

$5 \%$

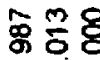

กั

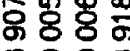

noom

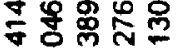

$+0-00$

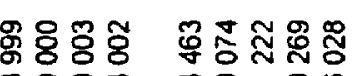

m०

2.0.

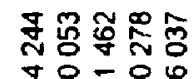

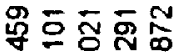

$\forall 0 \div 0$ w

뚱응응

ํㅜㅇ흥형

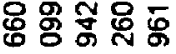
-000

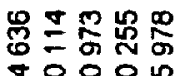

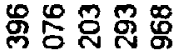

$\checkmark 0-0$ is

Oᄋ

00500

용요용

mo $0 \mathrm{~m}$

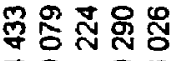

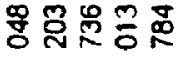

00000

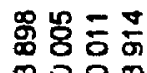

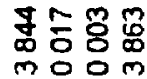

这ㅎㅎㅇ영

mo 0

总㤩示总可

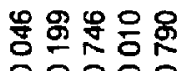

.000\%

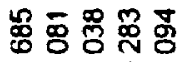

- $0-00$

융요영응음

00000

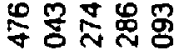

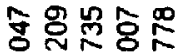

ํํㅇㅇํㅇ

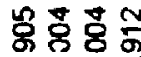

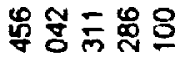

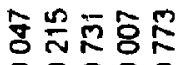

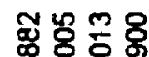

00000

웡 츄숭용

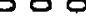

ํㅗㅇㅊํํํํํำ

00000

放 文に 


\begin{tabular}{|c|c|c|c|c|c|}
\hline & 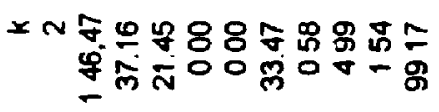 & 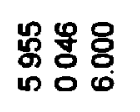 & 눙융융융 & 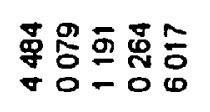 & 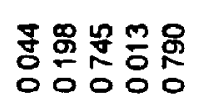 \\
\hline & 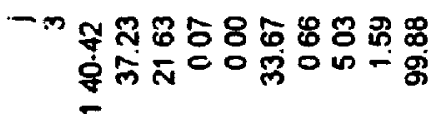 & 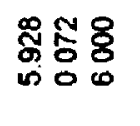 & 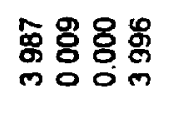 & 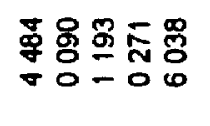 & 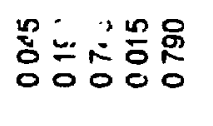 \\
\hline & 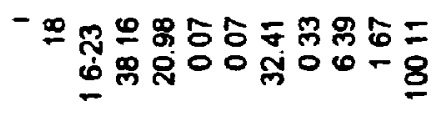 & 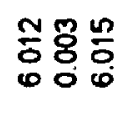 & 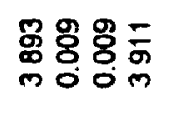 & 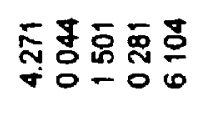 & 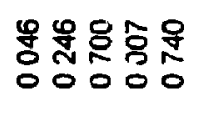 \\
\hline & 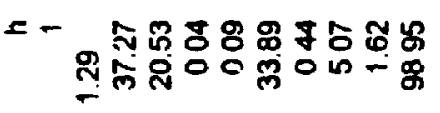 & 용요 & 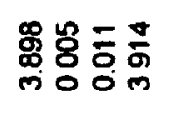 & 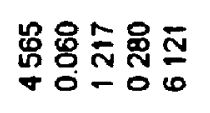 & 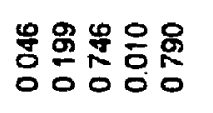 \\
\hline & 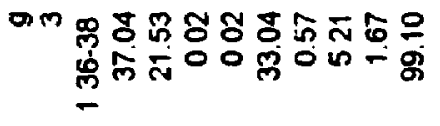 & 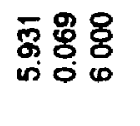 & 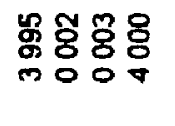 & 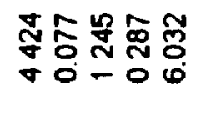 & 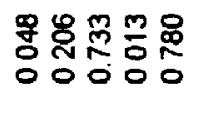 \\
\hline & 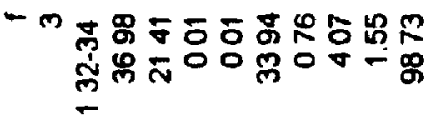 & 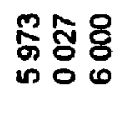 & 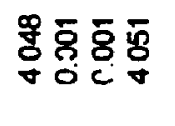 & 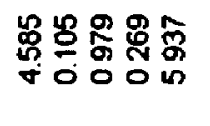 & 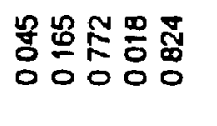 \\
\hline & 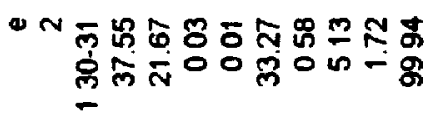 & 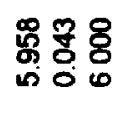 & 응용ㅇㅇㅇㅇㅁㅇ & 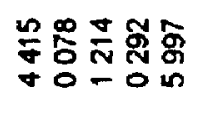 & 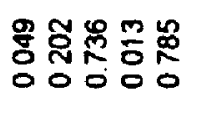 \\
\hline & 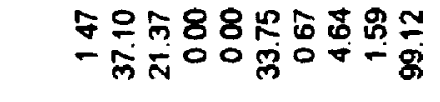 & 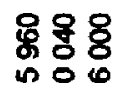 & 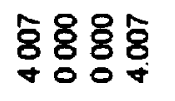 & 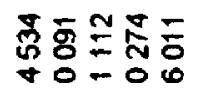 & 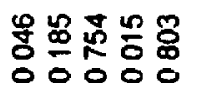 \\
\hline & 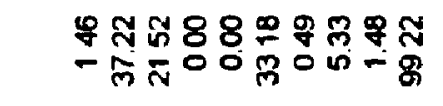 & 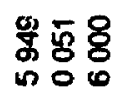 & 용융용 & 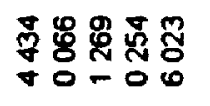 & 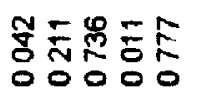 \\
\hline & 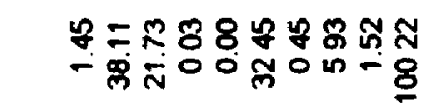 & 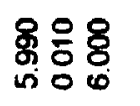 & 동용ㅇㅇㅇㅁㅇ & 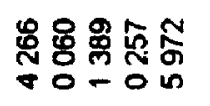 & 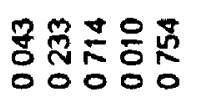 \\
\hline & 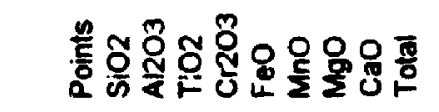 & $\bar{\omega}$ & $\bar{\alpha}$ & . & 出坫导竞 \\
\hline
\end{tabular}




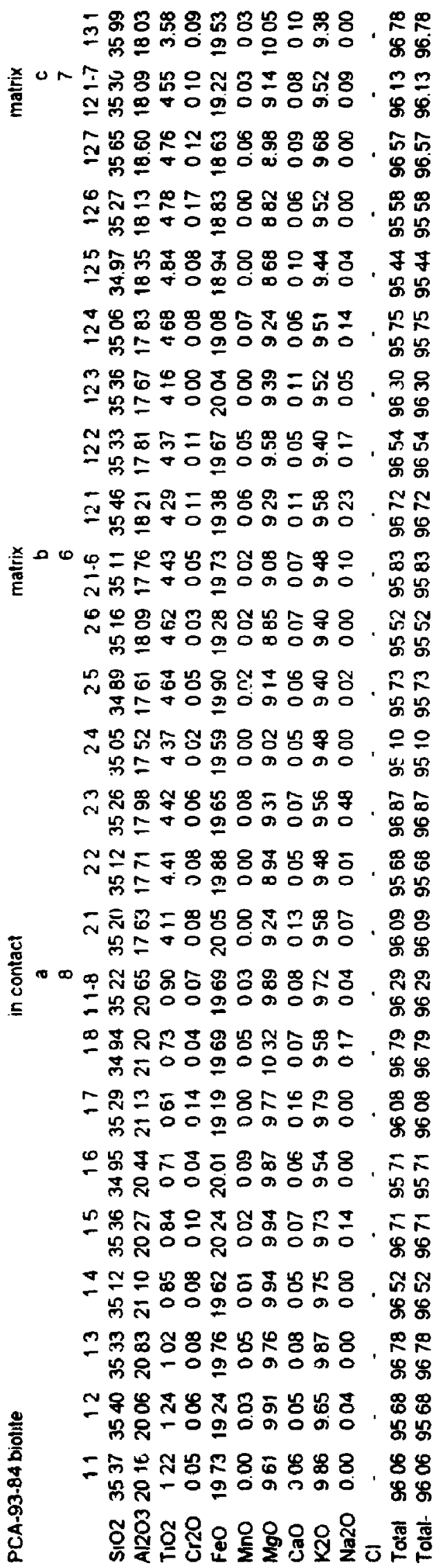

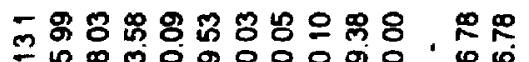

$2=$

\&

的

용

品

专

\%

员

요

品

号

禺

$\sim N$

80

另搹

สิธ

ตั

$2 \pi$

ช้ํㅇ

으으

岁甪

क

怘

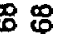

용

品最

옹

\%ั

a

कै 8

\& 8

\$

$\pi$

赑

下云

$8 \%$

กิธ

$\& \%$

电

$\mathscr{8} 8$

88

$8 \%$

$8 \%$

茟

i $\stackrel{\sim}{\infty}$

可

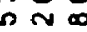

急然 8

․ N

品品웅

心 $\infty$

영

in $\infty$

词

in $\infty$

点正要

in $N$

禺

ज N

항ㅇㅇㅇ

in $N \infty$

卷思宫

in N

识若

in $\sim \infty$

응

in $\sim x$

웅융용

in

형ㅇㅇㅇ

in $\sim$

号宫员

in $N$

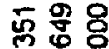

in $\sim$

윰 $\overline{8}$

is $\sim$

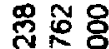

in $N$

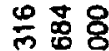

in $\infty$

确雾

in $\infty$

空怘 8

in $\infty$

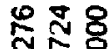

is $N$

항용

थ $N$

总 ำ 8

แ $N \infty$

通客

iñ

के

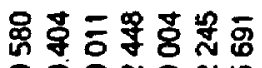

000 non

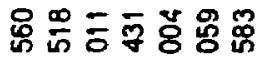

OOONONU

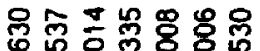

응응 웡유

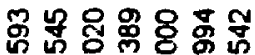

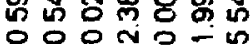

냉 ్ㅐㅇ응 용현

- 00 No-

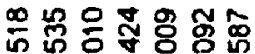

잉잉

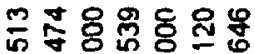

o 0 on

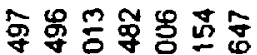

o o o vo n in

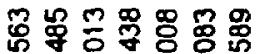

OODNON N

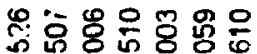

O O NON N

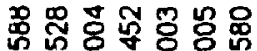

○。잉

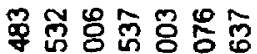

O O O NON N

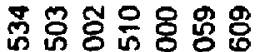

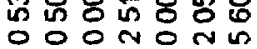

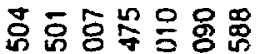

o o on

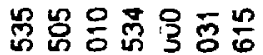

○0 ONON

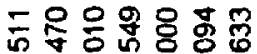

○。Nㅇㅇㅢ

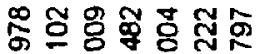

o o ono n in

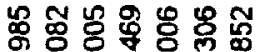

o o on 0 n

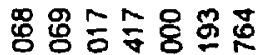

-00noni

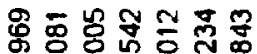

o o O N O N

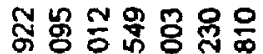

000 舟品

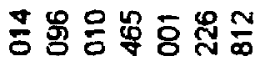

- o onon w.

品용용요용

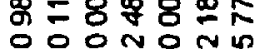

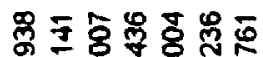

oO ONON n

형

otonon

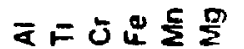

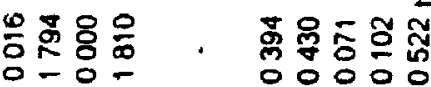

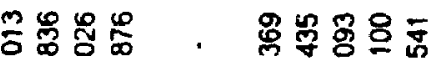

ㅇㅇ 0000

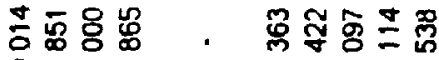

$0-0 \%$ 00000

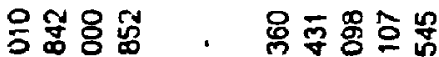

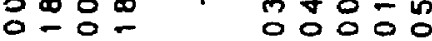

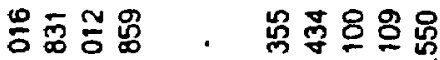

ㄴㅇ 00000

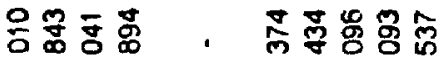

응ㅇㅇㅇㅇㅇ

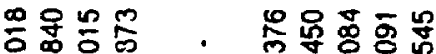

응 0000

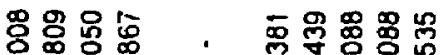

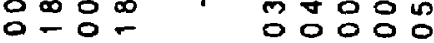

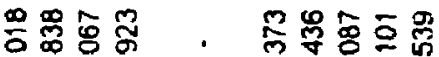

응 0000

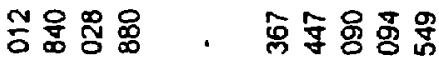

$0-0-\quad 00000$

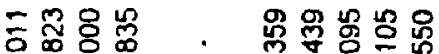

응 응응

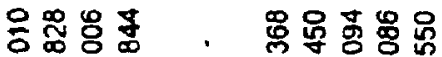

O-0 0000

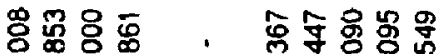

O-0

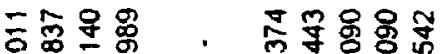

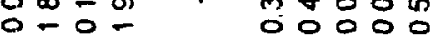

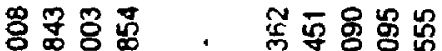

으응

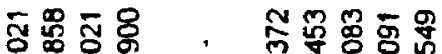

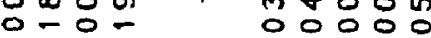

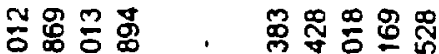

O-0-

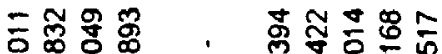

$000-\quad 00000$

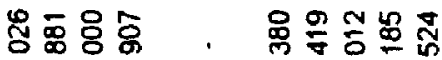

0-0- 0000

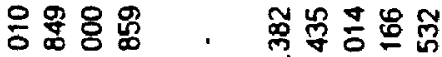

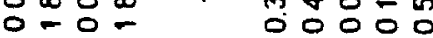

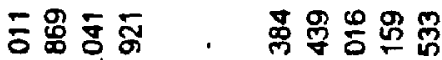

응 O응

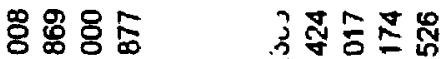

0-0- 응응

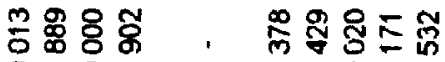

응

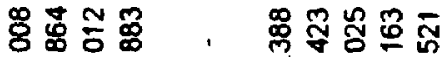

O-0- 00000

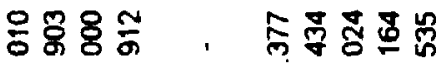

O-0-

$8 \times \frac{\pi}{2}$ 
ํㅡㄹ

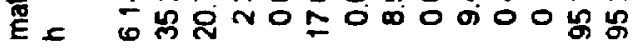

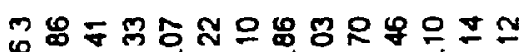

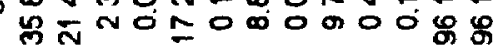

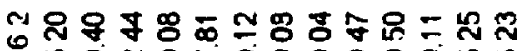

留只 N

бக

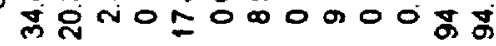

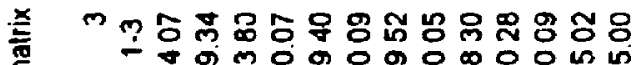

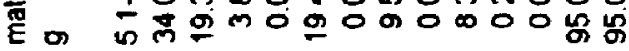

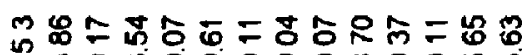

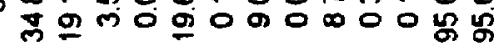

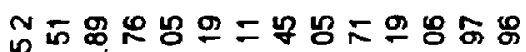

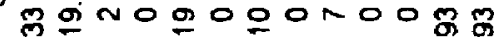

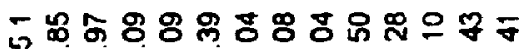

씅

ำ

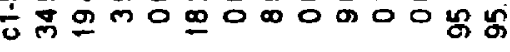

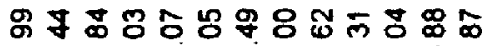

४ 户

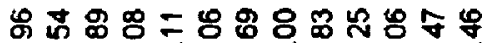

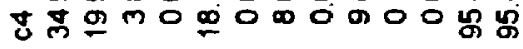

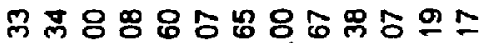

円

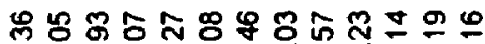

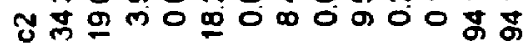

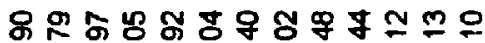

ঢ

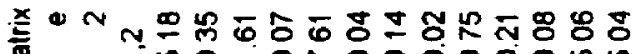

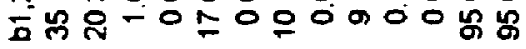

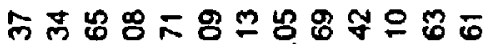

공유 응 0 응 000 냉

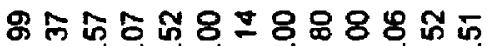

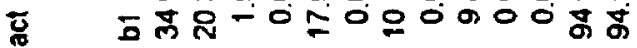

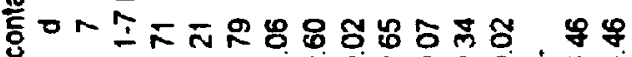

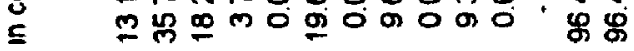

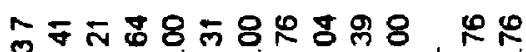

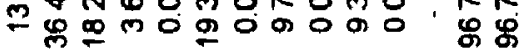

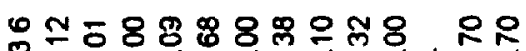

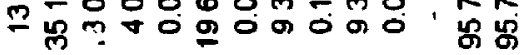

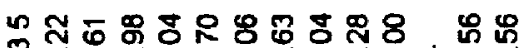

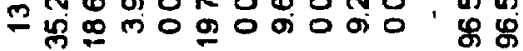

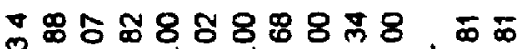

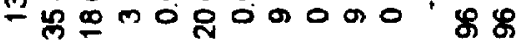

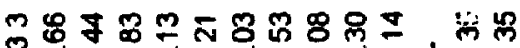

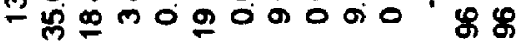

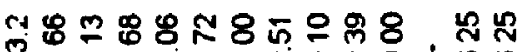

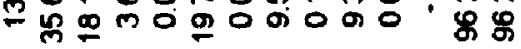

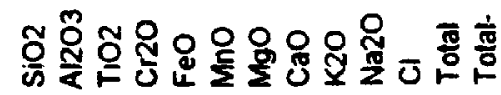

串 哭 8 ๓

急范号

in $N$

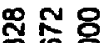

in $\sim$

중

แ $N$

융ㅇㅇ

in $\sim$

嵒云号

in 0

냉

क ⿻ 丨

กิ้ ํํㅇㅇㅛ

in

퐁

in

웅용

in $N$

웅 용용

in $\sim$

忞电

in 0

동용

in 你

용용요

แก

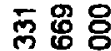

is $\sim$

离号号

in $\infty$

疍

in $\sim$

웡 8

羟

零

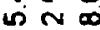

웅용

in $r 10$

总司号

ก $N$

兽

क N

象邻

in N

得员

in $N$

$\bar{\infty}$

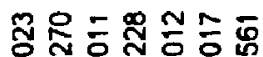
- O ONONm

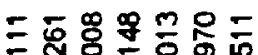
- O ONo-n

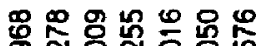
OOONON

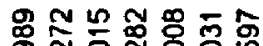
O 0 O त 0 N

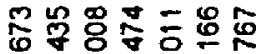
o o ond n

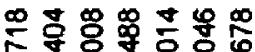

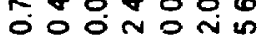

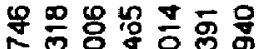
잉융 N

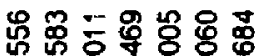
O O O non in

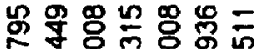
ㅇㅇㅇ응

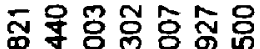
\%马ㅇㅇㅇㅇㅇㅇㅛ

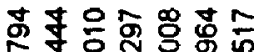

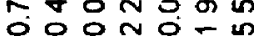

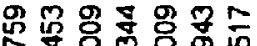

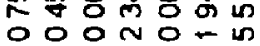

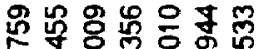
잉유요

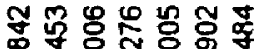
心

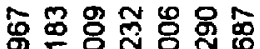

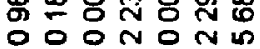

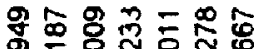

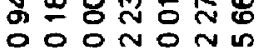
品品 즁용용 O O O N O N 영 웡 寽 용용 o. 0 on

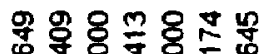
o o onon m

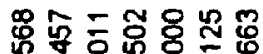
OO ONON n

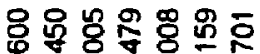
O O OnON m

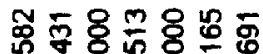
o d ond nu

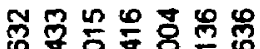

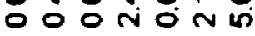

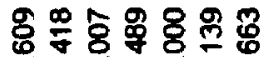

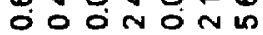

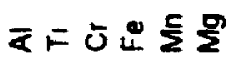

총ำ융

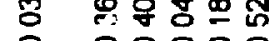

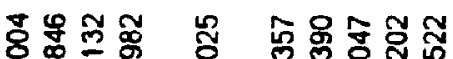

… - 0 O०OO०

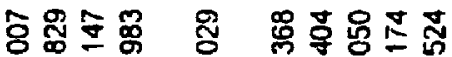

O-O- 0 O०000

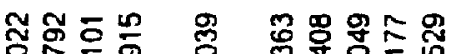

O- -0 0 0000

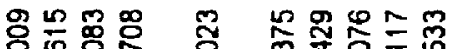

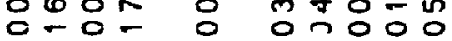

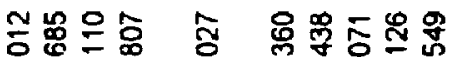

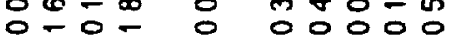

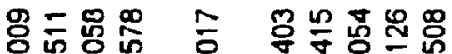

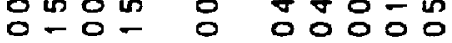

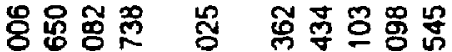

잉ㅇㅇㅇㅇㅇㅇㅇㅇ

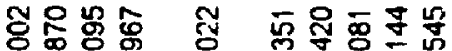

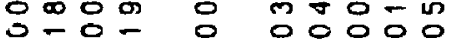

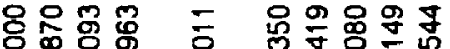

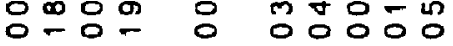

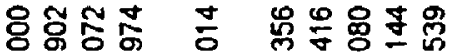

잉응 잉ㅇㅇ

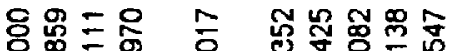

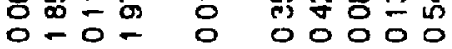

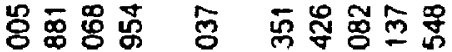

ㅇㅇㅇㅇㅇㅇㅇㅇㅇㅇㅇ

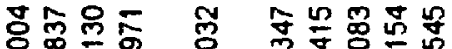

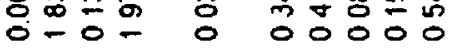

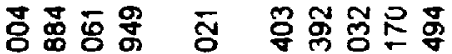

- - - 0 잉ㅇㅇ

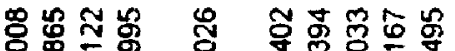

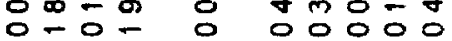

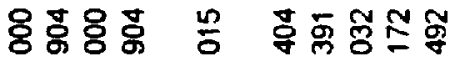

$0-0 \div 0$ 0 0000

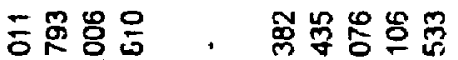

둥 0000

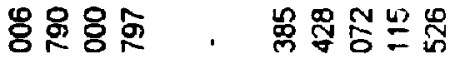

- - - 00000

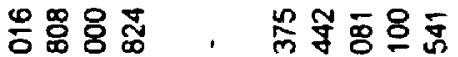

$0-0-00000$

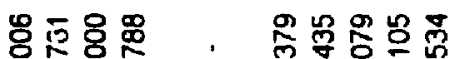

0-0- 00000

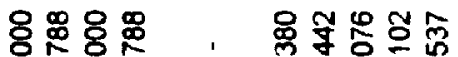

$0-0-00000$

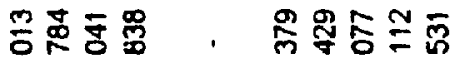

- - - 00000

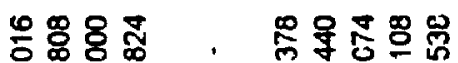

O-0- 00000

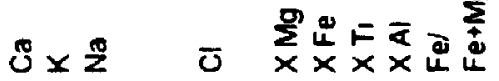




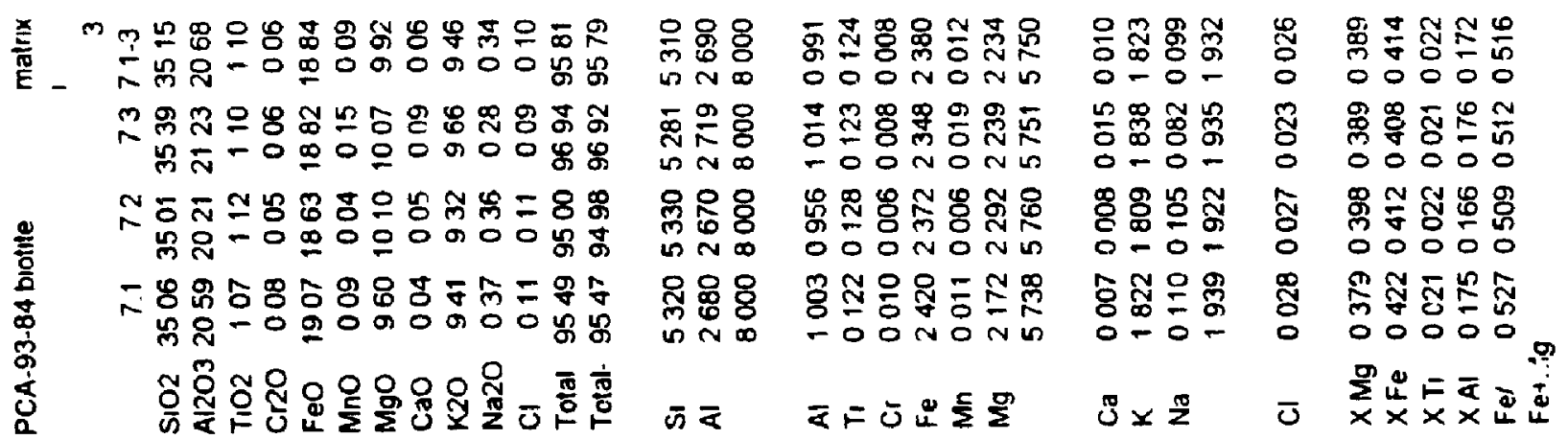




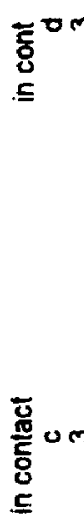

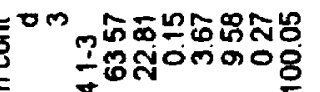

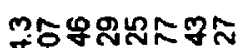

ธ่งกmOด

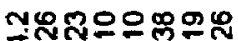

Bतกण

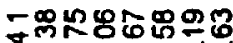

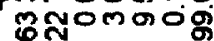

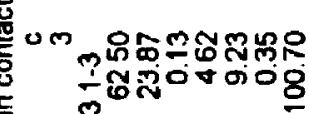

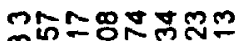

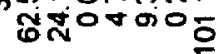

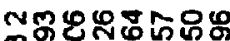

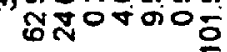

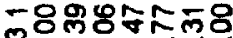

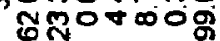

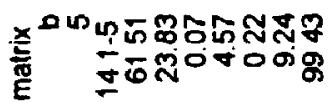

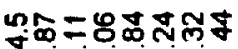

Tढ்

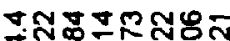

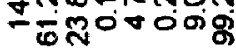

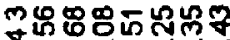

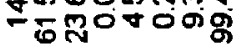

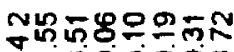

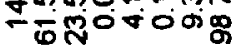

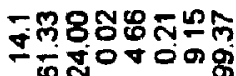

है

士

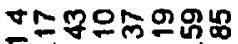

숭유응

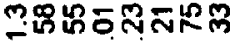

유-0 $=8$

ำกำกำ

๕ลํㅇำ

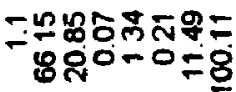

둥융유윻표

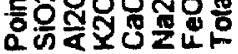

송

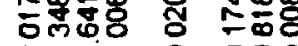

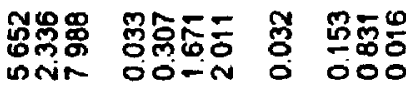

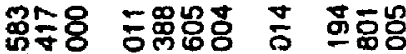

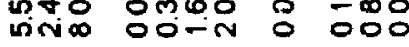

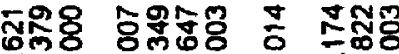

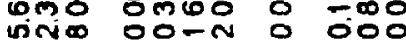

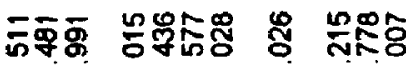

nnir oofn o coo

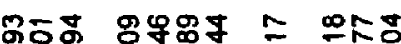

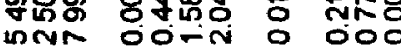

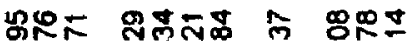

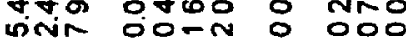

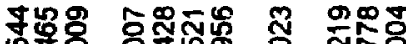

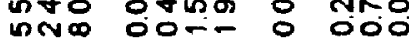

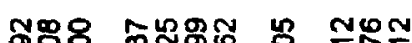

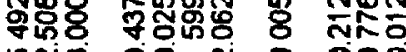

nNo 0O-N O 000

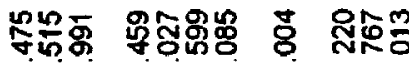

Unit ODFn O OOO

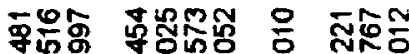

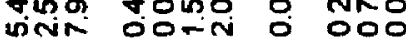

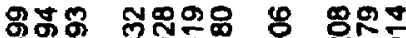

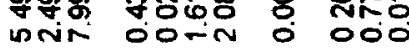

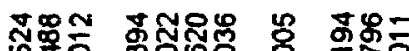
m.

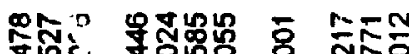
जNंक OO-N O 000

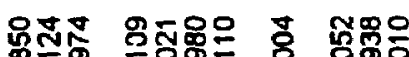

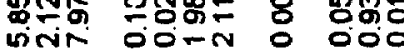

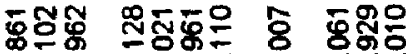

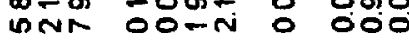

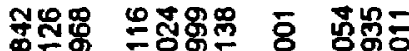
mกi OOON

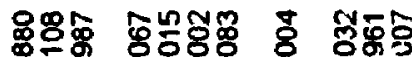
vint gonn o 000

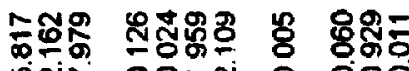
inNi oon o 000 कर $x$ ×ல் 푬-m

유뀽ำ둔 $\frac{1}{6} \tilde{D} \operatorname{din} 000$

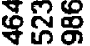

吾覃戺

ร

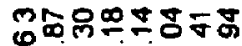

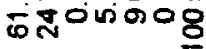

$00 \%$

OO-N

000

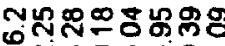

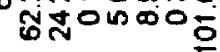

wer oOn

ลิ่กํํํㅇ

จ八

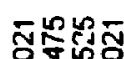

nNT oo-n

옹 깻ㅇㅇㅇ

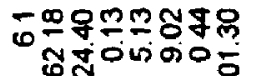

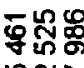

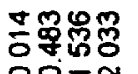

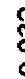

000

造

(n)

00-

路

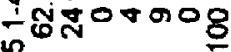

พับ

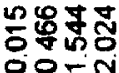

ถั

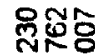

ชักำํำ

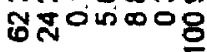

중 जñ

$\pm n=8$

용요

8

\%o

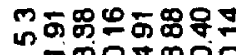

รู้

呵密品

जint oci-Ni

영

증ํㅗㅇ용

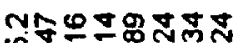

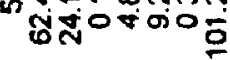

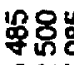

음웅ํํㅇㅇㅇ

8

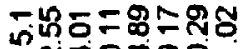

ner

OO-N

충ㅇㅇ

성ํण 005

gु̛ğ

궁훙ํํㅇ

8

ㅊํํ융

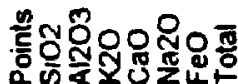

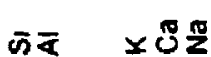

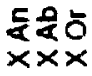




\begin{tabular}{|c|c|c|c|}
\hline 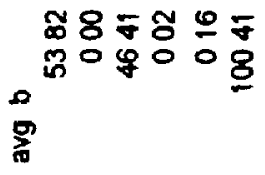 & 뚱응 & テ & : \\
\hline 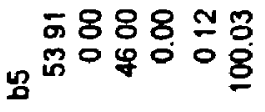 & 음융응 & 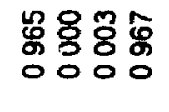 & 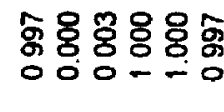 \\
\hline 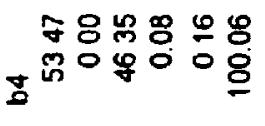 & 웅융응 & 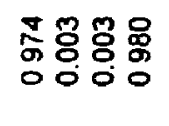 & 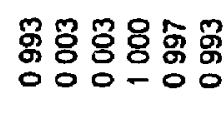 \\
\hline 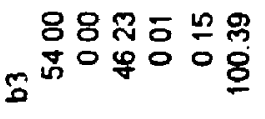 & 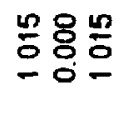 & 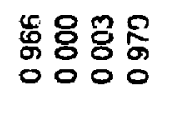 & 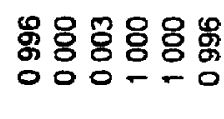 \\
\hline 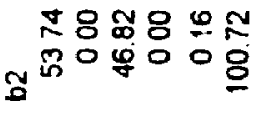 & ㅇㅇㅇㅇㅇㅇㅇㅁㅇ & 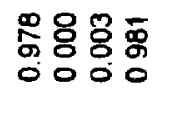 & 형응응용영 \\
\hline 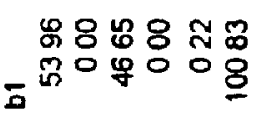 & 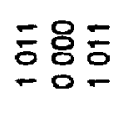 & 중융영 & 녕ㅇㅇㅇㅇㅇㅇㅇㅇㅇ용 \\
\hline
\end{tabular}

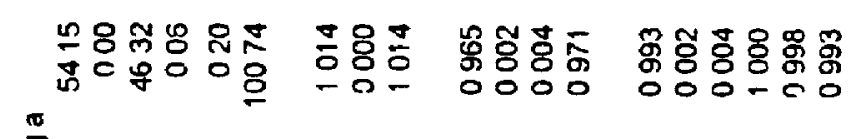

范

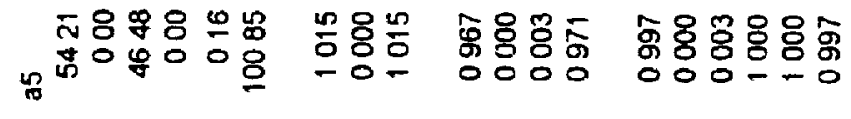

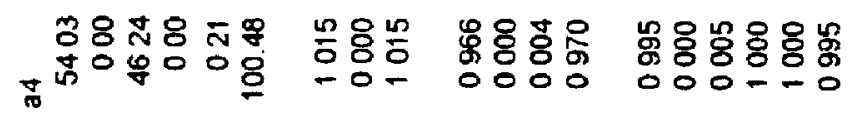

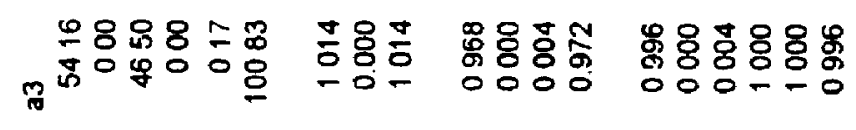

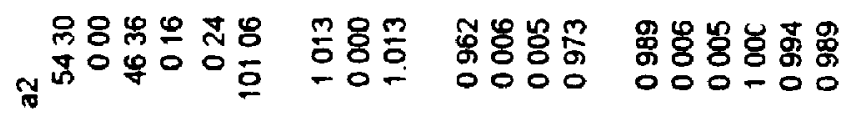

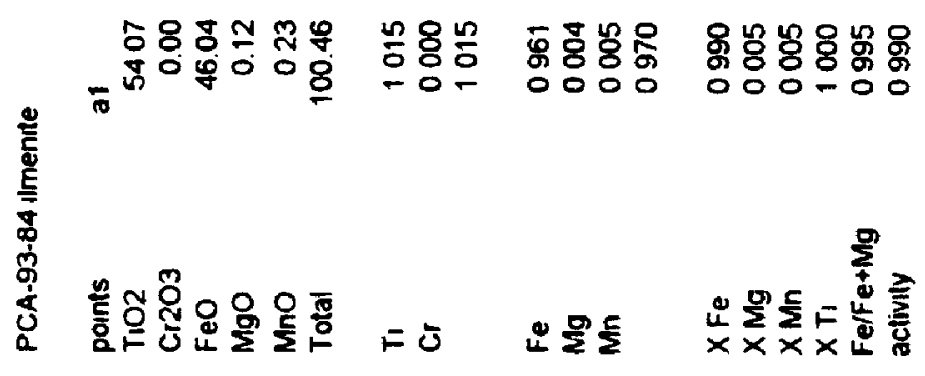




\begin{tabular}{|c|c|c|c|c|c|}
\hline$\nabla$ & 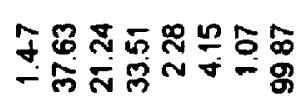 & 我客亯 & $\underset{\forall}{\stackrel{g}{g}}$ & 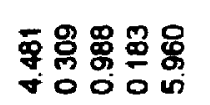 & $\begin{array}{l}\overline{8} \\
0 \\
0\end{array}$ \\
\hline 00 is & 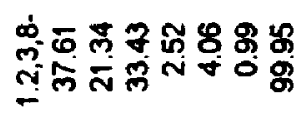 & 옹ํํ윰 & $\underset{\sigma}{\sigma}$ & 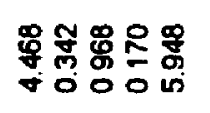 & 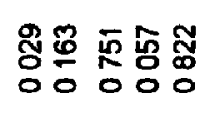 \\
\hline $\bar{x}^{2}-$ & 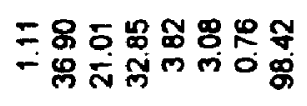 & 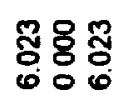 & $\begin{array}{l}\bar{\sigma} \\
\dot{q}\end{array}$ & 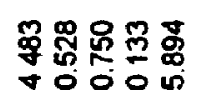 & 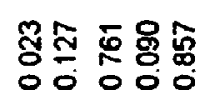 \\
\hline \multirow[t]{11}{*}{$\bar{x}^{\infty}$} & 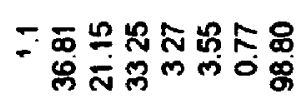 & 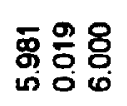 & $\underset{\mathscr{O}}{\mathscr{8}}$ & 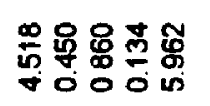 & 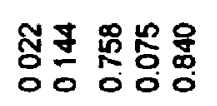 \\
\hline & 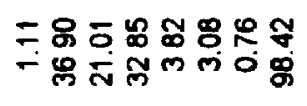 & 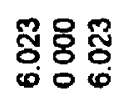 & $\underset{\dot{F}}{\dot{\sigma}}$ & 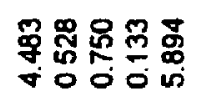 & 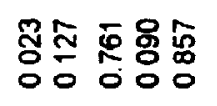 \\
\hline & 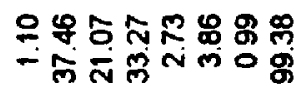 & 영 & 总 & 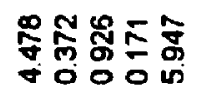 & 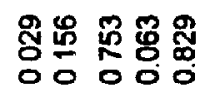 \\
\hline & 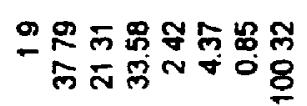 & 훙융 & $\underset{\mathbf{m}}{\stackrel{\mathbf{g}}{\mathbf{m}}}$ & 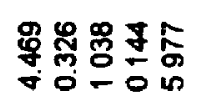 & 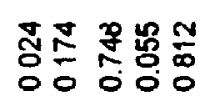 \\
\hline & 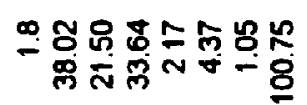 & 웅융유 & 음 & 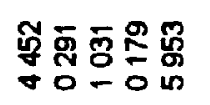 & 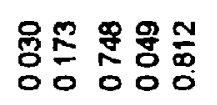 \\
\hline & 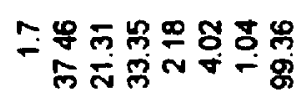 & 웅융 & 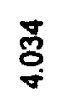 & 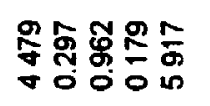 & 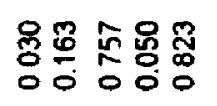 \\
\hline & 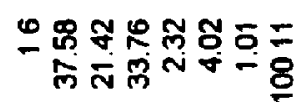 & 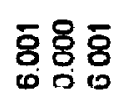 & $\underset{\delta}{\mathscr{\sigma}}$ & 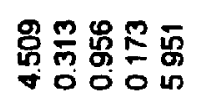 & 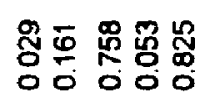 \\
\hline & 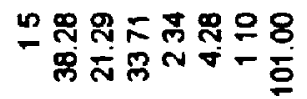 & 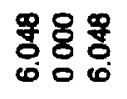 & $\underset{్}{\mathscr{\%}}$ & 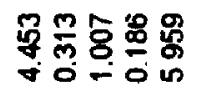 & 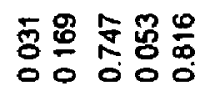 \\
\hline & 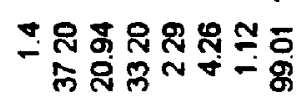 & 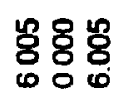 & 兽 & 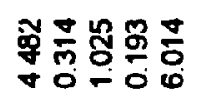 & 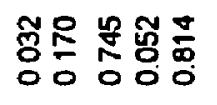 \\
\hline & 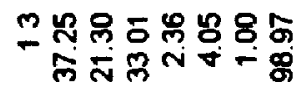 & 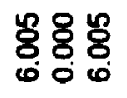 & S & 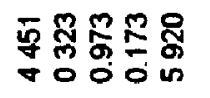 & 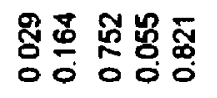 \\
\hline & 풍 & 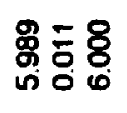 & 8 & 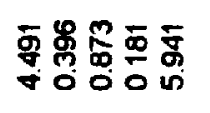 & 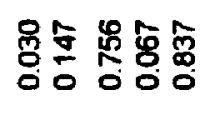 \\
\hline 동 & 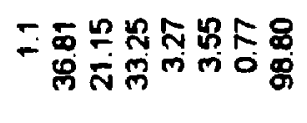 & 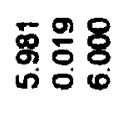 & $\underset{8}{8}$ & 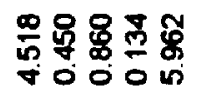 & 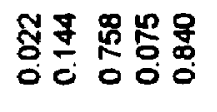 \\
\hline 2 & 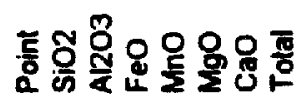 & कर & $\bar{\alpha}$ & $\mathbf{u}$ & \\
\hline
\end{tabular}




\begin{tabular}{|c|c|c|c|}
\hline 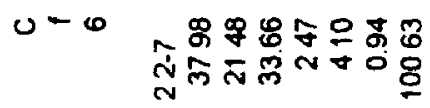 & 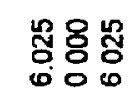 & 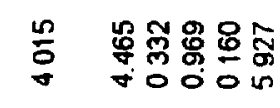 & 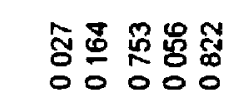 \\
\hline 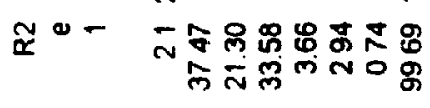 & 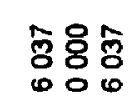 & 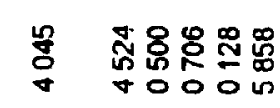 & 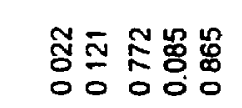 \\
\hline 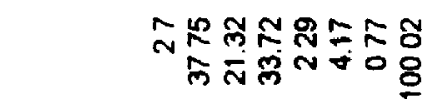 & 苛务 & 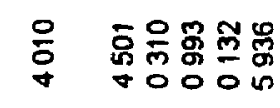 & 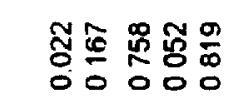 \\
\hline 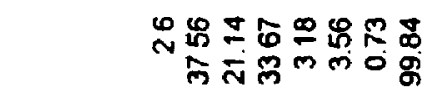 & 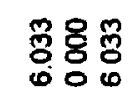 & 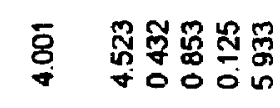 & 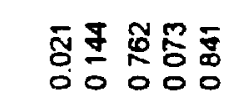 \\
\hline 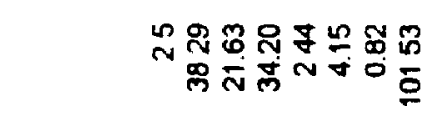 & 종융융 & 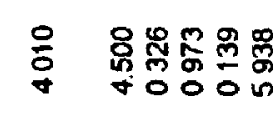 & 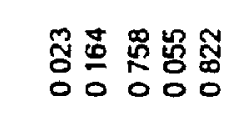 \\
\hline 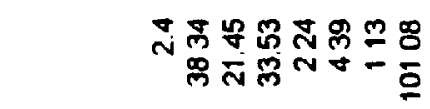 & 궁용 & 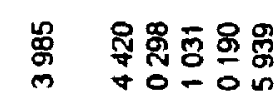 & 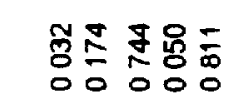 \\
\hline 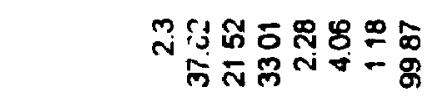 & 영영 & 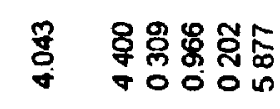 & 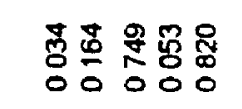 \\
\hline 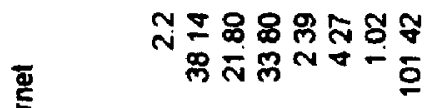 & $8 \overline{8}$ & 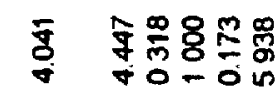 & 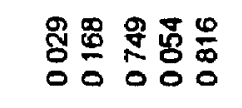 \\
\hline 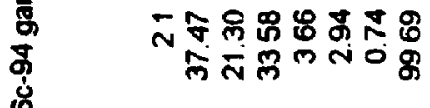 & 商客管 & 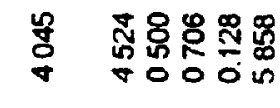 & 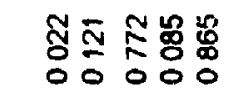 \\
\hline 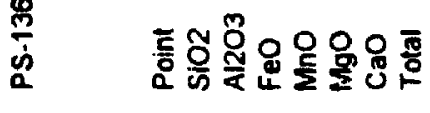 & & $\bar{a}$ & \\
\hline
\end{tabular}




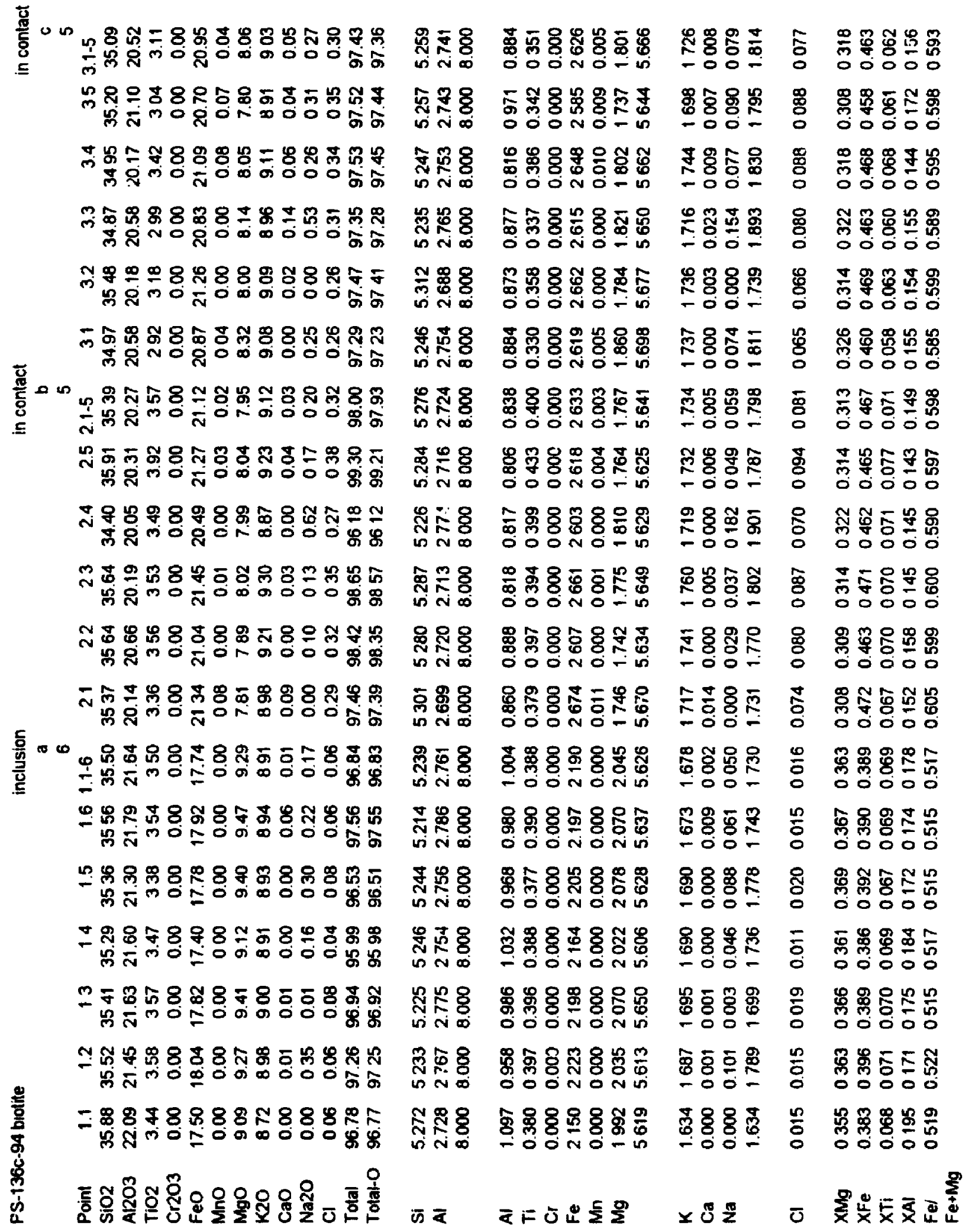




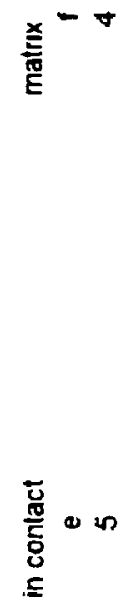

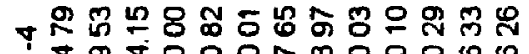

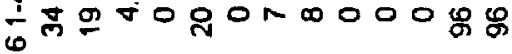

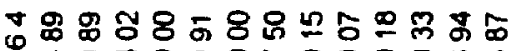

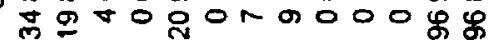

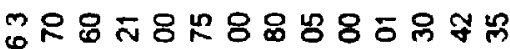

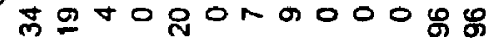

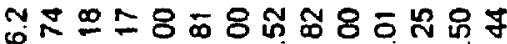

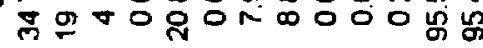

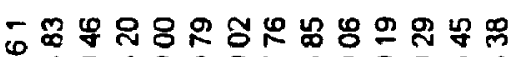

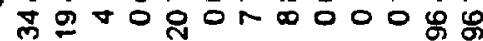

- ๓

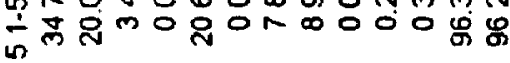

นกำำ

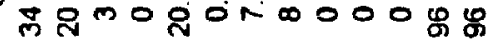

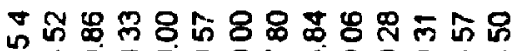

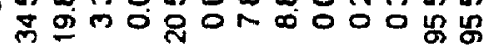

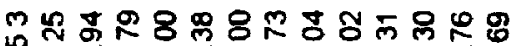

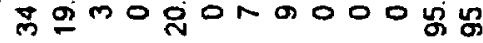

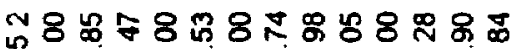

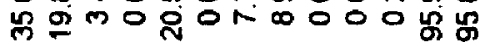

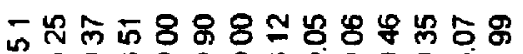

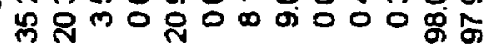

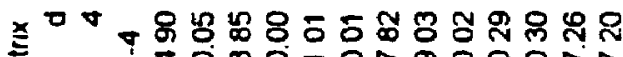

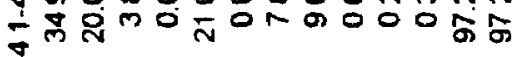

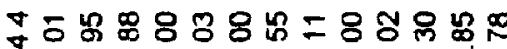

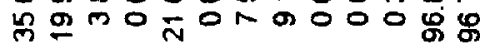

พำ

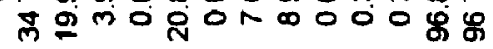

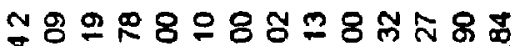

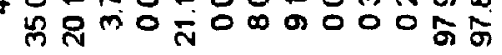

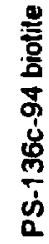

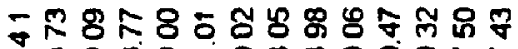

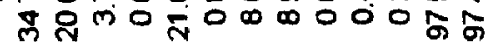

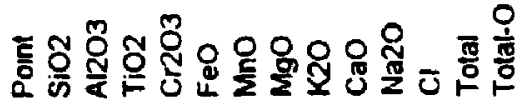

$\stackrel{\infty}{*} \mathbb{8}$

แn $N$

过色家

แ $\sim \infty$

웅ㅇㅇㅇ

แ. $\sim \infty$

음 앵 8

in

노용

แ $N$

융용

in $N$ D

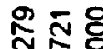

in $N$ 舟

요용

in $N \infty$

요요

is $N$ 我

的悉 8

in

的是 8

in $N \infty$

产负各

in $N$

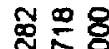

in $N$

急苂

in $N$ क

武喿䂞

in

윰 in $N$ D

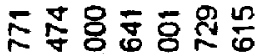
OOONOI U

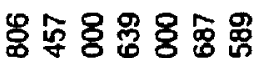

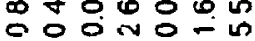

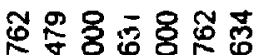
O O O NO- M

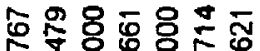

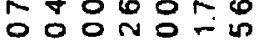

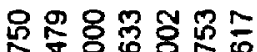
o o ono-n

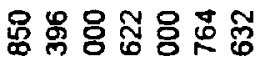
OOONO=-

-

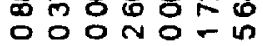

్ㅐㅁㅇㅇㅇㅇㅇㅇㅇㅛ O OONO-

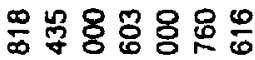
OOONO

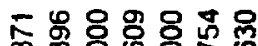
O

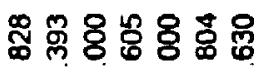

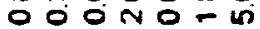

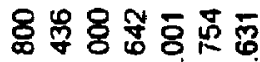
OOONOL

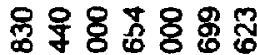

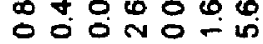

ㅇํํ 突 OOONOTW

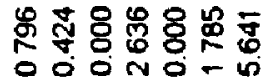

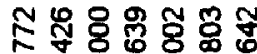

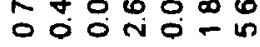

윰융용용

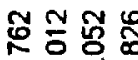
- 0 O-

운잉용옹 - $00-$

윰융용

읏응배ㅇㅛㅛ

-O0-

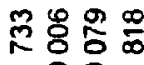

- 00 -

두요요용

- 00 -

츗웅 品

- 00 -

융용

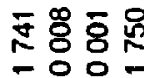

호유ㅇㅛㅛ

- 00 -

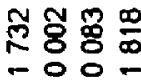

슝ㅇㅇㅇㅇㅇ용 등응

융용

- $00-$

욛융

총용 号号

둥

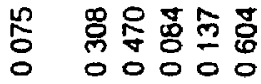

\&

号商尊总总 - 00000

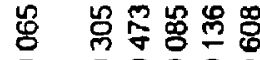
00000

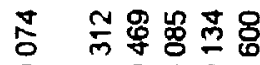
- 00000

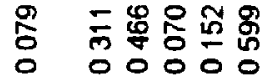

능

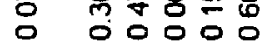

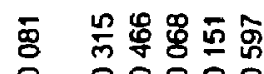
- 00000

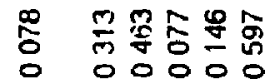

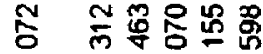
- 00000

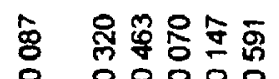

号 둥 尊令吉 O 00000

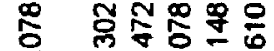
- 00000

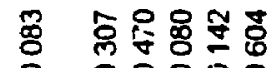
- 00000

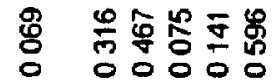

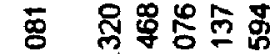
잉ㅇㅇ

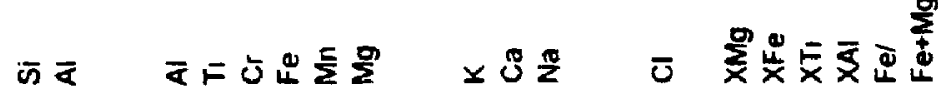




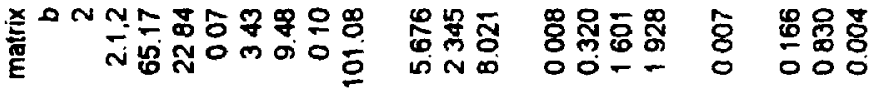

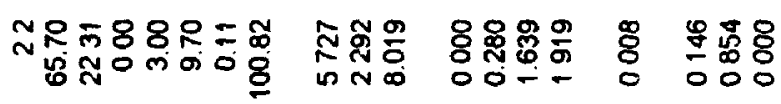

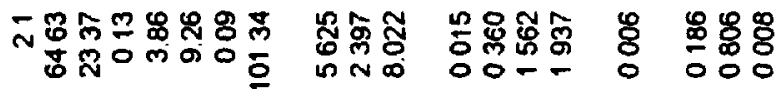

肴"

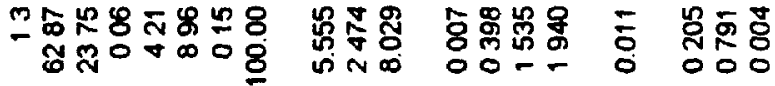

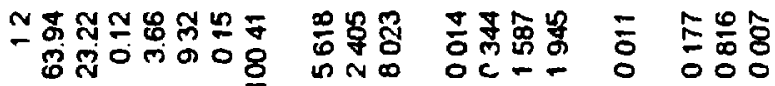

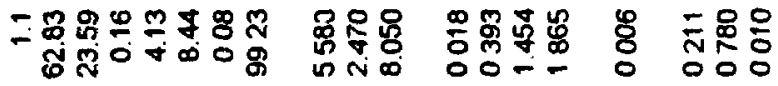

$\frac{n}{\frac{6}{0}}$

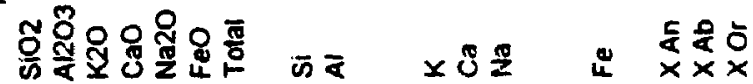




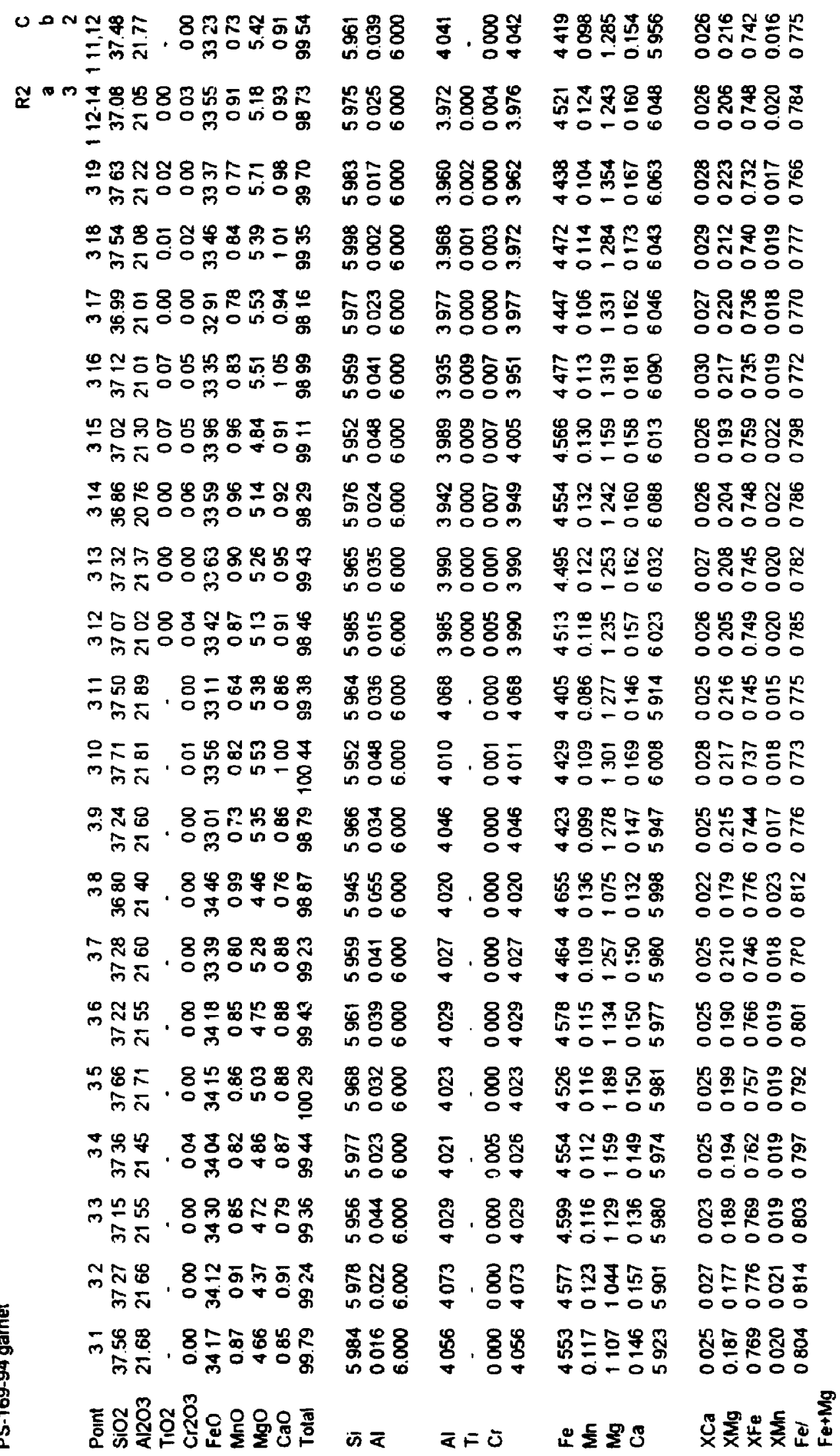




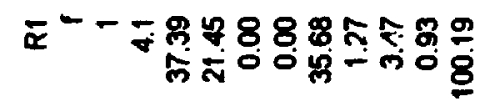

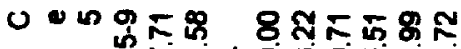

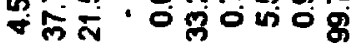

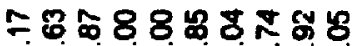

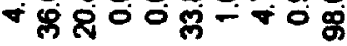

응

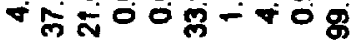

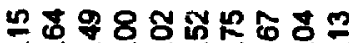

जिए人

죽요

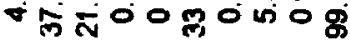

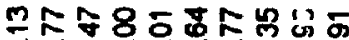

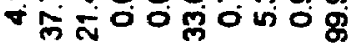

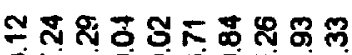

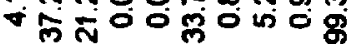

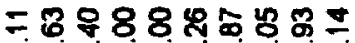

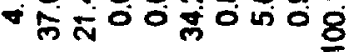

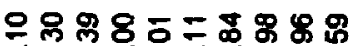

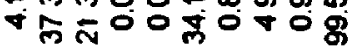

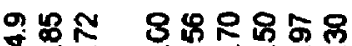

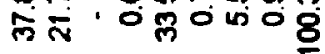

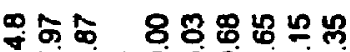

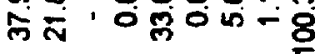

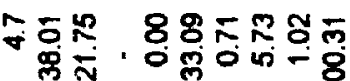

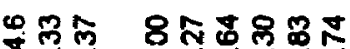

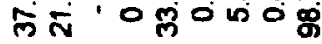

守的电 8

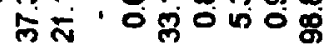

平米禺 $8=8 \%$

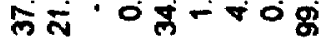

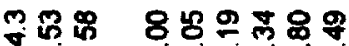

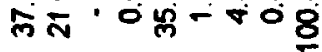

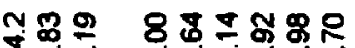

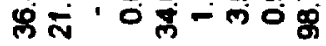

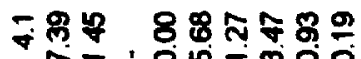

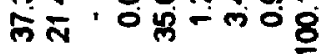

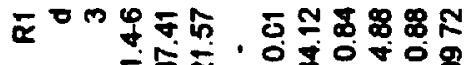

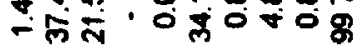

产

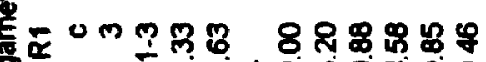

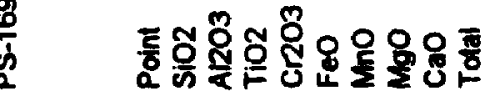

$\overline{8} 8$

รั

\$ํㅡㅇ

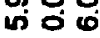

弯骂

ज० 0

$8 \%$

iso.

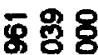

in 0

\%영

ำ

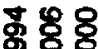

15.

유요

5ow

요요을

(5)

$8 \%$

เn.

융ำ

mo.

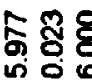

另홍

nก

\$:

两

(5)

娄

웅요

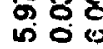

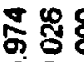

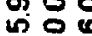

$\overline{8} 88$

no.

858

is

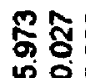

क व

б.8 8

帘.

$888 \%$

깅유

क्षे

ณ๐్రิ:

लํㅇㅇㅛ

웅ㅎㅇ응

웋웅훙후

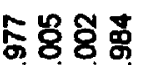

ल00

용용용

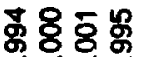

ल०0ल

市. 察:

융. 8

+

홍. 8

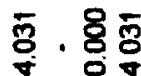

资.

$50 \mathrm{~m}$

둥. 80

$=8 \overline{0}$

\% 8

4.0

通.

ธ్ํ.

身.
당요

$+0 \%$

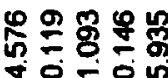

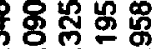

$\checkmark 0$ -

要费是용

\% -

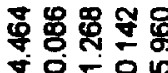

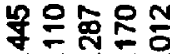

두응

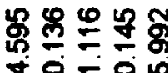

영응요용

ن.

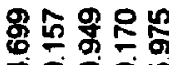

To0o in

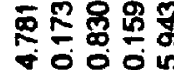

证两

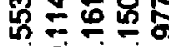

o- ón

형듕영

ธิ์

00000

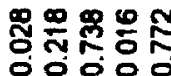

응ㅇㅇ

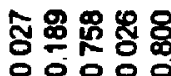

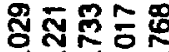

00000

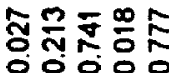

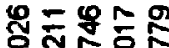

엉ㅇㅇㅇ

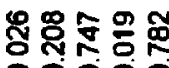

00000

옹옹옹응

웅ㅇㅇㅇ

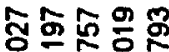

00000

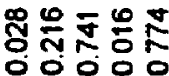

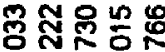

엉ㅇㅇ

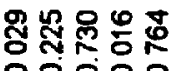

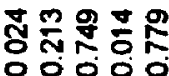

象요용

ำ

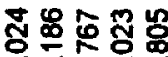

엉ㅇㅇ

종종ํㅇ응

OO $00^{\circ}$

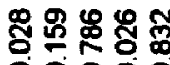

옹 종

ㅇํㅇㅇㅇㅇㅇㅇ

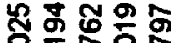

엉ㅇㅇㅇ

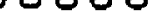

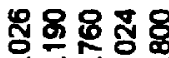

\section{㩆}

สํ

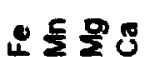

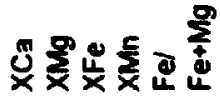




\begin{tabular}{|c|c|c|c|c|}
\hline 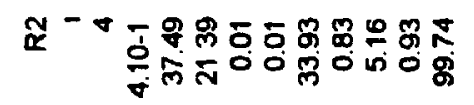 & 행요 & 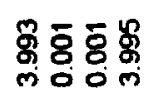 & 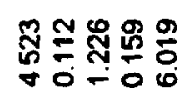 & 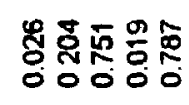 \\
\hline ᄃ & 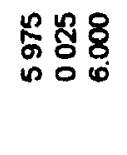 & 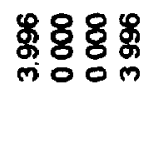 & $\frac{0}{\frac{9}{6}} \frac{9}{0} \frac{8}{0} \frac{0}{0}$ & 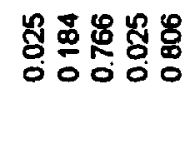 \\
\hline - F 媳 & 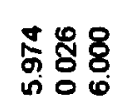 & 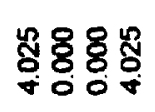 & 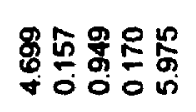 & 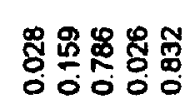 \\
\hline 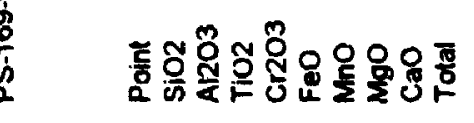 & & $=5$ & 5 & 89 \\
\hline
\end{tabular}




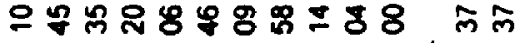

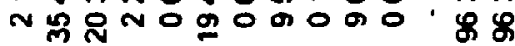

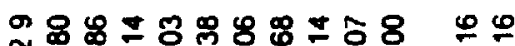
MONOSOOOOO

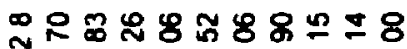

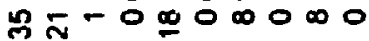

ก 承

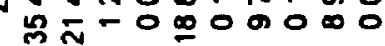

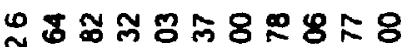

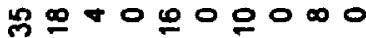

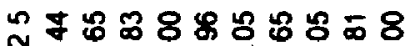
只 $9000=000$

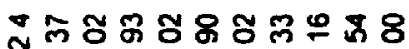

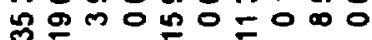

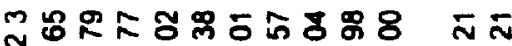

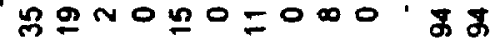

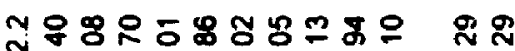
MNONO NODO. \&

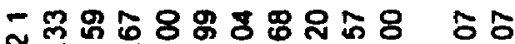
周으응 =0

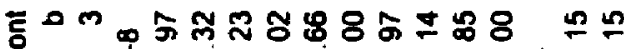

ํำ

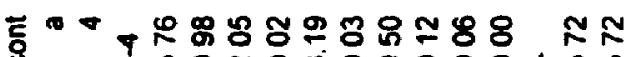

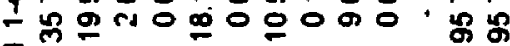

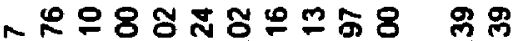

工出需

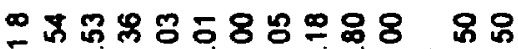

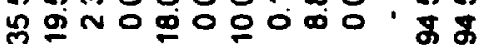

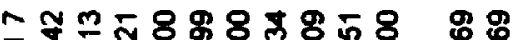

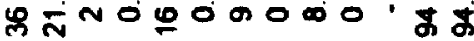

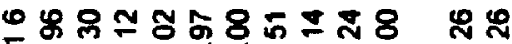

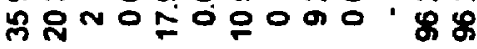

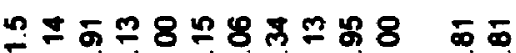

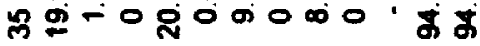

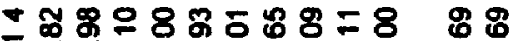

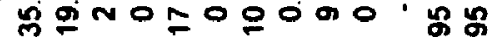

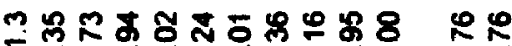

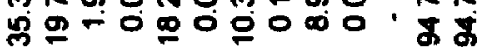

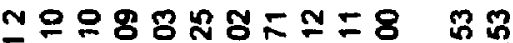

\& \& NO

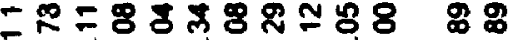

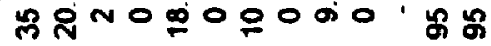

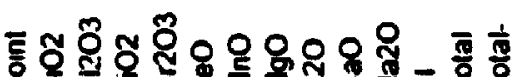

के

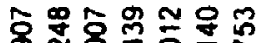

กิก

กิำ

กิธ่용

O- 0 -

흉용:

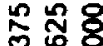

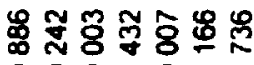

o o ononin

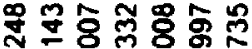

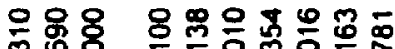

nNa -OONONin

กิ 8

ले

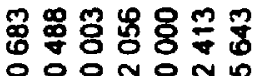

-

i

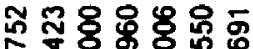

엉ㅇㅇㅇㅇㅇㅁㄴ

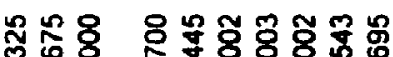

in $\infty$

OOONON

品 $\overline{8}$

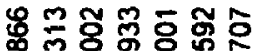

ก

00 O - ON

苛高8

in $\infty$

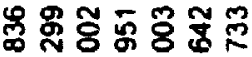

융웅

o o o- on m

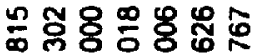

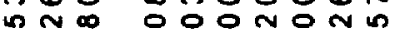

禺莒 8

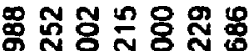

m

융용

แ $N \infty$

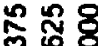

in $\sim$

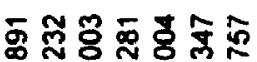

000 NON

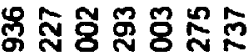

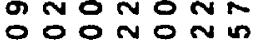

항ㅇㅇㅇ

舟

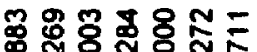
OOONONm

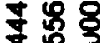

里 융융영

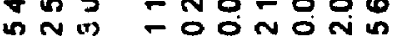

负高是 in $\sim$

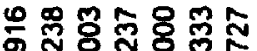
응젼ำ

t융

\%

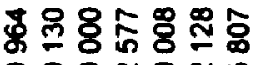

OOONON

茟号

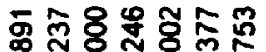

in $\infty$

OOONONE

总市

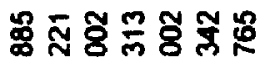

แn

O OnONL

祐

ऽN

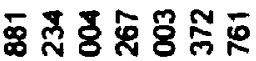

०0ำก

总表䒠

in

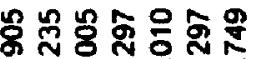

o O O N 0 N

형영용 궁

$0-0-$

웅횽용형

둉용

응음

엉 항

는

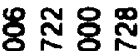

등

ธิธิ์

O-O-

용 8

응

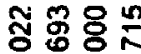

응 -

융 귝용 둔

0-0 -

총옹ㅇㅇ욤

응

겅 융ㅇㅇ 숭

O-O-

풍 \&्ठㅇ

능-

총 爷융

등

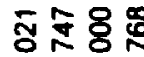

$0-0-$

능웡ㅇㅇㅇ

응응

똥쬬용

$0-0-$

응 용용

능 -

엉: 용요

-ㅡㄴ-

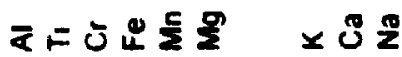

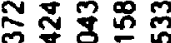

00000

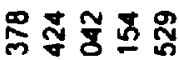

00000

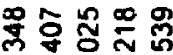

00000

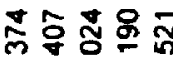

00000

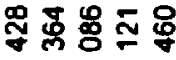

00000

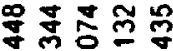

00000

㛚哭糸

00000

可㝵号 퐁

00000

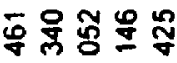

00000

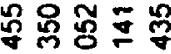

00000

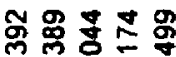

00000

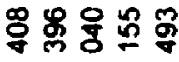

00000

馬 8 웅응

00000

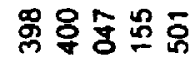

00000

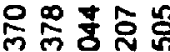

00000

항횽ㄱㅇ용

00000

号专号罢

00000

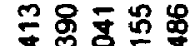

00000

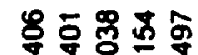

00000

국

00000

费守于战

00000

कर 


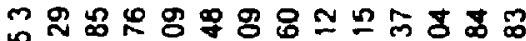

లొ

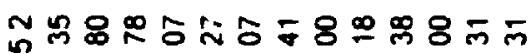

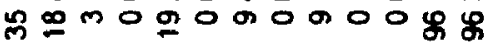

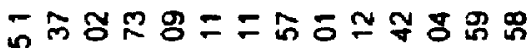

足可以

垔 $^{-n}$

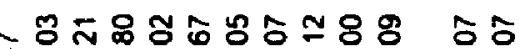

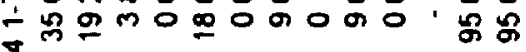

오용ㅇㄷㅇㅛ $8 \div 8=$

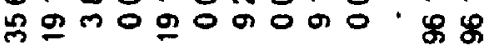

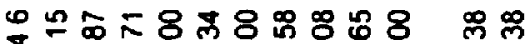

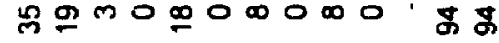

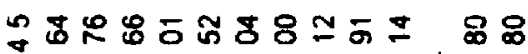

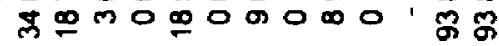

অ สั

స్

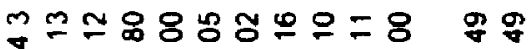

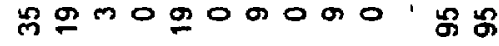

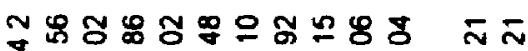

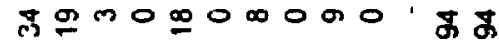

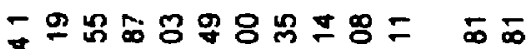

品900000000.

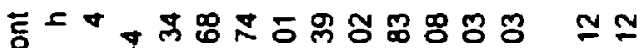

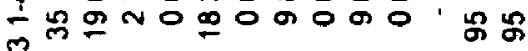

-

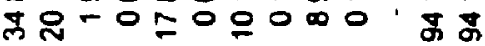

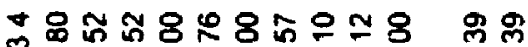

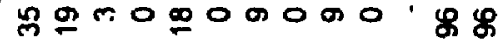

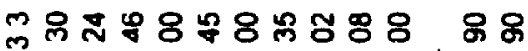

nूm

उ

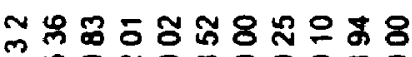

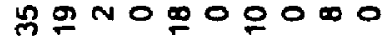

$8 \%$

ณ ะ

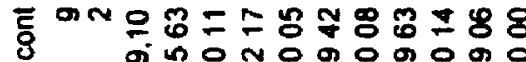

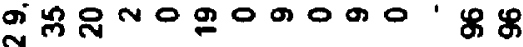

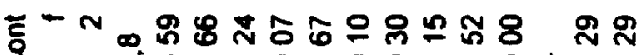

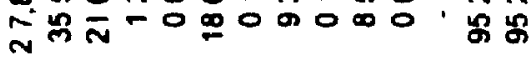

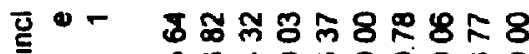

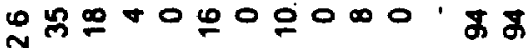

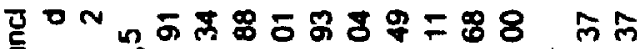

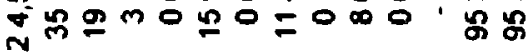

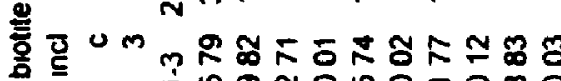

ํํㅇㅁำ

$\$ \&$

あ る

\$

龺

के

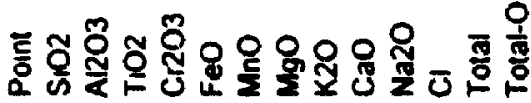

串용

in $N$

సิ

in $\infty$

遍 槵 号

in $N$

象芯

i $\sim \infty$

金总

ก $\sim$

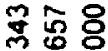

in $N$

总莙员

in $\infty$

沾芒

ก $\infty$

్ㅠ:

in $\infty$

율: 卷

แก $N$

옹ㅇㅇㅇㅇㅇ

in $\sim \infty$

幽骂

in $\sim$

今.

ก $N$

鬲总

ก

总得客

in $\infty$

앵융요

in $N$

第客各

in $\infty$

第罚 i

㻤

in $\infty$

需若

in

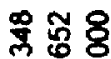

$n \sim \infty$

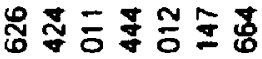

O0ㄱㅇㅛ

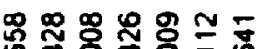

0ㅇㅇㅇㅇㅠ

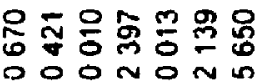

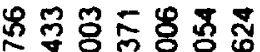

응엥유

籿等 8 \& $\overline{8} \overline{8}$

OOONON

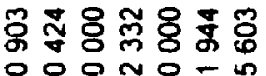

テ

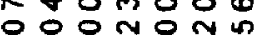

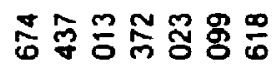

o o onon m

กี้

OOONON W

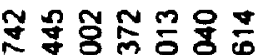

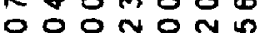

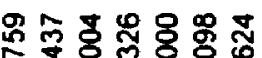

OOONON

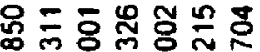

00N에

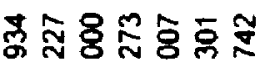

O O ONONn

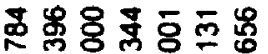

OOONON

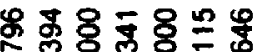

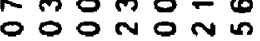

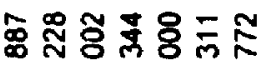

OOONONH

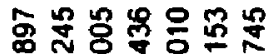

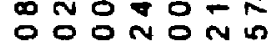

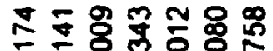
- O0 Non

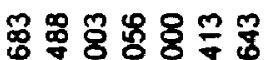

OOONONM

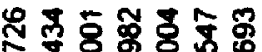

ㅇํㅇ융ㅇㅇ

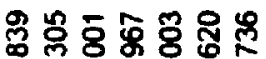

$000-0$ in

$\bar{s} \bar{\alpha}$

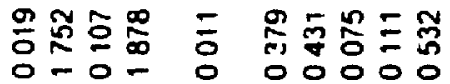

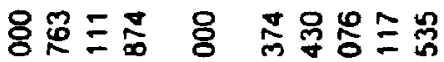

등 영ㅇㅇㅇ

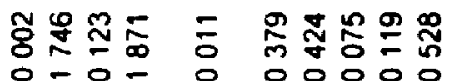

융공్ㅇㅇㅛ

$0-0$ -

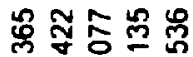

OOOOO

웅 学点

$0-0-$

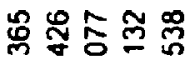

둥융

능

00000

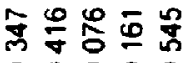

00000

六员产要

芦莳合監

00000

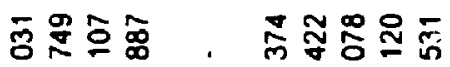

O- 0 00000

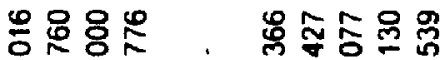

등 00000

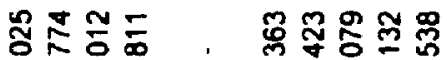

0- 잉

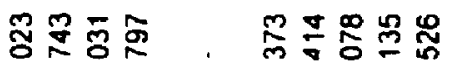

O-O- 00000

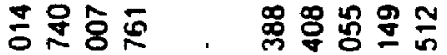

승 0000

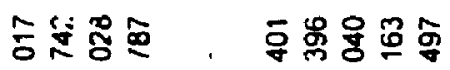

O-0- 00000

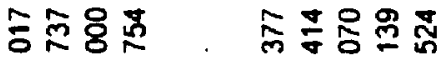

0-0- 00000

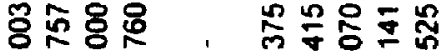

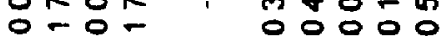

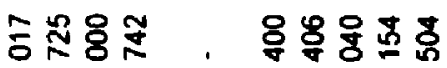

O-0 00000

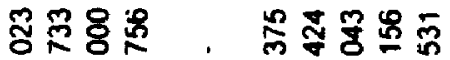

O-O- 00000

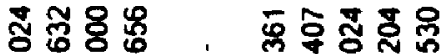

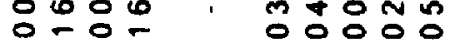

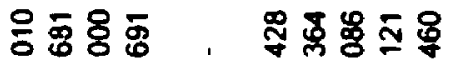

O-0- 00000

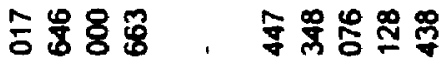

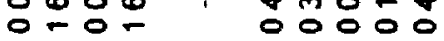

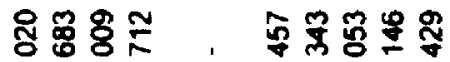

O음

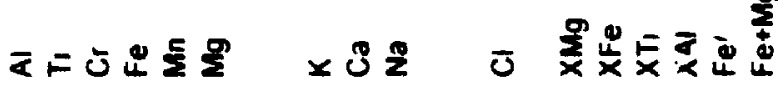




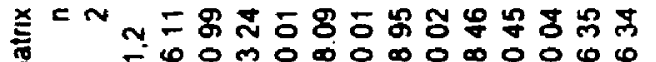
E б̆

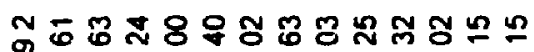

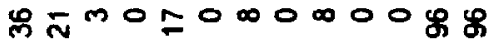

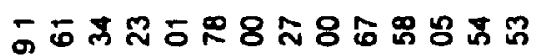
品沓

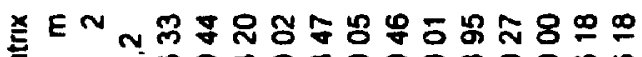
ह

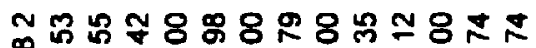

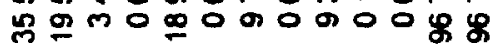

ธェ 的NOニO

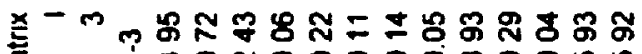

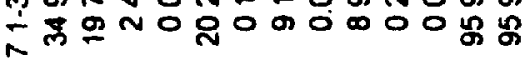

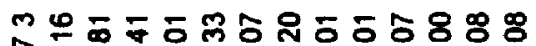

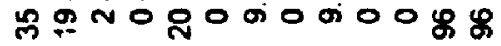

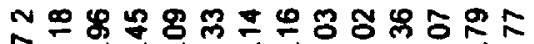

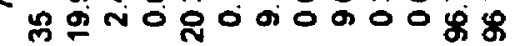

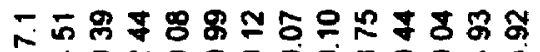

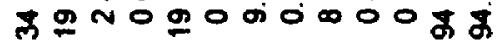

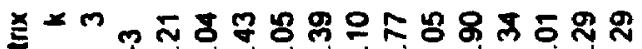
है 웅 8 ₹

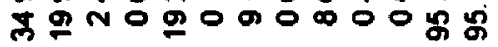

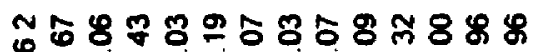

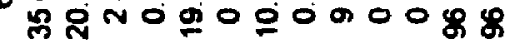

-

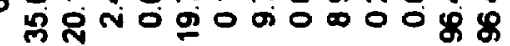

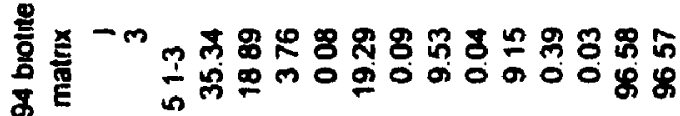
क क $\grave{b}$

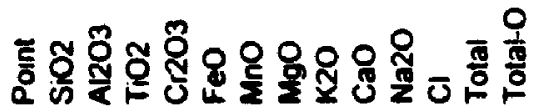

$\frac{10}{5}$ in 용용 ก $\sim \infty$ 융용 in $N$ ํํㅇㅇㅇ ถึ 㟒费 in $N$ 今ิ in $\sim$

ฝิํํㅇㅇㅇ 的 $\infty$

象迢 ก $N$

: 政

옥응 in $\infty$

蛋ํㅛㅇ in $\sim \infty$ 蛋 NN冓

N.:용 in $N$

运罂家 ज脑 司器䂞 N $\infty$

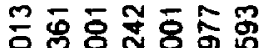
-

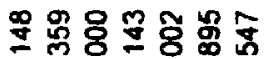
- 0 ONO

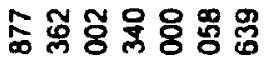
OOONON In

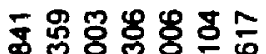

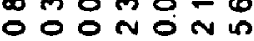

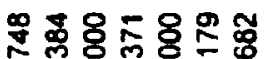
OOONON

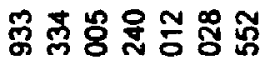
OOONON

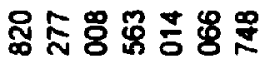
oOOnON

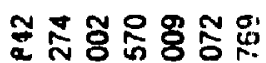
o OO nO N 踶

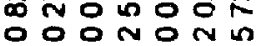

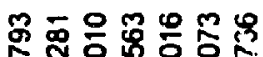
OOO N

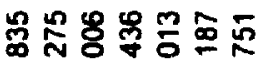
OOONON

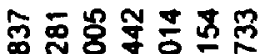

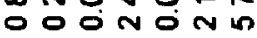

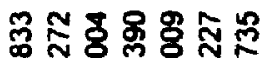
OOONON

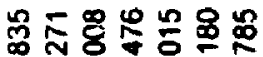

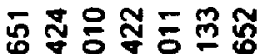

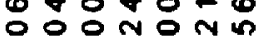

कर oㅇํㅇำ

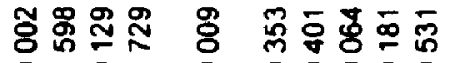
0-0- 000000

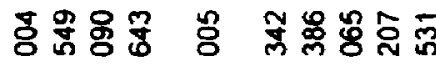
0-0-0 00000

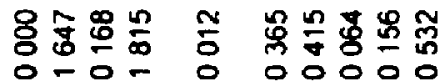

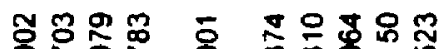

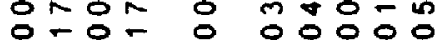

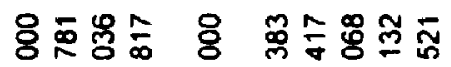
0-0-0 00000

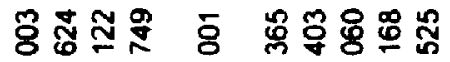
$0-0-000000$

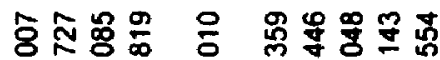
Oㅇㅇㅇ 0 영ㅇㅇㅇ

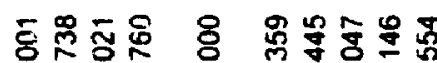
O-0- 0 00000

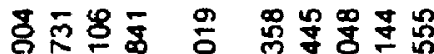
등ㅇㅇㅇ 융융

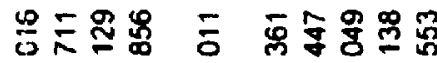

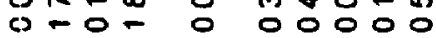

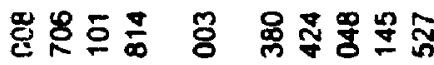
ㄷㅇ - 0 000 00

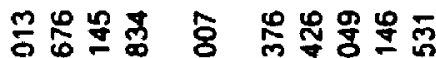

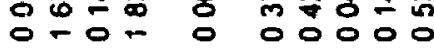

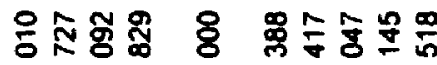
0-0- 000000

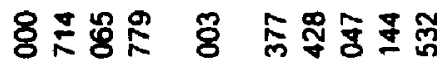
- -0- 0 00000

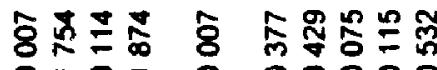
O-0- 0 00000 ストさ出定是 $\quad$ ×

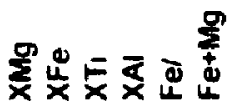




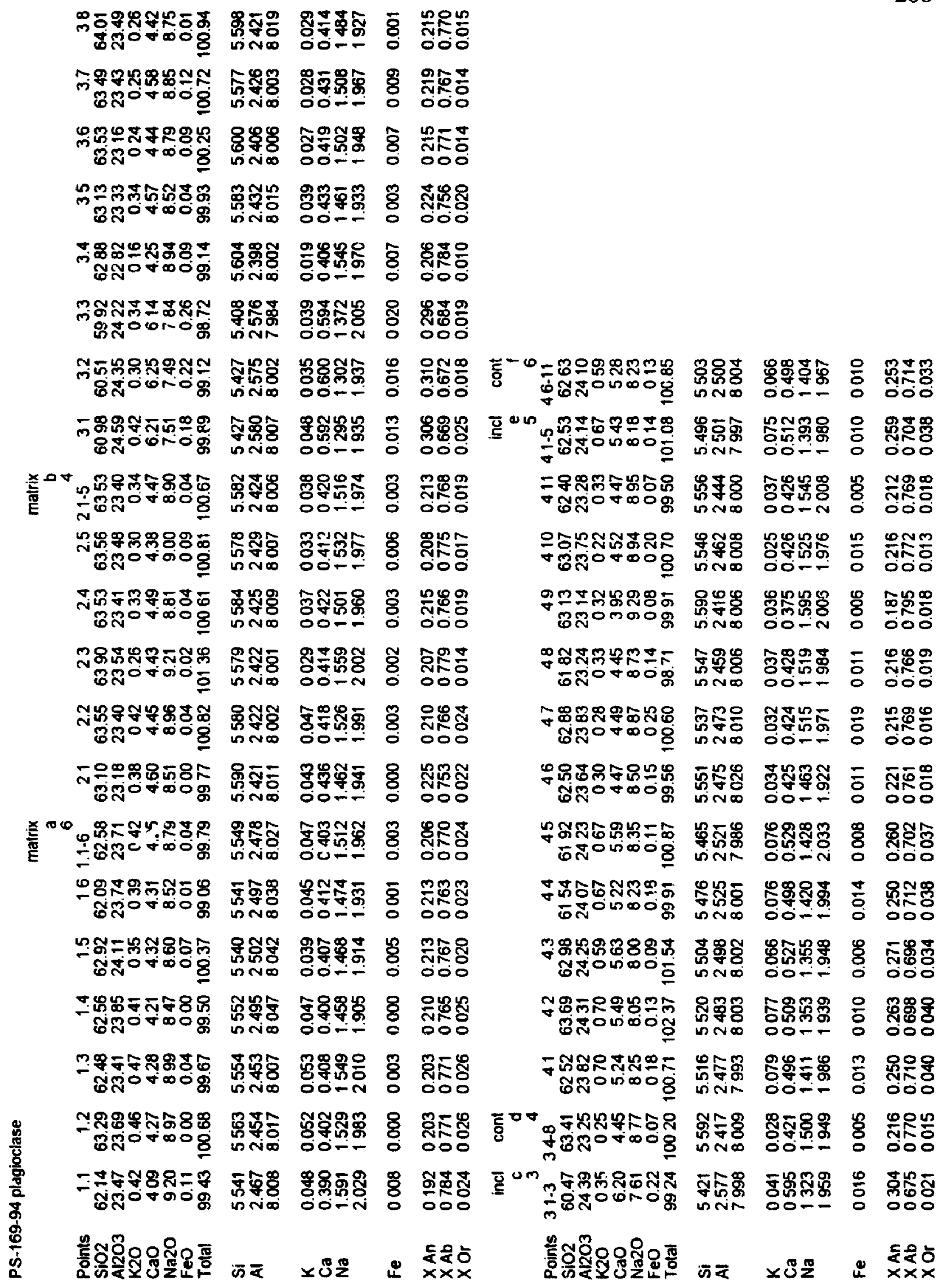




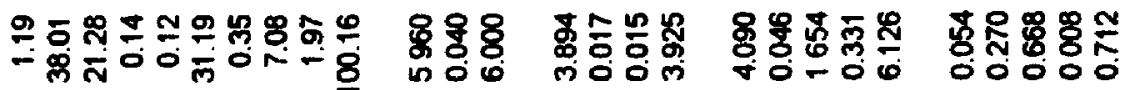

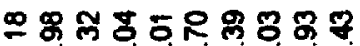

一

岁농용

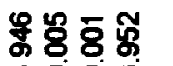

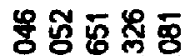

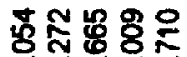

ニ

ํㅜㅇ

móㅇ.

$\div 0-0.0$

0000

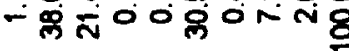

悉응푱

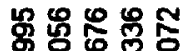

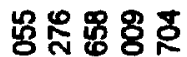

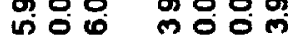

लि口- 0

00000

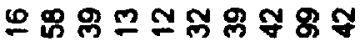

-

\% 용

춘도응

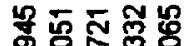

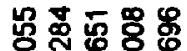

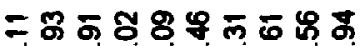

罗

응응

लं० 00

$\circ 0000$

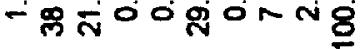

जi

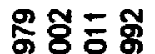

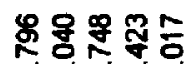

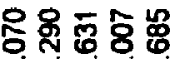

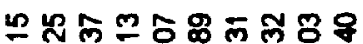

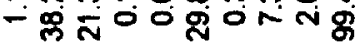

8

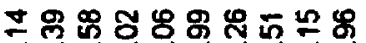

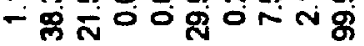

영동

m०ठ

웅ㅇㅇㅇ

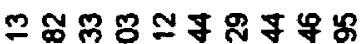

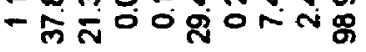

量实

냉융용

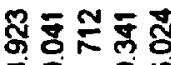

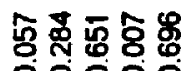

8ิ

mo-06

00000

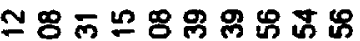

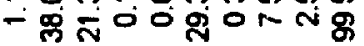

n० mo

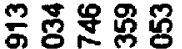

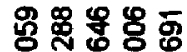

iं

mo $=00$

웅영

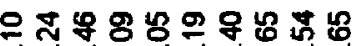

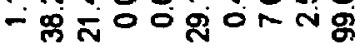

速 $\overline{8}$

令路동

总兽守电宁

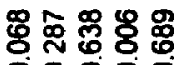

串웅융 쑝

mo 00

00000

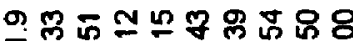

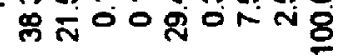

б 용용

तi $00 \mathrm{O}$

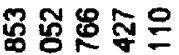

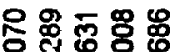

ஜㄷ요용

लi 000

융ㅇㅇㅇ

no 0 mo

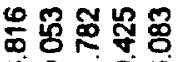

웅휴웡용

용

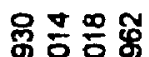

(i) -00

00000

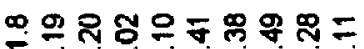

需

in o

ल웅엉

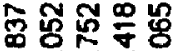

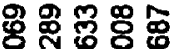

용 용

ช్ㅇํㅇํํㅇ

mo

00000

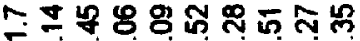

需

。

m

吕多员恋采

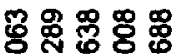

麦옹

ธ్ㅎㅇㅎㅇ용

00000

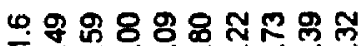

की

영 옹

लि

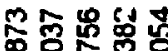

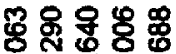

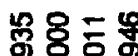

i응

Oㅇํㅇㅇㅇㅇ

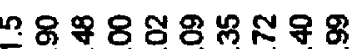

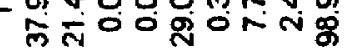

8

ल०

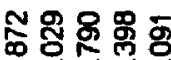

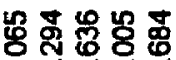

웅요유

ल० - 0

00000

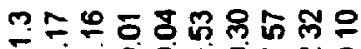

no 0

लㅇㅇㅇ

영 둥유용

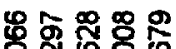
mó- 0 00000

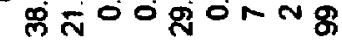

웅 8

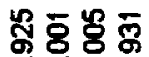

韋웡용 용

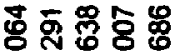

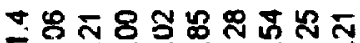

界

영응용

लㅇㅇㅇ

MO -00

00000

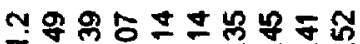

角

เ 0

్ㅐㅇㅇㅇ ్ㅇㅇ శ్

合苍界尽

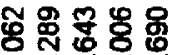

适

m $00 \mathrm{~m}$

i०

00000

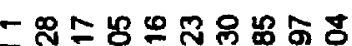

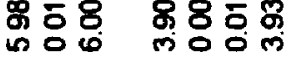

영웡 웡

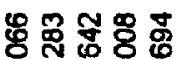

- 总

\& 8

응 웅형

o 000

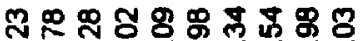

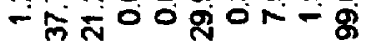

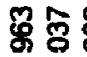

영용ํํำ

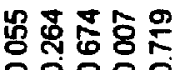

$28=$

- 0 - 0

0000

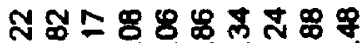

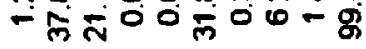

엻응

궁용

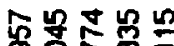

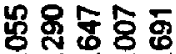

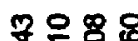

ल0 00

○० 000

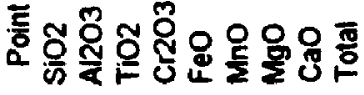

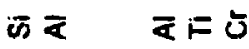

연돌로용

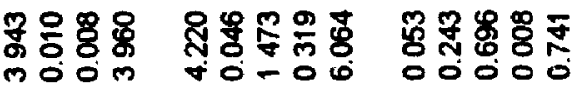

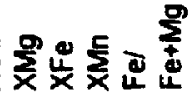




\begin{tabular}{|c|c|c|c|c|c|}
\hline \multicolumn{2}{|c|}{ 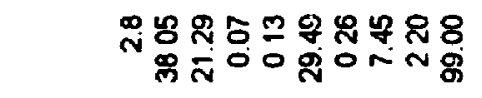 } & 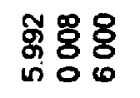 & 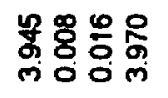 & 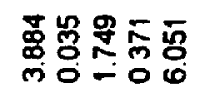 & 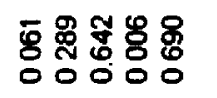 \\
\hline & 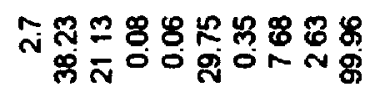 & 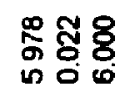 & 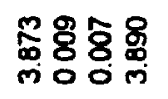 & 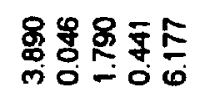 & 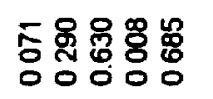 \\
\hline & 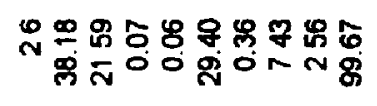 & 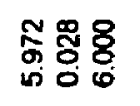 & 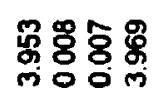 & 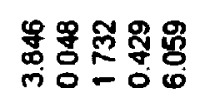 & 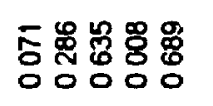 \\
\hline & 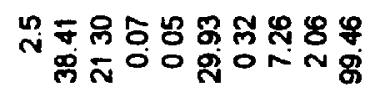 & 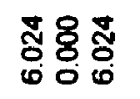 & 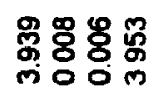 & 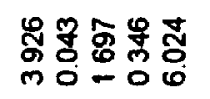 & 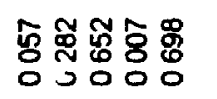 \\
\hline & 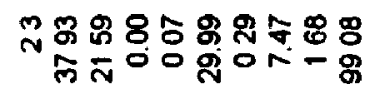 & 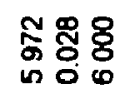 & 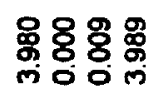 & 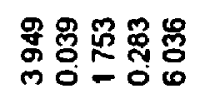 & 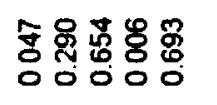 \\
\hline & 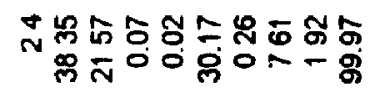 & 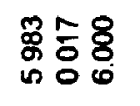 & 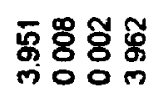 & 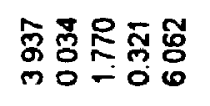 & 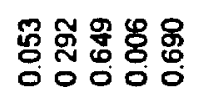 \\
\hline & N & 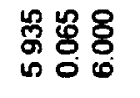 & 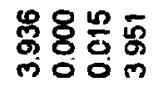 & 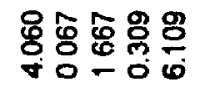 & 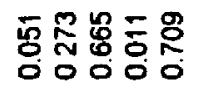 \\
\hline & 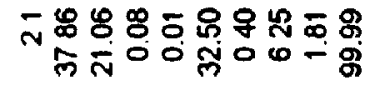 & 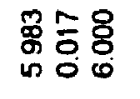 & 兽응흉효 & 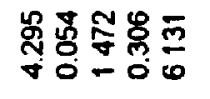 & 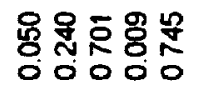 \\
\hline $\mathscr{X}$ & 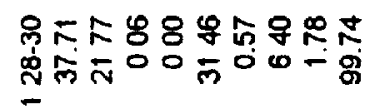 & 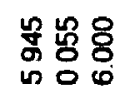 & 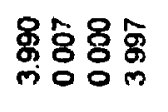 & 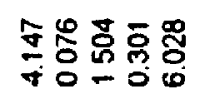 & 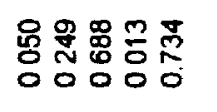 \\
\hline & 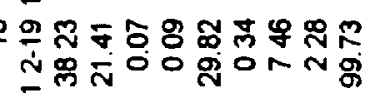 & 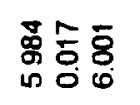 & 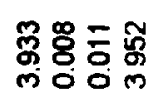 & 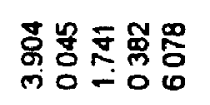 & 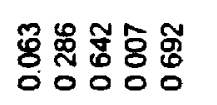 \\
\hline $\bar{\alpha}^{0}-$ & - & 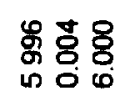 & 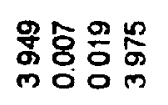 & 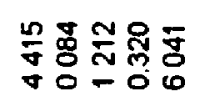 & 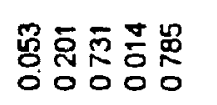 \\
\hline$\approx \pi \mathrm{m}$ & 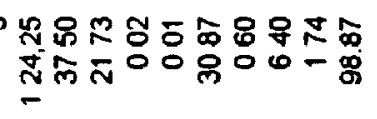 & 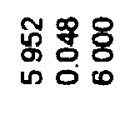 & 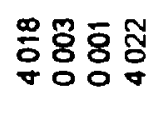 & 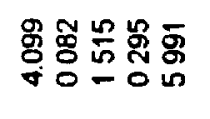 & 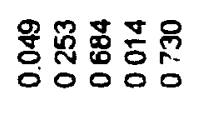 \\
\hline & 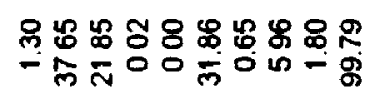 & 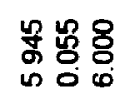 & 뚱ㅇㅇㅇㅇㅇㅇ윰 & 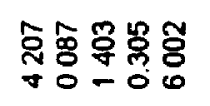 & 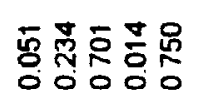 \\
\hline & 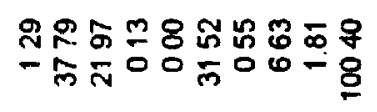 & 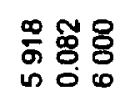 & 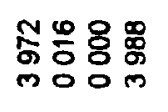 & 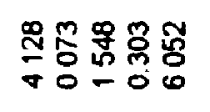 & 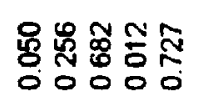 \\
\hline & 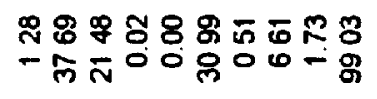 & 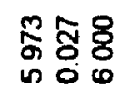 & 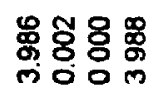 & 矛若否点品 & 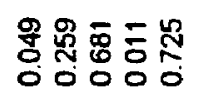 \\
\hline & 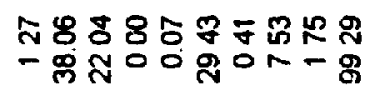 & 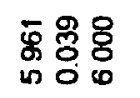 & 융잉영 & 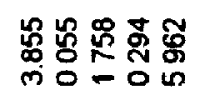 & 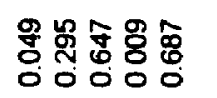 \\
\hline & 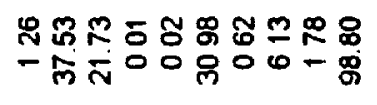 & 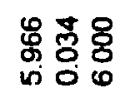 & 융ㅇㅇㅇ융 & 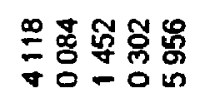 & 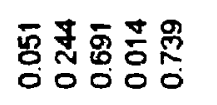 \\
\hline & 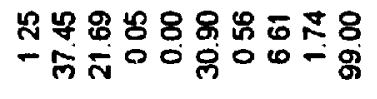 & 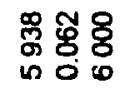 & 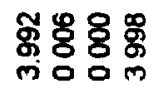 & 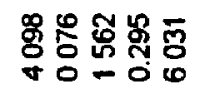 & 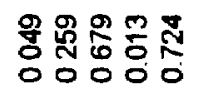 \\
\hline & 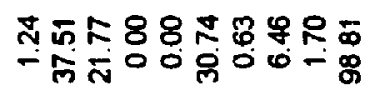 & 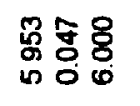 & प্口犬 & 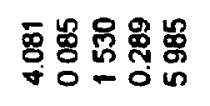 & 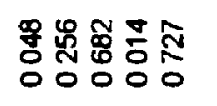 \\
\hline ฮ & స్ & 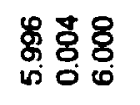 & 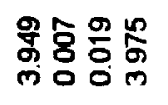 & 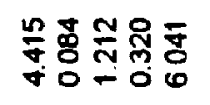 & 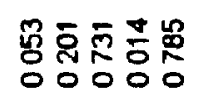 \\
\hline 宽 & 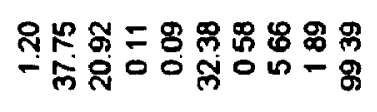 & 象 & 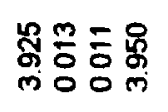 & 옹요ㅇㅝㅠ & 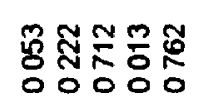 \\
\hline$\frac{\text { ণ }}{8}$ & 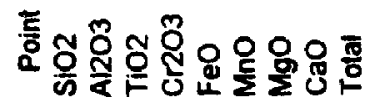 & $\bar{\infty} \bar{\alpha}$ & $<10$ & 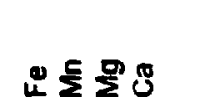 & 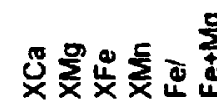 \\
\hline
\end{tabular}




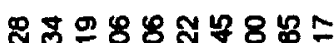

สู

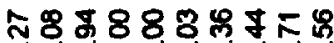

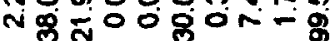

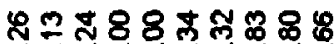

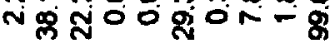

녕8

สำ 엉ำ

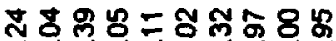

N然

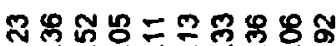

N

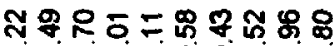

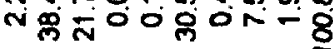

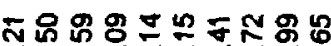

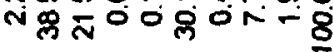

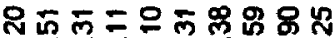

N

온

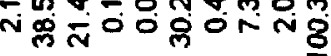

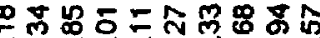

ज品

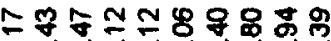

N

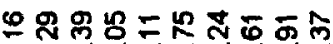

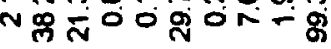

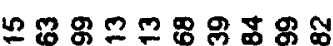

त苟

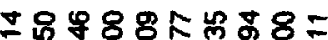

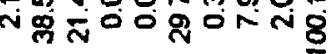

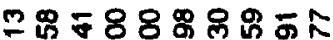

న

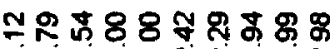

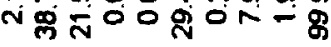

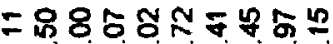

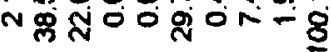

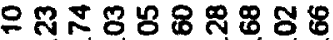

N

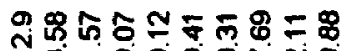

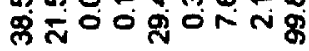

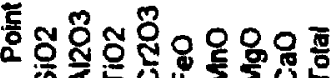

辛品

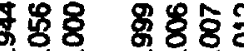

กㅇㅇㅇ

영영 응용

옹ำ

경영

กั 8

(2)

8

동요

in

品号突品

mo

กั

造势

ก잉

28

(5)

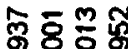

깅요

กั๊

영응형

กัธठ์

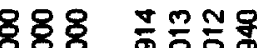

000 ก.

ร̄o

000

哭흠융

ํㅣㅇำ

횽용

जo

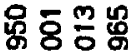

ㄱㅇㅇㅇㅛ

농영

은드용

잉요

iso

ธิธํํㅇำ

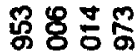

잉요

政

8 응

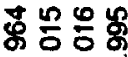

200

- 98

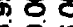

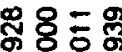

잉요

츙요

0.00

₹ $88 \overline{8}$

잉요

\%용

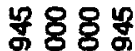

60

范 8

พ150

(1)

ํํㅇ융ำ

영영

กิธ

กิ่

옹요용

กั0.

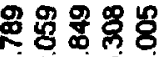

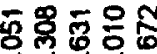

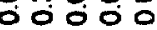

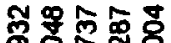
궁응

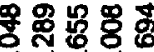

0000

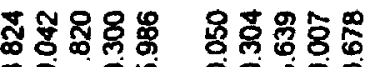

mo

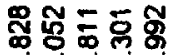

융중용응

0000

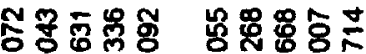

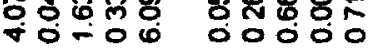

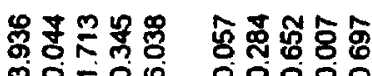

그응

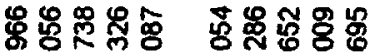

ก०-00 0000

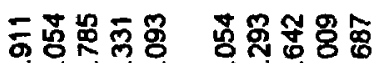

ก०-0० 0000

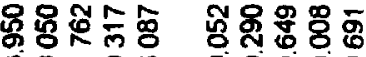

ก०-06 0000

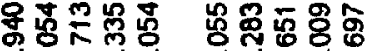

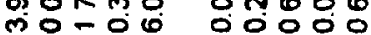

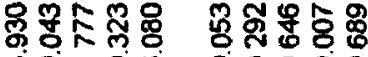

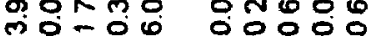

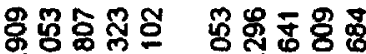

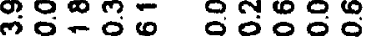

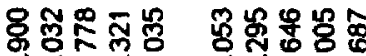

กั0-0० 00000

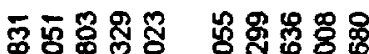

ल0-00 00000

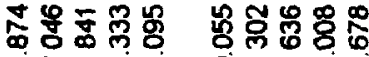

ஸิ㇒-0.0

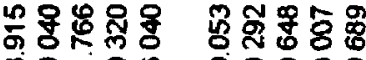

ल० -00 00000

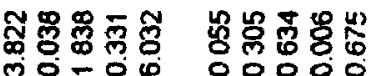

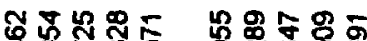

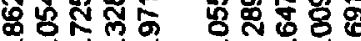

ल०

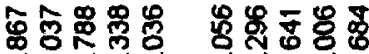

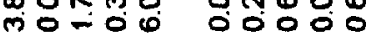

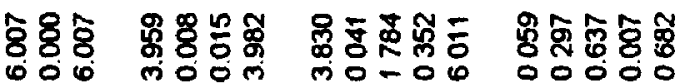

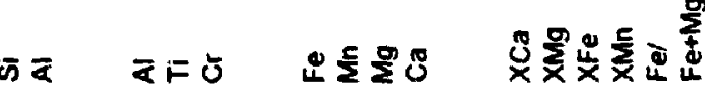




\begin{tabular}{|c|c|c|c|c|}
\hline 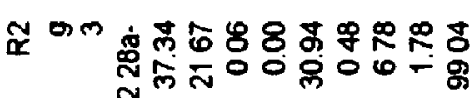 & 용ㅇㅇㅇㅇㅇㅇ & 옹홍영 & 葛 & 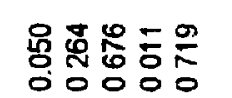 \\
\hline 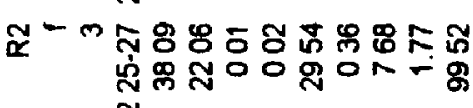 & 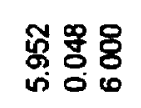 & 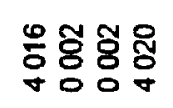 & 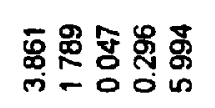 & 옹영영명영 \\
\hline 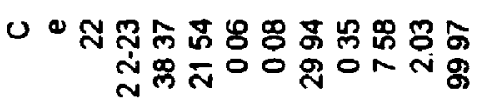 & 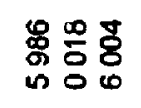 & 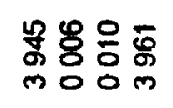 & 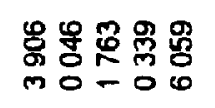 & 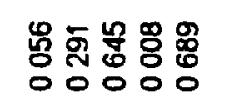 \\
\hline 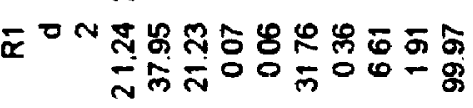 & 동ㅇㅇㅇㅇㅇㅇ & 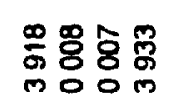 & 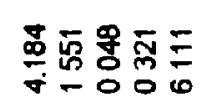 & 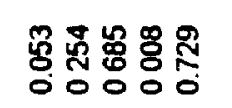 \\
\hline 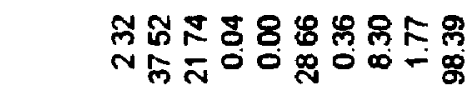 & 영: & 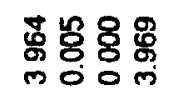 & 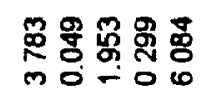 & 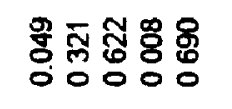 \\
\hline 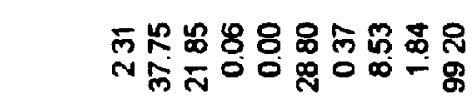 & 옹영영 & 言害哭器 & 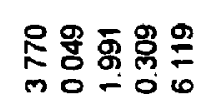 & 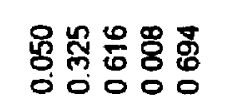 \\
\hline 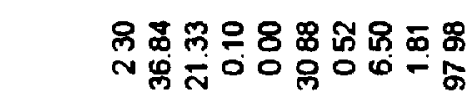 & 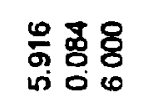 & 员产客客兽 & 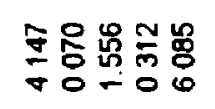 & 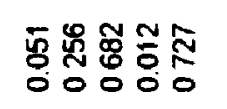 \\
\hline 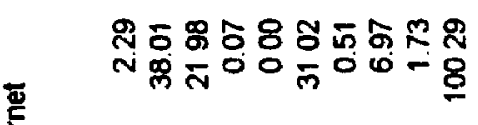 & 兽 & 泪哭哭各 & 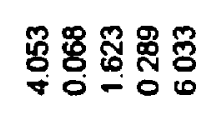 & 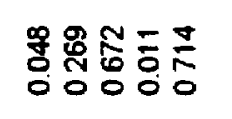 \\
\hline 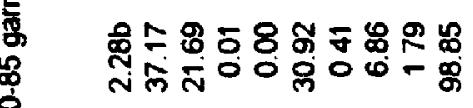 & 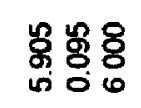 & 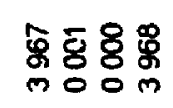 & 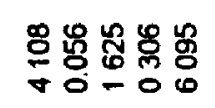 & 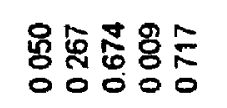 \\
\hline 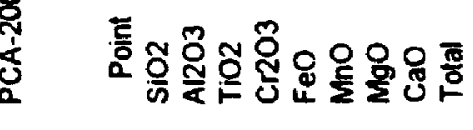 & & & & \\
\hline
\end{tabular}




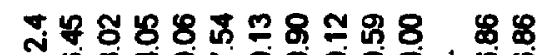

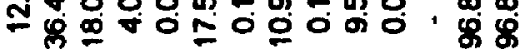

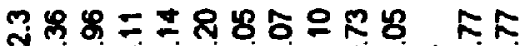
果=0

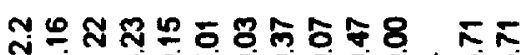

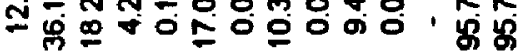
สํํำ

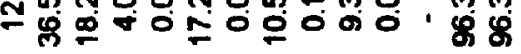

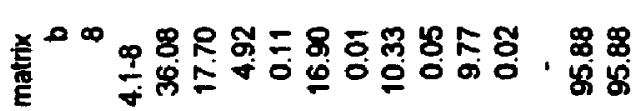

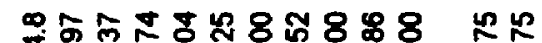
出

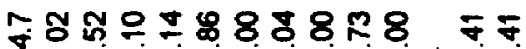

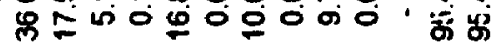

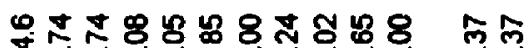

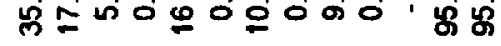

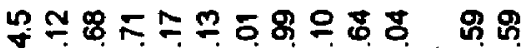
过出

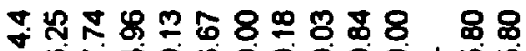

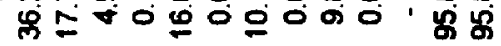

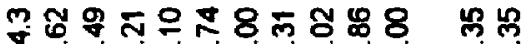
负

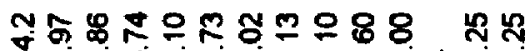
近

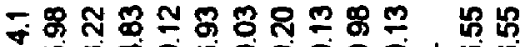
出

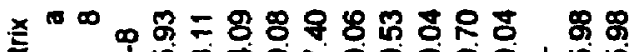
ำ

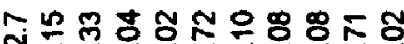

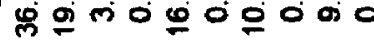

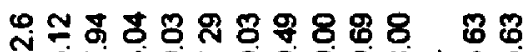

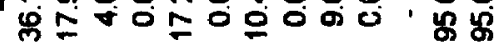

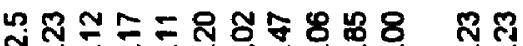

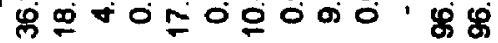
ㄴำ

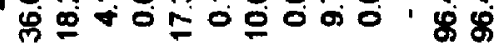

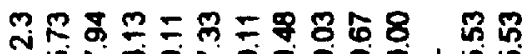
出

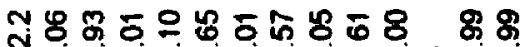
近行 స

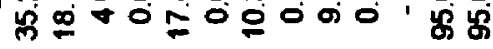

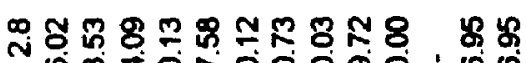

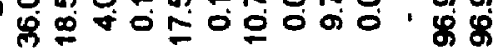

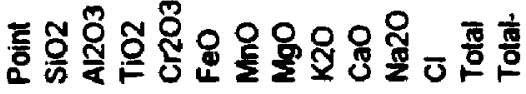

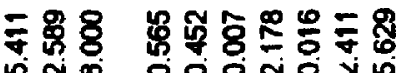
ज N $\infty$

$\overline{9} 8$ ง

电解 8 जก

手量 8 in

\& 8 ง

궁용

的

ชู่ิ์ 8 in

象

งก

융요

กึ่

भु당

in

可部 क

웅 जก

危余号

जि

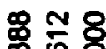
in

웅요용

in

ชูㅇํㅇ ज

芦总 8

的

要 งก

兽 की

ㅇํㅇㅇㅛ ज ल

密谟 iึ

웅 8

in $N$

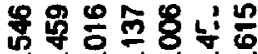

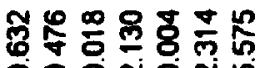
000 Non

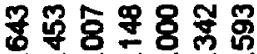

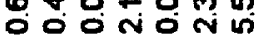

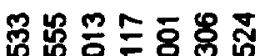

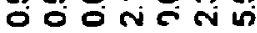

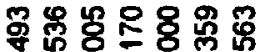
-0口ON口N

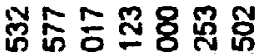
ㅇㅇㅇㅇำ

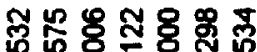
O०० NON

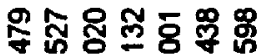
○。ํำ

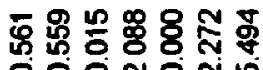
OOONON

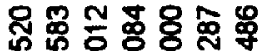
ㅇㅇㅇ유엥

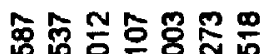
OOONON

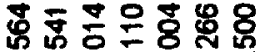

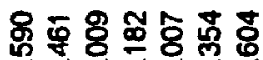

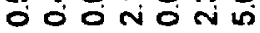

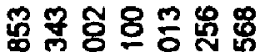

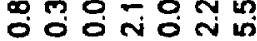

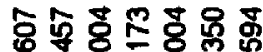

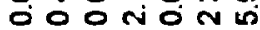

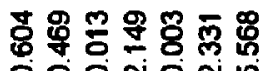
OOONON

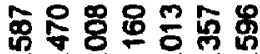

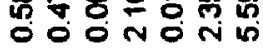

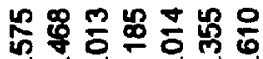
OOONON⿴囗十

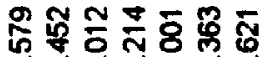
คํㅇㅇㅇํㅇ

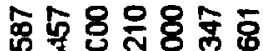
ट0口 Nonin

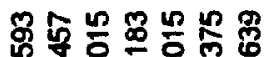
OOONON ऊ นึㅇㅇㅇㅇㅇำ

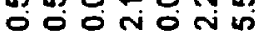

응응 $0-0-\quad 0000$

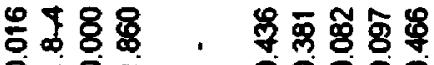

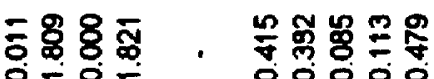

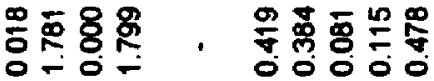

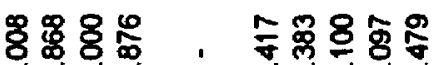
$0-0-100000$

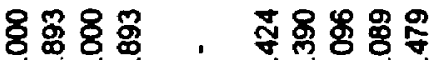
$0 \% 00000$ 8용용요영 $0-0-00000$

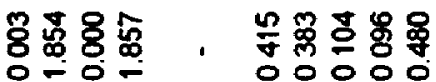

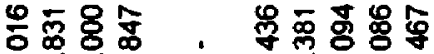
0-0. 00000

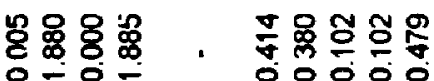

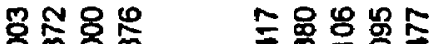
음잉.

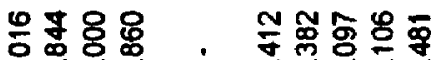
0 - -00000

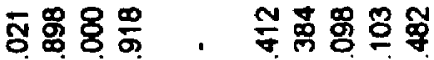
$0 \div 0 \div 0000$

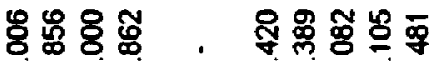
O-O- 00000

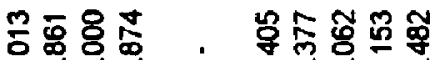
口-

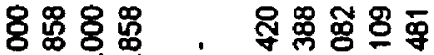
O-

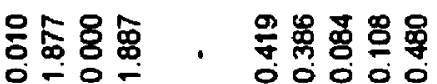

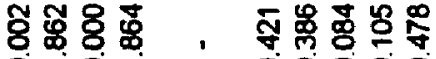

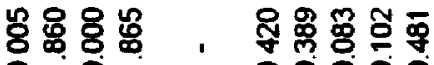
$0-0-10000$

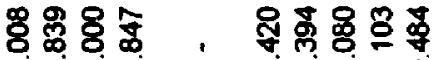
$0 \div 0 \div 0000$

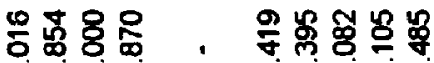
잉 O000

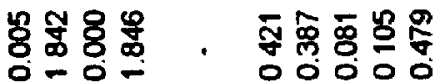

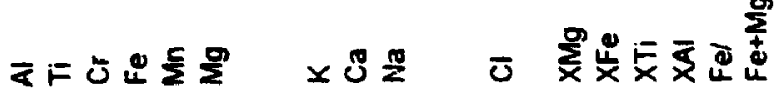


武

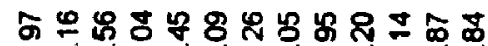
๙

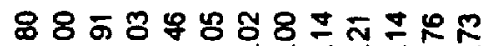

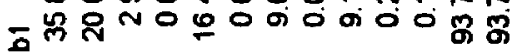

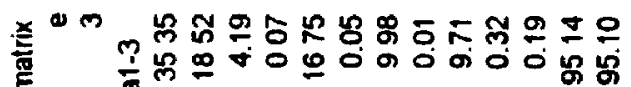

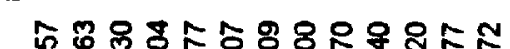
\%ู

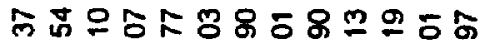
ช

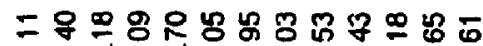

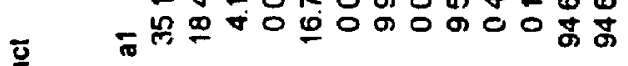

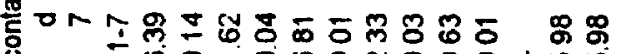
鸟可-

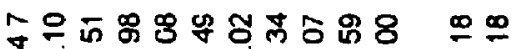

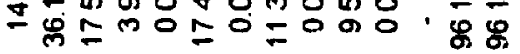

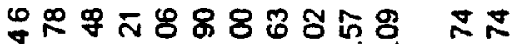

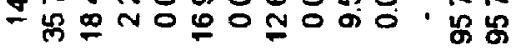

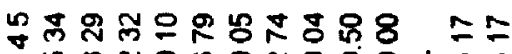

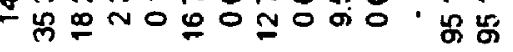

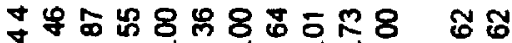
士心

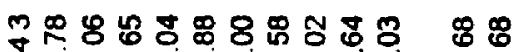

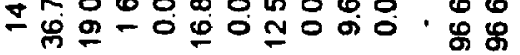

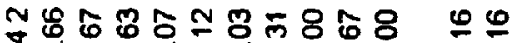
士

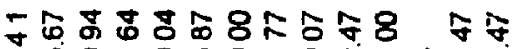

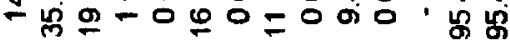

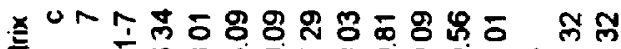

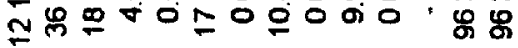

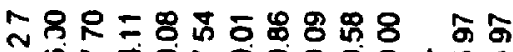

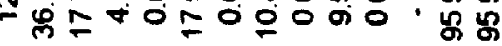

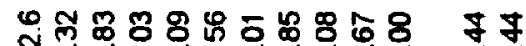

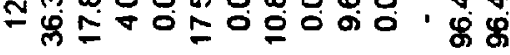

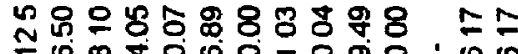

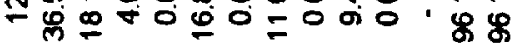

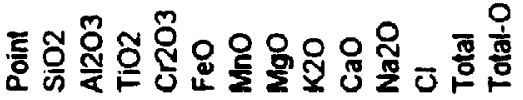

急象 的

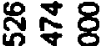
क त

寻查 8 เi

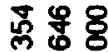
แ

웅용ㅇㅁ 的品

近 ஸึ

늄 뱅ㅇㅇㅇ is $N$

웝 品 옹 ஸ

\%ิํํㅇ 웅 ๑ $\infty$

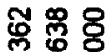
in

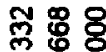
is $\infty$

学出员 的

旁莒号 in $N$

学宫 的 $N$

용 is 急 8 जin

矛嵒 แก 车要 8 แ $N$

雮 8 แ $\sim \infty$

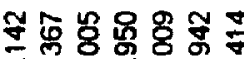
=00-0- in

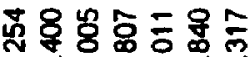
- 00 - 0 -

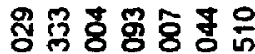
- 0 O non

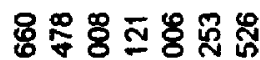
-0ำก N

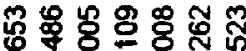

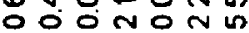

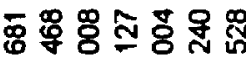
०0에에

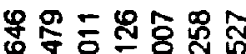

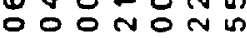

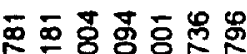

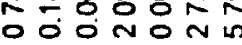

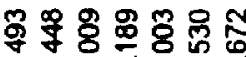
OOON口N

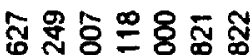

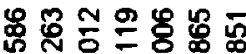
OOONON

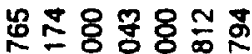
응응 응 少

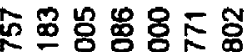

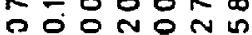

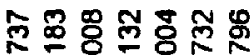
oOO NON

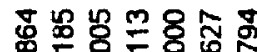

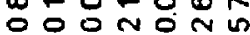

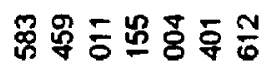
OOONON

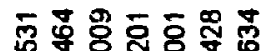
O OO ONON

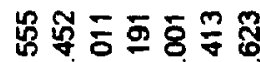
o o onon

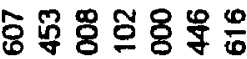
oO OnON

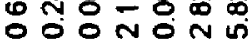

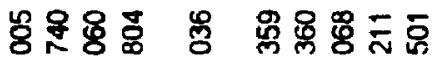
$0-0-000000$

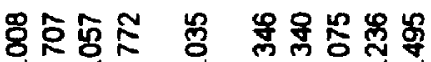
-0 0 00000

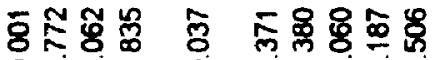
O-O- 0 00000

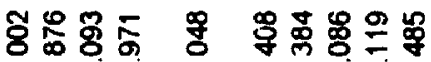
О- - - 00000

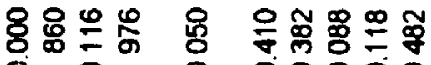

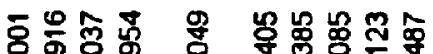
응응응 영융영

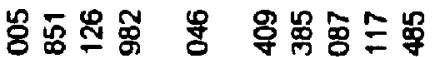
O-5- 0 OOO50

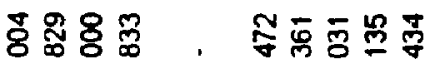
O-0- 00000

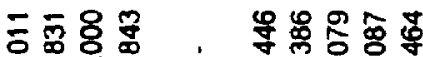
O- 0 00000

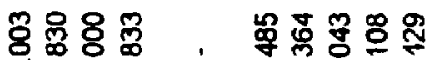
0-0ㄴ

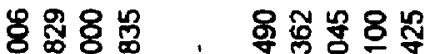
응ㄴㄷㅇㅇㅇㅇㅇㅇ

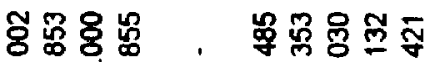
응 00000

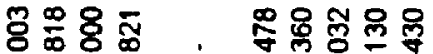

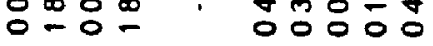

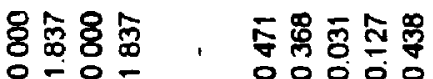

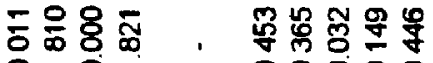
$0-0-00000$

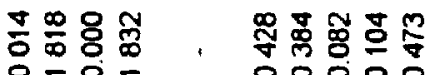

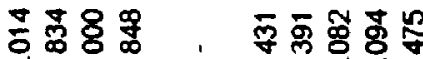
O-0- 00000

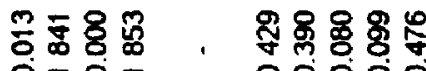
$0-0-00000$

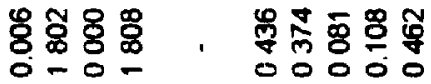
$\overline{\text { व }}$

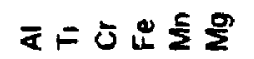

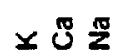

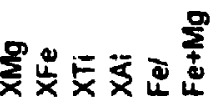




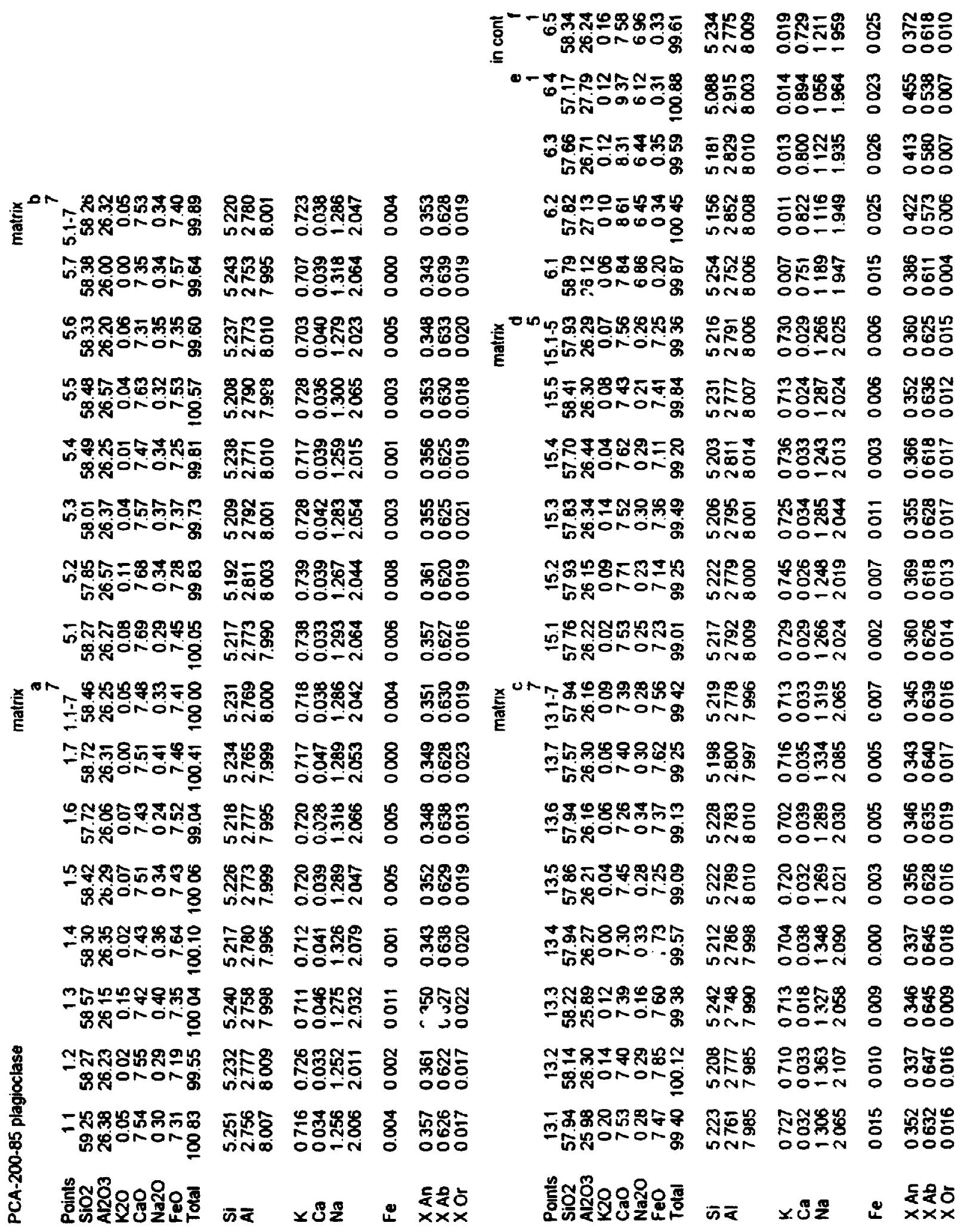




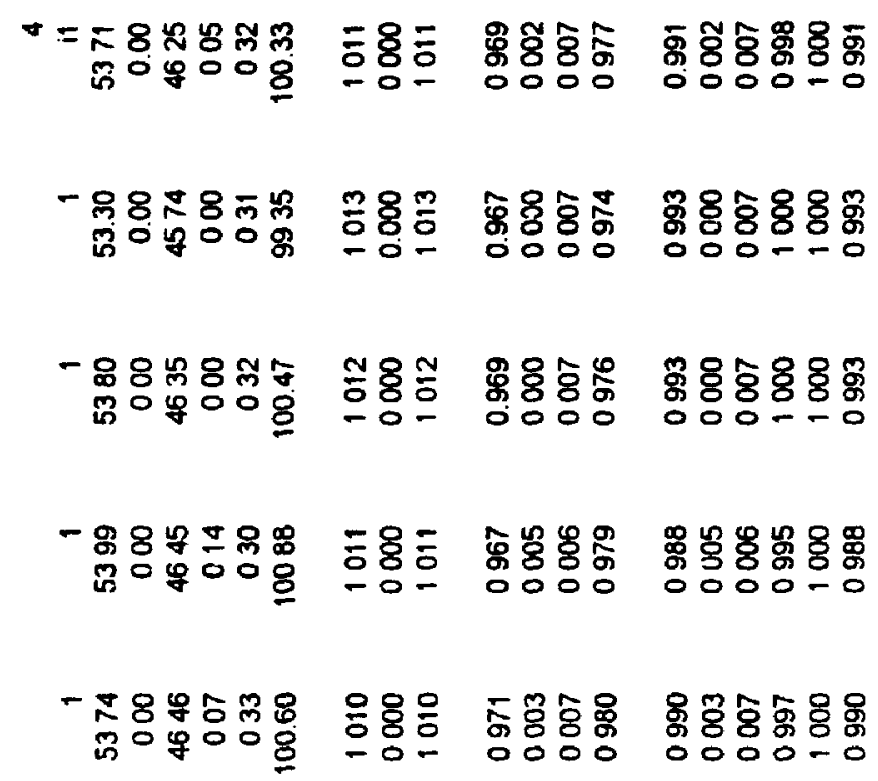

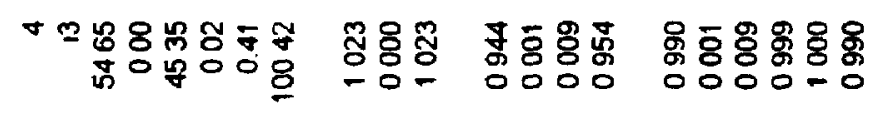

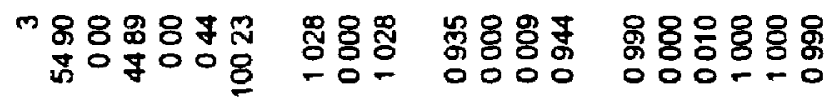

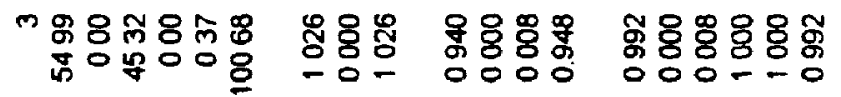

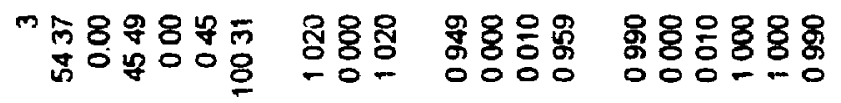

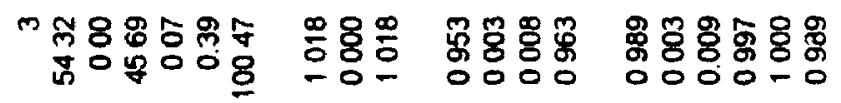

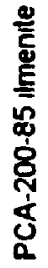

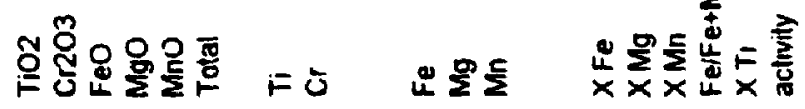




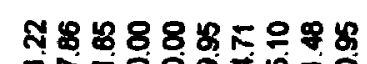

-

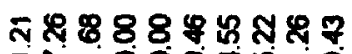

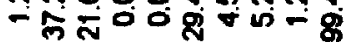

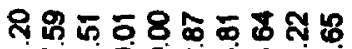

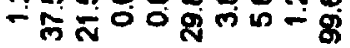

웅 78

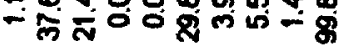

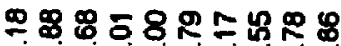

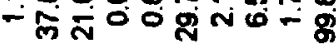

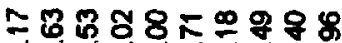

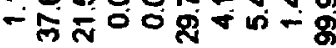

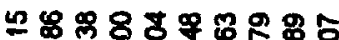

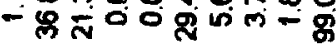

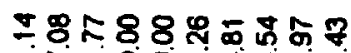

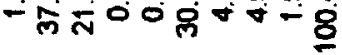

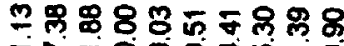

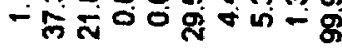

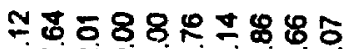

-

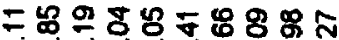

=

은 궁 $88 \% 6 \%$

一

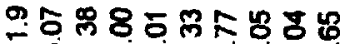

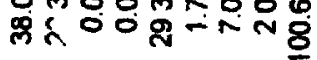

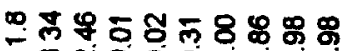

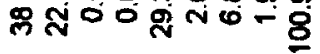

โ กิ

串 สํำ

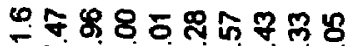

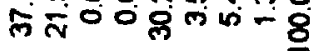

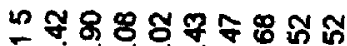

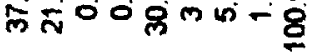

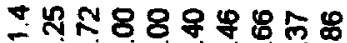

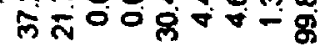

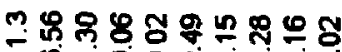

मूं

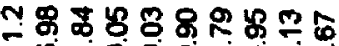

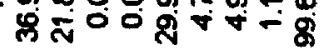

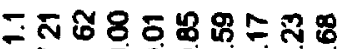

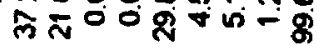

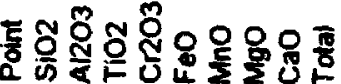

\section{将8}

जi 0

承客

जio.

$8 \overline{8} 8$

กํ.

$8 \%$

\& 8

\$

ज०

8.

공응

全용

जo 6

ริ종용

no 0

$8 \overline{8} 8$

जo

\%훙

s。

要용

50

음용

in

品

sio.

유요

in

용용요

ஸ。ㅇ․

용요

ง⿵门

옹용

กㅇ

సิ

5.

广⿻

150

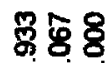

동요

के व
888

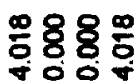

$8 \overline{8} 8$

ก००0

농 8 ก.

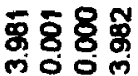

\$용 mó

응 88

+०0\%

5.88

mo응

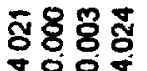

용 8

70 영

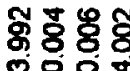

กิ8ชి

$40 \%$

농용ㅎㅁ응

옹형요울

काव

₹

0000

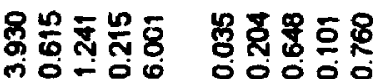

$8 \div$

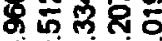

ํํㅇำ

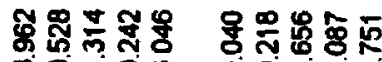
ल०

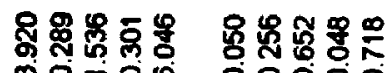

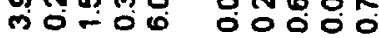

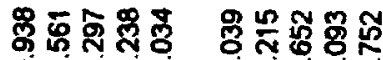
ก०-O० :

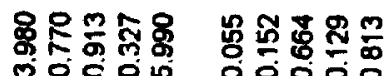

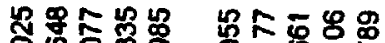

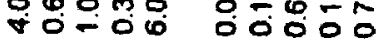

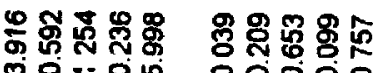

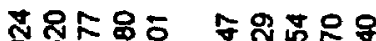

สํํำ ก०-06 00000

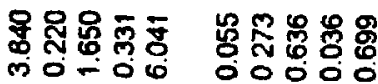

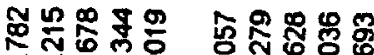

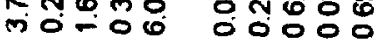

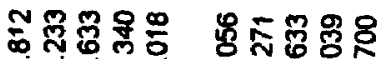
ก०००० 0000

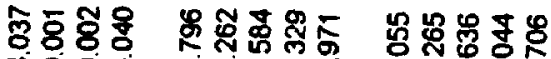

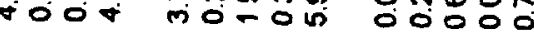

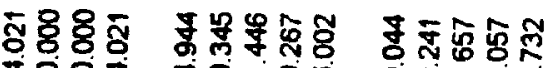


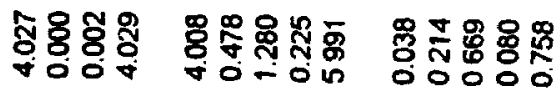

Nㅜ용

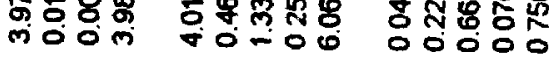

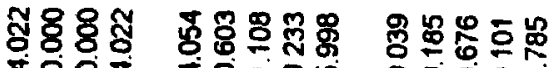

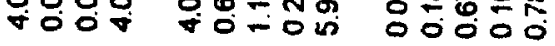

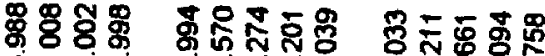
싱융

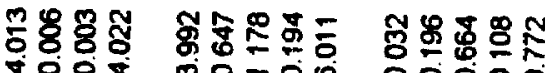

연

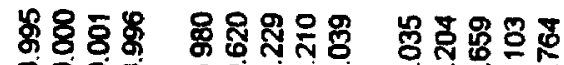

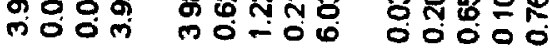

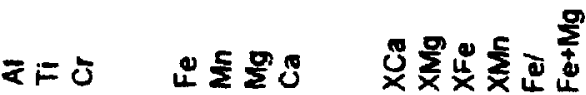




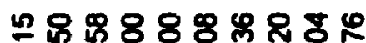

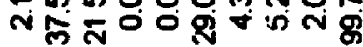

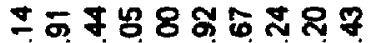

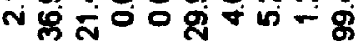

$m \mathbb{R}=8 \bar{\Phi}:=08$

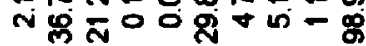

Nำ番 $88 \%$

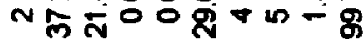

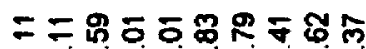

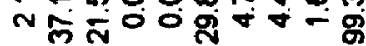

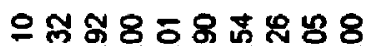

ฟमें

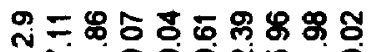

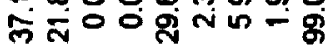

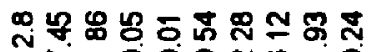

กักั

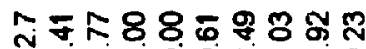

的云OO

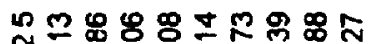

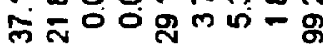

눈

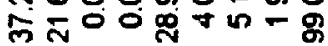

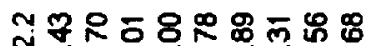

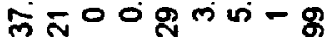

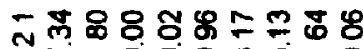

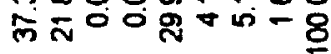

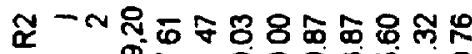

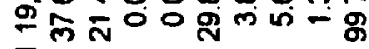

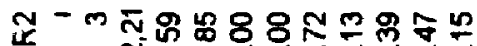

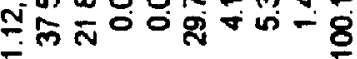

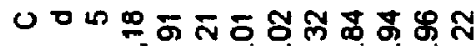

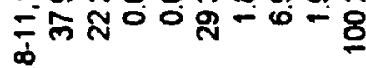
$\infty$

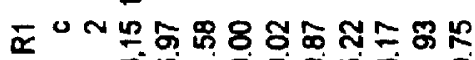

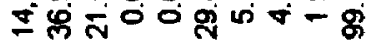

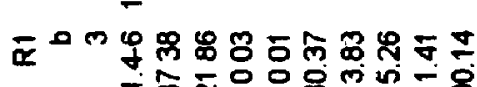

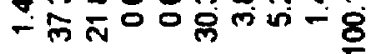

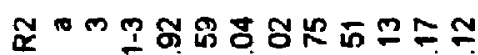

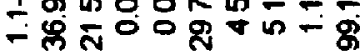

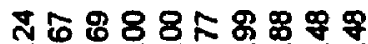

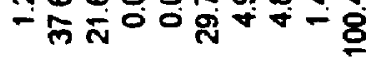

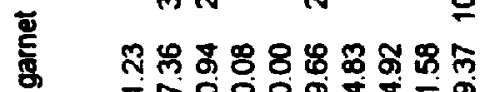

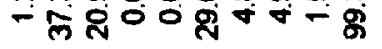

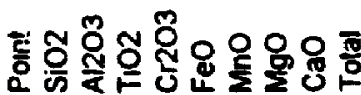

$8 \%$

जi

웅ㅇㅇ 8

เi०

Nㅜㅇ용용

जी 0

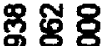

ㄱㅇㅇㅇㅛ

票要

형

ตㅇํㅇ

象

ऽ 6

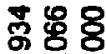

in 0

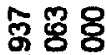

เก

ปั。

is 00

品品 8

जio 0

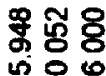

옹영용

no

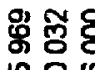

no

青品

no 0

รัฐ 8

no 0

สํํㅇㅇ

กิ००

สู่ํํㅇㅇ

is 0

중용

no 0

$\bar{\Phi}$ 学店

in 0

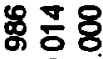

no

홍응해

तi $0 \mathrm{~m}$

๘区 ๘にう

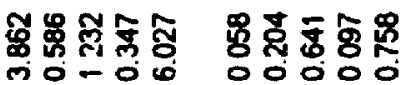

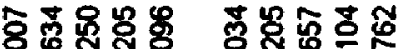

\%०-00 00000

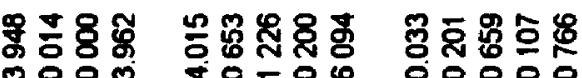

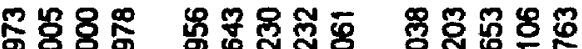

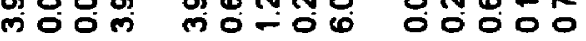

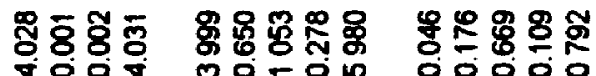

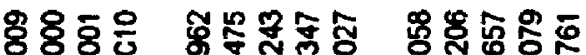

40.

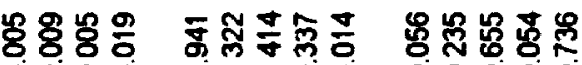

400 ल0-0.

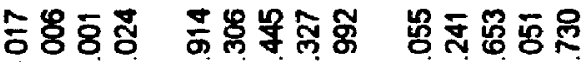

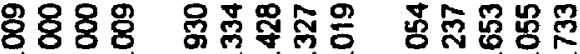

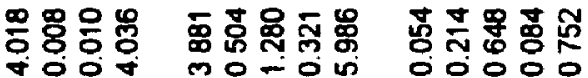

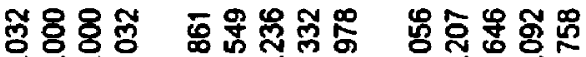

400\% mo-a ooodo

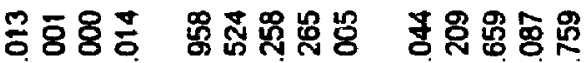

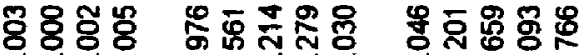

-oor mo-0 00000

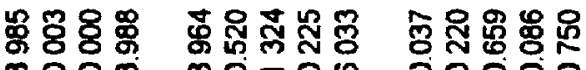

กำ

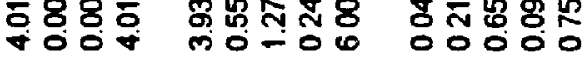

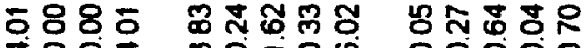

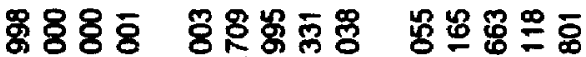

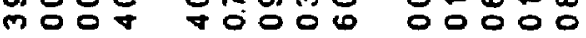

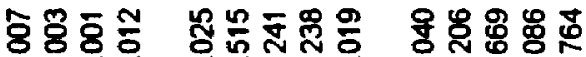

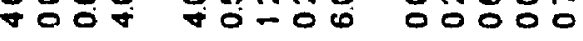

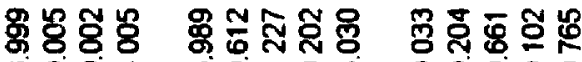
ก००

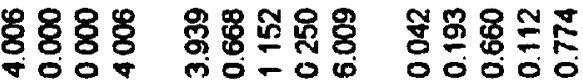

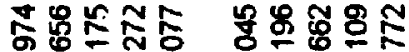

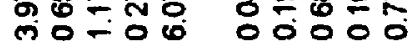

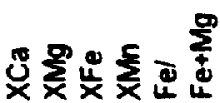


๔

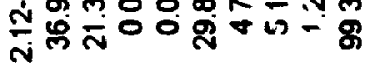

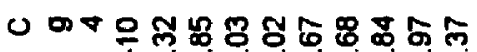

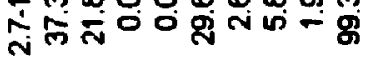

我一 N

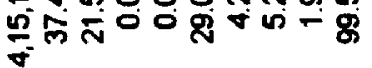
तi

ฮั ข

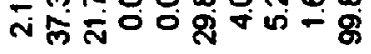

สำร ㅇํㅇา的的

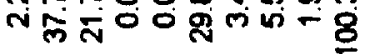

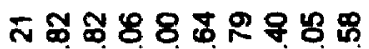

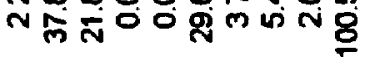

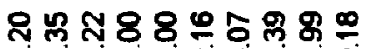
ง

으

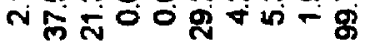

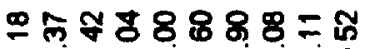

N於ล

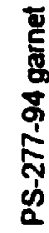

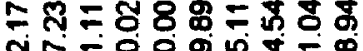

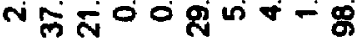

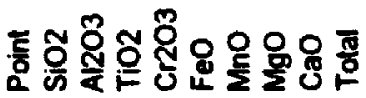

옹용 응응

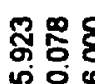

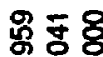
ज倇

क्रेष्ठ용

号莒 内i 응

员员

\$ 888 no mo m

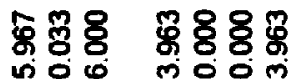

웅용 in 엉

\section{$\infty 88$}

\$용 mo \%용 용 in 0

絭 웅융용

응항융융

\$

용ㅎㅇㅇ웅

굥 용용 mo

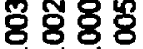
+. らを をにう

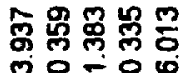

둥요

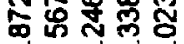

\$户山

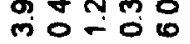

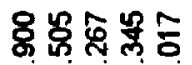
mo- 00

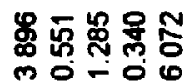

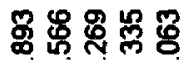

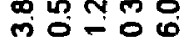

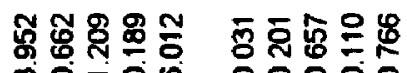

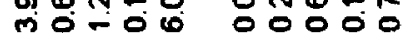
융형효용

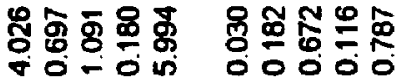

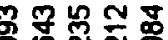
m응 mo-0

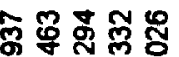

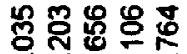
0000 结过

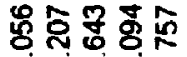
०0ㅇㅇㅇ

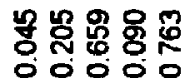

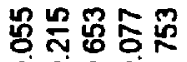
00000

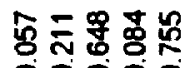
00000 号瓷管雨 令

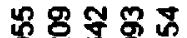
पू द्रें 00000

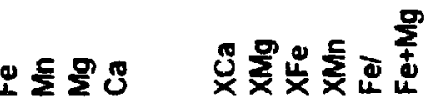




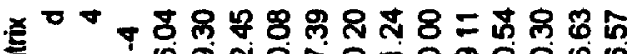

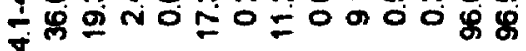

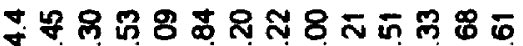
ल बं ำ

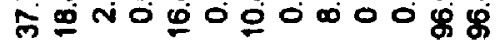

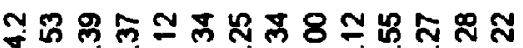
命

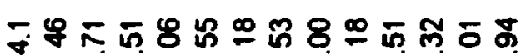

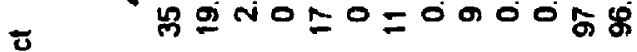

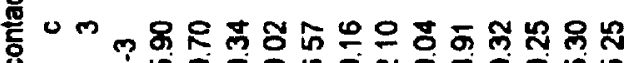

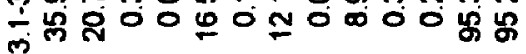

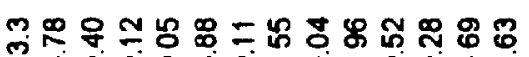

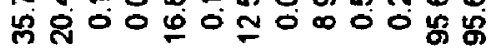

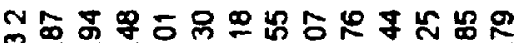
留

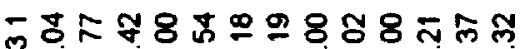

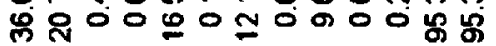

ํㅡㄹ n

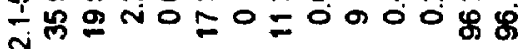

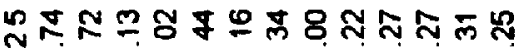
मू

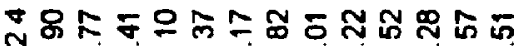

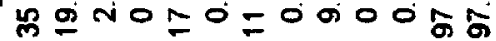

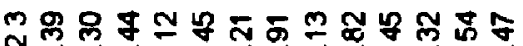

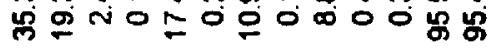

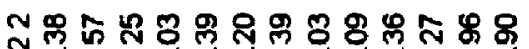
岁 은

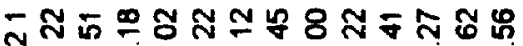
每

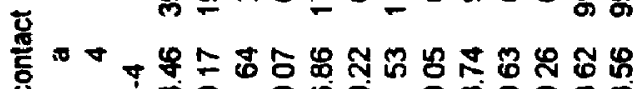

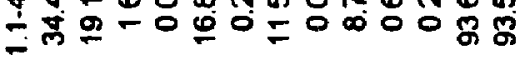

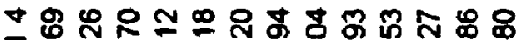
सं

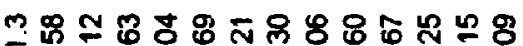
से क्ष०

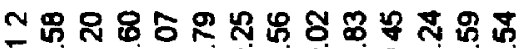

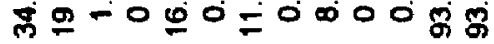

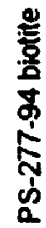
กำ- ๒

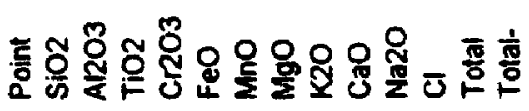

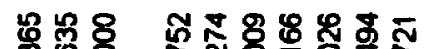
in

용 นึ

용 8

i $v$ क

용 फ

Nㅗㅇ 8

的 $N$

$\Re$ 今

un $\mathrm{N}$

苞 范 8

n $\mathrm{N}$

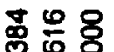

is

-

טi $N$

능

แก $\sim$

鬲 它

in

志串

in $N$

岀乌

in $\mathrm{N}$ 冓

总 용

Uก

항

in तi $\infty$

啘

un $\infty$

ิㅗ숑

in $\mathrm{N}$ io

角8 in

묭ㅇㅇ

is $\infty$

옹 용 的

\& ○。 0 NO N

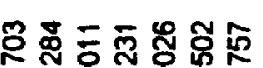

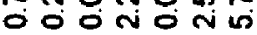

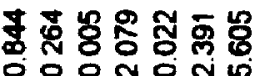

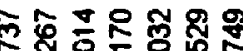

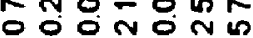

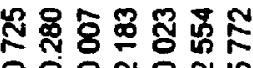
O O O N

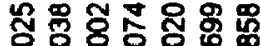
- O

员 o don

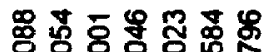

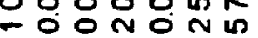

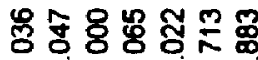
- OO

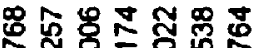

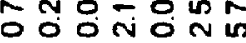

영 용용슝요 0 O O N O N U

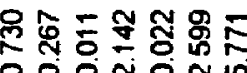

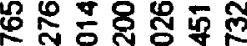

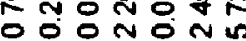

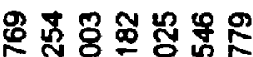
o o o Non Un

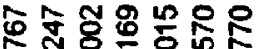
o OOO

용요용 odonom un

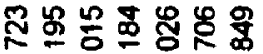
- OD N

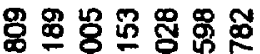
OOO NO N is

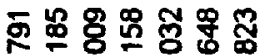
o o 0 N कर

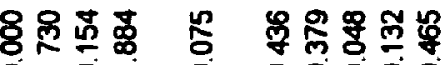
0 - 0 O 00000

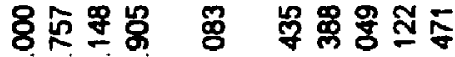
$0-0 \div 0$ 00000

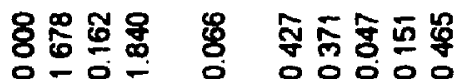

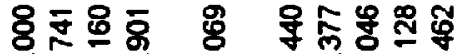

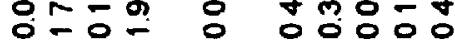

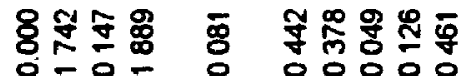

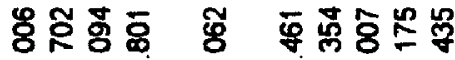
$0 \div 0 \div 0$ 00000

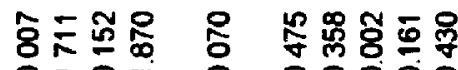

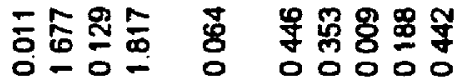

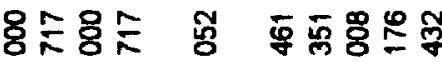
0 -0- 0 00000

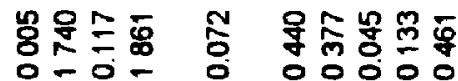

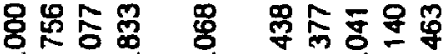
Oㄷㅇㅇ융ㅇㅇㅇㅇㅇㅇ

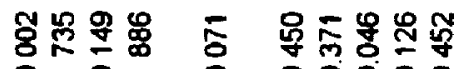
0-0-0 00000

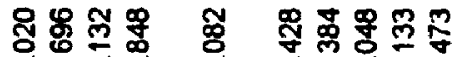
$0-0 \div 00000$

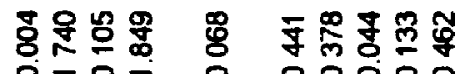

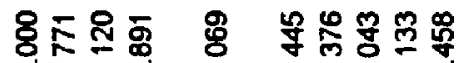
O-D-0 00000

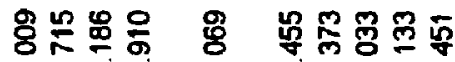
$0 \div 0 \div 0$ 00000

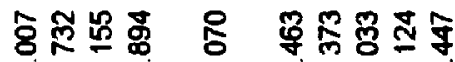
$0-0-0$ 0000

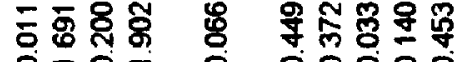

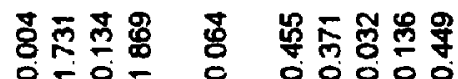

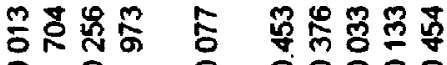
व-O- 0 O००00

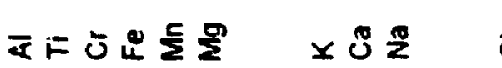




\begin{tabular}{|c|c|c|c|c|c|c|}
\hline , & 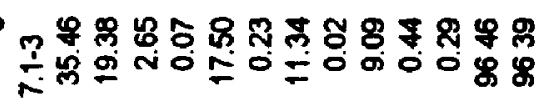 & 急듀용 & 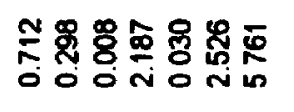 & 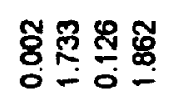 & $\stackrel{\mathbb{S}}{\mathscr{O}}$ & 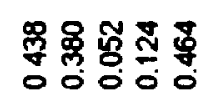 \\
\hline & 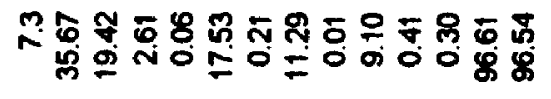 & 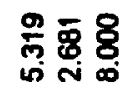 & 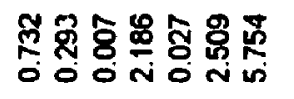 & 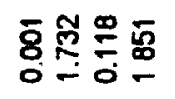 & $\stackrel{0}{0}$ & 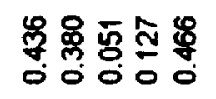 \\
\hline & 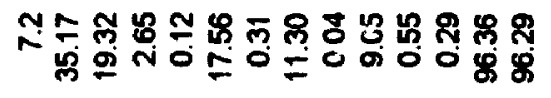 & 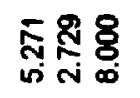 & 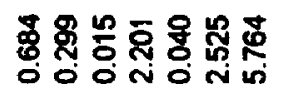 & 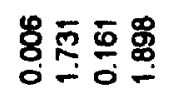 & $\frac{2}{0}$ & 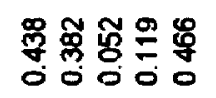 \\
\hline & 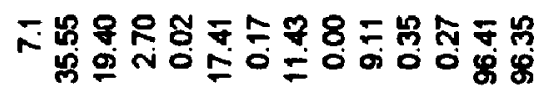 & 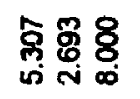 & 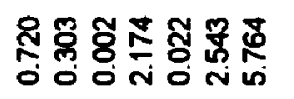 & 8 & $\oiint_{0}$ & 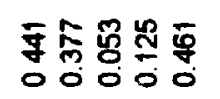 \\
\hline & 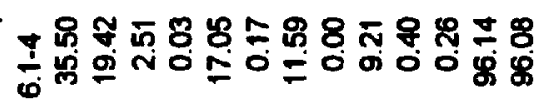 & 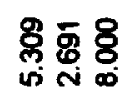 & 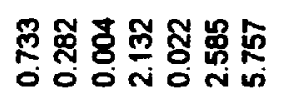 & 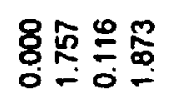 & $\widehat{\xi}$ & 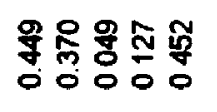 \\
\hline & 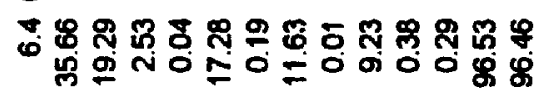 & 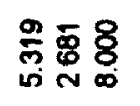 & 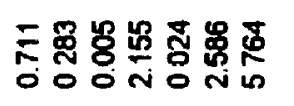 & 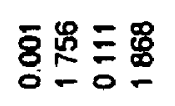 & $\frac{\mathbb{Z}}{0}$ & $\begin{array}{l}\frac{9}{7} \\
0 \\
0\end{array}$ \\
\hline & 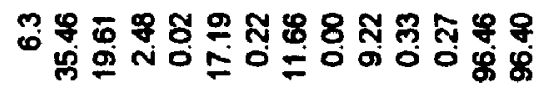 & 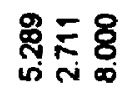 & 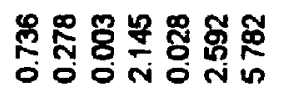 & 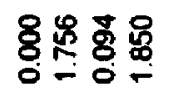 & $\underset{\xi}{\mathscr{\xi}}$ & 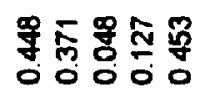 \\
\hline & 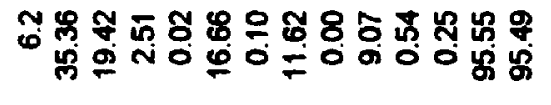 & 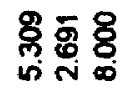 & 产 壱 & 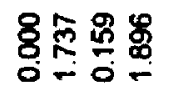 & 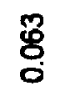 & 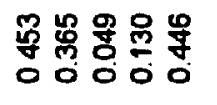 \\
\hline & 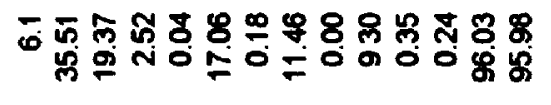 & 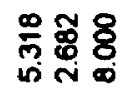 & 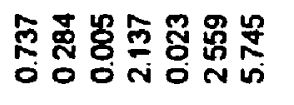 & 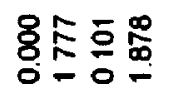 & $\bar{g}$ & 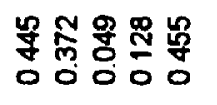 \\
\hline & 范 & 㧀志 & 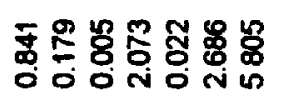 & 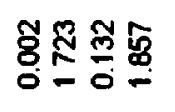 & 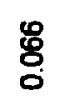 & 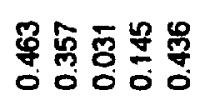 \\
\hline & 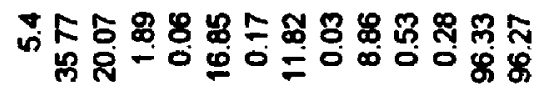 & 氕苾 & 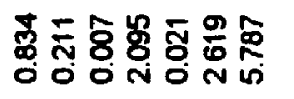 & 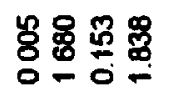 & $\underset{0}{\tilde{0}}$ & 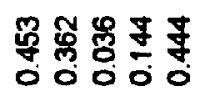 \\
\hline & 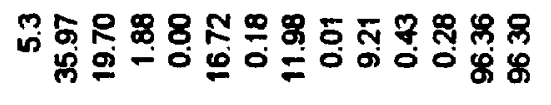 & 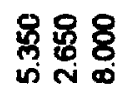 & 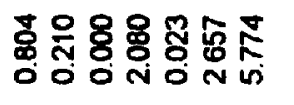 & 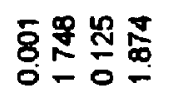 & $\bar{\delta}$ & 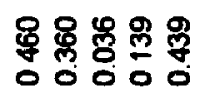 \\
\hline & 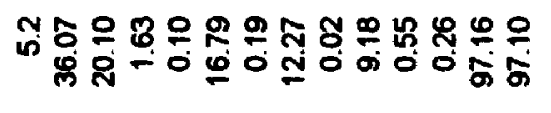 & 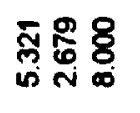 & 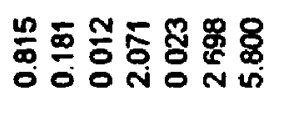 & 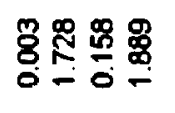 & 員 & 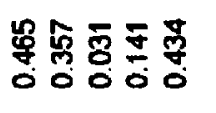 \\
\hline & 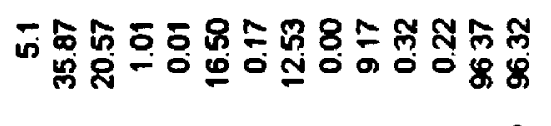 & 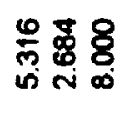 & 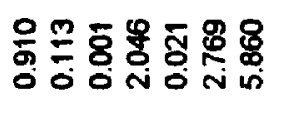 & 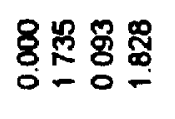 & 志 & 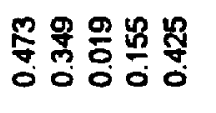 \\
\hline & 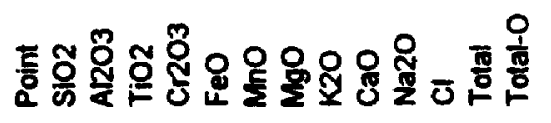 & க் & $\bar{\alpha}$ & & $\overline{0}$ & 모 \\
\hline
\end{tabular}




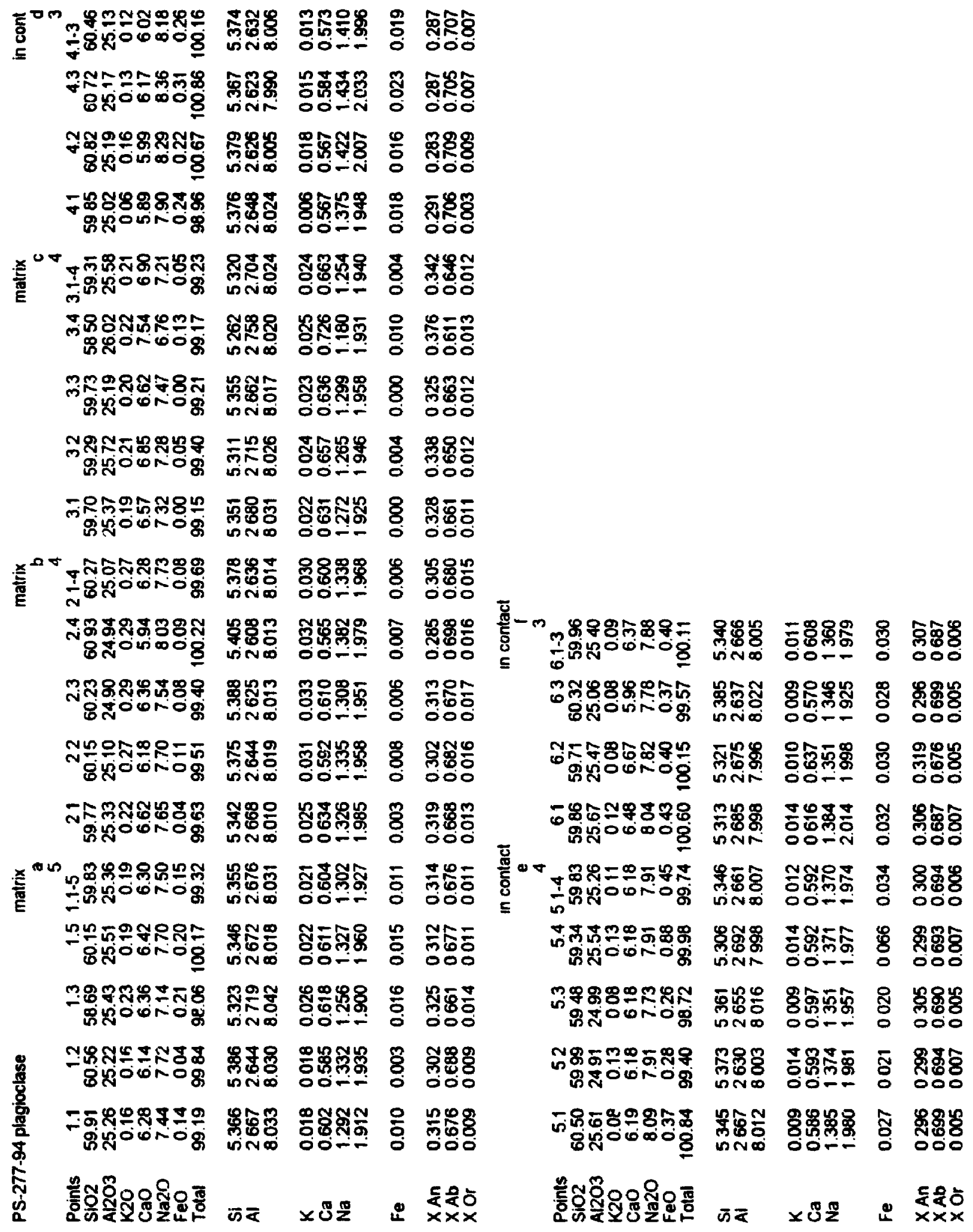




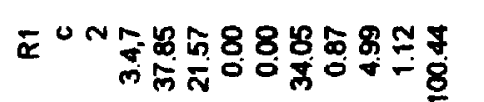

$=8 \% 8$ \%RF늠

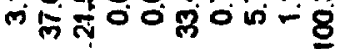

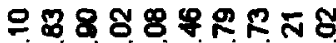

लं

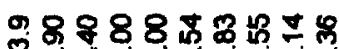

ले

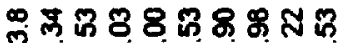

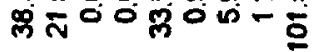

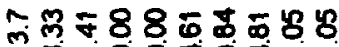

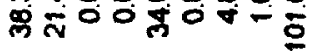

ஸั.

กิ่

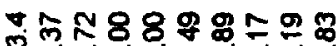

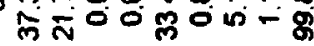

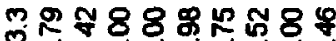

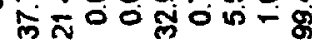

กู

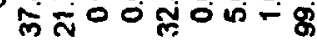

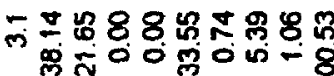

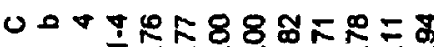

ज政0

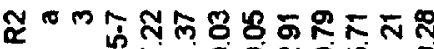

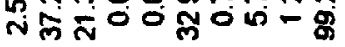

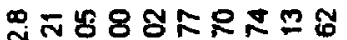

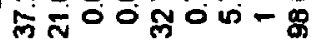

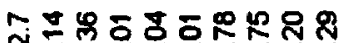

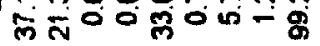

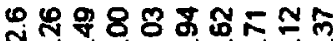

लित

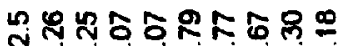

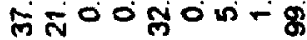

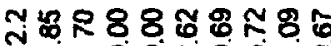

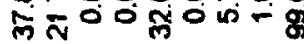

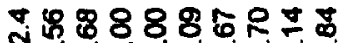

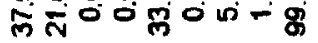

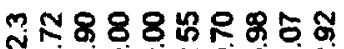

जिं

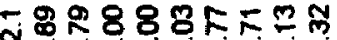

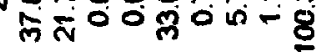

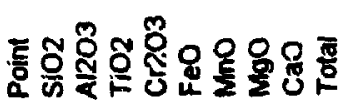

\$융ㅎํ

费费

भु. 8

जio.

多

象号 8

in

용용.

00

88.8

₹ิ 8

in 0

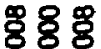

000

查

范8

由 0

部范

공용

in 0

ธิธี

जि०

$8 \overline{8}$

夏通

जि 0

苾高客

వ̄ㅇㅇㅇ

no 6

贸金迢

nก०

我果客

ஸо

종

is 0
\$88\%

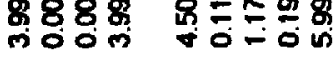

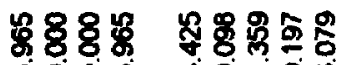

nं어

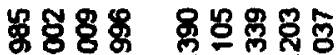

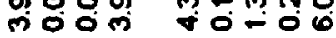

年8 8 \%

iि

సี⿻ㅇํ유

깅유

ㅊํㅇำ

ก००

$888 \%$

món

용요

$\checkmark 00 \div$

음 8

응

웅용영

造8

- 007

ํㅛㅇํ요

$40 \%$

888

๓о口冋

$\overline{8} 80$

mion

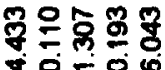

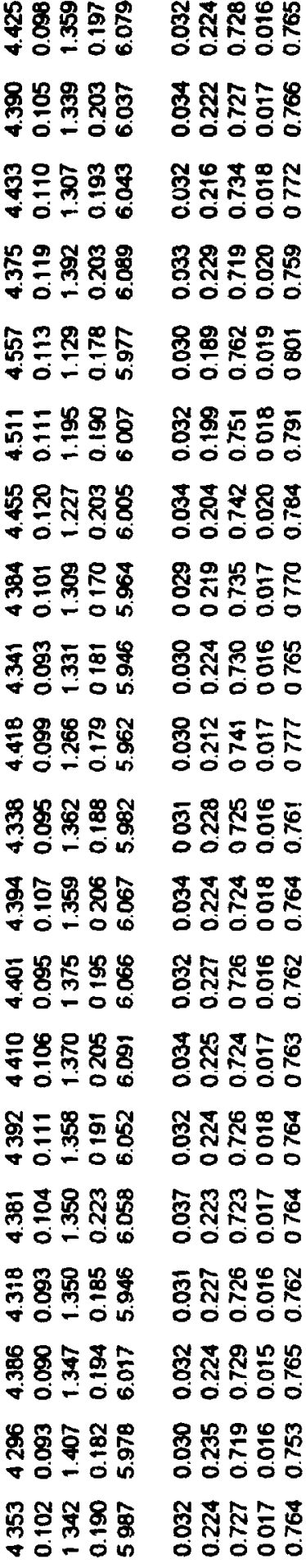

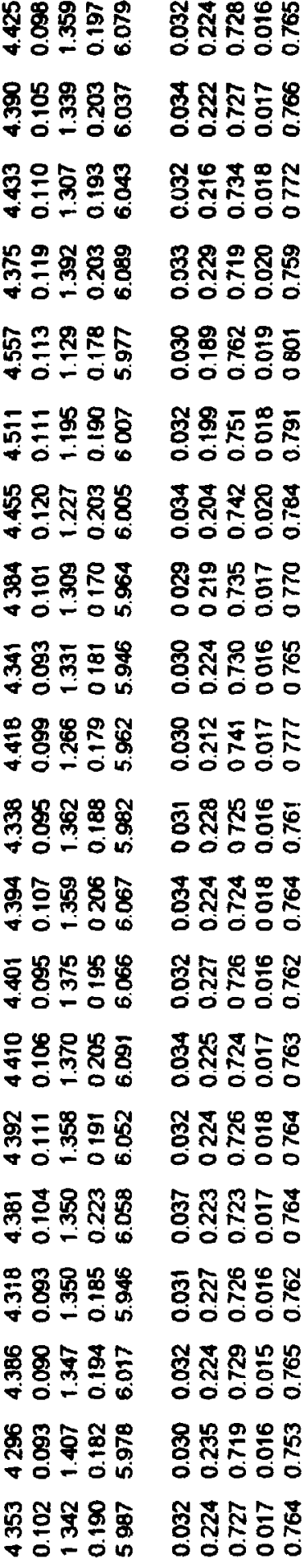

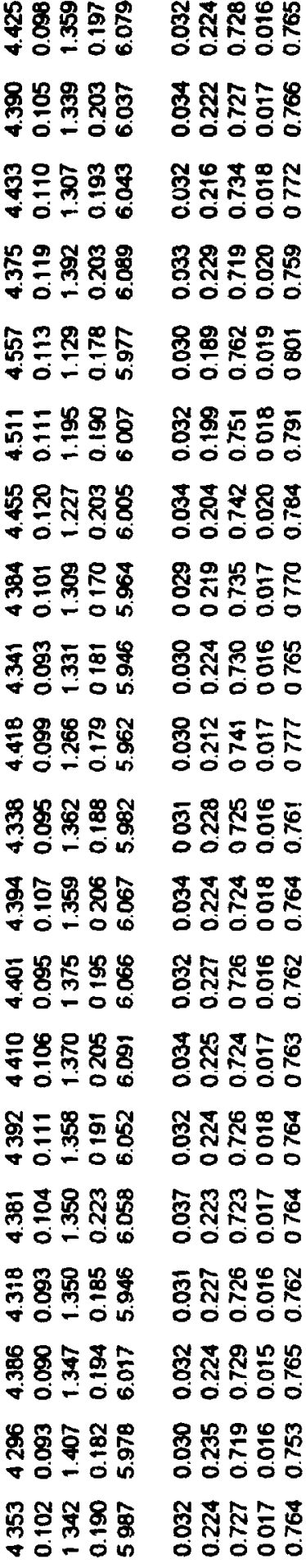

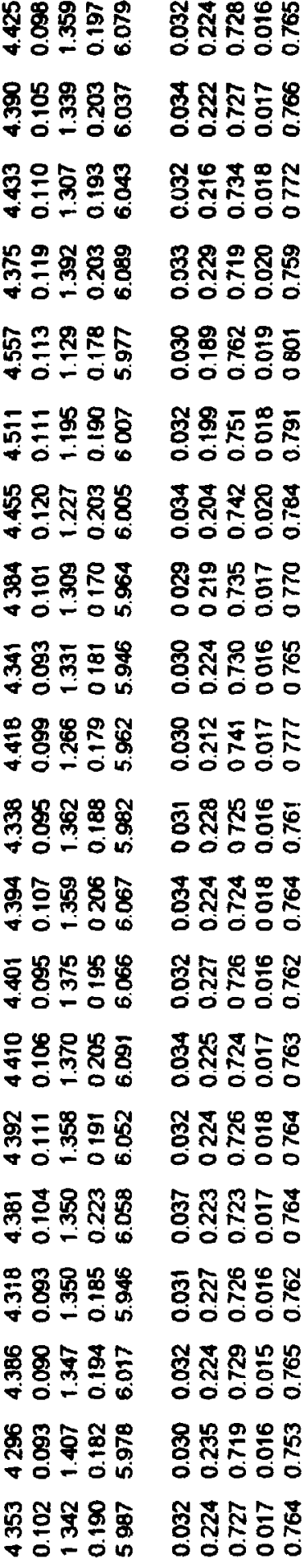

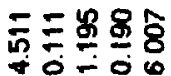

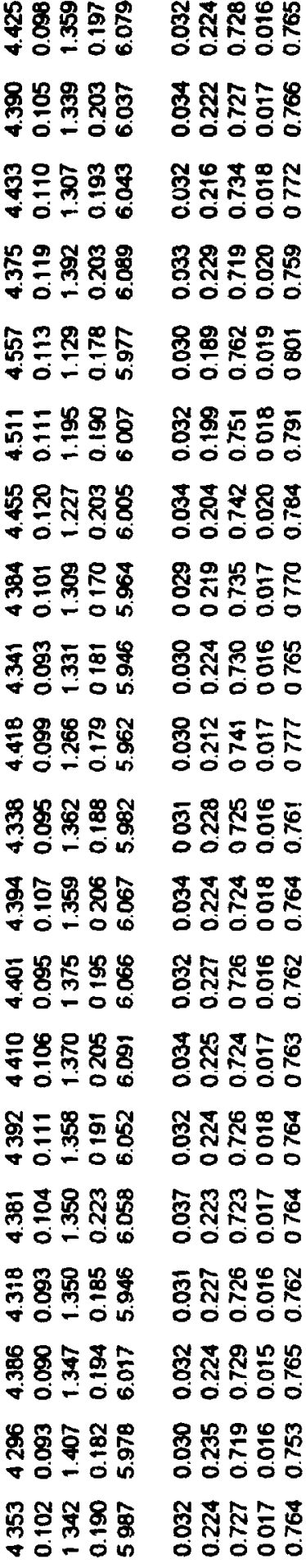

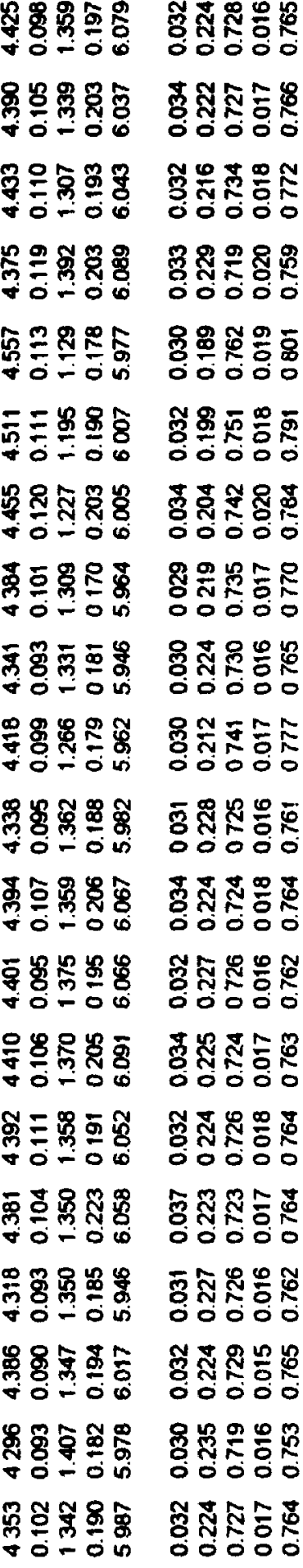

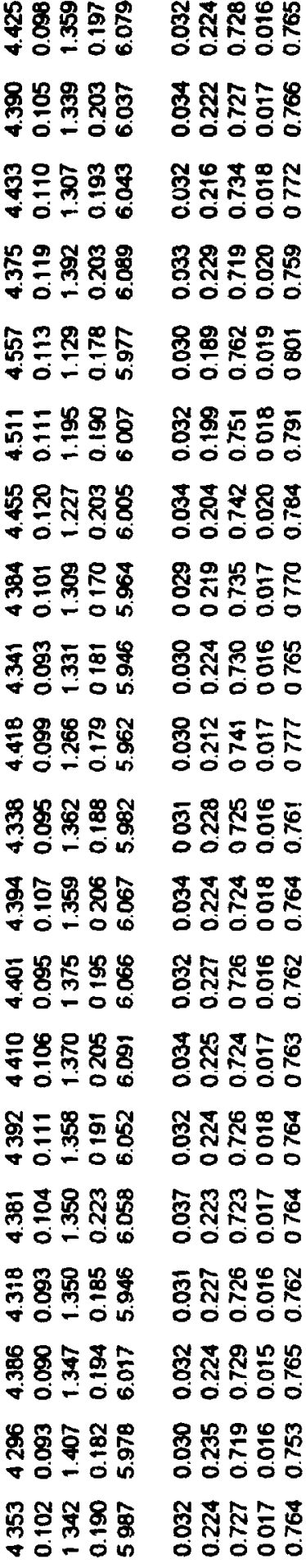

To- 0 क

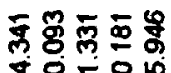

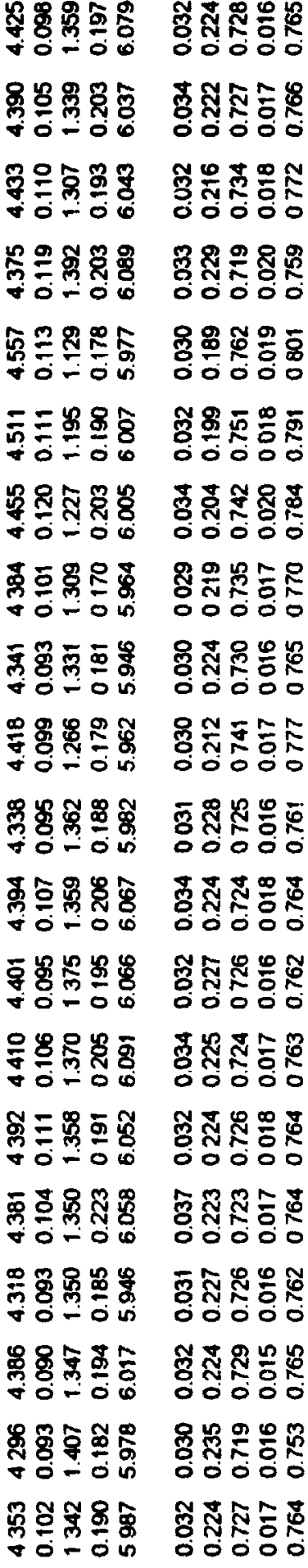

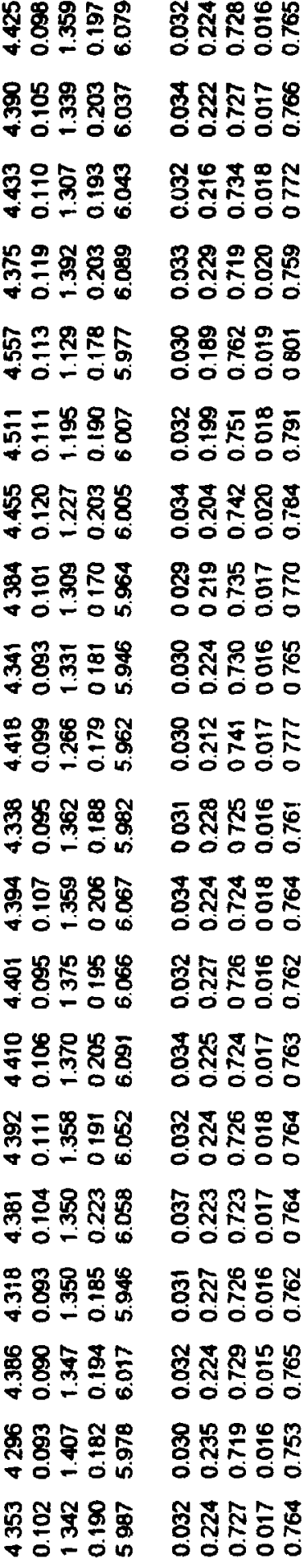

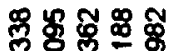

$70=0 \%$

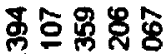

70.00

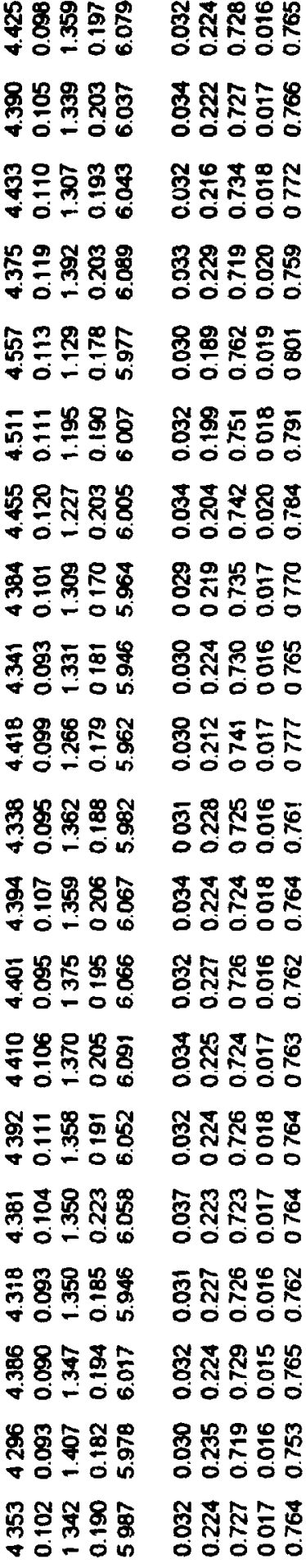

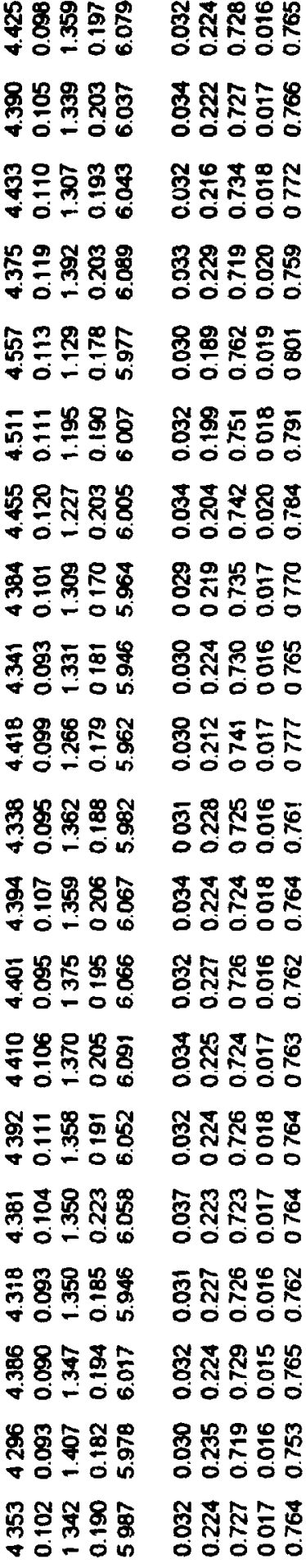

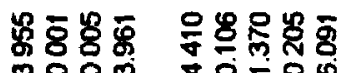

응

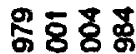

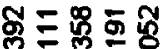

ก००0 $\checkmark 0-0 \%$

品8 8

गुO०

웅융융

4004

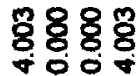

\$ٔ 88

\$5 88

응영

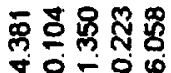

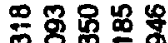

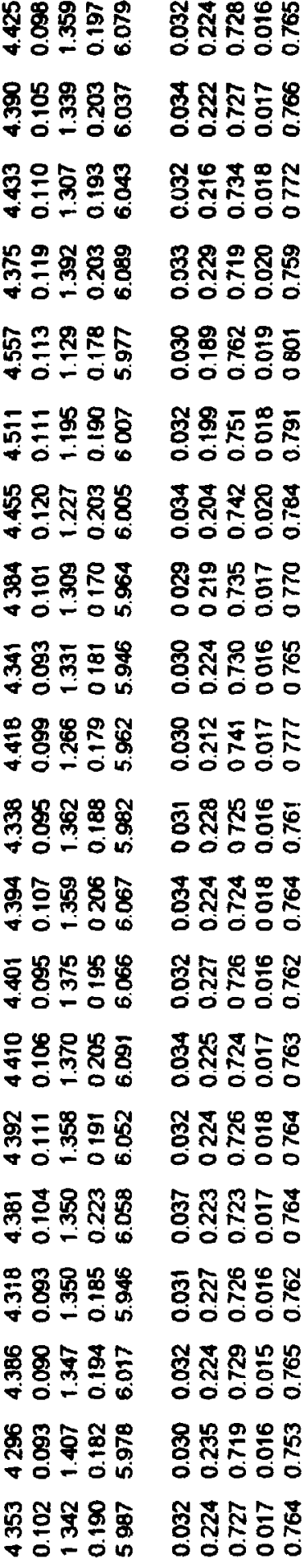

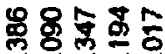

\%००००

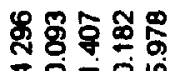

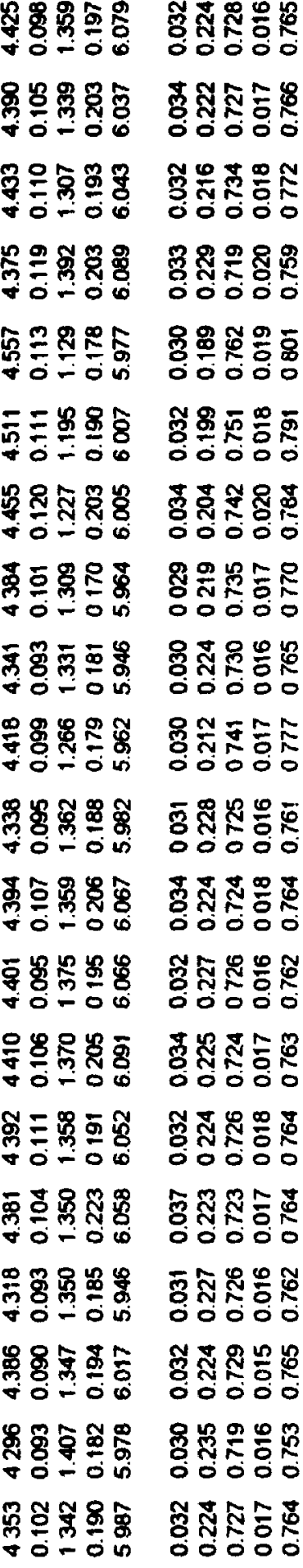

设

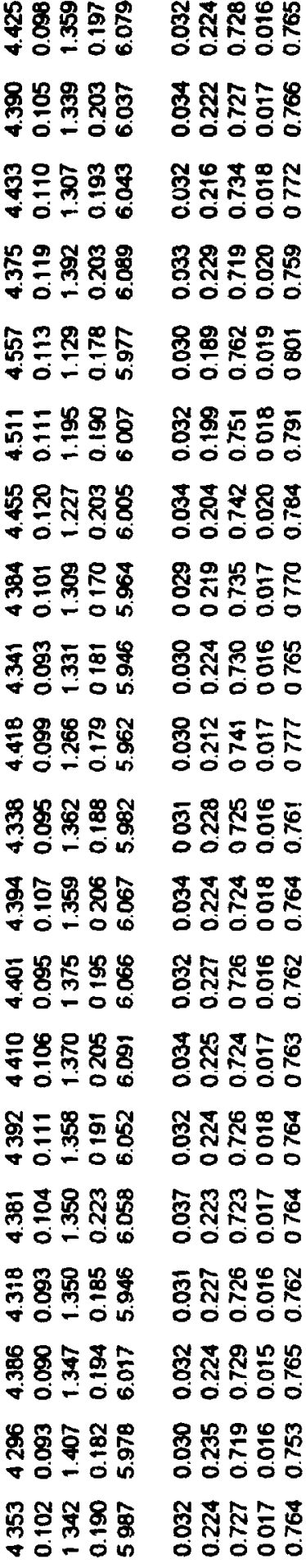

ํㅜㅇ응

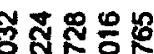

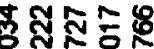

0000

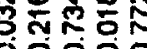

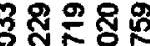

이음

列

요욤ำ

O००00

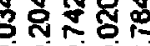

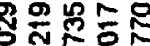

0000

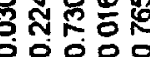

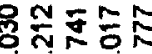

列

O0000

政 政

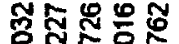
00000

和

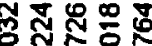

O응으

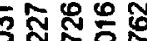

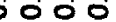

らを こたす 


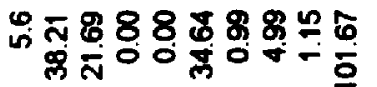

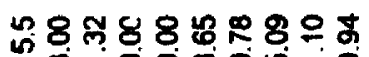

为

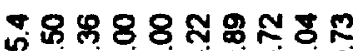

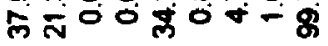

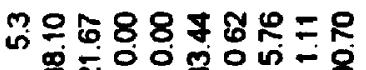

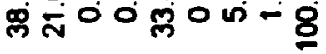

ㄱำ สุ 888 ณึ

需

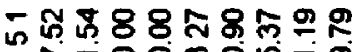

में

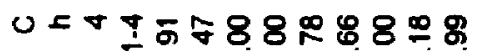

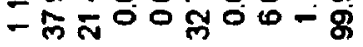

๔

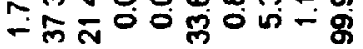

$\bar{x}^{-N}$ -

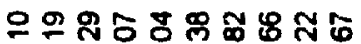

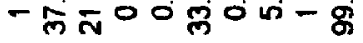

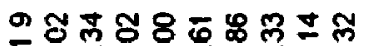

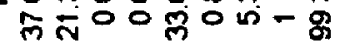

는 8

हี่

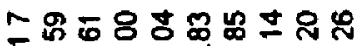

的的然的-8

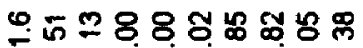

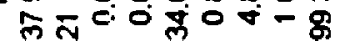

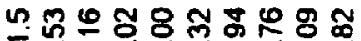

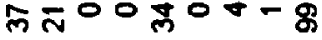

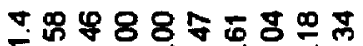

的云次

는요요용요

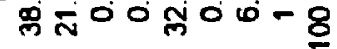

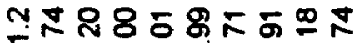

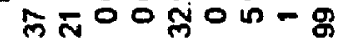

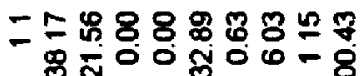

0.

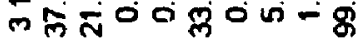

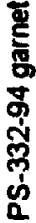

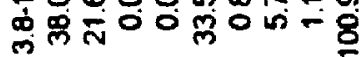

\$유 \$8 88 8

ज융

웅응

ஸ。

ஸึ。

槵

ำ

宛 등

no

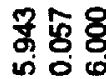

ธํㅇㅇ

000

영동

ㄱㅇㅇ

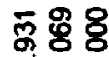

no 0

\% 88

กิㅇㅇ

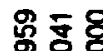

ㄱㅇㅇㅇㅠ

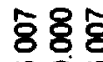

60

要迢

กํㅇㅇ

ลำㅇํㅇ

ก0.

858

no

影云

궁

雾 $\overline{8} 8$

ธ형

$00 \%$

แ 0

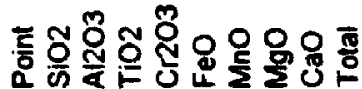

종

弯亏

ง⿵门人

罗点 8

落

no

$8 \overline{8}$

\$88

100

888

$\$ 88 \%$

100

\$8 80

多8 808

品 85

moom

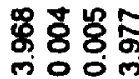

嵒 $\overline{8}$ 용

moom

品骂等

mo

$\overline{8} 80$

กั0

素要응

罗客骂

응영

棁 88

moom

5๊

응욤

8888

mo 0

ริ영융

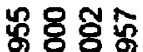

moom

夸各家苛

$8 \overline{8} 8$

ल०0

कं

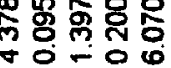

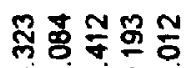

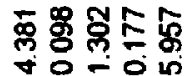

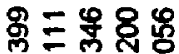

प5

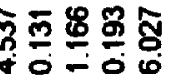

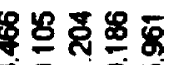

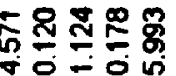

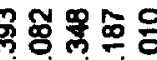

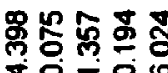

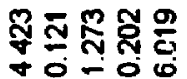

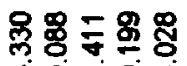

政造造

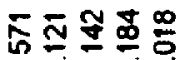

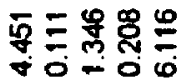

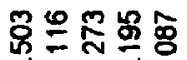

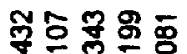

讷士口品。

T०-0。

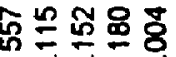

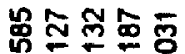

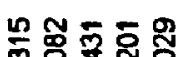

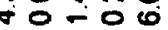

款要等砳

$\checkmark 0-06$

ำำำำ

응ㅇㅇㅇㅇ

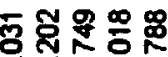

응응

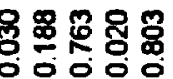

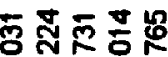

응ㅇㅇㅇ

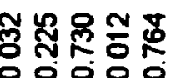

弱추요요

О응으

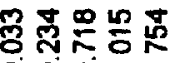

00000

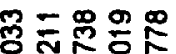

응ㅇㅇ

융으용용

00000

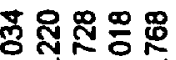

응응

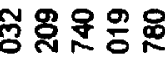

0000

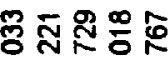

으응

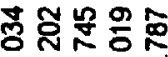

응ํํㅇํํ

융홍ㅁㅇ응

00000

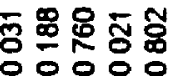

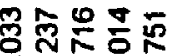

O०000

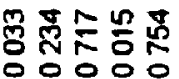

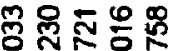

엉영

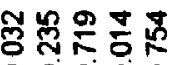

00000

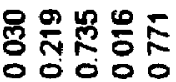

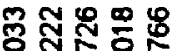

O000

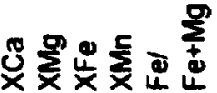




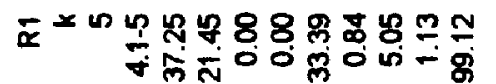

นูำ

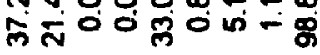

过为8 $80 \% 8 \%$

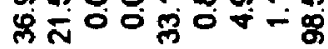

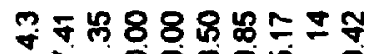

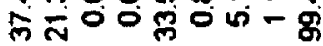

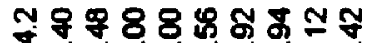

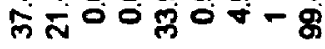

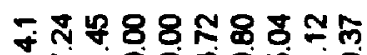

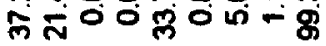

U-U I

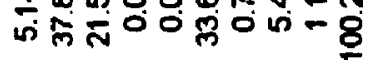

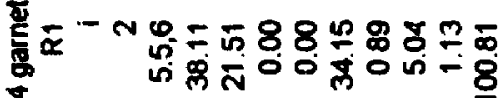

ఫ

ฉู่

ஜ்

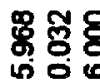
多染安

울동 sio. 옹ํㅀ용

กิ8 is

\section{量要客} ธก.

范安 ตั.

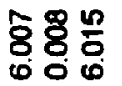

$\frac{0}{5} 88 \%$ 驾骂哭骂

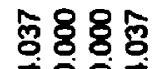
$+00 \div$

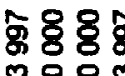
ระ8용

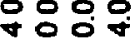
पु요용 帝

\$8: $8:$ no.

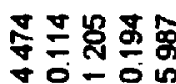

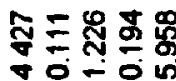

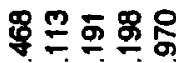
둥ㅇㅇ

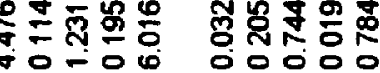
品토용

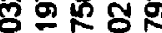
To-0 00000

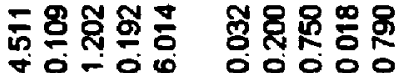

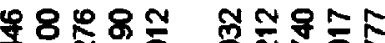

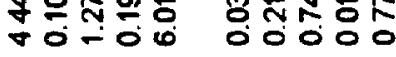

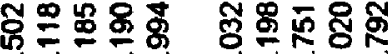

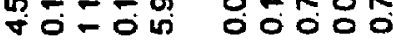

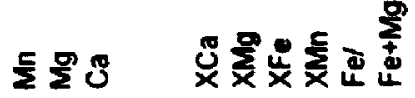




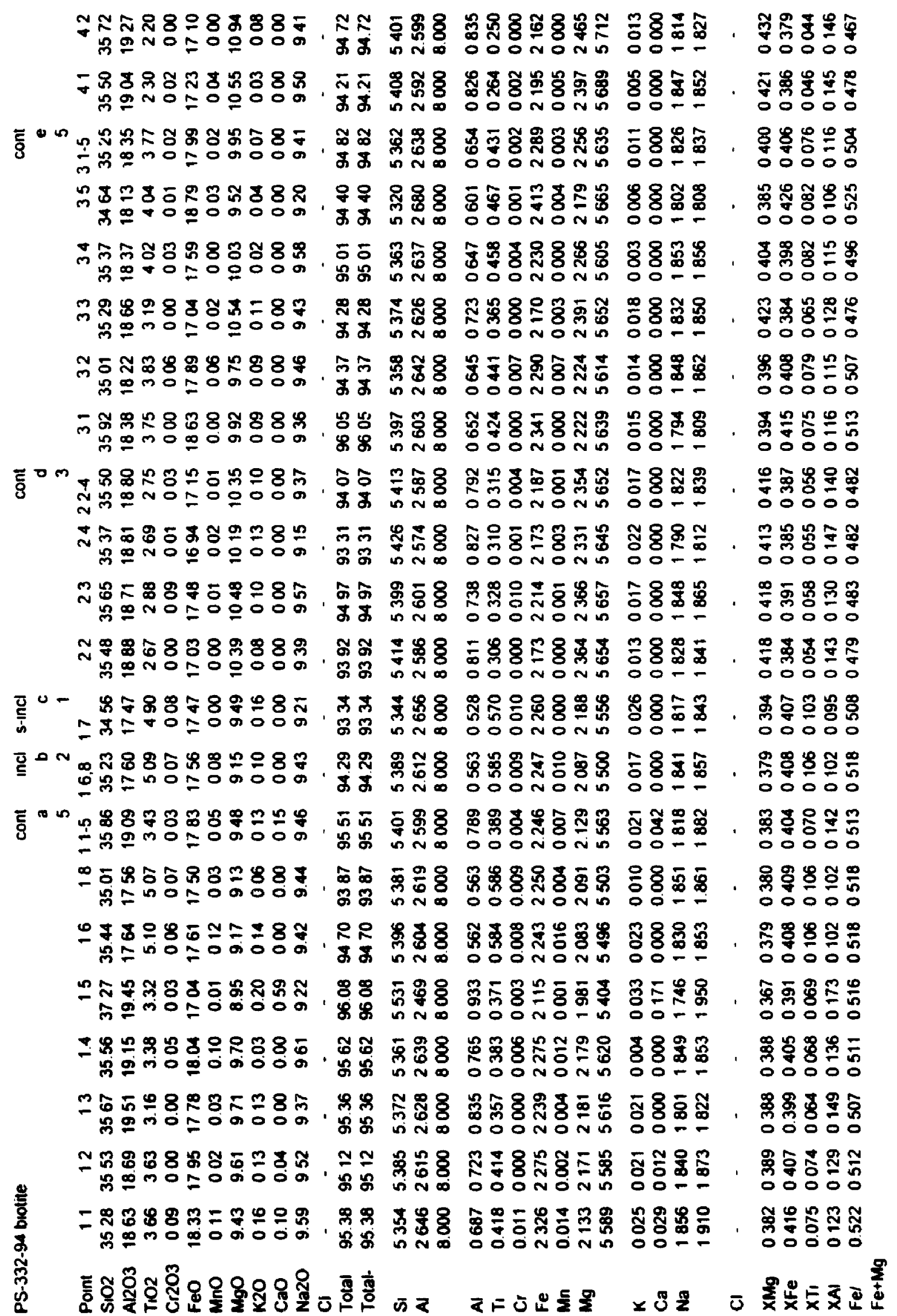




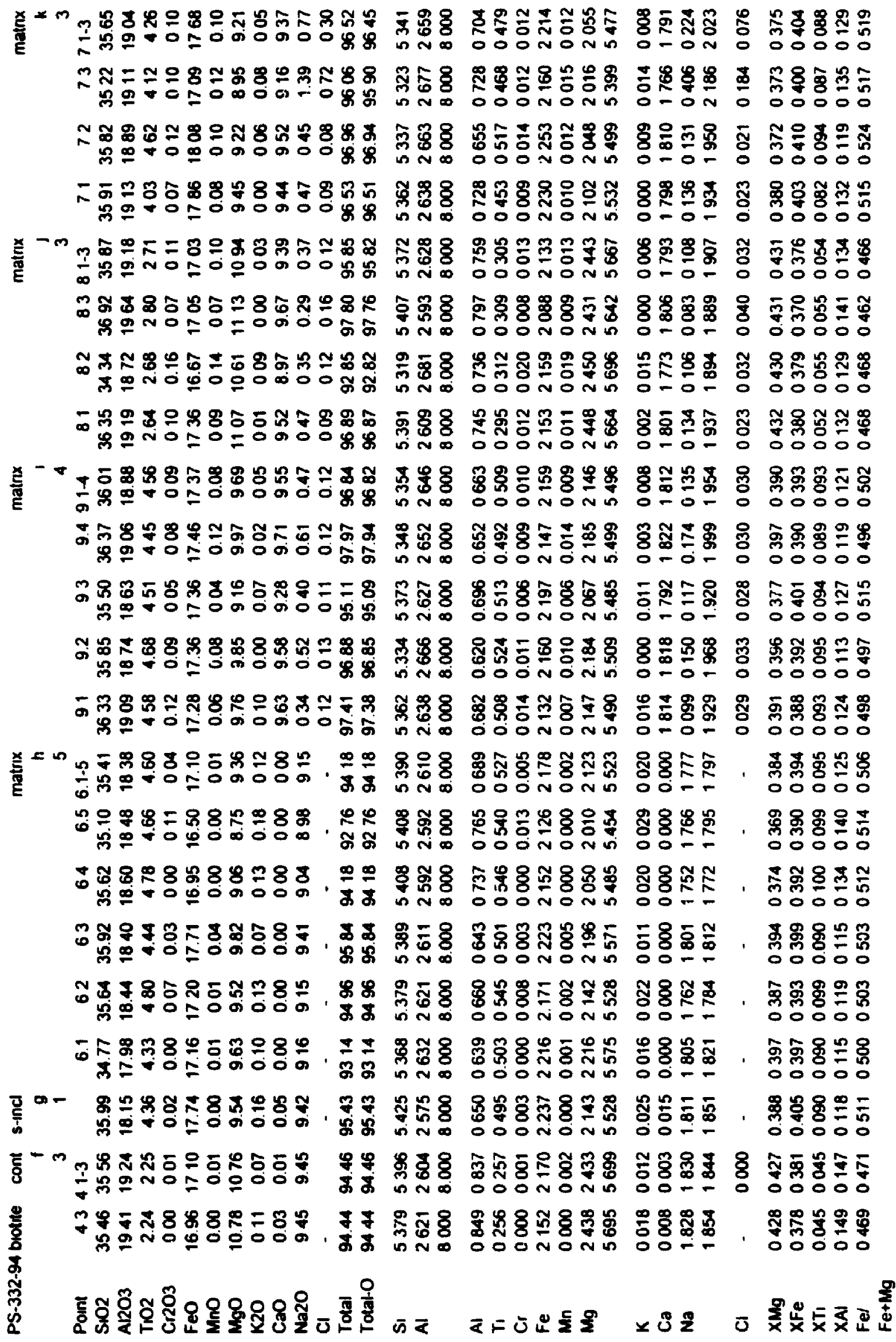




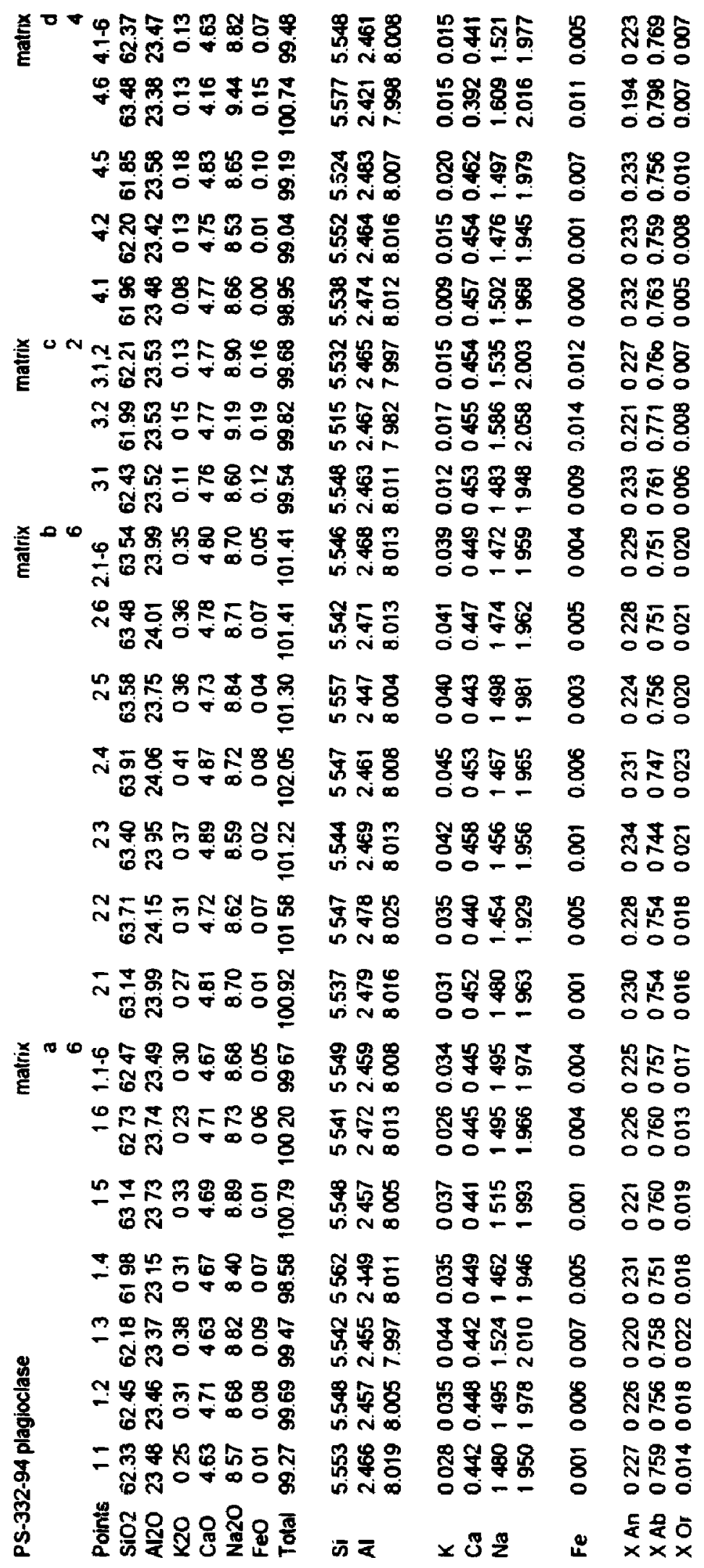




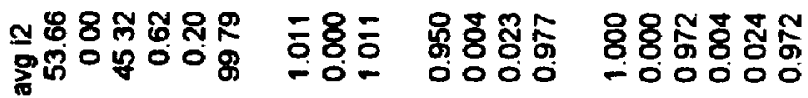

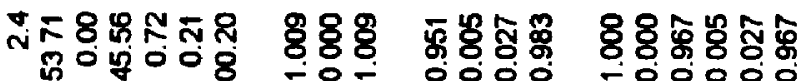

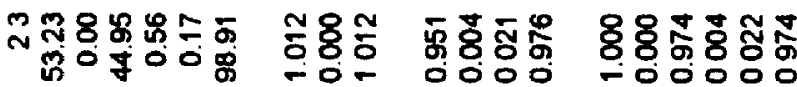

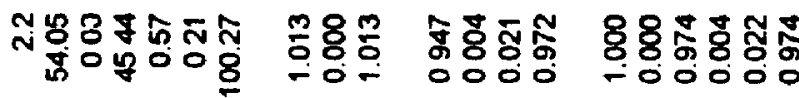

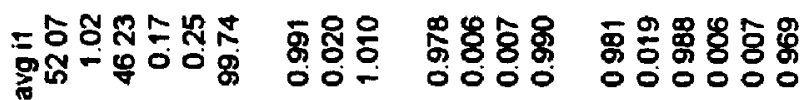

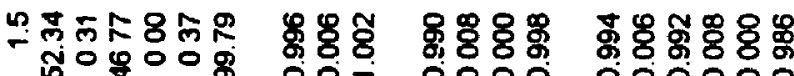

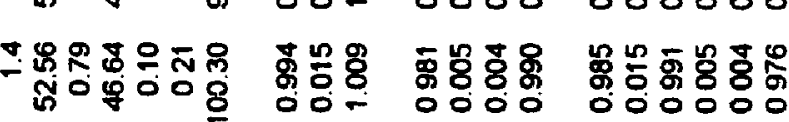

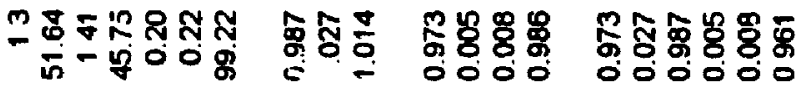

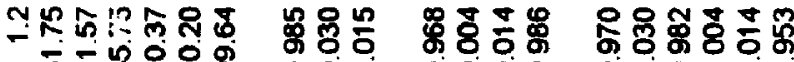

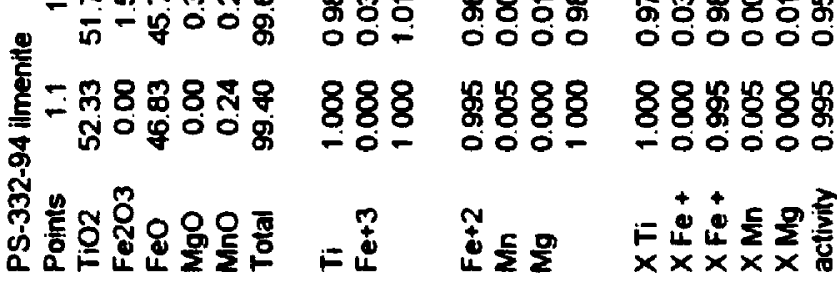


జ

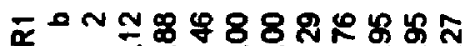

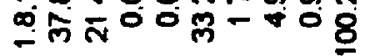

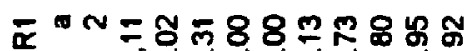

च लू

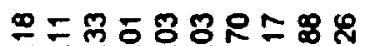

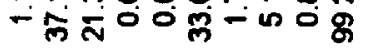

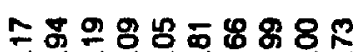

禺

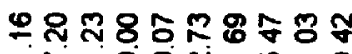

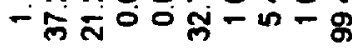

는

- मूं०० लूल ज०

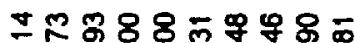

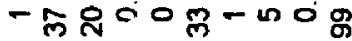

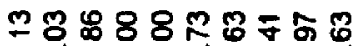

-

눙ำ

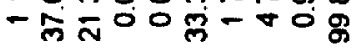

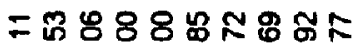

- 的

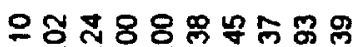

-

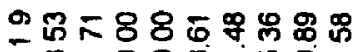

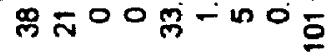

농ํำ

塄

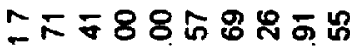

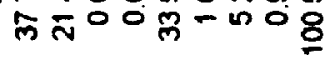

는 88 중ํำ

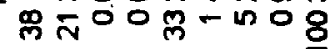

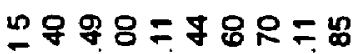

塄

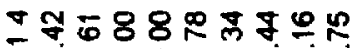

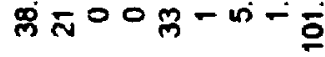

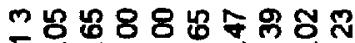

क्र

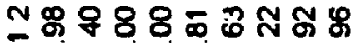

ติ่

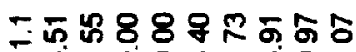

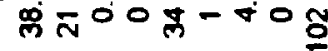

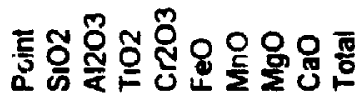

5ू용

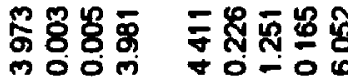

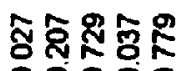

०0000

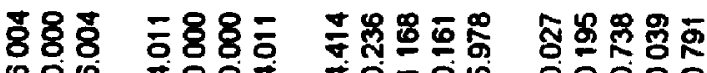

$88 \%$

00

$\$ 88$

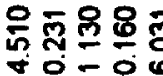

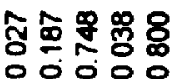

웧 $\overline{8} 8$

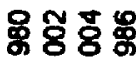

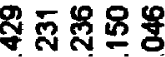

串蛋忿品

जि 0

중옹 \&

ชิำ

炎 影

no

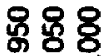

ज०

पू 8 용용

ल0

$\$ 8$

no․

옹용용응

moㅇ

옹융응

융용용용

흏 동 용

ऊc

용양용용

moㅇㅇ

ชูง ํํㅇํํำ

용ㅇㅇ

능용후

- 0 - 00

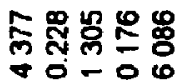

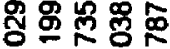

00000

000

용요용

下

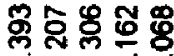

प०

守肎发吉喿

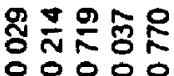

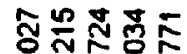

ㅇㅇㅇㅇㅇㅇ

꿍융 훙

000

앵양용영

잉요

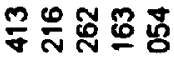

긍ㅇㅇ

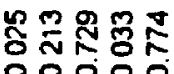

눙융 눙

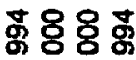

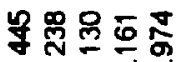

○ =동

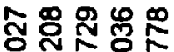

00000

000

moom

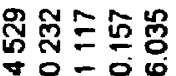

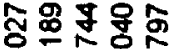

00000

형ㅇㅇ용

000

홍용용용

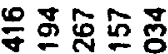

등ㄷㅇ

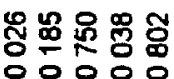

옹융잉

แn 00

옹융:

ioㅇ

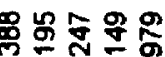

पo-0 n

융유ㅇㅠㅠ융

00000

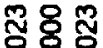

000

응융영

잉요

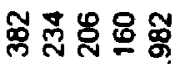

०0-0

串总点号尽

O0000

象듕융

is 0

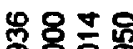

ㄱㅇㅇㅇㅣ

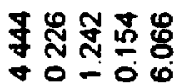

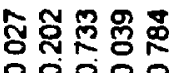

O 0

\%ํㅀㅇㅇㅇ

동유융ㅎ

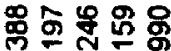

- O - 0 i

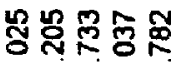

O000

以०6

n००

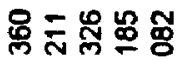

ิㅗㅇㅠㅠ용용

O०००00

$\checkmark 0-00$

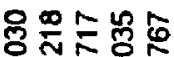

O응ㅇ

5융요

各审审帝

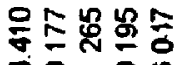

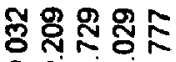

0-00 00000

罗

is 00

ก००m

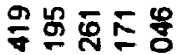

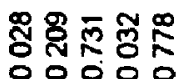

\& $88 \%$

m00

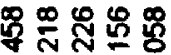

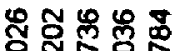

ํํㅇํํㅇ ำ

응요

\& $88 \%$

mㅇㅇㅇ

รำำํํㅇ

$+0=50$

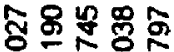

웅등응

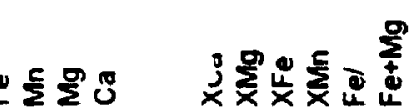




\begin{tabular}{|c|c|c|c|c|}
\hline 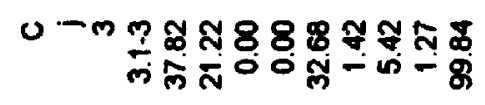 & $80 \%$ & 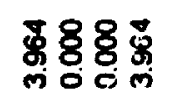 & 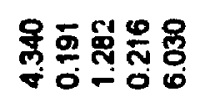 & 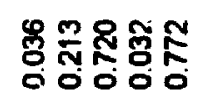 \\
\hline 户 & 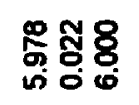 & 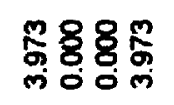 & 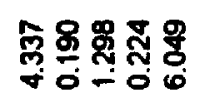 & 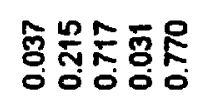 \\
\hline 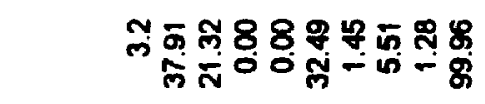 & $88 \%$ & \$్లిర్రి: & 햄영 & 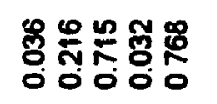 \\
\hline 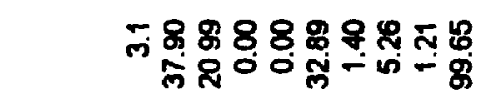 & 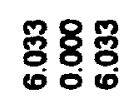 & 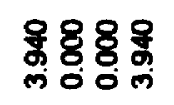 & 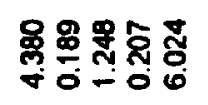 & 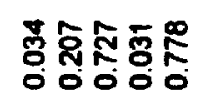 \\
\hline 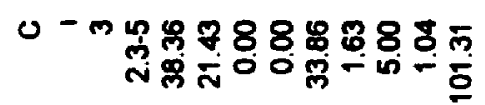 & : & 幽 $8080.0 \%$ & 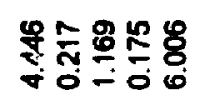 & 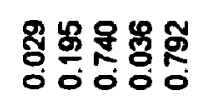 \\
\hline 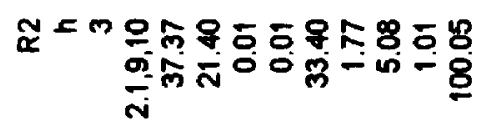 & 藏荢号 & 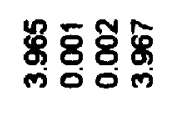 & 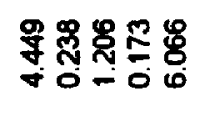 & 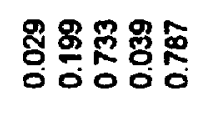 \\
\hline ON & 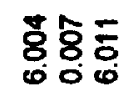 & 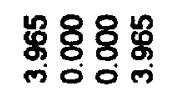 & 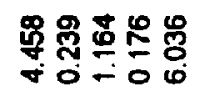 & 옹응융웅영 \\
\hline 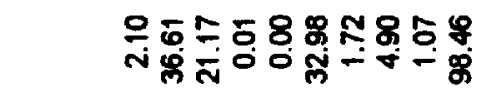 & 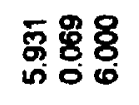 & 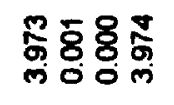 & 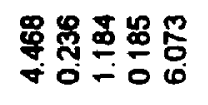 & 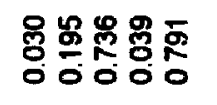 \\
\hline 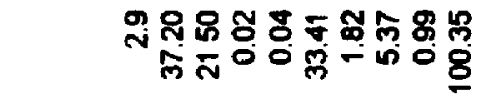 & 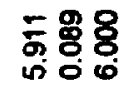 & 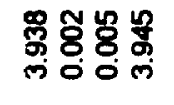 & 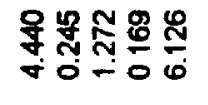 & 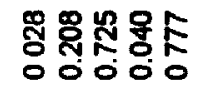 \\
\hline 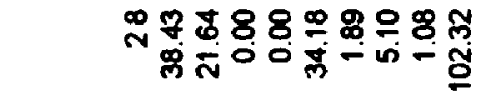 & 商高各 & థ్ల్లి & 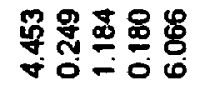 & 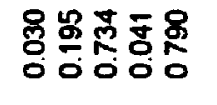 \\
\hline 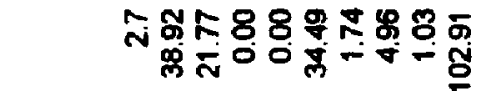 & ธ్ర0] & 象 & 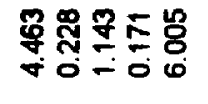 & 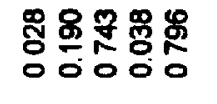 \\
\hline 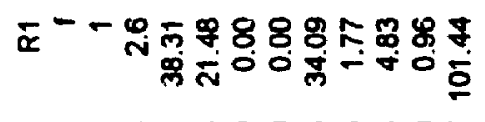 & 웅융융 & 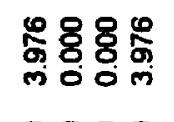 & 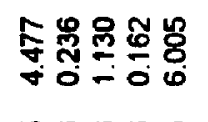 & 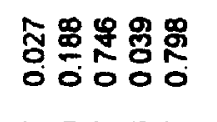 \\
\hline 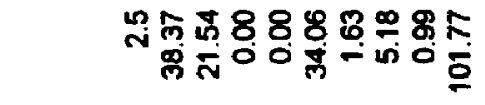 & 80808 & 율 & 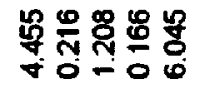 & 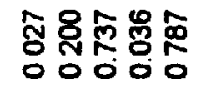 \\
\hline - స్ల & 옹 & 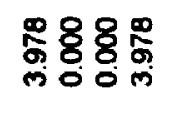 & 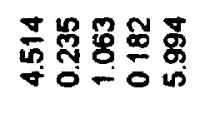 & 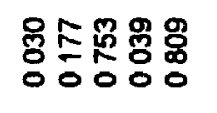 \\
\hline 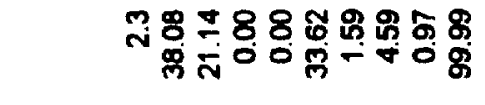 & 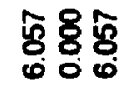 & 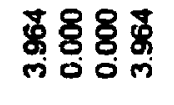 & 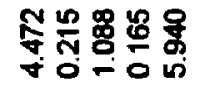 & 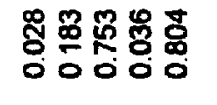 \\
\hline స䒠 & 要: & $\overline{\$}$ & 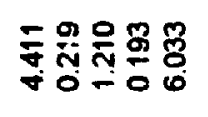 & 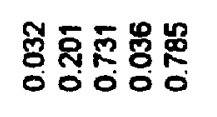 \\
\hline 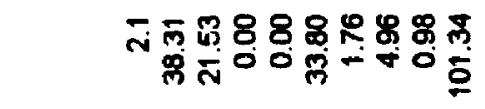 & 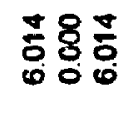 & 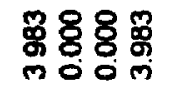 & 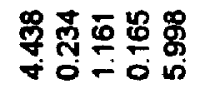 & 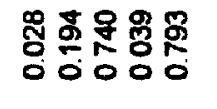 \\
\hline 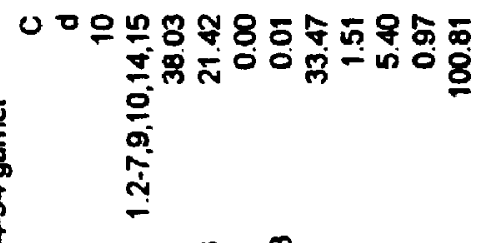 & $\begin{array}{ll}8 \\
8\end{array}$ & 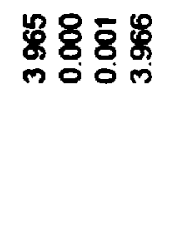 & 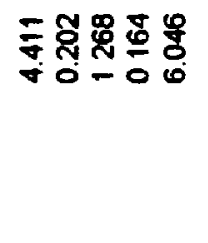 & 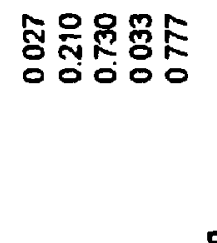 \\
\hline 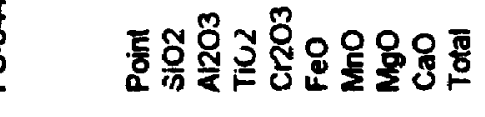 & & & 5 & $\frac{1}{2}$ \\
\hline
\end{tabular}




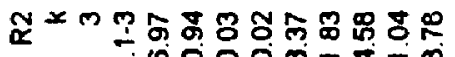

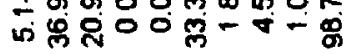

需芯号

$\$$

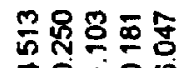

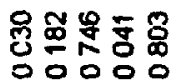

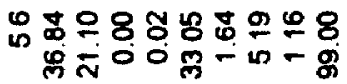

艿安客

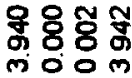

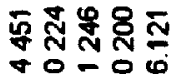

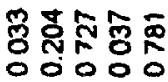

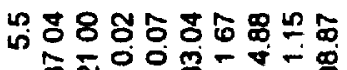

동융

舫:

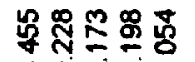

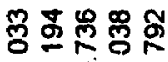

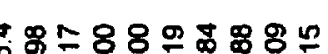

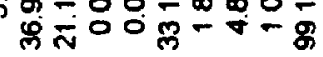

总品客

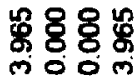

TO-OO

00000

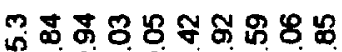

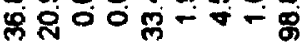

恋要遂

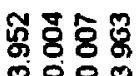

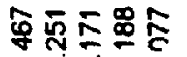

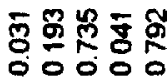

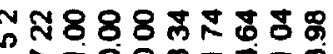

.

mo

今。유.

总迤客兽

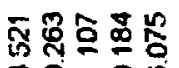

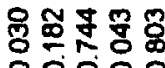

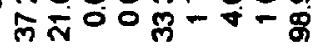

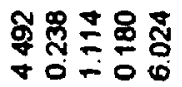

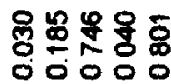

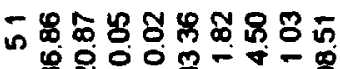

ถูํํ요

$\$$

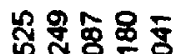

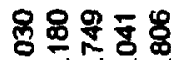

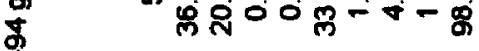

ก०० ल00

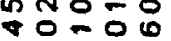

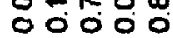

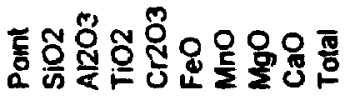

कर

$\triangle$ \ニ

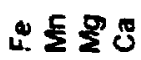

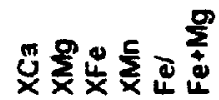




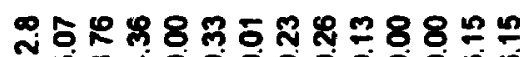

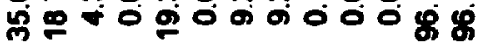

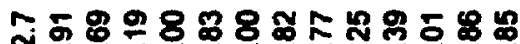
में0\%0

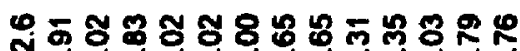
में कि 产。”

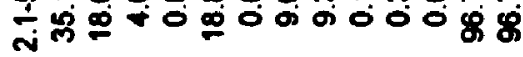
นึำ में

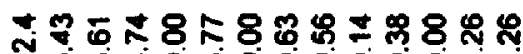

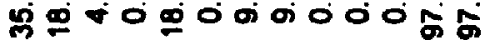

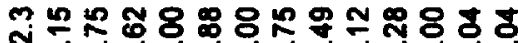
मूं०ण

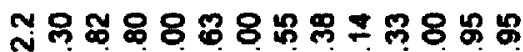
过宁

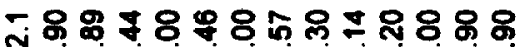
๖ लें

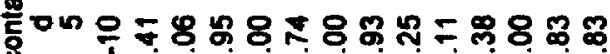

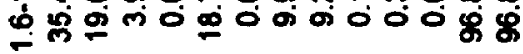

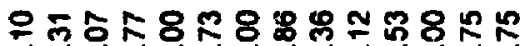

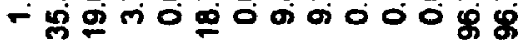

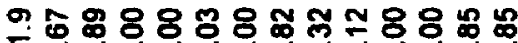

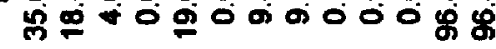
웅 में

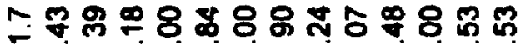

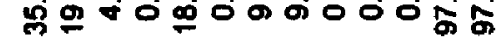
는

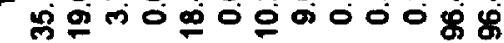

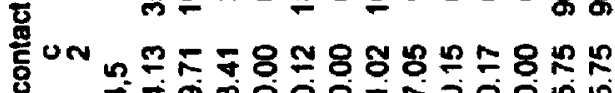

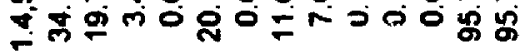
n= म्ल

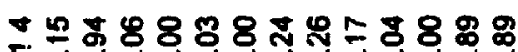

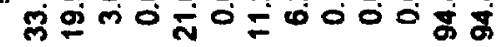
คธ

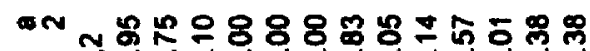
.

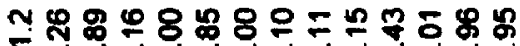

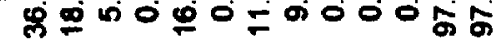

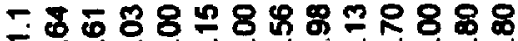
边

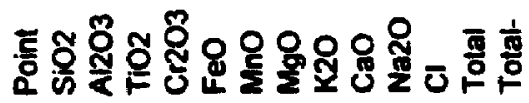

8 운 แก

费 ్ㅠ용 Sิ ñ

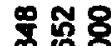
in

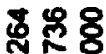
in 员负员 กิก

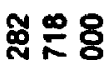
พं

赵果各 กู่

웅용 ง

苾品 8 งก่

承 ฟกำ

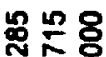
ธง

天

2 ก

옴ㅇㅇㅇ कल

路웅 जٓ⿻上丨

응응 in

용용 ตNe⿻

สำ जก N

옹유요 un

啉:

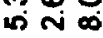

密둥 in $N$

解造 ธत网 总용 ल

\section{कब}

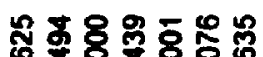
00 Non

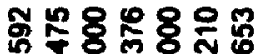

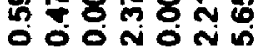

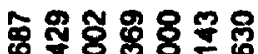
00

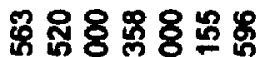
OOON口N

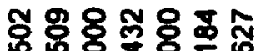
엉에에

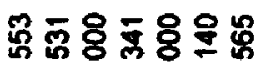
- OONONO

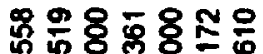

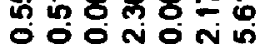

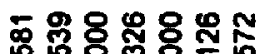
00 0 Non

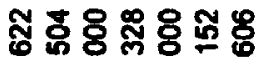
00口n

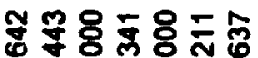
0.0

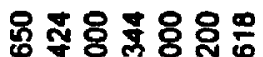
coonon

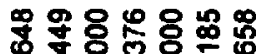
0о

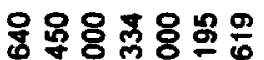
000nON

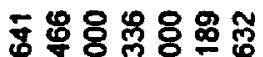
O유에

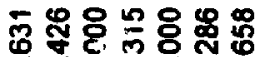
0ั0엉

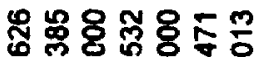
०。ำก

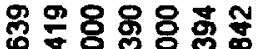
엉에윰

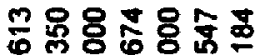
OOONONO

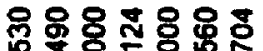

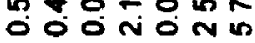

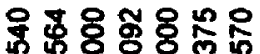
000 Non

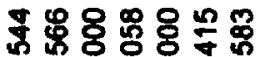
०0。 Non

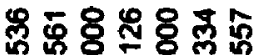

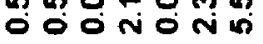

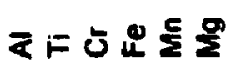

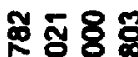
- 00

喿号풍 -0.

훙흐하 -0.

\&

- O0응

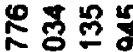
등영

응 용윻

- $00-$

영융훙응

등-

ڤ용영

- 00

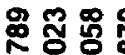

$-0$.

象电三

영두

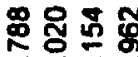

- O०

온융

등응

䞤步总

$-0$.

운 궁용요

$\div 0 \div$

กำ

응으

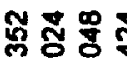

$\div 0$

密 $\overline{0}$

$\div 0$

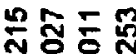

응응

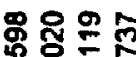

- O. -

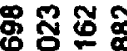

$-80$

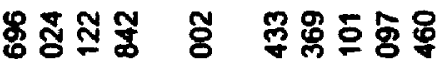

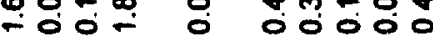

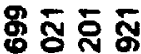

.०० -

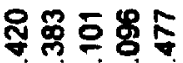

00000

$\times 808$

8 费盟骂三品

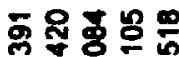

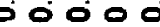

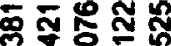
00000

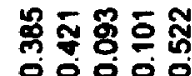

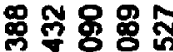
잉ㅇㅇ

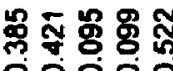

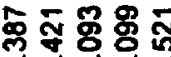

䒠合吉 00000

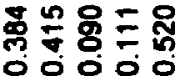

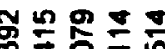
00000

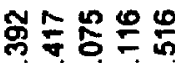

00000

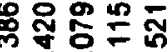
00000

可的最哲的 00000

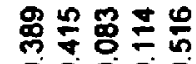
00000

훙웅옹픙 0000

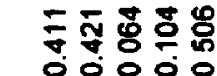

운융형융 00000

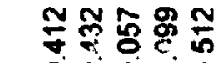
00000

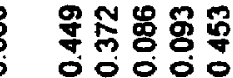

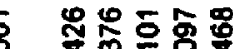

응 


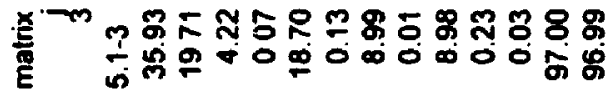

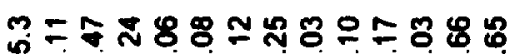
边 พื่ กับ 只

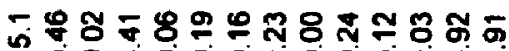

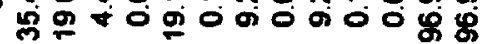

ำ है

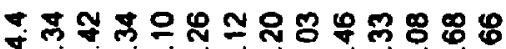

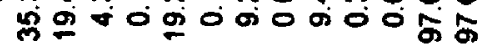

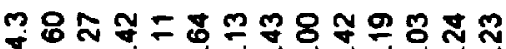

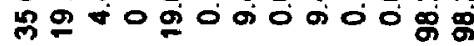

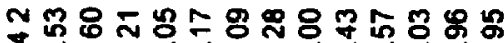

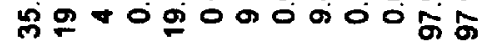

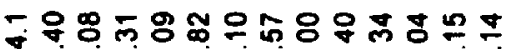

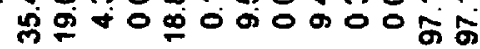
뜬ㅇำ

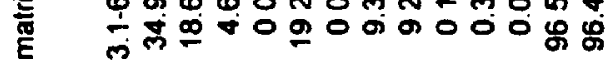

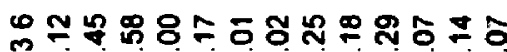

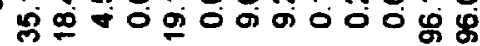
ณำ

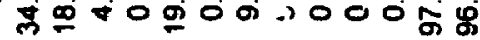

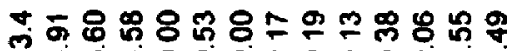

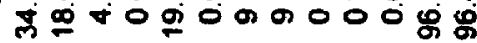
잉 票

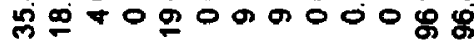
พ

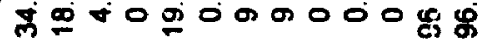

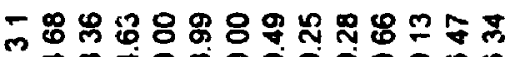
点

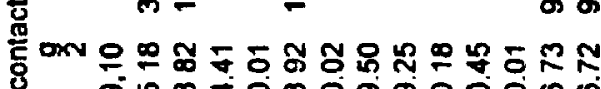
성

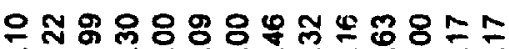

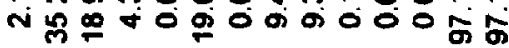

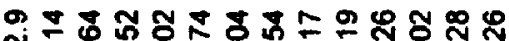

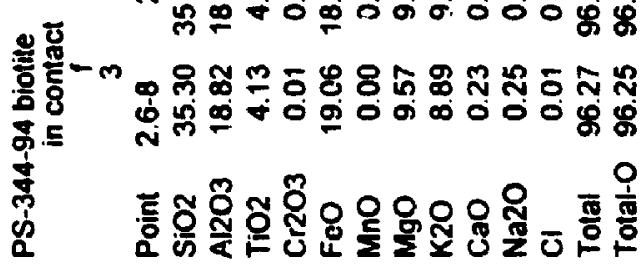

\%్ जิ ง 要 8 i ธ్ల. ஸึ

奠 8 8 in

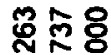
ஸे

员全员 ஸ่ 讯号 in $\infty$

路是 แ $N$ D

ำ เ

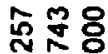
ง

호용 内

준용

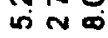

옹요 น त

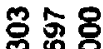
ธतก ㅎํㅇㅇㅛ

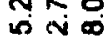

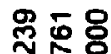
แ

กํำ ஸึ่ 둥요 में के

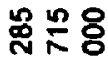
ธก ธิ i कर

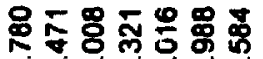

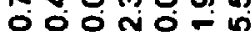

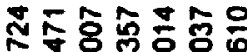

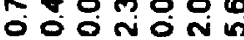

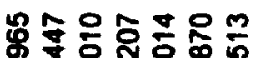

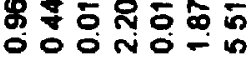

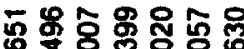

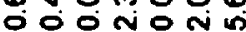

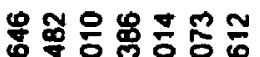
OOONON

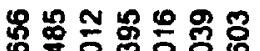
Oㅇㅇㅇ N

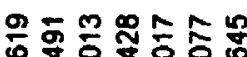
OOONON

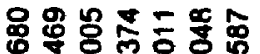
OO ON N

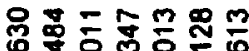
OO O Non

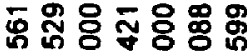
Oㅇㅇㅇㅇㅇㅇ 에

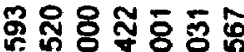

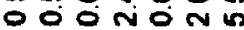

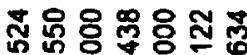

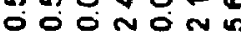

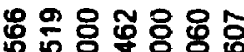
OOO non

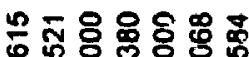
o o ononim

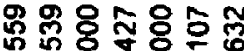
o o oñ nín

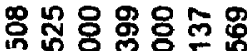
r =onon

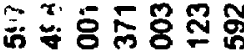

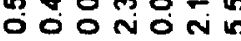

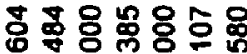

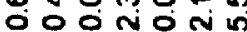

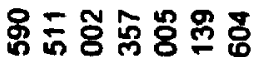
o o ononion

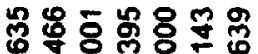

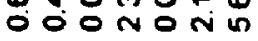

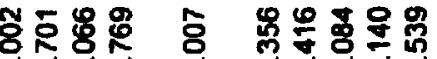
O-O

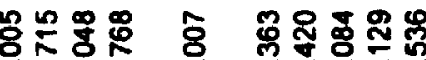

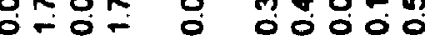

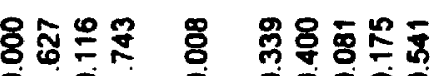
$0-0100000$ 审乐芦总 O-

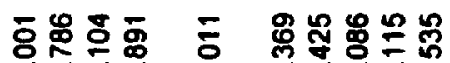
O-O- 0 00000

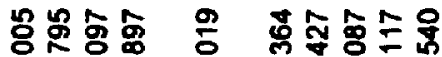
등ㅇㅇㅇ 융융ㅇ

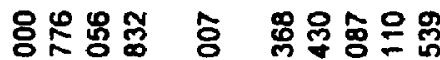
O- O- 00000

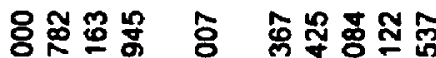

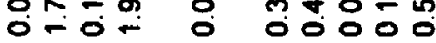
융영 卷 O- 0 - O 00000

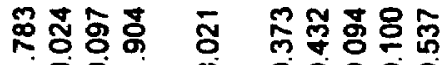
-OO- O 00000

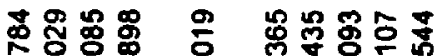
응융 웅

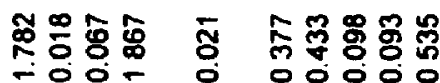

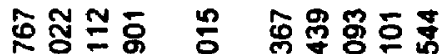
ㅍㅇㅇ듕 웅 영영영

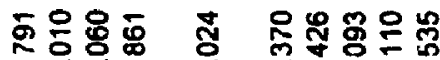
- 00- 00000

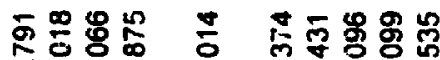
영잉 응

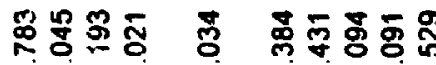

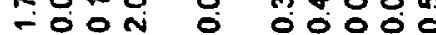

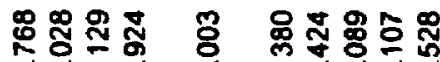
- 00- 0 00000

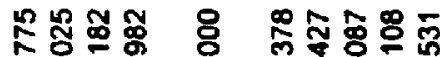
융뎡 응 형융영

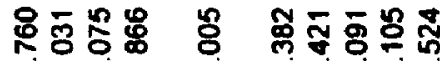
-OON O 00000

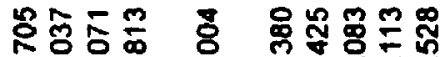
융융 융융융

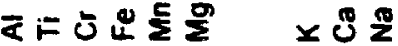




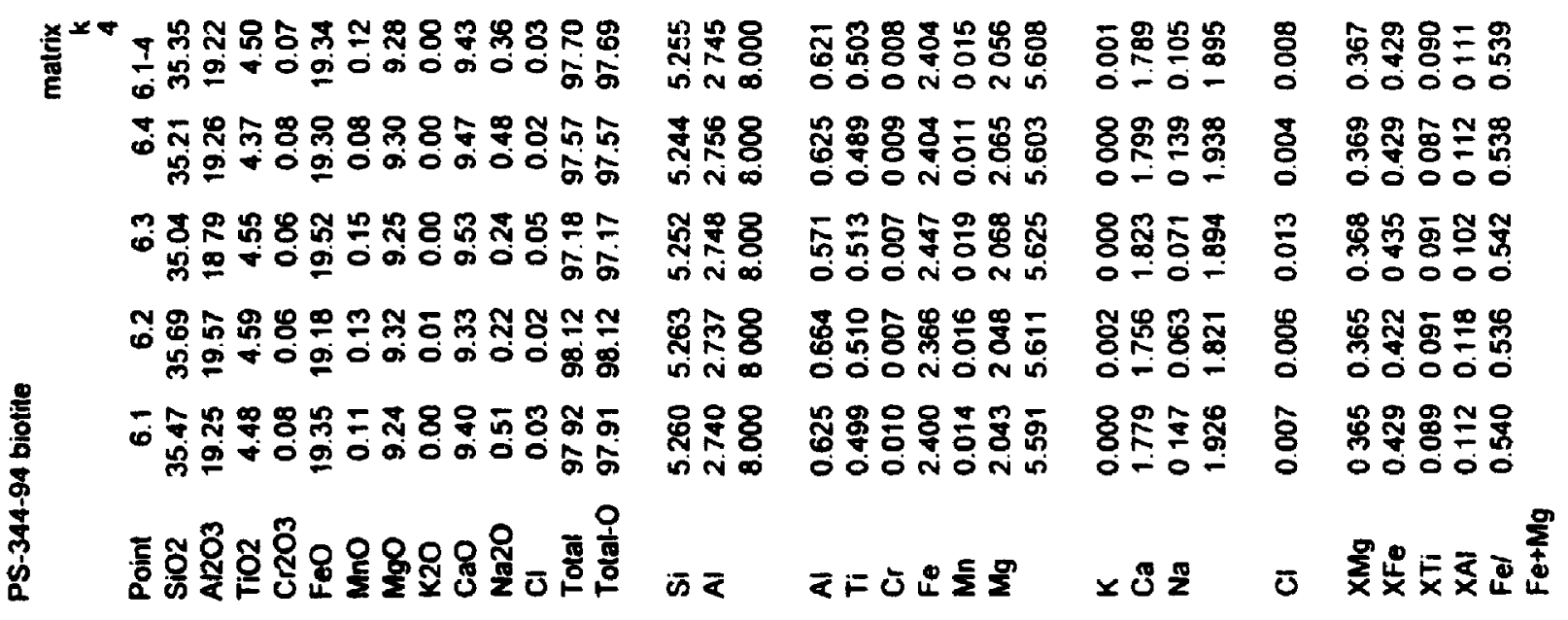




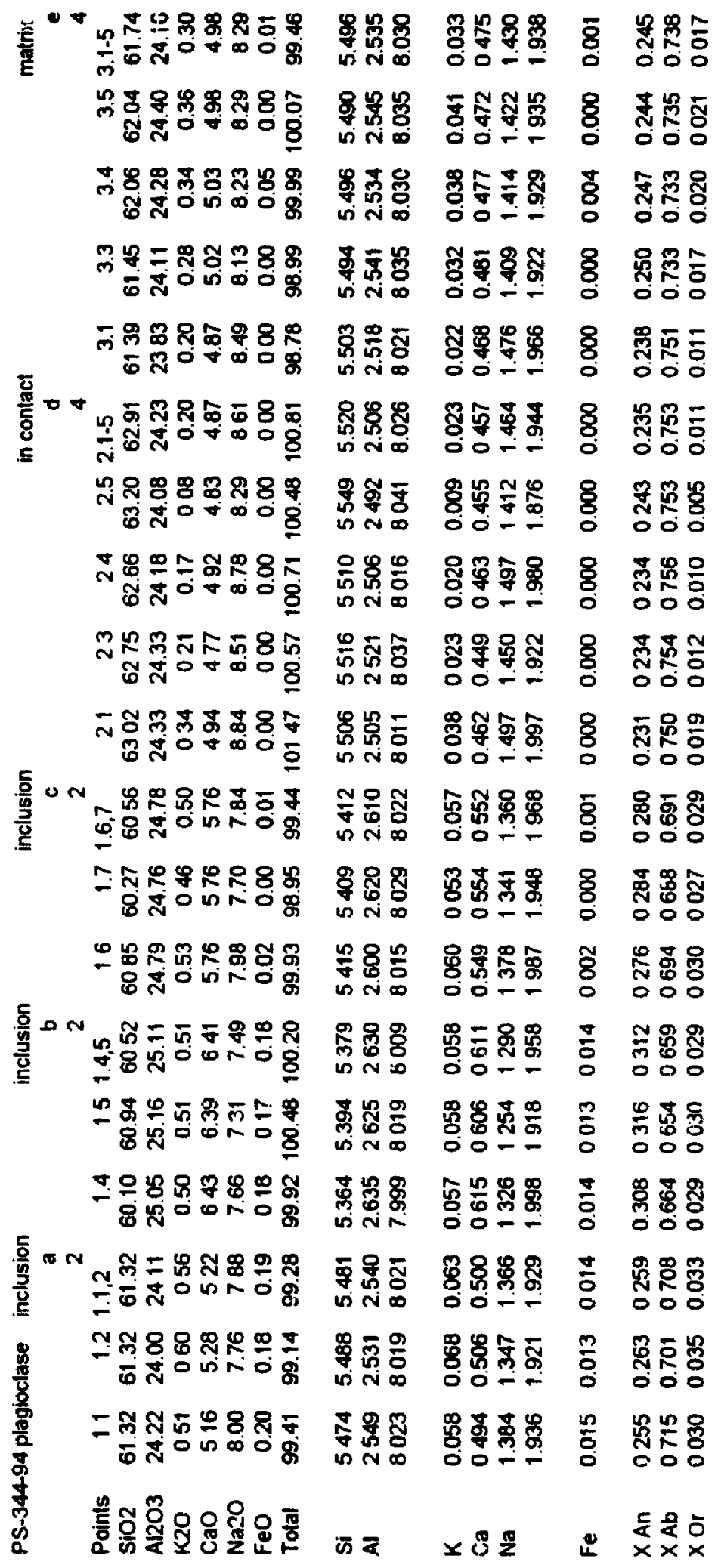




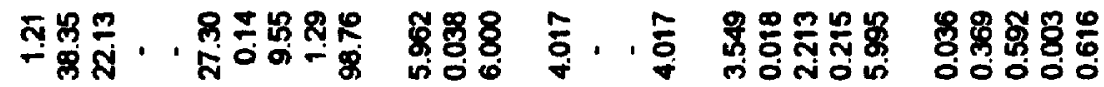

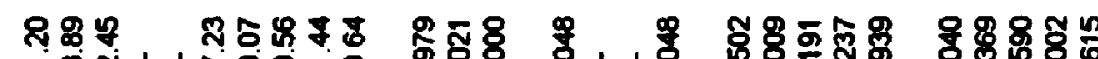
-

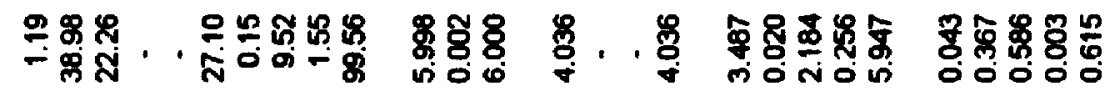

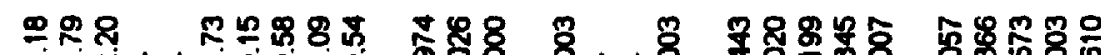

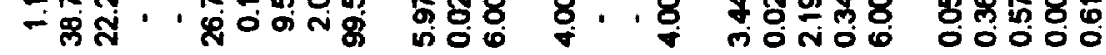

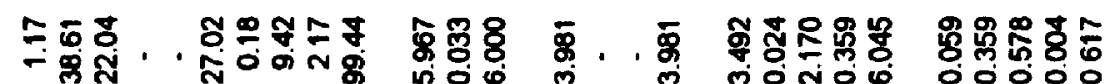

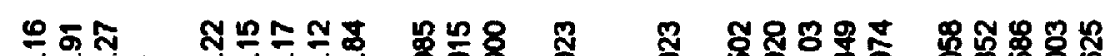
-

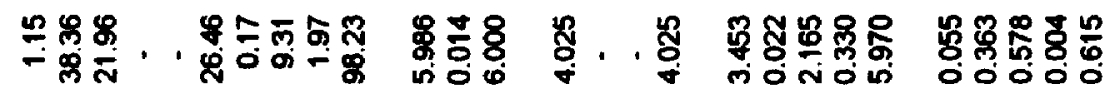

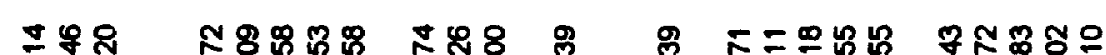

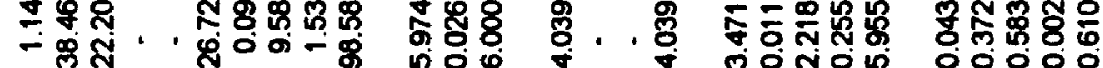
$m 8=88 \%$

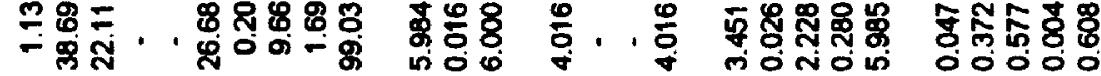

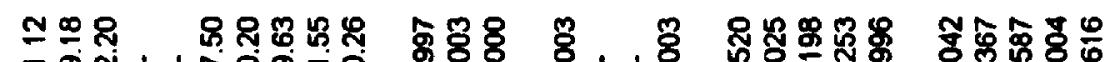

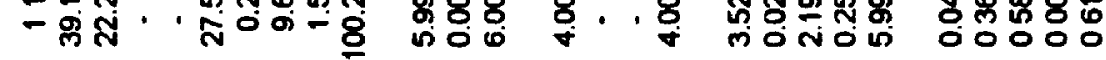

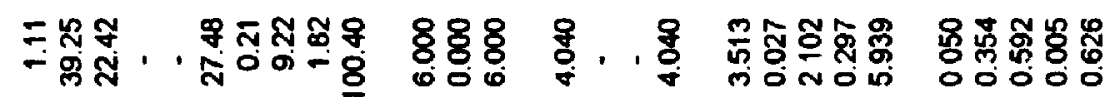

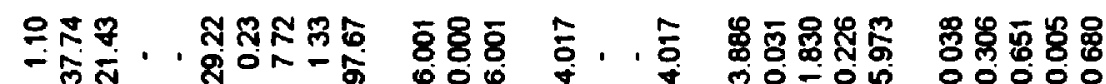

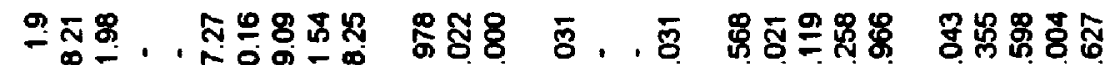

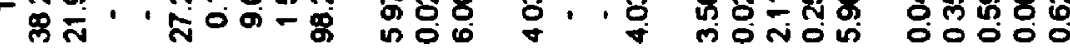

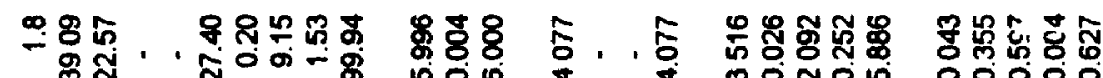

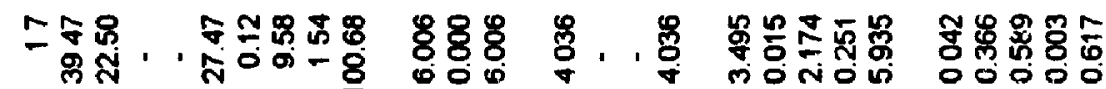

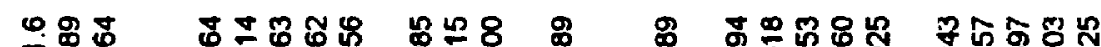
需

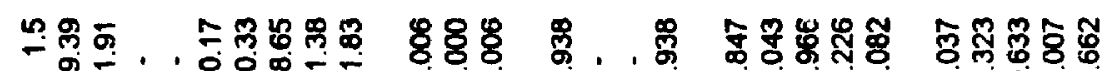

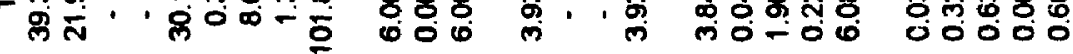

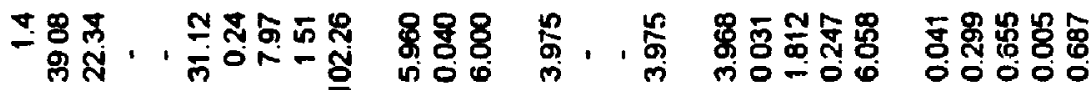

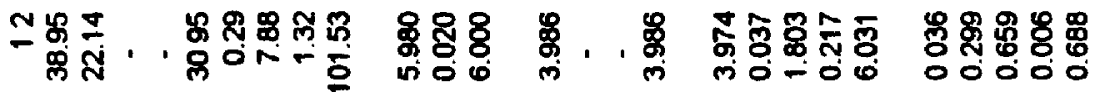

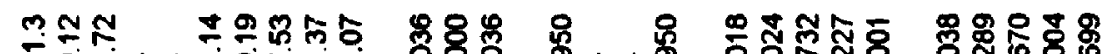

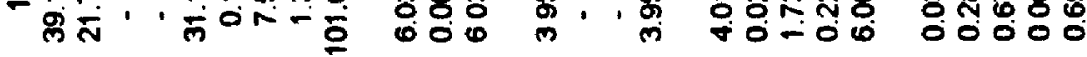

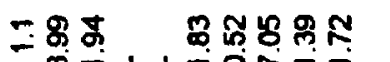
$8 \%$

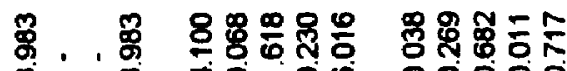

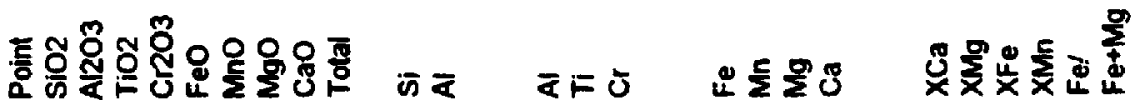




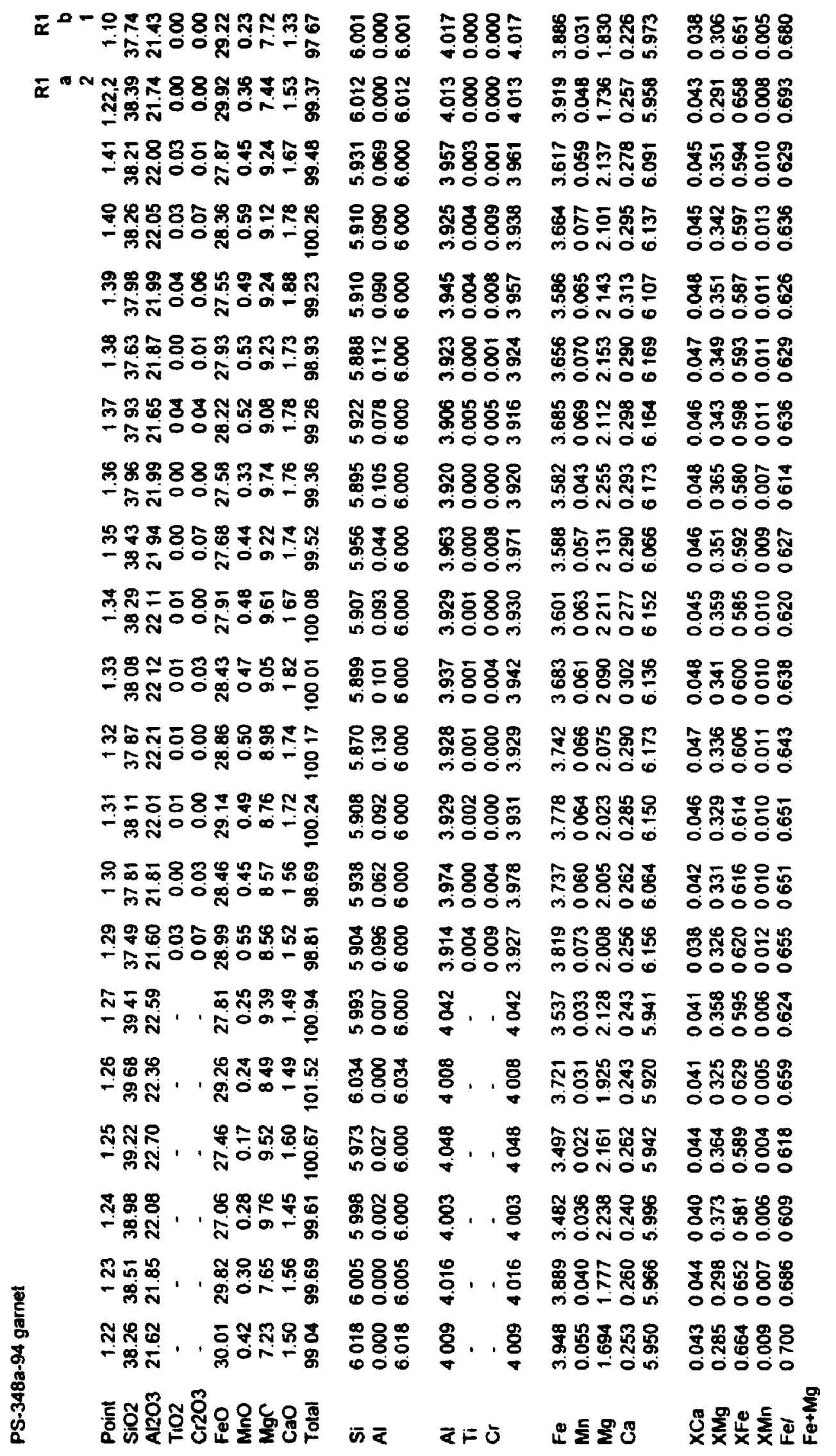


웅용ำ

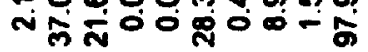

ニ员下本8 $7 \%$ ํㅜㅇ

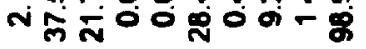

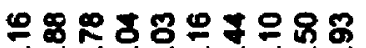

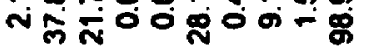

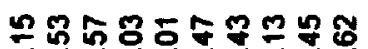

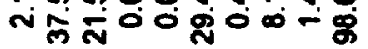

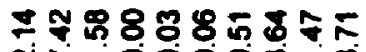

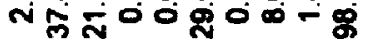

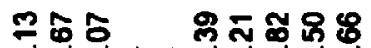

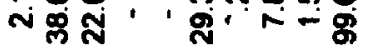

주용ำ

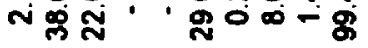

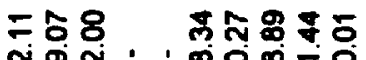

N忽 ' '

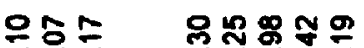

ง

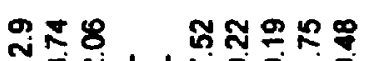

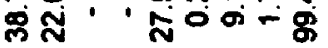

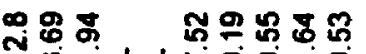

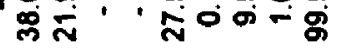

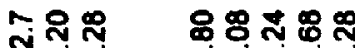

g

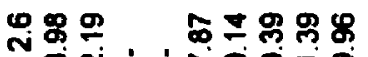

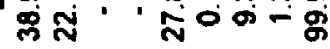

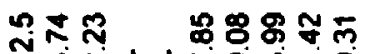

罚 ' Noळ-g

홍

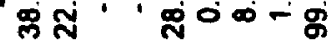

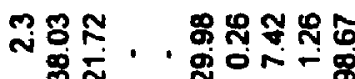

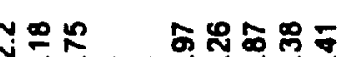

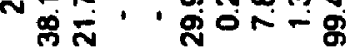

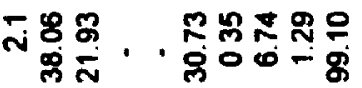

ט・テエ

更

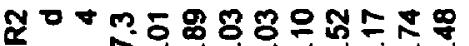

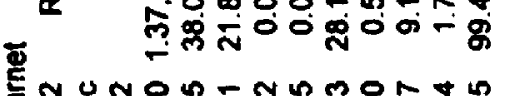

둔

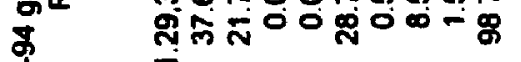

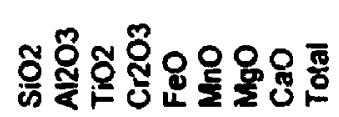

종

등요

$\$ \cong 8$

충융용

ชัํํㅇㅇㅇ

फ0 0

888

แ०००

กั่ำ

10.0

됴융

용용요

$8 \%$

0

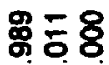

in

ถึํㅇㅇㅇㅇ

i⿵门人

ㅎㅇㅇㅇㅛ

000

行용

no.

象

nio

\$요

no.

$8 \overline{8} 8$

sio

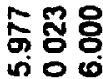

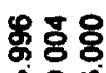

ino

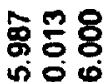

m

900

รัฐㅇํㅇ

กิด

\%్요유

लं०ूo

용요용

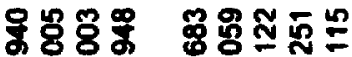

ल०ठำ

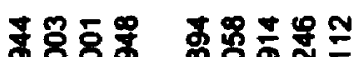

mo $0 \mathrm{~m}$

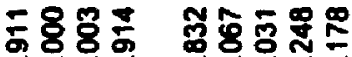

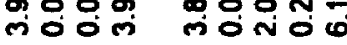

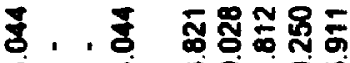

io-

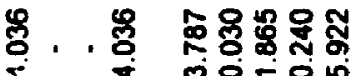

沓, 量

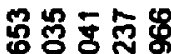

लं० तion

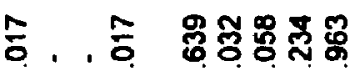

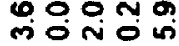

\&. .

莴颉串费

món

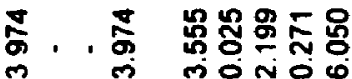

뚜응요유유

행응웅손

monon

声.

象융 ్ㅗ웛

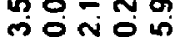

5. $\cdot$.

迢둥흉융

กับํํㅇ

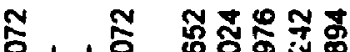

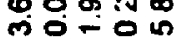

号, . 兽

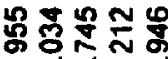

moto

㬝, , 惫

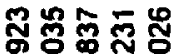

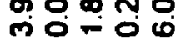

\& . .

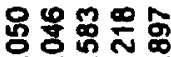

- 0 - 0 \%

: ㅇㅇㅇㅇㅇ용

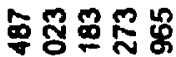

ionom

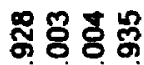

号总总是

엉 त̄

궁ㅎํ융

ल००ण

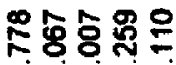

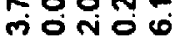

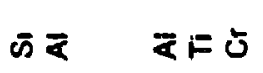

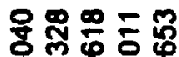

ร.8.:อ

궁형영

옹영웅웅형

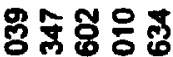

용옹

애음

엉영엉

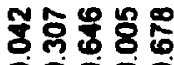

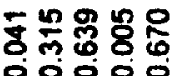

웅 궁당 웅

$\circ 0000$

용옹응 눙융

00000

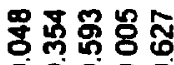

웅 总:

00000

ఫ융용용

용용뭉

0000

옹융융융유.

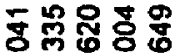
00000

号总骂造总

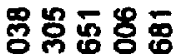

०000

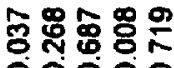

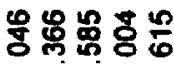

00000

운운 능

m以

00000

(1)

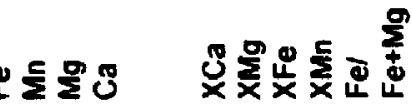

0000 
Uール

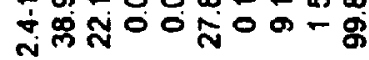

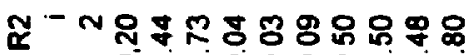
的策

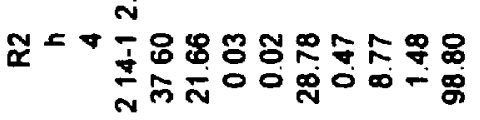

ส

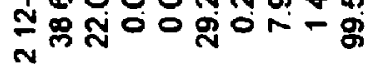

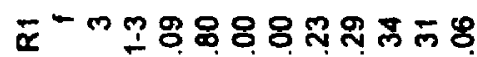
त̄

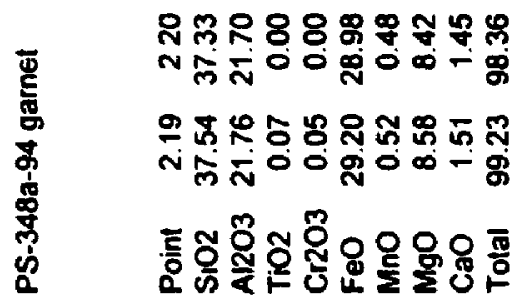

용ㅎㅇㅇㅎㅜ जo 罗 웅 nio 0 웅용융 ํํํํำ 형용 जio 6 웅ㅎㅇㅇㅇㅇ nO ळ no 0 $\bar{\varnothing}$
ชึㅇㅇㅇㅇㅇ 400 รั ल००

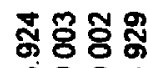
i०om 웅용융영 궁용용 중 유워

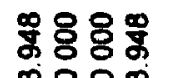
लि० 용 \&्ठㅇ ल0 ๘トす

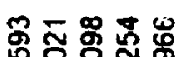

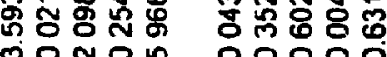

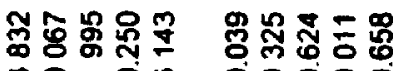
mo-06 0000

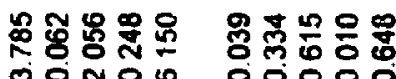

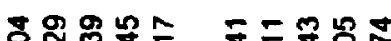

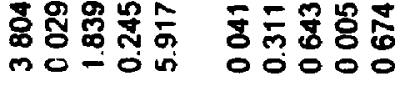

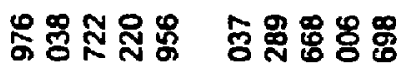
กำ-

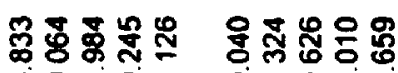
ल0-00 00000

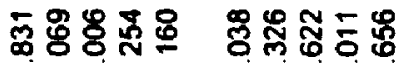
MONOO OOOOO

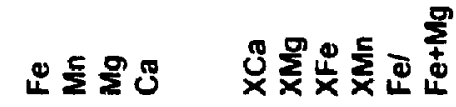




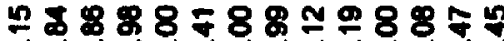

- मूल

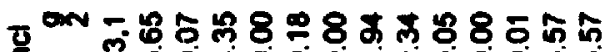

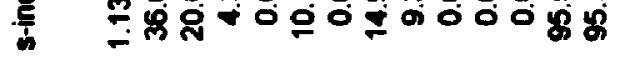

지요

-

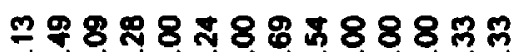

-

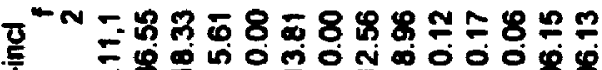

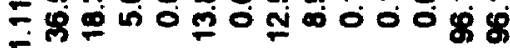

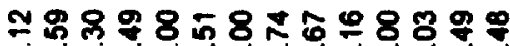

-

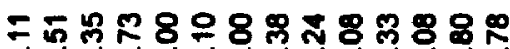

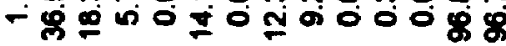

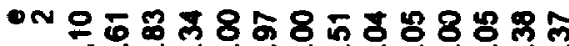

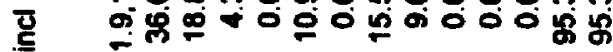

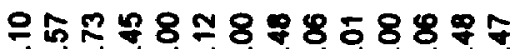

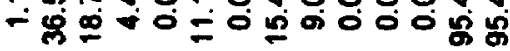

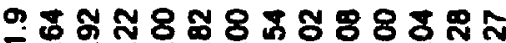

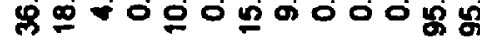

ON

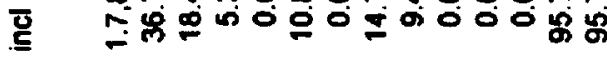

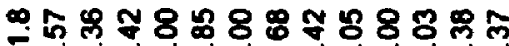

जू 以

₹

边

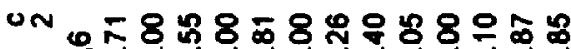

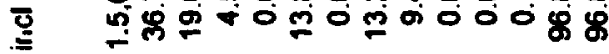

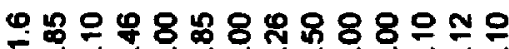

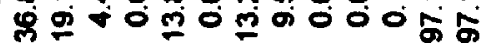

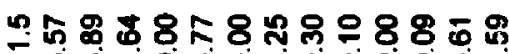

फ़

อN +

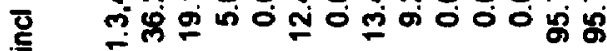

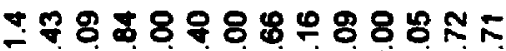

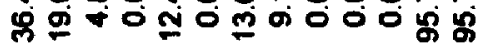

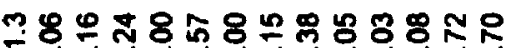

\&

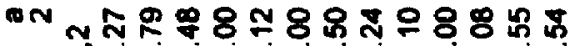

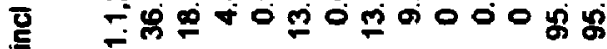

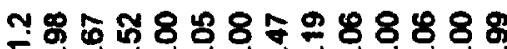

मूं

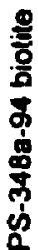

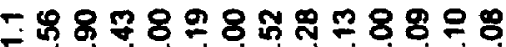

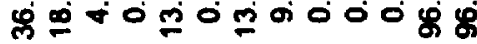

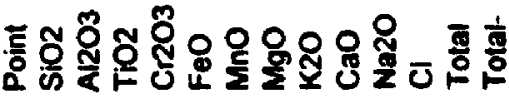

今ั0:

ஸें

- 8

i

串要 8

i

\$

内 त

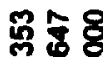

in

용

i

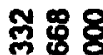

in

疋

in

중용

us

范客

जi तi को

윙 ठㅇ

ชิ

遍 8

in

in

市通8

内

웅융

뚱

I 88

जึ่

용용

in

종요

ज़ं

嘀采

ज嗐

号迨号

성

路 8

ज为

要送

तิ
牙客

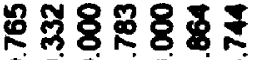

-00-0

สุร

○00-0 ल

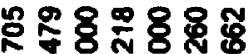

000 엉

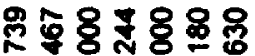

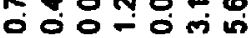

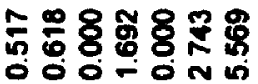

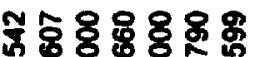

영응 n

-

- O0-0

蕒

엉응 ल엉

品

000 - 0 mi

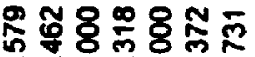

암용용요용

००ण О

\%

- o o-o ni

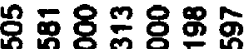

0 ㅇㅇㅇㅁㅇㅇ

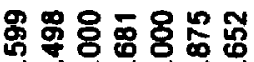

O0 O- O N

융 क्षे

융융 品

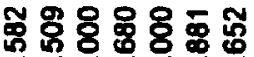

O OOO-ON N

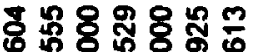

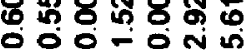

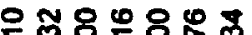

ติ

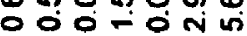

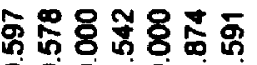

OOO-ON

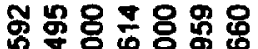

ㅇㅇㅇ응 웅

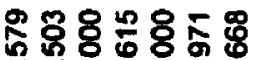

$000 \div$ 的

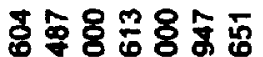

०००-0N

๘区

くたず゚完罗
000 - 0 m n

ㅊํㅇ융

응궁

용요

엉-

ํํㅇㅇㅇㅇㅇㅝ

$-009$

$588 \%$

응궁

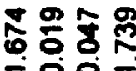

용요

잉응

총융

-0.

융용용

$-00=$

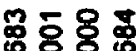

- 0 -

웅웅

응-

용둥요

둥응

象额

응응

영둥

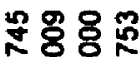

응

융ㅎㅇ융

-0ㄷ․

웅응

궁용

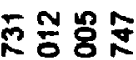

엉-

응융

-0ㅇ-

员骂용

-0.

웅 8

200

궁응

훙응

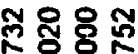

응

$\times 8 \frac{\pi}{2}$
웅옹영응

운ㅉㅗㅀㅀำ

응ㅇ

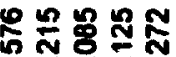

ชํํํํำ

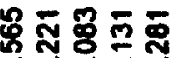

00000

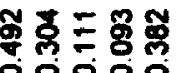

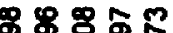

政

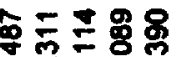

00000

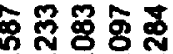

00000

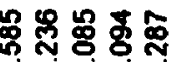

О००००

卷员可可副

00000

웅ํㅠ용영

00000

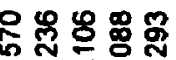

ОOODO

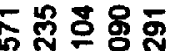

잉잉ㅇㅇ

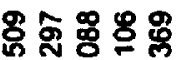

00000

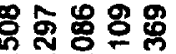

00000

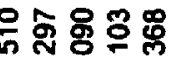

00000

충 충영응

00000

ซึ:

0000

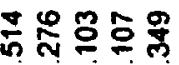

00000

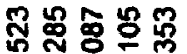

ํํㅇ응

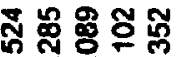

00000

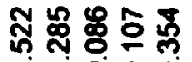

00000

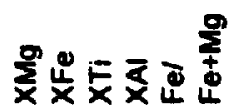




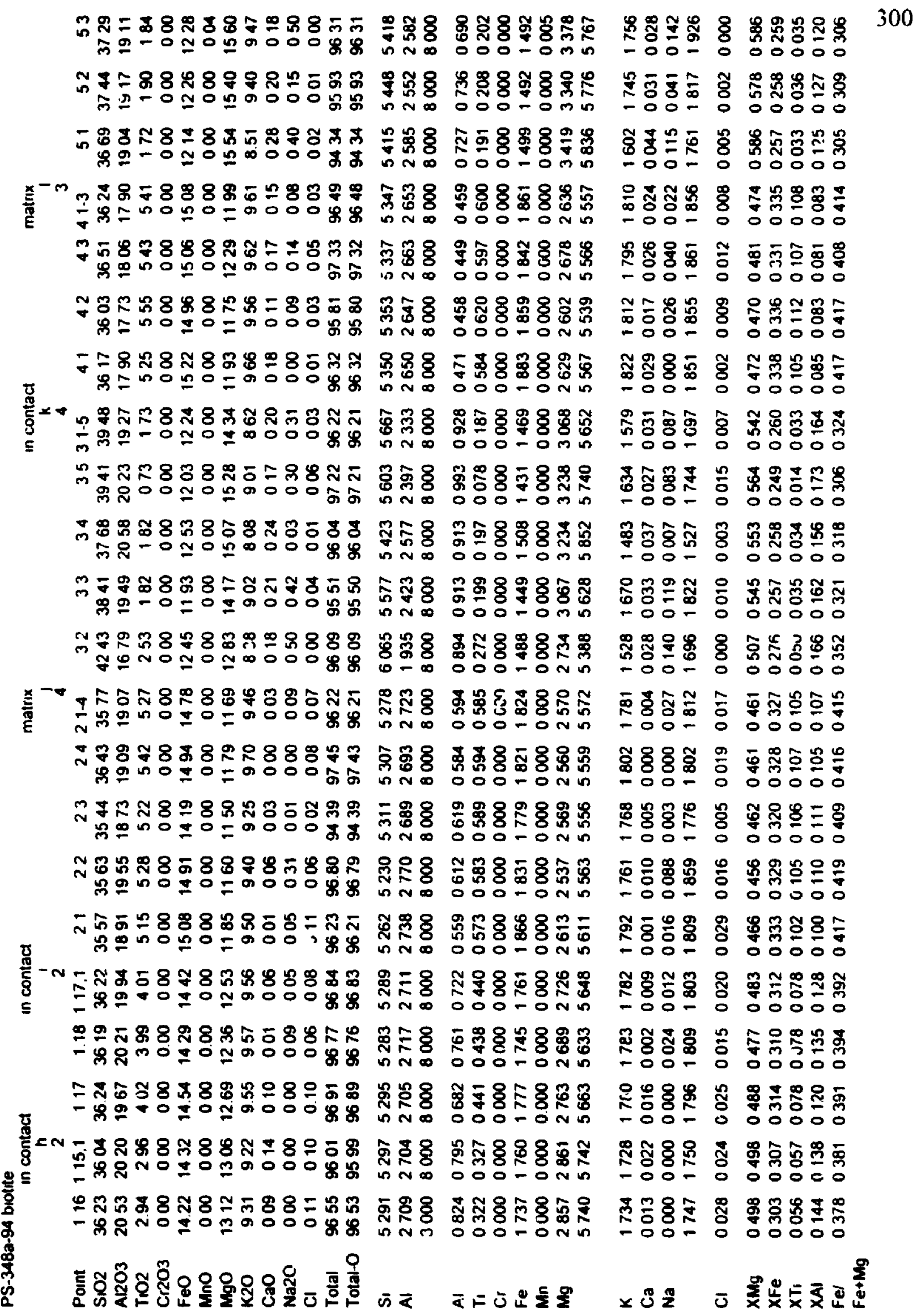




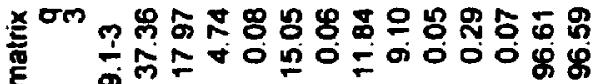
๓ 塄

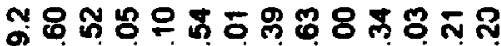

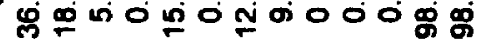

ธธ㇒

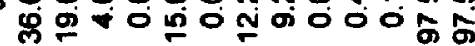

즌

E

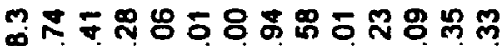

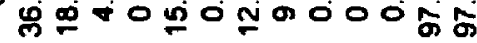

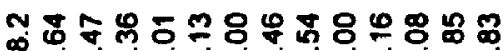

边

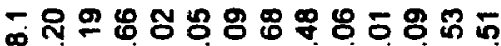

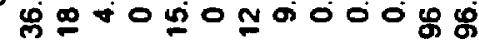

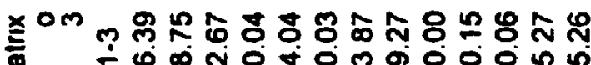

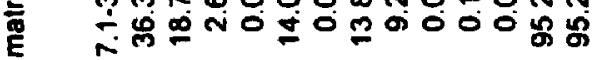

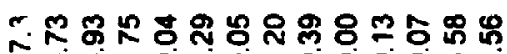

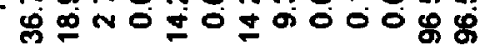

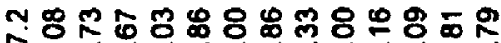

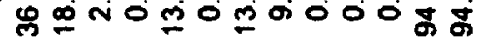

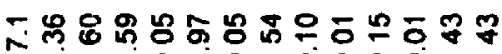

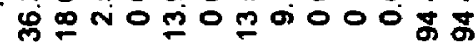

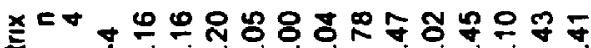

E

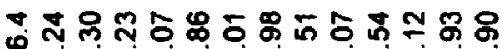

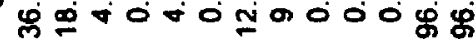

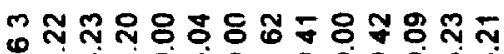

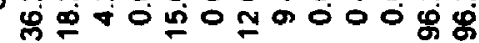

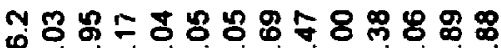

吕 =

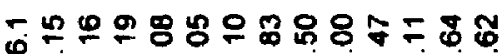

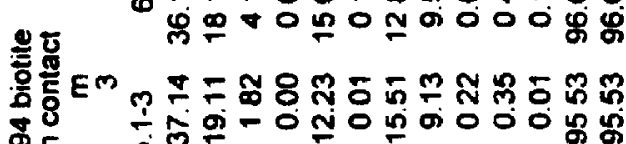

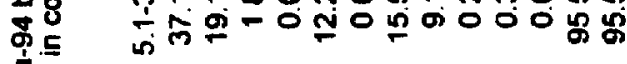

密

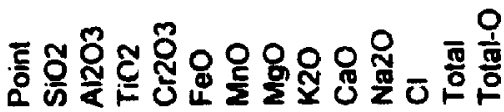

즁융

जง

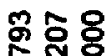

的

동용

जi i

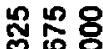

जิ $\infty$

象 ํํㅇ

ง

్ㅗㅇㅇㅛ

in

只 ํํㅇ

is तi क

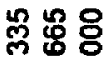

ง

色总品

ஸึ

ำㅇํㅇ

in $\infty$

ํํำ

ஸ

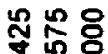

जा

立嵒

जi N

ณ

ஸึ

웅용

जin

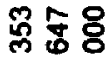

in

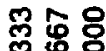
4 $N$

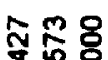

ज N

कर

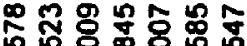
นึㅇㅇㅇํㅇ

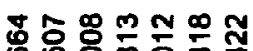

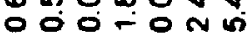

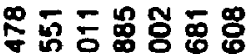

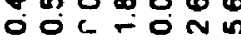

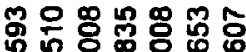

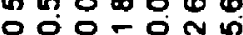

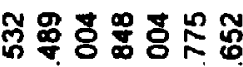
- OO - O N

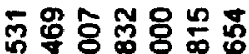

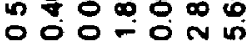
总骂 OOO-ON Ni

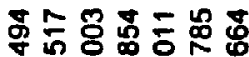
o o o - on m

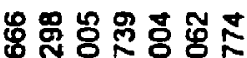

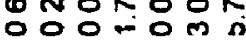

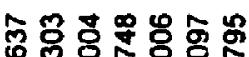
000-0m

도ㅇㅠㅛ요요 엉응등

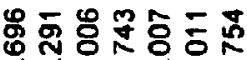
Oㅇㅇㅇㄷㅇㅇ

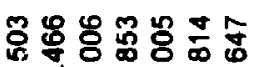
$000-0 \mathrm{~N}$ in

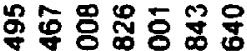

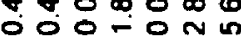

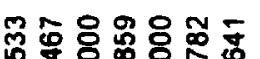
OOO-ON U

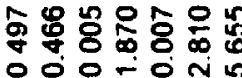

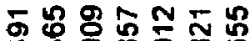

48000 O OO- -

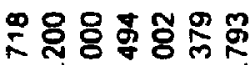

$000-0 \mathrm{~mm}$

둥ㅇㅇㅇ용묘

옹

융교융

웅응 号罟

-00 -

용ㅎㅇㅇ용

-00-

옹용

응

蛋若哭

- 00 -

홍용

- 00

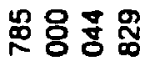

- 00 -

ผ 용횽 욧

- 00 -

ณํㅇㅇ용

등응

송요용요

- 00 -

용웧용

-00-

ํํㅇㅎํㅇㅊㅡ

응-

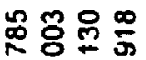

- 00 -

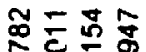

등으

솟요ㅇㅝㅛ

- 00 -

놋용응ㅇㅇ

- $00-$

용용 ్ㅛㅇ

ㄷㅇㅇ은

둥형용 膢

- 0 0 -

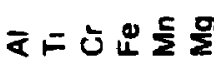

용

00000

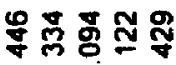
00000

里罢品兴 00000

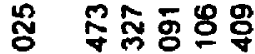
- 00000

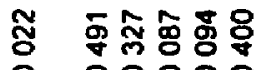

- 00000

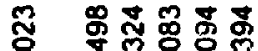
000.30

풍윰ㅁㅇㅇ므융 00000

귱츙형유 00000

융묘ㅇㅛㅠ 용 00000

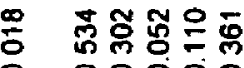
- 00000

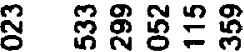
ธํㅇㅇㅇㅇ

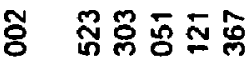
- 00000

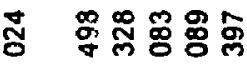
- 00000

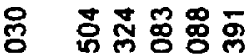
000000

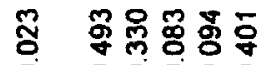
- 00000

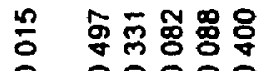
O 00000

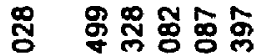
- 00000

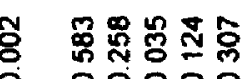

00000

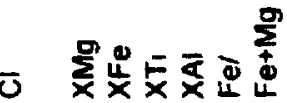




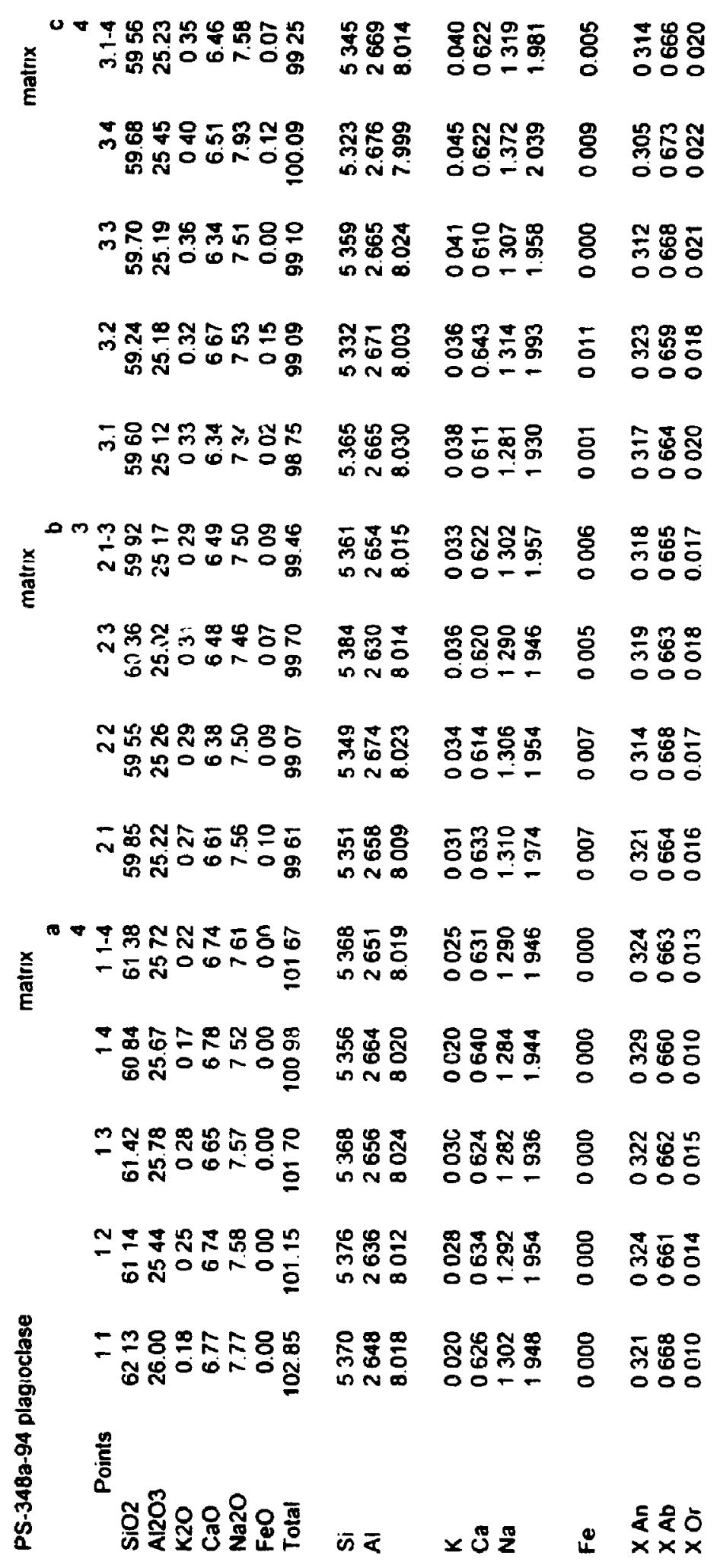




\section{Appendix D: Mineral assemblages of amphibolite gneisses}

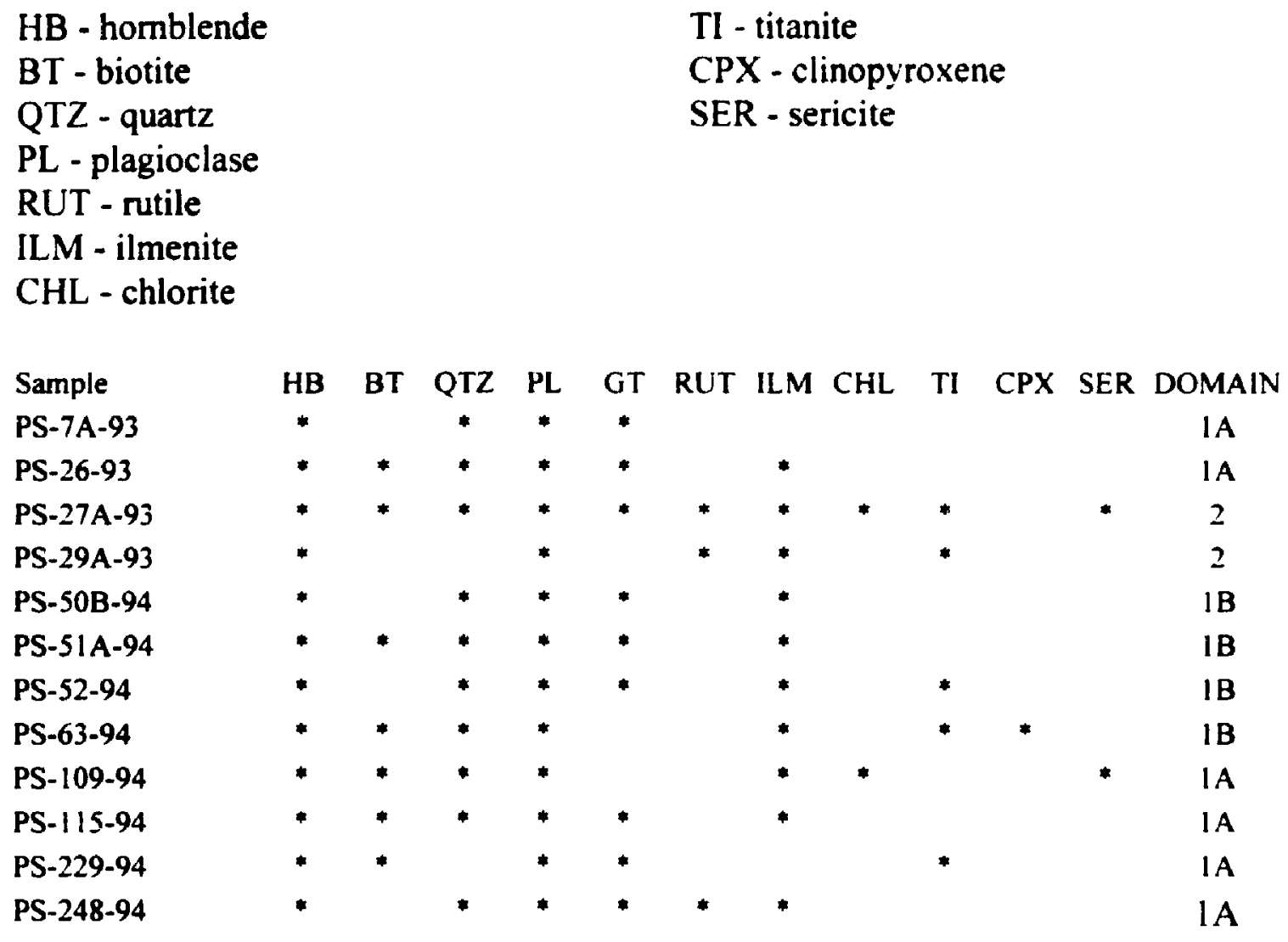


Appendix E: Mineral analyses for amphibolite gneisses 


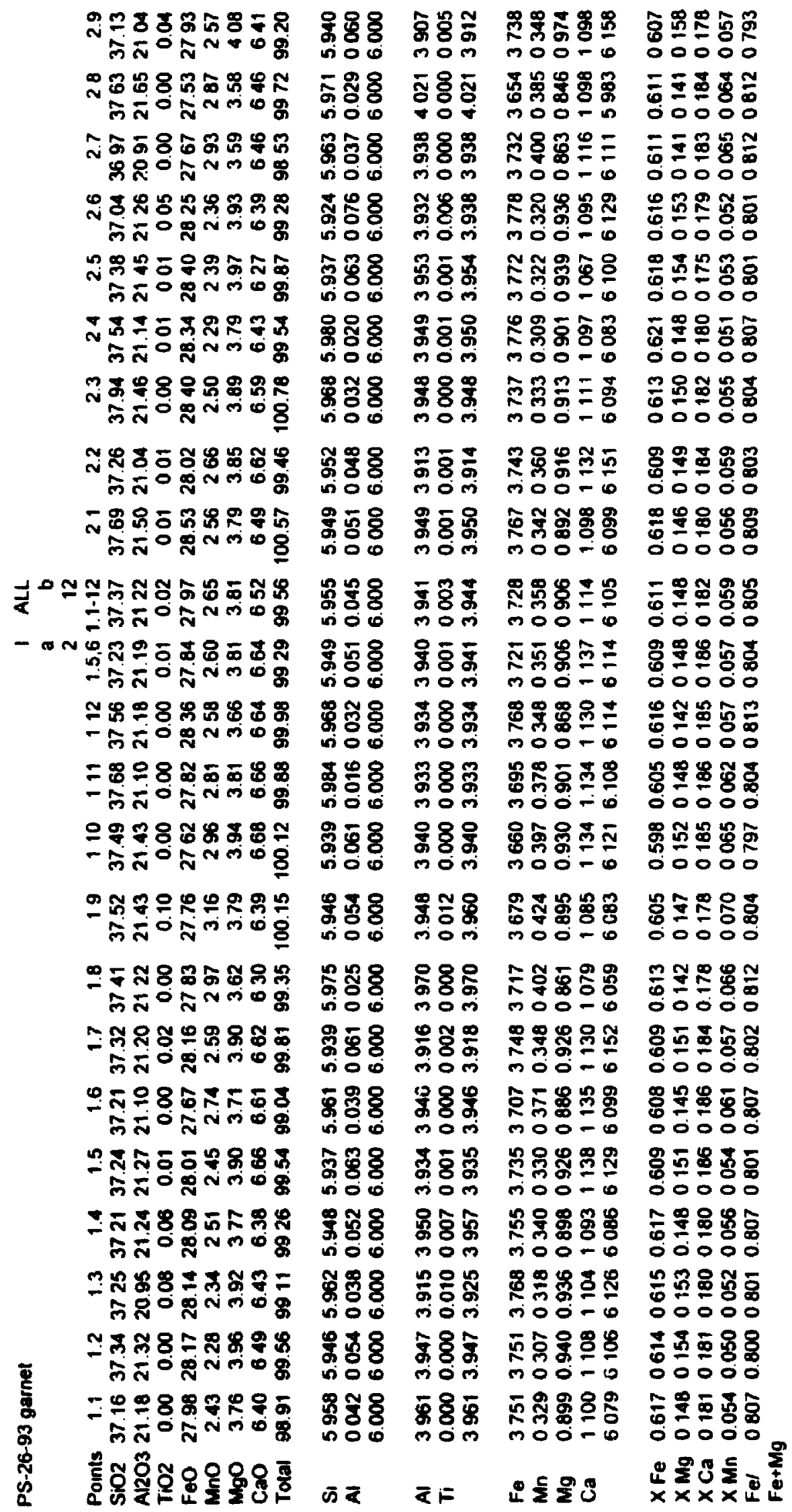




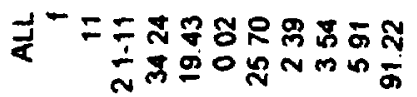

ำกำ N

๔

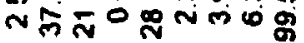

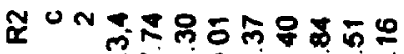

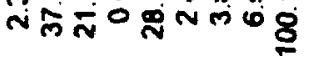

=

N

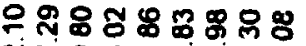

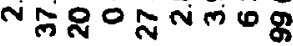

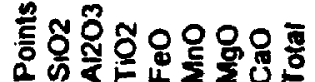

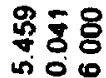

\%

5َ용ำ

$\therefore \%$

58

जo

공용

요

ธิ

un 00

ら̄

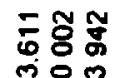

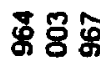

mom

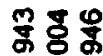

ल०

के

लि०

훙용요

กั

용 용

ज०

$\bar{\varepsilon}$

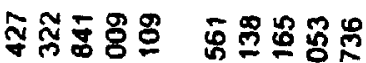

M0-600000

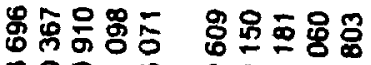

mo0-0 00000

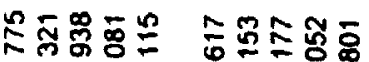

ल융

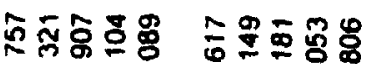

m00-0

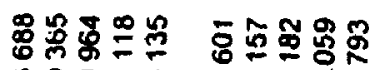

MDO-D 05000

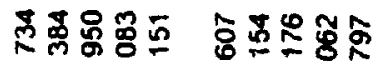

ल00-क 00000

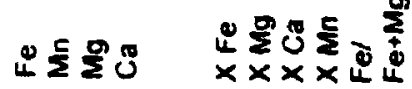




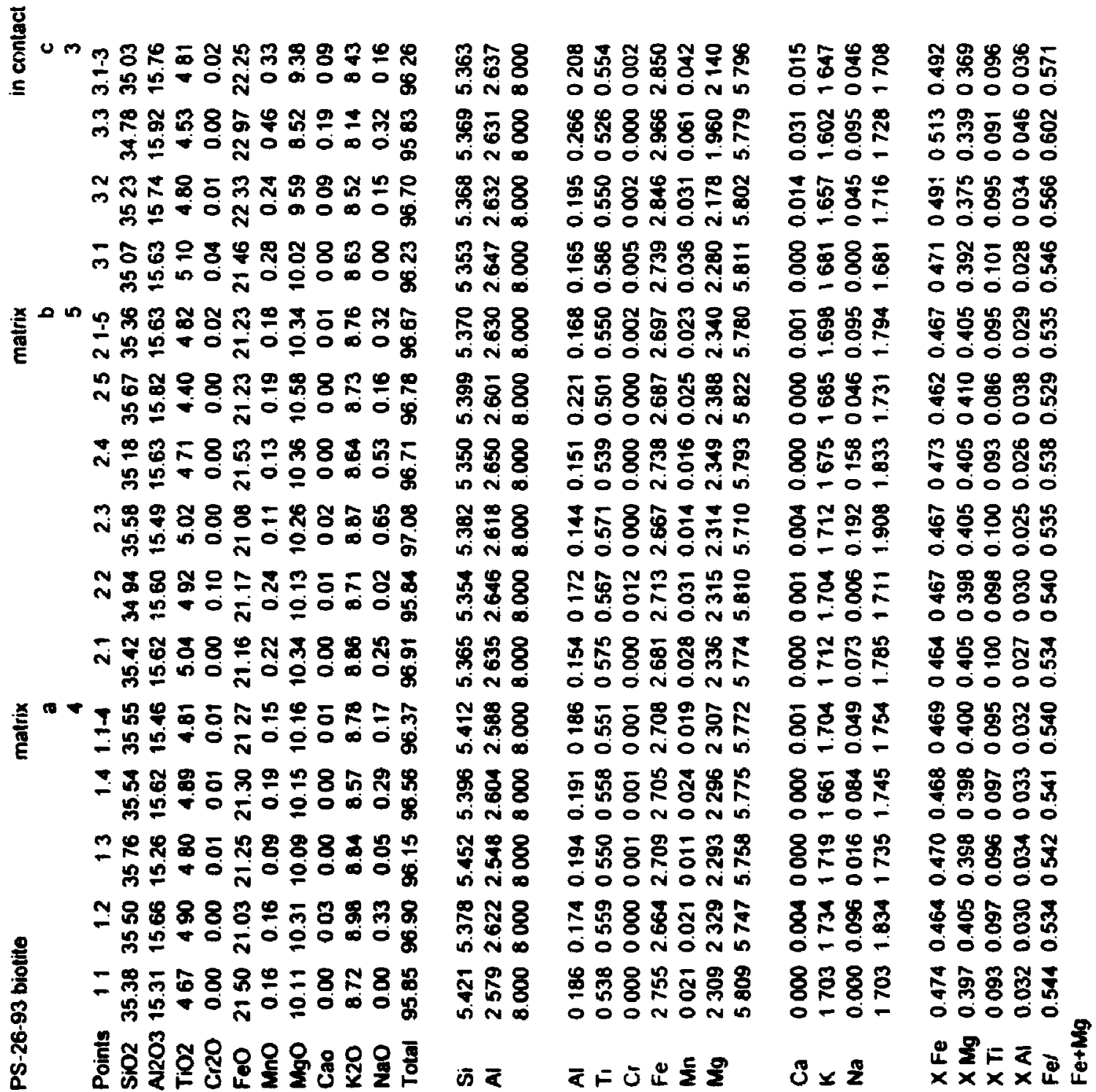




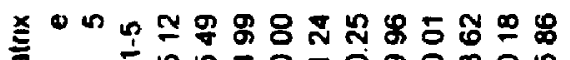

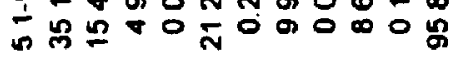

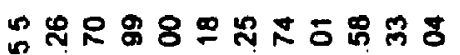
W

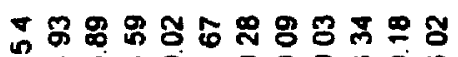
J กำ

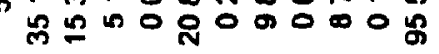

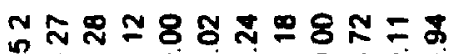
峞 ธก मू

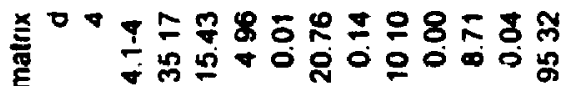
我品 $888 \%$ 出 근

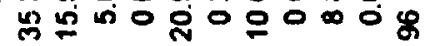

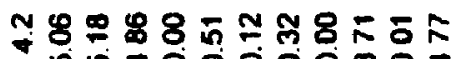
遍 以0 ธำส

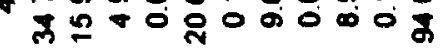

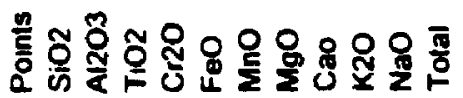

里牙 in $\sim \infty$

总 in 굴 吕 웅 ๓ $\times \infty$ \$ 8 in $\infty$ 횽효용 क त $\infty$ 商 in 웅 卷용 कू 要总 8 แ $N$

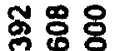
क ले 惫 8 in $N \infty$ ᄃ용요 is of क⿱亠乂

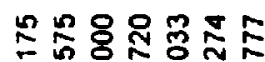
OOOONON

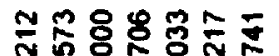
กั0ㅇํㅇำ

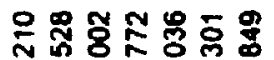

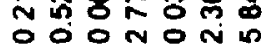

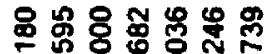
OO ONON

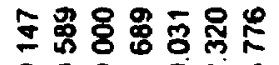
OOONON

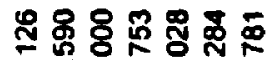
OOONON 용ㅎㅇ용

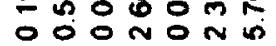

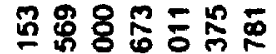

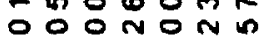

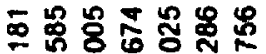
OO ONONA

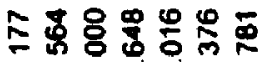
OOONONA

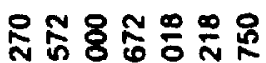
OOO NON くにう电是

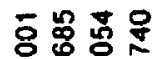
$0-0-$ 둥형둥 응응

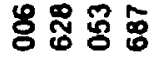
$0 \div 0 \div$ 哭농 0- 융유윯 0 - 0 -

号: 总 ㄴ웅응 등

8 등 -

ㅇํㅇ총ํำ 등 -

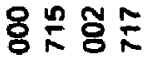
- - 0 -

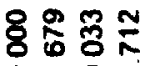
O-O $8 \times 2$

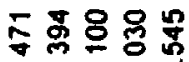
00000

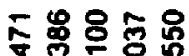
$\circ 0000$

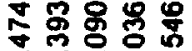
영 0

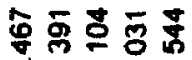
00000

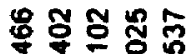
00000 ํํㅇ ิํำ 00000

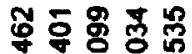
응ㅇㅇ

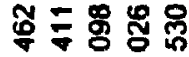
00000

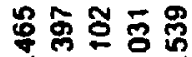
00000

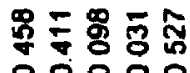
00000

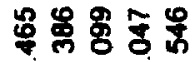
00000

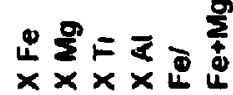




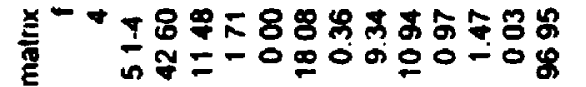

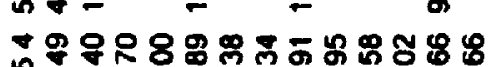
ปู =-ONO

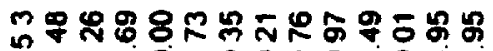

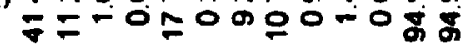
N

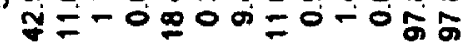

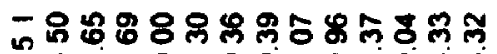
ఫु

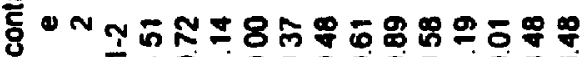

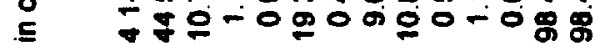

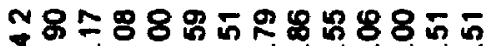

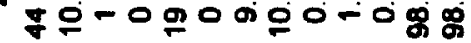

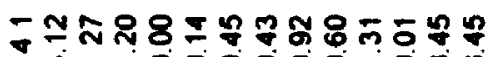
5 $\quad$ ป 产

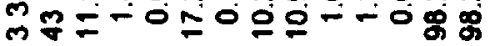

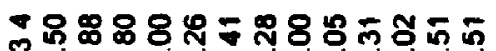

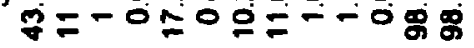

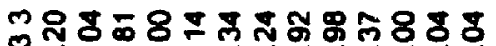
ᄃ

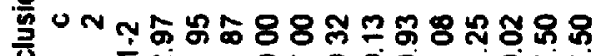

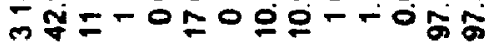

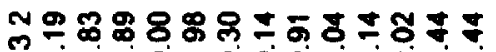

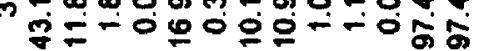

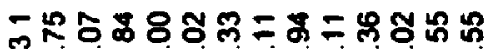

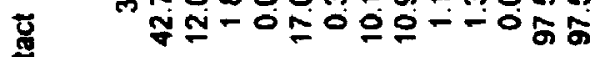
కั 5 NTE-ONODEO-O

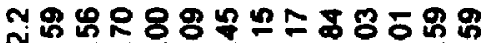
\% =-0900=0-0\%

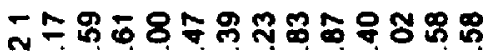
ㄱำ

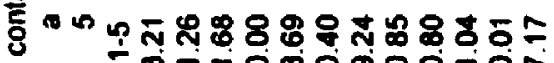

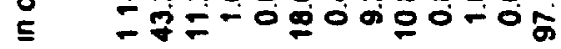

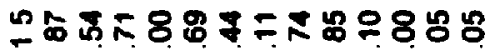

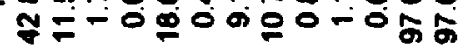

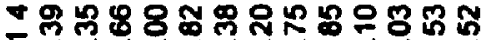
V =-0ळ0000-0\&\&

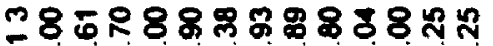

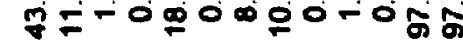

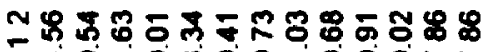

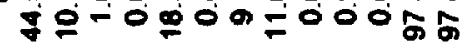

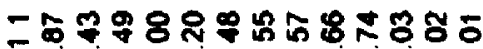
พู่ำ- $0000000 \%$

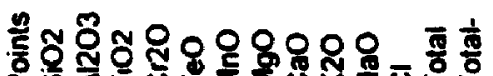

표요 प0 용 0 궁요 $0=\infty$

유용 $0-\infty$

응요 $0-\infty$

今े

$0 \div$

ํㅗㅇㅇㅇㅇ

$0-\infty$

웅웅웅

$0-\infty$

号嵒

$0-\infty$

뇨

$0 \div$

资是

$0=\infty$

कํํㅇㅇㅇ

$0-\infty$

\%

$\infty-\infty$

จำ

$0 \div$

858

$0-\infty$

芹尔宫

$0-\infty$

获步 8

$0-\infty$

웅

$0-\infty$

ํํㅇㅇㅛ

$\omega=\infty$

巻동

ऽ-

్ㅐ용ㅇㅇ

$0-\infty$

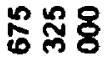

$0-\infty$

ํํㅇ둉ㅇ

$\infty-\infty$

कळ

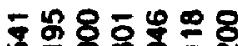

ธOOㅁNㄴN

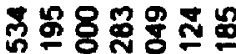

OOONONM

응용ㅎㅁ웅

OOONON

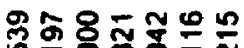

OOONON

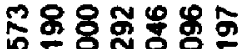

OOOONON

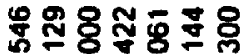

응스뉴

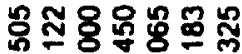

OOONON

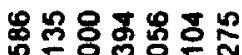

OOONON

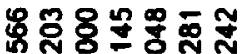
OOOONON

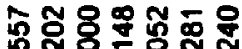
OOONON

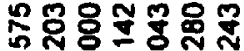
०0ำกㄴ.

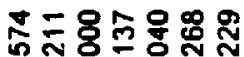

OOONON

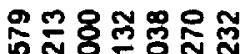

OOONON

范 OOONON

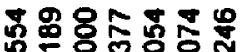
OOONON

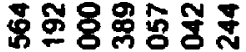

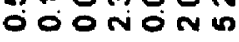

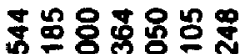
- OO№n

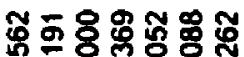
OOONONM

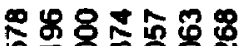
○́ㅇำ N

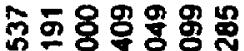
응응ํำ

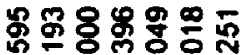
- OONON

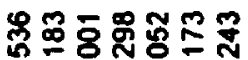
OOONON

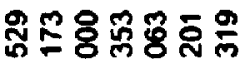
OOONON U.

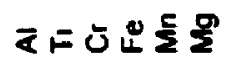

象哭骂

-0ㅇ

옹용

- $00 \mathrm{~N}$

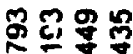

- $00 \mathrm{ON}$

요용

- $00 \mathrm{~N}$

표용

- $00 \mathrm{~N}$

눙ㅇㅇ형

-OÓ丶

운흐옹드

- OON

政票界

- $00 \mathrm{~N}$

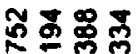

- 0 \%

논유욜

- OON

里思哥

- $00 \mathrm{~N}$

옷워요용

- OON

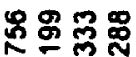

-OON

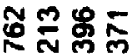
- $00 \mathrm{~N}$

声战

- 0 ơ

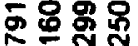

-OÓn

웅용

-00

요ㅇㅛㅠ

- $00 N$

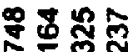

-0ㅇำ

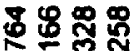

-OON

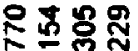

-OON

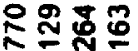

- 00 O

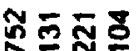

-OON

$8 \times 2$
ำํํำ 공

0000

육웅듕으

0000

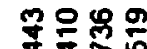

0000

용요

○000

융용్ㅆ

0000

务亭总岛

0000

융으영 웅

0000

落品

0000

우ㅇㅠㅛ용

0000

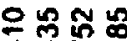

등유

융ํㅛ용

ํํㅇㅇ

웅等员是

0000

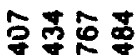

0000

음도용

0000

号量志㤐

0000

号总最量

0000

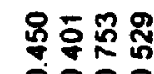

0000

우ㅇㅛㅛ용

0000

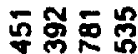

0000

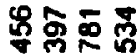

○。ㅇㅇ

भ్ㅠㅎㅠ 궁

0000

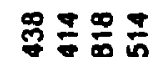

0000

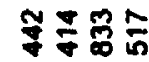

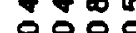

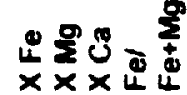




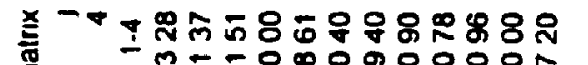
ธิ

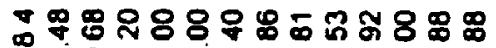

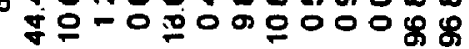

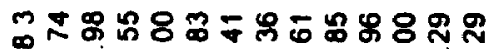

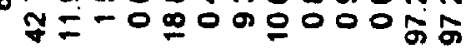

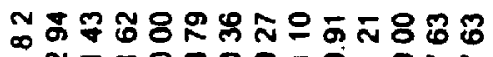

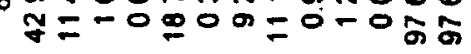

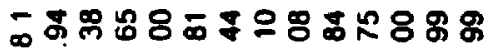
ฮ

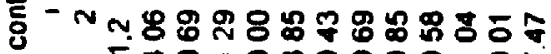
自은

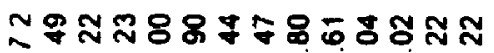

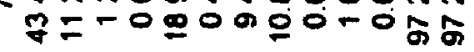

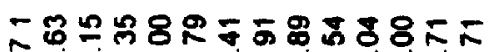

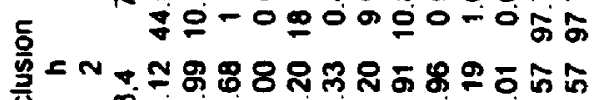

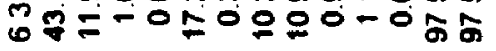

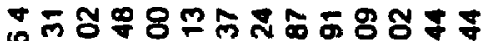

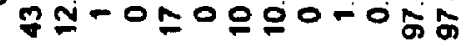

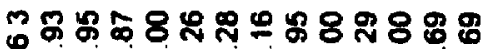

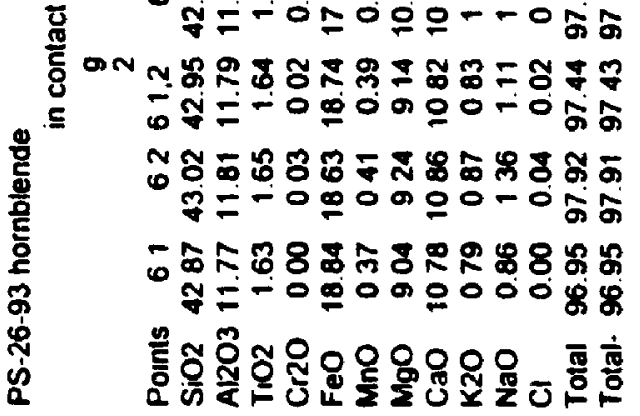

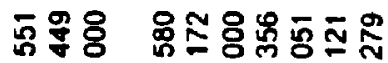
D- 000 ก0ก 果总 8 $0-\infty$ 표 8 ษ $\infty-\infty$

\$웅융

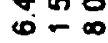
్ㅐㅁ중 $0-\infty$ 费步 $0=\infty$ 몽요 $0-\infty$

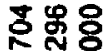
$\infty-\infty$

颍各 $0-\infty$ \%ㅇํㅇㅇㅠ $0-\infty$ 㛚家 $0-\infty$ 홍융 $0-\infty$ 웡 5이

훙요 $0-\infty$ らく

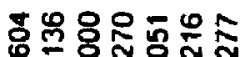
OOONON

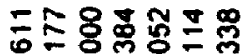
OOONON

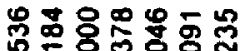
นO유에

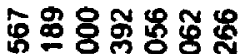
OOONON

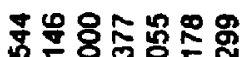
OOONON

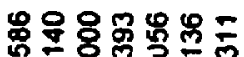
OOONON

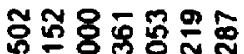
OOONON

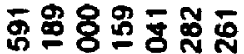
OOONON

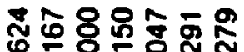
OOOONON W

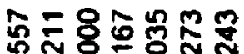
oOOnONm

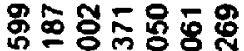
OOONON

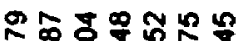
กㅇㅇㅇ ले OOONON

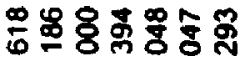
OOONONM テニゔ동

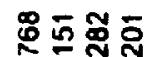
- $00 N$ 융유유 -OON

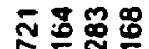
- 00 N 㐘躡屏 ธัด ํㅗㅇ - $00 \mathrm{~N}$ 송으융용 -OON กำ늉 โ行

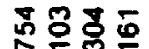
-OON

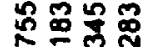
-00 䨤里号 ํํㅇ주ㅇㅠㅛ - $00 \mathrm{~N}$

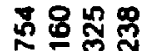
-0N

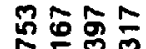
-00 N ํㅗำํํ용 - $00 \mathrm{~N}$ $8 \times \frac{\pi}{2}$

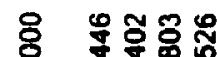
0000 윤ํㅠ 8 깅영

융 용용 - 0000

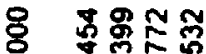
- 0000

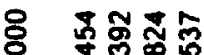
0000 \% - 0000

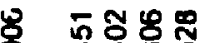
० 0000

于우운 子รั - 0000

궁 으요ㅇㅛㅛ ○ ㄷㅇㅇㅇ 훙ㅎㅀ 00000 8 ํㅗㄱ - 0000

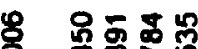
- 0000

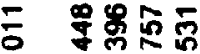
: ㅇํㅇㅇㅠ

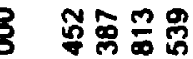

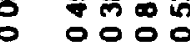

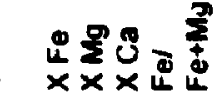




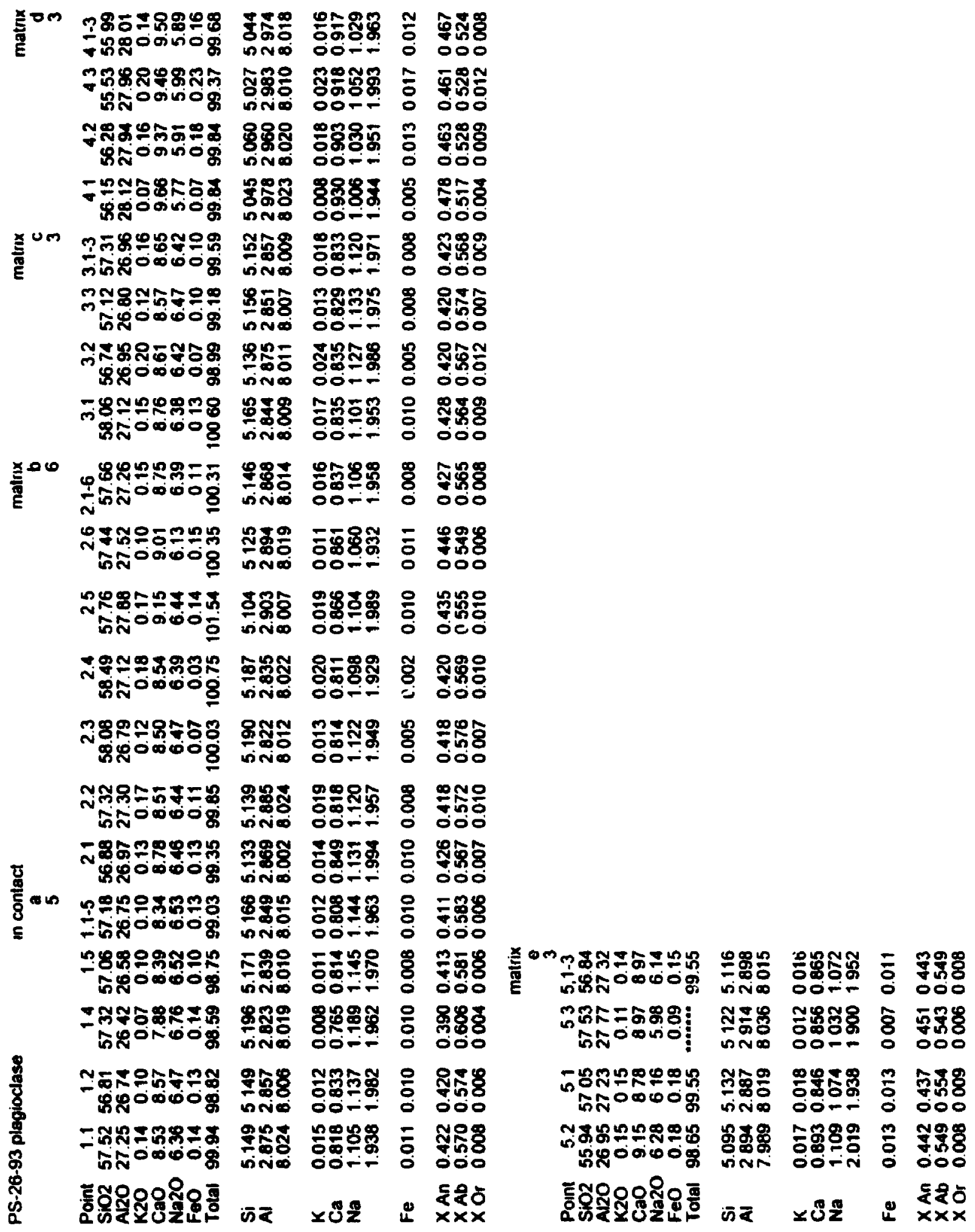




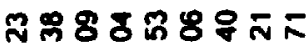

的只口品

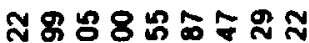

पूก

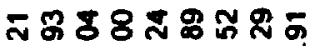

边

กิ 8888 ำ

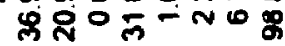

우요ำ กิ

กंก

중 8 б 8 \%

的品

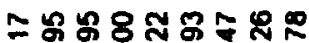

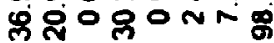

으우요요요유

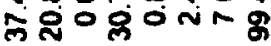

등ํㅇํำำ

กัล

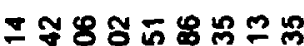

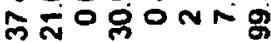

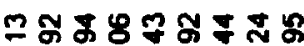

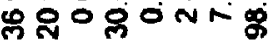

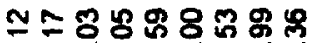

ติ

二

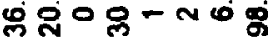

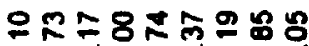

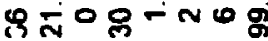

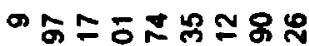

乌ั

종요용

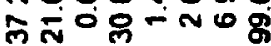

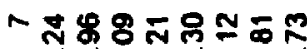

लं

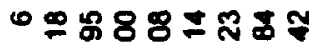

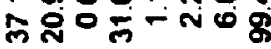

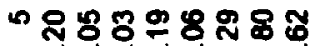

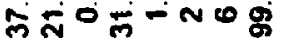

- 488\%용

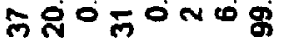

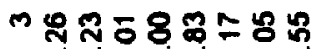

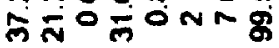

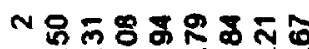

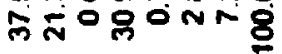

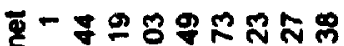

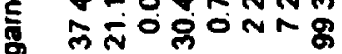

8

\$ั

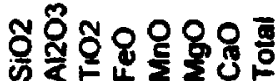

象둥

जo

总安8

no

형요

no 0

\$ి్ㅠㅇ

ऽ००

ํㅠㅇ

no 0

妾 8

ज०

동

जi 0

웅융

000

象둥

응

888

000

必塄

no 0

ํํㅇ용

in 0

홍ㅎㅇ

00 :

₹ิ路

- 0

串争

in 0

둥용

no 0

\$용

i 80

巻둥용

แ००

ํํㅇ

जi

\%횽ㅇㅇ

n 0

웅둥웅

内่

离 Бุ

no

\&̊요용

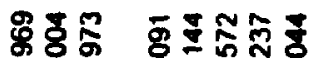

ल०

5ิ

ल०

ఫ్ర్ 8

mom

죵중

음

్ํㅇํํ

क्षण

$\overline{8} 8 \overline{8} \overline{8}$

mom

号 8

mom

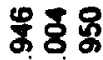

mom

용요

ल०

若 ซิ

nom

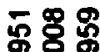

ก요

$8 \%$

กo.

速这:

no

象客察

ก0

劳 $\overline{8}$ 兽

mom

융

लि०

品=罾

nड़

\$8:

ल0

\% 8

in

$88 \%$

ión

$\overline{8} \overline{8} 8$

아

쟁ㅇㅎㅇ형

mon

ㅇํㅇㅇㅠ

三요용

$\div 00=0$

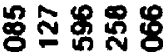

- $00-0$

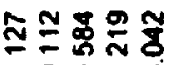

-00-

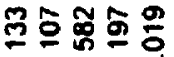

- $00-0$

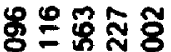

- $00-6$

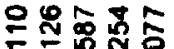

ธ으응

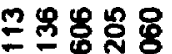

- ธำ-

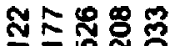

$\checkmark$ ㅇㅇㅇ

쫋ㅊㅀㅇㅛ

-50 -

초흥요용

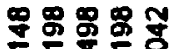

TOO-

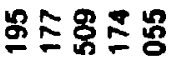

+00-0

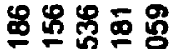

-00-0

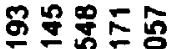

-OO- 0

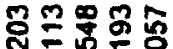

$\checkmark 00=0$

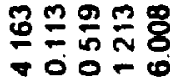

응둥츙유

- $00-\omega$

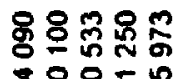

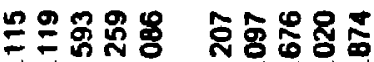

$00-0$ 00000

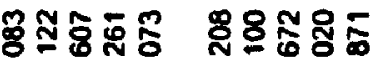

- $00-000000$

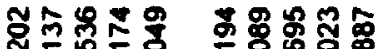

-00-0 00000

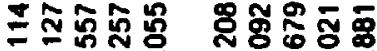

प00-0 00000

00000

-00-

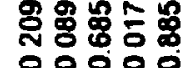

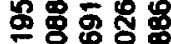

00000

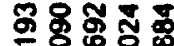

00000

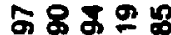

ㄷㅇㅇㅇㅇㅇㅇㅇ

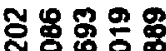

응ㅇㅇㅇㅇㅛ

도은ㄷํㅇ용

00000

0000

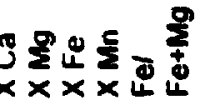




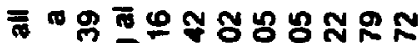

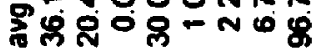

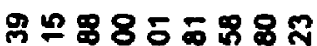

放0

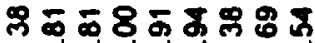

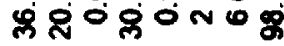

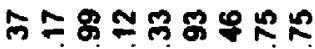

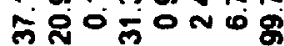

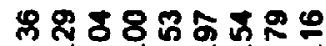

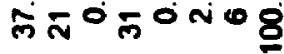

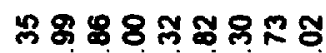

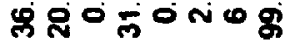

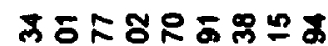

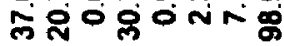

กำษำ

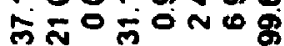

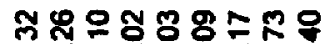

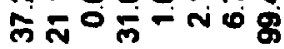

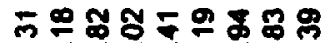

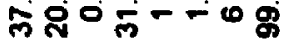

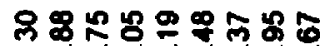
舟里

สรธด

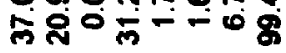

으웅유요

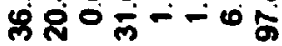

స $8.888 \%$

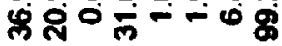

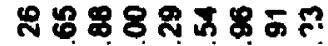

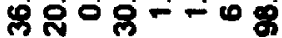

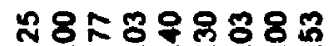

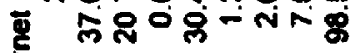

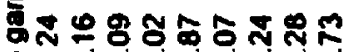

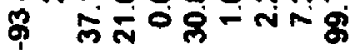

$\stackrel{8}{8}$

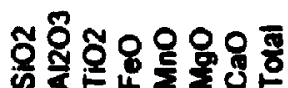

용ำ

이

용요

जi 0

중

ก่ 06

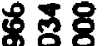

in 6

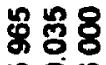

ก०

邹官

용웅

so

유8

noㅇㅇ

\%

no०

ริ8\%

000

\%8:

00

$\overline{8} 88$

no 0

58

n.

กิิ

ก.

죵

กิ응

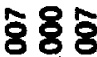

ㄷ․

용요

of 0

is 0

க்
$8 \widetilde{8}$

लำ क्ष

융요유.

mom

พิ융

mo

害器

mom

กิ๊

ก व

\& 8

iom

₹政里

nom

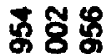

nom

\& 8 多

nom

$\overline{8} 8$

nom

\%

io

$\$ 8 \%$

लि० ल

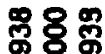

mo.

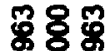

mom

棁 8 \%

iom

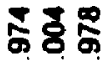

लि

$8 \%$

क ल

₹=
당

트융요

- $00-0$

용응요잉

ن०0=-

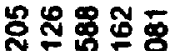

roo- 0

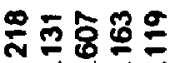

-00-0

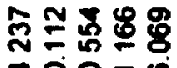

$00-0$

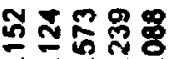

-00-1

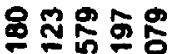

-00-0

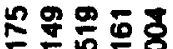

-00-0

욱후웡용

$+00-0$

\$8 중

-0。-

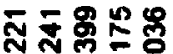

-00-

웅즘응

-

ธิธ

- Oठㄷㅇㅇㅇ

뜬충ํํㅇ

ํํㅇำำ

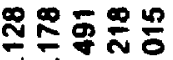

-00-0

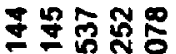

Too-o

눈돌올용
눙

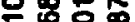

00000

สํㅇㅇㅇㅛ

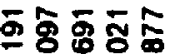

00000

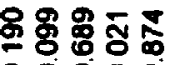

00000

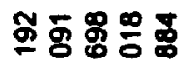

00000

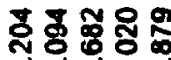

0000

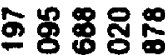

00000

象里

00000

농훟ํㅇㅎํ 응ㅇㅇㅇㅇㅇ

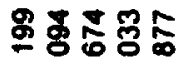

00000

엉 8 영용

00000

용요용응

0000

송ㅎㅇㅇㅇㅇㅇ

응ㅇㅇㅇ응

융ㅎㅇ영영

응ㅇㅇ

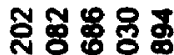

00000

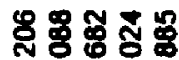

00000

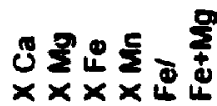

응으 


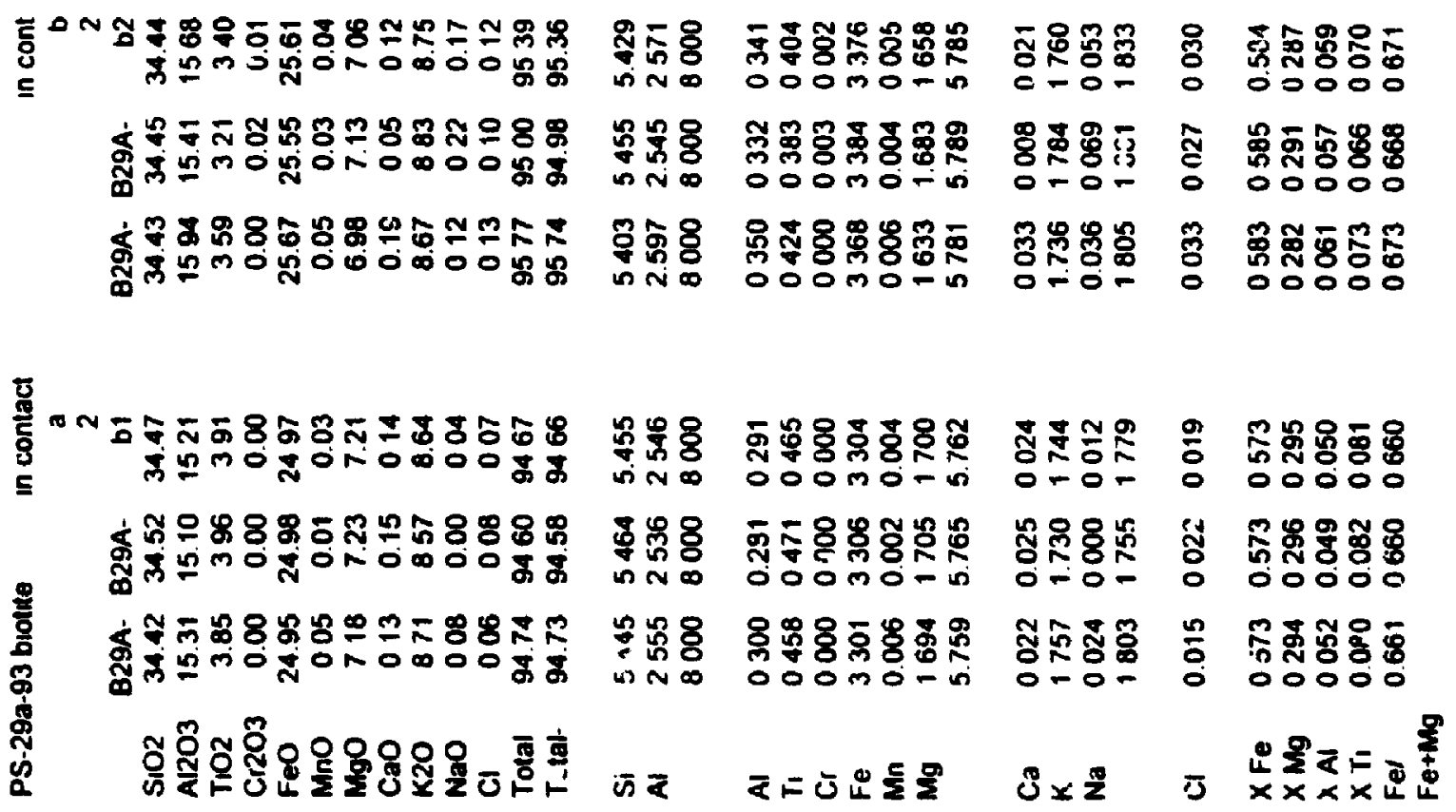




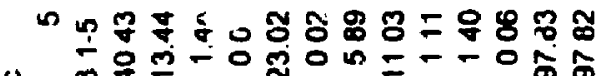

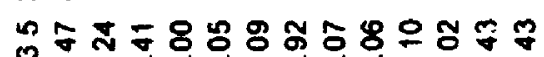
으에

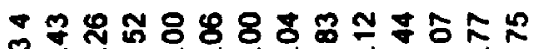

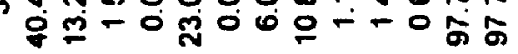

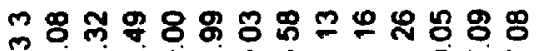

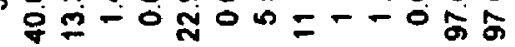
Nㅜㅇㅇㅠ8

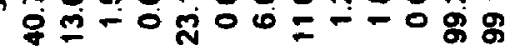
一品ヘヘ \%ั

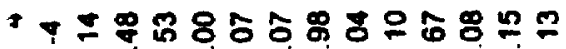

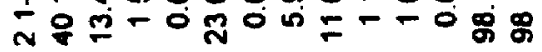

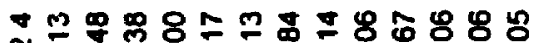

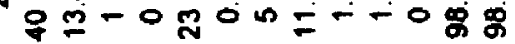

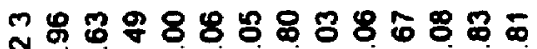

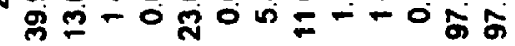

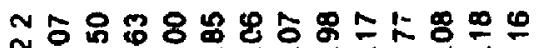
ำ

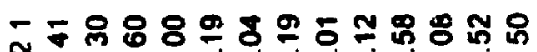

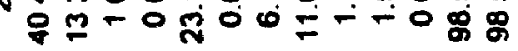

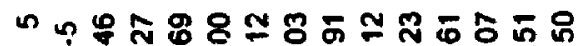

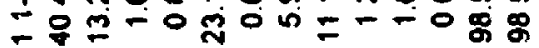

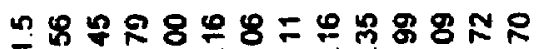

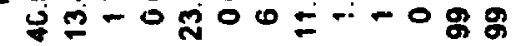

ำกัง ำ-

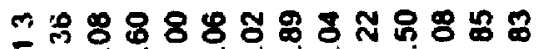
ㅅำ

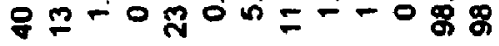
$=7$

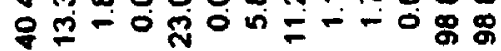

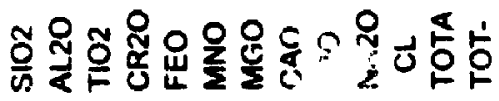

影的

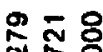
$\infty=\infty$

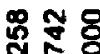
$0-\infty$ 尊造 $0-\infty$ 융요 - -

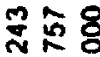
$\infty-\infty$

要贯 $0-\infty$ 뾰요 $0-\infty$

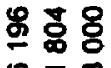

要蒠 - $-\infty$

동 $0-\infty$

융응 $0=\infty$

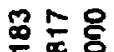
- $-\infty$

훈용 $0 \div-\infty$

要送 제 政害

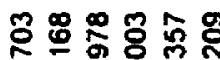
OONO-

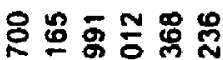
OONO-

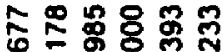
OOnO-口

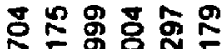
은은 ำำํํㅇำ 은응

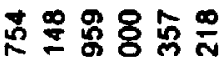
OONO-W

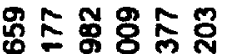
०Oกั0-

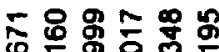
○0

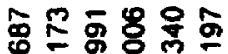
OONO-m

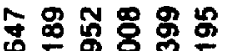
OONOT ธิธ ㅁำ 题里象氛 응 告응

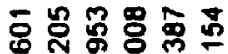
OONO-

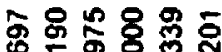
OONO-

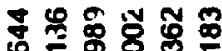
OONO-

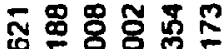
$0-\infty$ 옹용 $0-\infty$ के दे 으으

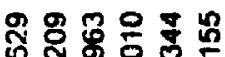
응 \&下出交导

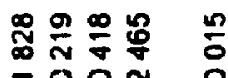

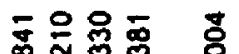
-OON

昶尽 ్ㅜㅇ -OO N

适可骂? - $00 \mathrm{~N}$ 苾芦品哭 - 0 0

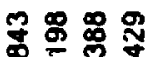
-OON

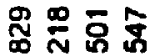
-00

올 요욤 - ํํㅇำ 兽只家志 -OON क雨路 -00

음 $\bar{x}$ -OO

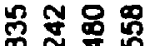
응 品 可 - $00 \mathrm{~N}$ 的雚罢 - $02 N$

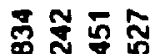
- OO

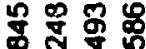
-O요

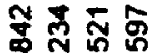
๓ำㅇำ

Sิ

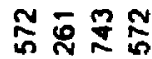
००00

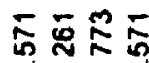
0000

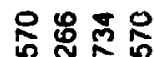
०000

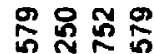
०000 的总总它 0000

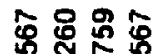
0000 눙 的空元 0000 会步命 ด0००

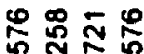
OOOO

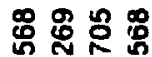
0000

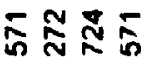
०००० 눙 的然露 $\circ 000$

คี $\circ 000$ 동ำ 0000 ธิ 0000 贾石器要 응응 会胥是占 잉

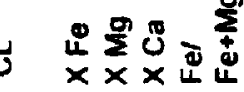




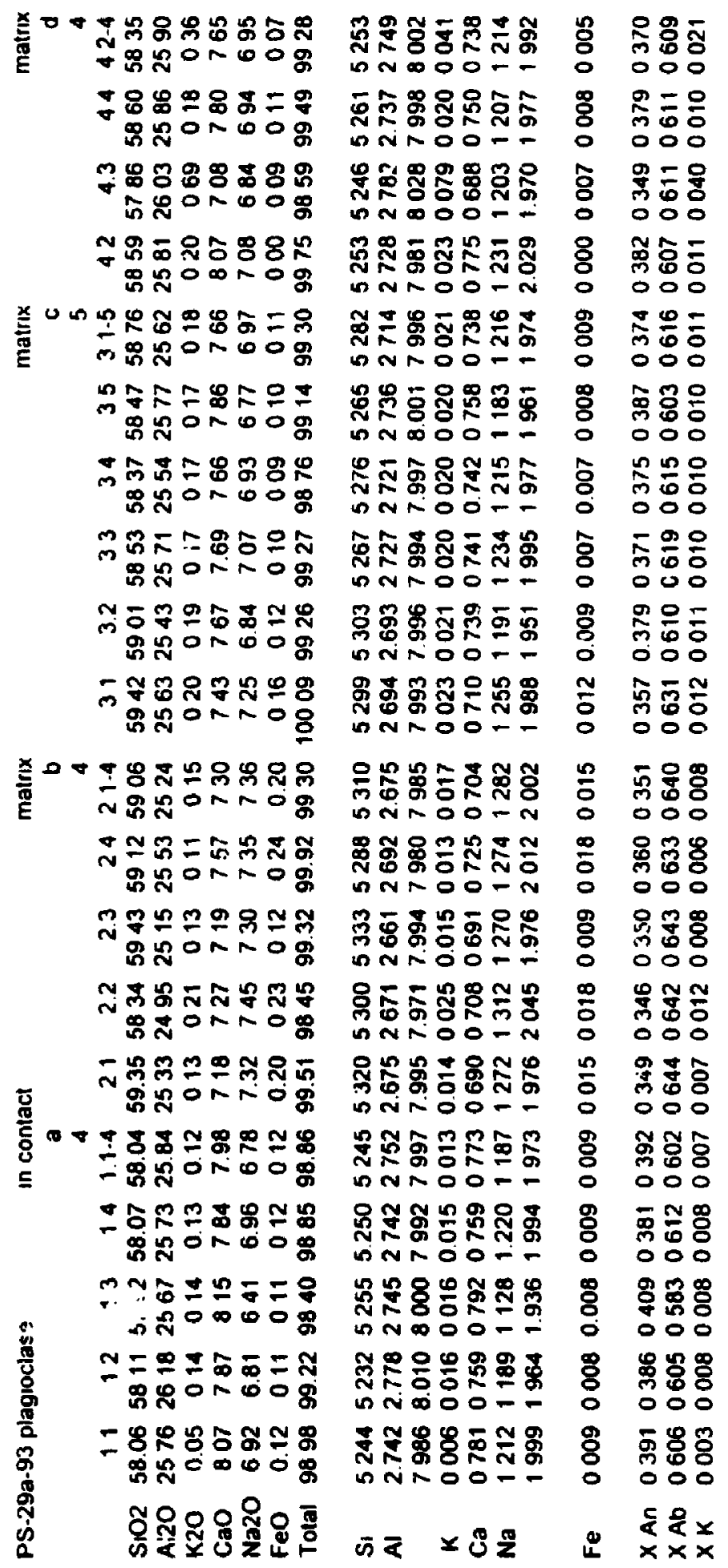




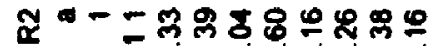
的。たー

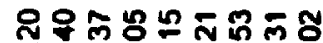

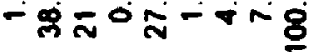

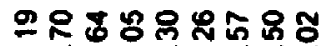

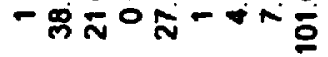

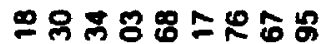

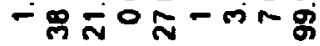

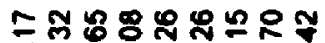

-

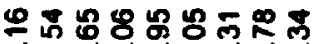

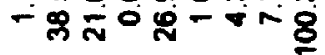

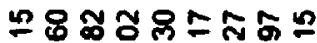

一贯公公一市

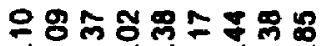

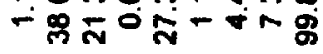

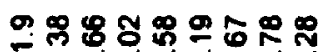

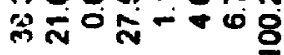

舟安万贯士

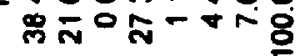

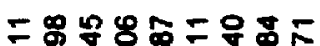

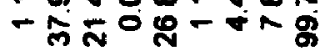

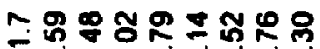

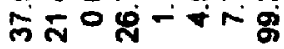

ำ

一贯

융융요요

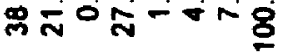

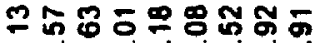

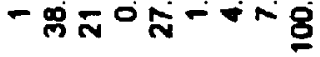

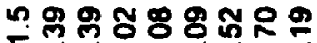

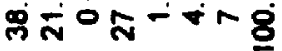

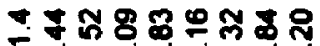

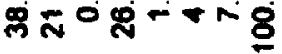

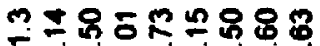

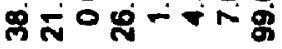

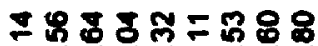

- 㐘六心

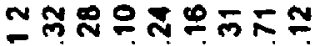

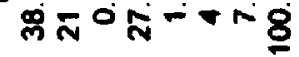

二网品

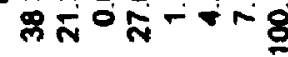

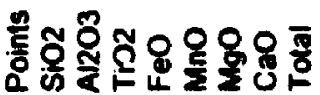

웅융융

ㅊํㅇ용

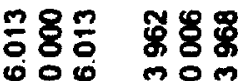

궁요 808

ติ๊o

जi 0

$\overline{8}$ 으음

mor

$\overline{5} \overline{0} \bar{\sigma}$

क

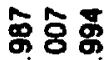

लिख

勇 8

जo:

影要

nom

ฐ๊

ஸे

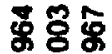

iั

웅용

60

离合

8 实

กㅇํㅇ

品

응

옹영

is 0

용요

6

형

no

888

60

$n 8$

잉

ㄷㅎㅇํํㅇ

000

808

000

క్ర8

60

응용

000

5용응

000

距客 लि

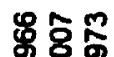
nom

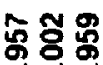

ल0

$\overline{8} 8$

野

लं

$\$$

mon

용요

mom

옥홍 ion

券安弯

तิ

สิธิ์

लं०

需言最

nom

勇骂

iom

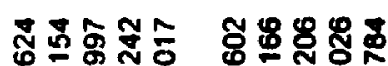
moo-

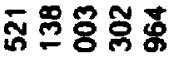

mo-

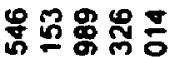

noo-

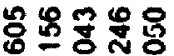

긍ㄱ응

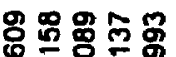

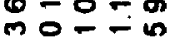

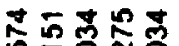

กํ-응

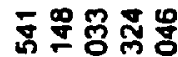

mo- -

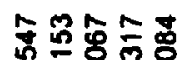

mor-

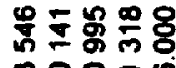

moo-6

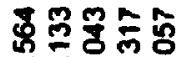

no- -

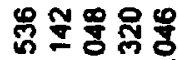

mo--

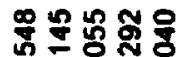

mo-n

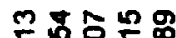

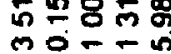

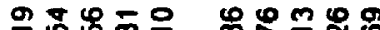

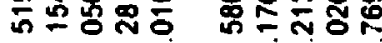

ज०--6 0000०

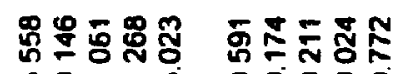
กО-7

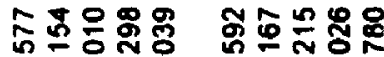
ก0-응

ㄷํㅇํํ

జิ

कर

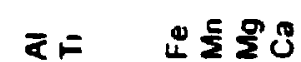

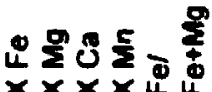




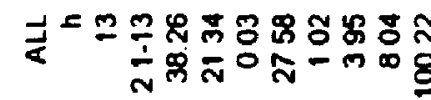

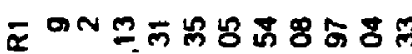
त्ल

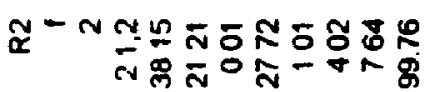

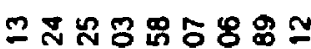

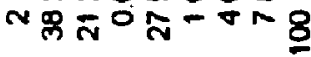

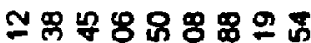

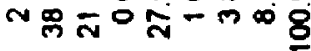

$=848$ 능

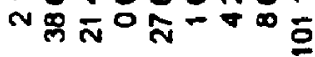

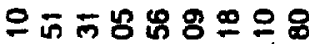

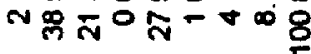

จำธ

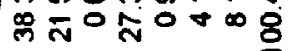

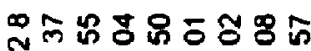

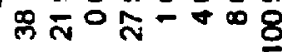

กษ

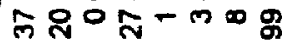

요요 思思思思

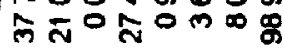

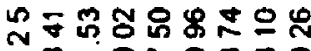

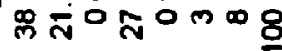

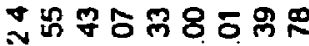

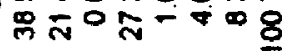

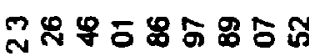

กึ

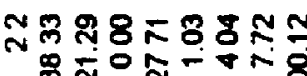

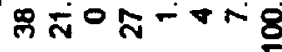

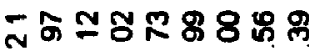

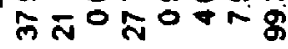

บ4ำำำำำำำ

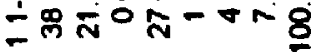

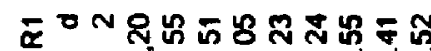

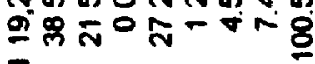

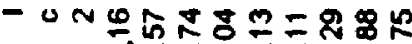

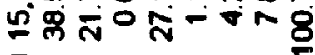

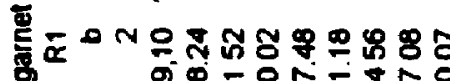

品

ที่

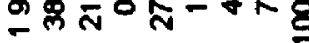

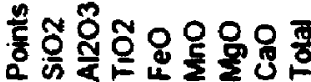

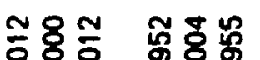

000

웅용

000

용융

$\frac{20}{5} 8 \frac{5}{5}$

¿요으

둥요요

눙 8

\% รั

no 0

\%용요

000

88

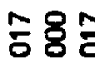

00

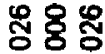

100

둥용

888

0

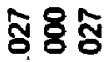

60

옹응

8

600

옿용

1000

象菖=

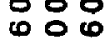

ริํㅇ 8

00

总最品

mom

용ํㅇ

mo m

\& 8

สิ8

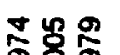

两品

용 용

mom

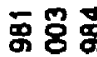

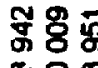

옹 8

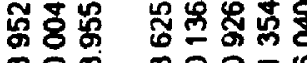

mom noo-

ํㅜㅇํำํำ

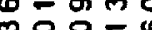

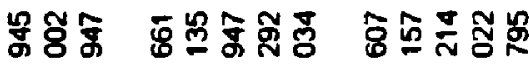

mom no०-0 00000

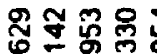

กิ-

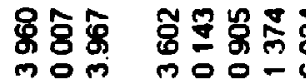

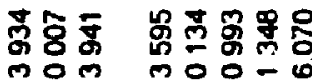

m mó잉

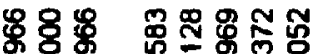

mom क्लि0-0

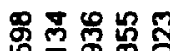

क०० =

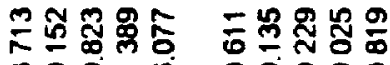

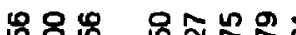

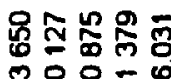

लिल ल00-

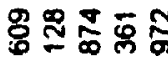

ल0 - in

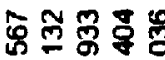

- $00-0$

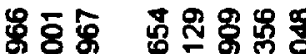

mom ल00-0

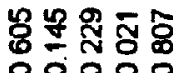

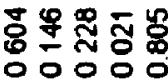

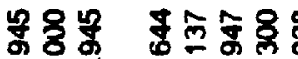

mom $m 00-0$

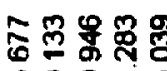

mom noo-

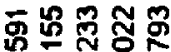

O००००

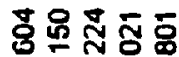
00000

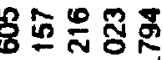
00000

ำำํำ 00000

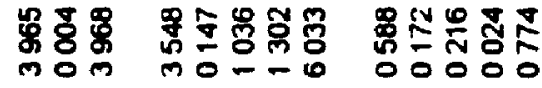

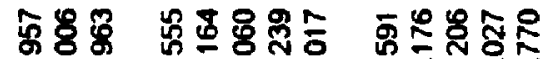
mom mo-- 00000

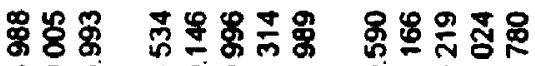
mO

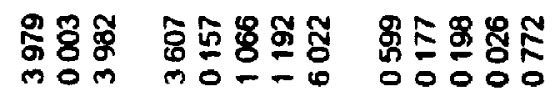

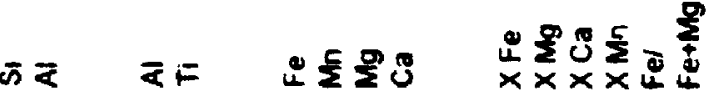




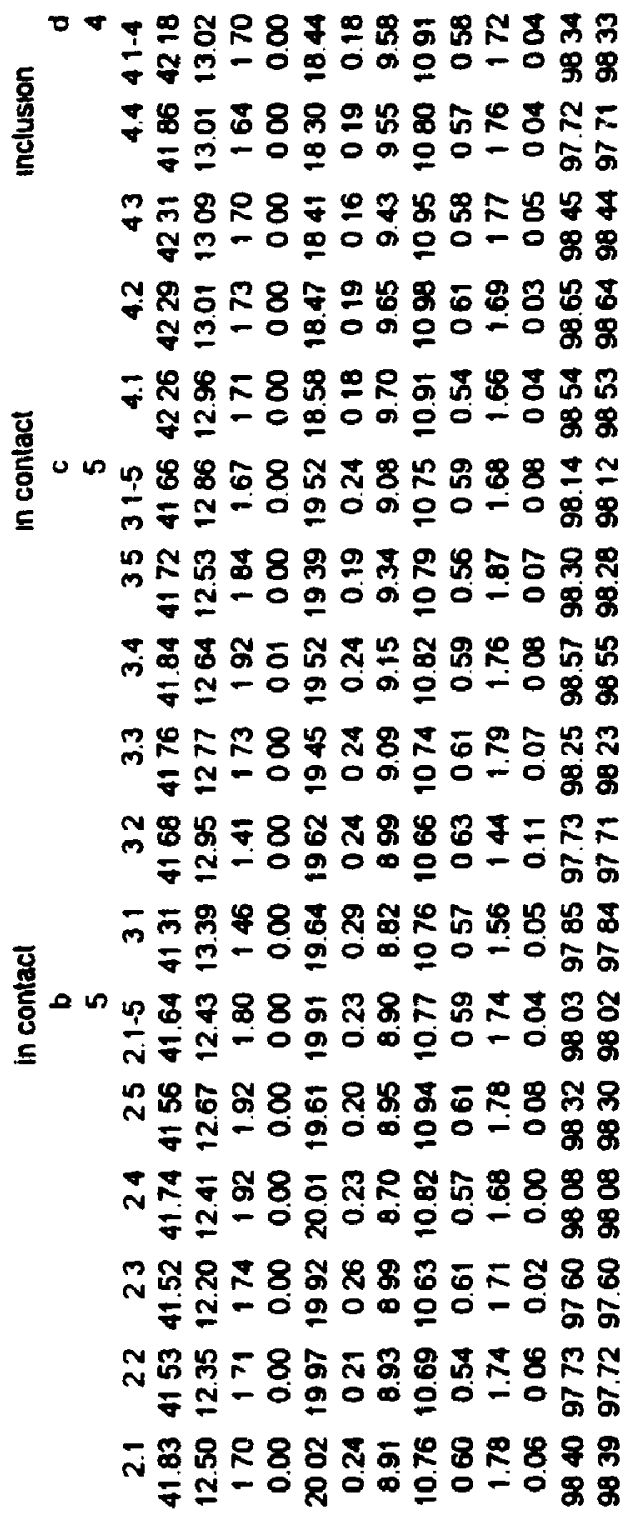

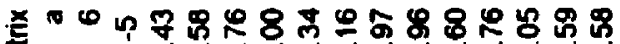

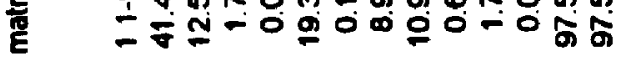

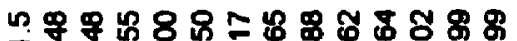

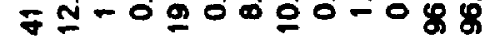

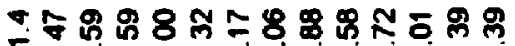
-

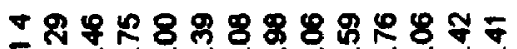

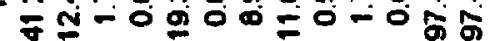
ำำ8 - $N-0$ - 0 O 농 -

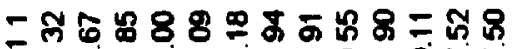

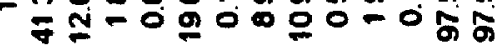

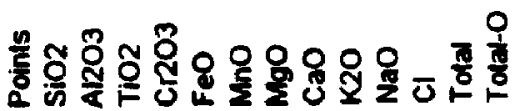

论 $\overline{8} 8$

ᄃ⿱ 口8 8



鬲央 8

$0-\infty$

$\frac{n}{8} 8$

$0 \div \infty$

尽 88

$0 \div-\infty$

क용

$0 \div$

용ㅇㅇ

$0 \div-\infty$

多

$0-\infty$

\$ 88

$0-\infty$

긍

$0 \div-$

ญ용

$0-\infty$

n. 88

$0-\infty$

ํํำ

$0-\infty$

电里

$0-\infty$

ธิธ

$0-\infty$

สำ

$0 \div-\infty$

ํํㅇ

응

8 88

$0=\infty$

年量 8

$0-\infty$

ต용

$0-\infty$

总导 8

胥禺8

$0-\infty$

ลิํํㅇ

$0-\infty$

鿖蛋

$0 \approx \infty$

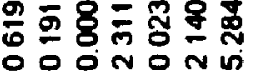

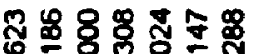

응

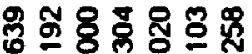

ODONONU

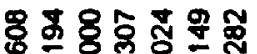

OOONON

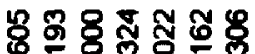

OOONONG

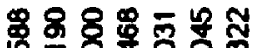
०0№Nm

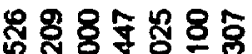
응ํำ

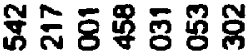
- OO NO N U

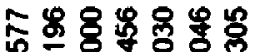

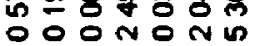

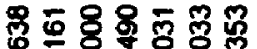

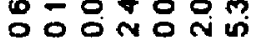

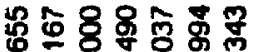
응 영

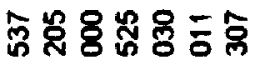
- o a nonu

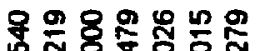
O 0 O N $\mathrm{N}$ N

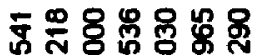
OOO NO- is

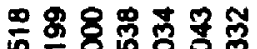
○00 No rin

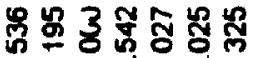
o O O No n Un

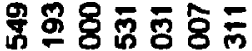
$000 N 0$ in

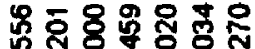

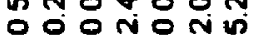

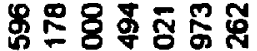

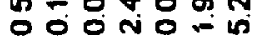

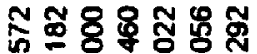
o o d n n in 융듀웡 웡응 웡 o $00 \mathrm{No}$ Ni in

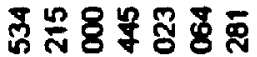
o o o no n Un

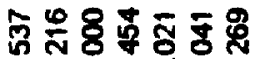
o 00 o 0 in

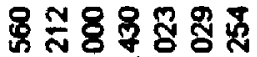
o o onon

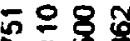

- $00 \mathrm{~N}$

บ응ํㅇ

- $00 \mathrm{~N}$

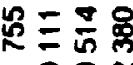

- $00 \mathrm{~N}$

费三费兽

- $00 \mathrm{~N}$

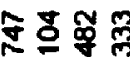

-OON

구ㄴㅡㅡ뭉

-OON

与웡

-0ㅇำ

傮可可

这

올 땡요

=용

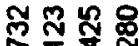

-004

온음 㝵음

-00

员哭的芯

-OO N

ํํำ ํㅗำ

-

울응 용

-OON

员里免

-OO N

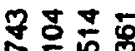

-0ั0

온음 용

- O0 N

용으응 용

โ-ํํㅇ

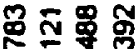

-0ㅇㅅㅡ

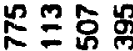

-0에

㖉武手

-О人

里里员孚

- $00 \mathrm{~N}$

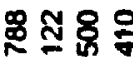

$-00 \mathrm{~N}$

$-00 \mathrm{~N}$
क्ष

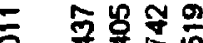
응응

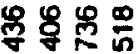
0000 为各㗈 ०00。

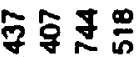
0000 罗金是思 0000 款总和 0000 ธํํ요 O००。

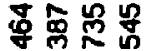
○000 造造是 잉

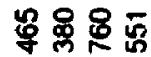
0000 㝵总员品 ㅇㅇㅇ 웅 - O००

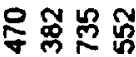
○000 急合专总 0000 웅 0000 용요 ㅇoㅇ ผ 0000 鱼总品管 0000 声罂影品 采茴品 最量事步 0000

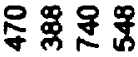
0000

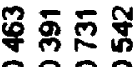
0000 8 稌果 0000

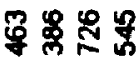
0000

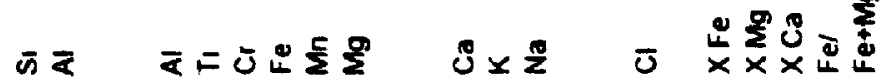




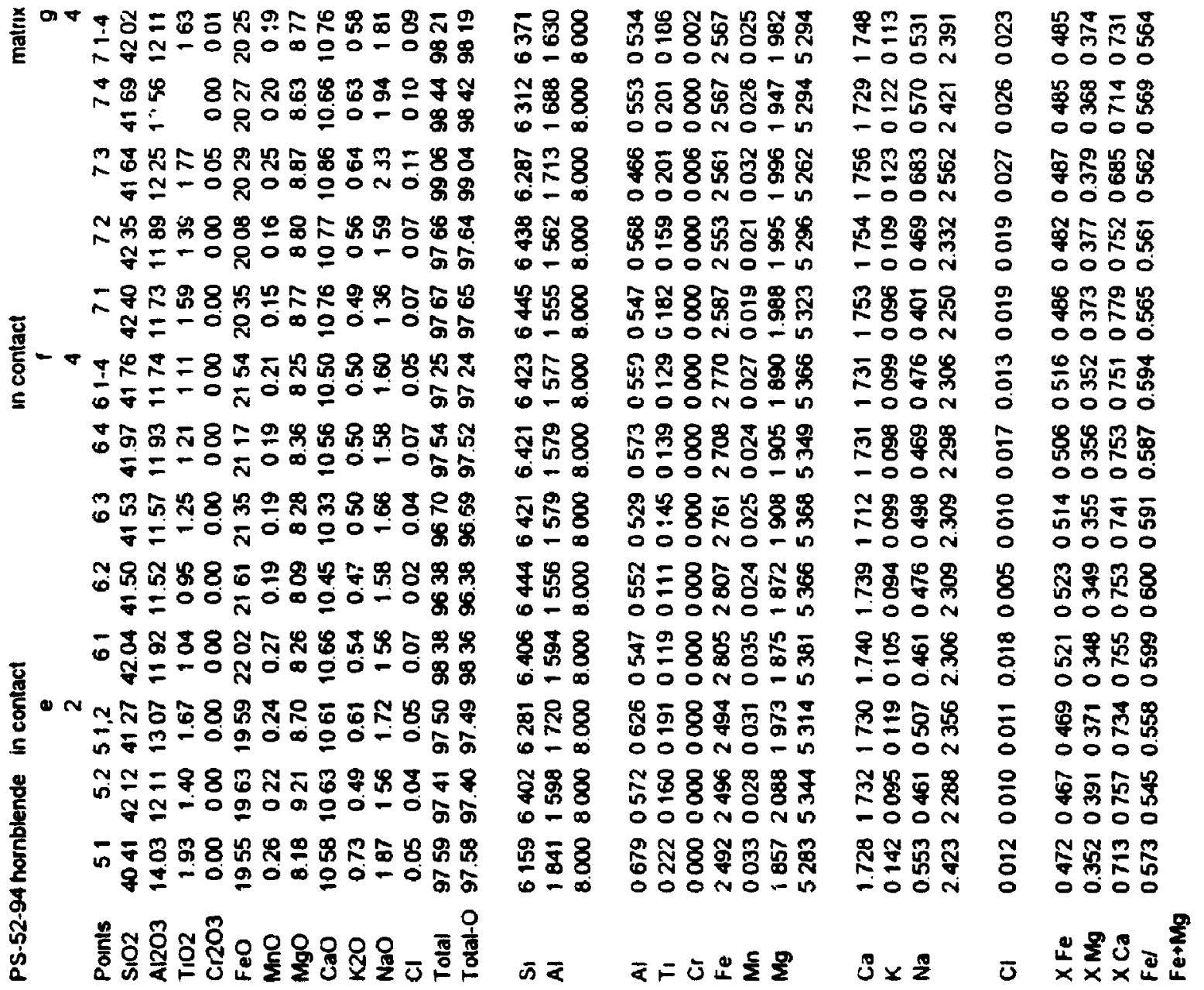




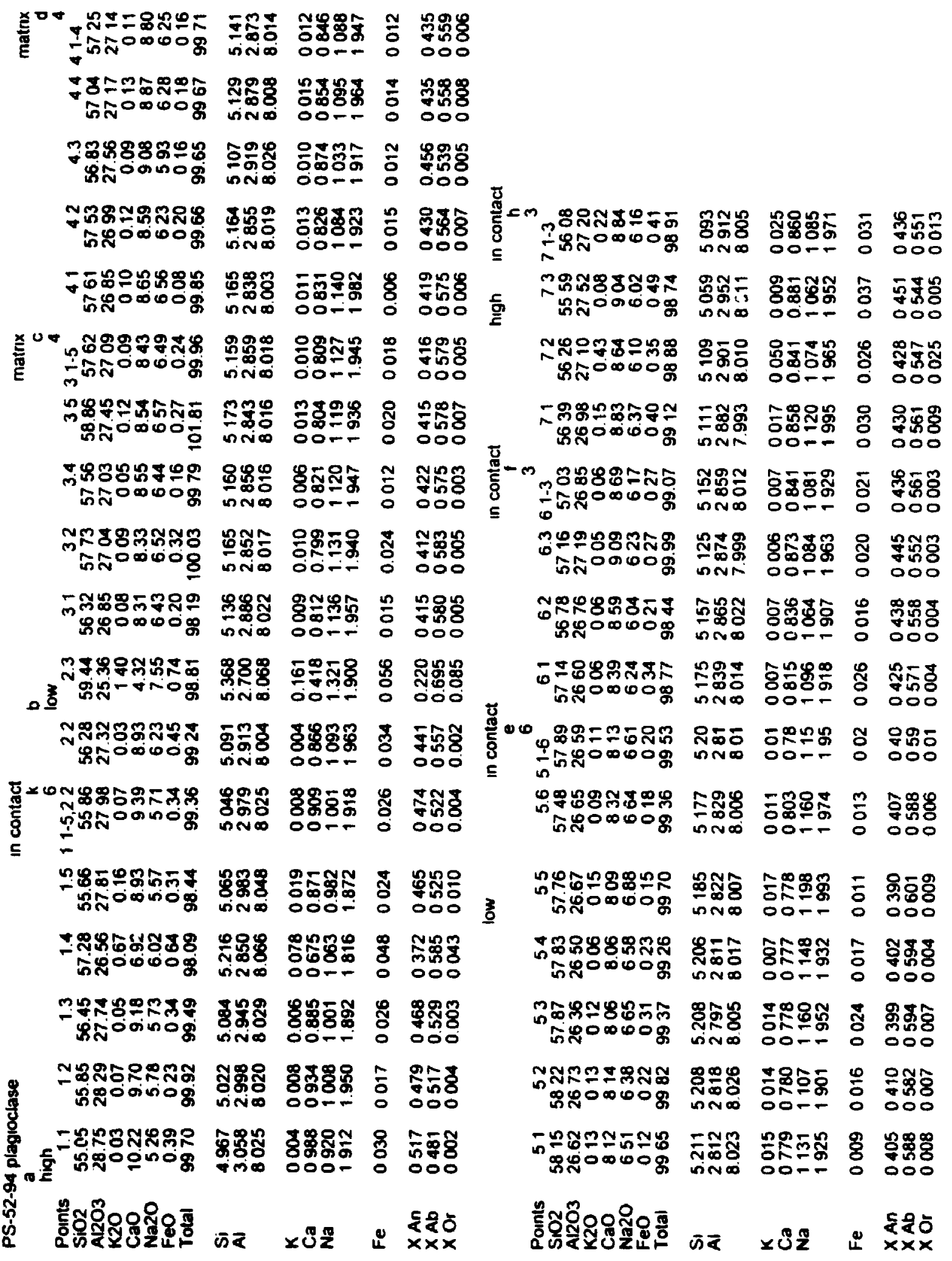




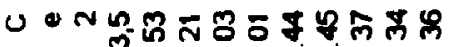

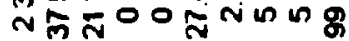

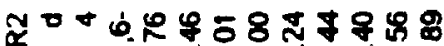

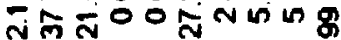

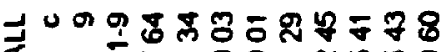

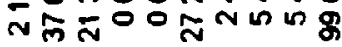

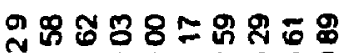

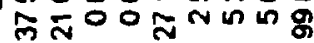

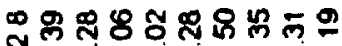

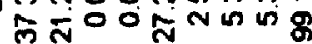
ลัญ ลิ ลั0 กัN

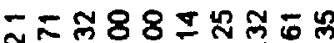

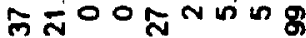
ำำ8

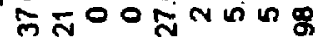

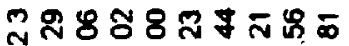
กิ-

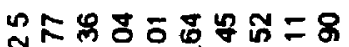

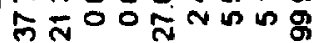

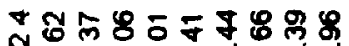

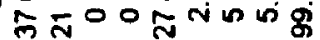

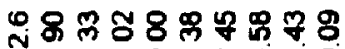

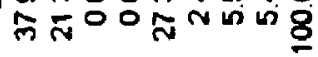

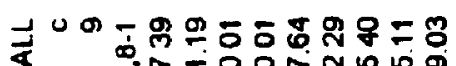

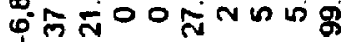
$\div$

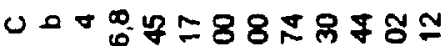

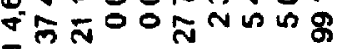

そั・ニ

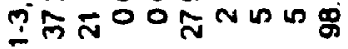

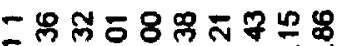

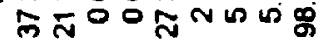

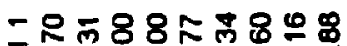

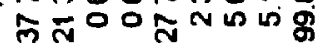

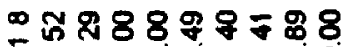

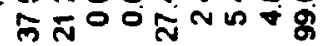

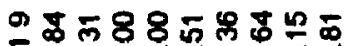

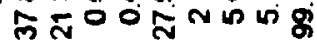

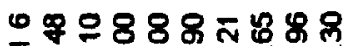

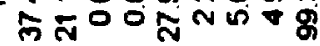

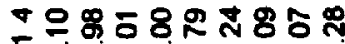

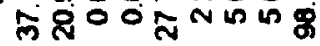

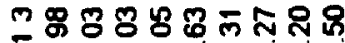
पू⿱

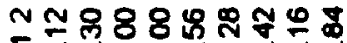

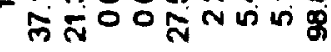
二ร888ำ 8 ส กิ-

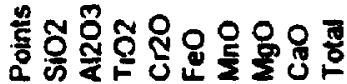

경웅 8 in 0

¥궁

जo 0

巻 $\overline{5} 8$

मी० 6

范客条

ज० 0

$8 \overline{8} 8$

๑3

no 6

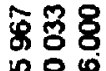

象定

in 0

多号 8

is 0

囱豆 8

no 0

ิㅗㅇㅇㅠ

เ 0 \%

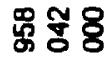

no 0

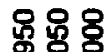

is 0

通得 8

500

공용

is

需号 8

जo

눙 8

in 0

$\$ 88$

no 0

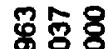

in 00

홓ㄷㅇㅇ

no 0

50

जo 0

รึํํㅇㅇㅇㅇ

is 0.

옹용

जo 0

ํํㅇㅇ

in 0

$\overline{\bar{\alpha}}$
응효 mo

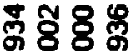
ํㅏㅇ응

谷行 n०० ₹ ल00

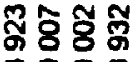
mo

잉용ㅎㅇ 유 mo

굥요

궁융

ल엉

б용

moom

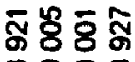

ल००m

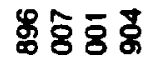

ल०0

б

ल०0

갱효

ल०0

్ㅎㅎㅇㅛ

농용

लㅇㅇㅇㅛ

호용

웅

응이

\%용요

ल००

สีร $8 \%$

ल00m

홍요

ल० $0 \mathrm{~m}$

충용용

ल0

б효으

ल००

굥용

$500 \mathrm{~m}$

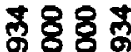

ल유

๙ニう
塄然范客声 ल0 - 00

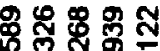

กิ

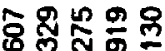

ल0 - 0

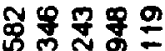

ल0 - 0

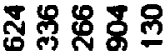

mo-0

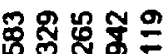

ल0 - 00

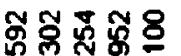

mo-0

塄

no- 0

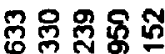

ก0-0

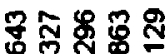

กo-0

꿍율윰응

mo-0

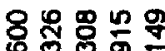

no-0 0

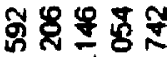

00000

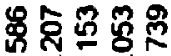

00000

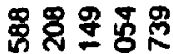

00000

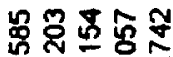

00000

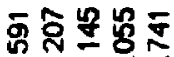

00000

品起落落

00000

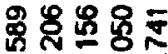

00000

8 象星员总

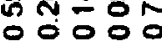

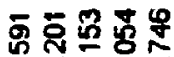

00000

费资量急总

0000

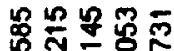

00000

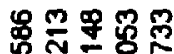

00000

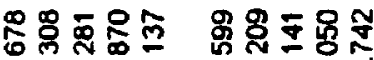

no-0. 00000

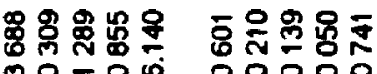

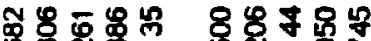

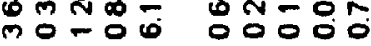

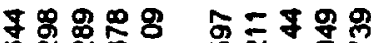

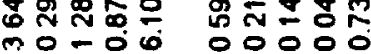

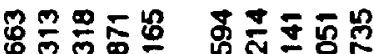

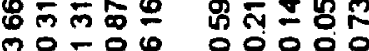

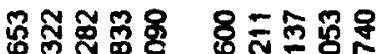

mo-0. 00000

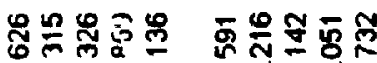

mo-0o 00000

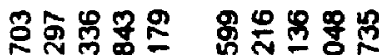

ก०-0\% 00000

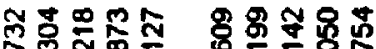

mo-00 00000

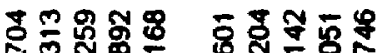

mo-0 00 00

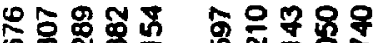

กำ-

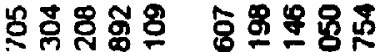

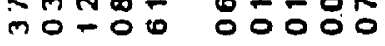

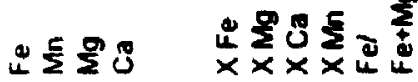




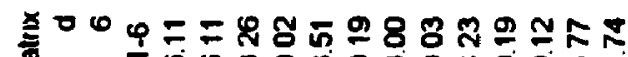

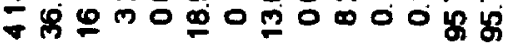
๑8 म

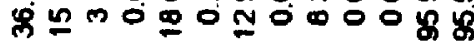

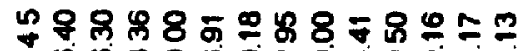

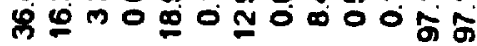

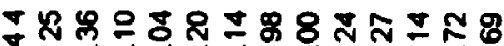

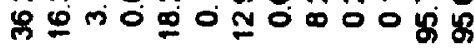

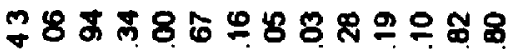
边 ㄱํำ

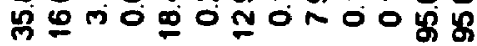

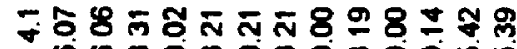

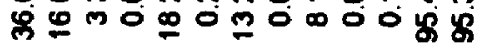

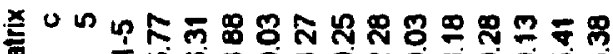

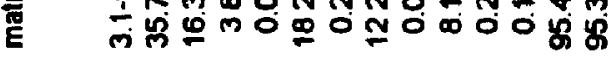
舟ธㄷำ

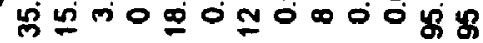

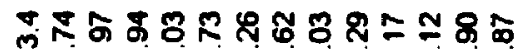

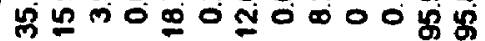

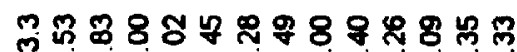

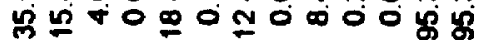

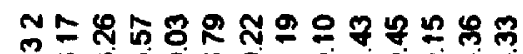

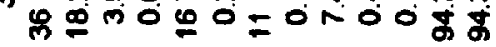

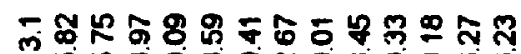

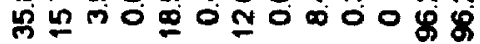

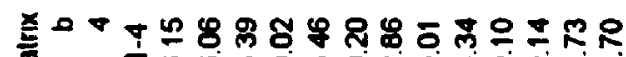

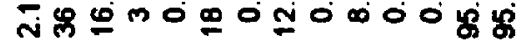

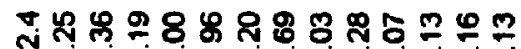
乌广

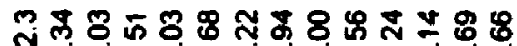

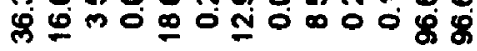
츄음

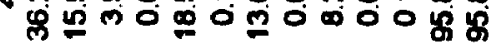

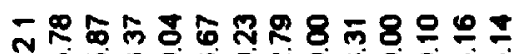

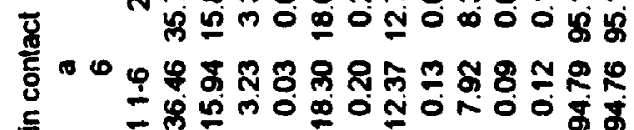
士

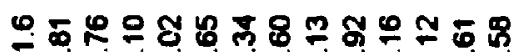

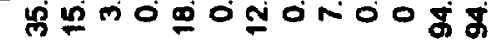

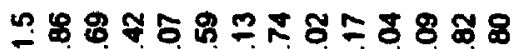

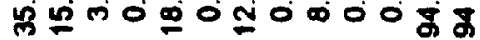

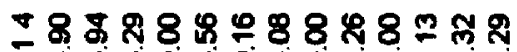

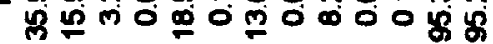

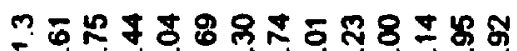

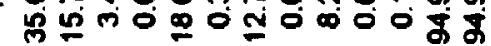

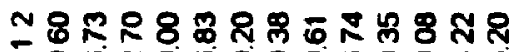

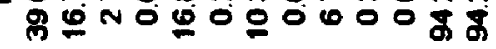

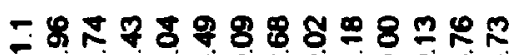

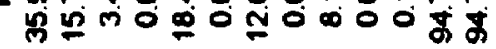

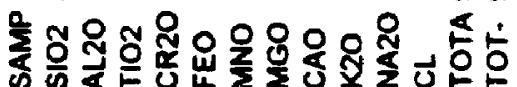

จ용

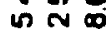
乎㖞 8 जi $\infty$ 용 8 ติ

\% 的早

88 แ $\sim \infty$

学范 8

ज

争 88

in

可忽 8

ต

눙 8

ஸे

5. 88

in

్ㅐㅇㅇㅇㅇ

in

茟落

เง

驾 8

क त

于

जि

ริํํ요

的空

羿骂

जा

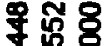

แ

స్ํ용

ज N

స్ํํ유

ก

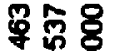

ก

学家

क त

勇怒

कก

\%

क

- 88

แ

융

ज实

क事
象多 8 ०००त跔

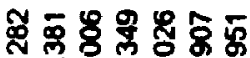
ด०

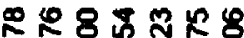
กุ

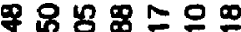

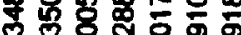
o 0 ond n n

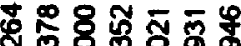
○ㅇำ

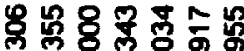

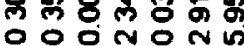

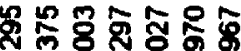
ㅇํㅇำ

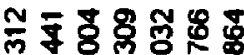
o o on

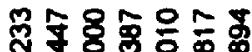

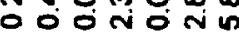

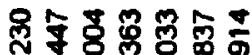
- OO N N N n

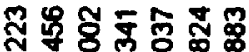
OOONON

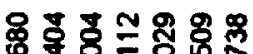
- OONON

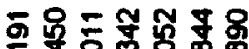
○ 0 O

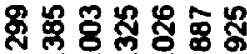
○。 ONON

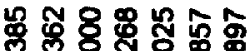
○ O

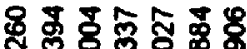
○유유

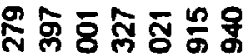

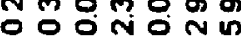

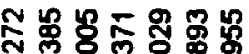
O O O O N

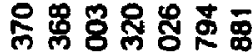
OOO ONON

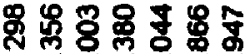
○OONON

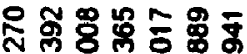
०Oำ N సิ OOONON

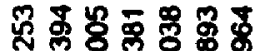
O OO NON n \% 8 \% OOONON

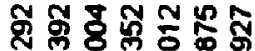
0 OONON

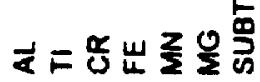

号员䒓 응$8 \% 8 \%$ $0-0-$

8 多管栗 는

요용요용 응 -

否两范思 ㄴ-口资勇客者 드80 는

管卷要要 으-

$8 \frac{0}{6} \overline{5}$ $0-0$ 웅용영 $0-0$ -

융융동유 - - -

등 㞧量占 $0-0$ -

ธ유융 늠

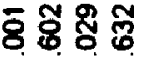
응응

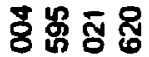
응응

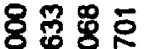
응 -

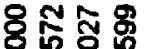
음능응 8 웅용용 응응

สํㅇํㅠㅇํㅇ ถ ㄴ- -

콩훙ㅎㅇ응 응$8 \%$

8 용용 눙ริ8 8 $0-0-$ 영웡으원 $0 \div 0=$ 808 응

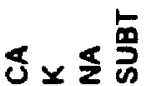

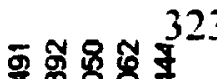
ㅇํㅇㅇㅇ

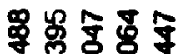
00000

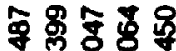
00000 궁요영 00000

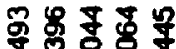
0000

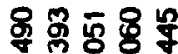
00000

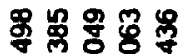
00000

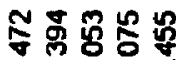
00000 연운웅영 0000 多 웅용영 00000 多䍙塄号急 ○0000

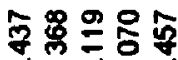
00000

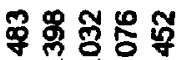
○0000

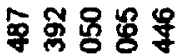
00000

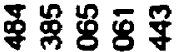
00000

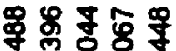
00000

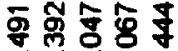
00000

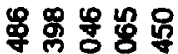
00000 学实多莳 00000

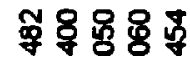
00000

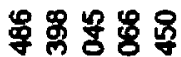
00000

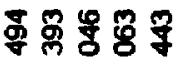
00000

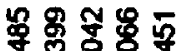
00000

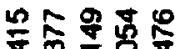
00000

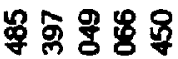
이이

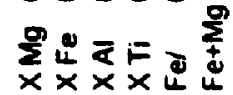


ำำ

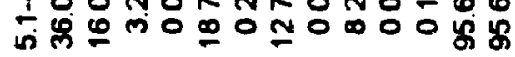

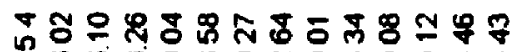

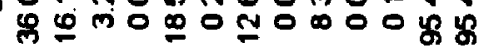

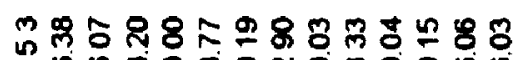

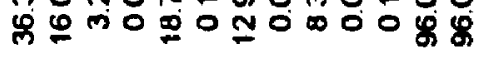

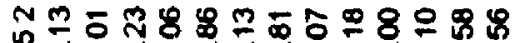
过员 Б官 $8=8 \%$ 命ำ

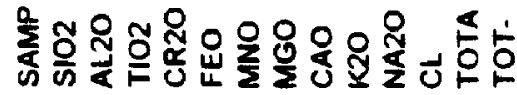

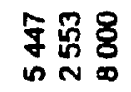

牙含要 แก

㪍总 ง 要高各 的 范它 in $N$ क范

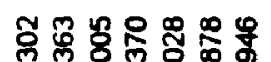

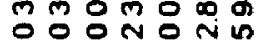
颌的号员员冬

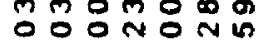

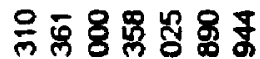

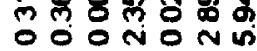

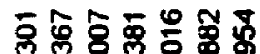
Oด口

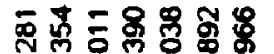
OOO NON H

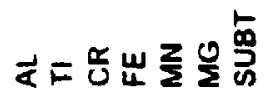

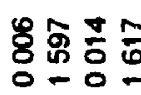

홍용 몽 응응

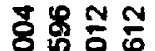
ㄴํㅇ응

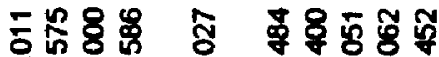

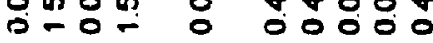

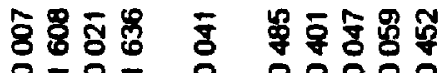

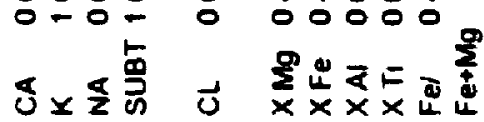




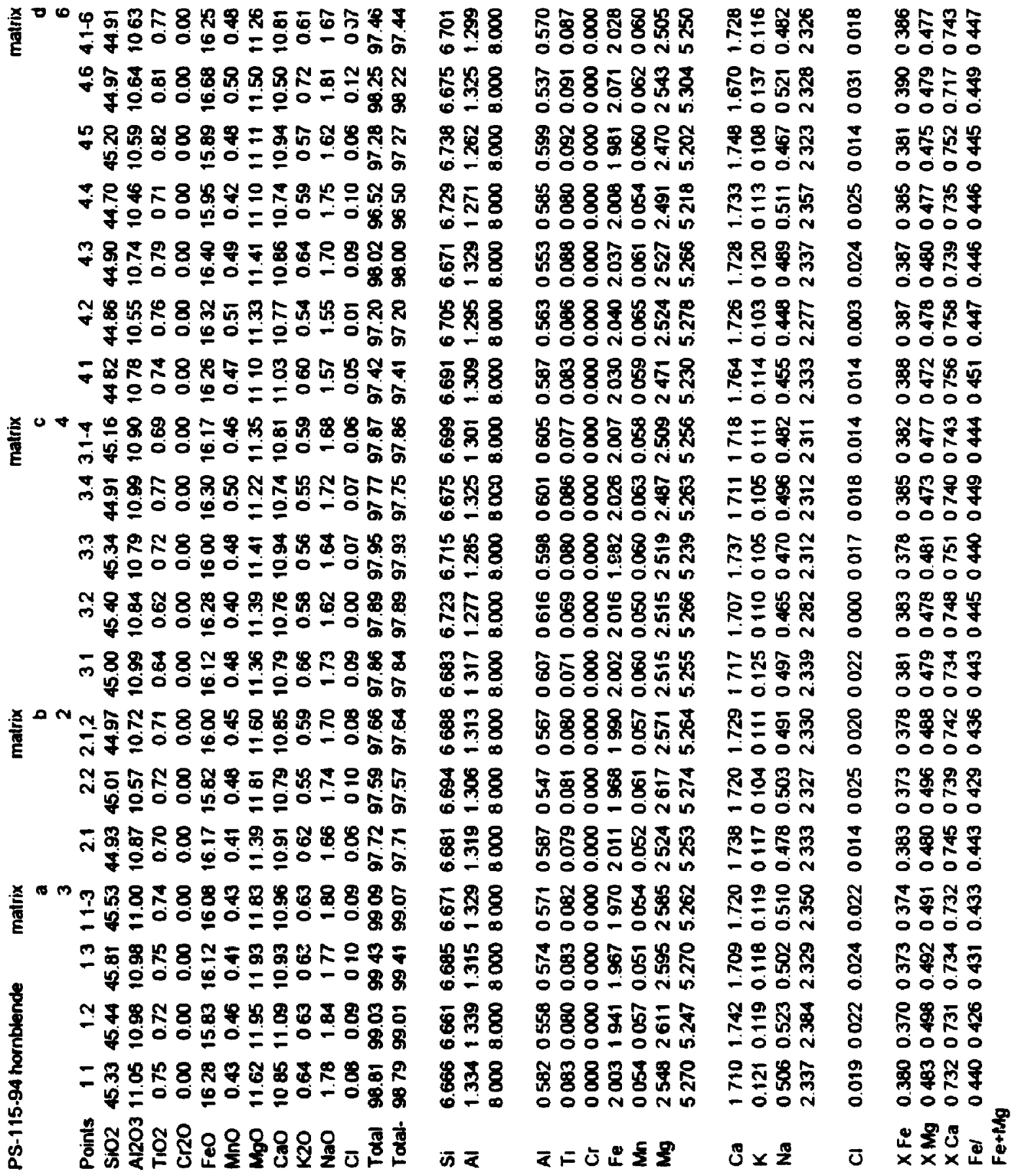




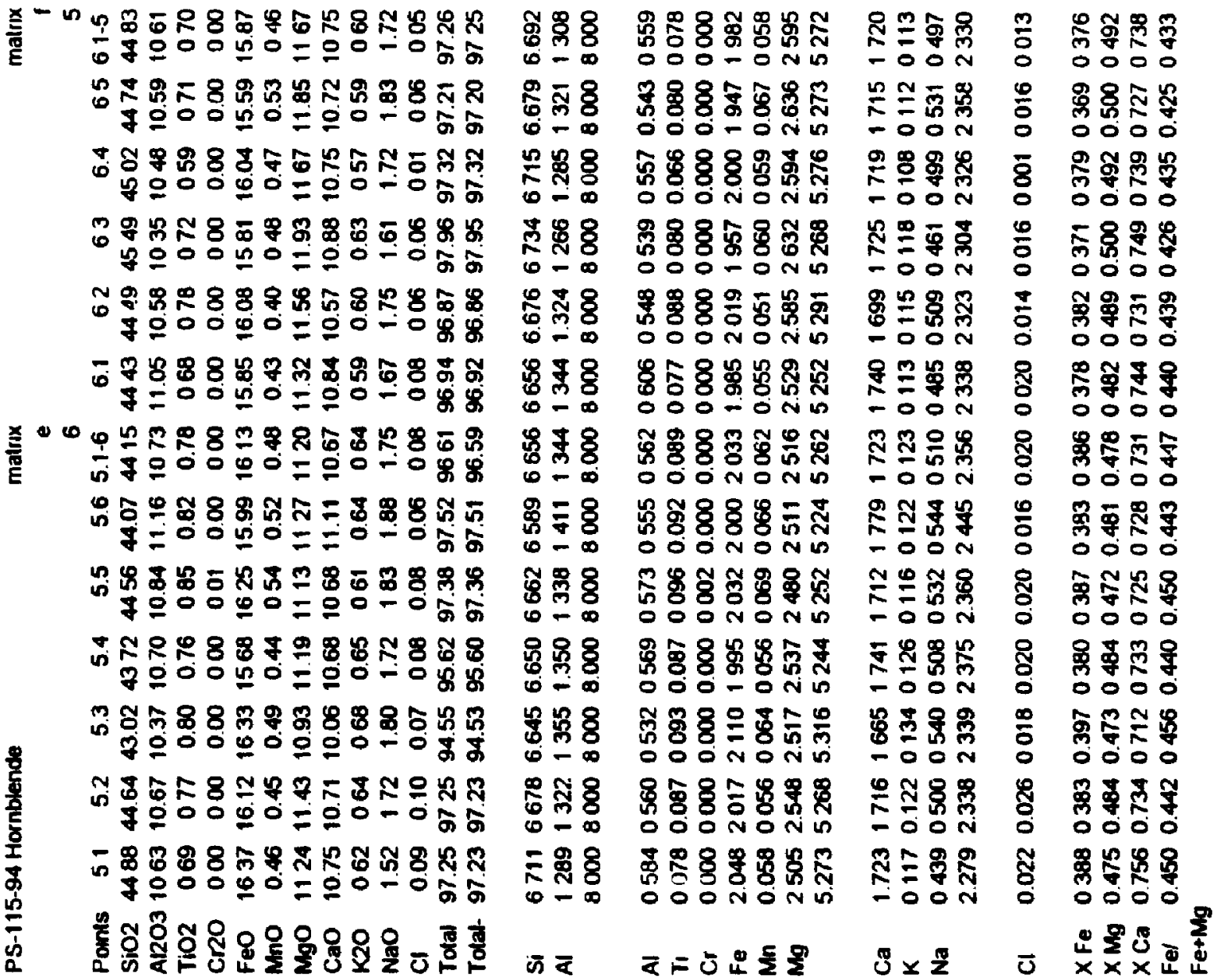




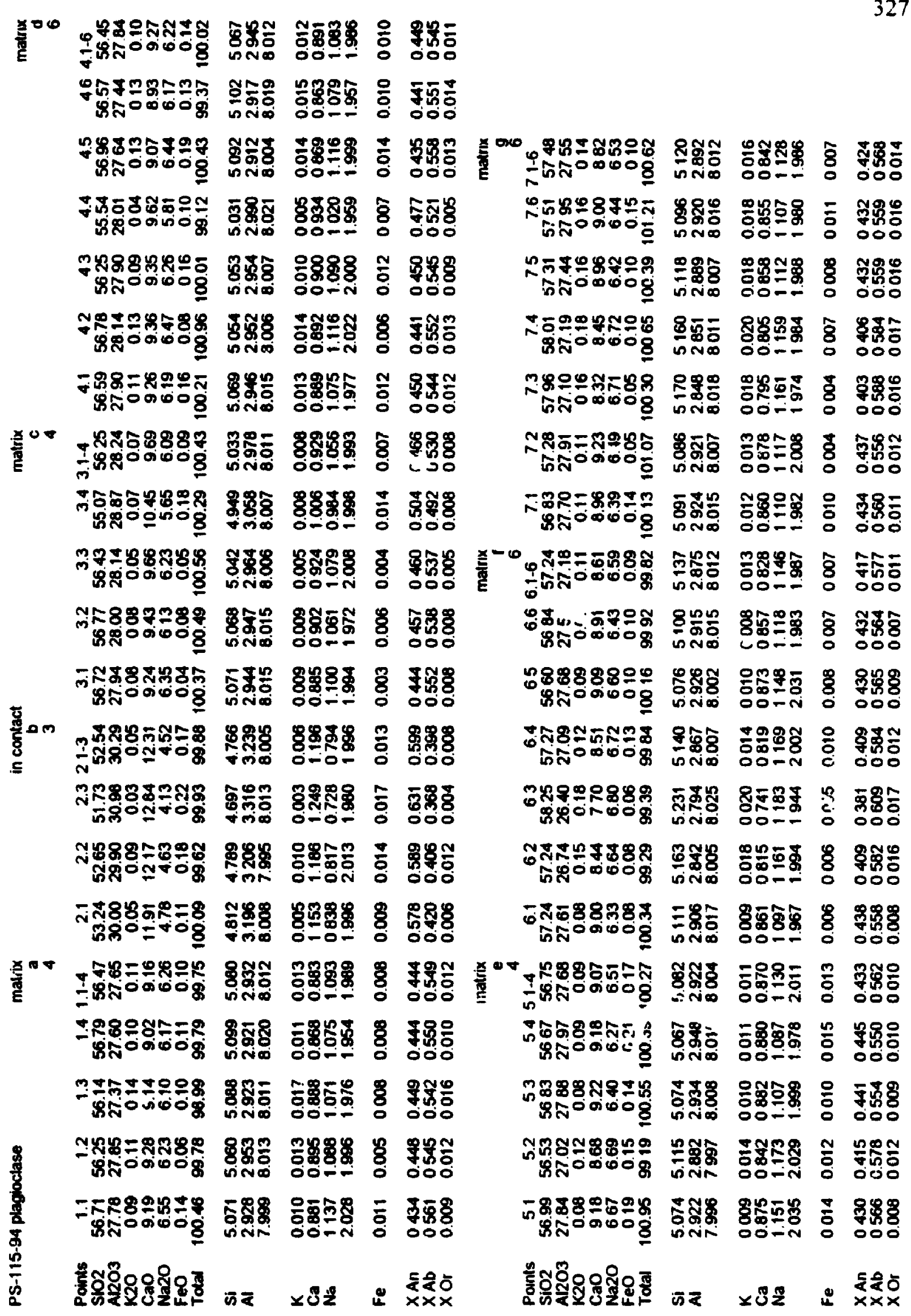




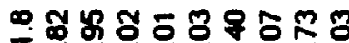

\& $80 \%$ -

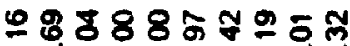

一於ล

ำกำ 8 กษ

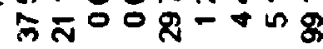

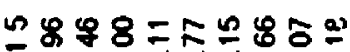

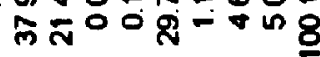

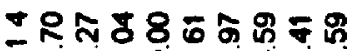

ตับ

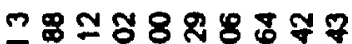

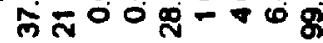

ㄱํำ88889중

需

二영 $88=8988$

निं

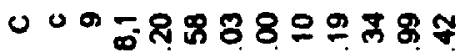

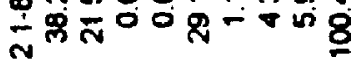

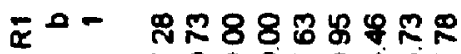

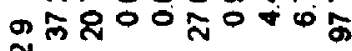

สำ

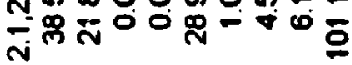

으 88588 요융

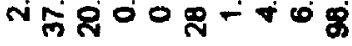

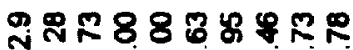

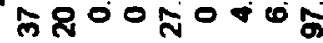

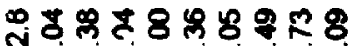

出 00 क्ष-10

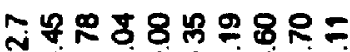

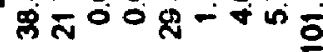

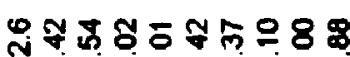

角

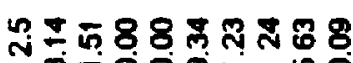

कृ

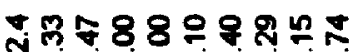

的००

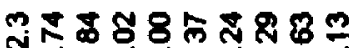

की

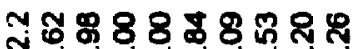

旋

సं요용 888 का

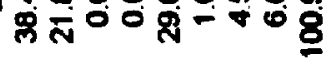

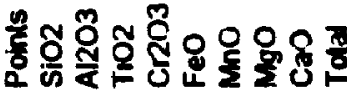

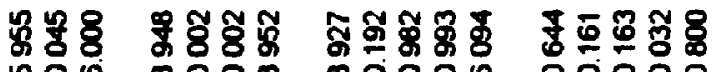

no inom noo०o 00000

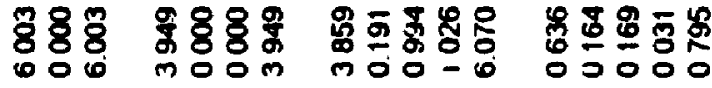

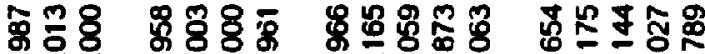

जo moom not-co 00000

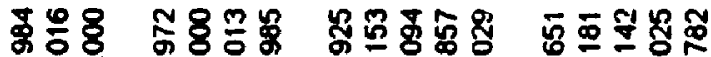

以0

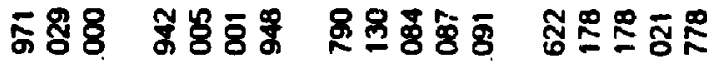

no moom mo--0 00000

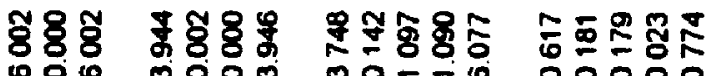

疋

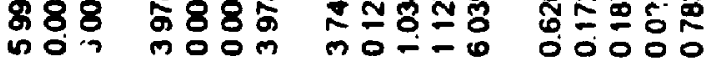

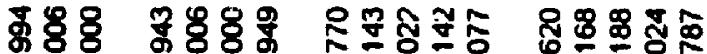

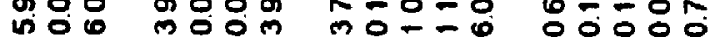

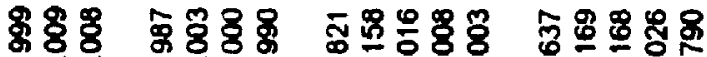

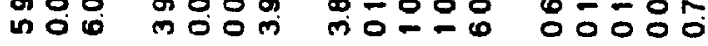

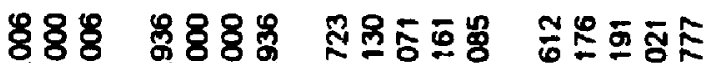

00 लㅇㅇํ

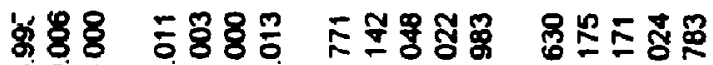

जo ToO mo- n 00000

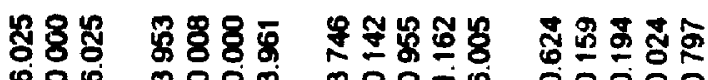

moom moo-i 00000

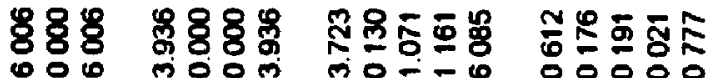

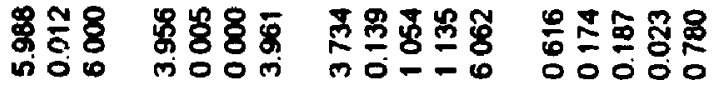

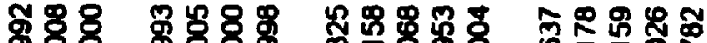

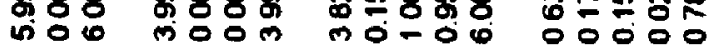

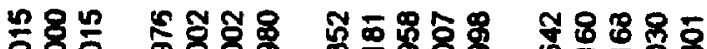

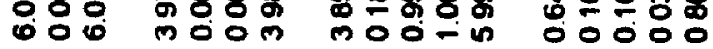

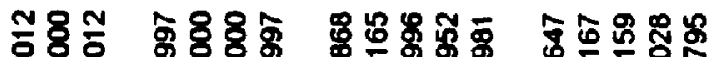

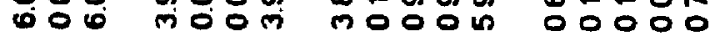

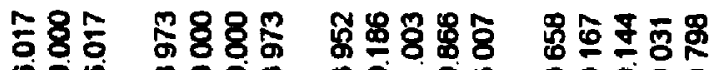

$000 \mathrm{mo0m}$ mo-00 00000

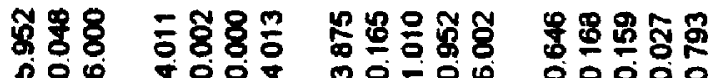

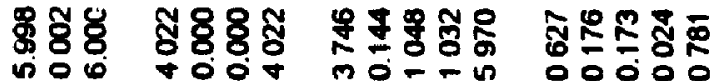

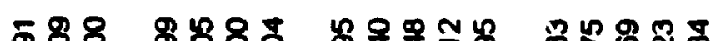

雨各总

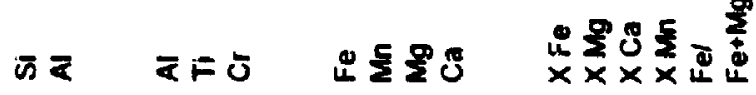




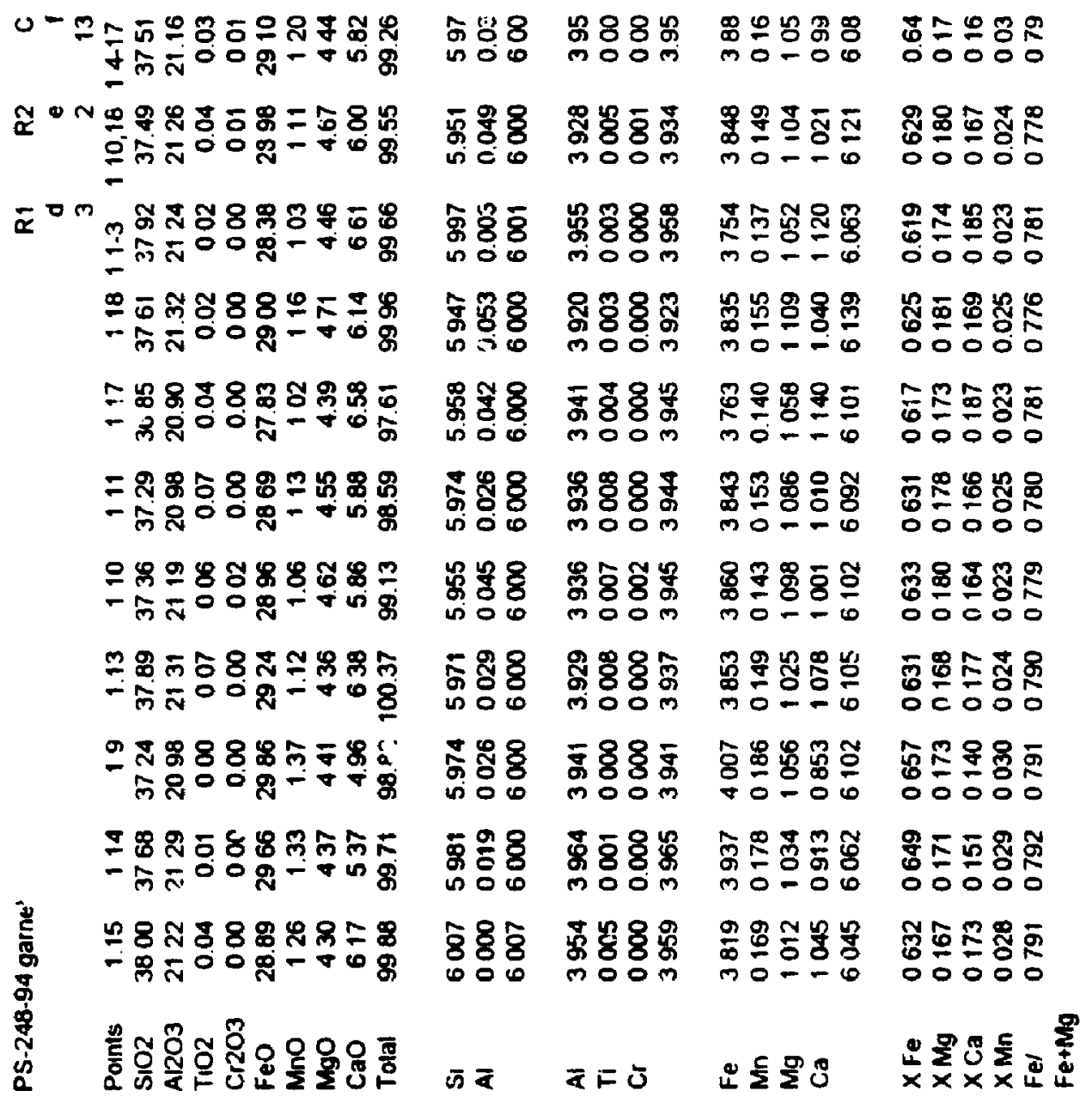




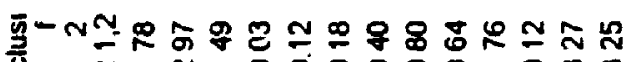

อ 西合8 $\simeq \mp \cong-0900 \div 0-0$ 思

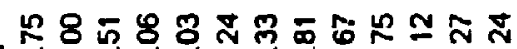
ᄃ

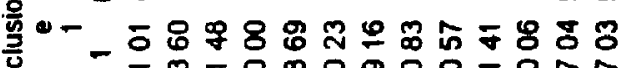

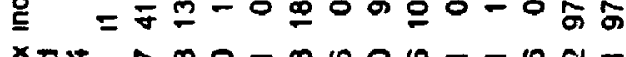

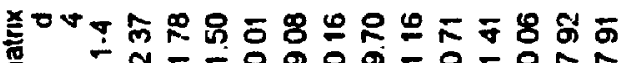
ह

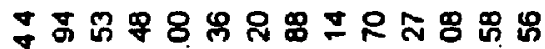

ᄀ

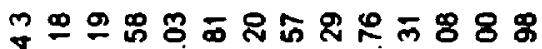

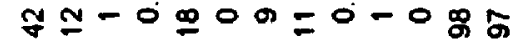

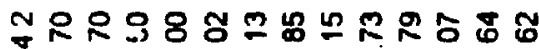

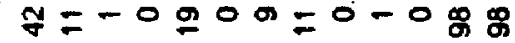

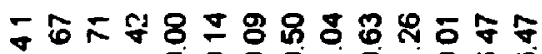

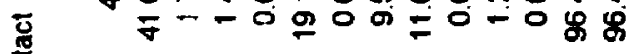

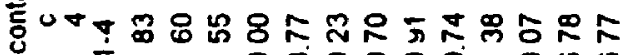
든

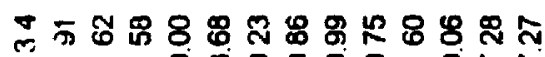

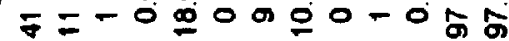

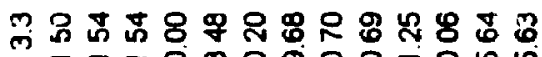

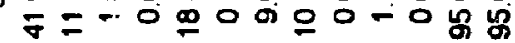
깄 $\bar{F}=-0 \Phi 00=0-0 \% \&$

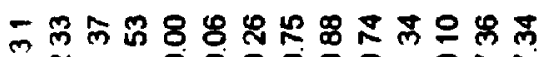

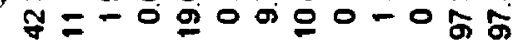

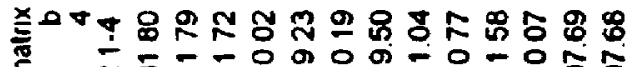
N

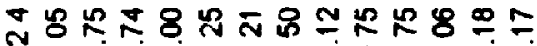
年 $=-0000=0-0$ \%

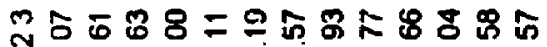

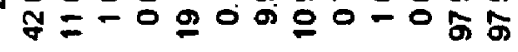

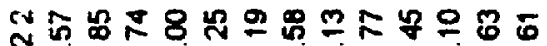

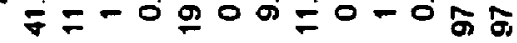

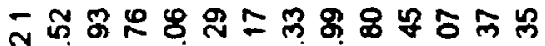

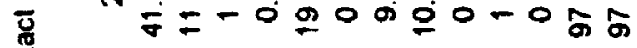

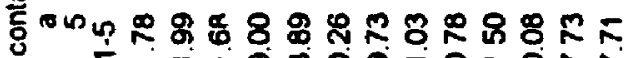

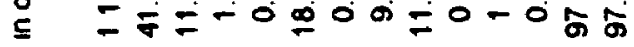

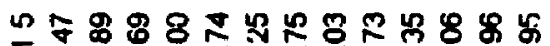
$\bar{T}=-0 ஹ 0 \sigma=0-08 \%$ ป男สำ8 8 ส

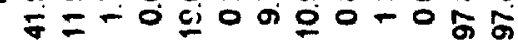

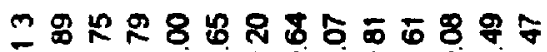

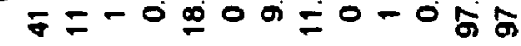

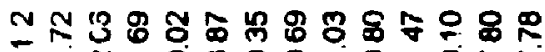
-

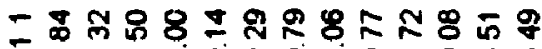

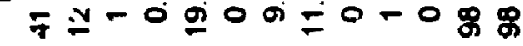

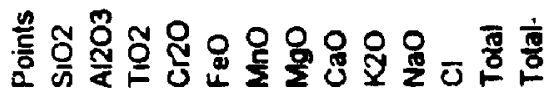

䗆 옹

$0-\infty$

58

๑ $-\infty$

용 8

$\infty-\infty$

ํㅛㅇ

$0 \div-\infty$

용 8

$0 \div$

냉 8

$\dot{0}-\infty$

点

$\infty \div$

₹

总界 8

$0-\infty$

용요용

$\omega-\infty$

要

$0-\infty$

음 8

$\infty-\infty$

曷 莒 8

$\infty-\infty$

홍

$0-\infty$

คำ

6

용

ஸ๐

공 8

$0 \div \alpha$

总

$0-\infty$

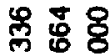

$0 \div-\infty$

웅용요

$0 \div$

ลู 8

$0-\infty$

芷 ్ㅜㅇ

$0 \div \infty$

ถึ 8

$\omega-\infty$

요용

कृ

용

호 :

$0-\infty$

के

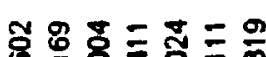

.0.0n

卷造 8 종ํำ

응ㅇㅇㅇำ

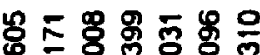

ㄷㅇㅇㅇㅇㅇㅇㅇ i

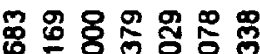

응융융

员옹훙ㅇㅇ용

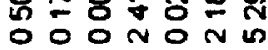

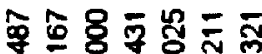

o o ono n n

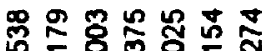

00 ONON in

웡용용ㅎㅁ웡

o 00 n

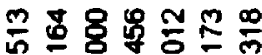

o o on 0 n

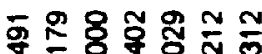

O O ONON n

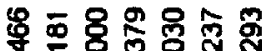

- 0 ono n in

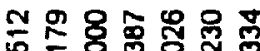

O 0 O NO N

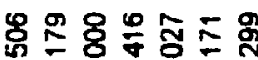

0 0 O N

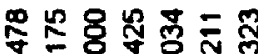

o 00 n

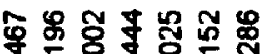

o o o in o n n

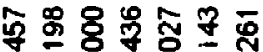

0 0 O N 0 . in

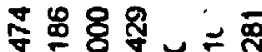

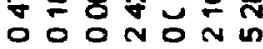

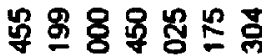

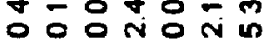

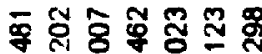

- co n o n in

产

O 0 O NO $\mathrm{N}$ in

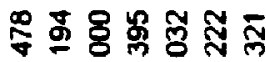

o 00 no n m

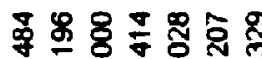

o o ono n m

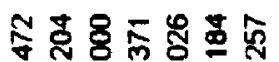

o o ono n in

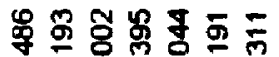

0 0 O N 0 n

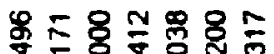

ot 0 Non

テトさ兄京

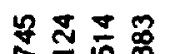

$=0$ 范

ํำ ำ

ㄷํㅇำ

品星

등

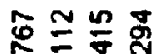

I0

品点思

- :

ถูำ 号

- $00 \mathrm{O}$

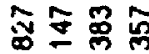

- $00 \%$

몽중

- $00 \mathrm{~N}$

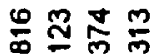

- $00 \%$

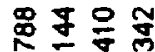

- OON

罗等尔

- $00 \mathrm{~N}$

成点兽

- б0

욤용 号

- 000

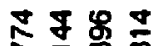

- 0 ơ

量员导㠻

- $00 \mathrm{O}$

兽 曹孚

- 00

융 용

등

음요용

- 융

요용

- 0 o

สํำ

- 00

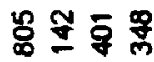

- $00 \mathrm{~N}$

노융

- 00

负

- OON

ริ

- 00

영 웅

든

$8 \times 2$
ㅇํㅇㅎㅠ 융

0000

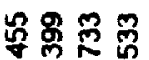

- 000

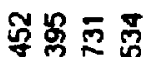

0000

导嵒只离

○000

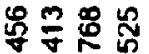

0000

每告要

0000

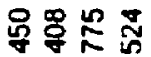

0000

莳零市㤏

0000

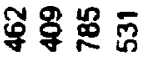

0000

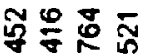

CO00

ำำำำ

0000

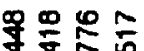

0000

昌은 융

0000

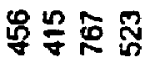

0000

ชํㅇํㅇํㅇ

0000

争守羿

0000

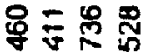

0000

㐿号界蛋

0000

等守芯务

0000

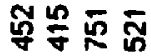

0000

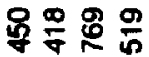

0000

结专节

0000

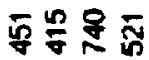

0000

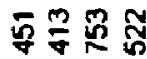

0000

娄零蛋

0000

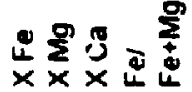




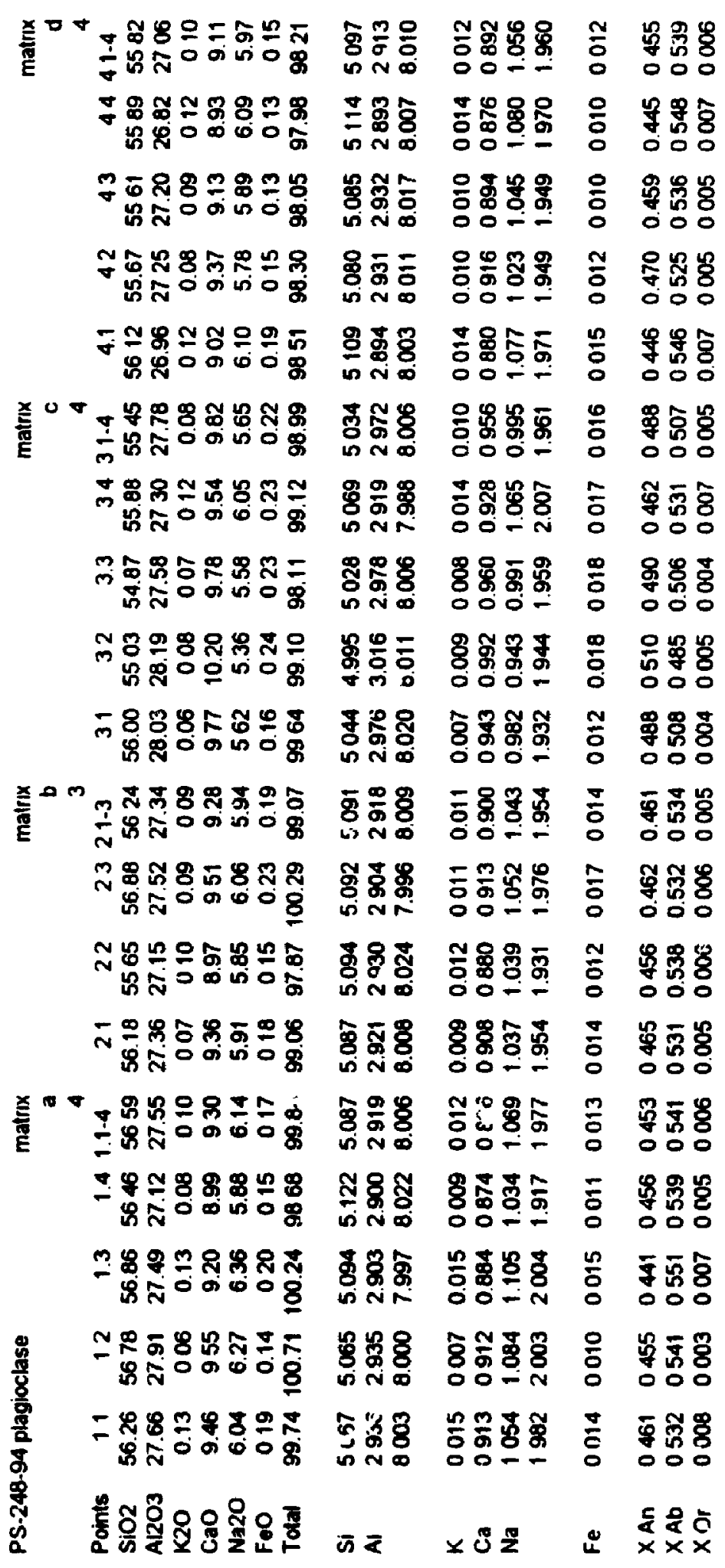


Appendix F: Mineral assemblages of marbles and calc-silicate gneisses

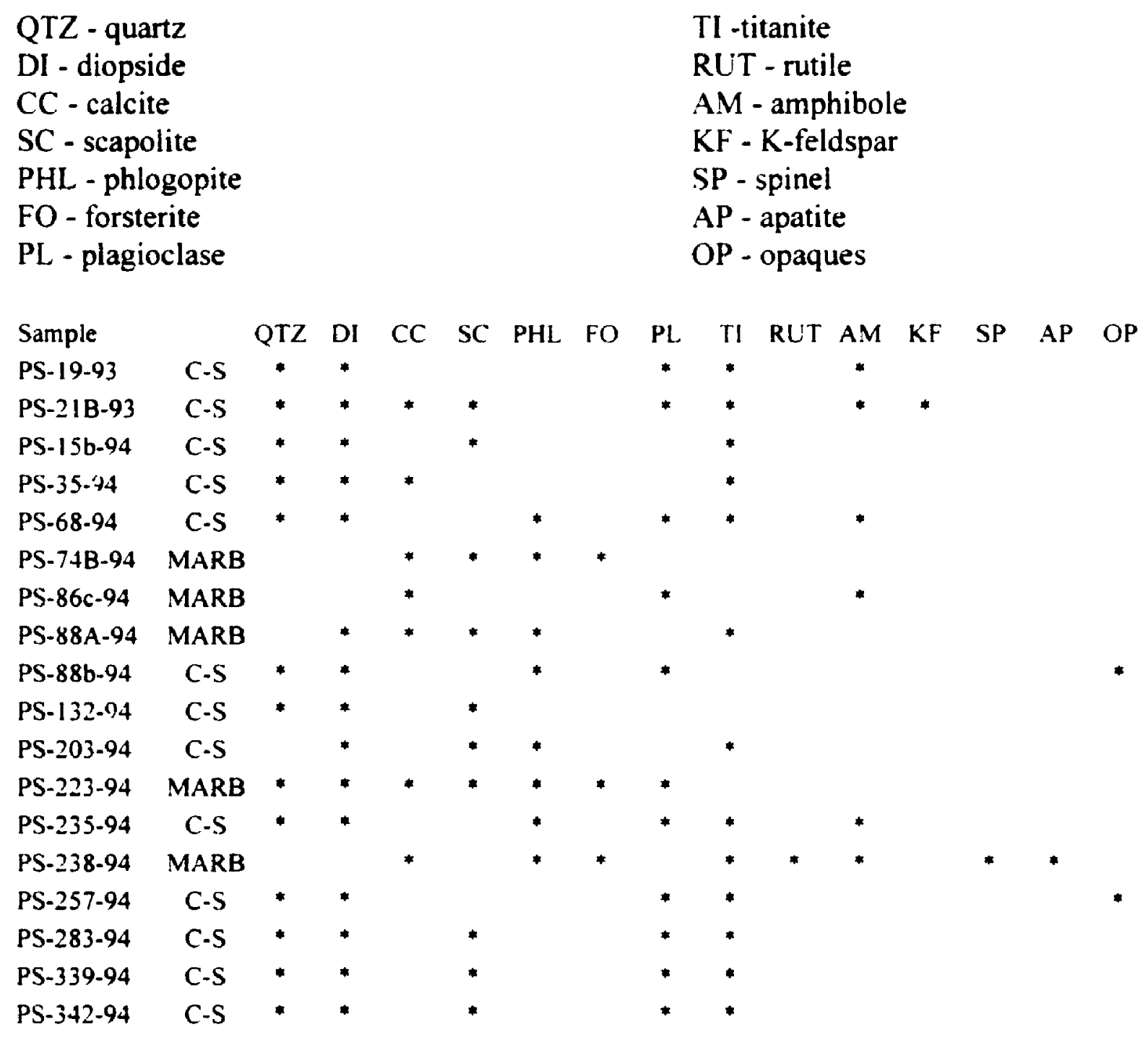


Appendix G: Mineral analyses for marbles and calc-silicate gneisses 


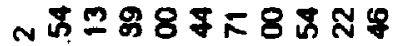

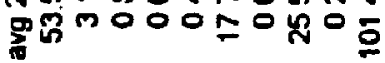

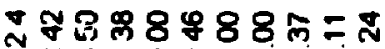
4. กำํํำ

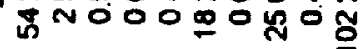
을

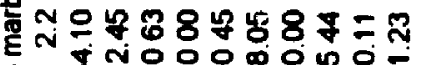

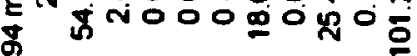
釆 तु้

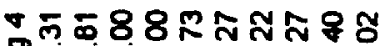

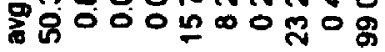

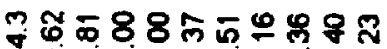

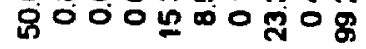
궁주요

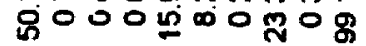

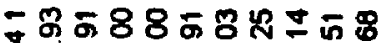

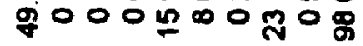

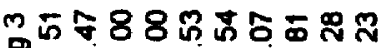

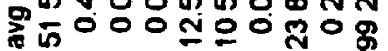

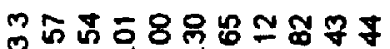

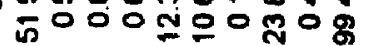

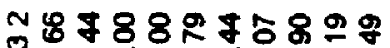

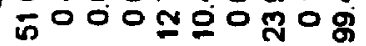

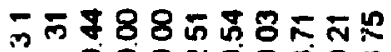
网 00 응ำ

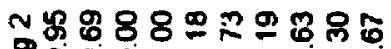
藏品0

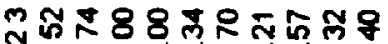

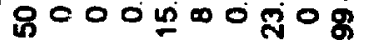
テ 号000

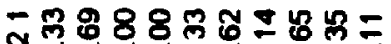
ज行 -

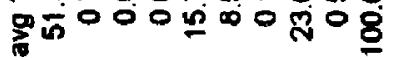
즌동ำ

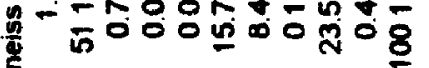

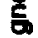

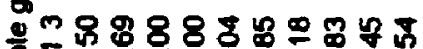
落

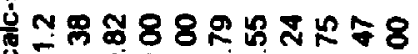
的00

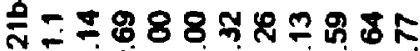
it

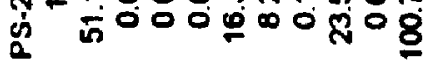

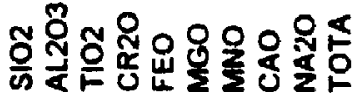

$8 \overline{8} \overline{8}$

-

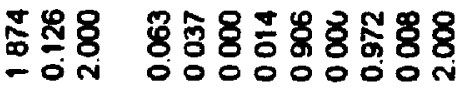

종

$-0$

電 8

$-0 \mathrm{~N}$

䅀要

-。

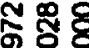

잉요

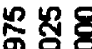

$-20$

귱요

-O

\%

-ON

舫

-

용ํㅠ

$-0 \mathrm{~N}$

훙

\$.

$-0 \mathrm{O}$

宓司

-ON

5.

- 융

융요

응

융요

$-0 \mathrm{~N}$

कूळ

-ON

홍유

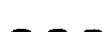

요ㅇㅛㅛ

$-0 \mathrm{~N}$

옹효

9. ర్ㅇㅇ

ถิธิธีช

잉

I\& 8

क융

-O N

कळ 00000000

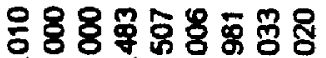

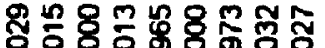
응응영용

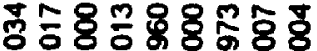
응ㅇㅇㅇㅇㅇㅇㅇㅇ

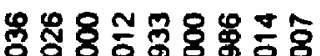
$00000000 \mathrm{~N}$

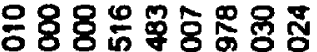
응ㅇㅇㅇㅇㅇㅇㅇ

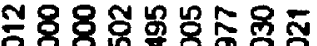
ㅇㅇㅇㅇㅇㅇㅇㅇㅇㅇ

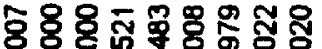

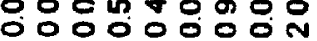

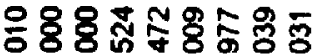
00000000 ก

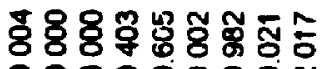
$00000000 \mathrm{~N}$

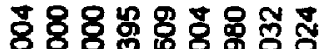
$00000000 \mathrm{~N}$

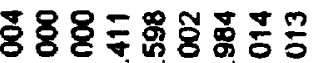
O000000

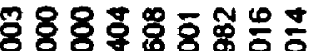
o o o o o. 0 o o o

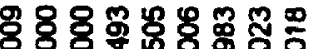
- 0000000 N

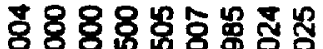

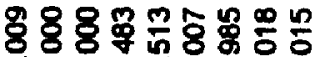
00000000 엉

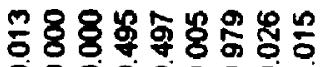
$00000000 \mathrm{~N}$

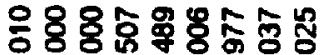
00000000 N

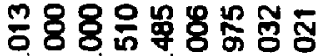

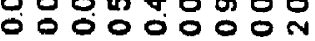
$00000000 \mathrm{~N}$

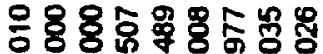
응응ㅇํㅇㅇำ

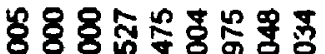

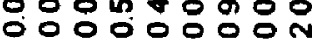

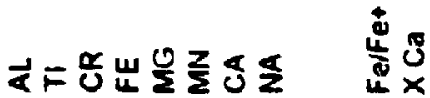

용

ㄷㅇㅇ

홍

뎡

훙

00

乐急

00

点采

00

은 옹

00

怘

00

웅 똥

00

兽覃

00

용

00

嵒寽

00

황요

00

吕寽

00

号是

00

多 9

00

艿

00

in

00

罗票 00

용

옹

ㄷํ 


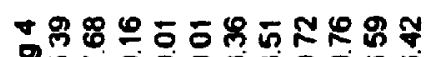
政 太 눙ㄷㅇㅇㅛ Jก̃ 我员

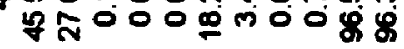

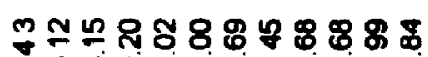
马ू

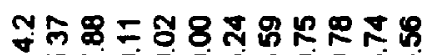

IN NOOOONOO\&

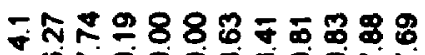

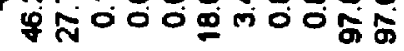

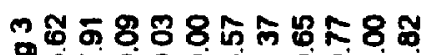

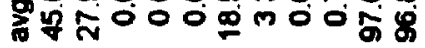

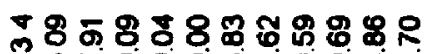

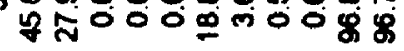

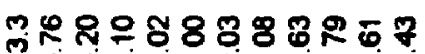

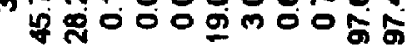

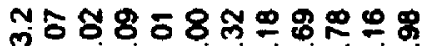

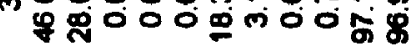
的

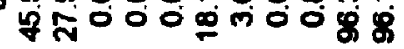

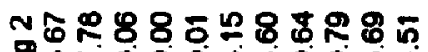

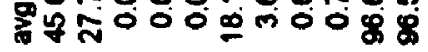

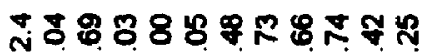
भกत००

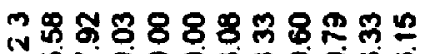
पु N भล⿵人一

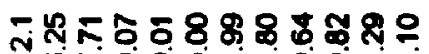

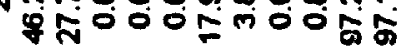

一

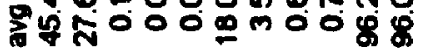

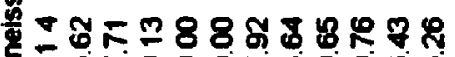

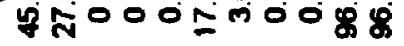

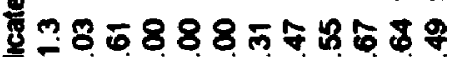

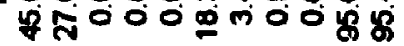

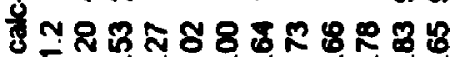
T

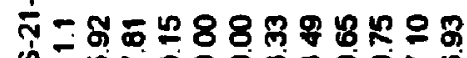
ะ

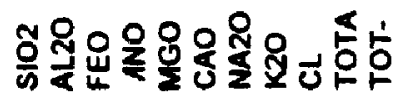

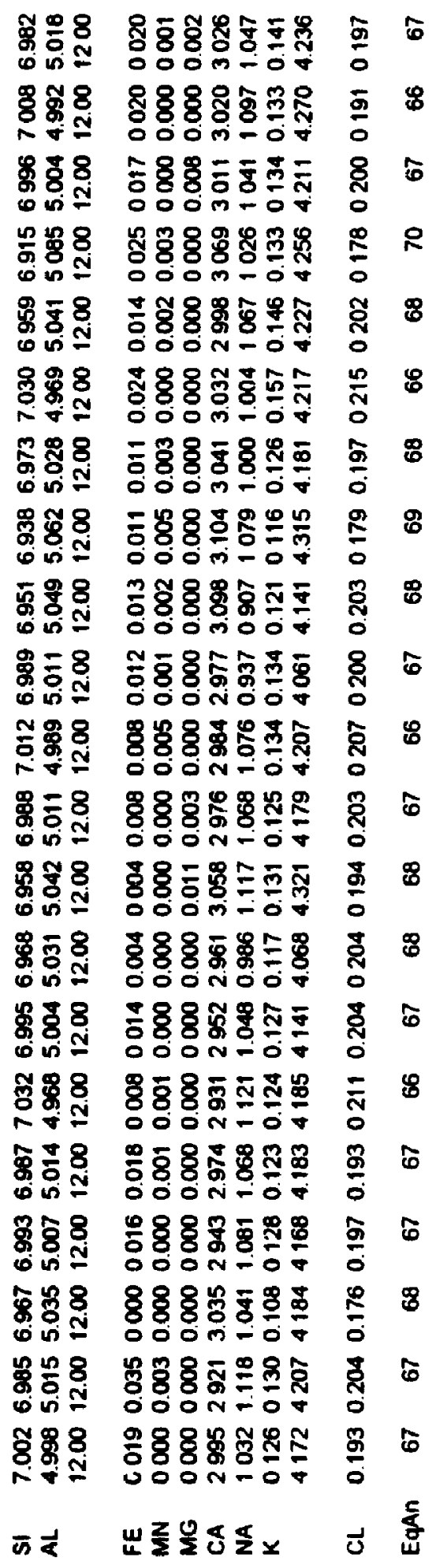




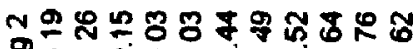

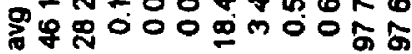

츙ำ

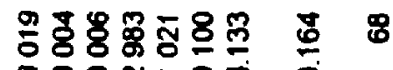

คNミニ

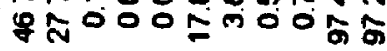

N

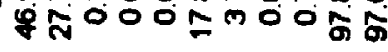

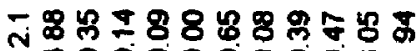

子

- จ

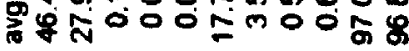

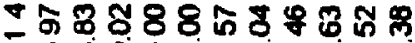

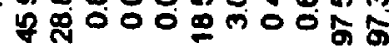

H

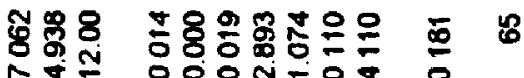

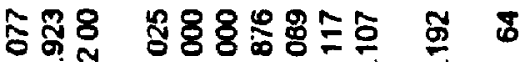

ำ

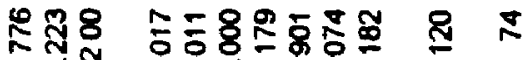

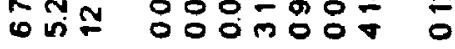

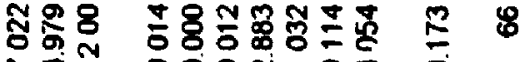

NT2000n-0,

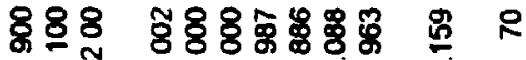

ำ大

UI

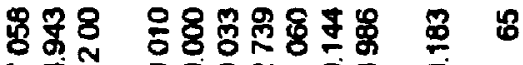

-

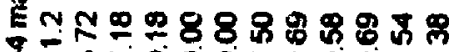

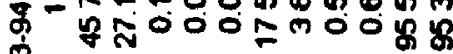

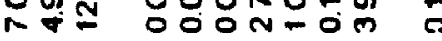

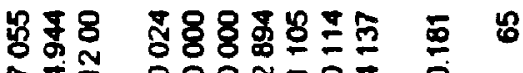

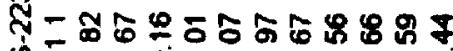

品 8 N $000=000 \%$

song

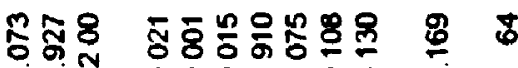




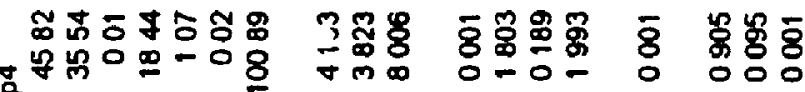

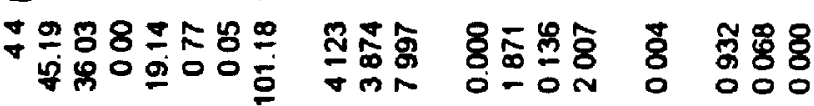

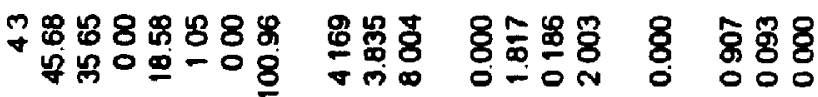

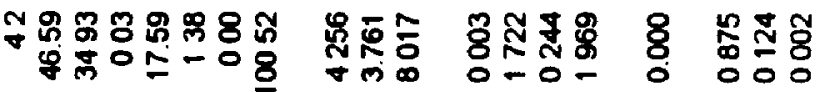

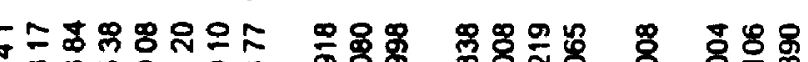

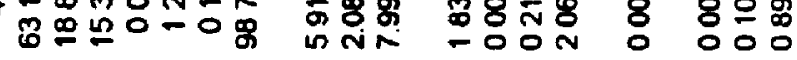

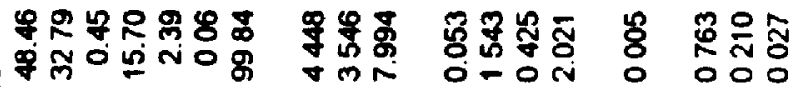

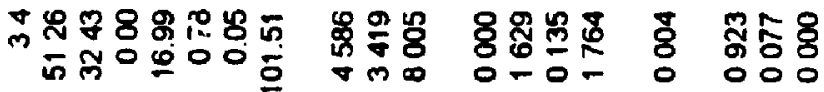

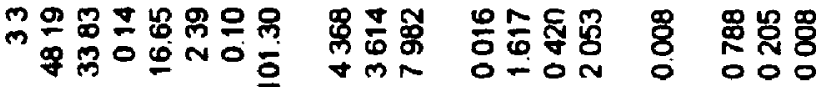

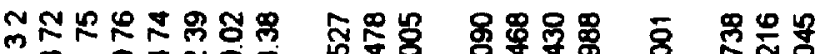

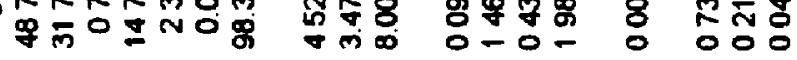

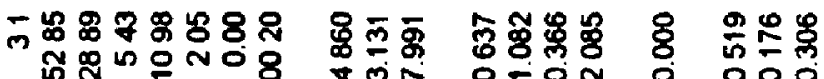

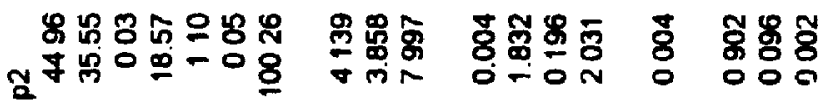

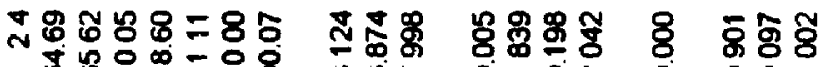

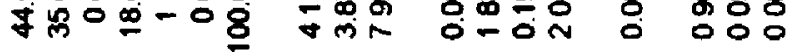

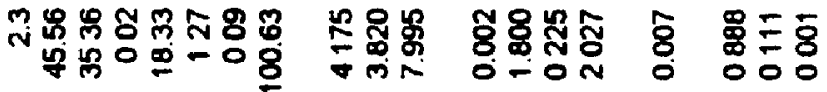

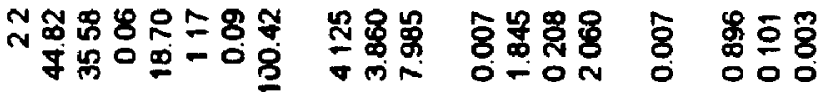

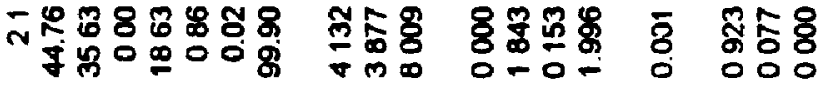

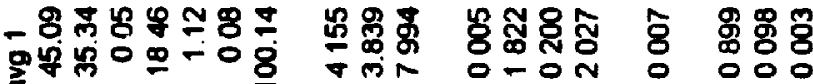

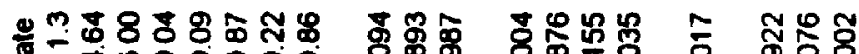

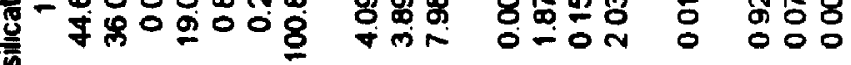

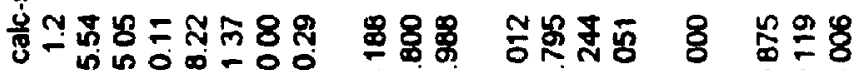

ำ

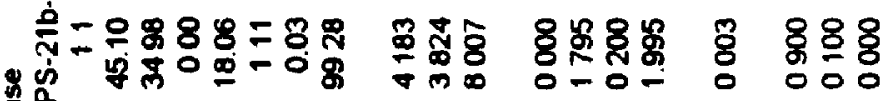

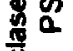

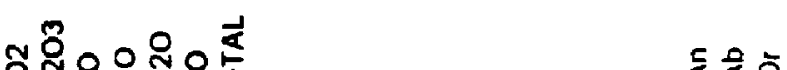

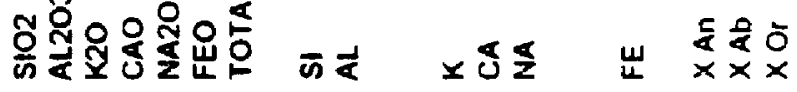




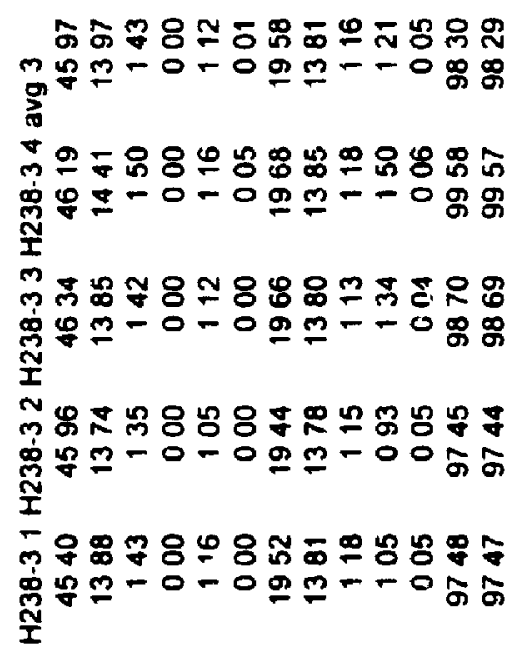

$\frac{2}{g} \frac{m}{6} 8$

$\infty-\infty$

\section{串解}

$0=\infty$

울용

$0 \div \infty$

స ํํำ

$\omega-\infty$

유용

$\omega-\infty$

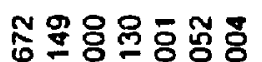
$00000 \%$

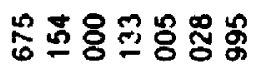

0000074

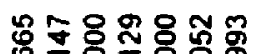

0000000

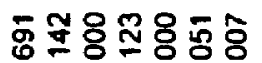
ำ000ง

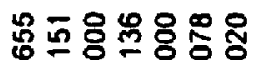
$00000 \%$ in

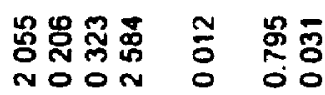

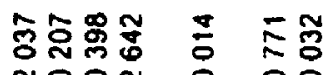

NOON OOO

홍응용영 웅

NOONODO

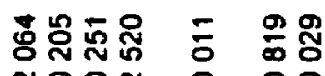

NOON OOO

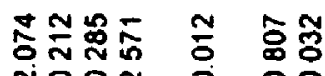
noon o o

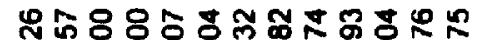

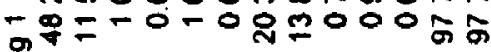
要

ำง

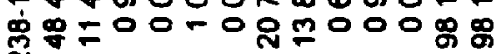
ํ.

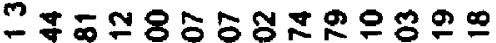

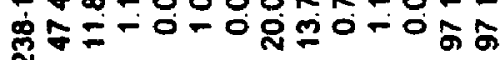
บ

은

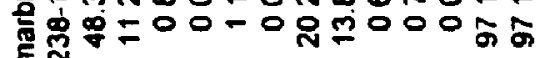
E

节

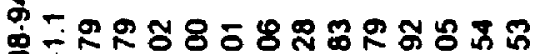

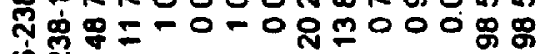
ம்

$\frac{\text { 뭉 }}{\frac{8}{2}}$
용 용

$0-\infty$

$5 \frac{9}{8} 8$

ธ용

₹思

$0-\infty$

도용

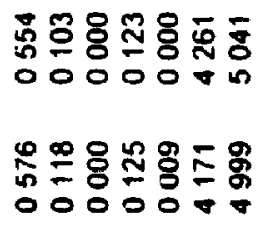

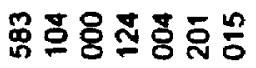

$00000 \%$

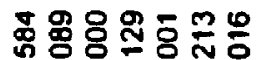

$00000 \%$ m

능용ㅎํ용 $000007 \mathrm{~m}$

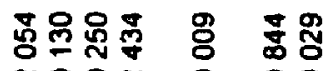

NOON O OO

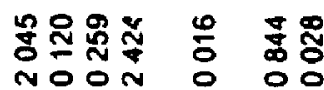

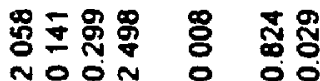

옹종용

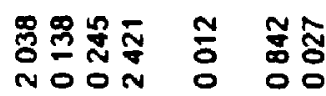




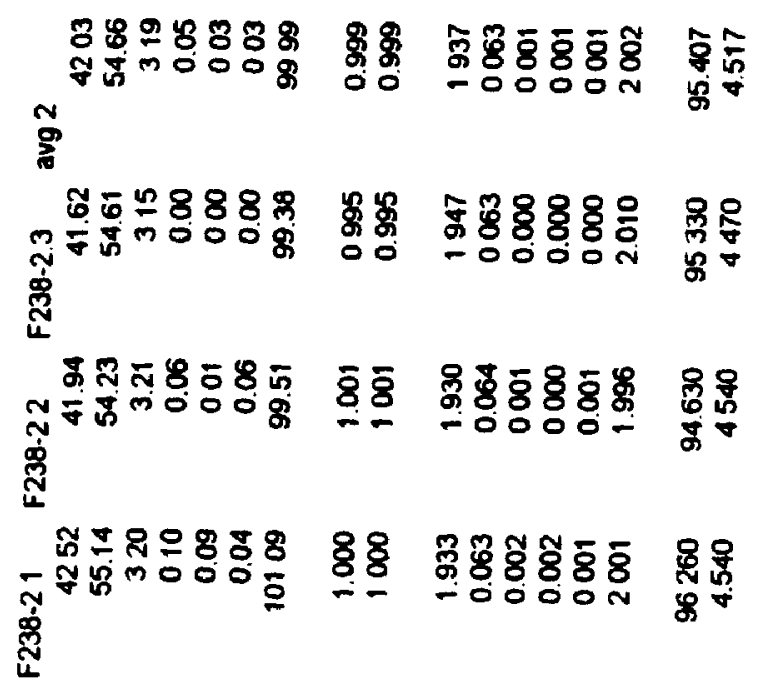

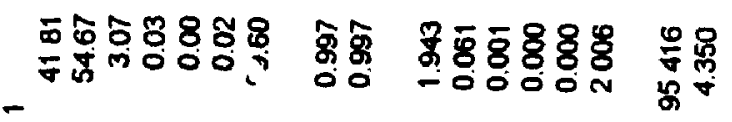
क्ष

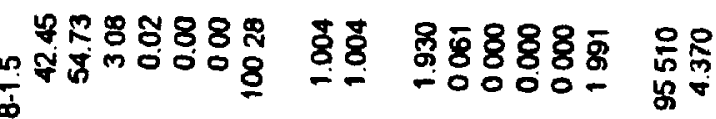

旁

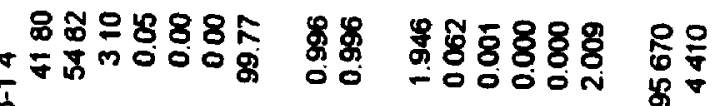

密

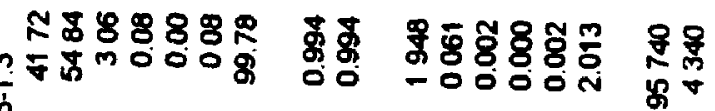

密

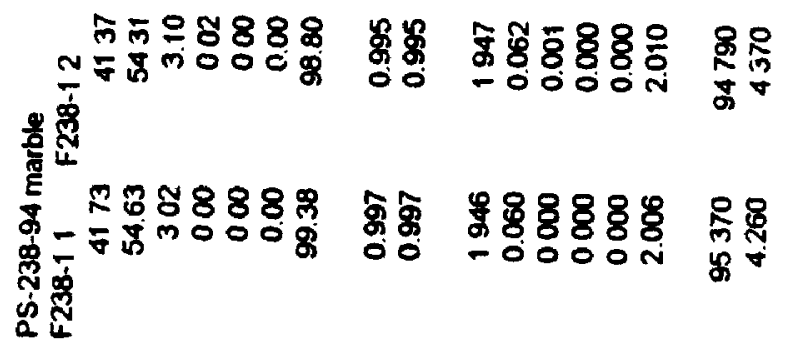

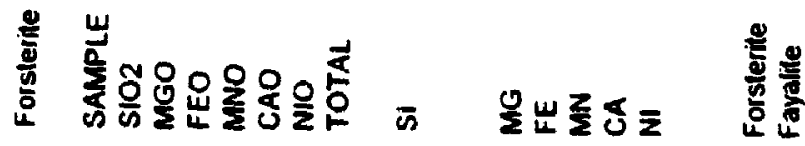




\section{Appendix H: Mineral assemblages of ultramafic schists}

\begin{tabular}{|c|c|c|c|c|c|c|c|}
\hline Sample & OL & $\mathrm{TR}$ & $\mathrm{CPX}$ & OPX & PHL & SER & CHR LOCATION \\
\hline PS-7B-93 & * & * & * & & * & * & RINDA RIDGE (DOMAIN IA) \\
\hline PS-7C-93 & * & $*$ & & $*$ & * & & RINDA RIDGE (DOMAIN IA) \\
\hline PS-7D-93 & * & * & & & * & * & RINDA RIDGE (DOMAIN IA) \\
\hline PS- $30-93$ & * & * & $*$ & $*$ & $*$ & * & * NORTH OF SLOCAN (HWY 6) \\
\hline PS-146-93 & * & * & * & * & & & RINDA RIDUE (DOMAIN IA) \\
\hline
\end{tabular}



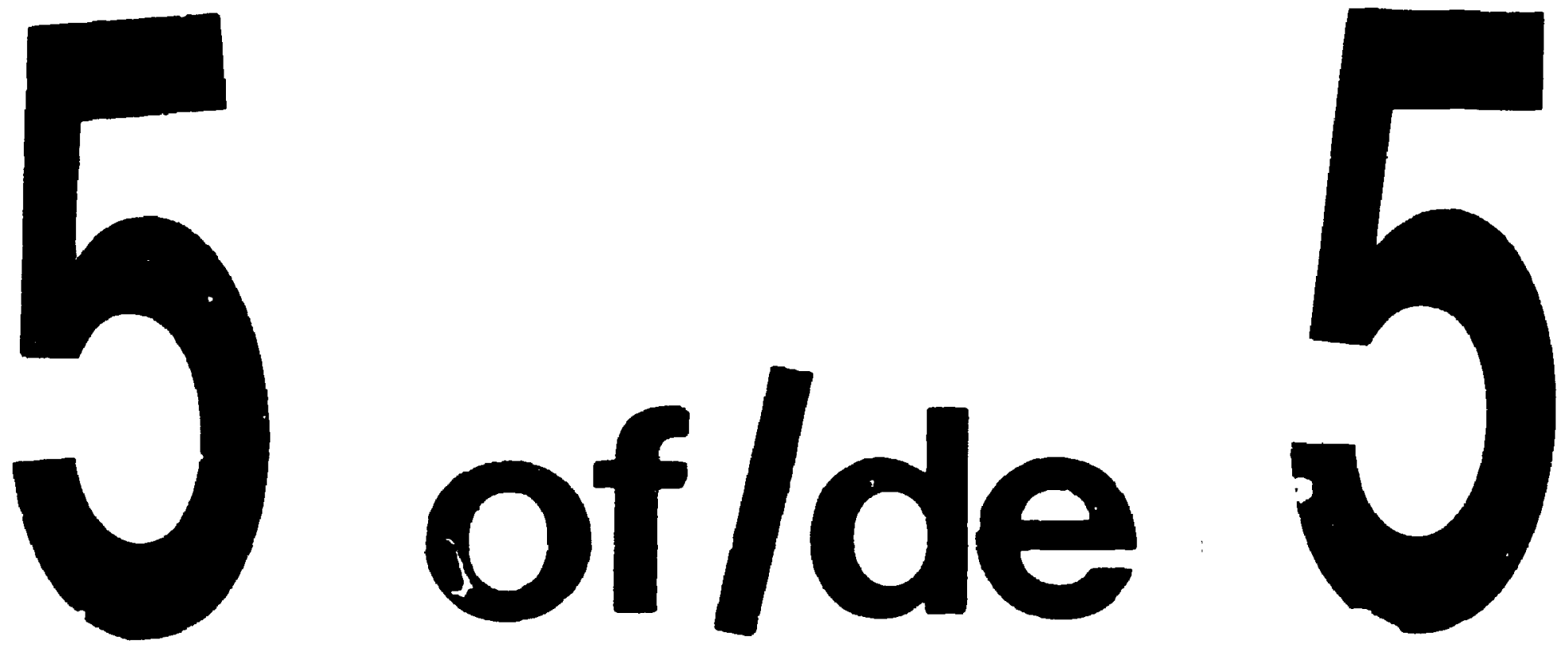

PM-1 3', " 4 " PHOTOGRAPHIC MICROCOPY TARGET NaS 1010a ANSI/ISO \#2 EOUIVALENT

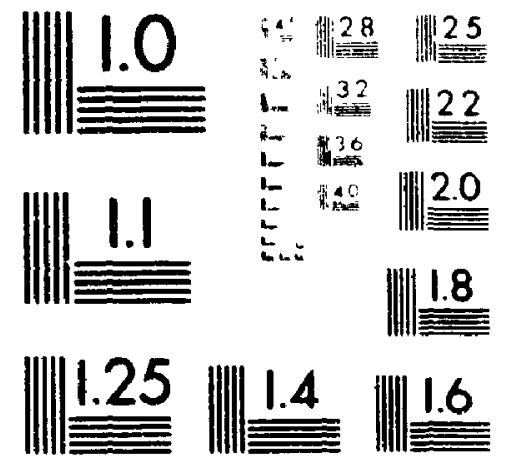

PRECISION ${ }^{\text {SM }}$ RESOLUTION TARGETS 


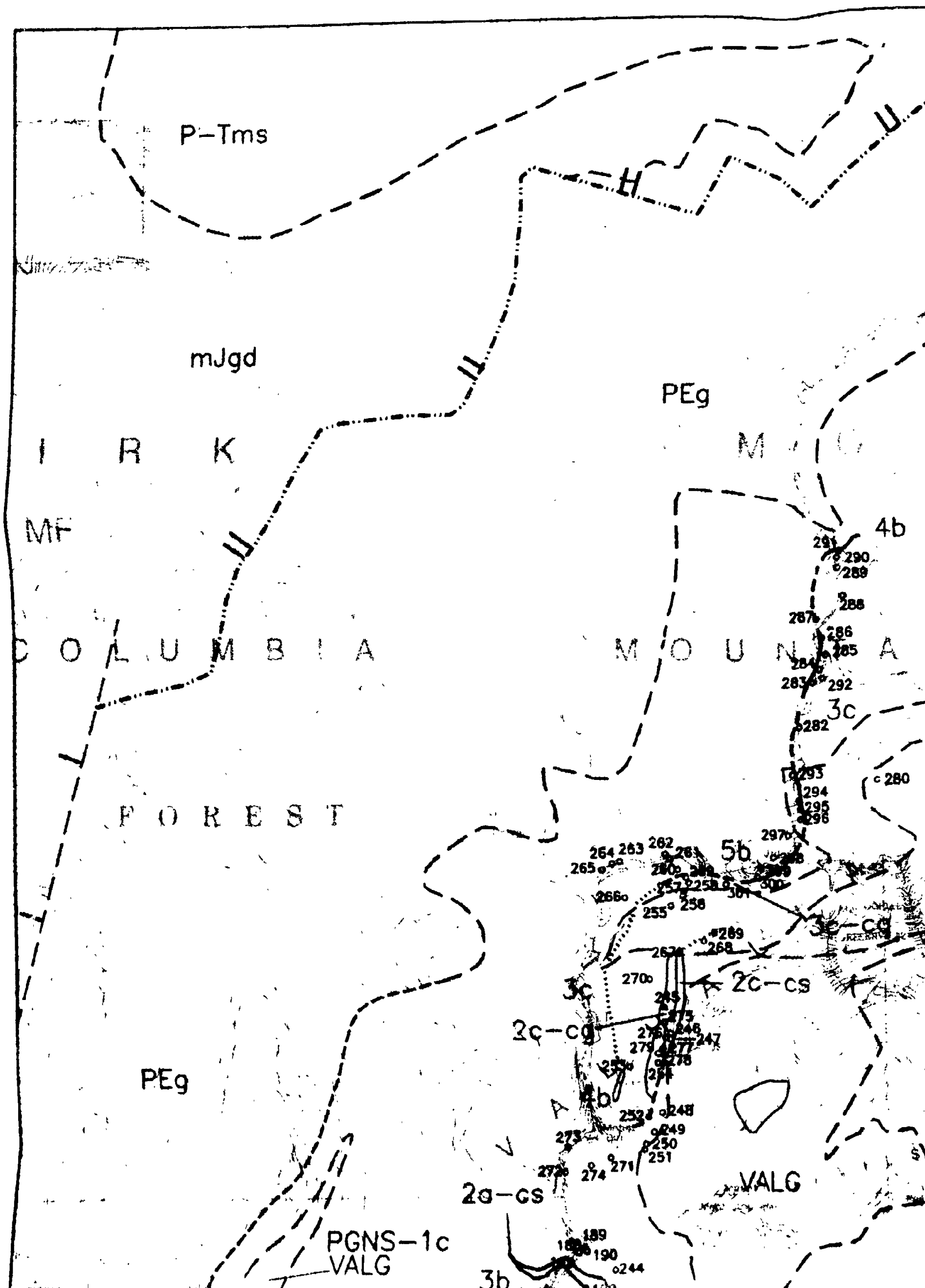




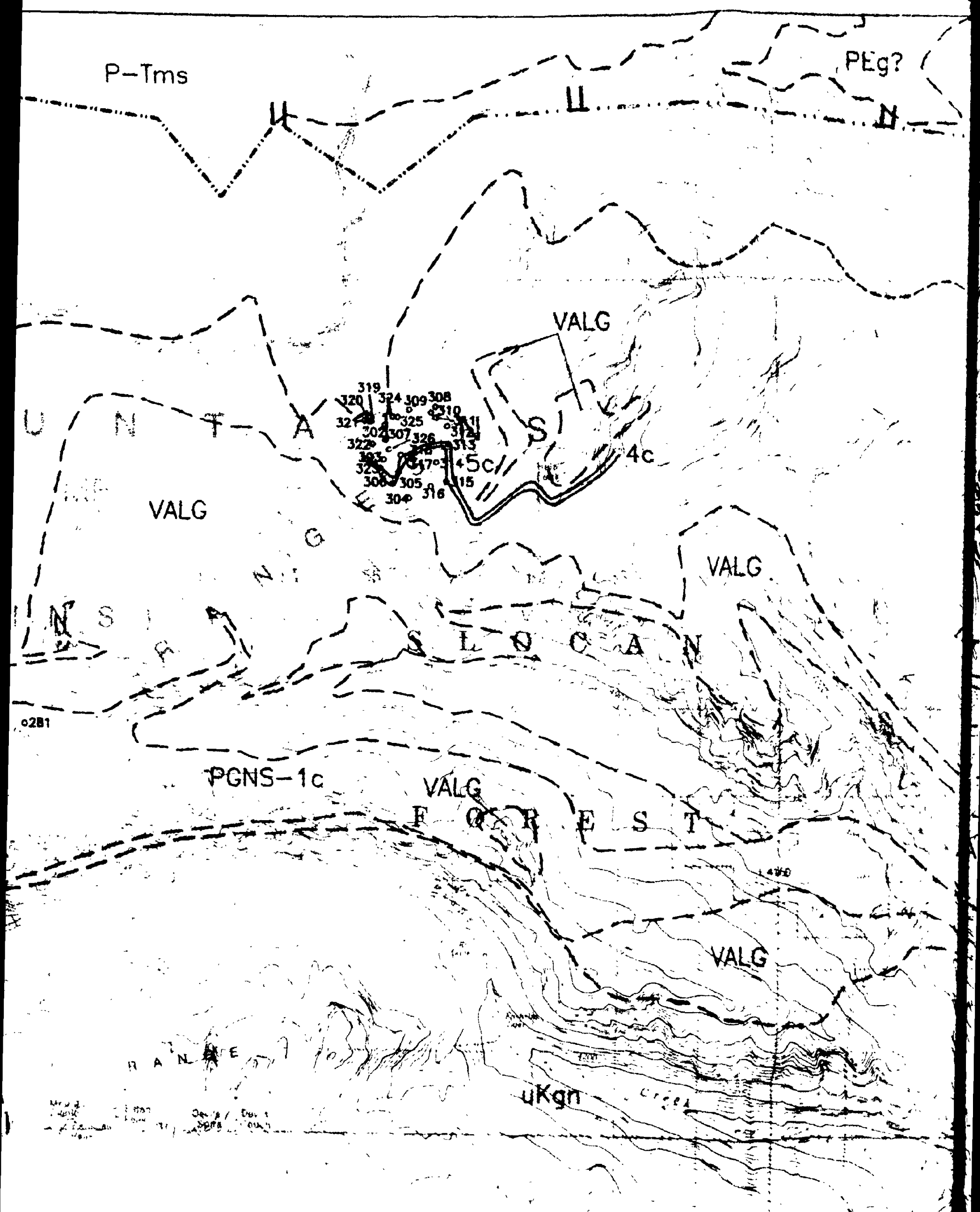




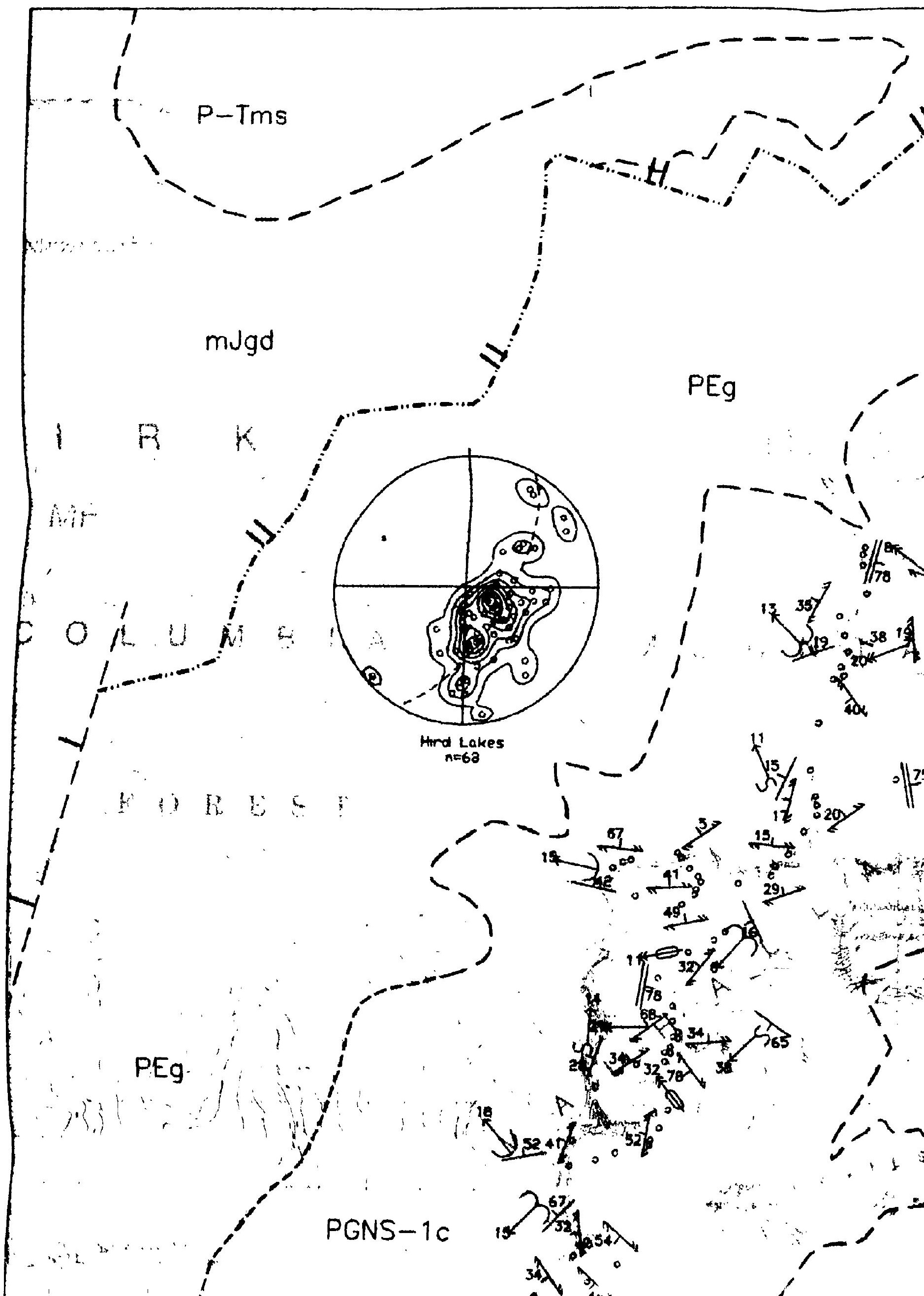




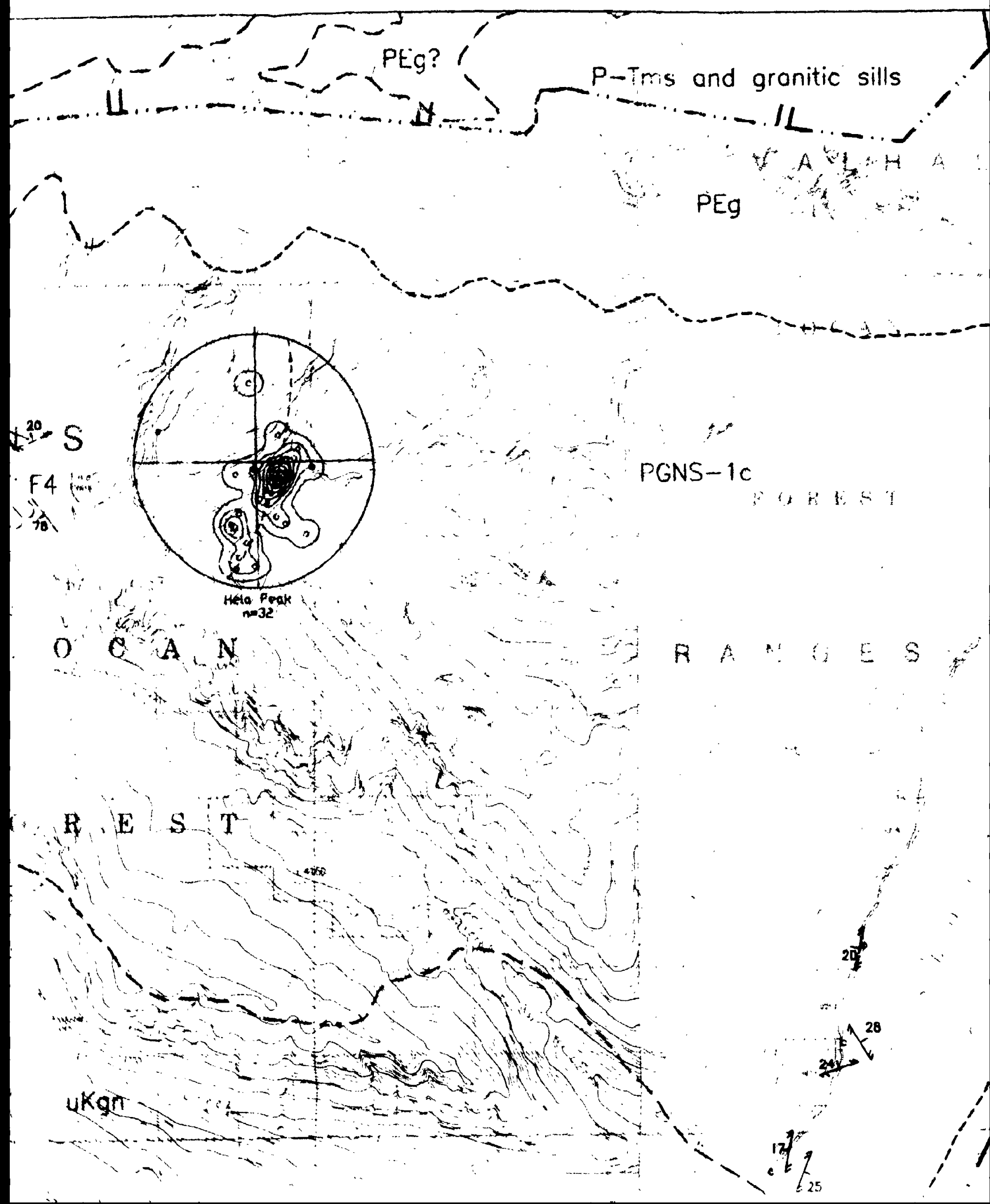




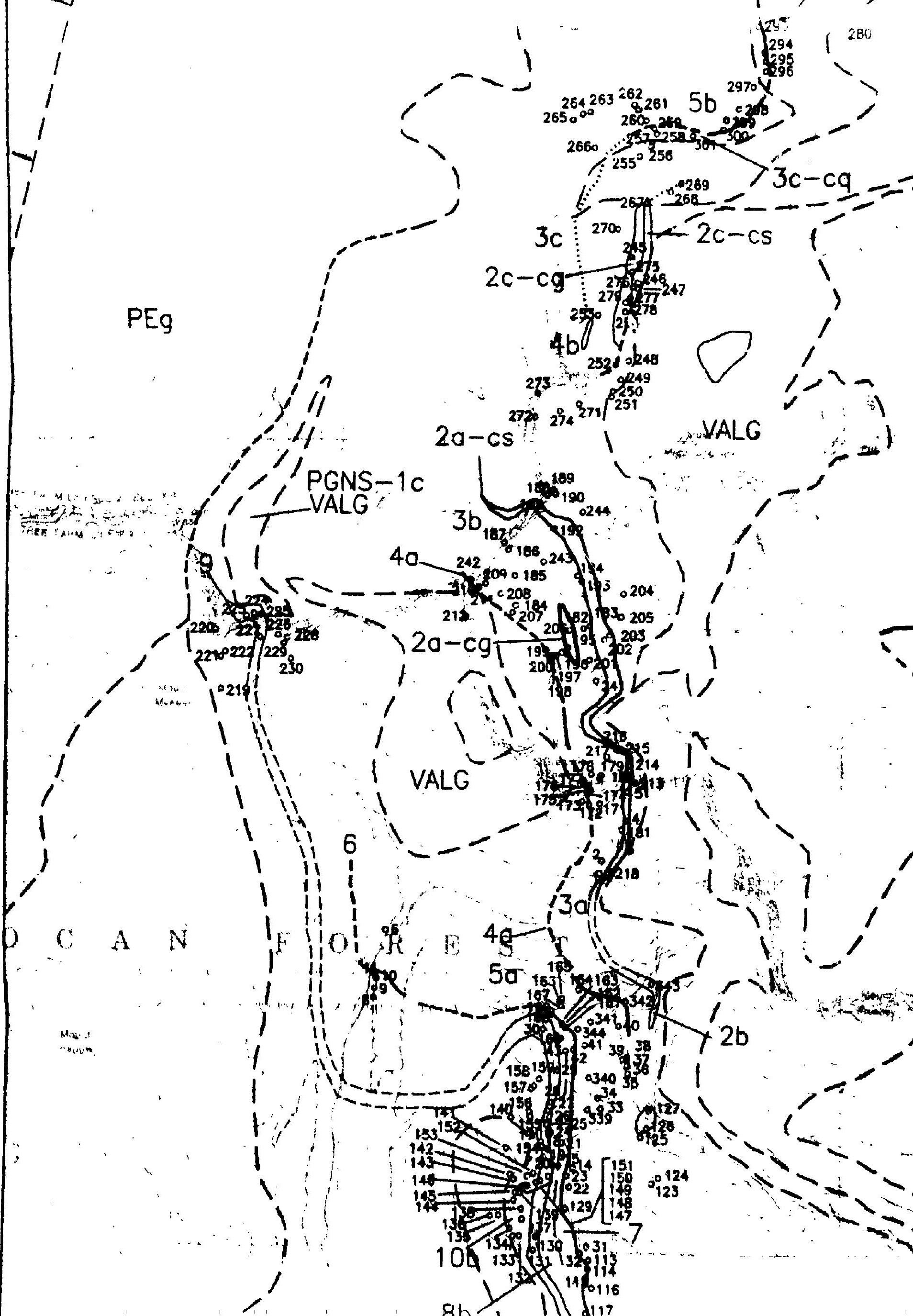




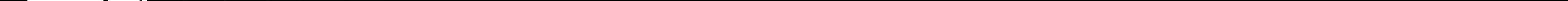




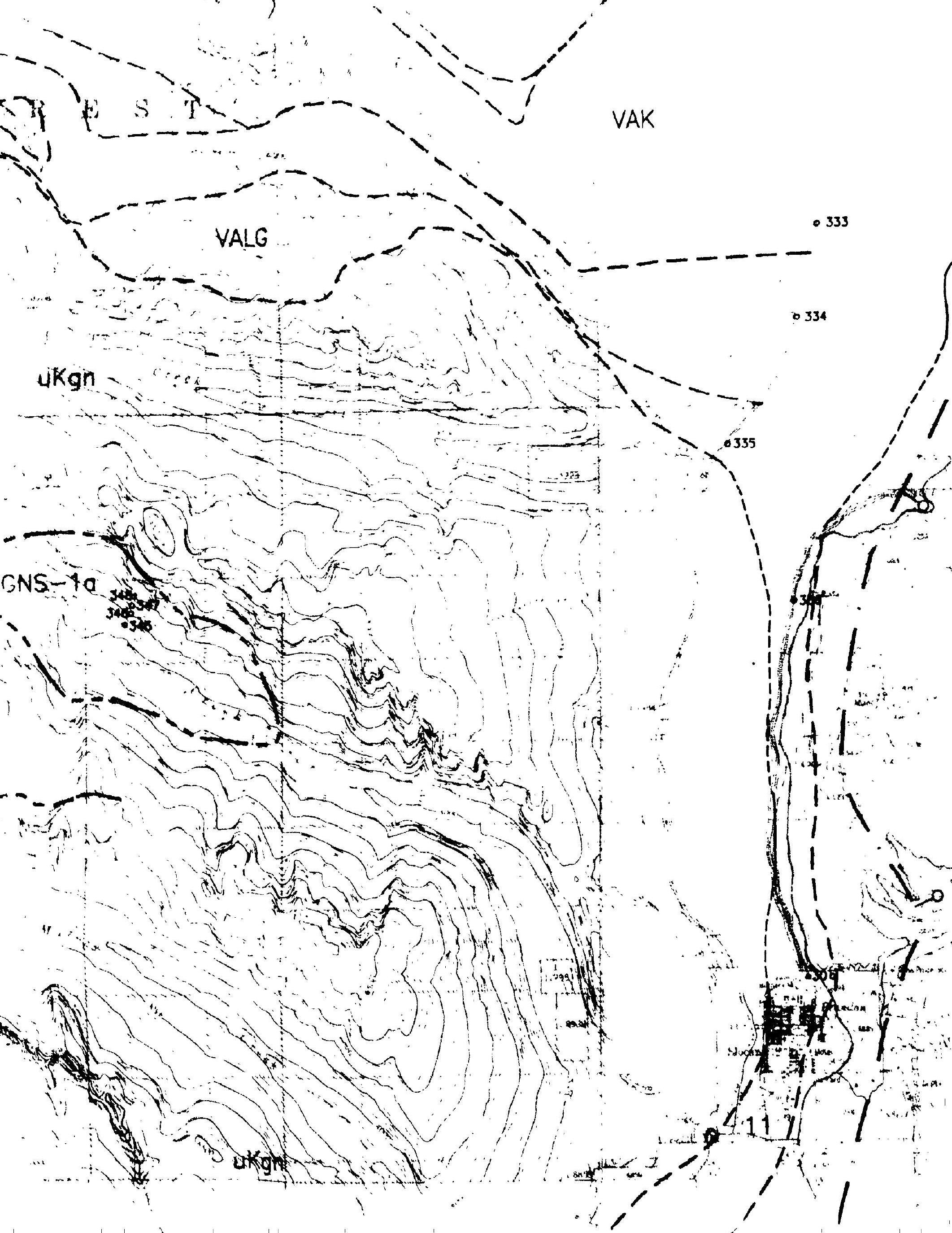





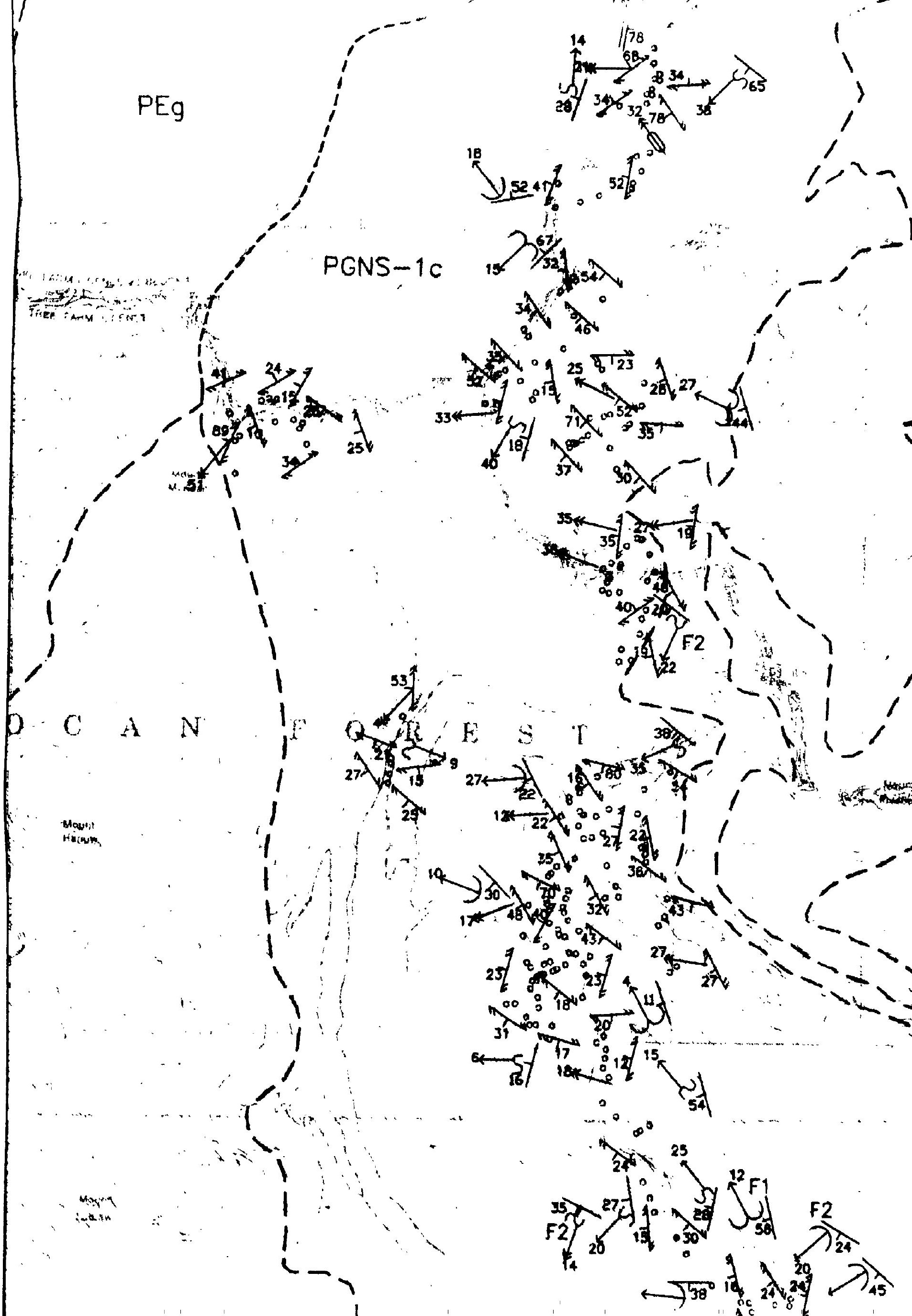




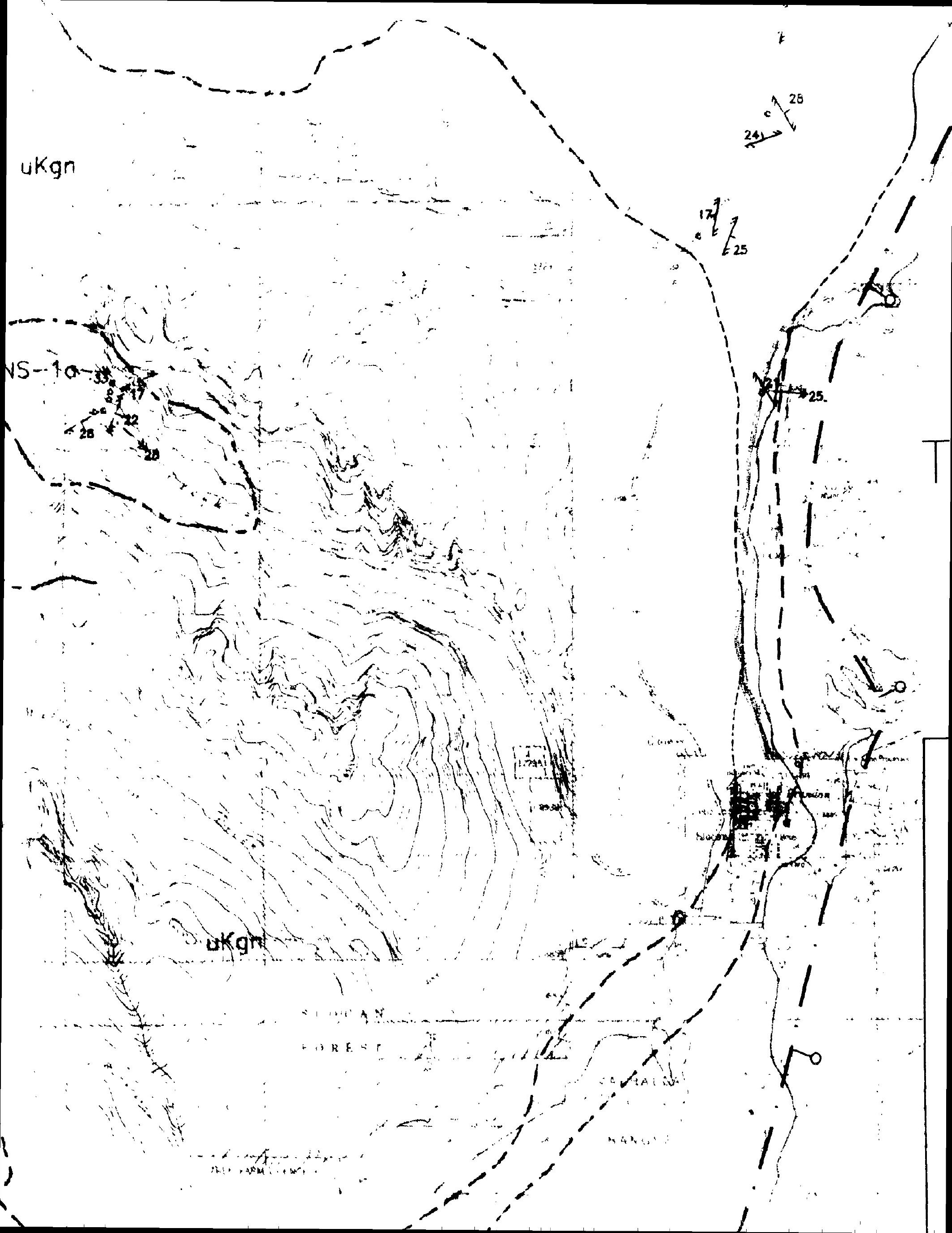




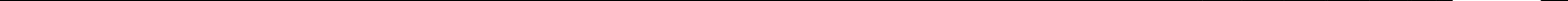




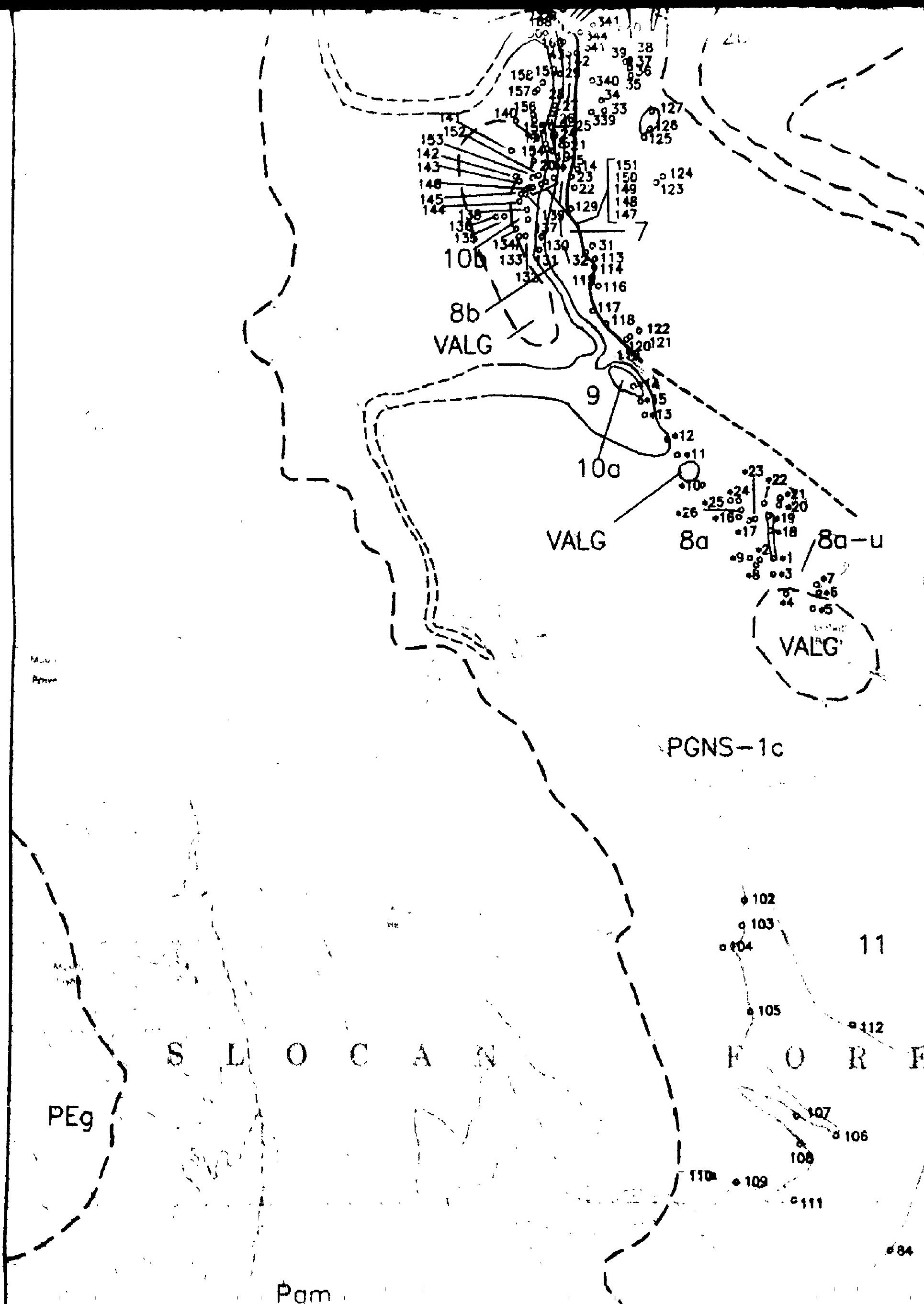


Eocene - Poleocene

PEg Lodybird granite - leucocratic biotite quartz monzonite to granite

Poleocene

Fgm Airy quartz monzonite - $K$-feldspar megacrystic biotite $+/-$ rornblende quartz monzonite

\section{Cretoceous}

uKgn Mulvey granodiorite - mielonocratic biotite-hornblende ougen gronodioritic gneiss with leucratic veins

Middle Jurassic

mugd Nelson batholith and sotellites

Paleozoic and Triassic

P-Tmis Nemo Lokes belt: polydeformed semipelite and pelite, calcoreous schist, amphibolite and ultramafic rocks

Neoproterozoic - Paleozoic?

PGNS-1c Vathulls assemblage (paragneiss)

11 Undivided rocks: pelitic schist, migmatitic schist, amphibolite gneiss, misrble $\left(m_{1}\right)$, colc-silicate greiss, quartzite and ultramufic rocks

10a Quartzite, psommite

10b Semipelitic schist, psammite, argillaceous quartzite

9 Rindo marble

80 Rindo ridge composite unit: marble, colc-silicote gneiss, psommite

semipelitic schist to pelitic schist, amphibolite gneiss. ultramafic rocks $(8 a-u)$

$8 \mathrm{~b}$ Marble, calc-silicate gneiss, psammite to pelitic schist

7 Semipelitic to pelitic schist, psommite

6 Bannock Burn marble

5a Hoder Creek composite unit: psommite, pelitic schist, argillaceous quartzite, marble, calc-silicate gneiss, amphibolite gneiss

5b Semipelitic to pelitic schist, psammite

sc Semipelitic schist, psomimite

4a Marble, calc-silicate gneiss

$4 b$ Demers marble

4c Marble, siliceous marble

3o Quortzite

3b Semipelitic to pelitic schist. migmatitic schist, 


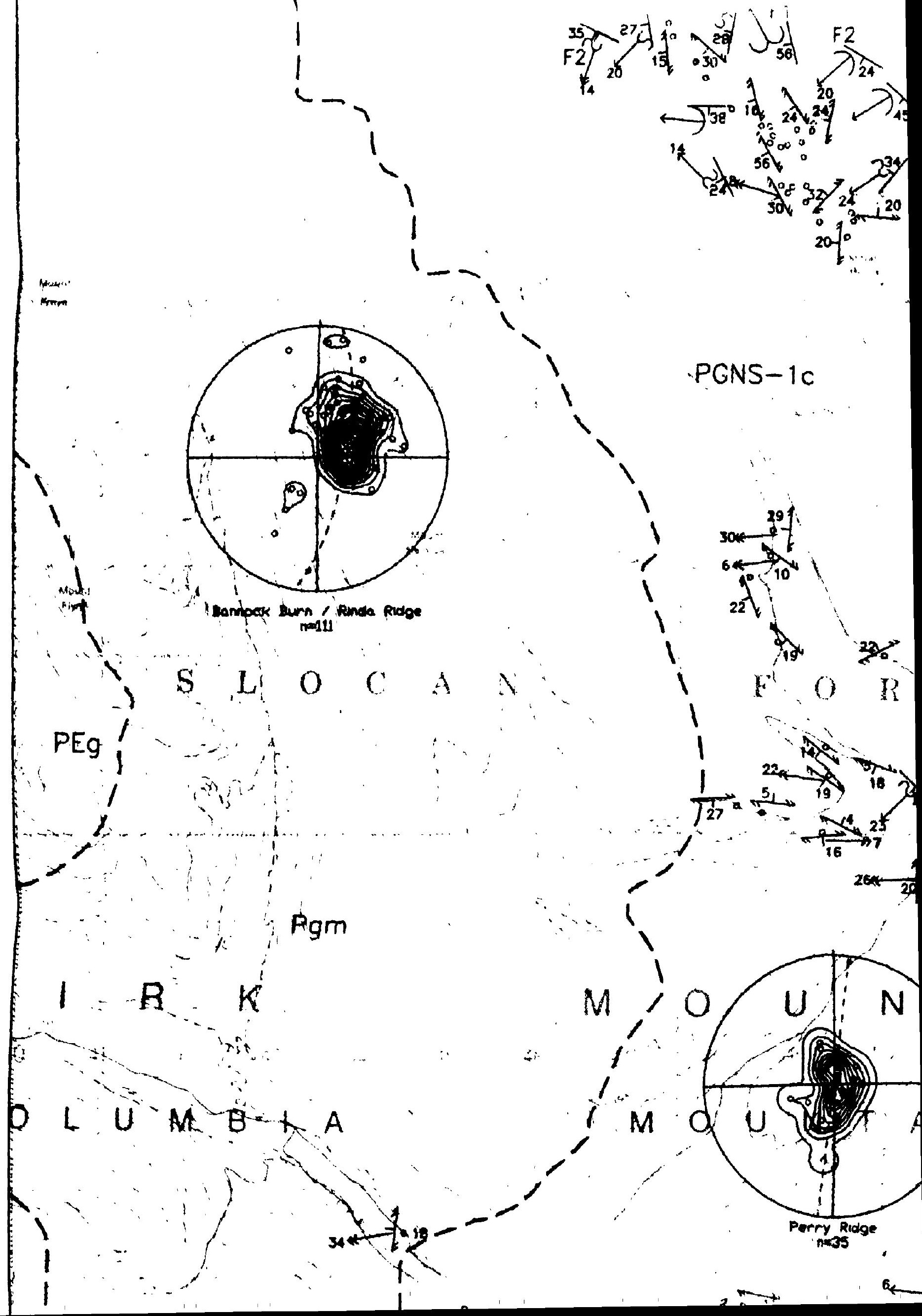



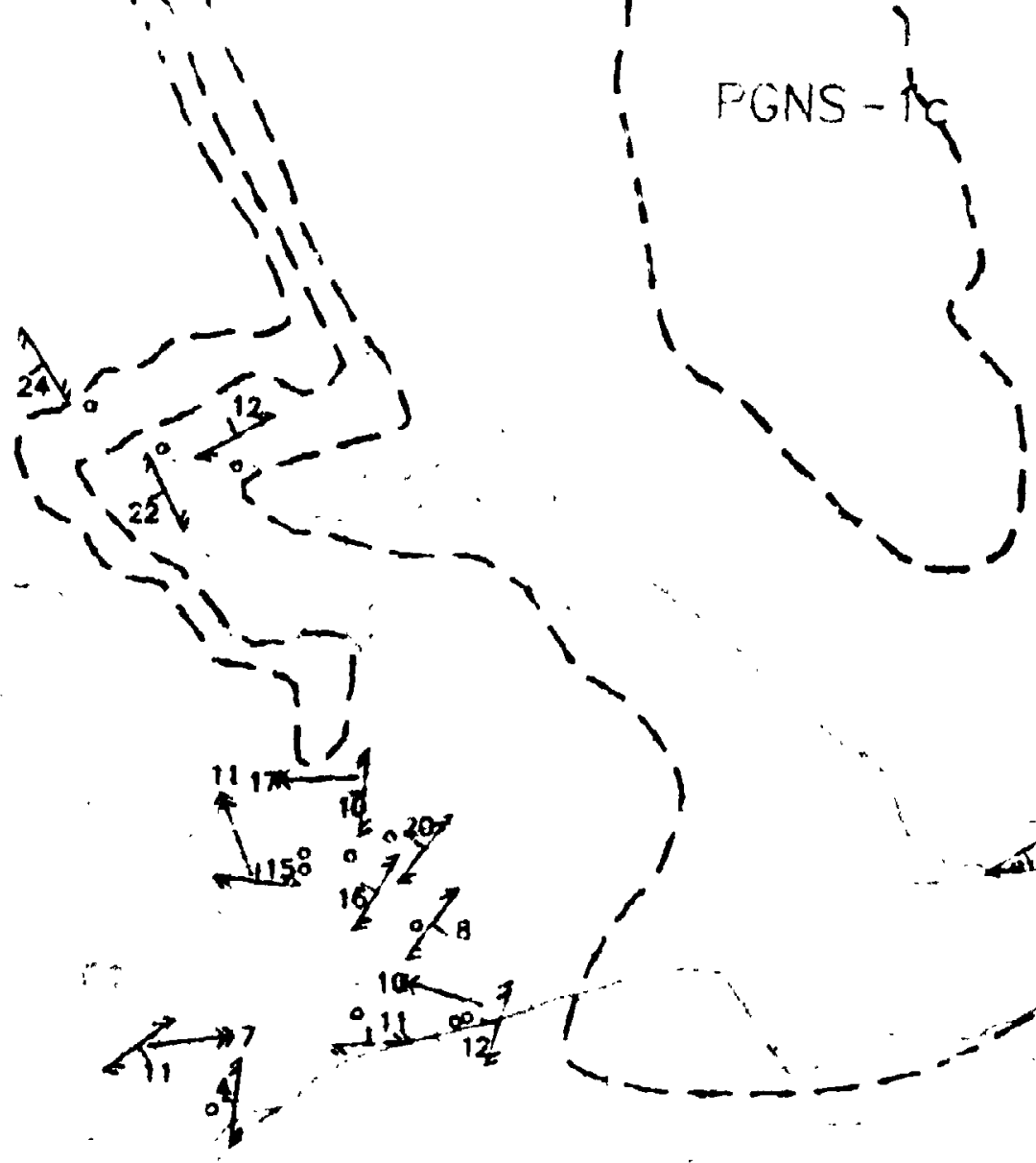

,

$\sqrt{5}$ 


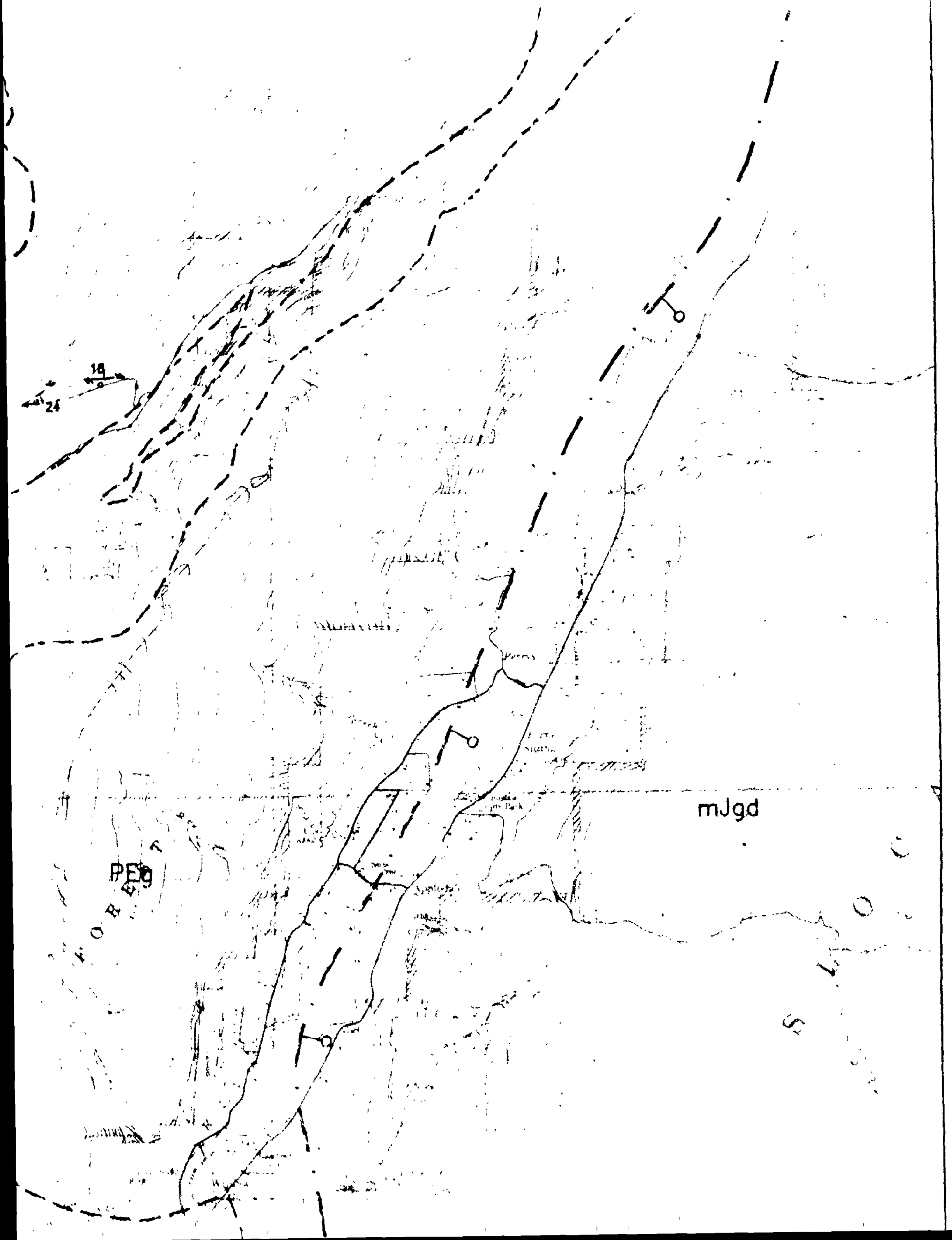


Middle Jurassic

mugd Nelsor batholith and satellites

Poleozoic orid Triossic

P-Trms Nems Lakes belt: polydeformed semipelite and pelite. calcareous schist, amphibolite and ultramafic rocks

Neoproternzoic - Poleozoic?

PGNS-1c Valtallo assembloge (paragneiss)

PGNS-1b (age uncertain)

PGNS-10 (age uncertain)
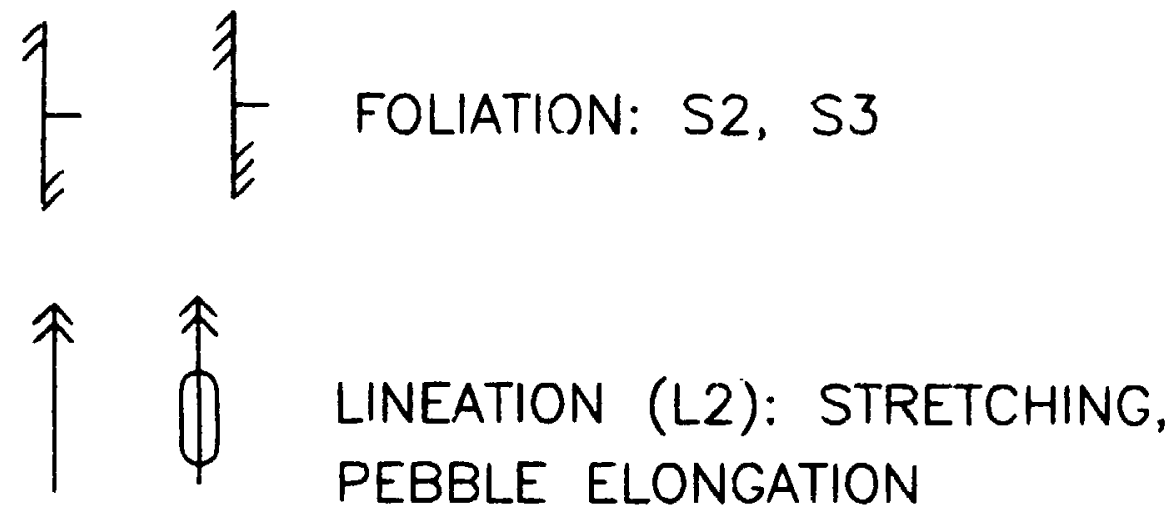

AXIAL PLANE

$\uparrow \uparrow \uparrow \uparrow \uparrow$

FOLD AXIS: S, U, W, Z

\section{ALL FOLDS ARE THIRD GENERATION EXCEPT WHERE NOTED}

$\|$

MAFIC DYKE

Stereograms represent poles to $S 2$ foliation planes for oreas noted. 


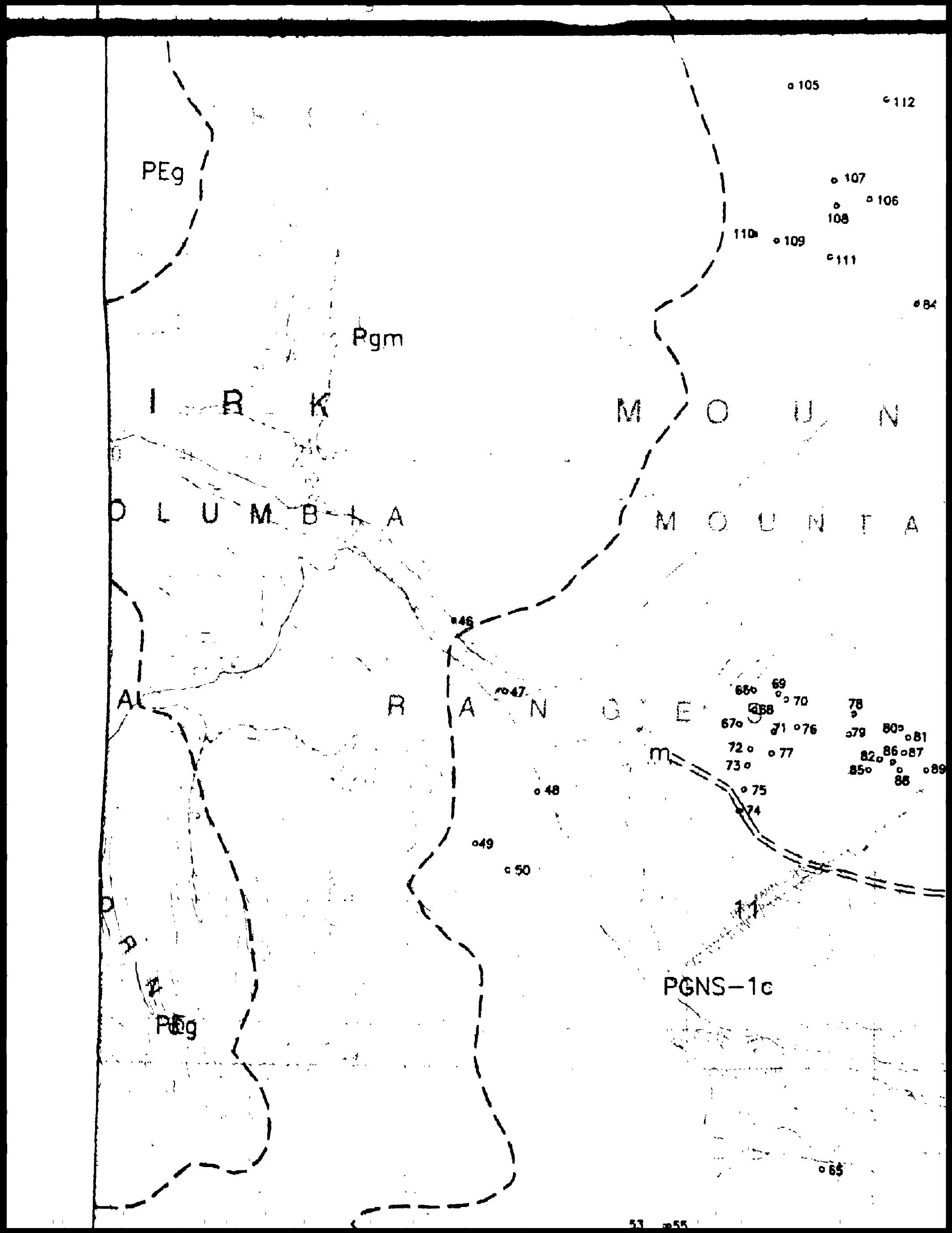




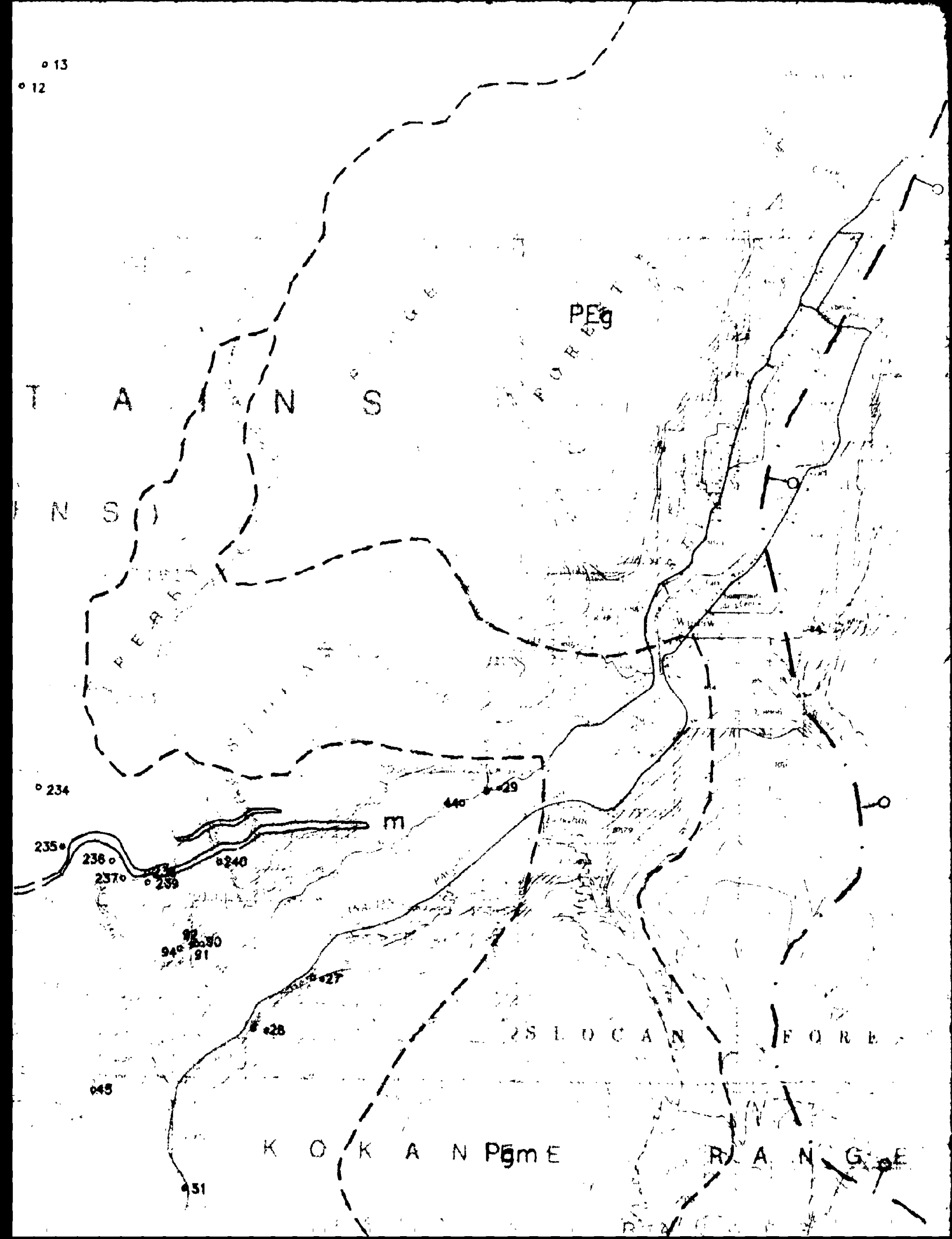




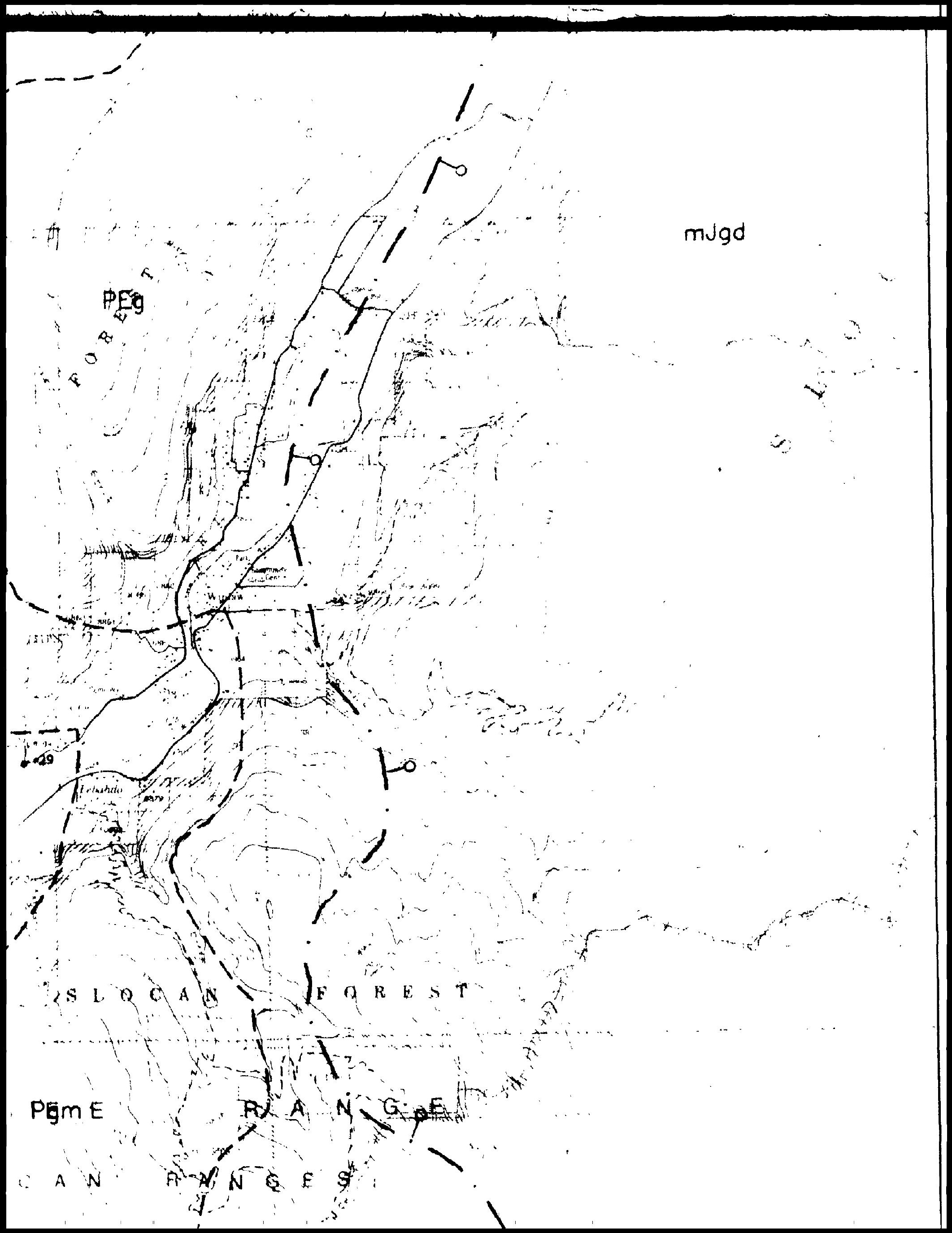


50 Hoder Creek composite unit: psommite, pelitic schist, orgillaceous quartzite, marble, cals-silicate gneiss, omphibolite gneiss

$5 b$ Semipelitic to pelitic schist, psommite

5c Semipelitic schist. psomimite

4a Marble, calc-silicate gneiss

4b Demiers miarble

$4 c$ Marble. siliceous marble

3a Quortzite

3b Semipelitic to pelitic :chist, migmatitic schist, orgillaceous quortzite

3c Quartzo-feldspothic gneiss, pelitic schist, colcareous quortzite $(3 c-c q)$

2a Hird Lokes calc-silicate (2a-cs) - quartzite cobble metaconglomerate $(2 a-c g)$

2b Marble

1 Quartzo - feldspathic gneiss

VAK Volhalla Assernblage intrude by $K$-feldspor megocrystic

VALC Vuit'alla Assembloge comprising $>75 \%$ leuccgranite

Poragneiss (age uncertain)

PGNS- 16

PGNS-1a<smiles>IC1CCCCC1</smiles>

GEOLOGICAL CONTACT: MAPPED. ASSUMED

SLOCAN LAKE FAULT: MAPPED, ASSUMMED

Lo

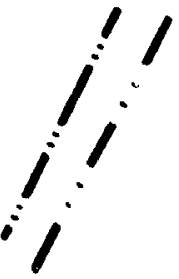

VALKYR SHEAR ZONE: MAPPED, ASSUMED 


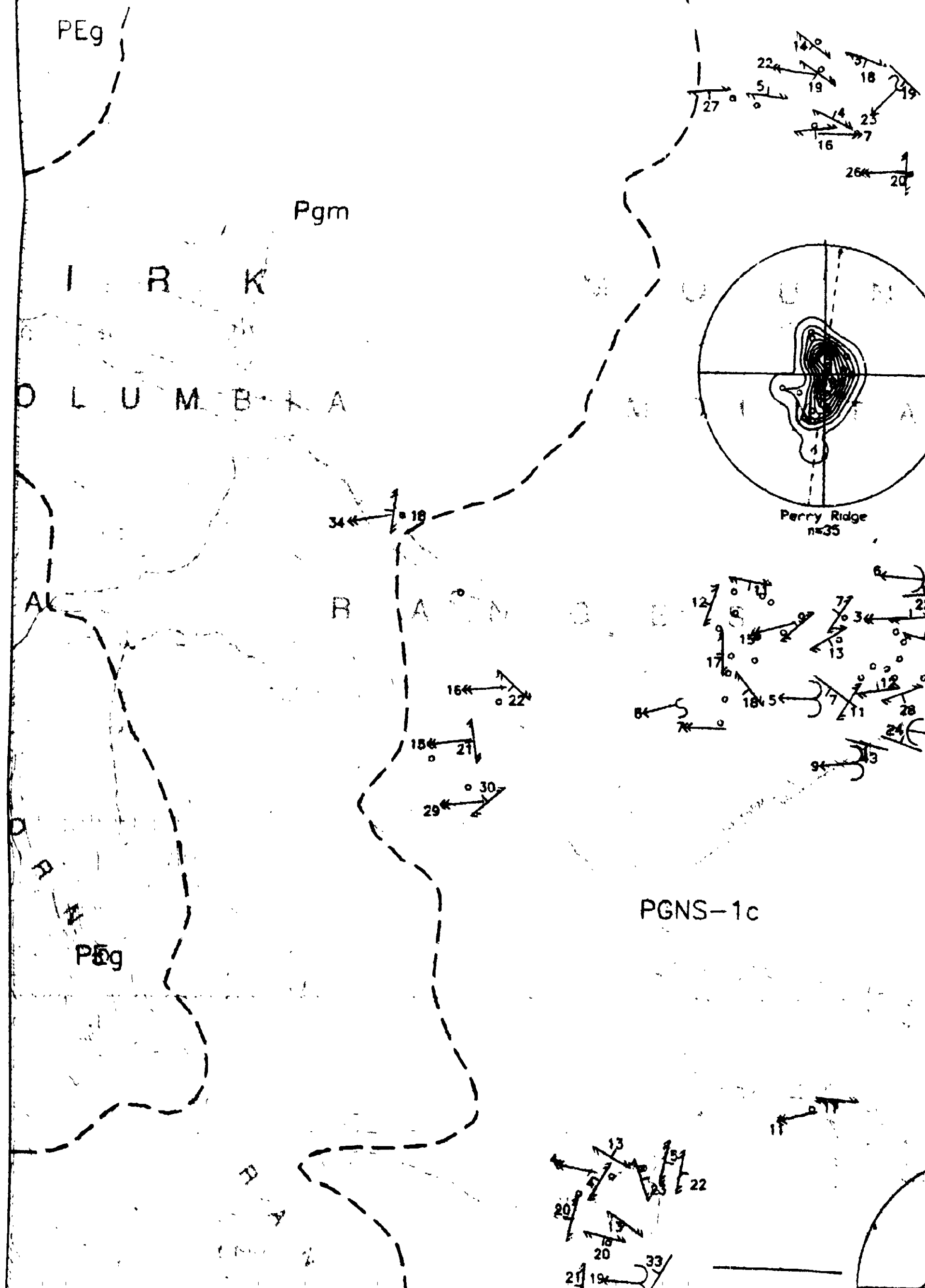




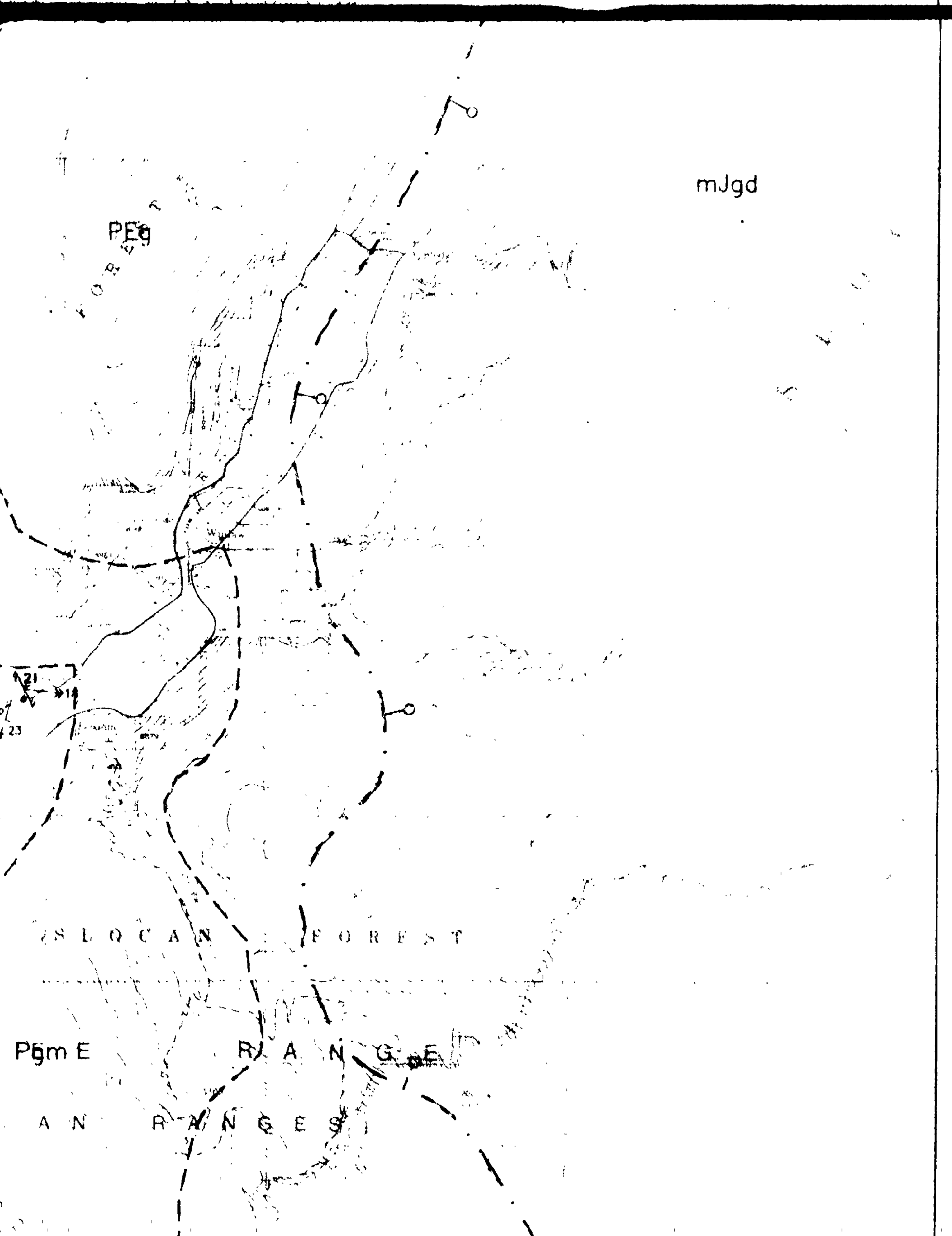




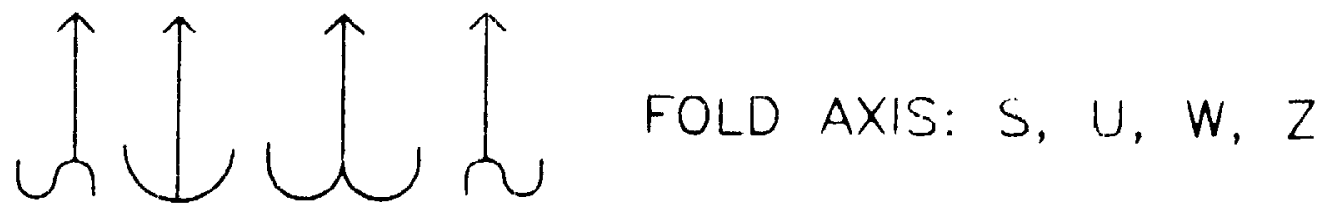

ALL FOLDS ARE THIRD GENERATION EXCEPT WHERE NOTED

$\sharp$

MAFIC DYKE

Stereograms represent poles to $\mathrm{S} 2$ foliation planes for areas noted.

CONTACT: MAPPED, ASSUMED

SLICAN LAKE FAULT: MAPPED, ASSUMED

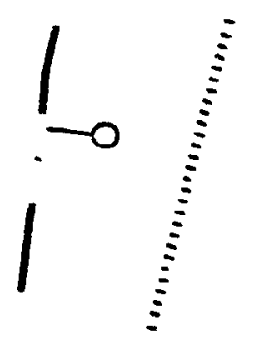

VALKYR SHEAR ZUNE: MAPPED, ASSUMED 


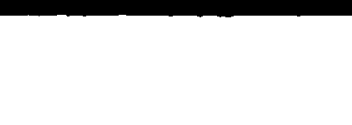

045

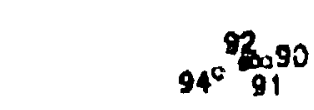

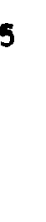

.
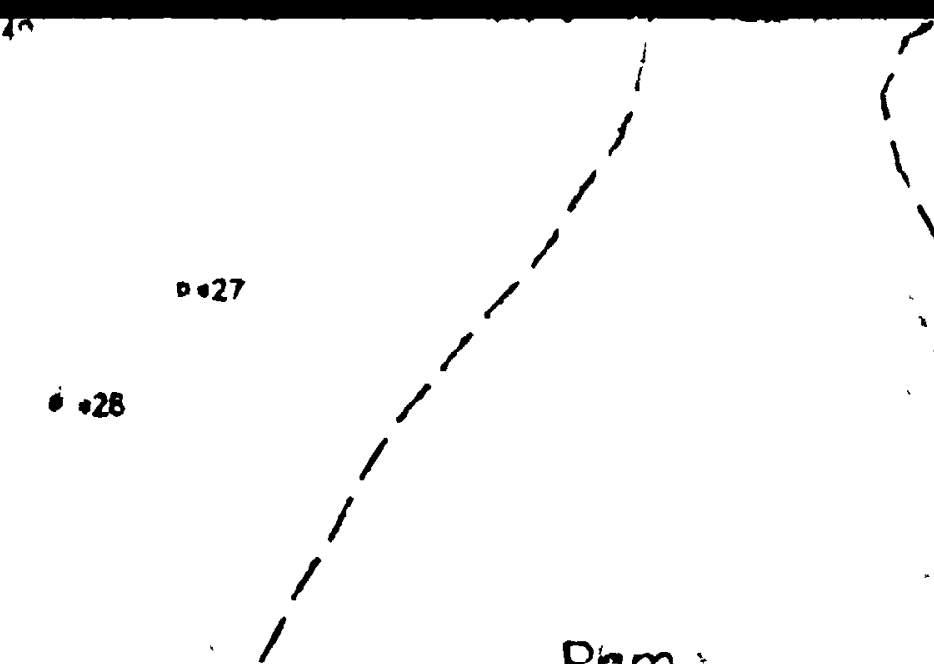

as

PGNS-1C

Pgm

Pgm:
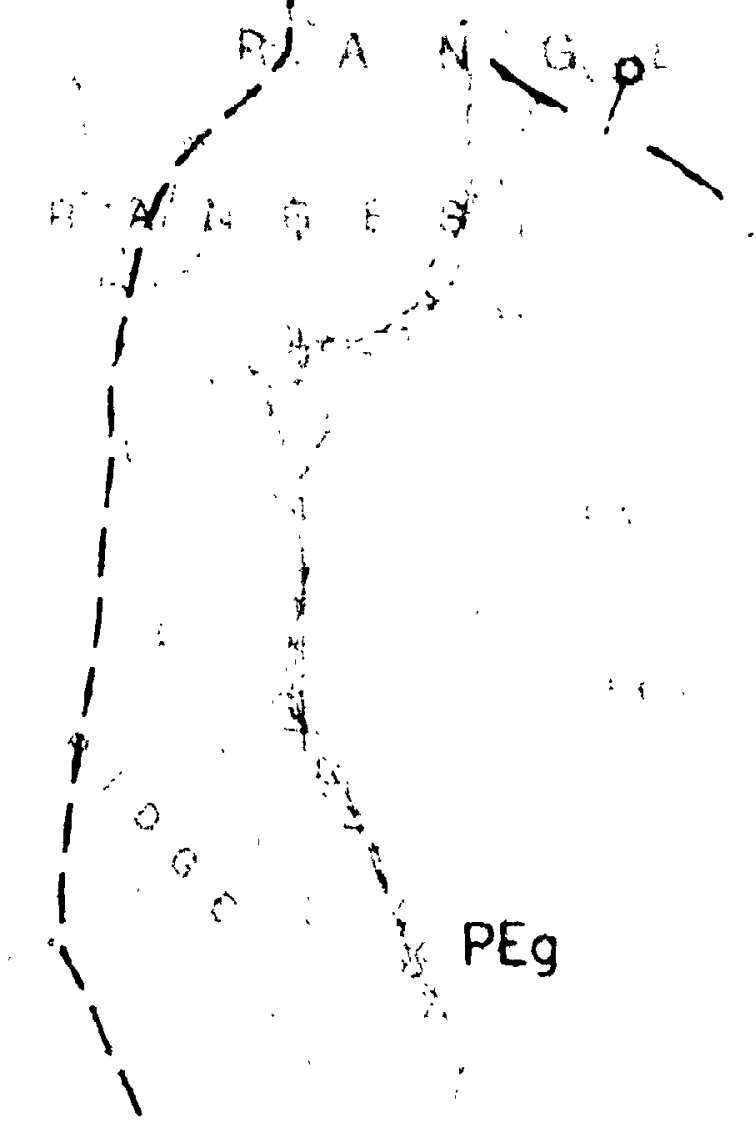


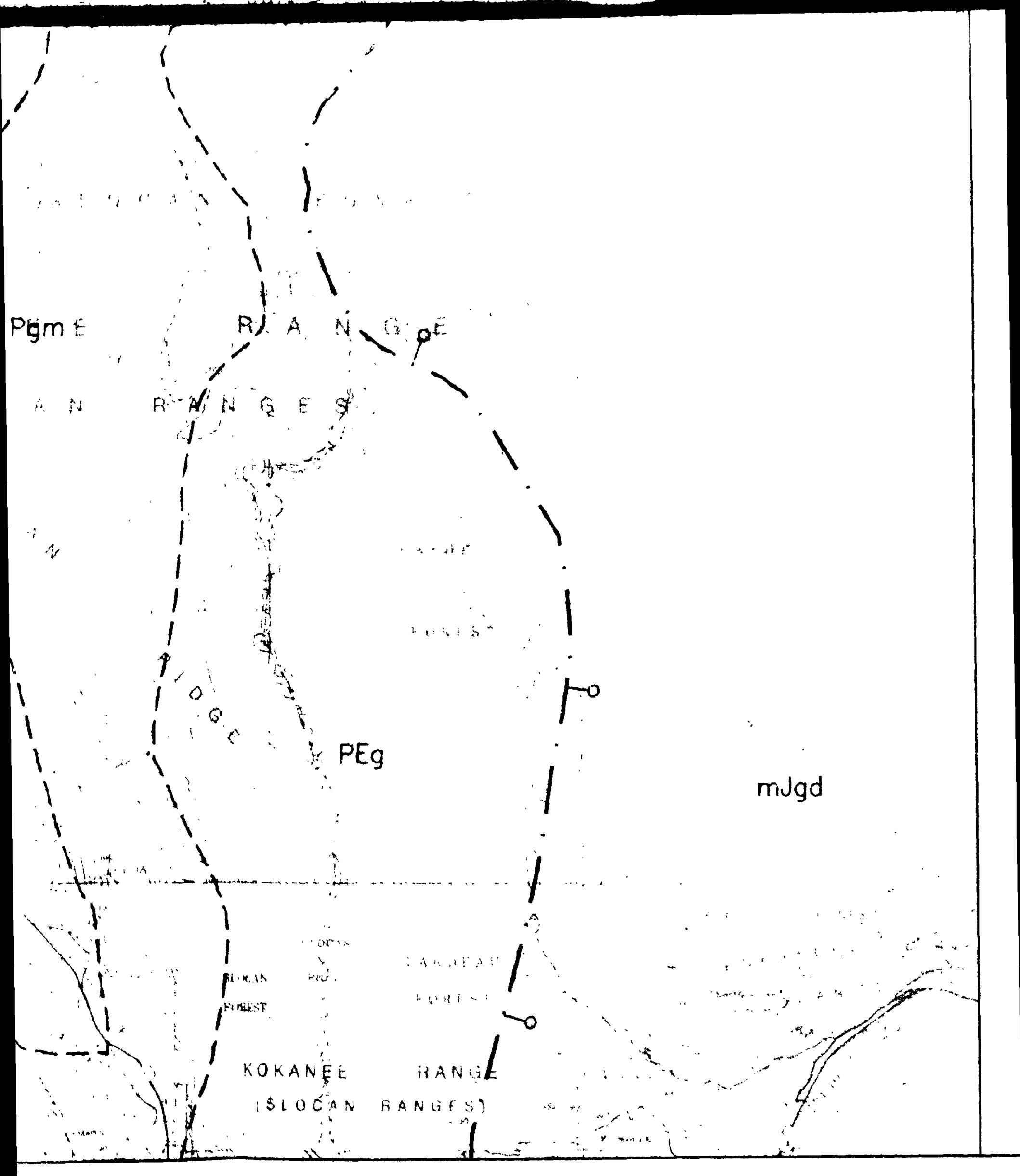




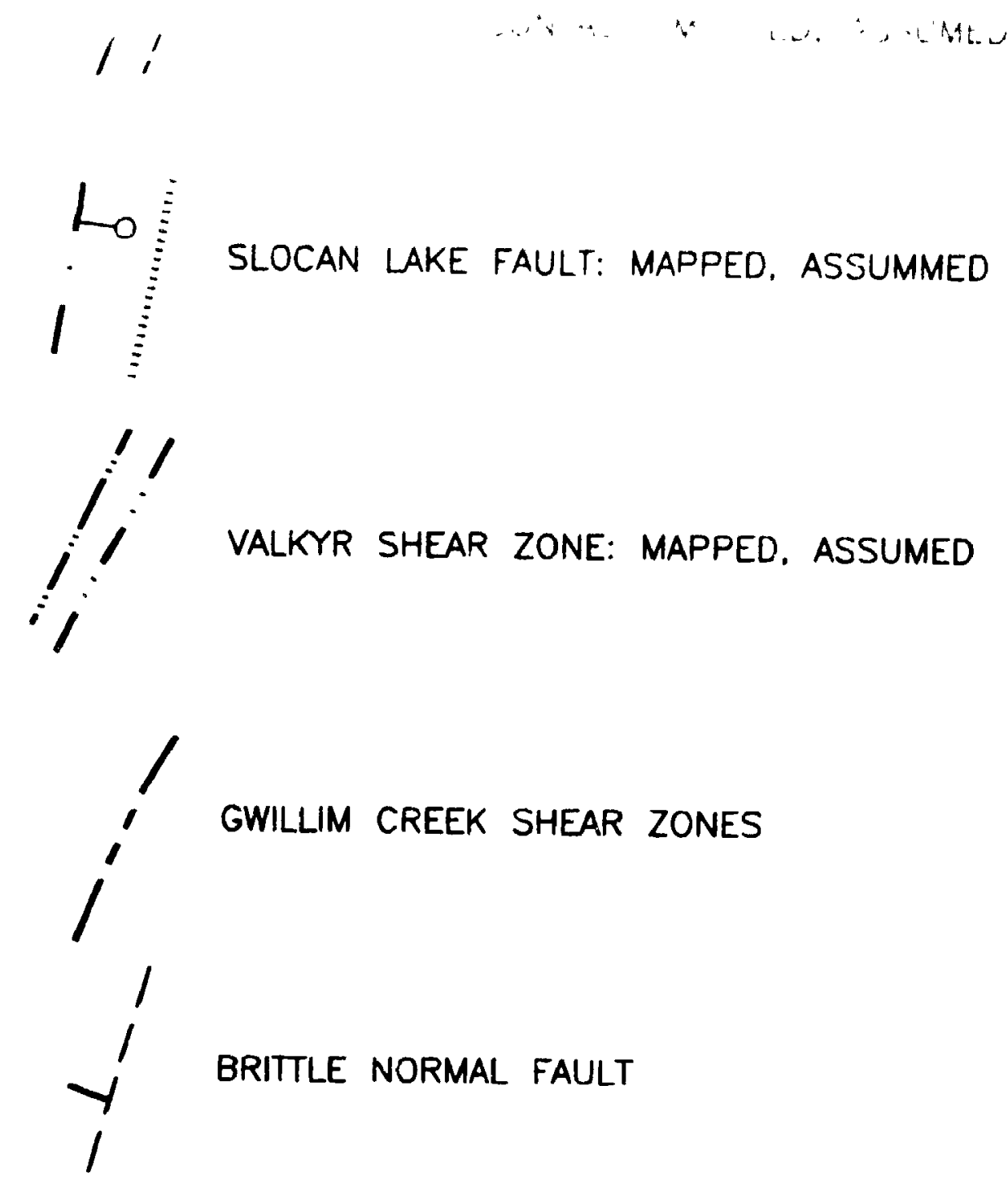

60 Station number year 1994

-29 Station number yecr 1993

Mop is modified from Reesor (1965). Porrish (1981, 1984) and Corr (1986).

SCALE 1:50000

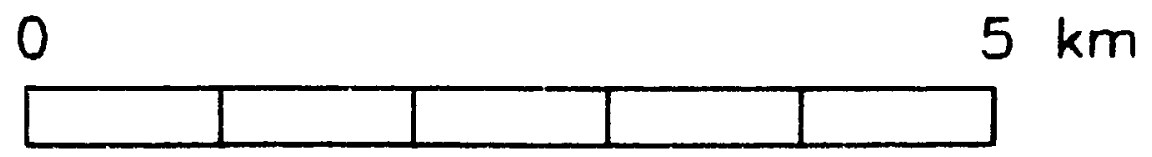




\section{$r r$}

Ec S $\quad T_{1}-21$

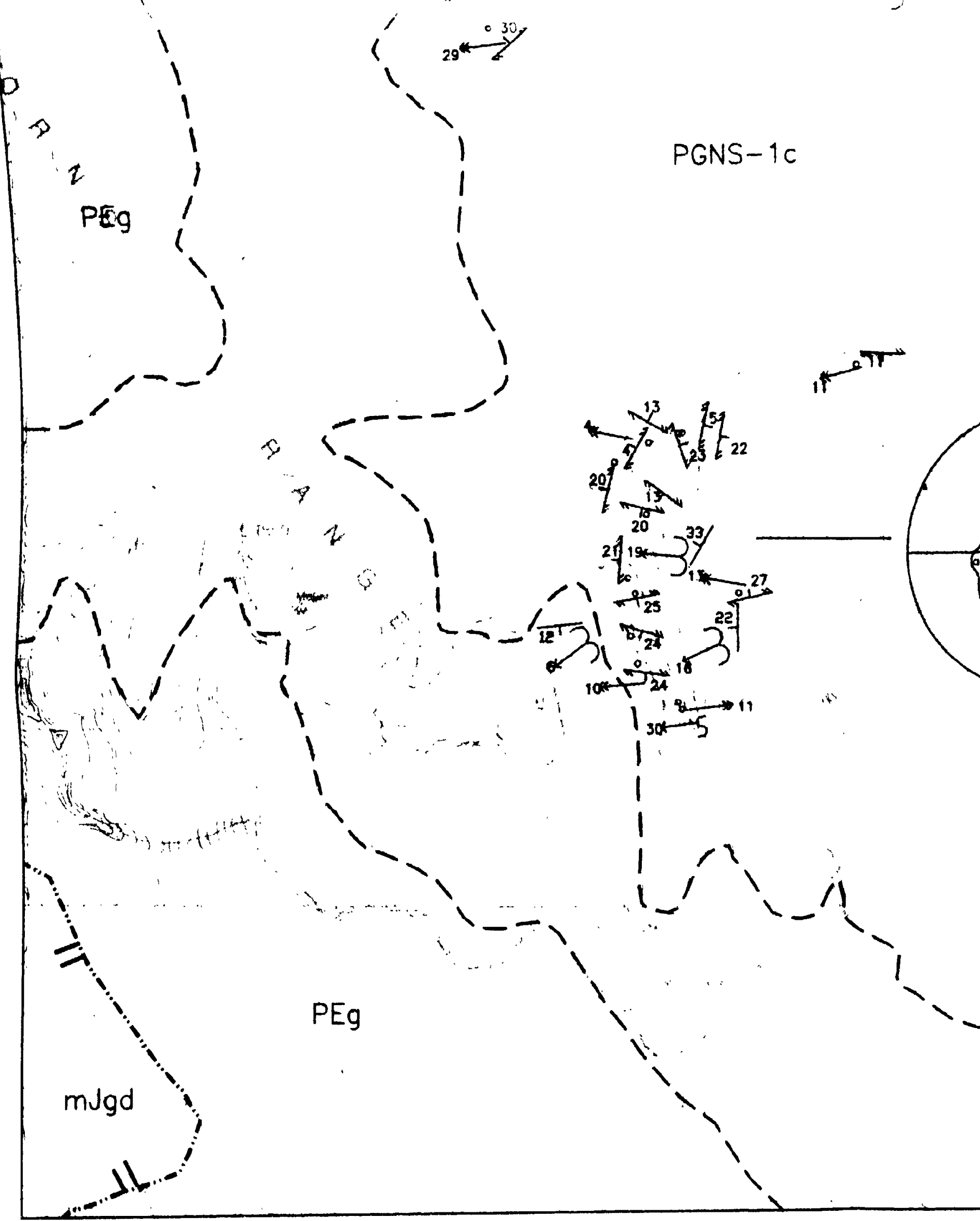




\section{SLUCAN LAKE FAULT: MAPPED, ASSUMED}

VALKYR SHEAR ZDNE: MAPPED, ASSUMED

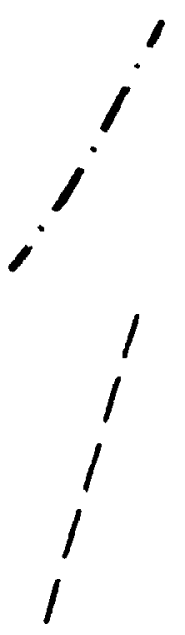

GWILLIM CREEK SHEAR ZUNES

BRITTLE NORMAL FAULT

- STATION LOCALITY

SCALE $\quad 1: 50000$

0

$5 \mathrm{~km}$

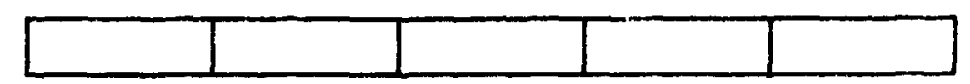


A Noth Valhalla Dome

5
4
3
2
1
0
1

Passmore Dome

${ }^{5}$

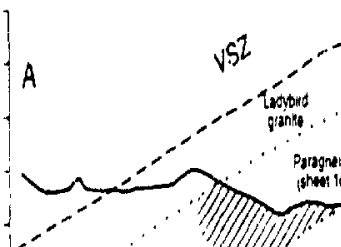

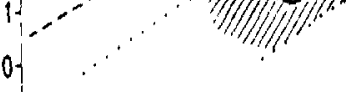

$\mathrm{km}$ domain $1 \mathrm{a}$

domain 3

\section{not it}

(a)

\section{B}
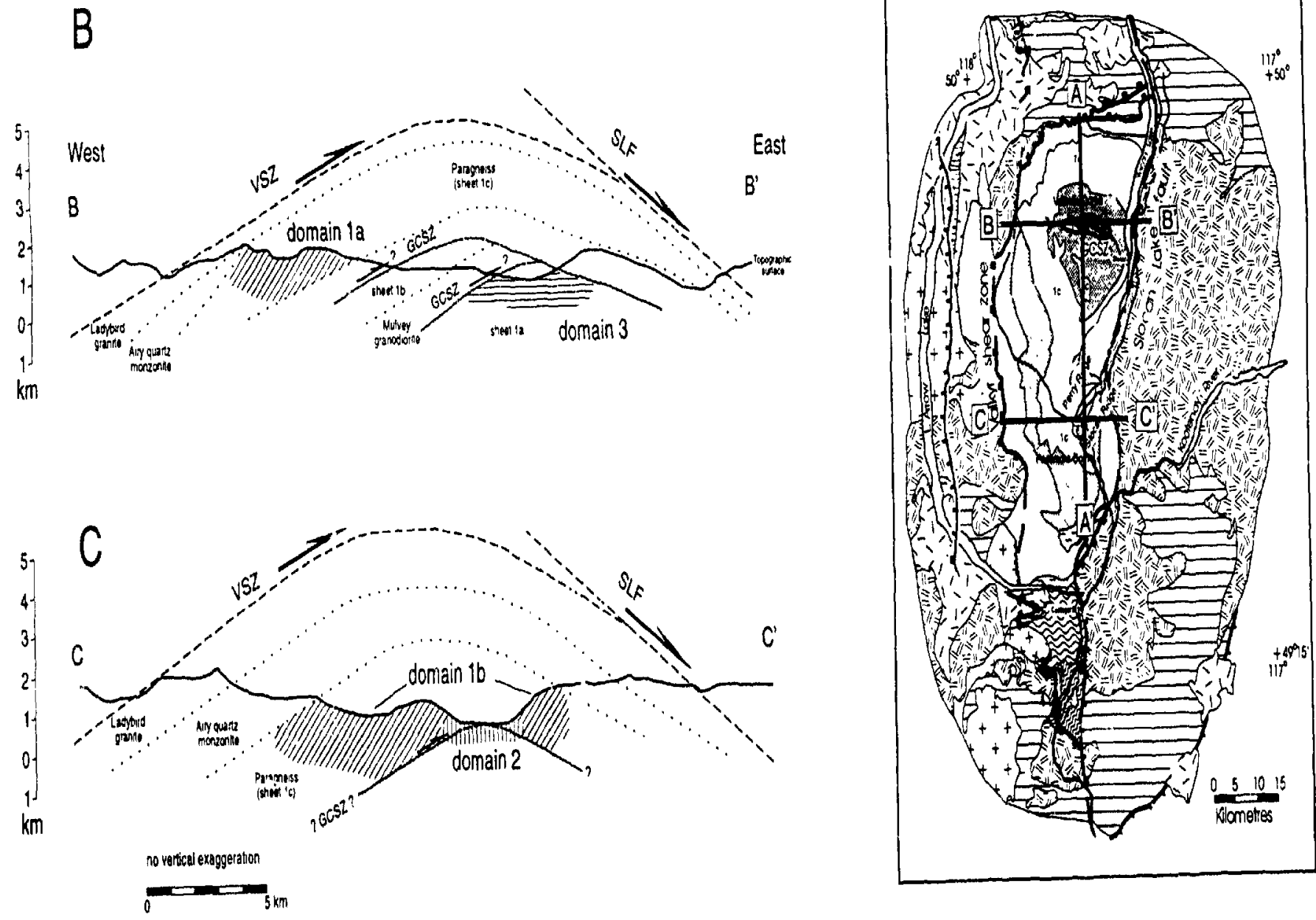

Cross-sections $A, B, C$ through the 'valinalla compiex. 

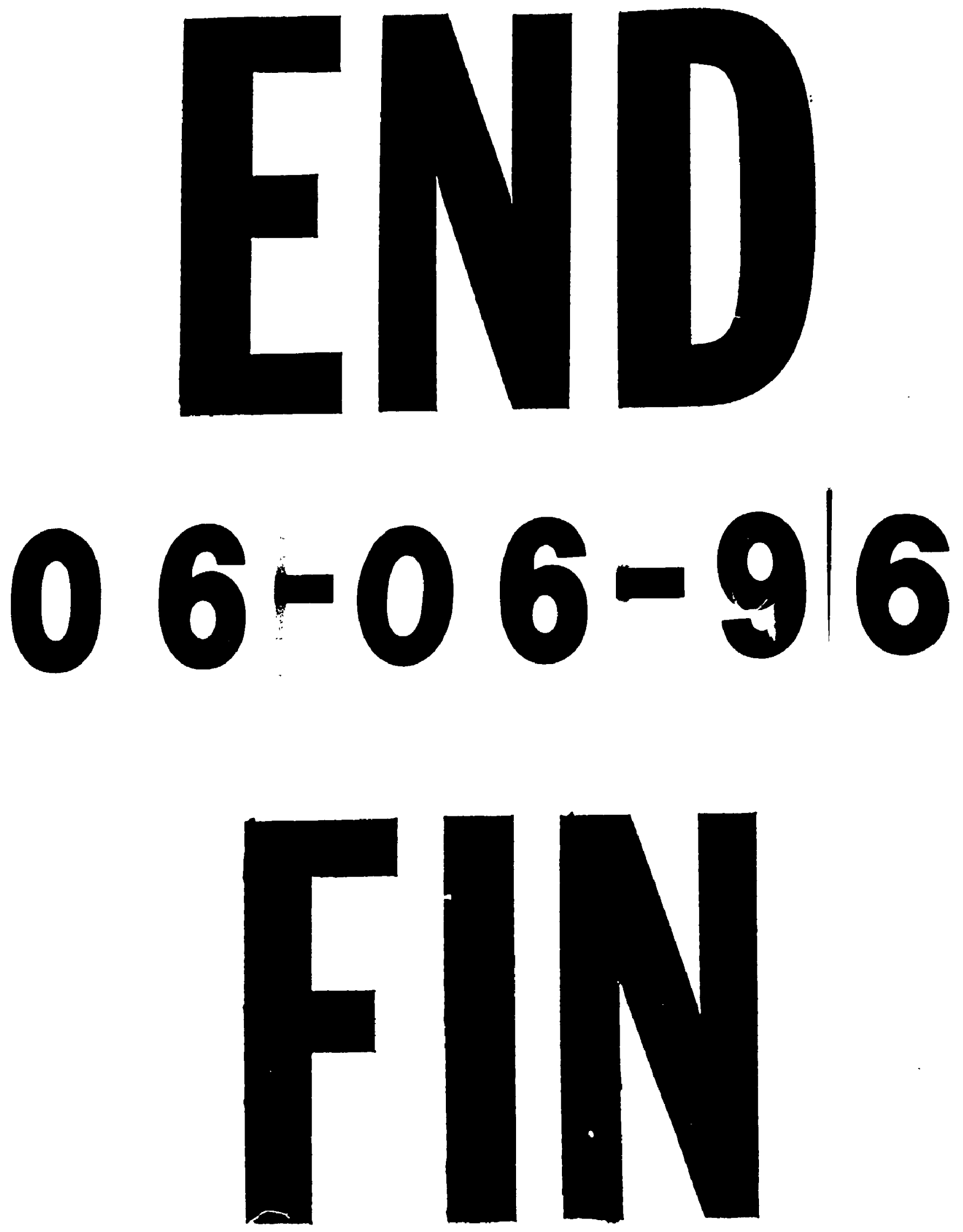